\title{
DOE/PC/90550 -TI6
}

\section{INTEGRATED DRY $\mathrm{NO}_{x} / \mathrm{SO}_{2}$ EMISSIONS CONTROL SYSTEM SODIUM-BASED DRY SORBENT INJECTION TEST REPORT}

(Test Period: August 4, 1993 to July 29, 1995)

DOE Contract Number DE-FC22-91PC90550

\author{
Prepared by \\ R. A. Smith \\ G. H. Shiomoto \\ L. J. Muzio \\ Fossil Energy Research Corp. \\ Laguna Hills, CA \\ T. Hunt \\ Public Service Company of Colorado \\ Denver, CO
}

Prepared for

Public Service Company of Colorado

Denver, CO

Draft: November 1994

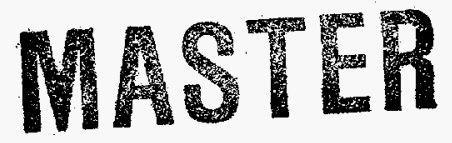

Revised: January 1997

Final: April 1997

Patents Cleared by Chicago on May 23,1997

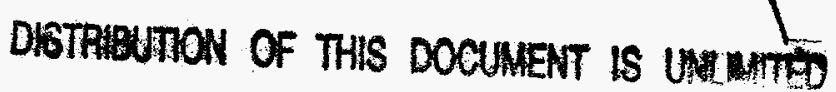




\section{DISCLAIMER}

This report was prepared by Fossil Energy Research Corp. and Public Service Company of Colorado pursuant to a cooperative agreement funded partially by the U. S. Department of Energy and neither Fossil Energy Research Corp. nor any of its subcontractors, nor the U. S. Department of Energy, nor any person acting on behalf of either:

(a) Makes any warranty or representation, express or implied, with respect to the accuracy, completeness, or usefulness of the information contained in this report, or that the use of any information, apparatus, method, or process disclosed in this report may not infringe privately owned rights; or

(b) Assumes any liabilities with respect to the use of, or for damages resulting from the use of, any information, apparatus, method, or process disclosed in this report.

Reference herein to any specific commercial product, process, or service by trade name, trademark, manufacturer, or otherwise, does not necessarily constitute or imply its endorsement, recommendation, or favoring by the U.S. Department of Energy. The views and opinions of authors expressed herein do not necessarily state or reflect those of the U. S. Department of Energy. 


\section{DISCLAIMER}

Portions of this document may be illegible electronic image products. Images are produced from the best available original document. 


\section{ABSTRACT}

The DOE sponsored Integrated Dry $\mathrm{NO}_{x} / \mathrm{SO}_{2}$ Emissions Control System program, is a Clean Coal Technology III demonstration, being conducted by Public Service Company of Colorado. The test site is Arapahoe Generating Station Unit 4, a $100 \mathrm{MWe}$, down-fired utility boiler buming a low sulfur Western coal. The project goal is to demonstrate up to 70 percent reductions in $\mathrm{NO}_{x}$ and $\mathrm{SO}_{2}$ emissions through the integration of: 1) down-fired low$\mathrm{NO}_{\mathrm{x}}$ burners with overfire air; 2) Selective Non-Catalytic Reduction (SNCR) for additional $\mathrm{NO}_{x}$ removal; and 3) dry sorbent injection and duct humidification for $\mathrm{SO}_{2}$ removal.

This report documents the sixth phase of the test program, where the performance of dry sorbent injection with sodium compounds was evaluated as $\mathrm{a} \mathrm{SO}_{2}$ removal technique. Dry sorbent injection was performed "in-duct" downstream of the air heater (ahead of the fabric filter), as well as at a higher temperature location between the economizer and air heater. Two sodium compounds were evaluated during this phase of testing: sodium sesquicarbonate and sodium bicarbonate. In-duct sodium injection with low levels of humidification was also investigated. This sixth test phase was primarily focused on a parametric investigation of sorbent type and feed rate, although boiler load and sorbent preparation parameters were also varied.

The in-duct injection of sodium sesquicarbonate achieved the target 70 percent $\mathrm{SO}_{2}$ emission reduction at normalized stoichiometric ratios ranging from approximately 1.6 to 2.2. (The stoichiometric ratio is 2 moles of sodium per mole of sulfur). The data exhibit day-to-day variations which were attributable to the sorbent feed system which utilized volumetric screw feeders.

Sodium bicarbonate injection ahead of the fabric filter showed variable $\mathrm{SO}_{2}$ removal characteristics which were attributed to the relatively low temperatures at the fabric filter inlet (i.e., on the order of 230 to $270^{\circ} \mathrm{F}$ ). Injection of sodium bicarbonate at the air heater inlet, where the temperatures were on the order of $600^{\circ} \mathrm{F}$, showed more consistent $\mathrm{SO}_{2}$ removals. Under these conditions, sodium bicarbonate yielded a 70 percent $\mathrm{SO}_{2}$ removal at a $2 \mathrm{Na} / \mathrm{S}$ ratio of approximately 1.1 . 
A byproduct of the sodium $/ \mathrm{SO}_{2}$ chemistry is the oxidation of $\mathrm{NO}$ to $\mathrm{NO}_{2}$, which may result in plume visibility. The $\mathrm{NO}_{2}$ production with sodium sesquicarbonate was less than with sodium bicarbonate. However, on some occasions, a faint visible plume was observed. The test program showed that $\mathrm{NO}_{2}$ levels were not only dependent on the type and amount of sodium compound injected, but also on the fabric filter cleaning cycle. After each cleaning cycle, the $\mathrm{NO}_{2}$ emissions increased markedly.

A long-term test of nominally four months was conducted with sodium sesquicarbonate injection ahead of the fabric filter. A rolling average $\mathrm{SO}_{2}$ removal of 40 percent was easily maintained for the duration of the test. Average $\mathrm{NO}_{2}$ emissions during this test were 6.7 ppm and there were no occurrences of a brown plume at the stack.

A four-week 70 percent removal test with sodium sesquicarbonate injection ahead of the fabric filter fell just short of the goal, with an average $\mathrm{SO}_{2}$ removal of 67.9 percent. System availability during this test was only 94 percent primarily due to a 32-hour period when neither of the two sorbent injection systems were in service. The average $\mathrm{NO}_{2}$ emissions during this test were $15.2 \mathrm{ppm}$, and a faint brown plume was visible on several occasions. 


\section{ACKNOWLEDGEMENTS}

The authors would like to thank Mr. Jim Love, Arapahoe Plant Manager, and his maintenance and operating staff for the exceptional cooperation they have provided during this project. Special thanks are also deserved by Mr. Jerry L. Hebb, Mr. Tom Arrigoni, and Mr. Tim Mcllvried of PETC DOE, whose contributions are greatly appreciated. We are all saddened by Tom Arrigoni's and Tim Mcllvried's deaths in the USAir accident. The advice and technical assistance provided by Mr. Jeff Stallings and Ms. Barbara Toole-O'Neil at EPRI have also been of great assistance throughout the project. Last, but definitely not least, is our appreciation to the many PSCo Engineering and Construction personnel and other contractors who have made the Integrated Dry $\mathrm{NO}_{x} / \mathrm{SO}_{2}$ Emissions Control System a success. 
TABLE OF CONTENTS

Section

Page

DISCLAIMER $\ldots \ldots \ldots \ldots \ldots \ldots \ldots \ldots \ldots \ldots \ldots \ldots \ldots \ldots$ i

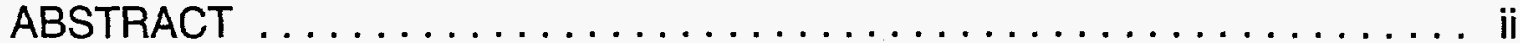

ACKNOWLEDGEMENT $\ldots \ldots \ldots \ldots \ldots \ldots \ldots \ldots \ldots \ldots \ldots \ldots \ldots \ldots$ iv

TABLE OF CONTENTS $\ldots \ldots \ldots \ldots \ldots \ldots \ldots \ldots \ldots \ldots \ldots \ldots$

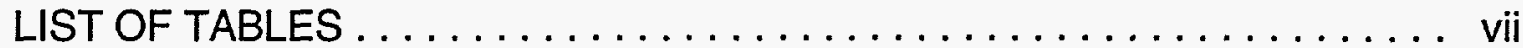

LIST OF FIGURES $\ldots \ldots \ldots \ldots \ldots \ldots \ldots \ldots \ldots \ldots \ldots \ldots \ldots \ldots \ldots \ldots \ldots \ldots$ viii

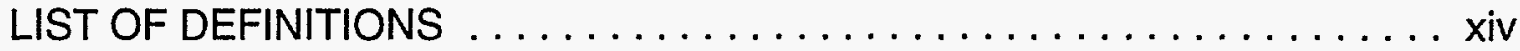

EXECUTIVE SUMMARY $\ldots \ldots \ldots \ldots \ldots \ldots \ldots \ldots \ldots \ldots \ldots \ldots$ ES-1

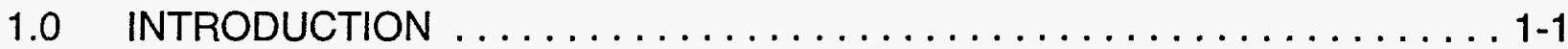

2.0 PROJECT DESCRIPTION . . . . . . . . . . . . . . . . . . . 2 .

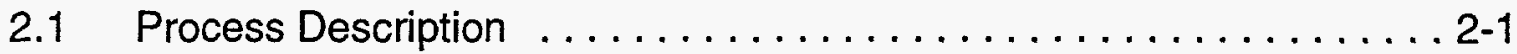

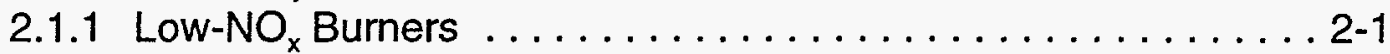

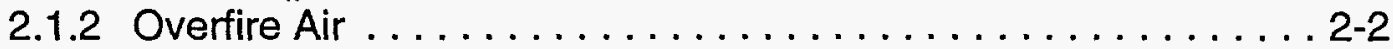

2.1.3 Selective Non-Catalytic Reduction ............... 2-3

2.1.4 Dry Sorbent Injection System .................. $2-3$

2.1.5 Humidification ............................ 2-5

2.2 Project Participants . . . . . . . . .

3.0 DRY SORBENT INJECTION AND HUMIDIFICATION

SYSTEM DESCRIPTION . . . . . . . . . . . . . . . . . . . 3-1

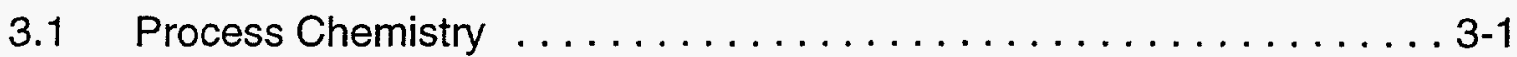

3.2 Existing Boiler Equipment $\ldots \ldots \ldots \ldots \ldots \ldots \ldots \ldots \ldots \ldots \ldots \ldots \ldots \ldots$

3.3 Humidification System ......................... 3-9

3.4 Dry Sorbent Injection (DSI) System $\ldots \ldots \ldots \ldots \ldots \ldots \ldots \ldots \ldots .11$

3.4.1 Dry Sorbent Storage and Handling . . . . . . . . . . . . 3-14

3.4.2 Fabric Filter Inlet Sorbent Injection . . . . . . . . . . . 3-16

3.4.3 Air Heater Inlet/Economizer Exit Sorbent Injection . . . . . . . . 3-16

3.5 Operational Problems . . . . . . . . . . . . . . . . . . $3-18$

3.5.1 Sorbent Storage and Handling Problems . . . . . . . . . 3-20

3.5.2 Sorbent Injection Problems . . . . . . . . . . . . . . 31

3.5.3 Determining Sorbent Feed Rate . . . . . . . . . . . . . . 3-21

3.5.4 Sorbent Pulverizer Problems . . . . . . . . . . . . . . 3-22

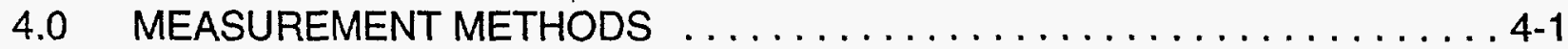

4.1 Gas Analysis Instrumentation $\ldots \ldots \ldots \ldots \ldots \ldots \ldots \ldots \ldots \ldots \ldots$ 4-1

4.2 Gas Sampling System . . . . . . . . . . . . . . . . . 4-3

4.3 Approach to Saturation $\ldots \ldots \ldots \ldots \ldots \ldots \ldots \ldots \ldots \ldots \ldots .4 .10$ 


\section{TABLE OF CONTENTS (Continued)}

Section

Page

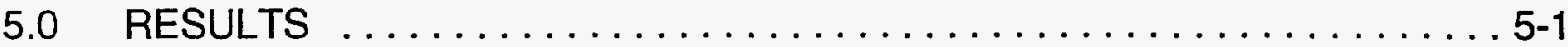

5.1 Sorbent Characteristics $\ldots \ldots \ldots \ldots \ldots \ldots \ldots \ldots \ldots \ldots \ldots \ldots \ldots$ 5-1

5.2 Sodium Sesquicarbonate $\ldots \ldots \ldots \ldots \ldots \ldots \ldots \ldots \ldots \ldots .5$

5.2.1 Injection of Sodium Sesquicarbonate at the FFDC Inlet . . . . 5-5

5.2.2 Sodium Sesquicarbonate Injection at the Air Heater Inlet . . . 5-24

5.3 Sodium Bicarbonate . . . . . . . . . . . . . . . . . . . . 5-27

5.3.1 Injection of Sodium Bicarbonate at the FFDC Inlet $\ldots \ldots \ldots 5-27$

5.3.2 Sodium Bicarbonate Injection at the Air Heater Inlet . . . . . . 5-35

5.4 Solids Analysis . . . . . . . . . . . . . . . . . . . . 5-50

5.4 .1 Sodium Sesquicarbonate $\ldots \ldots \ldots \ldots \ldots \ldots \ldots \ldots \ldots$. 50

5.4 .2 Sodium Bicarbonate ................... 5-63

6.0 LONG TERM LOAD FOLLOWING TEST RESULTS $\ldots \ldots \ldots \ldots \ldots \ldots \ldots .6$ 6-1

7.0 DISCUSSIONS AND CONCLUSIONS $\ldots \ldots \ldots \ldots \ldots \ldots \ldots \ldots \ldots .7 .1$

8.0 REFERENCES $\ldots \ldots \ldots \ldots \ldots \ldots \ldots \ldots \ldots \ldots \ldots \ldots \ldots \ldots \ldots \ldots$ 8-1

\section{Appendices}

A Synopsis of the Colorado School of Mines, Bench-Scale Study of Sodium $/ \mathrm{SO}_{2} / \mathrm{NO}_{\mathrm{x}}$ Chemistry

B Detailed Data Summary for Parametric Tests 


\section{LIST OF TABLES}

Number

Page

4-1 Gas Species Measured by Perkin Elmer MCS 100 Analyzer . . . . . . . . . 4-1

4-2 CEM RATA Results $\ldots \ldots \ldots \ldots \ldots \ldots \ldots \ldots \ldots \ldots \ldots \ldots \ldots$

5-1 Sorbent Characteristics $\ldots \ldots \ldots \ldots \ldots \ldots \ldots \ldots \ldots \ldots \ldots \ldots \ldots \ldots$

5-2 Effect of Sodium Sesquicarbonate Feed Rate on Pulverizer

Performance (Pulverizer Speed: 4000 rpm) $.5-2$ 


\section{LIST OF FIGURES}

Number

Page

ES-1 Comparison of $\mathrm{SO}_{2}$ Removals for Injection of Sodium Sesquicarbonate (Fabric Filter Inlet) and Sodium Bicarbonate (Air Heater Inlet) ........ ES-3

1-1 Arapahoe Unit 4 Integrated Dry $\mathrm{NO}_{x} / \mathrm{SO}_{2}$ Emissions Control System . . . . . 1-2

2-1 Conceptual Temperature Window for the SNCR Process $\ldots \ldots \ldots \ldots \ldots 2-4$

3-1 Calculated Decomposition Times for Sodium Bicarbonate $\ldots \ldots \ldots \ldots \ldots$ 3-5

3-2 Side View of Equipment Downstream of the Arapahoe Unit 4 Air Heater . . . 3-7

3-3 Top View of Equipment Downstream of the Arapahoe Unit 4 Air Heater . . . . 3-8

3-4 Simplified Diagram of the Humidification Injection and Control System . . . . 3-10

3-5 Humidification and Sorbent Injection Grids (East Half) $\ldots \ldots \ldots \ldots \ldots$ 3-12

3-6 Humidification Nozzles and Sorbent Injector $\ldots \ldots \ldots \ldots \ldots \ldots \ldots . . \ldots \ldots \ldots$

3-7 Simplified Diagram of One Sorbent Handling System

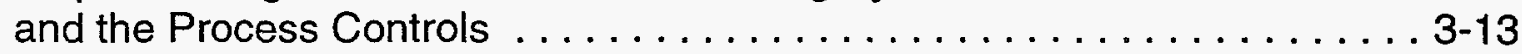

3-8 Approximate Locations of Duct Injectors from the A and B Side Sorbent Systems ............................. 3-17

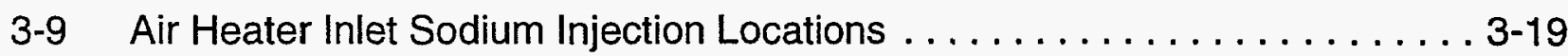

3-10 Air Heater Inlet Sodium Injector Design . . . . . . . . . . . . . . 3-19

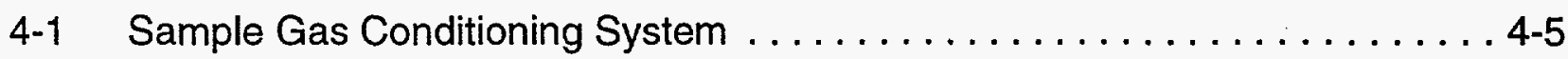

4-2 Economizer Exit Sampling Locations . . . . . . . . . . . . . . . .

4-3 Air Heater Exit Sampling Locations $\ldots \ldots \ldots \ldots \ldots \ldots \ldots \ldots \ldots \ldots .4 .9 \ldots$

4-4 Fabric Filter Outlet Duct Sampling Location $\ldots \ldots \ldots \ldots \ldots \ldots \ldots \ldots$ 4-9 


\section{LIST OF FIGURES (Continued)}

Number

Page

5-1 Sodium Sesquicarbonate Particle Size Distribution for Raw and Pulverized Samples . .........................

5-2 Sodium Bicarbonate Particle Size Distribution for Raw and Pulverized Samples

5-3 $\quad \mathrm{SO}_{2}$ Removal Versus Time for Sodium Sesquicarbonate Injection Ahead of the FFDC (Test 818)

5-4a $\mathrm{SO}_{2}$ Removal as a Function of $2 \mathrm{Na} / \mathrm{S}$ Ratio for Sodium

Sesquicarbonate Injection Ahead of the FFDC $\ldots \ldots \ldots \ldots \ldots \ldots \ldots .8$

5-4b Utilization as a Function of $2 \mathrm{Na} / \mathrm{S}$ Ratio for Sodium

Sesquicarbonate Injection Ahead of the FFDC $\ldots \ldots \ldots \ldots \ldots \ldots \ldots$

5-5 Comparison of $\mathrm{SO}_{2}$ Removals for Sodium Sesquicarbonate Injection Ahead of the Arapahoe Unit 4 FFDC to Previous Full-Scale Demonstrations (Cameo and Nixon data from Muzio, et al., 1984 and Fuchs, et al., 1989) . . . . . . . . . . . 5-9

5-6 Effect of Using One and Two Sorbent Preparation Systems on $\mathrm{SO}_{2}$ Removal (Sodium Sesquicarbonate Injection Ahead of the Fabric Filter) ........ 5-11

5-7 Effect of Pulverizer Speed on $\mathrm{SO}_{2}$ Removal for Sodium

Sesquicarbonate Injection Ahead of the FFDC

5-8 Compartment-by-Compartment Gaseous Measurements for

Sodium Sesquicarbonate Injection Ahead of the FFDC (Test 641) . . . . 5-14

5-9 $\mathrm{NO}_{x}$ Removal and $\mathrm{NO}_{2}$ Emissions Versus Time for Sodium

Sesquicarbonate Injection Ahead of the FFDC (Test 818) . . . . . . 5-16

5-10 Compartment-by-Compartment $\mathrm{No}$ and $\mathrm{NO}_{2}$ for Sodium

Sesquicarbonate Injection Ahead of the FFDC (Test 641)

5-11 Change in $\mathrm{NO}_{x}$ and $\mathrm{NO}_{2}$ for Sodium Sesquicarbonate for

FFDC Inlet Injection (Test 641)

5-12 Summary of $\mathrm{NO}_{2}$ Emissions with Sodium Sesquicarbonate Injection

$5-21$ 


\section{LIST OF FIGURES (Continued)}

Number

Page

5-13 Summary of $\mathrm{NO}_{\mathrm{x}}$ Removals with Sodium Sesquicarbonate

Injection Ahead of the FFDC

5-14 Effect of Humidifiction Approach to Saturation Temperature on $\mathrm{SO}_{2}$

Removal for Sodium Sesquicarbonate Injection Ahead of the FFDC

5-15 Compartment-by-Compartment Gaseous Measurements for

Sodium Sesquicarbonate Injection with Humidification

Ahead of the FFDC (Test 665)

5-16 Comparison of $\mathrm{SO}_{2}$ Removals for Sodium Sesquicarbonate

Injection at Air Heater Inlet and FFDC Inlet Locations . . . . . . . . . 5-26

5-17 Comparison of Response Time Characteristics for Sodium

Sesquicarbonate Injection at Air Heater Inlet and

FFDC Inlet Locations $(2 \mathrm{Na} / \mathrm{S}=1.0)$

5-18 $\mathrm{SO}_{2}$ Removal Versus Time for Sodium Bicarbonate Injection

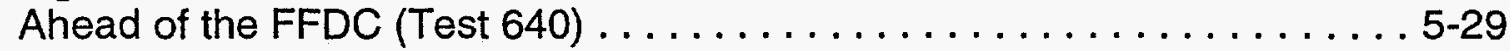

5-19 Inlet and Exit FFDC Temperatures Versus Time for Sodium

Bicarbonate Injection Ahead of the FFDC (Test 640)

5-20 $\mathrm{SO}_{2}$ Removal, FFDC Exit Temperature, and 2Na/S Ratio Versus Time

for Sodium Bicarbonate Injection Ahead of the FFDC (Test 763)

5-21 $\mathrm{SO}_{2}$ Removal as a Function of $2 \mathrm{Na} / \mathrm{S}$ Ratio for Sodium

Bicarbonate Injection Ahead of the FFDC (Test 763)

5-22 Utilization as a Function of $2 \mathrm{Na} / \mathrm{S}$ Ratio for Sodium

Bicarbonate Injection Ahead of the FFDC (Test 763)

5-23 Compartment-by-Compartment $\mathrm{SO}_{2}$ Removals for Sodium

Bicarbonate Injection Ahead of the FFDC (Test 763)

5-24 $\mathrm{NO}_{x}$ Removal and $\mathrm{NO}_{2}$ Emissions Versus Time for Sodium

Bicarbonate Injection Ahead of the FFDC (Test 763)

5-25 $\mathrm{SO}_{2}$ Removal Versus Time for Sodium Bicarbonate Injection

Ahead of the Air Heater (Test 792, $2 \mathrm{Na} / \mathrm{S}=1.0$ ) 


\section{LIST OF FIGURES (Continued)}

Number

Page

5-26 $\mathrm{SO}_{2}$ Removal as a Function of $2 \mathrm{Na} / \mathrm{S}$ Ratio for Sodium

Bicarbonate Injection Ahead of the Air Heater . . . . . . . . . . . . 5-39

5-27 Utilization as a Function of $2 \mathrm{Na} / \mathrm{S}$ Ratio for Sodium

Bicarbonate Injection Ahead of the Air Heater . . . . . . . . . . . . 5-39

5-28 Compartment-by-Compartment $\mathrm{SO}_{2}$ Removals for Sodium

Bicarbonate Injection Ahead of the Air Heater (Test 792) . . . . . . . 5-41

5-29 $\mathrm{NO}_{x}$ Removal and $\mathrm{NO}_{2}$ Emissions Versus Time for Sodium

Bicarbonate Injection Ahead of the Air Heater (Test 792) . . . . . . . . 5-43

5-30 Compartment-by-Compartment NO Removals for Sodium

Bicarbonate Injection Ahead of the Air Heater (Test 792) . . . . . . . . 5-45

5-31 Compartment-by-Compartment $\mathrm{NO}_{2}$ Emissions for Sodium

Bicarbonate Injection Ahead of the Air Heater (Test 792) . . . . . . . . 5-46

5-32 Summary of $\mathrm{NO}_{2}$ Emissions with Sodium Bicarbonate Injection $\ldots \ldots \ldots$ 5-48

5-33 $\mathrm{NO}_{2}$ Emissions and FFDC Cleaning with

Sodium Bicarbonate $(2 \mathrm{Na} / \mathrm{S} 0.9$ to 1.1$) \ldots \ldots \ldots \ldots \ldots \ldots \ldots .48$

5-34 Summary of $\mathrm{NO}_{x}$ Reduction with Sodium Bicarbonate Injection . . . . . . 5-49

5-35a Sodium Analysis Results for Sodium Sesquicarbonate Injection

Samples Collected Before FFDC Cleaning (Test 641)

5-35b Sodium Analysis Results for Sodium Sesquicarbonate Injection

Samples Collected After FFDC Cleaning (Test 641) . . . . . . . . . . 5-52

5-36a Sulfate Analysis Results for Sodium Sesquicarbonate Injection

Samples Collected Before FFDC Cleaning (Test 641) .......... . 5-54

5-36b Sulfate Analysis Results for Sodium Sesquicarbonate Injection

Samples Collected After FFDC Cleaning (Test 641) ... . . . . . . 5-54

5-37a Utilization Calculations for Sodium Sesquicarbonate Injections

Samples Collected Before FFDC Cleaning (Test 641) ......... 5-55

5-37b Utilization Calculations for Sodium Sesquicarbonate Injection

Samples Collected After FFDC Cleaning (Test 641) ............ . 5-55 


\section{LIST OF FIGURES (Continued)}

Number

Page

5-38 Utilization Calculations for Sodium Sesquicarbonate Injection Samples Collected During Air Toxics Tests $(2 \mathrm{Na} / \mathrm{S}=1.5) \ldots \ldots \ldots \ldots .58$

5-39 Compartment-by-Compartment Utilization Calculations and $\mathrm{SO}_{2}$ Removals for Sodium Sesquicarbonate Injection (Test 704) . . . . 5-59

5-40 Compartment-by-Compartment 2Na/S Ratio Calculations for Sodium Sesquicarbonate Injection (Test 704)

5-41 Flue Gas Velocity Distribution at FFDC Inlet for Sodium Sesquicarbonate Injection (Test 705)

5-42 Total Particulate Loading (Flyash and Sorbent) at FFDC Inlet for Sodium Sesquicarbonate Injection (Test 705)

5-43 Sodium Analysis Results for Samples Collected at FFDC Inlet for Sodium Sesquicarbonate Injection (Test 705)

5-44 Calculated Sodium Distribution at FFDC Inlet for Sodium Sesquicarbonate Injection (Test 705)

5-45a Sodium Analysis Results for Sodium Bicarbonate Injection Ahead of the FFDC (Test 763)

5-45b Sulfate Analysis Results for Sodium Bicarbonate Injection Ahead of the FFDC (Test 763) $5-64$

5-46 Utilization Calculations for Sodium Bicarbonate Injection Ahead of the FFDC (Test 763) . . . . . . . . . . . . . . . . 5-66

6-1 Long-Term Test with Sodium Sesquicarbonate Injection at $40 \%$ $\mathrm{SO}_{2}$ Removal, Hourly Averages for November, 1994

6-2 Long-Term Test with Sodium Sesquicarbonate Injection at $40 \%$

$\mathrm{SO}_{2}$ Removal, Hourly Averages for December, 1994

6-3 Long-Term Test with Sodium Sesquicarbonate Injection at $40 \%$ $\mathrm{SO}_{2}$ Removal, Hourly Averages for January, 1995 


\section{LIST OF FIGURES (Continued)}

Number

Page

6-4 Long-Term Test with Sodium Sesquicarbonate Injection at $40 \%$

$\mathrm{SO}_{2}$ Removal, Hourly Averages for February, $1995 \ldots \ldots \ldots \ldots \ldots \ldots$. $6-6$

6-5 Long-Term Test with Sodium Sesquicarbonate Injection at $40 \%$

$\mathrm{SO}_{2}$ Removal, Hourly Averages for March, 1995

6-6 Daily Averages for Long-Term Test with Sodium

Sesquicarbonate Injection at $40 \% \mathrm{SO}_{2}$ Removal $\ldots \ldots \ldots \ldots \ldots \ldots 6-8$

6-7 Hourly Averages for Long-Term Test with Sodium

Sesquicarbonate Injection at $70 \% \mathrm{SO}_{2}$ Removal $\ldots \ldots \ldots \ldots \ldots \ldots 6-10$

6-8 Hourly Averages for Long-Term Test with Sodium Sesquicarbonate

Injection at $75 \% \mathrm{SO}_{2}$ Removal (January 2-11, 1996) 


\section{LIST OF DEFINITIONS}

B\&W Babcock \& Wilcox

CEM Continuous Emission Monitor

DCS Distributed Control System

DOE U.S. Department of Energy

$\mathrm{DRB}-\mathrm{XCL}^{\circledR} \quad$ Dual Register Burner - Axially Controlled Low-NO

DSI Dry Sorbent Injection

EPRI Electric Power Research Institute

FERCo Fossil Energy Research Corp.

FFDC Fabric Filter Dust Collector

ID

Induced Draft (fan)

LNB Low- $\mathrm{NO}_{\mathrm{x}}$ Burner

MMD Mass Mean Diameter

MWe MegaWatts (electrical)

OFA OverFire Air

ppm Parts Per Million

ppmc Parts Per Million Corrected to 3 percent $\mathrm{O}_{2}$ level, dry

PSCo Public Service Company of Colorado

psig Pounds per Square Inch Gauge

RATA Relative Accuracy Test Audit

SCFH Standard Cubic Feet per Hour, measured at 1 atmosphere and $60^{\circ} \mathrm{F}$

SNCR Selective Non-Catalytic $\mathrm{NO}_{x}$ Reduction

$\mathrm{T}_{\mathrm{app}} \quad$ Approach to Saturation Temperature

$2 \mathrm{Na} / \mathrm{S} \quad$ Sodium-to-Sulfur Ratio 


\section{EXECUTIVE SUMMARY}

This test report summarizes the technical activities and results for one phase of a Department of Energy sponsored Clean Coal Technology III demonstration of an Integrated Dry $\mathrm{NO}_{x} / \mathrm{SO}_{2}$ Emissions Control System for coal-fired boilers. The project is being conducted at Public Service Company of Colorado's Arapahoe Generating Station Unit 4 located in Denver, Colorado. The project goal is to demonstrate up to 70 percent reductions in $\mathrm{NO}_{x}$ and $\mathrm{SO}_{2}$ emissions through the integration of existing and emerging technologies, including: 1) down-fired low- $\mathrm{NO}_{x}$ burners with overfire air; 2) Selective NonCatalytic Reduction (SNCR) for additional $\mathrm{NO}_{x}$ removal; and 3) dry sorbent injection and duct humidification for $\mathrm{SO}_{2}$ removal.

Due to the number of technologies being integrated, the test program has been divided into the following test activities:

- Baseline tests with the original combustion system

- Baseline tests with the original combustion system and SNCR

- $\quad$ Low-NO $\mathrm{N}_{\mathrm{x}}$ Burner (LNB)/Overfire Air (OFA) tests

- $\quad$ LNB/OFASNCR tests

- $\quad$ LNB/OFAVCalcium Injection tests

- $\quad$ LNB/OFA/Sodium Injection tests

- $\quad$ LNB/OFA/SNCR Dry Sorbent Injection tests (integrated system)

- $\quad$ Air Toxics Characterization.

This report presents the results of the sodium injection tests performed after the combustion system retrofit on the Arapahoe Unit 4 boiler. The $\mathrm{SO}_{2}$ removal performance of the sodium sorbents was evaluated with the in-duct dry injection system. The primary injection location was downstream of the air preheater, at the inlet of the fabric filter dust collector. Two sodium-based dry sorbents were tested, sodium sesquicarbonate and sodium bicarbonate. Unlike the previously tested calcium sorbents, the sodium compounds were processed through an attrition mill prior to injection, in order to reduce the particle size and increase the $\mathrm{SO}_{2}$ removal effectiveness. Humidification was briefly tested with sodium sesquicarbonate by atomizing water into the flue gas, and cooling the average gas temperatures closer to the saturation point. Both sodium reagents were injected at two locations: in-duct (downstream of the air heater ahead of the fabric filter) 
and at a higher temperature location at the inlet to the air heater. Parametric testing of the sodium injection system was conducted during the period of August 4, 1993 to May 27, 1994.

The primary operating parameter for the sodium injection processes was the normalized stoichiometric ratio, which is the amount of sorbent injected relative to the mass flow of sulfur in the flue gas. The chemical reactions require two molecules of sodium to react with each molecule of sulfur $\left(\mathrm{SO}_{2}\right)$, therefore the normalized stoichiometric ratio is expressed as $2 \mathrm{Na} / \mathrm{S}$, where a unity value is equivalent to the stoichiometric concentration. Parametric variations of the $2 \mathrm{Na} / \mathrm{S}$ ratio, sorbent type, and boiler load were performed for the sodium injection tests. In the cases when humidification was utilized, the primary operating variable was the approach to saturation temperature of the flue gas. Saturation temperatures of the flue gas ranged from 112 to $118^{\circ} \mathrm{F}$, depending on boiler operating conditions. During these tests, the humidification system was used to vary the approach to saturation from 50 to $90^{\circ} \mathrm{F}$.

With a nominal $2 \mathrm{Na} / \mathrm{S}$ ratio of 2.0 , the $\mathrm{SO}_{2}$ removals with in-duct sodium sesquicarbonate injection ranged from 64 to 78 percent (Figure ES-1). Altematively, the $2 \mathrm{Na} / \mathrm{S}$ ratio required for 70 percent $\mathrm{SO}_{2}$ removal ranged from 1.6 to 2.2. Sorbent utilization decreased with increasing sorbent injection rates, as shown by the tapering off of the $\mathrm{SO}_{2}$ removals as the $2 \mathrm{Na} / \mathrm{S}$ ratio was increased to higher levels. The scatter apparent in Figure ES-1 represents day-to-day variations that were seen in the process. It is believed that a large portion of these variations resulted from day-to-day changes in sorbent feed rate and not the effectiveness of the process at a given $2 \mathrm{Na} / \mathrm{S}$ ratio. Since the sorbent feed was based on the calibration of a volumetric screw system, any loss in feed capacity could not be readily detected. This would cause the actual $2 \mathrm{Na} / \mathrm{S}$ to be lower than the set point.

The in-duct injection of sodium bicarbonate showed erratic results which are attributed to the relatively low flue gas temperatures at the fabric filter inlet (i.e., approximately 230 to $270^{\circ} \mathrm{F}$ ). Since the operating temperatures for the duct and baghouse at Arapahoe 


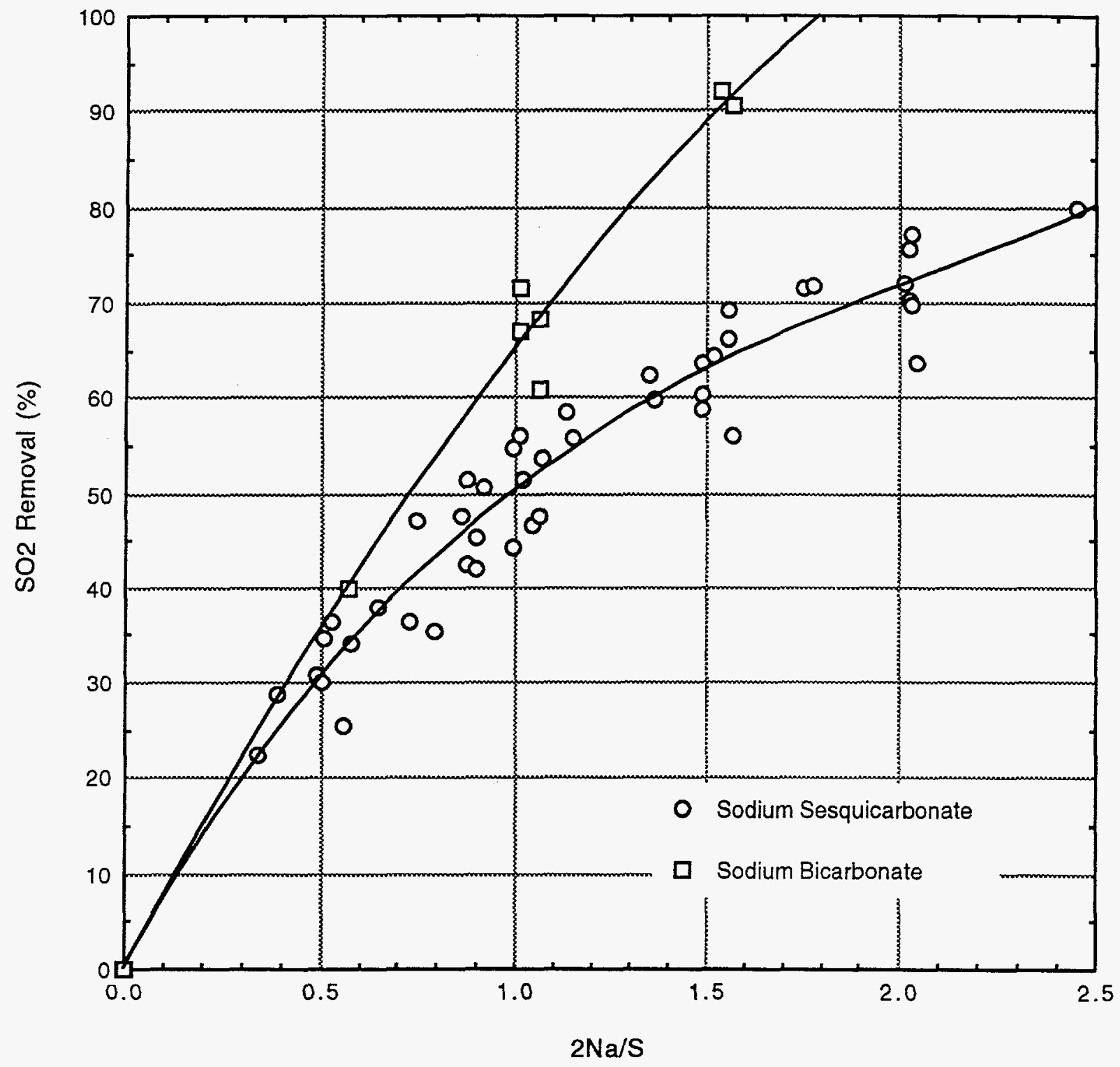

Figure ES-1. Comparison of $\mathrm{SO}_{2}$ Removals for Injection of Sodium Sesquicarbonate (Fabric Filter Inlet) and Sodium Bicarbonate (Air Heater Inlet) 
Unit 4 were on the low side for optimum use of sodium bicarbonate, modifications were made and additional testing was conducted with injection at the economizer exit (air heater inlet) during April and May 1994. These results were more consistent than those for duct injection and showed that a $2 \mathrm{Na} / \mathrm{S}$ ratio of approximately 1.1 was required for a 70 percent $\mathrm{SO}_{2}$ removal (Figure ES-1).

There were no apparent problems associated with the use of the sodium injection system with regard to the operation of the boiler or any cold-end equipment. Chronic problems with sorbent feed, injection system deposit formation and plugging, and sorbent pulverizer operation were encountered throughout the test program. However, all of these problems are deemed manageable by routine maintenance procedures.

In addition to determining the $\mathrm{SO}_{2}$ removals achievable with the injection of sodium sesquicarbonate and sodium bicarbonate, the evaluation of the impact of the sodium compounds on $\mathrm{NO}_{2}$ emissions and $\mathrm{NO}_{x}$ removal was also an important element of this test phase. One of the more interesting observations from the current test program was the process dynamics of $\mathrm{NO}_{2}$ formation with sodium injection. Time-resolved measurements showed that the $\mathrm{NO}_{2}$ emissions were not only dependent on the amount of sodium injected but also on the cleaning cycle of the Arapahoe Unit 4 fabric filter. With both sodium sesquicarbonate and sodium bicarbonate, the $\mathrm{NO}_{2}$ emissions were found to increase markedly just after a cleaning cycle. This suggests that there is an interaction between the $\mathrm{NO}_{2}$ and the fly ash. This was further confirmed by measurements made in each individual fabric filter compartment which showed that the $\mathrm{NO}_{2}$ levels were not just a function of the $\mathrm{SO}_{2}$ removal in each compartment, but also appeared to be related to the amount of fly ash collected in each compartment. This phenomena accounts for the high degree of variability in $\mathrm{NO}_{2}$ emissions and $\mathrm{NO}_{x}$ reductions reported not only in this test program, but in previously reported full-scale sodium injection demonstrations (Fuchs, et al., 1989; Muzio, et al., 1984).

In terms of the levels of $\mathrm{NO}_{2}$ produced, sodium sesquicarbonate produced $\mathrm{NO}_{2}$ levels of nominally $10 \mathrm{ppm}$ at a nominal $2 \mathrm{Na} / \mathrm{S}$ ratio of 2.0 (although there were a few occasions 
where the $\mathrm{NO}_{2}$ level reached almost $30 \mathrm{ppm}$ ). The $\mathrm{NO}_{2}$ levels with sodium bicarbonate injection were generally higher. At a nominal $2 \mathrm{Na} / \mathrm{S}$ ratio of $1.0, \mathrm{NO}_{2}$ levels with sodium bicarbonate injection were nominally $20 \mathrm{ppm}$ with levels occasionally reaching $50 \mathrm{ppm}$. No plume coloration was noted with sodium sesquicarbonate injection, although some plume coloration was observed with sodium bicarbonate injection when $\mathrm{NO}_{2}$ levels exceeded $35 \mathrm{ppm}$.

At injection rates providing $70 \% \mathrm{SO}_{2}$ removal, both sodium sorbents resulted in $\mathrm{NO}_{\mathrm{x}}$ removals of nominally 10 percent. These levels are consistent with those reported in the previous full-scale demonstrations mentioned above (Fuchs, et al., 1989; Muzio, et al., 1984).

After completion of the parametric tests with both sorbents, a long-term test of nominally four months duration was conducted with sodium sesquicarbonate injection ahead of the fabric filter. During this test, the control system was set to achieve a 40 percent $\mathrm{SO}_{2}$ removal. Daily average $\mathrm{SO}_{2}$ removals of 40 percent were easily achievable during the four-month period, although there were brief periods when the sodium injection system was off-line due to minor problems with plugging the sorbent transport lines or system maintenance requirements.

Following completion of the four-month test, a second long-term test was run with a $\mathrm{SO}_{2}$ removal setpoint of 70 percent. This test was run for four weeks and ended when Arapahoe Unit 4 was taken off-line for a scheduled 10-week outage. At the end of the test, the rolling average $\mathrm{SO}_{2}$ removal was 67.9 percent, just short of the goal of 70 percent. A number of mechanical problems resulted in a system availability of only 94 percent for the four-week test. During this high $\mathrm{SO}_{2}$ removal test period there were a few occasions when the $\mathrm{NO}_{2}$ levels caused a slightly visible plume. This was the first time that a visible $\mathrm{NO}_{2}$ plume was encountered during the test program. 


\subsection{INTRODUCTION}

This report presents the results from one phase of the Public Service Company of Colorado (PSCo) and the Department of Energy (DOE) sponsored Integrated Dry $\mathrm{NO}_{x} / \mathrm{SO}_{2}$ Emissions Control System program. The DOE Clean Coal Technology III demonstration program is being conducted by Public Service Company of Colorado at PSCo's Arapahoe Generating Station Unit 4, located in Denver, Colorado. The intent of the demonstration program at Arapahoe Unit 4 is to achieve up to 70 percent reductions in $\mathrm{NO}_{\mathrm{x}}$ and $\mathrm{SO}_{2}$ emissions through the integration of existing and emerging technologies, while minimizing capital expenditures and limiting waste production to dry solids that are handled with conventional ash removal equipment. The technologies to be integrated are: 1) a downfired low-NO $\mathrm{NO}_{\mathrm{x}}$ burner system with overfire air; 2) Selective Non-Catalytic Reduction (SNCR) with urea and ammonia-based compounds for additional $\mathrm{NO}_{x}$ removal; and 3) dry sorbent injection (calcium- and sodium-based compounds) and duct humidification for $\mathrm{SO}_{2}$ removal. Figure 1-1 shows a simplified schematic of the integrated system as implemented at Arapahoe Unit 4.

During the demonstration program, these emissions control systems are being optimized and integrated with the goal of achieving up to 70 percent reductions in $\mathrm{NO}_{\mathrm{x}}$ and $\mathrm{SO}_{2}$. It is anticipated that the emissions control system will achieve these reductions at costs lower than other currently available technologies. It is also anticipated that these technologies will integrate synergistically. For example, an undesirable side effect of sodium-based sorbent injection for $\mathrm{SO}_{2}$ control has been oxidation of $\mathrm{NO}$ to $\mathrm{NO}_{2}$, resulting in plume colorization. Pilot-scale testing, sponsored by the Electric Power Research Institute (EPRI), has shown that the presence of $\mathrm{NH}_{3}$ can reduce the $\mathrm{NO}_{2}$ emissions from sodiumbased dry sorbent injection. In the integrated system, the byproduct $\mathrm{NH}_{3}$ emissions from the urea injection system will serve to minimize $\mathrm{NO}_{2}$ formation. 


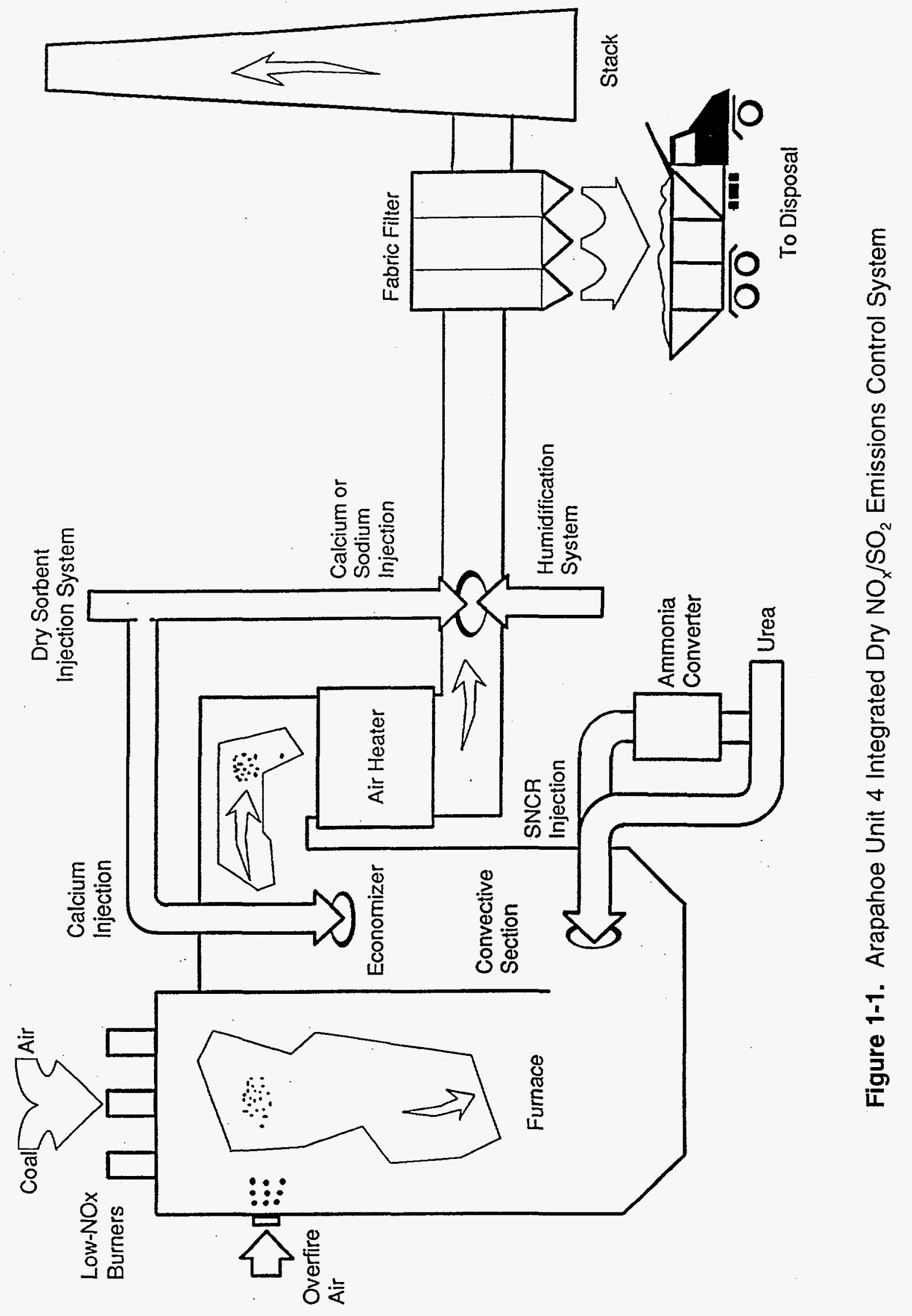


Due to the number of technologies being integrated, the test program has been divided into the following test activities:

- Baseline tests of the original combustion system. These results provide the basis for comparing the performance of the individual technologies as well as that of the integrated system. (completed, Shiomoto, et al., 1992)

- Baseline combustion system/SNCR tests. Performance of urea and aqueous ammonia injection with the original combustion system. (completed, Smith, et al., 1993)

- Low-NO burner (LNB)/overfire air (OFA) tests. (completed, Smith, et al., 1994a)

- LNB/OFA/SNCR tests. $\mathrm{NO}_{x}$ reduction potential of the combined low-NO combustion system and SNCR. (completed, Smith, et al., 1994b)

- $\quad$ LNB/OFA/calcium-based sorbent injection. Economizer injection and duct injection with humidification. (completed, Shiomoto, et al., 1994)

- $\quad$ LNB/OFA/sodium injection. $\mathrm{SO}_{2}$ removal performance of sodium-based sorbents. (subject of this report)

- Integrated Systems test. $\mathrm{NO}_{x}$ and $\mathrm{SO}_{2}$ reduction potential of the integrated system using LNB/OFA/SNCR/Dry Sorbent Injection (with calcium- or sodium-based reagents).

In addition to the investigation of $\mathrm{NO}_{x}$ and $\mathrm{SO}_{2}$ emissions, the test program also investigated air toxic emissions. Air toxic emission levels were measured during the testing of the low-NO ${ }_{x}$ combustion system, and during the LNB/OFASNCR tests with urea. Air toxics emission levels were also measured during the calcium injection tests, and additional tests were conducted during the sodium injection tests to determine the potential air toxics removal of these two pollution control technologies. The air toxics test results will be documented in separate Environmental Monitoring Reports.

This report presents the results of the dry sorbent injection tests with sodium-based sorbents. These tests included the use of sodium sesquicarbonate and sodium 
bicarbonate injection both downstream and upstream of the air heater. A limited number of sodium sesquicarbonate injection tests were performed with the use of the humidification system to enhance $\mathrm{SO}_{2}$ removal. 


\subsection{PROJECT DESCRIPTION}

The following subsections will describe the key aspects of all the technologies being demonstrated as a part of the Integrated Dry $\mathrm{NO}_{x} / \mathrm{SO}_{2}$ Emissions control system program. The project participants and their relative roles are also reviewed.

\subsection{Process Description}

The Integrated Dry $\mathrm{NO}_{x} / \mathrm{SO}_{2}$ Emissions Control system consists of five major control technologies that are combined to form an integrated system to control both $\mathrm{NO}_{\mathrm{x}}$ and $\mathrm{SO}_{2}$ emissions. $\mathrm{NO}_{\mathrm{x}}$ reduction is accomplished through the use of low- $\mathrm{NO}_{\mathrm{x}}$ burners, overfire air, and SNCR, while dry sorbent injection (using either calcium- or sodium-based reagents) is used to control $\mathrm{SO}_{2}$ emissions. Flue gas humidification will be used to enhance the $\mathrm{SO}_{2}$ removal capabilities of the calcium-based sorbents. Each of these technologies is discussed briefly below.

\subsubsection{Low-NO Burners}

$\mathrm{NO}_{x}$ formed during the combustion of fossil fuels consists primarily of $\mathrm{NO}_{x}$ formed from fuelbound nitrogen, and thermal $\mathrm{NO}_{\mathrm{x}} \cdot \mathrm{NO}_{\mathrm{x}}$ formed from fuel-bound nitrogen results from the oxidation of nitrogen which is organically bonded to the carbon in the fuel. Thermal $\mathrm{NO}_{x}$ forms when nitrogen in the combustion air dissociates and oxidizes at flame temperatures. Thermal $\mathrm{NO}_{x}$ is of primary importance at temperatures in excess of $2800^{\circ} \mathrm{F}$.

To reduce the $\mathrm{NO}_{x}$ emissions formed during the combustion process, Babcock and Wilcox (B\&W) Dual Register Bumer-Axially Controlled Low-NO ${ }_{x}\left(\mathrm{DRB}-\mathrm{XCL}{ }^{\circledR}\right)$ burners were retrofit to the Arapahoe Unit 4 boiler. Most low- $\mathrm{NO}_{x}$ burners reduce the formation of $\mathrm{NO}_{x}$ through the use of air staging, which is accomplished by limiting the availability of air during the early stages of combustion. This lowers the peak flame temperature and results in a reduction in the formation of thermal $\mathrm{NO}_{x}$. In addition, by reducing the oxygen availability in the initial combustion zone, the fuel-bound nitrogen is less likely to be converted to $\mathrm{NO}_{\mathbf{x}}$, but rather to $\mathrm{N}_{2}$ and other stable nitrogen compounds. The B\&W DRB-XCL ${ }^{\circledR}$ burner 
achieves increased $\mathrm{NO}_{\mathrm{x}}$ reduction effectiveness by incorporating fuel staging in addition to air staging. Fuel staging involves the introduction of fuel downstream of the flame under fuel-rich conditions. This results in the generation of hydrocarbon radicals which further reduce $\mathrm{NO}_{\mathrm{x}}$ levels. The fuel staging is accomplished through the design of the coal nozzle/flame stabilization ring on the burner. Additionally, combustion air to each burner is accurately measured and regulated to provide a balanced fuel and air distribution for optimum $\mathrm{NO}_{x}$ reduction and combustion efficiency. Finally, the burner assembly is equipped with two sets of adjustable spin vanes which provide swirl for fuel/air mixing and flame stabilization.

\subsubsection{Overfire Air}

Low-NO $\mathrm{N}_{\mathrm{x}}$ burners and overfire air reduce the formation of $\mathrm{NO}_{x}$ by controlling the fuel/air mixing process. While low- $\mathrm{NO}_{x}$ burners control the mixing in the near-burner region, overfire air controls the mixing over a larger part of the furnace volume. By diverting part of the combustion air to a zone downstream of the burner, initial combustion takes place in a near stoichiometric or slightly fuel rich environment. The remaining air necessary to ensure complete combustion is introduced downstream of the primary combustion zone through a set of overfire air ports, sometimes referred to as $\mathrm{NO}_{\mathrm{x}}$ ports. Conventional single-jet overfire air ports are not capable of providing adequate mixing across the entire furnace. The B\&W dual-zone $\mathrm{NO}_{x}$ ports, however, incorporate a central zone which produces an air jet that penetrates across the furnace and a separate outer zone that diverts and disperses the air in the area of the fumace near the $\mathrm{NO}_{x}$ port. The central zone is provided with a manual air control disk for flow control, and the outer zone incorporates manually adjustable spin vanes for swirl control.

The combined use of the low- $\mathrm{NO}_{\mathrm{x}}$ burners and overfire air ports was expected to reduce $\mathrm{NO}_{x}$ emissions by up to 70 percent. 


\subsubsection{Selective Non-Catalytic Reduction}

$\mathrm{NO}_{x}$ reduction in utility boilers can also be accomplished by Selective Non-Catalytic Reduction (SNCR). This process involves the injection of either urea or ammonia (anhydrous or aqueous) into the combustion products where the gas temperature is in the range of 1600 to $2100^{\circ} \mathrm{F}$. In this range, $\mathrm{NH}_{2}$ is released from the injected chemical which then selectively reacts with $\mathrm{NO}$ in the presence of oxygen, forming primarily $\mathrm{N}_{2}$ and $\mathrm{H}_{2} \mathrm{O}$. An SNCR system is capable of removing 40 to 50 percent of the NO from the flue gas stream.

Urea and ammonia each have their own optimum temperature and range within which $\mathrm{NO}_{\mathrm{x}}$ reduction can occur. An example of such a temperature "window" is shown conceptually in Figure 2-1. At temperatures above the optimum, the injected chemical will react with $\mathrm{O}_{2}$ forming additional $\mathrm{NO}_{\mathrm{x}}$, thereby reducing the $\mathrm{NO}_{\mathrm{x}}$ removal efficiency. At temperatures below the optimum, the injected chemical does not react with NO, resulting in excessive $\mathrm{NH}_{3}$ emissions (referred to as ammonia slip). Chemical additives can be injected with the urea to widen the optimum temperature range and minimize $\mathrm{NH}_{3}$ emissions.

The SNCR chemical of primary interest for the present program is urea. The urea is generally injected into the boiler as a liquid solution through atomizers. The atomizing medium can be either air or steam, although air is used in the current installation. The urea and any additives are stored as a liquid and pumped through the injection atomizers. At Arapahoe Unit 4, a system has also been installed to catalytically convert the urea solution to ammonium compounds. The urea solution can be either injected directly into the furnace or processed through the catalytic system prior to injection.

\subsubsection{Dry Sorbent Injection System}

The dry sorbent injection (DSI) system consists of equipment for storing, conveying, pulverizing and injecting calcium- or sodium-based reagents into the flue gas between the 


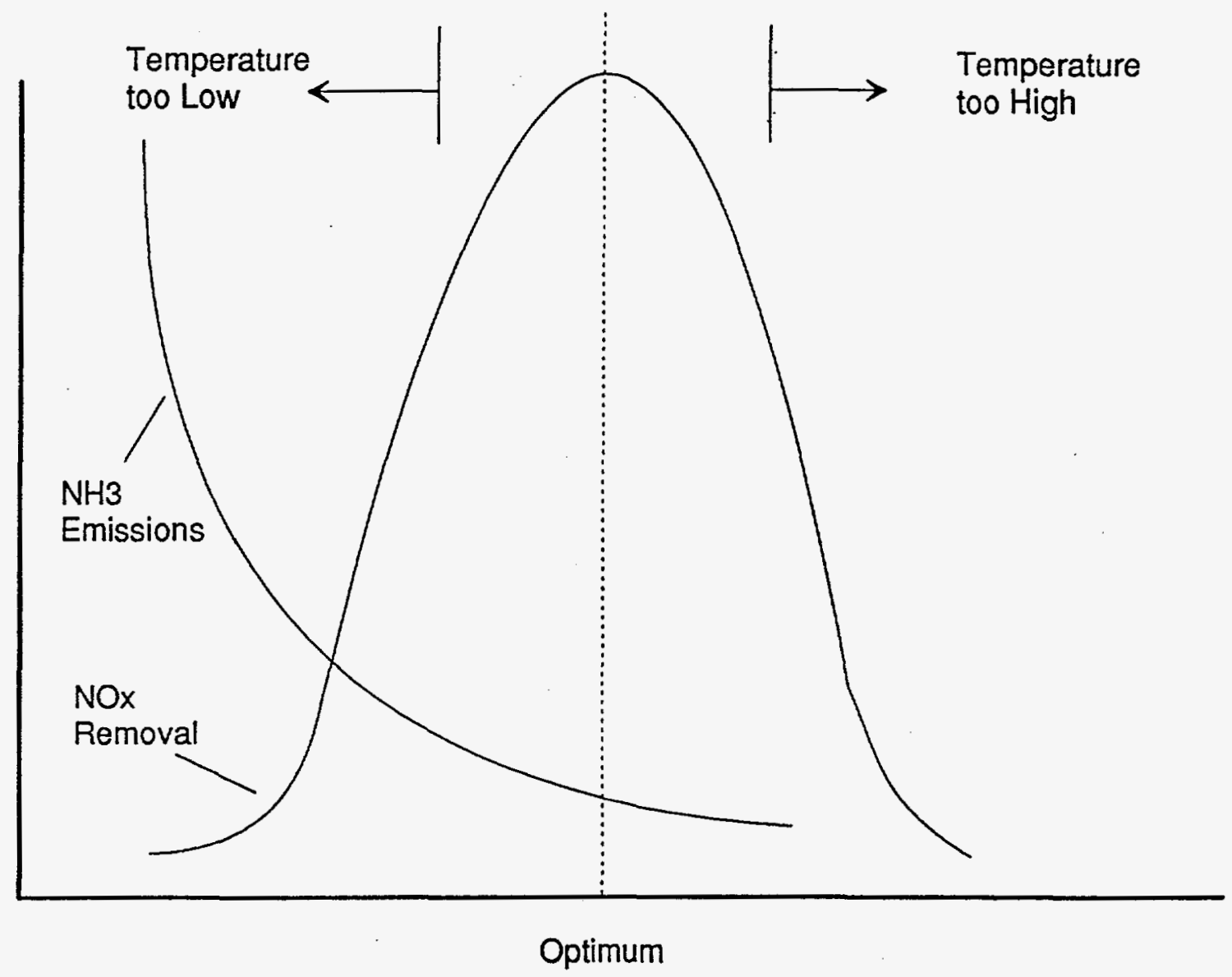

Temperature

Figure 2-1. Conceptual Temperature Window for the SNCR Process 
air heater and the particulate removal equipment, or calcium-based reagents upstream of economizer. The $\mathrm{SO}_{2}$ formed during the combustion process reacts with the sodium- or calcium-based reagents to form solid sulfites and sulfates. These reaction products are collected in the particulate removal equipment together with the flyash and any unreacted reagent, and then removed for disposal. The system is expected to remove up to 70 percent of the $\mathrm{SO}_{2}$ when using sodium-based products while maintaining high sorbent utilization.

Although sodium-based DSI systems reduce $\mathrm{SO}_{2}$ emissions, $\mathrm{NO}_{2}$ formation has been observed in some applications. $\mathrm{NO}_{2}$ is a red/brown gas; therefore, a visible plume may form as $\mathrm{NO}_{2}$ in flue gas exits the stack. Previous pilot-scale tests have shown that ammonia slip from urea injection reduces the formation of $\mathrm{NO}_{2}$ while removing the ammonia which would otherwise exit the stack.

In certain areas of the country, it may be more economically advantageous to use calciumbased reagents, rather than sodium-based reagents, for $\mathrm{SO}_{2}$ removal. $\mathrm{SO}_{2}$ removal using calcium hydroxide $\left(\mathrm{Ca}(\mathrm{OH})_{2}\right)$ involves dry injection of the reagent into the furnace at a point where the flue gas temperature is approximately $1000^{\circ} \mathrm{F} . \mathrm{Ca}(\mathrm{OH})_{2}$ materials can also be injected into the flue gas ductwork downstream of the air heater, but at reduced $\mathrm{SO}_{2}$ removal effectiveness.

\subsubsection{Humidification}

The effectiveness of calcium hydroxide in reducing $\mathrm{SO}_{2}$ emissions when injected downstream of the air heater can be increased by flue gas humidification. Flue gas conditioning by humidification involves injecting water into the flue gas downstream of the air heater and upstream of any particulate removal equipment. The water is injected into the duct by dual-fluid atomizers which produce a fine spray that can be directed downstream and away from the duct walls. The subsequent evaporation causes the flue gas to cool, thereby decreasing its volumetric flowrate and increasing its relative and absolute humidity. It is important that the water be injected in such a way as to prevent it 
from wetting the duct walls and to ensure complete evaporation before the gas enters the particulate removal equipment or contacts the duct tuming vanes. Since calcium hydroxide is not as reactive as the sodium-based reagents, the presence of water in the flue gas, which contains unreacted reagent, provides for additional $\mathrm{SO}_{2}$ removal. Up to 50 percent $\mathrm{SO}_{2}$ removal is expected when $\mathrm{Ca}(\mathrm{OH})_{2}$ is used in conjunction with flue gas humidification.

\subsection{Project Participants}

PSCo is the project manager for the project, and is responsible for all aspects of project performance. PSCo has engineered the DSI system and the modifications to the flyash system, provided the host site, trained the operators, provided selected site construction services, start-up services and maintenance, and is assisting in the testing program.

EPRI provided technical assistance and advice on many of the technologies and also contributed to the project funding. B\&W was responsible for engineering, procurement, fabrication, installation, and shop testing of the low- $\mathrm{NO}_{\mathrm{x}}$ burners, overfire air ports, humidification equipment, and associated controls. They are also assisting in the testing program, and will provide for commercialization of the technology. NOELL, Inc. was responsible for the engineering, procurement and fabrication of the SNCR system. Fossil Energy Research Corp. is conducting the testing program. Western Research Institute is characterizing the waste materials and recommending disposal options. Colorado School of Mines conducted bench scale research on the mechanism and chemical kinetics of $\mathrm{NO}_{2}$ formation reaction with dry sorbent injection. (Appendix A contains a synopsis of their work.) Stone \& Webster Engineering is assisting PSCo with the engineering efforts. Cyprus Coal and Amax Coal are supplying the coal for the project, while Coastal Chemical, Inc. is providing the urea for the SNCR system. 


\subsection{DRY SORBENT INJECTION AND HUMIDIFICATION SYSTEM DESCRIPTION}

The dry sorbent injection (DSI) system consists of a redundant system designed for the delivery of calcium- or sodium-based materials into the duct work between the air heater and the fabric filter. The redundant system includes two separate systems, including storage silos, feeders, pulverizers, and delivery systems. In addition to the fabric filter inlet injection location, temporary injectors were also installed at the air heater inlet to inject sodium bicarbonate at higher flue gas temperatures.

The DSI and the duct humidification systems were added to the existing Arapahoe Unit 4 boiler and flue gas duct work, and required no major modifications beyond adding access into the existing flow system. The original Unit 4 electrostatic precipitators had been removed and a new reverse-gas fabric filter and induced draft (ID) fans were installed in 1985. A retrofit fabric filter dust collector (FFDC) was relocated in back of the common stack for Units 3 and 4 and required a long duct that connected the fabric filter inlet with the existing air heater exit. This air heater exit duct provided the site for both duct sorbent injection and humidification.

\subsection{Process Chemistry}

Details of the chemical and physical processes which occur between sodium-based sorbents and $\mathrm{SO}_{2}$ are currently not well understood. In terms of the chemical processes, it is generally thought that $\mathrm{SO}_{2}$ reacts directly with sodium carbonate $\left(\mathrm{Na}_{2} \mathrm{CO}_{3}\right)$. Previous work (Muzio, et al., 1984), proposed that the overall chemical mechanisms for the two sorbents investigated during the current phase of testing are as follows:

Sodium Bicarbonate

$$
\begin{aligned}
& 2 \mathrm{NaHCO}_{3}-\cdots>\mathrm{Na}_{2} \mathrm{CO}_{3}+\mathrm{H}_{2} \mathrm{O}+\mathrm{CO}_{2} \\
& \mathrm{Na}_{2} \mathrm{CO}_{3}+\mathrm{SO}_{2}+1 / 2 \mathrm{O}_{2}-\cdots>\mathrm{Na}_{2} \mathrm{SO}_{4}+\mathrm{CO}_{2}
\end{aligned}
$$


Sodium Sesquicarbonate

$$
\begin{aligned}
& \mathrm{NaHCO}_{3} \cdot \mathrm{Na}_{2} \mathrm{CO} \cdot 2 \mathrm{H}_{2} \mathrm{O}--->\mathrm{NaHCO} \cdot \mathrm{Na}_{2} \mathrm{CO}_{3}+2 \mathrm{H}_{2} \mathrm{O} \\
& 2\left(\mathrm{NaHCO}_{3} \cdot \mathrm{Na}_{2} \mathrm{CO}_{3}\right)-\cdots>3 \mathrm{Na}_{2} \mathrm{CO}_{3}+\mathrm{H}_{2} \mathrm{O}+\mathrm{CO}_{2} \\
& \mathrm{Na}_{2} \mathrm{CO}_{3}+\mathrm{SO}_{2}+1 / 2 \mathrm{O}_{2}-\cdots>\mathrm{Na}_{2} \mathrm{SO}_{4}+\mathrm{CO}_{2}
\end{aligned}
$$

The sodium bicarbonate mechanism consists of two steps. In the first, a high surface area $\mathrm{Na}_{2} \mathrm{CO}_{3}$ particle is created through the release of $\mathrm{H}_{2} \mathrm{O}$ and $\mathrm{CO}_{2}$. This decomposition is followed by the sulfation reaction, with the final product being sodium sulfate $\left(\mathrm{Na}_{2} \mathrm{SO}_{4}\right)$. There has also been speculation that overall utilization is enhanced by having the decomposition step and sulfation step occur simultaneously in the duct. During the current test series, it was found (consistent with previous studies) that the overall reaction between sodium bicarbonate and $\mathrm{SO}_{2}$ was highly sensitive to flue gas temperature. As will be addressed in more depth below, it is believed that the endothermic decomposition step (Eqn. 3-1) is likely responsible for this temperature sensitivity.

The mechanism for sodium sesquicarbonate consists of three steps, where the last two are very similar to the two discussed above for the sodium bicarbonate mechanism. It is speculated that the initial release of the two water molecules (Eqn. 3-3) "opens-up" the pore structure of the particle, thereby allowing reaction to occur between $\mathrm{SO}_{2}$ and the $\mathrm{Na}_{2} \mathrm{CO}_{3}$ component. This initial dehydration reaction is thought to occur at relatively low temperatures (on the order of $120^{\circ} \mathrm{F}$ ). Results of the current series of tests seem to confirm this hypothesis, as they have shown that the overall reaction between sodium sesquicarbonate and $\mathrm{SO}_{2}$ occurs rapidly and is relatively insensitive to flue gas temperature.

As the sodium compounds react with $\mathrm{SO}_{2}$, there are parallel reactions that result in the oxidation of $\mathrm{NO}$ to $\mathrm{NO}_{2}$ with some $\mathrm{NO}_{x}$ removal. While the $\mathrm{NO}_{x}$ removal is an added benefit of the dry sorbent injection process, the oxidation of $\mathrm{NO}$ to $\mathrm{NO}_{2}$ can pose operational problems. NO is a colorless gas which has no impact on plume visibility. 
However, $\mathrm{NO}_{2}$ is a brown gas that, depending on the concentration, can result in plume visibility. In the case of particulate matter, a plume becomes visible due to light scattering from the particulate matter in the plume. The mechanism is different for the coloration due to $\mathrm{NO}_{2} . \mathrm{NO}_{2}$ in the plume tends to absorb all visible wavelengths of light except the red wavelengths. The transmitted red wavelengths then give the plume its brownish appearance. The amount absorbed depends on the product of the $\mathrm{NO}_{2}$ concentration times the diameter of the plume. Lindau (1991) proposed the following equation to calculate the effect of $\mathrm{NO}_{2}$ on opacity:

$$
\begin{array}{ll}
\text { Opacity }(\%)= & 100\left[1-\exp \left(-0.000101 \times \mathrm{NO}_{2} \times \mathrm{D}\right)\right] \\
& \mathrm{NO}_{2}=\mathrm{NO}_{2} \text { concentration, ppm } \\
& \mathrm{D}=\text { stack diameter, feet }
\end{array}
$$

For a utility boiler stack diameter of 20 feet, an $\mathrm{NO}_{2}$ concentration of $25 \mathrm{ppm}$, the above equation predicts an opacity of $5 \%$. This is only an approximate value, as plume visibility is extremely complex and depends not only on the stack diameter and $\mathrm{NO}_{2}$ concentration, but also on the background conditions, view angle relative to the sun, etc.

The detailed chemistry resulting in $\mathrm{NO}$ to $\mathrm{NO}_{2}$ oxidation and $\mathrm{NO}_{x}$ removal is not well understood. The most comprehensive study of the $\mathrm{Na} / \mathrm{SO}_{2} / \mathrm{NO}_{x}$ interaction was performed by Verlaetent et al., 1993. They proposed the following reaction sequence to explain the removal of $\mathrm{SO}_{2}$ and $\mathrm{NO}_{x}$ with sodium bicarbonate.

$$
\begin{aligned}
& \mathrm{NaHCO}_{3}+\mathrm{SO}_{2}--->\mathrm{NaHSO}_{3}+\mathrm{CO}_{2} \\
& 2 \mathrm{NaHSO}_{3}--->\mathrm{Na}_{2} \mathrm{~S}_{2} \mathrm{O}_{5}+\mathrm{H}_{2} \mathrm{O} \\
& \mathrm{Na}_{2} \mathrm{~S}_{2} \mathrm{O}_{5}+2 \mathrm{NO}+\mathrm{O}_{2}-\cdots>\mathrm{NaNO}_{2}+\mathrm{NaNO}_{3}+2 \mathrm{SO}_{2} \\
& 2 \mathrm{NaHSO}_{3}+2 \mathrm{NO}+\mathrm{O}_{2}--->\mathrm{NaNO}_{2}+\mathrm{NaNO}_{3}+2 \mathrm{SO}_{2}+\mathrm{H}_{2} \mathrm{O}
\end{aligned}
$$

In this mechanism, $\mathrm{SO}_{2}$ behaves as a catalyst by helping the formation of sodium-nitrogen compounds. It is believed that the product $\mathrm{NaNO}_{2}$ is unstable, and is therefore rapidly converted to carbonate and nitrate via the following reactions:

$$
\begin{aligned}
& \mathrm{NaNO}_{2}+1 / 2 \mathrm{CO}_{2}+1 / 2 \mathrm{O}_{2}-\cdots>1 / 2\left(\mathrm{Na}_{2} \mathrm{CO}_{3}\right)+\mathrm{NO}_{2} \\
& \mathrm{NaNO}_{2}+1 / 2 \mathrm{O}_{2}-\cdots \mathrm{NaNO}_{3}
\end{aligned}
$$


It must be emphasized that the above mechanisms are only speculation and must be more completely evaluated prior to acceptance. However, these mechanisms provide a preliminary framework on which to structure explanations regarding differences in $\mathrm{SO}_{2}$ and NO removal, as well as $\mathrm{NO}_{2}$ production, characteristics of the two sodium-based sorbents.

In a parallel effort to the full-scale tests at Arapahoe Unit 4, a fundamental study of the sodium/SO $/ \mathrm{NO}_{2}$ chemistry was conducted by the Colorado School of Mines (Lai, et al., 1994). The goal of the study was to gain a better understanding of the detailed chemistry in terms of $\mathrm{SO}_{2}$ and $\mathrm{NO}_{x}$ removal, as well as $\mathrm{NO}_{2}$ formation. The study involved both bench-scale experiments and chemical kinetic modeling. The results of this study support Verlaetent, et al.'s (1991) mechanism involving $\mathrm{Na}_{2} \mathrm{~S}_{2} \mathrm{O}_{5}$ as the key intermediate (Eqn. 3-7). A synopsis of this study is provided in Appendix $A$ of this report.

As mentioned above, the overall temperature dependence of the sodium bicarbonate- $\mathrm{SO}_{2}$ reactions are thought to be due to the thermal decomposition of sodium bicarbonate. This decomposition was studied by Keener, et al., 1985, using a shrinking core model. Using this model the decomposition time is given by:

$$
\begin{aligned}
t= & \frac{\rho_{A} M_{c} d_{s}}{2 k M_{A}} \\
\rho_{a}= & \text { density of sodium bicarbonate } \\
M_{c}= & \text { molecular weight of } \mathrm{CO}_{2} \\
d_{s}= & \text { initial diameter of sodium bicarbonate particle, } \mathrm{cm} \\
M_{A}= & \text { molecular weight of sodium bicarbonate } \\
\mathrm{k}= & \text { reaction rate constant } \\
= & A e^{-E / R T} \\
& A=4.91 \times 10^{4} \mathrm{~g} / \mathrm{cm}^{2} \mathrm{sec} \\
& E=20,500 \mathrm{cal} / \mathrm{g}-\mathrm{mole} \\
& T=\text { temperature, }{ }^{\circ} \mathrm{K}
\end{aligned}
$$

Using this model, the decomposition time for sodium bicarbonate is shown as a function of particle size and temperature in Figure 3-1. The figure shows that below $300^{\circ} \mathrm{F}$, decomposition time increases dramatically as the temperature decreases. For instance, at $250^{\circ} \mathrm{F}$, a 20 micron sodium bicarbonate particle will require 45 minutes to completely decompose. 


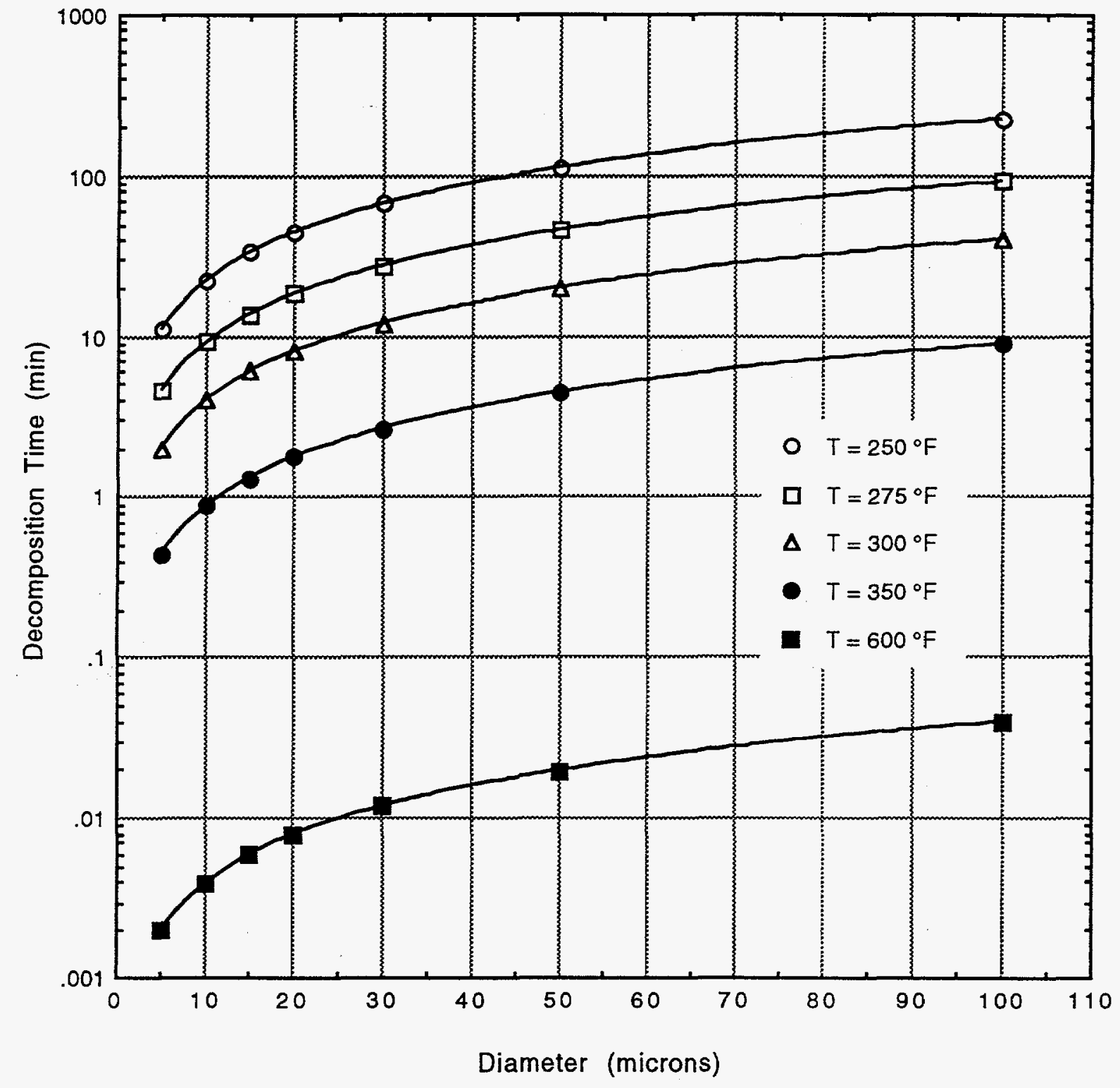

Figure 3-1. Calculated Decomposition Times for Sodium Bicarbonate 


\subsection{Existing Boiler Equipment}

Arapahoe Unit 4 utilizes a single tubular air heater for heating the secondary air. The boiler flue gases exit the air heater in a single, short and very wide duct. The air heater exit duct work immediately transitions into a narrower and taller duct. Figures 3-2 and 3-3 show the side and top views of duct/FFDC/stack arrangement for Unit 4. The air heater exit is approximately 150 feet from the inlet of the fabric filter, while the transition duct accounts for 36 feet of the total. Flow diverting vanes are used in the transition duct, while flow straighteners are used in the duct immediately downstream of the transition point. The balance of the duct is 114 feet long and has moderate changes in profile and elevation into the fabric filter. The location of the duct sorbent and humidification injection is just downstream of the flow straighteners, approximately 103 feet from the fabric filter inlet, where the duct is $17^{\prime} 3^{\prime \prime}$ wide by 9' 9" tall. Approximately halfway to the fabric filter, the duct work transitions into a $15^{\prime}$ ' wide by 11 ' 6 " tall duct. 'In this second transition duct, a single, vertical air foil is installed near the center of the duct, to divert gas from the west to the east side of the duct. According to plant personnel, this air foil is intended to eliminate ash drop-out or deposition on the bottom of the duct on the east side. This air foil is part of the existing boiler equipment and was not installed as part of the test program. The air foil assembly also includes a horizontal stiffener that connects the center of the foil with the west wall. After this second transition point and the air foil location, the duct starts to rise up to the fabric filter inlet elevation. As the duct rises in elevation, it also gradually changes to conform with the 12' wide by 14 ' tall fabric filter inlet dimensions.

The Arapahoe Unit 4 FFDC is an Ecolaire Environmental Company reverse gas fabric filter with 12 compartments and is designed for a gas flow of 600,000 acfm at $290^{\circ} \mathrm{F}$. These compartments are arranged in a 2 wide by 6 long pattern around the centrally located inlet duct. Each compartment consists of 252 woven fiberglass bags that are 12 inches in diameter and 34 feet long. The original operating pressure drop was specified as 6.6 inches of $\mathrm{H}_{2} \mathrm{O}$ at the design conditions, although the operating practice at the plant initiates 


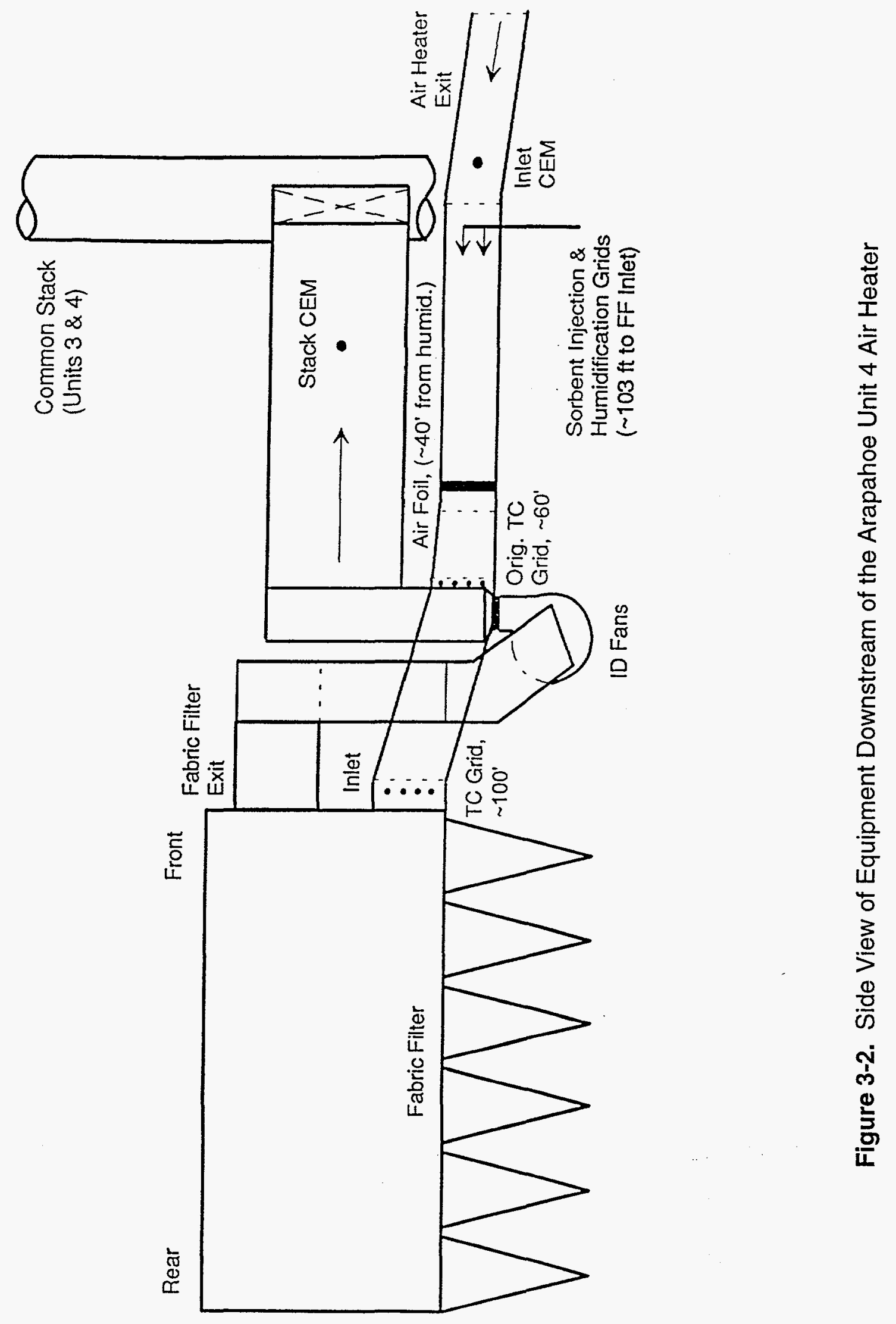




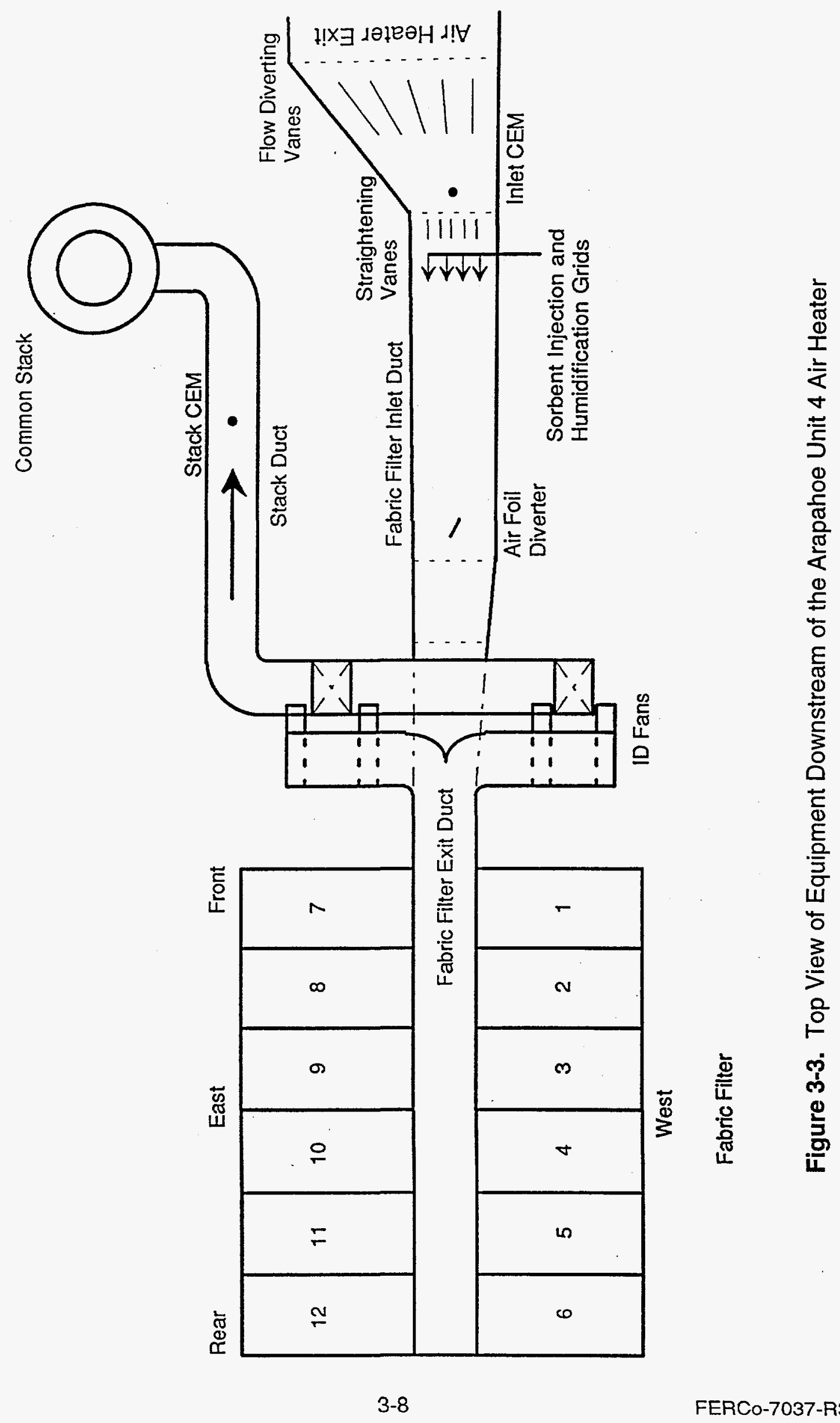

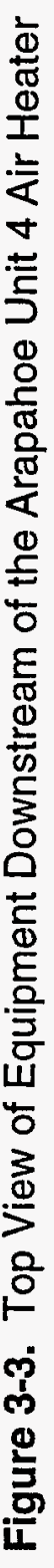


a cleaning cycle when the pressure drop reaches 4.0 inches of $\mathrm{H}_{2} \mathrm{O}$. At full load, normal $\mathrm{O}_{2}$ levels, and with all compartments in service, the fabric filter pressure drop decreases to approximately 2 inches of $\mathrm{H}_{2} \mathrm{O}$ immediately after a cleaning cycle. Each compartment gas inlet pulls flue gas from the bottom of the fabric filter inlet duct into the upper level of the ash hoppers, just below the tube sheet. The flue gases flow up into the bags and the clean gas exits into a common duct located near the top of the compartments. Poppet valves and dampers control the gas flow and cleaning for each compartment.

After the cleaned flue gases exit the fabric filter, the duct splits for the two ID fans, then recombines into a single duct to return back to the common stack for Units 3 and 4 . The single duct between the ID fans and the stack was used for all gas sampling at the fabric filter exit or "stack" location, since the common stack was not suitable for monitoring Unit 4.

\subsection{Humidification System}

The humidification system was used primarily for the tests with calcium-based sorbents (Shiomoto, et al., 1994), although a number of tests were conducted with sodium sesquicarbonate injection as well. Humidification lowers the flue gas temperature by spraying a finely atomized water spray from an array of atomizers. The humidification system installed at Arapahoe Unit 4 includes a set of atomizer lances installed in the duct, a variable speed water pump, two large atomizing air compressors, a thermocouple grid to monitor the gas temperatures, and a humidification process control system (Figure 3-4). The humidification grid is located in the air heater exit duct, just downstream of the flow straighteners and near the beginning of a long straight run of duct (Figure 3-2). This location is also the site of the sorbent injectors for the duct injection system.

The humidification atomizers are a dual-fluid design, utilizing high pressure air. Six atomizers are arranged on each lance, with air and water supplied from a common lance header. Each lance incorporates an aerodynamic shell around the atomizers that is 


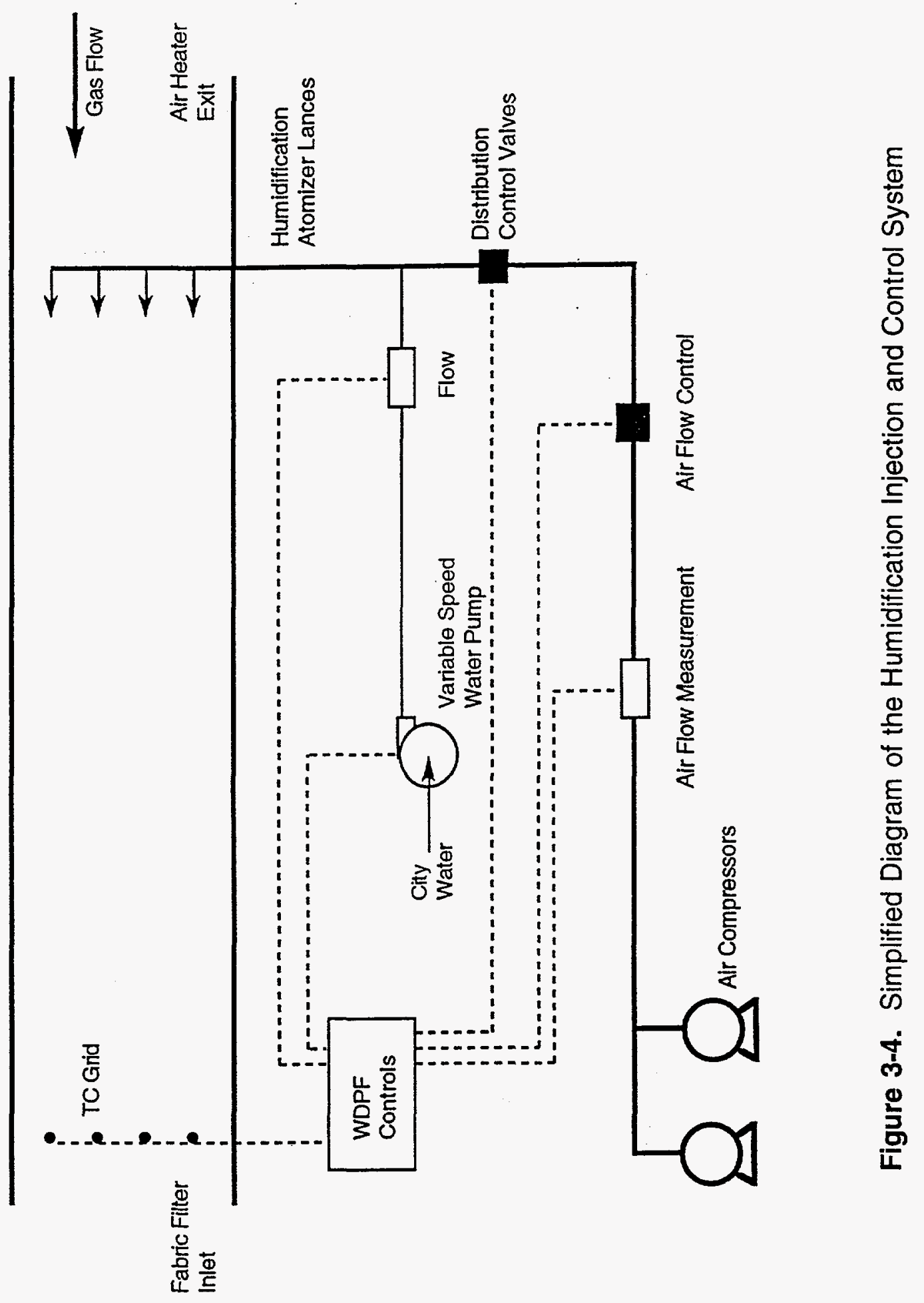


purged with clean gas (fabric filter outlet gas is used at Arapahoe Unit 4) to prevent ash deposition when the humidification system is not in use. A set of seven lances is installed into both the east and west side walls of the duct, for a total of 84 atomizers arranged in a 12 wide by 7 high grid (Figures 3-5 and 3-6). City water is supplied to common headers and controlled with a variable speed pump. A magnetic flow meter and temperature indicator provide the signals supplied to the system controls. The water is filtered to prevent plugging of the atomizers. A detailed description of the system is contained in a prior test report (Shiomoto, et al., 1994).

A grid of 12 flue gas thermocouples located downstream of the lances and just upstream of the fabric filter inlet monitors the effects of the humidification system. Although the average gas temperature is used for control, each individual thermocouple is monitored. Alarms and water shut off controls are provided for both the individual and average temperatures. The humidified fabric filter inlet gas temperature is controlled by modulating the water flow rate with the variable speed pump.

\subsection{Dry Sorbent Injection (DSI) System}

The DSI system at Arapahoe Unit 4 utilizes two identical preparation and injection systems to provide the required capacity at high sorbent flow rates and redundancy at lower flow rates. These two systems are entirely separate up to and including the sorbent injectors in the duct. This system initially allowed sorbent injection at either the fabric filter inlet or the economizer inlet by manual piping changes. The economizer injection location was utilized during the calcium-based sorbent injection tests. As will be discussed in the presentation of the results, it became apparent during the current phase of testing that it would be desirable to inject the sodium compounds ahead of the air heater, at the economizer exit. This modification used the existing economizer injection piping.

Figure 3-7 shows one of the two sorbent preparation and injection systems. Each system includes a storage silo, variable speed screw feeder, rotary air lock, blower for conveying air, pulverizer to grind the sorbent, distributor to split the sorbent stream, and injectors. 


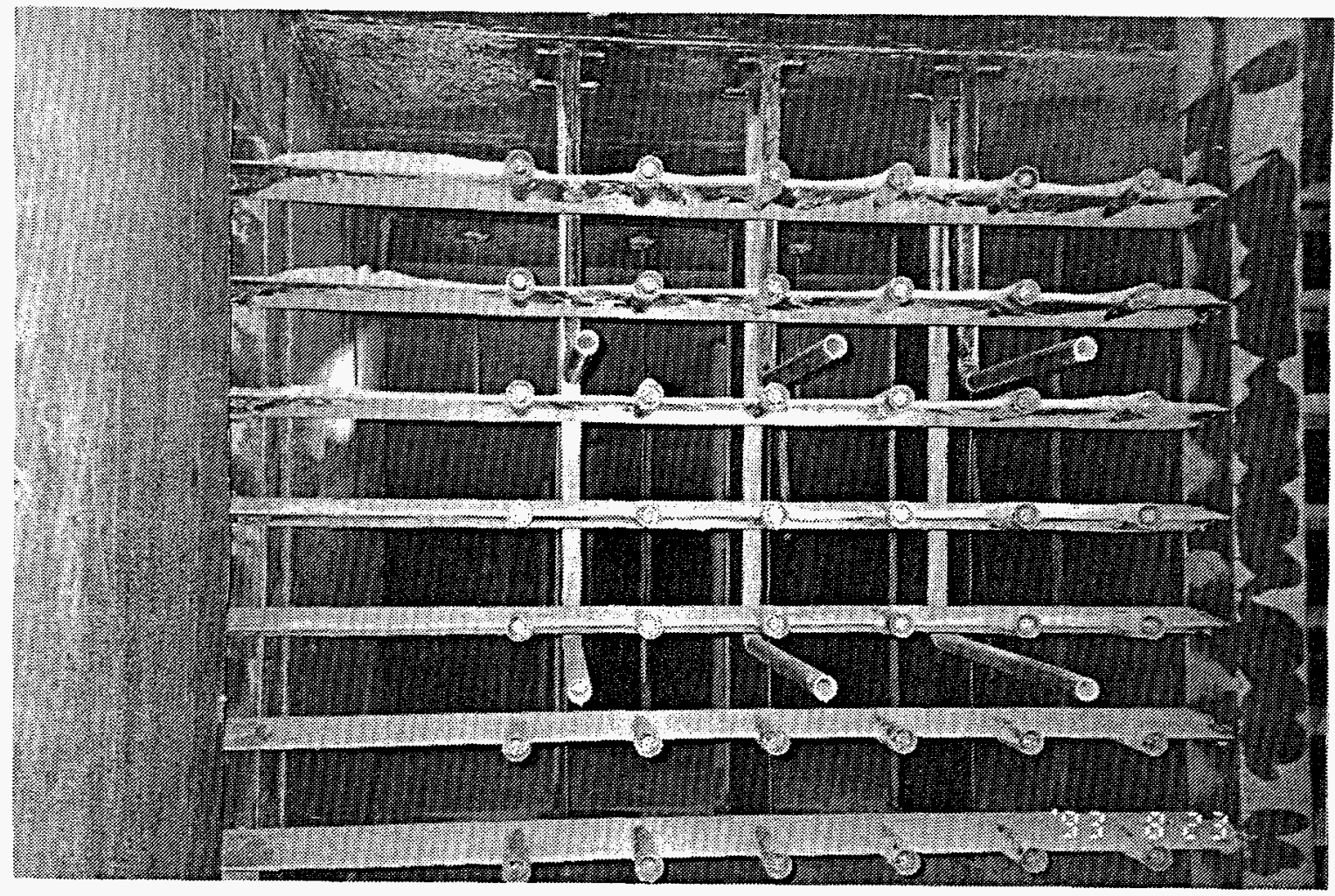

Figure 3-5. Humidification and Sorbent Injection Grids (East Half)

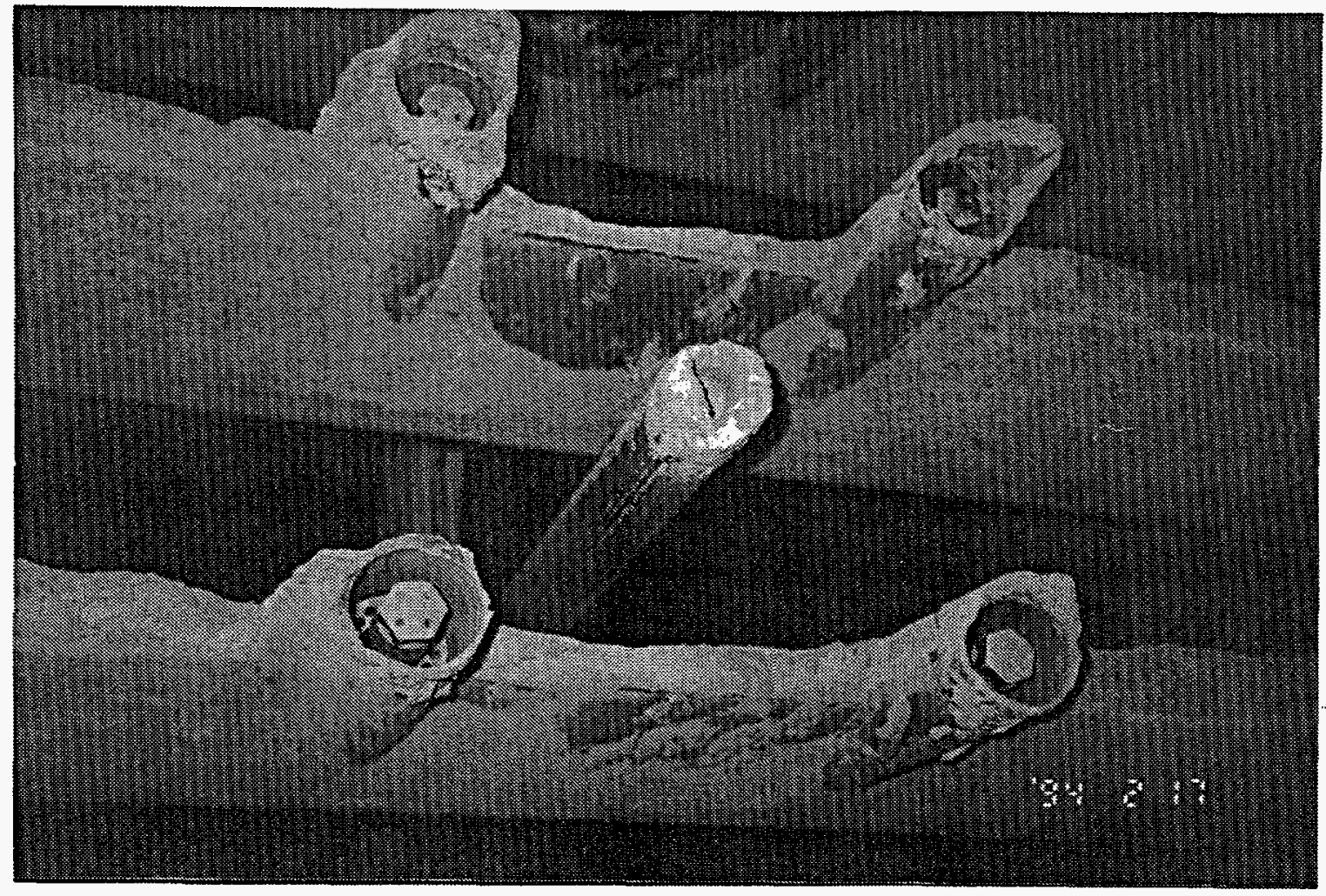

Figure 3-6. Humidification Nozzles and Sorbent Injector 


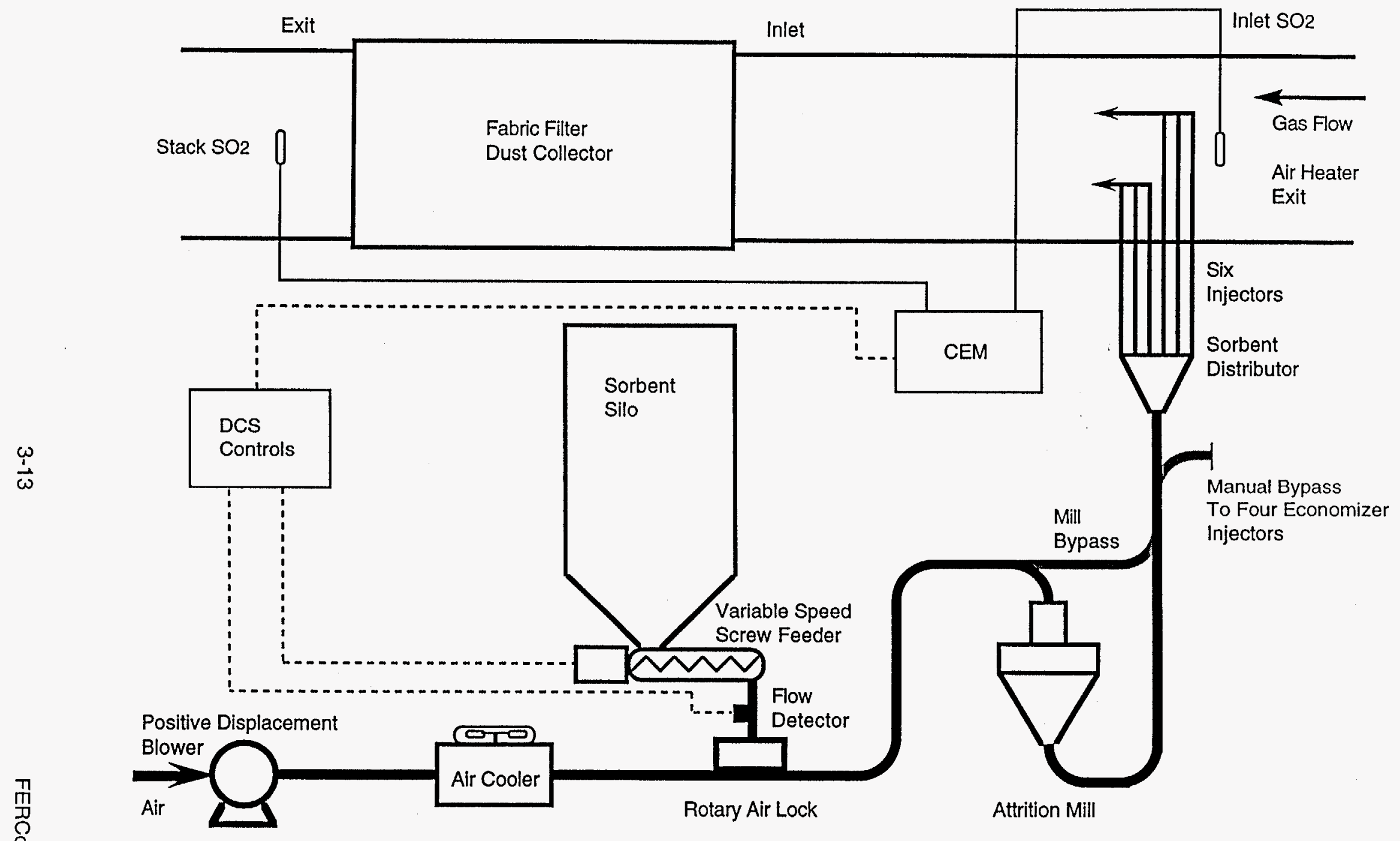

Figure 3-7. Simplified Diagram of One Sorbent Handling System and the Process Controls 


\subsubsection{Dry Sorbent Storage and Handling}

The two sorbent preparation and injection systems (labeled A and B for the testing) are identical in capacity and operation. Each has separate controls and can be independently operated from a control screen on the Arapahoe Unit 4 Distributed Control System (DCS). The following paragraphs describe one of the two identical systems.

Sorbent is stored in a silo with a capacity of approximately 6100 cubic feet. Dry sorbents are transported by truck and pneumatically loaded into the top of the silo. The silo is vented at the top through a small fabric filter system which prevents fugitive dust emissions. An ultrasonic level indicator provides continuous silo level measurements.

A slide gate is installed at the bottom of the silo hopper to allow isolation from the feeder when necessary. Directly below the slide gate is a variable speed screw feeder. The volumetric screw feeder provides the sorbent flow control for the system and can be operated with local controllers in the sorbent preparation building or from a screen on the DCS. The feeder can be operated in either a manual or automatic mode. In the manual mode, the operator sets a constant screw speed. In the automatic mode, an $\mathrm{SO}_{2}$ removal setpoint is input and the control system varies the feed rate to maintain the setpoint $\mathrm{SO}_{2}$ removal. The automatic control system also incorporates a trim control to limit $\mathrm{NO}_{2}$ emission levels to less than a user defined setpoint. For the majority of the current test phase, the feeder speed was manually set to obtain the desired stoichiometric ratio.

The screw feeder delivers sorbent directly into the top of a rotary air lock which provides the necessary isolation between the sorbent feed and the conveying air systems. The air lock is used for isolation, not feed rate control, and therefore is operated at a constant rotational speed. The air lock is vented to relieve the higher pressure from the conveying air and help prevent pressurization of the bottom of the silo and screw feeder. The vent line extends up to the top of the silo and into the fabric filter venting system. A flow detection probe installed between the exit of the screw feeder and the inlet of the air lock 
is used to detect the loss of sorbent flow. When properly calibrated for sorbent type, this probe determines loss of flow and displays an alarm on the DSI control screen.

The conveying air piping passes just below the rotary air lock, which allows the sorbent to drop into and be dispersed within the air. The air is supplied from a positive displacement blower that operates at a constant speed and air flow rate (nominally $660 \mathrm{cfm}$ at $9 \mathrm{psig}$ ). Blower air pressure is monitored to determine if plugging occurs or if sorbent flow is abnormal. The air supply pressure is limited to $10 \mathrm{psig}$ by a relief valve installed downstream of the blower. An air-to-air heat exchanger installed downstream of the blower cools the conveying air whenever the sorbent pulverizers are in use. This heat exchanger cools the air to reduce the mill exit air temperature and prevent sorbent overheating. After the air cooler, the air flows under the rotary air lock and picks up the sorbent flow. After the sorbent and air are mixed, the flow can be directed either into or around the Entoleter attrition mill via manual piping changes. The attrition mill was bypassed during the previous series of tests with calcium-based sorbents (Shiomoto, et al., 1994). During the current series of tests, the mill was placed in service in order to increase the fineness of the sodium-based sorbent.

After exiting the mill, the sorbent and carrier air are piped to one of the injection locations at either the fabric filter inlet or the air heater inlet. The piping at each injection location is very similar, although the number of injectors differs. Most of the testing was performed at the fabric filter inlet location. A distributor is installed on the top of the air heater exit duct to split the sorbent flow to each injector. A single pipe supplies the sorbent from the preparation system and the flow is evenly split into six injection streams. At the outlet of the distributor is a separate ball valve on each line that is used to isolate each injector from the system. The piping for injection at the air heater inlet is identical, except that a distributor divides the flow into four streams instead of six. 


\subsubsection{Fabric Filter Inlet Sorbent Injection}

The duct injection location at the fabric filter inlet was utilized for the majority of the tests with sodium-based sorbents. This location (shown in Figure 3-2) is located at the air heater exit just downstream of the flow straighteners. Injection at this location provides approximately 103 feet of duct work prior to entry into the fabric filter. Immediately after the sorbent and humidification injection location, the duct remains relatively constant in cross section for roughly one-half of the distance to the fabric filter.

The sodium-based sorbents are injected into the flue gas stream at the same plane as the humidification system through a grid of 12 nozzles arranged in a 2 high by 6 wide array. Injection nozzles from each of the two systems (A and B) are interspersed within the grid, so that operation with only a single system provides sorbent injection across the entire duct. The $A$ and $B$ systems alternate injectors in a checkerboard fashion within the 12 point grid. Each system comprises six injectors, three on each side of the duct (Figure 3-8).

Each injector is fabricated from a two-inch pipe, which enters the air heater exit duct from the top and turns 90 degrees within the duct. The exit of each injector is oriented downstream such that the sorbent-conveying air flow and flue gas flow are in the same direction. The injection nozzles are located at the exit plane of the humidification atomizers, and between two adjacent humidification lances in the vertical direction (Figures 3-5 and 3-6).

\subsubsection{Air Heater Inlet/Economizer Exit Sorbent Injection}

Early tests showed that the flue gas temperatures at the fabric filter inlet were too low (i.e., $<290^{\circ} \mathrm{F}$ ) for good performance with sodium bicarbonate injection. In an effort to enhance performance, the injection location was temporarily moved to the air heater inlet where the flue gas temperatures are nominally $650^{\circ} \mathrm{F}$. The piping utilized to inject calcium hydroxide at the economizer during the previous phase of testing (Shiomoto, et al., 1994), was modified to supply the temporary injectors at the air heater inlet. 


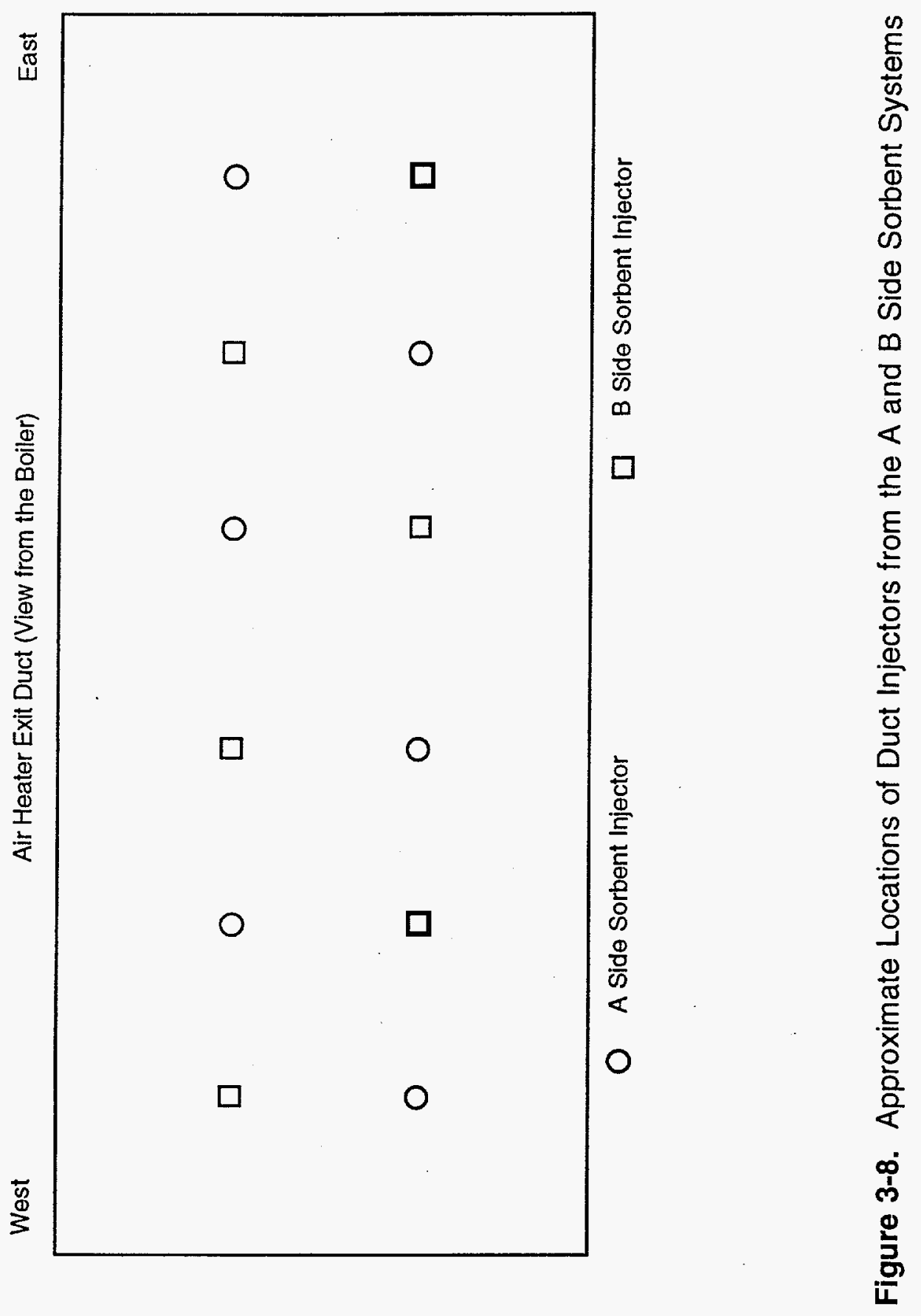


Changing the injection location from the fabric filter inlet to the economizer required plant maintenance personnel to remove and reinstall different piping connections to redirect the sorbent flow. In the economizer injection configuration, distributors for the $A$ and $B$ sorbent systems are located on opposite sides of the boiler, with the A system being on the west and the $B$ system on the east. This configuration required that both systems be in operation in order to inject $\mathrm{Ca}(\mathrm{OH})_{2}$ into the east and west halves of the boiler. During the current phase of testing, the economizer injection piping for the B system was utilized to supply the temporary injectors at the air heater inlet. The flow distributor remained in its original location, on the east side of the boiler, and two-inch diameter flexible hoses transported the sorbent up nearly one-half the height of the boiler, and across the width of the air heater inlet/economizer exit duct to the four new injectors.

Existing two-inch pipe ports were utilized for the temporary injectors. These ports are normally used for the economizer exit gas sample probes (described in the following section), and required removal of the probes when the injectors were in use. A total of six ports are available at the economizer exit sample location (Figure 3-9) and of these, the four center ports were used for the temporary injectors. With this injection pattem, only the center portion of the short and wide ( 7 feet tall by 40 feet wide) economizer exit/air heater inlet duct would be treated by the sorbent. However, it would provide the most optimum coverage available for this temporary injection system. The four injectors were fabricated from 1-1/2-inch diameter pipe and were built to fit inside the existing ports (Figure 3-10). Sorbent flowed through the 1 -inch diameter probe tip as well as through $3 / 4$-inch orifices on the probe side walls.

\subsection{Operational Problems}

Operational problems encountered during the sodium-based sorbent injection program are described briefly in this section. This experience is documented in order to avoid these problems in the future system designs. The problems to be discussed may be characteristic of this specific system design that could be improved with modifications. 


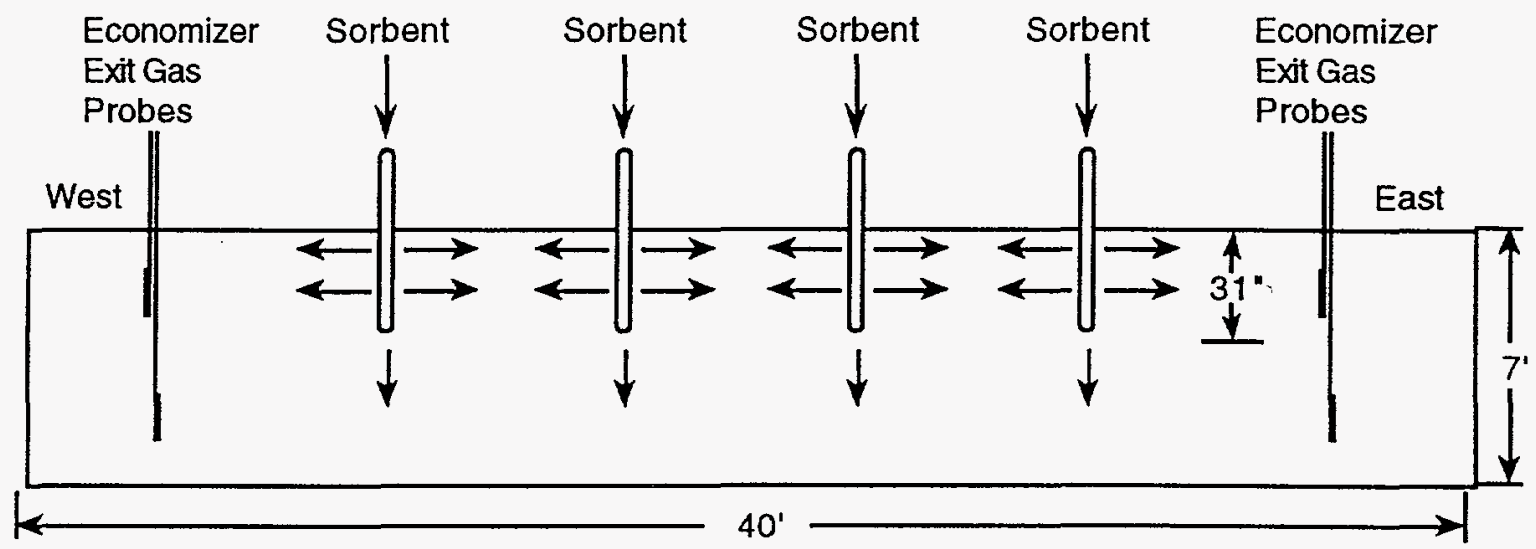

Figure 3-9. Air Heater Inlet Sodium Injection Locations

3/4" Dia. $\quad 3 / 4^{*}$ Dia.

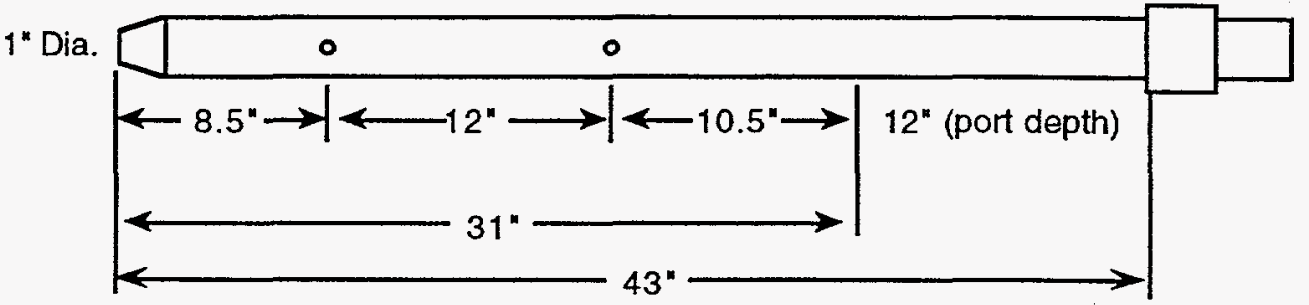

Figure 3-10. Air Heater Inlet Sodium Injector Design 
Other problems encountered with the use or limitations of sorbent injection will be covered in later sections with the test results.

\subsubsection{Sorbent Storage and Handling Problems}

A number of problems, which resulted in erratic or loss of sorbent flow, were encountered with the sorbent handling. Problems of this nature were observed with both calcium- and sodium-based sorbents. Storage problems in the silo which prevented smooth flow into the screw feeder were encountered periodically. Rat holes down the center of the silo would impede flow by allowing the feeder to run dry, despite a considerable amount of sorbent collecting on the hopper slopes. Vibrators on the silo hopper were only moderately successful at improving the sorbent flow, as was an "air cannon" installed on one silo hopper. Beating the hopper walls with a sledgehammer was frequently employed with some success. The majority of these problems were related to product contamination from moisture or other chemicals that occurred during transport. Some problems were simply due to the difficulty in flowing these bulk materials and were a consequence of the specific material handling properties.

Air leakage through the rotary air lock is suspected as a significant problem which can cause erratic feed rates. Air leakage from the carrier air can pressurize the outlet of the screw feeder and the bottom of the silo. Sufficient sorbent levels in the silo may reduce the problem; however, pressurizing the feeder and sorbent bed can disrupt the material flow and cause erratic or significantly reduced flow rates. A different air lock and/or improved venting system may reduce these problems. One additional problem suspected with the air lock venting concems the loss of sorbent carried away with vent air. With very fine materials, a significant portion of feed material may be lost to the vent system before the sorbent is added to the carrier air. This leads to feed rate calibration errors whenever screw feeder calibrations are performed at atmospheric pressure without the air lock in service. During the current program, revised feeder calibration procedures were instituted to resolve these difficulties, although the root cause is the rotary air lock leakage. This 
problem was primarily encountered with the fairly fine sodium sesquicarbonate (and calcium hydroxide) and was not a major issue with the sodium bicarbonate.

\subsubsection{Sorbent Injection Problems}

Plugging of individual injectors or the distributor has been a recurring problem with all sorbents. Hard deposits within the piping may be the result of aerodynamic impaction on turns or flow irregularities, or may perhaps be formed by contact with moisture in the flue gas or other ambient sources. In most cases, disassembly and removal of the hard deposits by hand was required. Injection transport lines were also periodically filled or flushed with water to soften and remove the deposits. A few deposits have been noted at the sorbent injector tips located in the fabric filter inlet, although none of the injector tips have been entirely closed off. Water was not used to clean the sorbent injector lines entering the duct. Additional problems were encountered with plugging of the long hoses running to the air heater inlet injectors. However, these problems may have been due to the temporary nature of this injector location design. In addition, sorbent types were changed quite frequently during the current phase of testing, and it is not certain to what extent this may have contributed to the plugging problems.

\subsubsection{Determining Sorbent Feed Rate}

One other issue of interest is the ability to accurately determine the sorbent feed rate during the short-term parametric tests. The project is intended as a full-scale commercial demonstration, with the equipment design reflecting a commercial configuration. In actual long-term operation, the control system would be set at a percent $\mathrm{SO}_{2}$ removal efficiency, and the feed rate adjusted automatically. Overall sorbent utilization would then be determined on an integrated basis over a relatively long time period. As such, a commercial system would not necessarily require gravimetric feeders to determine the instantaneous sorbent feed rate.

The lack of an instantaneous gravimetric sorbent feed rate posed some problems in determining the stoichiometric ratio ( $2 \mathrm{Na} / \mathrm{S})$ for the short-term tests. In order to determine 
the feed rate, calibration of the screw feeder was done two ways. As mentioned previously, a calibration was performed by shutting off the rotary air lock, and opening up an access port above the rotary air lock. The feeder was then calibrated with the discharge at atmospheric pressure. This raised a concern that when operating in the normal mode, the back pressure from leakage past the rotary air lock, or the sorbent loss through the vent line, could affect the feed rate relative to the atmospheric calibration. To check this, a second calibration was performed while the system was on-line. For this calibration procedure, the ball valve for an individual injector downstream of the sorbent distributor was turned off. A fabric filter bag was attached to the flexible hose downstream of the valve, and then a sorbent sample was collected and weighed from each injector line. Typically, this procedure yielded a feed rate approximately 10 to 20 percent less than the atmospheric calibration of the screw feeder for the sodium sesquicarbonate. For sodium bicarbonate, there was no measurable difference in the calibrations. All data presented in this report are based on the injector calibration procedure.

\subsubsection{Sorbent Pulverizer Problems}

The injection of sodium-based sorbents requires the use of the high-speed attrition mills in the sorbent feed system. The mills are installed downstream of the rotary air lock in the transport line prior to being conveyed to the fabric filter inlet or air heater inlet injection locations. These mills operate by impacting the sorbent particles on stationary and rotating pins as the sorbent flows through the mill. The rotating pins are mounted on a 200 pound disk operating at high speed. As a result of the high rotational speeds and the physical characteristics of the sorbents, mill vibration problems were often encountered.

Initially, the mills were operated at a speed of $5700 \mathrm{rpm}$. However, this proved to be too close to a second resonant frequency which caused significant vibrational problems that could not be resolved. The mill speeds were temporarily reduced to $4000 \mathrm{rpm}$, while awaiting parts that would eventually allow operation at $5000 \mathrm{rpm}$. During this period, rebalancing of each mill was also performed to reduce the vibration to reasonable levels. 
The majority of the sodium injection tests were conducted with the $5000 \mathrm{rpm}$ speed. Unless otherwise noted, all results presented in this report are from operation at $5000 \mathrm{rpm}$.

While lowering the mill speed reduced the problems, vibration remains a chronic, but manageable problem of the sorbent preparation and feed systems. By passing sorbents through the mills, deposits on the rotating parts would accumulate, and typically the mill vibration levels would gradually increase throughout a test day. When the vibration limits were exceeded, the mill and sorbent feed systems would trip off. Generally, a mill shut down and restart sequence was the only action required to dislodge the material and restore the mill vibration to acceptable levels. In some cases however, a water wash of the mill was necessary to remove soluble sodium deposits.

During one period of extended 24-hour operation, a catastrophic failure of the "B" mill occurred while injecting sodium bicarbonate. The failure was caused by tramp stainless steel delivered with the reagent. The non-magnetic stainless steel was not captured by the magnetic separator installed to protect the pulverizer. The net effect of the failure included a bent shaft, a broken mill case, shattered grinding element pins, uprooted anchor bolts, and numerous electrical and plumbing components that were apparently broken by excessive vibration. To prevent non-magnetic metal from entering the mill, a screen was installed downstream of the rotary air lock and upstream of the mill. 


\subsection{MEASUREMENT METHODS}

This section describes the measurement methods used to determine the system operating conditions and the $\mathrm{SO}_{2}$ reductions resulting from the sorbent injection processes.

\subsection{Gas Analysis Instrumentation}

An Altech 180 continuous emission monitoring (CEM) system was purchased as part of the Integrated Dry $\mathrm{NO}_{x} / \mathrm{SO}_{2}$ Emissions Control System and installed during the low- $\mathrm{NO}_{x}$ combustion system retrofit. The CEM system utilizes a Perkin Elmer MCS 100 infrared gas analyzer which is capable of continuously analyzing eight gas species simultaneously, using either gas filter correlation or single beam, dual wavelength techniques.

The analyzer cycles through and measures all eight gas species in approximately 22 seconds. In that time, two readings are made for each gas species to be measured. The first reading is a reference value at a known wavelength and gas concentration (either 0 or 100 percent), and the second is a measured reading to determine the quantity of the desired species in the sample stream. Table 4-1 provides a listing of the full-scale range, measurement technique, and interfering species for each of the gases measured.

Table 4-1

Gas Species Measured by Perkin Elmer MCS 100 Analyzer

\begin{tabular}{|l|l|l|l||}
\hline $\begin{array}{c}\text { Measured } \\
\text { Species }\end{array}$ & \multicolumn{1}{|c|}{ Range } & \multicolumn{1}{|c|}{ Measurement Technique } & \multicolumn{1}{|c|}{$\begin{array}{c}\text { Interfering } \\
\text { Species }\end{array}$} \\
\hline $\mathrm{NO}$ & $0-800 \mathrm{ppm}$ & Gas Filter Correlation & $\mathrm{H}_{2} \mathrm{O}$ \\
$\mathrm{CO}$ & $0-400 \mathrm{ppm}$ & Gas Filter Correlation & $\mathrm{H}_{2} \mathrm{O}$ \\
$\mathrm{SO}_{2}$ & $0-800 \mathrm{ppm}$ & Single Beam Dual Wavelength & $\mathrm{NH}_{3}, \mathrm{H}_{2} \mathrm{O}$ \\
$\mathrm{NO}_{2}$ & $0-100 \mathrm{ppm}$ & Single Beam Dual Wavelength & $\mathrm{NH}_{3}, \mathrm{SO}_{2}, \mathrm{H}_{2} \mathrm{O}$ \\
$\mathrm{CO}_{2}$ & $0-20$ volume \% & Single Beam Dual Wavelength & $\mathrm{H}_{2} \mathrm{O}$ \\
$\mathrm{H}_{2} \mathrm{O}$ & $0-15$ volume \% & Single Beam Dual Wavelength & $\mathrm{None}$ \\
$\mathrm{N}_{2} \mathrm{O}$ & $0-100 \mathrm{ppm}$ & Single Beam Dual Wavelength & $\mathrm{CO}, \mathrm{CO}_{2}, \mathrm{H}_{2} \mathrm{O}$ \\
$\mathrm{NH}_{3}$ & $0-100 \mathrm{ppm}$ & Gas Filter Correlation & $\mathrm{CO}_{2}, \mathrm{H}_{2} \mathrm{O}$ \\
\hline
\end{tabular}


Using the gas filter correlation technique, the system takes a reference reading at a known wavelength and a known concentration of gas, usually 100 percent. The system then takes another reading at the same wavelength for the sample gas and records the energy absorbed by the sample. The relative difference in energy is then representative of the concentration in the sample gas.

Likewise in the single beam, dual wavelength method, a reference reading is taken at a wavelength where the desired species does not absorb energy (zero percent reference). The system then takes a measured reading at a wavelength where the desired species is known to absorb energy. The relative difference in energy is again representative of the concentration of the species in the sample stream.

Once the ratio of reference to measure energy is calculated, the energy level is corrected to account for interferences via reference tables for each specific gas. After correction for interferences, the data is zero adjusted, converted to the appropriate units, calibration corrected, and output for display and recording.

Since $\mathrm{O}_{2}$ is not infrared active, the CEM system also contains an Ametek $\mathrm{O}_{2}$ analyzer. The sample cell is a zirconium oxide, closed-end tube with electrodes of porous platinum coated onto the inside and outside of the tube. The cell produces a millivolt signal proportional to the relative difference of $\mathrm{O}_{2}$ inside and outside of the cell. The millivolt signal is converted to percent $\mathrm{O}_{2}$, scaled ( 0 to 25 percent), and then displayed and recorded.

All CEM analyzer and sampling system functions, including a daily automatic calibration sequence, are controlled by the MCS 100 programmable logic controller. The measured gas concentration data is displayed on a dedicated 486-based computer, which also provides data logging, manipulation and reporting capabilities. 
A Relative Accuracy Test Audit (RATA) was performed on March 5, 1993, in order to verify the accuracy of the CEM system. The audit was performed by TRC Environmental Corp. in accordance with the requirements established in 40 CFR, Part 60, Appendices A and F. Complete documentation of the audit is contained in a separate report (Smith, et al., 1994a), and the results are summarized in Table 4-2.

Table 4-2

CEM RATA Results

\begin{tabular}{|l|c|}
\hline \multicolumn{1}{|c|}{ Parameter } & Relative Accuracy \% \\
\hline $\mathrm{CO}_{2}(\%$, wet) & 2.64 \\
Moisture (\%) & 7.86 \\
$\mathrm{O}_{2}(\%$, wet) & 17.81 \\
NO (ppm, wet) & 1.53 \\
NO (lb/MMBtu, wet $\left.{ }^{\star}\right)$ & 5.93 \\
NO (ppm, dry) & 1.02 \\
\hline
\end{tabular}

* Calculated on an $\mathrm{O}_{2}$ basis

Acceptance criteria for RATA evaluation of component instruments of the CEM is 20 percent. Based upon the results, all individual parameters were found to be within the acceptance criteria.

\subsection{Gas Sampling System}

As shown in Table 4-1, the MCS 100 is configured to measure $\mathrm{NH}_{3}$. Although this feature was not utilized during the current series of tests, this capability imposes some special requirements upon the design of the CEM sampling system. In order to maintain the sample integrity, the entire sampling system (probe, sample line, pump, flowmeter, and sample cell) must be maintained at $230^{\circ} \mathrm{C}\left(445^{\circ} \mathrm{F}\right)$. Due to these heat tracing requirements, the CEM system is configured to sample from only two different single-point 
locations. The first location is at the exit of the air heater in the duct leading to the fabric filter. The second location is in the duct leading to the common stack for Units 3 and 4. The air heater exit location is at a point just upstream of the flow straightening vanes and the sorbent injection/humidification lances (see Figure 3-2). The air heater exit location is used to determine the initial boiler exit gas conditions, while the stack or fabric filter outlet sample location is used for the determination of effects after the humidification and/or sorbent injection. Calculation of the $\mathrm{SO}_{2}$ removal between the air heater exit and the stack locations includes correction for dilution from ambient air inleakage, as well as the additional dilution effects resulting from the vaporization of the humidification water, if used.

In order to obtain a representative composite gas sample from the boiler, as well as provide the ability to look at discrete areas of the flue gas flow, Fossil Energy Research Corp. provided a sample gas conditioning system which would allow sampling from additional unheated sample probes. Although the MCS 100 was utilized as the gas analysis instrumentation, the measurement of $\mathrm{NH}_{3}$ at the additional sampling locations was not possible due to the lack of high temperature heat tracing. A schematic of the sample gas conditioning system is shown in Figure 4-1. The system can accommodate up to 24 individual sample lines. Up to 12 of these can be composited together and then analyzed. Each of the individual sample streams is dried in a refrigerated dryer where the gas is cooled and the moisture is dropped out in a trap. Each stream then passes through a metering valve and rotameter, after which all the streams are blended together in a manifold and directed to a pair of sample pumps. The rotameters are used to balance the individual flows in order to provide an accurate composite blend. Down-stream of the pumps, a portion of the composited sample is diverted to a final pass through the condenser (where the increased pressure aids in the removal of any remaining moisture), through a final particulate filter, and then to the Altech CEM for analysis. 


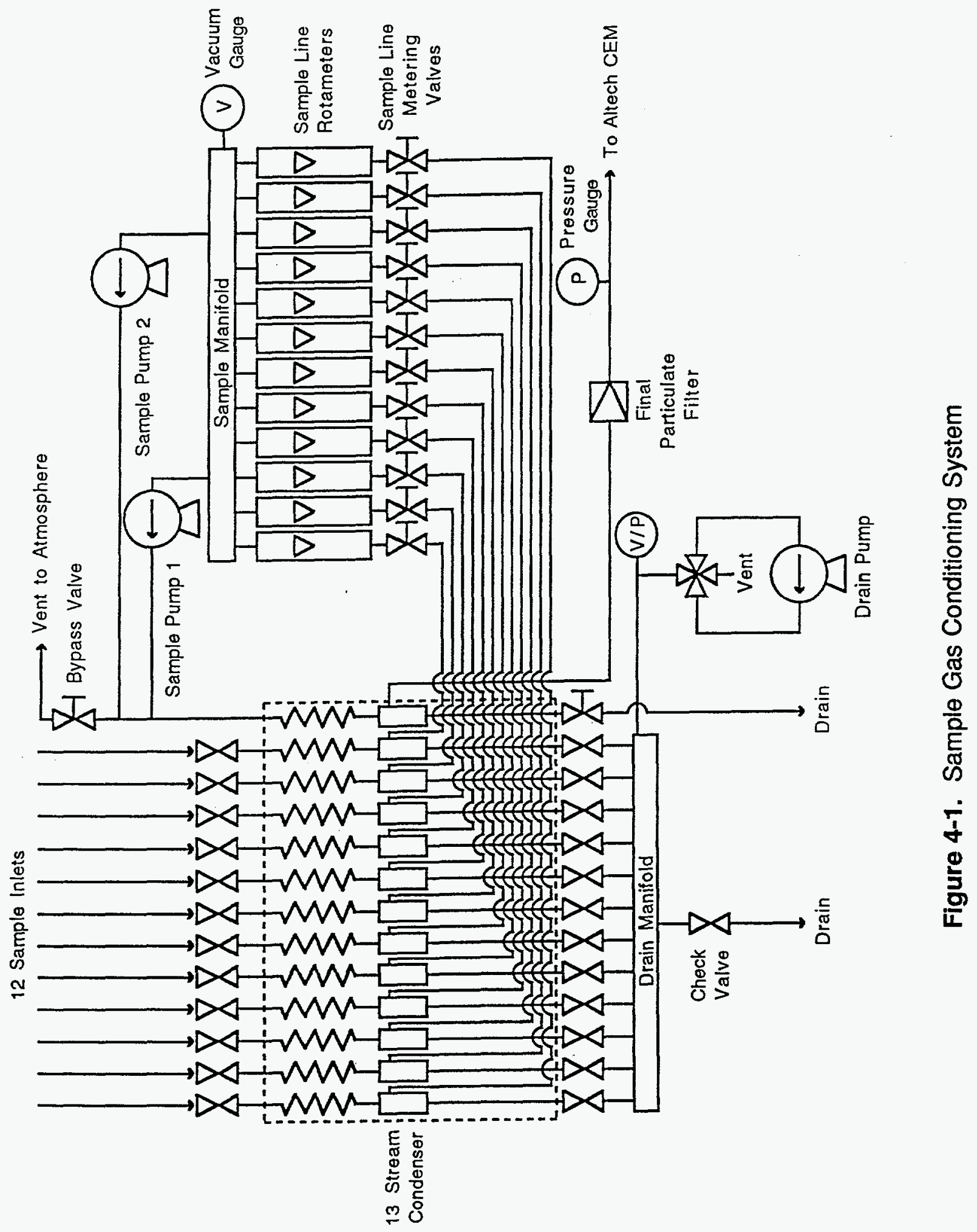


The locations of the unheated sample probes during the current phase of testing were identical to those used for the previous phases of the test program, namely: twelve at the exit of the economizer, six at the exit of the air heater, and one in the fabric filter outlet duct leading to the stack. Additional sample locations were provided for the individual fabric filter compartment outlet gas and the fabric filter inlet gas measurements that were performed manually.

The sample probe grid in the horizontal duct at the economizer exit is shown in Figure 4-2. Although this duct is 40 feet wide, it is only 7 feet deep, so an array of 12 probes positioned two high by six wide was deemed adequate to obtain a representative gas sample. The short probes were located at one-fourth of the duct depth, and the longer probes at threefourths of the duct depth. This spacing vertically divided the duct into equal areas. The use of two probe depths also provided the opportunity to ascertain any vertical stratification of gas species within the duct. Individual sample probes consisted of stainless steel tubing with sintered metal filters on the ends. The sample lines which transported the gas to the sample conditioning system consisted of polyethylene tubing which was heat traced and insulated to prevent freezing during the winter months.

Figure 4-2 also shows the location of the four PSCo $\mathrm{O}_{2}$ probes at the economizer exit which are used for boiler trim control. The PSCo equipment uses in situ probes that determine the $\mathrm{O}_{2}$ concentration on a wet basis. These probes (numbered $A, B, C$ and $D$ ) were located approximately three feet upstream of the Fossil Energy Research Corp. (FERCo) grid, and very near probe numbers 3, 5, 7 and 9 . Two additional sampling ports were available at the economizer exit which were used for limited $\mathrm{SO}_{3}$ measurements during the baseline burner and LNB/OFA tests.

The importance of the position of the 12-point grid relative to the four $\mathrm{PSCo}_{2}$ probes was realized during the baseline burner tests when it was found that the average $\mathrm{O}_{2}$ measured from the grid was nominally one percent higher than the average indicated in 


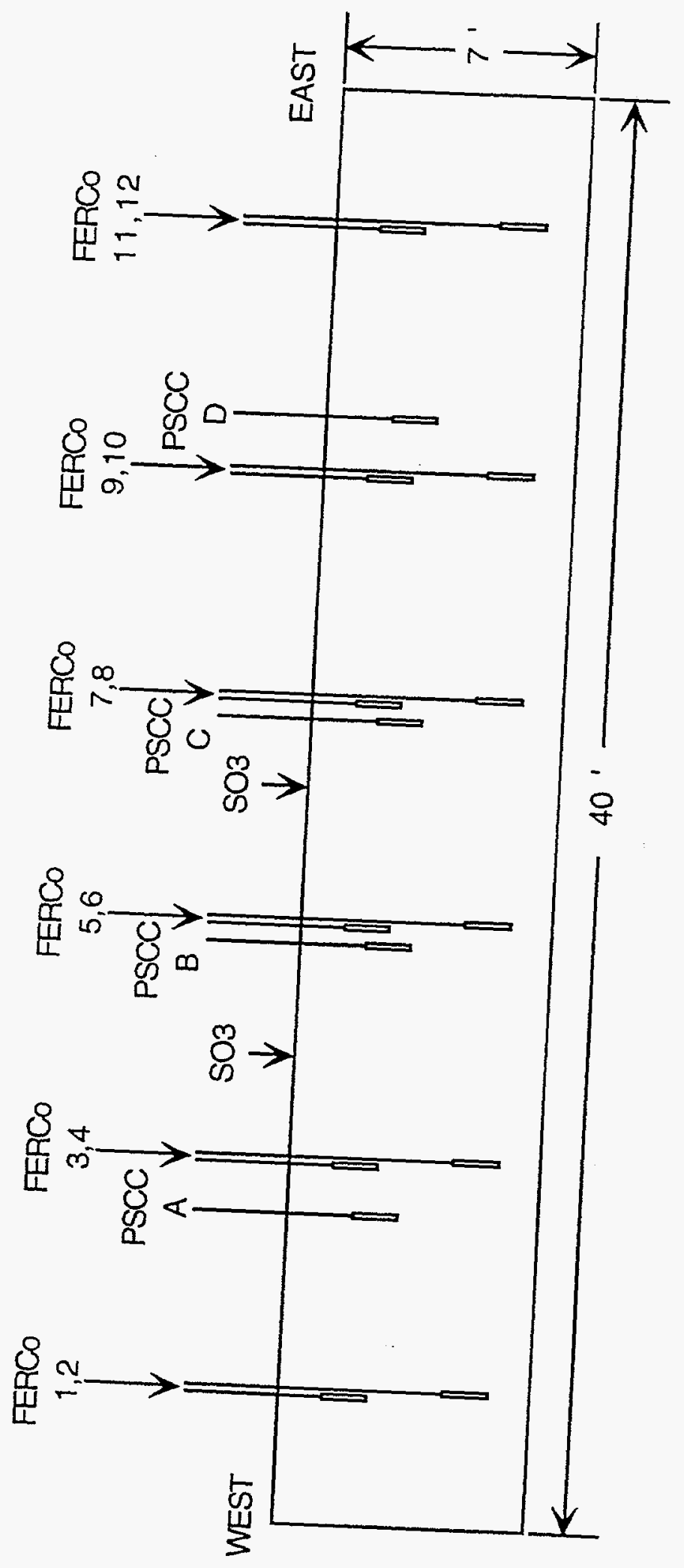

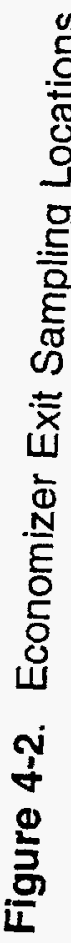


the control room. This difference was attributed to the inability of the four PSCo probes to detect the elevated $\mathrm{O}_{2}$ levels along the east and west sides of the duct which resulted from air in-leakage.

The economizer grid probes were not used to determine the $\mathrm{SO}_{2}$ emissions reductions from the sorbent injection or humidification processes; however, the grid was used to determine the actual boiler $\mathrm{O}_{2}$ levels and used in the calculations for total flue gas flow. This measurement point was also used for accurate determination of average boiler $\mathrm{NO}_{\mathrm{x}}$ emissions. Additional gas sample probes were installed at the air heater exit and the stack (fabric filter outlet duct) locations. The probes with unheated sample lines at the air heater exit were used during the tests with sodium bicarbonate injection at the air heater inlet, in order to provide an indication of the $\mathrm{SO}_{2}$ removal occurring upstream of the fabric filter. Only six probes were utilized at this test location. Figure 4-3 shows the location of the probes at the air heater exit. These sample probes and tubing were similar to the installation at the economizer exit. The staggered probes were installed at one-fourth and three-fourths duct depths, similar to the economizer exit. The figure also shows the location of the heated probe for the CEM system at the air heater exit. This probe was not in the same plane as the six-point grid, but approximately 3 feet upstream. At the stack sampling location, the heated probe for the CEM system was approximately 20 feet upstream of the unheated probe installed during the baseline burner tests. Only a single probe was used for both the CEM and the unheated probe locations since both were downstream of the fabric filter and induced draft fans where little stratification of the flue gas stream was expected. Figure 4-4 shows the installation of the heated CEM probe in the fabric filter outlet duct.

Along with the gas sample locations for the Altech and the FERCo systems, additional gas measurements were obtained from the individual fabric filter compartments. A separate fabric filter gas sample stream was added to the FERCo sample system and subsequently analyzed with the Altech CEM. Since accurate $\mathrm{SO}_{2}$ emissions would be required from the 


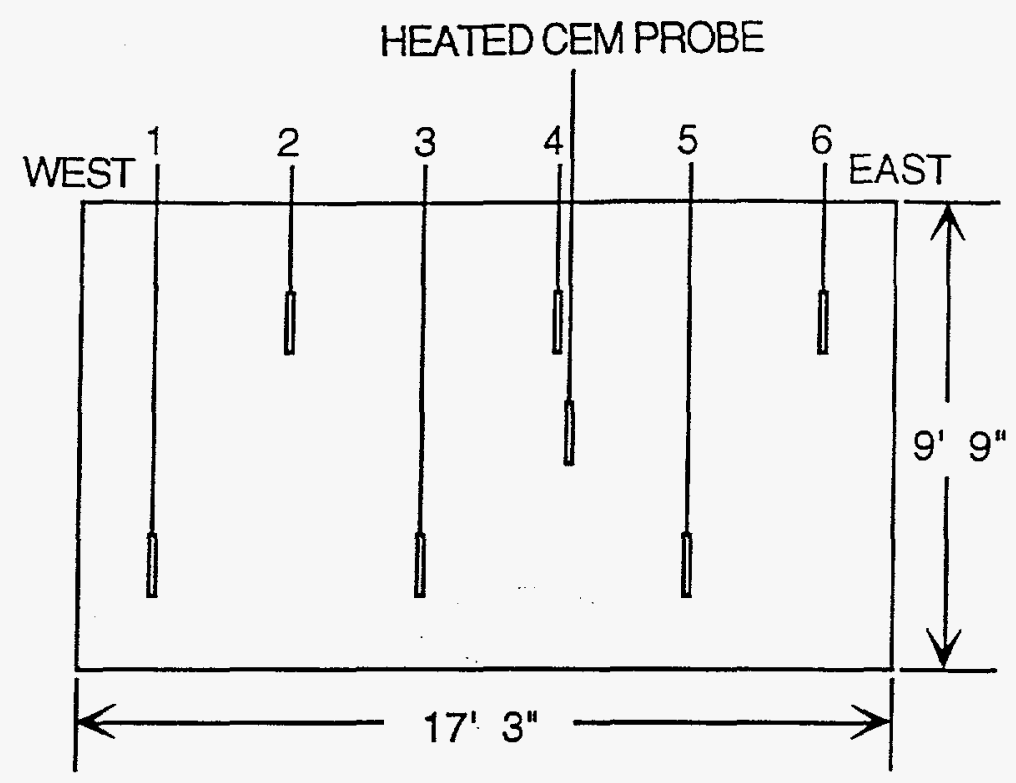

Figure 4-3. Air Heater Exit Sampling Locations

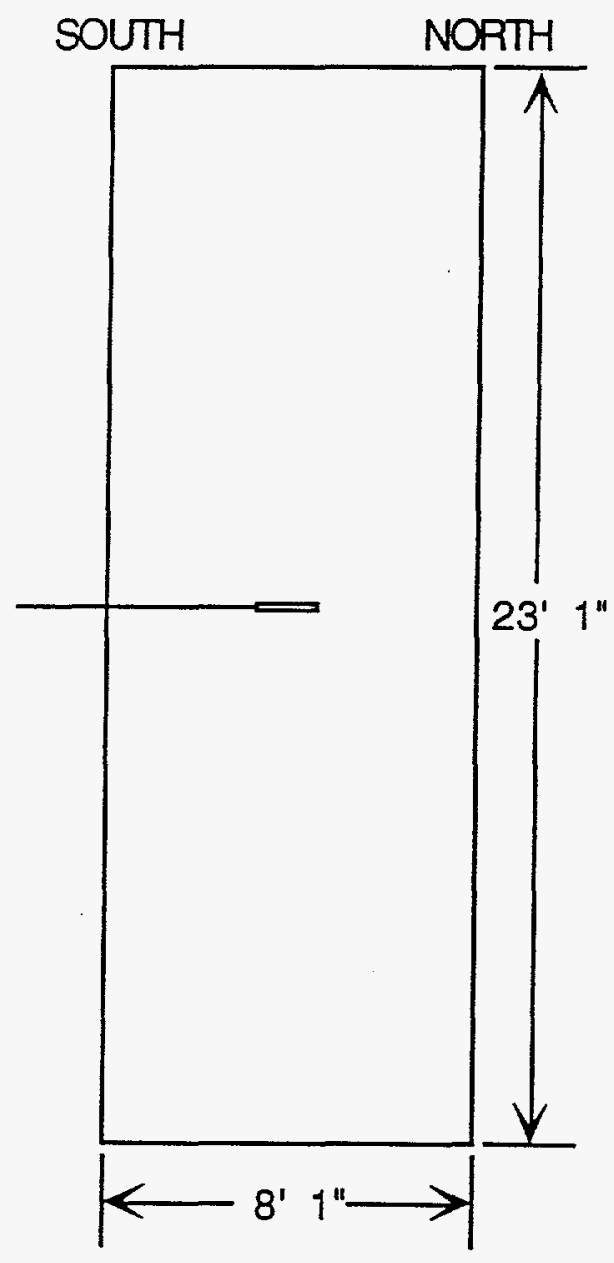

Figure 4-4. Fabric Filter Outlet Duct Sampling Location 
fabric filter compartment samples, a non-bubbling condenser and water dropout were added to the sample line just outside of the compartment sample location. Initially, existing pressure taps installed for monitoring compartment pressure drop were used to obtain a compartment gas sample from the top of the tube sheet on the clean gas side. During a boiler outage, a Teflon line was installed in the top of each compartment that was used to pull a sample from the center of the compartment clean gas outlet opening. A fitting was installed on the door of each compartment to access this compartment gas sample. The compartment gas samples were acquired manually and required that the sample line and water dropout be moved and reconnected for each compartment during this measurement. This data was utilized to analyze the $\mathrm{SO}_{2}$ removals and indirectly determine the sorbent distribution on a compartment-by-compartment basis. A comparison between the CEM stack sampling location and the average of the compartment samples showed very good agreement, and indicated that the compartment gas sampling technique was valid.

\subsection{Approach To Saturation}

The measurement of flue gas temperature and approach to saturation is a key variable in characterizing the humidification and $\mathrm{SO}_{2}$ removal process with calcium- or sodium-based sorbents. The use of a thermocouple grid should have permitted an accurate gas temperature measurement, given sufficient residence time for evaporation and an even distribution of water, and uniform flue gas and flow temperature. However, problems with partially wet thermocouples resulted in low gas temperature indications that affected the evaluation of the actual operating conditions. Correctly evaluating the actual flue gas and approach temperatures was considered a high priority item for evaluating the test results. While modifications to the measurement system improved performance, the grid could not accurately indicate the dry bulb temperatures with high humidification rates.

Several means of verifying the actual flue gas temperature and the amount of humidification were used: 1) monitoring the steady state baghouse outlet temperature, 2) measuring the flue gas wet bulb temperatures, and 3) adiabatic energy calculations of the 
humidification process. All of these verifications indicated that the equilibrium fabric filter exit temperatures were higher than the average measurement by the thermocouples at the inlet grid during steady state tests at high humidification rates (Shiomoto, et al., 1994). Four additional thermocouples were installed in the ID fan inlet ducts to provide a better means of monitoring fabric filter outlet temperature. While the fabric filter outlet temperature would be sufficient for steady state tests, it is not adequate for load following or short term tests and cannot be used to adjust or control the humidification process.

Psychometric calculations were performed to model the humidification process and verify the water flow rate and the average fabric filter inlet grid temperature measurements. These are described in the report documenting the testing with calcium-based sorbents (Shiomoto, et al., 1994). During the test program, the psychometric calculations were relied upon to determine the humidification system operation point and to determine the flue gas approach temperature. Errors from the fabric filter inlet grid were unavoidable at high water flow rates, but the set point temperatures could be biased to provide the desired test conditions while maintaining automatic controls for the water injection. In this report, the calculated approach temperatures were utilized for determining the humidification process operation and for all data interpretation. However, the humidification data summary in the appendix includes the calculated dry bulb temperature as well as the measured values throughout the system. 


\subsection{RESULTS}

This section presents the results of the short-term parametric sorbent injection tests. In presenting these results, the chemical and physical properties are presented first (Subsection 5.1). This is followed by a discussion of the results with sodium sesquicarbonate at both the FFDC inlet and air heater inlet (Subsection 5.2) and a similar discussion of the sodium bicarbonate results (Subsection 5.3). Subsection 5.4 presents the results of the solids analyses performed on fly ash/sorbent samples collected from the FFDC hoppers during injection tests with both sorbents. Finally, Subsection 5.5 presents the results of two parametric tests with sodium sesquicarbonate which were performed during an alternate coal test burn on Arapahoe Unit 4 in November, 1995. A detailed data summary for the short-term parametric tests is contained in Appendix B.

\subsection{Sorbent Characteristics}

The sodium sesquicarbonate used during the test program was obtained from Solvay Minerals, Inc., Green River, WY. The sodium bicarbonate was obtained from NaTec Resources, Inc., Houston, TX (solution-mined in Western Colorado). The chemical composition and physical characteristics of the two materials are shown in Table 5-1.

Table 5-1

Sorbent Characteristics

\begin{tabular}{||l|l|l||}
\hline Material & Sodium Sesquicarbonate & Sodium Bicarbonate \\
\hline Chemical Formula & $\mathrm{NaHCO}_{3} \cdot \mathrm{Na}_{2} \mathrm{CO}_{3} \cdot 2 \mathrm{H}_{2} \mathrm{O}$ & $\mathrm{NaHCO}_{3}$ \\
\hline Supplier & Solvay Minerals, Inc. & NaTec Resources, Inc. \\
\hline Composition: & & \\
$\mathrm{Na}_{2} \mathrm{CO}_{3}$ & $45.8 \%$ & - \\
$\mathrm{NaHCO}_{3}$ & $36.3 \%$ & $99.5 \%$ \\
\hline Percent Na by Weight & $29.8 \%$ & $27.2 \%$ \\
\hline Bulk Density & $49 \mathrm{lb} / \mathrm{ft}^{3}$ & $64 \mathrm{lb} / \mathrm{ft}^{3}$ \\
\hline
\end{tabular}


Samples of the raw (unpulverized) and pulverized materials were submitted to Leeds \& Northrup Co. for particle size analysis (Microtrac). The mass mean diameter (MMD) particle size for the raw and pulverized sodium sesquicarbonate samples were 27.8, 17.0, and 15 microns, respectively. The particle size distributions for the three samples are shown in Figure 5-1. The pulverized samples were collected when the mill was operating at speeds of 4000 and $5000 \mathrm{rpm}$. It should be noted that the particle sizes are determined after the sample is ultrasonically dispersed in a liquid medium. Thus, there may be some differences between the measurements and the actual particle size from the pulverizer.

Pulverized sodium bicarbonate samples were analyzed for mill speeds of both 4000 and $5000 \mathrm{rpm}$. The results (Figure 5-2) showed that the MMD's for the raw, $4000 \mathrm{rpm}$, and $5000 \mathrm{rpm}$ samples were $61.5,24.3$, and 18.8 microns, respectively. The data indicate that the reduced pulverized speed resulted in a slightly larger particle size distribution.

In addition to pulverizer speed, the particle size is dependent on the mass flowrate through the mill. Early in the test program, there was a concern that the grinding efficiency of one of the mills was degraded at higher sorbent feed rates. Table 5-2 shows the MMD's measured for samples collected at four different sodium sesquicarbonate feed rates while operating the mill at $4000 \mathrm{rpm}$. The results indicate that at a pulverizer speed of $4000 \mathrm{rpm}$, particle size was not adversely affected over the range of sorbent feed rates investigated.

Table 5-2

\section{Effect of Sodium Sesquicarbonate Feed Rate on Pulverizer Performance}

(Pulverizer Speed: 4000 rpm)

\begin{tabular}{|c|c|c||}
\hline $\begin{array}{c}\text { Feed Rate } \\
\text { (lb/min) }\end{array}$ & $\begin{array}{c}\text { 2Na/S Ratio } \\
\text { (nom. @ full load) }\end{array}$ & $\begin{array}{c}\text { MMD } \\
\text { (microns) }\end{array}$ \\
\hline 16.1 & 0.43 & 15.1 \\
25.5 & 0.65 & 19.6 \\
49.0 & 1.26 & 19.8 \\
58.4 & 1.91 & 17.0 \\
\hline
\end{tabular}




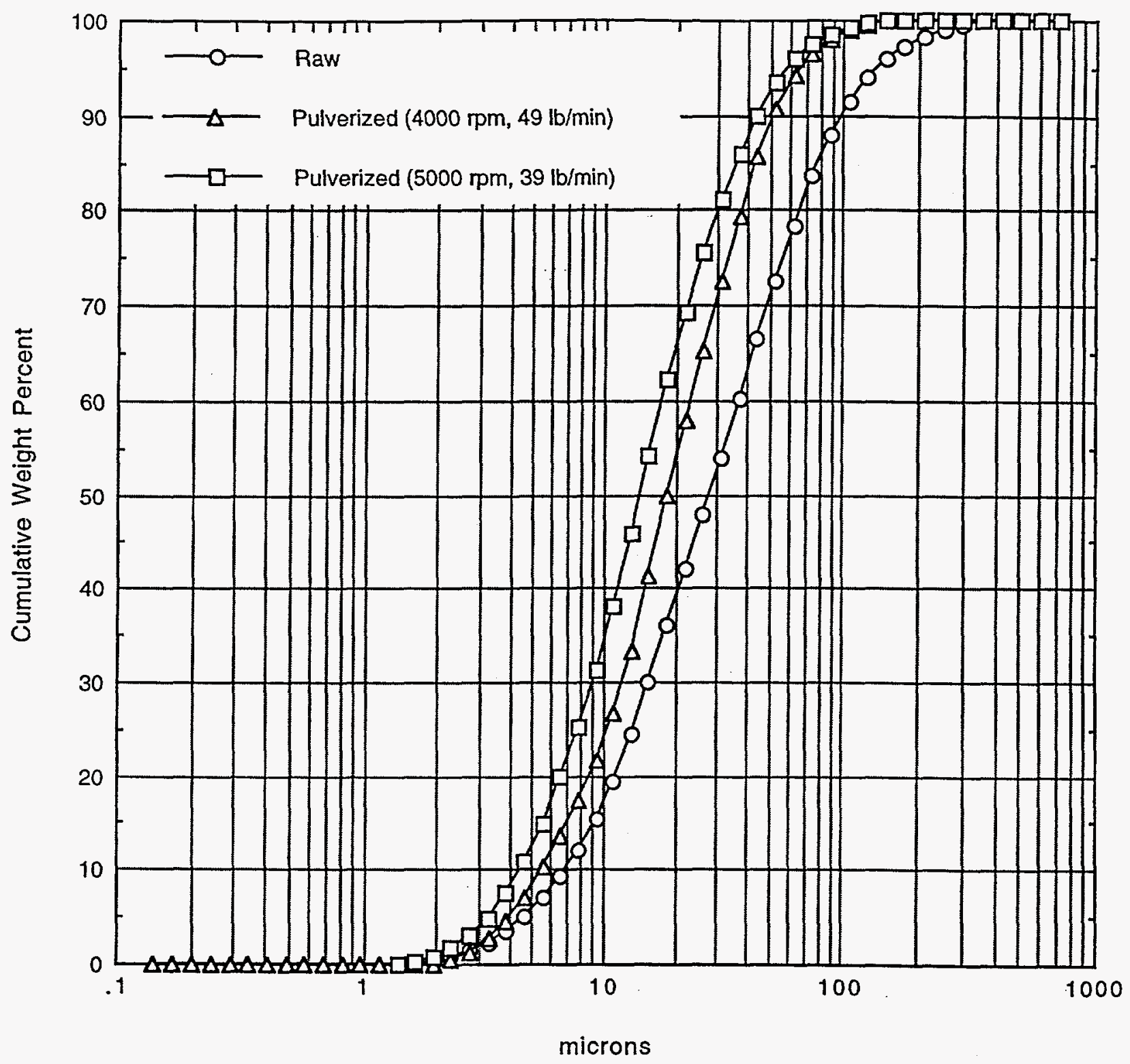

Figure 5-1. Sodium Sesquicarbonate Particle Size Distribution for Raw and Pulverized Samples 


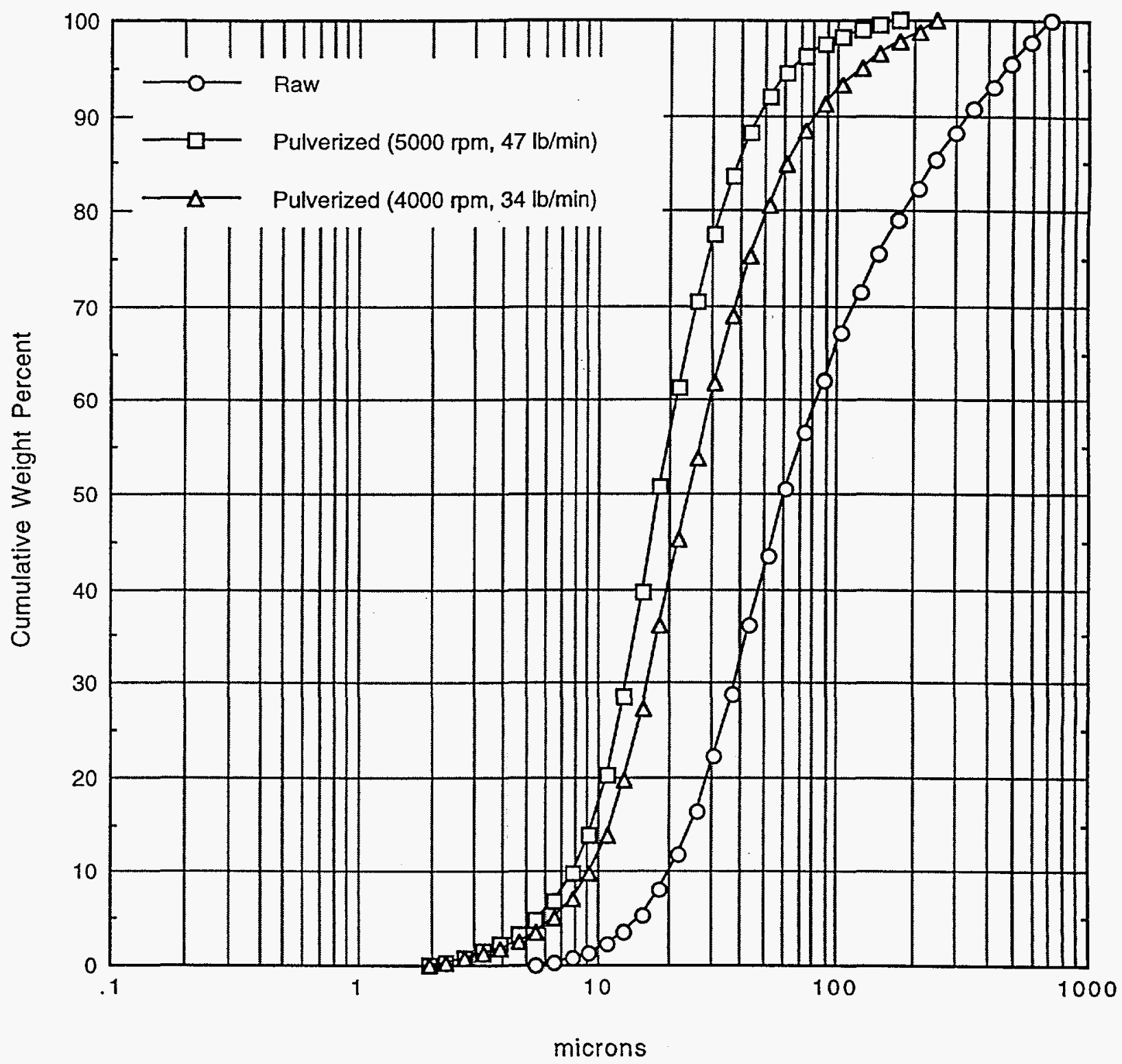

Figure 5-2. Sodium Bicarbonate Particle Size Distribution for Raw and Pulverized Samples 


\subsection{Sodium Sesquicarbonate}

The sodium sesquicarbonate tests were performed at two different injection locations. A majority of the tests were run with sorbent injection ahead of the FFDC, and without humidification. Although little effect was expected, a few tests were also run with humidification, since the equipment was already in place from the previous phase of testing with calcium-based sorbents (Shiomoto, et al., 1994). After the testing with sodium sesquicarbonate was complete, tests began with sodium bicarbonate at the same location. These tests showed that the flue gas temperatures at the FFDC inlet were too low for good $\mathrm{SO}_{2}$ removal performance with this sorbent. The injection location was then moved to a hotter region at the air heater inlet. After completion of the sodium bicarbonate tests at the new injection location, a short series of tests were also run with sodium sesquicarbonate in order to assess the effect of the higher injection temperature with this sorbent. The results of the sodium sesquicarbonate tests at the two injection locations are discussed separately in the following subsections.

\subsubsection{Injection of Sodium Sesquicarbonate at the FFDC Inlet}

A. $\mathrm{SO}_{2}$ Removal. The tests with sodium sesquicarbonate injection ahead of the FFDC showed the $\mathrm{SO}_{2}$ removal process to be very well-behaved with good day-to-day repeatability. Figure 5-3 shows the $\mathrm{SO}_{2}$ removal measured at the exit of the FFDC over a seven-hour time period, where a single injection condition was maintained throughout the duration of the test. The nominal $2 \mathrm{Na} / \mathrm{S}$ ratio for this particular test was 1.5 , and the boiler load was 107 MWe. Previous tests at PSCo's Cameo Station (Muzio, et al., 1984) and at City of Colorado Springs' Nixon Station (Fuchs, et al., 1989), have shown that the reactions between the sodium sesquicarbonate and $\mathrm{SO}_{2}$ are relatively rapid. Even during a fabric filter cleaning cycle there is only a temporary drop in $\mathrm{SO}_{2}$ removal. Therefore, an effort was made during the current series of tests to measure the $\mathrm{SO}_{2}$ removal immediately before and after each FFDC cleaning cycle in order to fully characterize this behavior. The $\mathrm{SO}_{2}$ removals measured "before cleaning" are shown in Figure 5-3 as dark symbols. In general, the results with sodium sesquicarbonate showed that once injection had begun, 


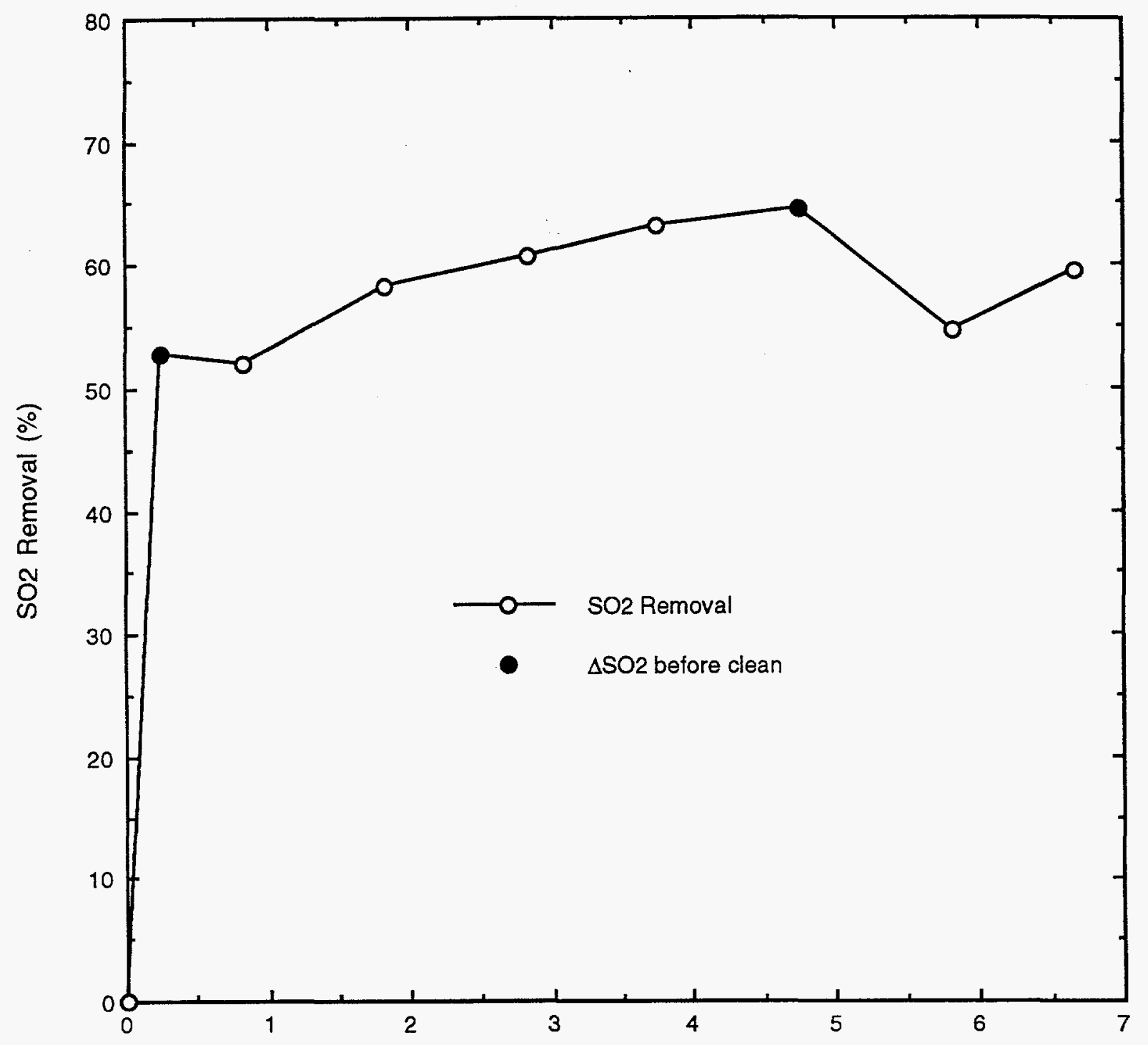

Time from Start of Test (hrs)

Figure 5-3. $\mathrm{SO}_{2}$ Removal Versus Time for Sodium Sesquicarbonate Injection Ahead of the FFDC (Test 818) 
$\mathrm{SO}_{2}$ removals increased rapidly and leveled-out in a relatively short amount of time. For the test shown in Figure 5-3, a cleaning cycle began only 15 minutes after sorbent injection was initiated. In that short amount of time, the $\mathrm{SO}_{2}$ removal had already reached 53 percent. There was no appreciable decrease in removal after this first cleaning cycle, and then a slow increase up to nominally 65 percent removal before the second cleaning. The decrease in $\mathrm{SO}_{2}$ removal at the six-hour mark (nominally 10 percent) is typical of the response that was seen after each cleaning cycle with sodium sesquicarbonate injection at the FFDC inlet.

The steady-state $\mathrm{SO}_{2}$ removal and utilization results of many tests like the one shown in Figure 5-3 are presented as a function of sorbent injection rate (expressed in terms of $2 \mathrm{Na} / \mathrm{S}$ ratio) in Figures 5-4a and 5-4b. Variations in boiler load were expected to have little effect on $\mathrm{SO}_{2}$ removal, and the data confirm this expectation. At nominal $2 \mathrm{Na} / \mathrm{S}$ ratios of 1.0 and $2.0, \mathrm{SO}_{2}$ removals range from 44 to 56 percent and 64 to 78 percent, respectively. Alternatively, the $2 \mathrm{Na} / \mathrm{S}$ ratios required to achieve the target $\mathrm{SO}_{2}$ removal of 70 percent ranged from 1.6 to 2.2 .

Along with boiler load, flue gas temperature was expected to have little effect on $\mathrm{SO}_{2}$ removal for sesquicarbonate injection ahead of the FFDC. As will be discussed in the presentation of the sodium bicarbonate results, the FFDC outlet temperature at Arapahoe Unit 4 routinely varies from 230 to $280^{\circ} \mathrm{F}$ depending on load, time of day, and ambient temperature. There was no effect seen over this temperature range during the current series of sesquicarbonate tests.

The $\mathrm{SO}_{2}$ removals shown in Figure 5-4a for $2 \mathrm{Na} / \mathrm{S}$ ratios up to 1.0 are comparable to those reported in the previous studies performed at Cameo and Nixon. As the $2 \mathrm{Na} / \mathrm{S}$ ratio is increased further, the results from the current study begin to fall below those of the earlier work. Figure 5-5 shows a comparison of the two sets of results. It was thought that the difference may have been due to a deterioration of the grinding performance of the 


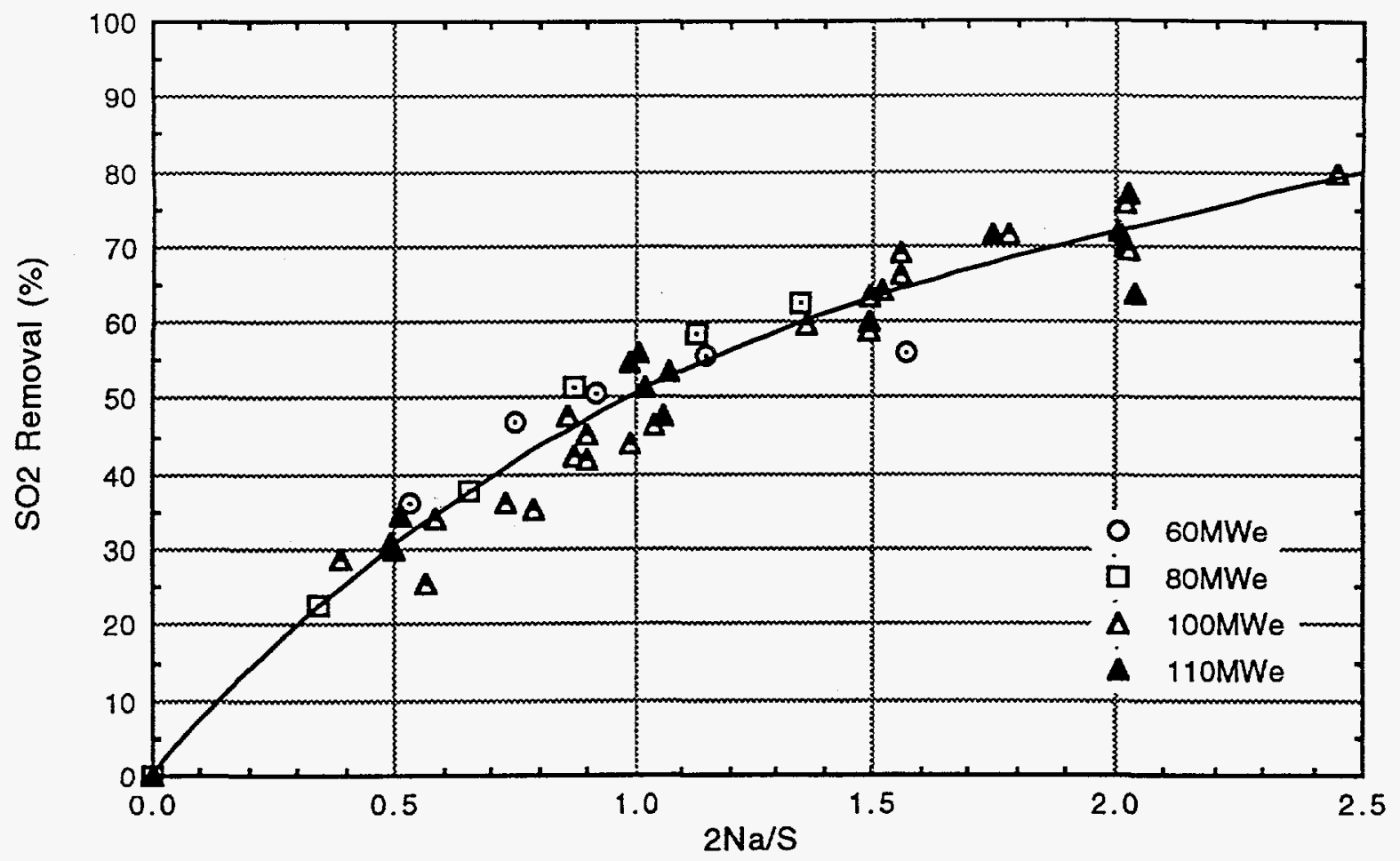

Figure 5-4a. $\mathrm{SO}_{2}$ Removal as a Function of $2 \mathrm{Na} / \mathrm{S}$ Ratio for Sodium Sesquicarbonate Injection Ahead of the FFDC

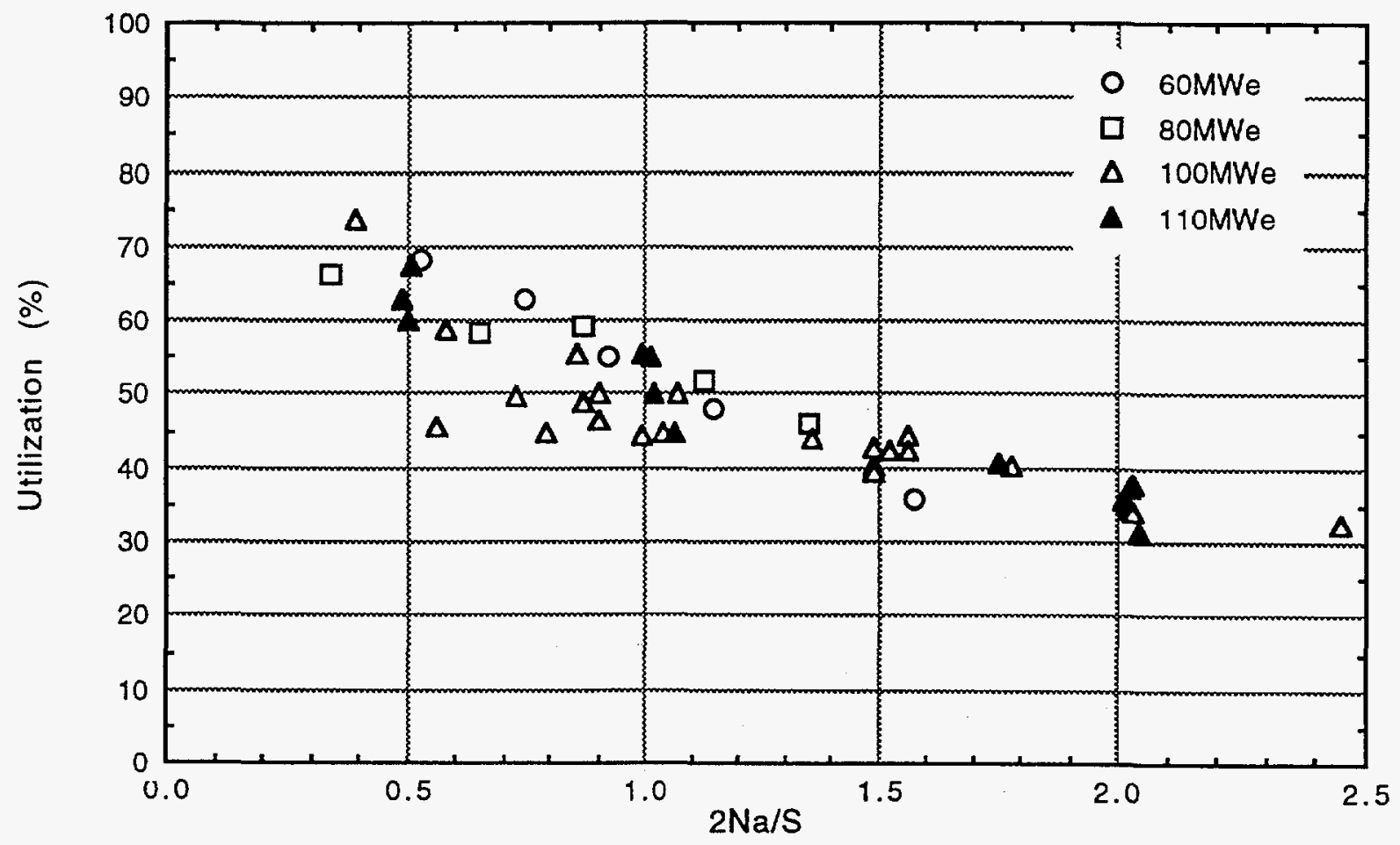

Figure 5-4b. Utilization as a Function of $2 \mathrm{Na} / \mathrm{S}$ Ratio for Sodium Sesquicarbonate Injection Ahead of the FFDC 


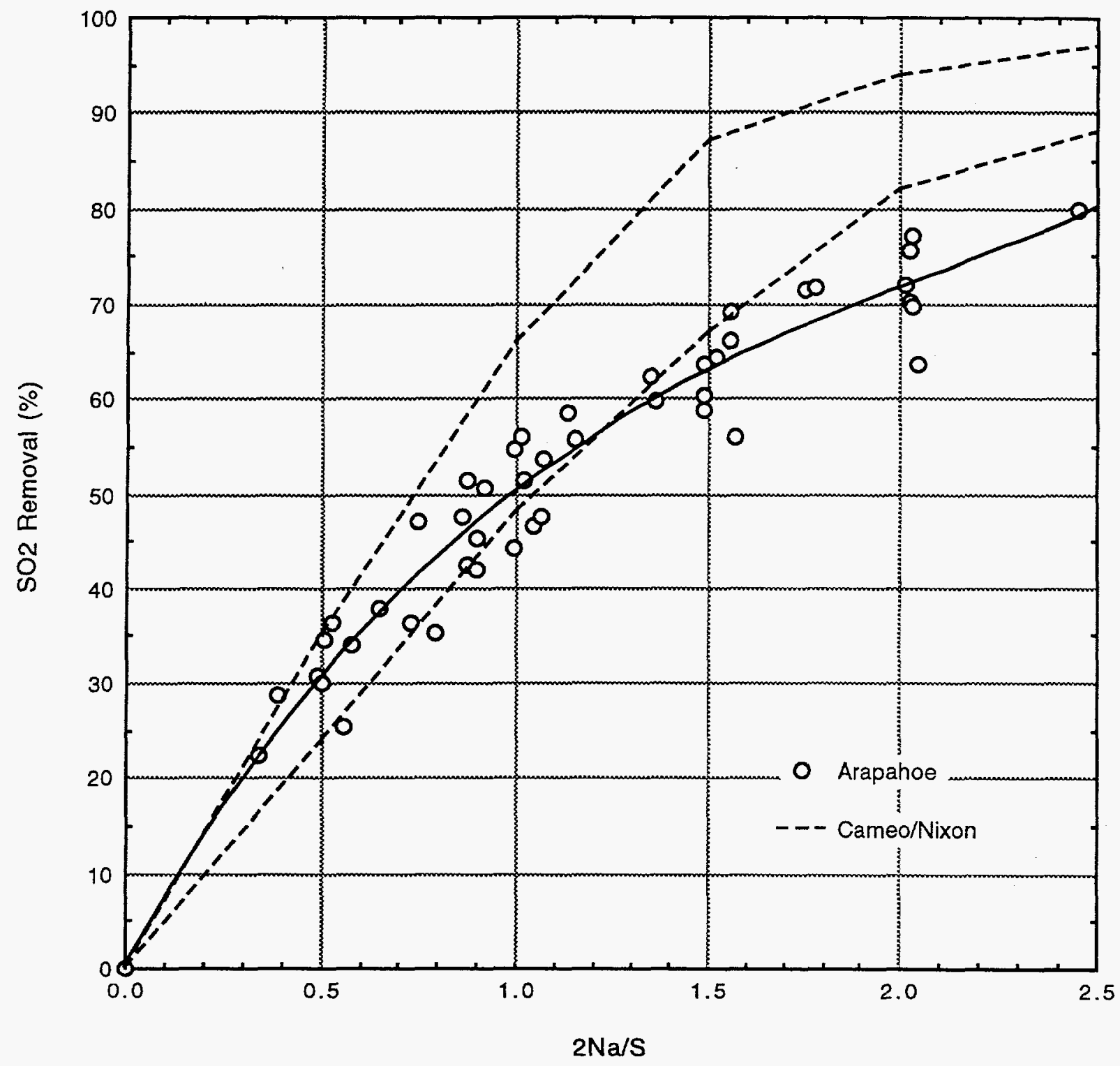

Figure 5-5. Comparison of $\mathrm{SO}_{2}$ Removals for Sodium Sesquicarbonate Injection Ahead of the Arapahoe Unit 4 FFDC to Previous Full-Scale Demonstrations (Cameo and Nixon data from Muzio, et al., 1984 and Fuchs, et al., 1989) 
Arapahoe DSI pulverizers at the higher sorbent feed rates; however, the test results shown in Table 5-2 do not support this hypothesis.

Further, tests were conducted using either one or both of the sorbent feed systems to achieve a given $2 \mathrm{Na} / \mathrm{S}$ ratio. If particle size degraded with pulverizer throughput rate, then a test using both systems to achieve a given $2 \mathrm{Na} / \mathrm{S}$ ratio should produce smaller sorbent particles and higher $\mathrm{SO}_{2}$ removal. With two sorbent preparation systems, each mill would only have one half of the throughput. Also, the distribution within the duct should improve as the material would be injected through twelve pipes instead of six. Figure 5-6 shows the $\mathrm{SO}_{2}$ removals obtained when one and two of the sorbent feed systems were used. As can be seen, the results in Figure 5-6 do not show any clear difference between using one or two sorbent preparation systems.

The data in Figures 5-4a and 5-4b show that $\mathrm{SO}_{2}$ removals and utilizations are consistent over time, with the 60, 80 and 100 MWe collected in September 1993 being comparable to the $110 \mathrm{MWe}$ data which was collected nearly eight months later in May 1994. The latter set of data was collected during the Integrated Systems (LNB/OFA/SNCR/sodiumbased DSI) phase of tests which immediately followed the phase of testing described in this report. Since the results in Figures $5-4 a$ and $5-4 b$ are repeatable, it is not believed that the differences between the Cameo/Nixon and Arapahoe results at higher feed rates are due to process variability at the current installation.

One variable which was expected to have an effect on process performance was sorbent particle size. Figure 5-7 shows the effect of pulverizer speed on $\mathrm{SO}_{2}$ removal for sesquicarbonate injection ahead of the FFDC. As discussed in Section 3, the sorbent pulverizers were installed with operating speeds of $5700 \mathrm{rpm}$. Before testing began, however, it was discovered that this speed was very near a critical frequency. Therefore, a decision was made to reduce the speed to $5000 \mathrm{rpm}$. Due to the 3-week lead time for the new drive sheaves, the pulverizers were run for a short time at $4000 \mathrm{rpm}$ (these sheaves were readily available). The data in Figure 5-7 show that the higher pulverizer 


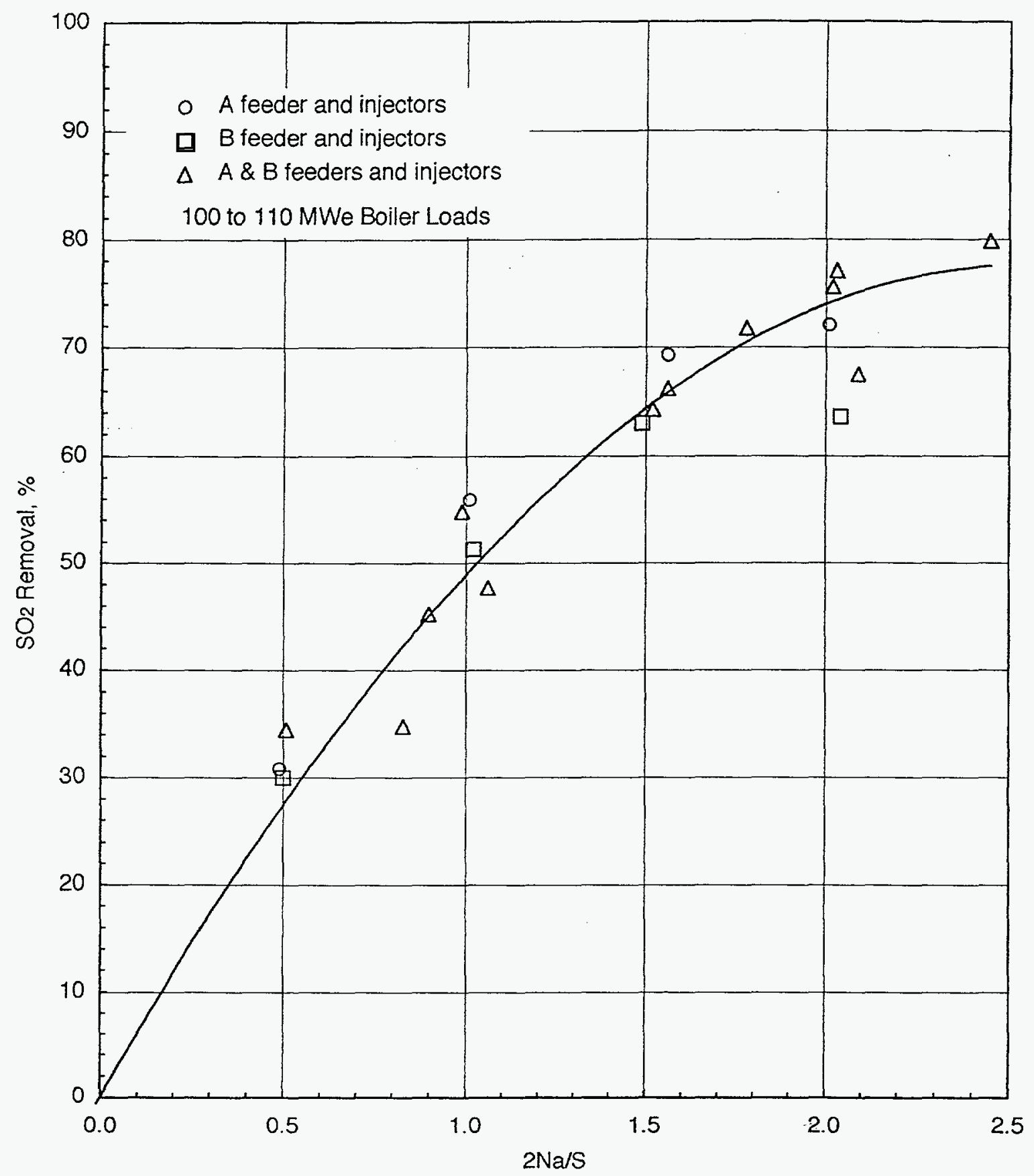

Figure 5-6. Effect of Using One and Two Sorbent Preparation Systems on $\mathrm{SO}_{2}$ Removal (Sodium Sesquicarbonate Injection Ahead of the Fabric Filter) 


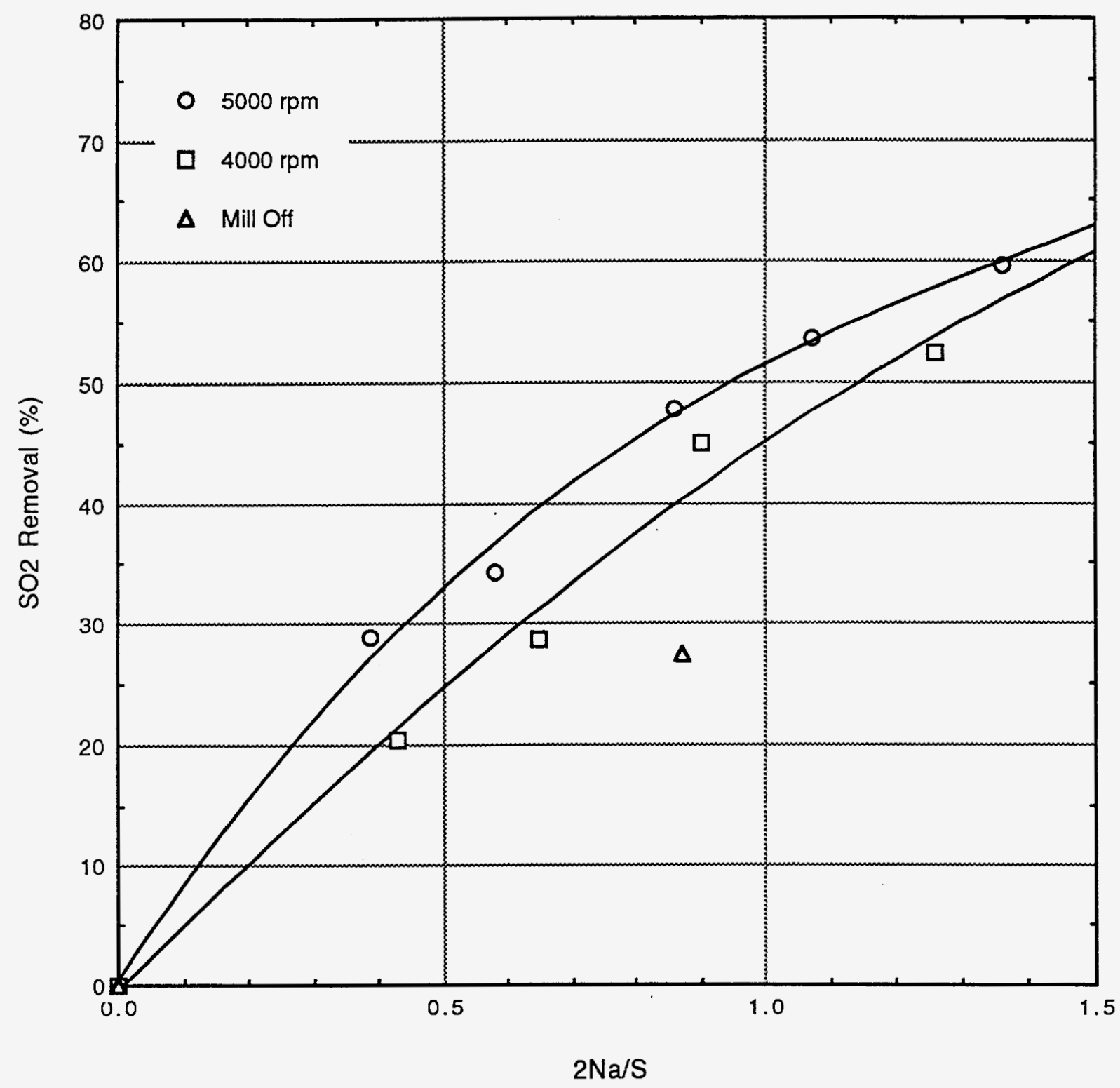

Figure 5-7. Effect of Pulverizer Speed on $\mathrm{SO}_{2}$ Removal for Sodium Sesquicarbonate Injection Ahead of the FFDC 
speed results in approximately a net seven percent increase in $\mathrm{SO}_{2}$ removal at a nominal $2 \mathrm{Na} / \mathrm{S}$ ratio of 1.0. The "mill off" point is a single test run at the end of a test day when the speed was $5000 \mathrm{rpm}$. After the final "mill on" test was finished, the mill was turned off while the sorbent feed continued. A data point was taken after the mill had stopped rotating (which can take 10 to 15 minutes due to the weight of the grinding disk). Recall that at $4000 \mathrm{rpm}$, the mill reduces the particle MMD from nominally 28 to 17 microns (Figure 5-1). The data in Figure 5-6 show that this size reduction results in an increase in $\mathrm{SO}_{2}$ removal from approximately 27 to 48 percent at a nominal $2 \mathrm{Na} / \mathrm{S}$ ratio of 0.9 .

During the final test at $4000 \mathrm{rmm}$, gaseous emission measurements were made at the exit of each FFDC compartment in an effort to characterize the distribution of sorbent in the fabric filter. Figure 5-8 shows the results of the compartment-by-compartment measurements. The results indicate that the highest levels of $\mathrm{SO}_{2}$ removal (45 to 58 percent) occur in the second and third compartments on each side of the baghouse, indicating that the majority of the sorbent is deposited in these areas. The peak on each side is followed by a rapid decrease down to 10 to 15 percent removal in the rear compartments. These results also show that, with the exception of the final two compartments, removals in the east compartments are nominally 10 to 15 percent higher than the removals in the corresponding west-side compartments. This difference is likely due to a bias in the injection system, which resulted from partial plugging of some of the east-side injectors. Also, note in Figure 5-8 that the average of the compartment-bycompartment $\mathrm{SO}_{2}$ removals is in good agreement with the overall $\mathrm{SO}_{2}$ removal across the FFDC (35.1 percent compared to 35.5 percent). This suggests that the gas flow rates through each compartment are relatively equal.

Another possible explanation for the differences between the current $\mathrm{SO}_{2}$ removal results at Arapahoe and the prior demonstrations at Cameo and Nixon (Muzio, et al., 1984, and Fuchs, et al., 1989) is the compartment-by-compartment variations in $\mathrm{SO}_{2}$ removal shown in Figure 5-8. However, reviewing the compartment-by-compartment $\mathrm{SO}_{2}$ removals 

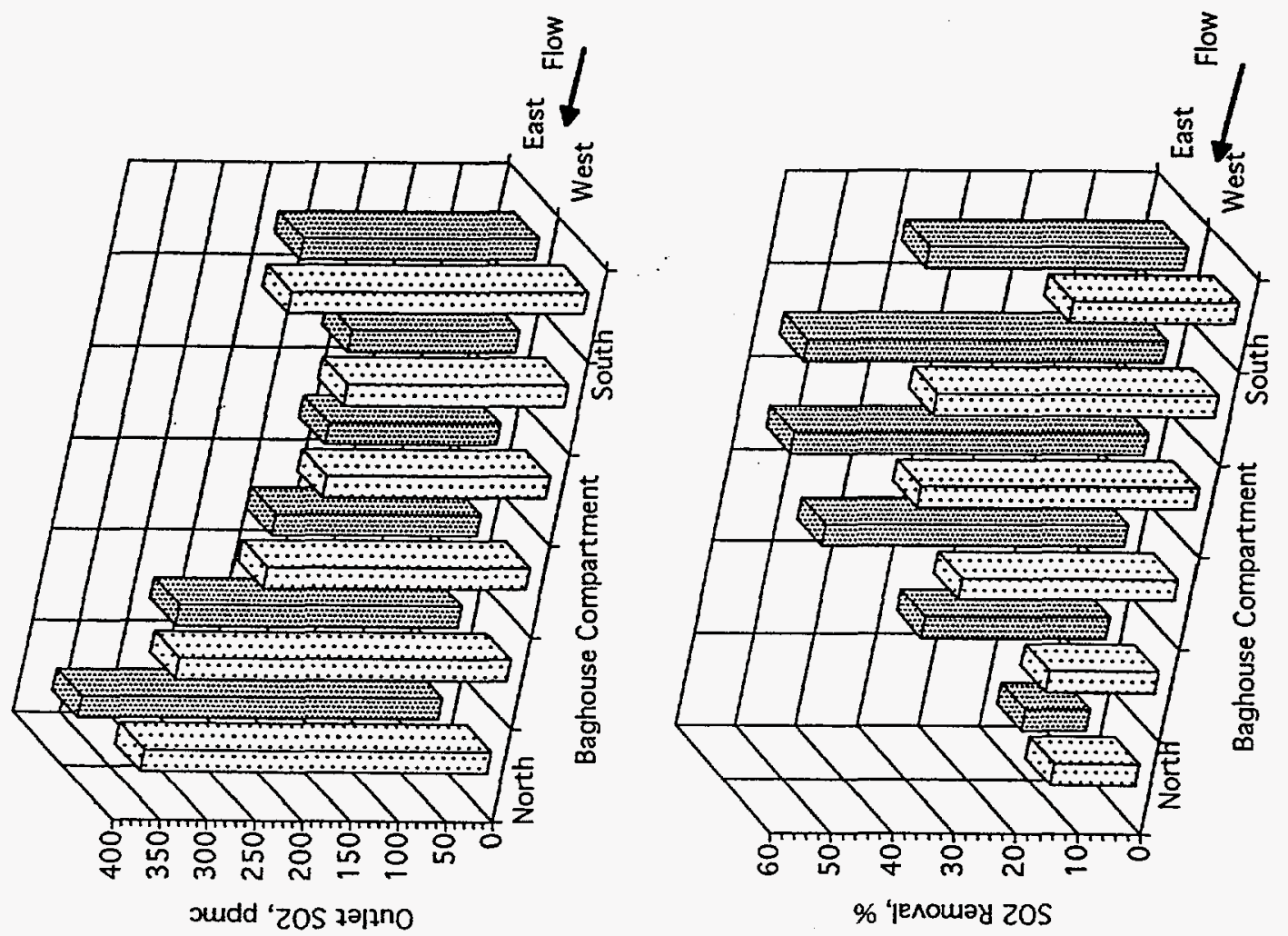

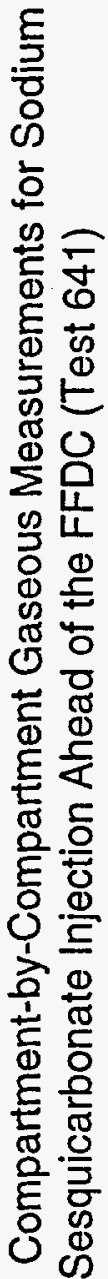

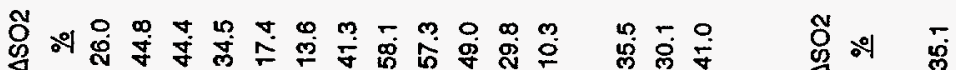
范

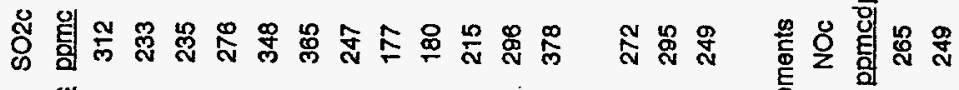

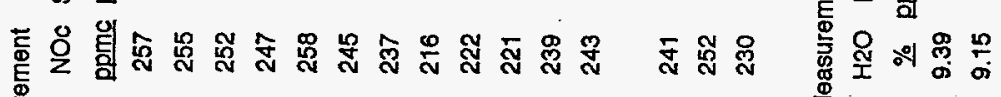

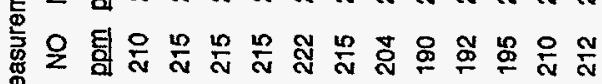

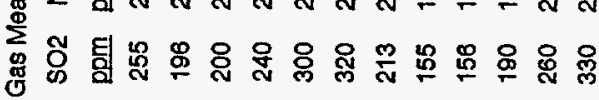

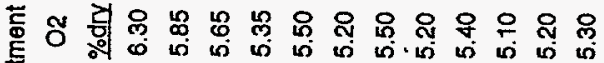
蛋 彥

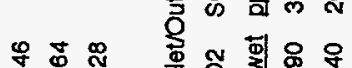

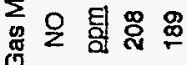
害䛯 官 일 
measured during the Cameo demonstration (Muzio, et al., 1984) show a higher degree of variability than seen in Figure 5-8. This suggests that compartment by compartment sorbent distribution does not explain the differences in the current Arapahoe results and prior Cameo/Nixon results.

B. $\mathrm{NO}_{x}$ Removals and $\mathrm{NO}_{2}$ Emissions. The previous work at Cameo and Nixon (Muzio, et al., 1984, Fuchs, et al., 1989) showed that in addition to the removal of $\mathrm{SO}_{2}$, sodiumbased sorbents also remove a small amount of $\mathrm{NO}_{\mathrm{x}}$ as well as oxidize a portion of the $\mathrm{NO}$ to $\mathrm{NO}_{2}$. During the current test program, $\mathrm{NO}_{\mathbf{x}}$ removals and $\mathrm{NO}_{2}$ emissions were characterized with both sorbents. Before presenting the sodium sesquicarbonate results in terms of the overall $\mathrm{NO}_{\mathrm{x}}$ removals and $\mathrm{NO}_{2}$ emissions measured as a function of the sorbent injection rate, it is of interest to first look at some of the time-resolved data from a typical test.

Figure 5-9a shows both the $\mathrm{NO}_{x}$ removal and $\mathrm{NO}_{2}$ emission traces recorded during the 7-hour test with sodium sesquicarbonate injection ahead of the FFDC shown in Figure 5-3. The $\mathrm{NO}_{2}$ trace shows an interesting trend that was not reported previously, where $\mathrm{NO}_{2}$ emissions increase sharply after each cleaning cycle. After the initial increase, there is a slow decrease in $\mathrm{NO}_{2}$ emissions until the second cleaning cycle begins. This behavior was also seen during the long-term sodium bicarbonate injection tests, and will be discussed in more detail during the presentation of those results. It is currently thought that this behavior is due to an interaction between $\mathrm{NO}_{2}$ and the fly ash on the bags. The peak $\mathrm{NO}_{2}$ level achieved during the test shown in Figure 5-9a was 12 ppm. However, data points immediately after each cleaning indicate an increasing trend, and it is possible that the peak level would have been higher if the test had been run for a longer time period. The $\mathrm{NO}_{x}$ removals shown in Figure 5-9a also indicate an increasing trend with time, but unlike the $\mathrm{NO}_{2}$ emissions, there does not appear to be an effect of FFDC cleaning cycle. The range of 10 to 15 percent $\mathrm{NO}_{\mathrm{x}}$ removal shown in Figure $5-9 \mathrm{a}$ is consistent with the levels observed during the previous Cameo and Nixon studies. 


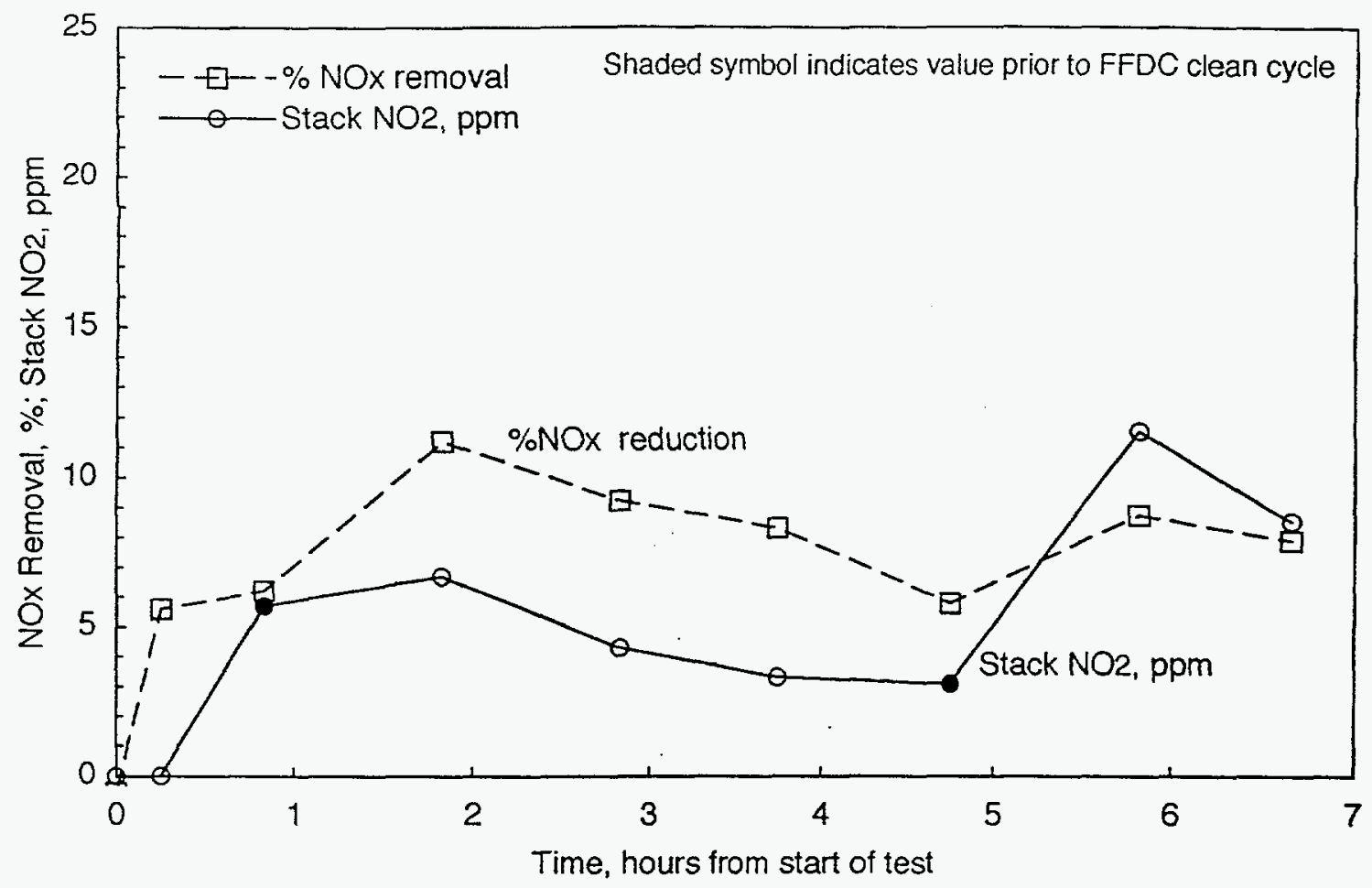

(a) $\mathrm{NO}_{2}$ and $\mathrm{NO}_{x}$ Removal

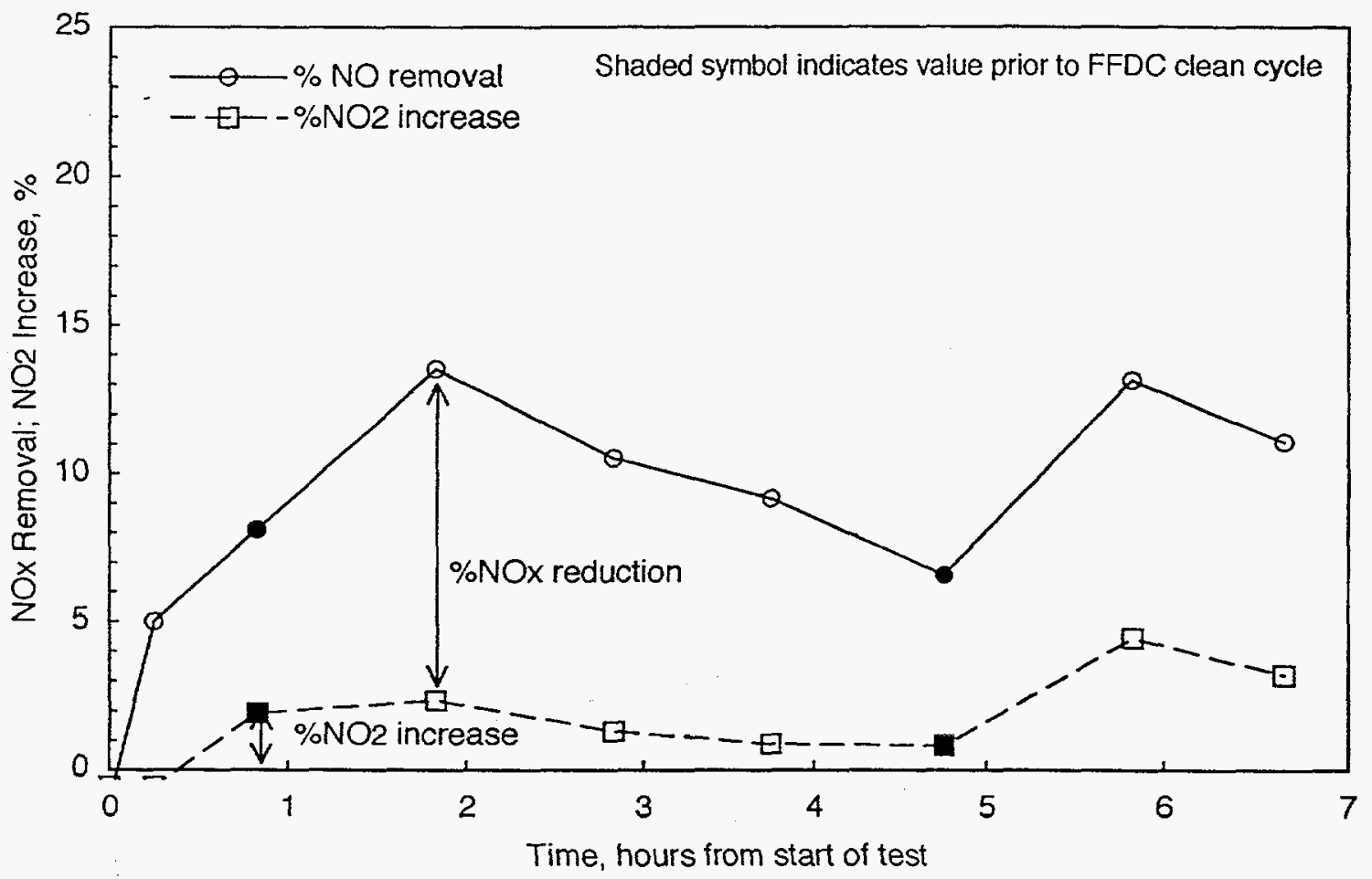

(b) Partitions of the Change in NO Between $\mathrm{NO}_{2}$ and $\mathrm{NO}_{x}$ Removal

Figure 5-9. $\mathrm{NO}_{\mathrm{x}}$ Removal and $\mathrm{NO}_{2}$ Emissions Versus Time for Sodium Sesquicarbonate Injection Ahead of the FFDC (Test 818) 
The increase in $\mathrm{NO}_{2}$ levels following a fabric filter cleaning cycle is quite interesting and, as mentioned above, have not been reported in previous studies of dry sodium injection. The effect is due to an interaction between $\mathrm{NO}_{2}$ and the fly ash on the bags. More specifically, it is currently thought that the interaction is with the carbon in the fly ash. Following the low $\mathrm{NO}_{x}$ combustion system retrofit, while the carbon content of the ash did not increase, it did appear to change physically. Even though the ash carbon contents were still at the pre-retrofit levels, the ash visually appeared black. This suggests the possibility that the low $\mathrm{NO}_{x}$ combustion system may form some small soot particles that coat the ash particles. This fine coating of carbon on the ash could be more reactive than an equivalent amount of carbon more uniformly distributed through an ash particle. How the fly ash, or fly ash carbon, interacts with the sodium generated $\mathrm{NO}_{2}$ is currently not known. Possible mechanisms include physical absorption of the $\mathrm{NO}_{2}$ by the carbon, catalytic oxidation of $\mathrm{NaNO}_{2}$ to $\mathrm{NaNO}_{3}$, or catalytic reduction of $\mathrm{NO}_{2}$ to $\mathrm{NO}$. The specific mechanism is currently not known; and the results indicate that the overall $\mathrm{NO}_{x}$ chemistry associated with dry sodium injection is even more complex than outlined in Section 3.

Figure 5-9a showed the $\mathrm{NO}_{2}$ levels and $\mathrm{NO}_{x}$ removal that occurred during the 7-hour test with sodium sesquicarbonate. As discussed in Section 3.1, the chemical mechanism is thought to involve the formation of an unstable intermediate sodium compound, $\mathrm{NaNO}_{2}$, which will further react to release $\mathrm{NO}_{2}$, or oxidize to form solid $\mathrm{NaNO}_{3}$. The latter resulting in $\mathrm{NO}_{\mathrm{x}}$ removal. It is of value to look at how the $\mathrm{NO}$ that reacts is partitioned between $\mathrm{NO}_{2}$ and $\mathrm{NO}_{x}$ removal. This is shown in Figure 5-9b for the data in Figure 5-9a. In Figure 5-9b, the total height of the line plotted with the "circles" represents the total change in NO due to the sodium reactions. The dotted line represents the conversion of $\mathrm{NO}$ to $\mathrm{NO}_{2}$. For instance, for the data point just before 2 hours, the total change in NO was about $13 \%$; the $\mathrm{NO}_{\mathrm{x}}$ removal was $11 \%$, and the increase $\mathrm{NO}_{2}$ emissions represents only $2 \%$ of the initial $\mathrm{NO}_{\mathrm{x}}$. Even after the second cleaning cycle, which started just before 5 hours, when the $\mathrm{NO}_{2}$ increased from $3 \mathrm{ppm}$ to $11 \mathrm{ppm}(2 \%$ to $5 \%)$, the majority of the change in NO resulted in $\mathrm{NO}_{\mathrm{x}}$ removal. 
Figure 5-10 shows the compartment-by-compartment $\mathrm{NO}_{x}$ removals and $\mathrm{NO}_{2}$ levels for the same test shown in Figure 5-8. As with the $\mathrm{SO}_{2}$ removals shown in Figure 5-8, the $\mathrm{NO}_{x}$ removal and $\mathrm{NO}_{2}$ levels are higher on the east side of the fabric filter. In fact, the $\mathrm{NO}_{2}$ levels are less than $1 \mathrm{ppm}$ exiting the compartments on the west side. As with Figure 5-9b, Figure 5-11 shows the partitioning of the $\mathrm{NO}$ that reacts between $\mathrm{NO}_{2}$ and $\mathrm{NO}_{\mathrm{x}}$ removal. For all compartments, the vast majority that reacts results in $\mathrm{NO}_{x}$ removal, rather than $\mathrm{NO}_{2}$ emissions.

Figure 5-12 summarizes the $\mathrm{NO}_{2}$ emission measurements as a function of injection rate $(2 \mathrm{Na} / \mathrm{S})$ for all of the sodium sesquicarbonate tests performed during the current study. The figure includes data for injection ahead of the FFDC as well as ahead of the air heater. (The air heater data will be discussed in a later section.) Although the data exhibit an increasing trend with injection rate, there is a large amount of scatter where the $\mathrm{NO}_{2}$ emissions range from approximately 5 to $25 \mathrm{ppm}$ at a nominal $2 \mathrm{Na} / \mathrm{S}$ ratio of 2.0 . As discussed above, the $\mathrm{NO}_{2}$ emissions depend not only on the injection rate, but also on the FFDC cleaning cycle (i.e., the amount of flyash on the bags). It should be noted that there has been no attempt to either filter or correlate the data in Figure 5-12 with respect to cleaning cycle timing.

Figure $5-13$ summarizes the $\mathrm{NO}_{\mathrm{x}}$ removals with sodium sesquicarbonate injection ahead of the FFDC. As was seen for $\mathrm{NO}_{2}$ emissions, there is a significant amount of scatter in the data, with a very slightly increasing trend with injection rate. $\mathrm{NO}_{\mathrm{x}}$ removals range from 2 to 18 percent at a nominal $2 \mathrm{Na} / \mathrm{S}$ ratio of 2.0 .

C. Sodium Sesquicarbonate Injection with Humidification. A limited number of sodium sesquicarbonate tests were run with humidification in order to see if the $\mathrm{SO}_{2}$ removals would increase. Five tests were run with an approach to saturation temperature of approximately $60^{\circ} \mathrm{F}$, and one each with approaches of 50 and $90^{\circ} \mathrm{F}$. The results of these tests are compared to the $\mathrm{SO}_{2}$ removals without humidification in Figure 5-14. It should 
Test 641, 2Na/S=0.88, NaSC, Mill Speed $4000 \mathrm{rpm}$ $9 / 7 / 93$

\section{Compartment Gas Measurement}

* O2 NO NO2 NOx NOxc NOxn $\triangle \% N O x \Delta \% N O 2$

- \%dry ppm ppm ppm ppmc _ \% \% $\%$

$\begin{array}{lllllllll}1 \text { sw } & 6.30 & 210 & 1.0 & 211 & 258 & 1.07 & 7.1 & 0.4\end{array}$

$\begin{array}{lllllllll}2 w & 5.85 & 215 & 0.4 & 215 & 256 & 1.06 & 8.0 & 0.2\end{array}$

$\begin{array}{llllllllll}3 w & 5.65 & 215 & 0.2 & 215 & 252 & 1.04 & 9.2 & 0.1\end{array}$

$\begin{array}{lllllllll}4 w & 5.35 & 215 & 0.3 & 215 & 248 & 1.02 & 10.9 & 0.1\end{array}$

$\begin{array}{llllllllll}5 w & 5.50 & 222 & -0.2 & 222 & 258 & 1.08 & 7.4 & -0.1\end{array}$

$\begin{array}{lllllllll}6 \mathrm{nW} & 5.20 & 215 & -0.1 & 215 & 245 & 1.01 & 11.9 & 0.0\end{array}$

$\begin{array}{lllllllll}7 \mathrm{se} & 5.50 & 204 & 0.0 & 204 & 237 & 0.98 & 14.8 & 0.0\end{array}$

$\begin{array}{llllllllll}8 \theta & 5.20 & 190 & 1.5 & 192 & 218 & 0.90 & 21.5 & 0.6\end{array}$

$\begin{array}{lllllllll}9 \theta & 5.40 & 192 & 5.1 & 197 & 227 & 0.94 & 18.2 & 2.1\end{array}$

$\begin{array}{llllllllll}10 e & 5.10 & 195 & 4.4 & 199 & 228 & 0.93 & 18.8 & 1.8\end{array}$

$\begin{array}{lllllllll}11 \theta & 5.20 & 210 & 0.5 & 211 & 240 & 0.99 & 13.7 & 0.2\end{array}$

$\begin{array}{llllllllll}12 n \theta & 5.30 & 212 & -0.1 & 212 & 243 & 1.00 & 12.6 & 0.0\end{array}$

$\begin{array}{ccccccccc}\text { Ave } & 5.46 & 208 & 1.1 & 209 & 242 & 1.00 & 12.8 & 0.4 \\ \text { West } & 5.64 & 215 & 0.3 & 216 & 253 & 1.04 & 8.1 & 0.1 \\ \text { East } & 5.28 & 201 & 1.9 & 202 & 232 & 0.96 & 18.6 & 0.8\end{array}$

Average Inlet/Outlet Gas Measurements

Probe 02 NO NO2 H2O NOX NOXC $\triangle$ NOX

\%wet ppm ppm \% ppm ppmed \%

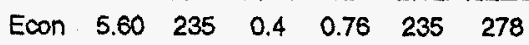

$\begin{array}{llllllll}\text { Stack } & 5.40 & 189 & 0.0 & 9.15 & 189 & 249 & 10.5\end{array}$
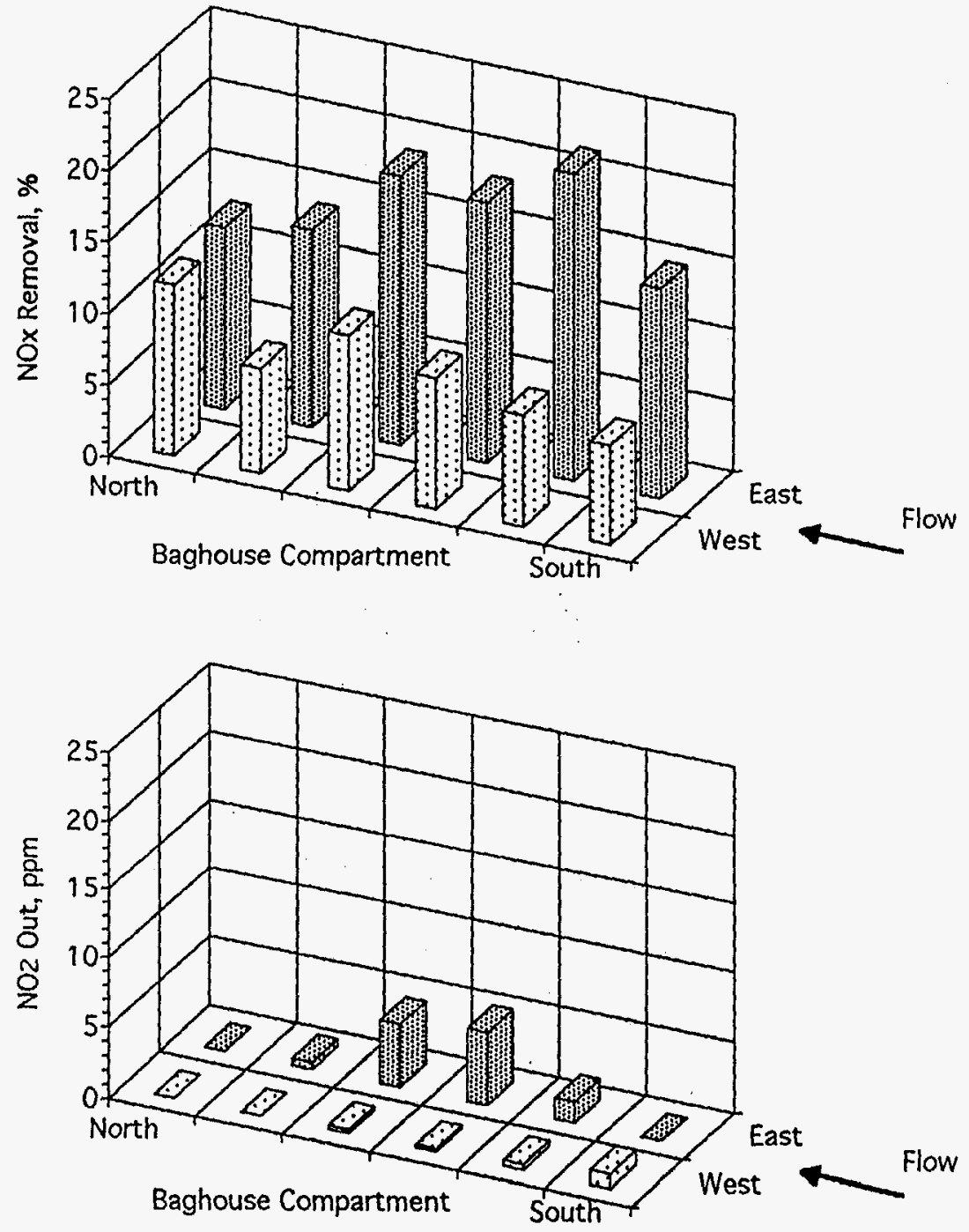

Figure 5-10. Compartment-by-Compartment $\mathrm{NO}$ and $\mathrm{NO}_{2}$ for Sodium Sesquicarbonate Injection Ahead of the FFDC (Test 641) 


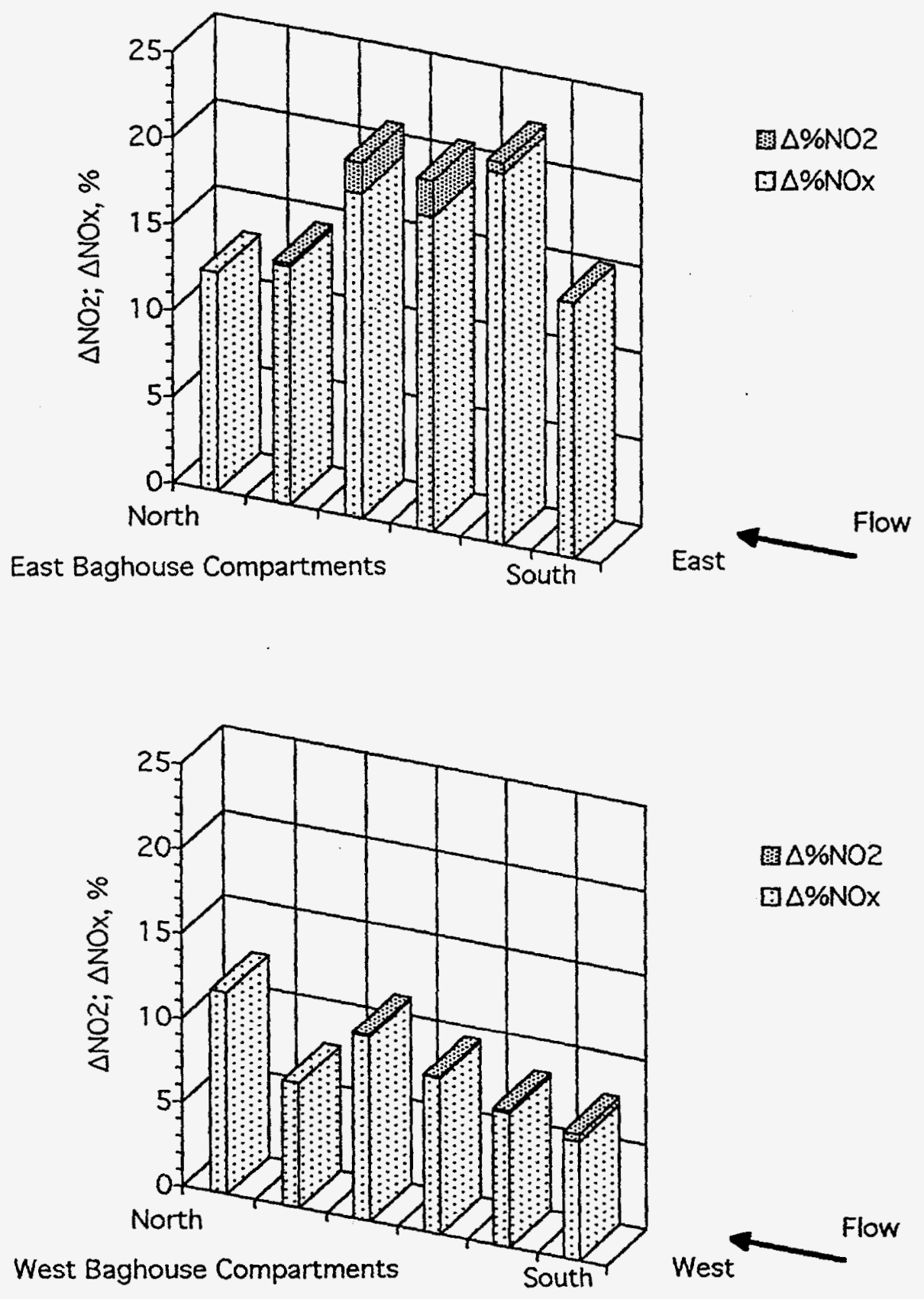

Figure 5-11. Change in $\mathrm{NO}_{\mathrm{x}}$ and $\mathrm{NO}_{2}$ for Sodium Sesquicarbonate for FFDC Inlet Injection (Test 641) 


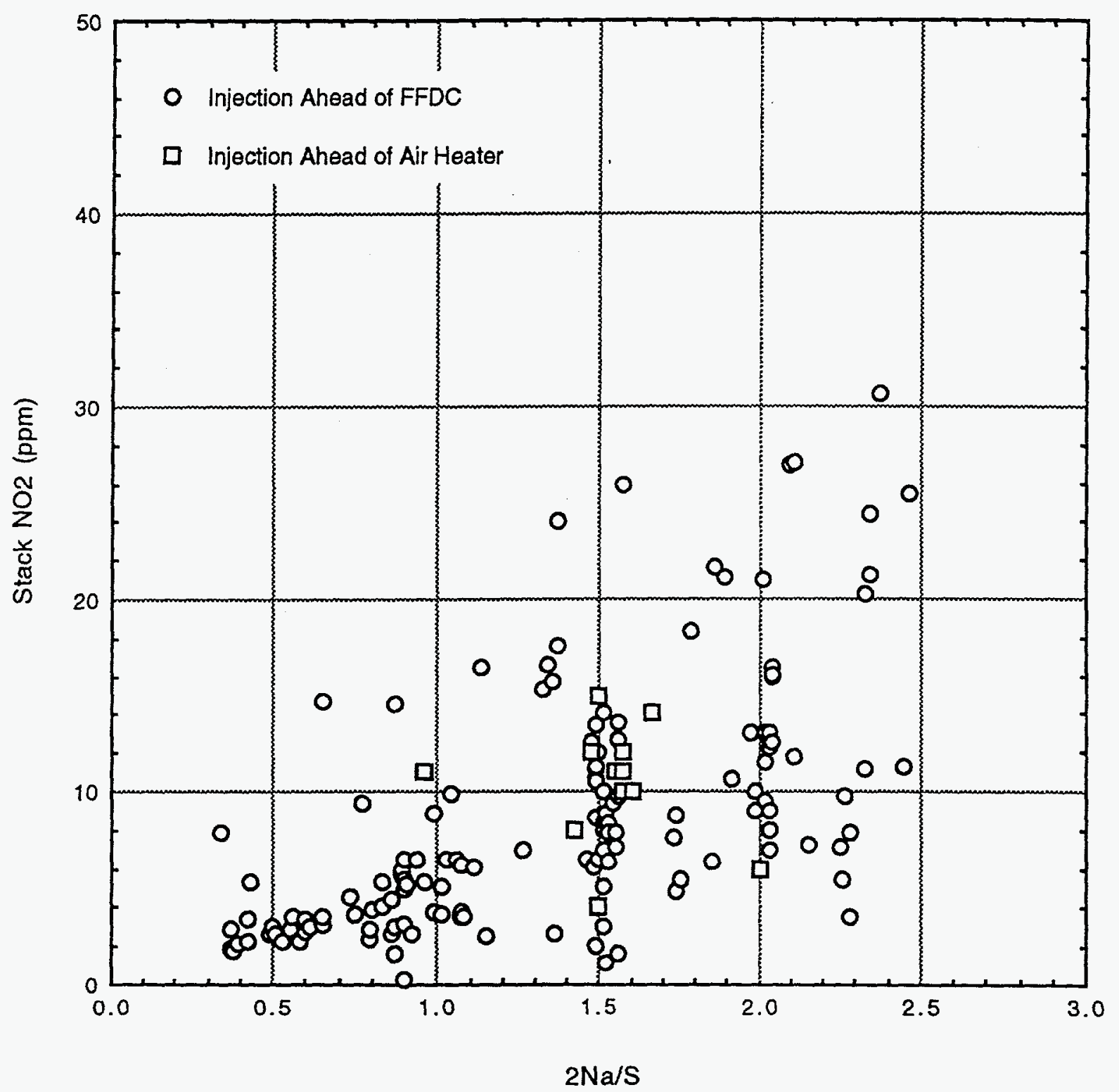

Figure 5-12. Summary of $\mathrm{NO}_{2}$ Emissions with Sodium Sesquicarbonate Injection 


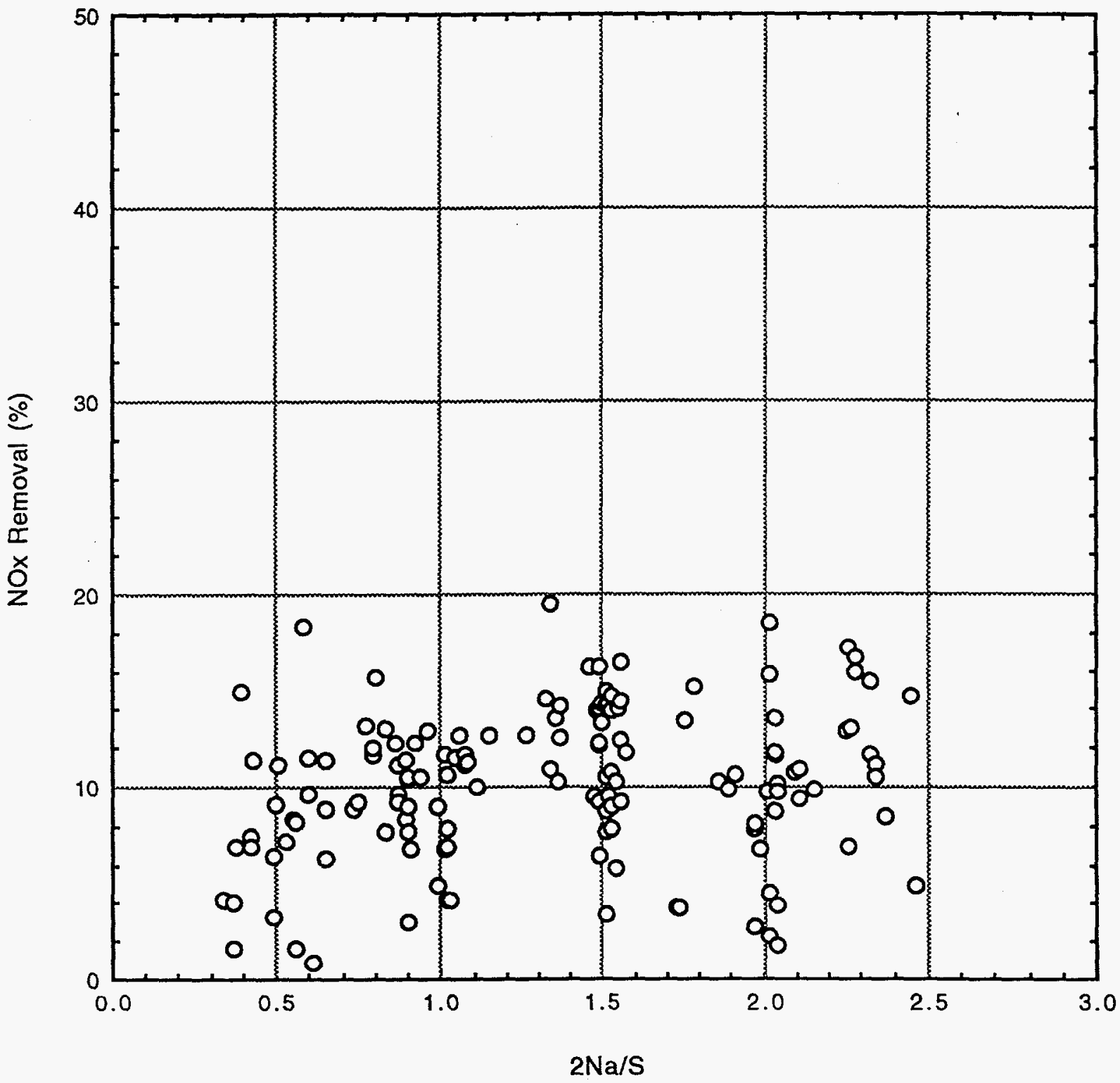

Figure 5-13. Summary of $\mathrm{NO}_{x}$ Removals with Sodium Sesquicarbonate Injection Ahead of the FFDC

$5-22$

FERCo-7037-R337 


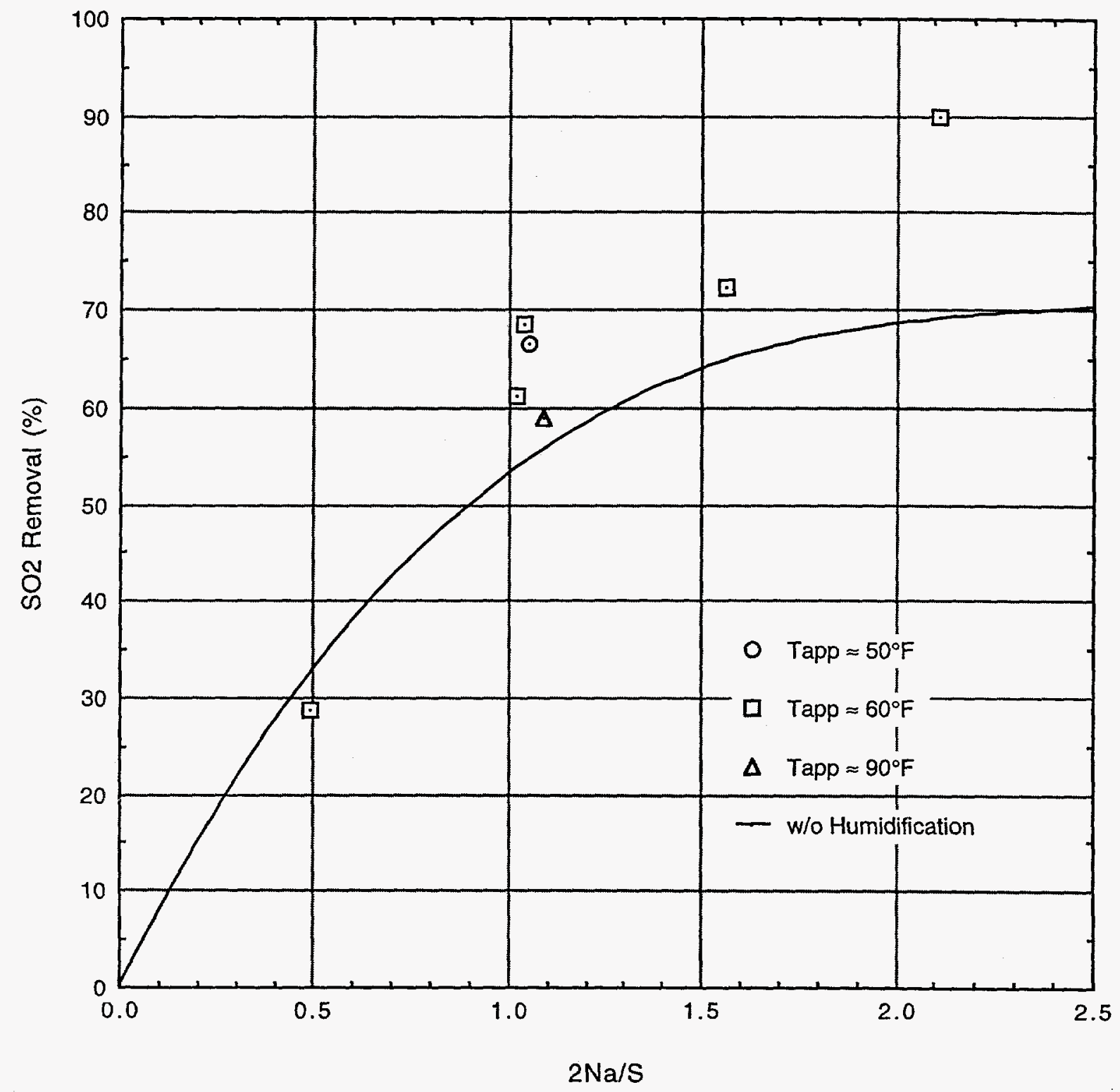

Figure 5-14. Effect of Humidification Approach to Saturation Temperature on $\mathrm{SO}_{2}$ Removal for Sodium Sesquicarbonate Injection Ahead of the FFDC 
be noted that the "without" points shown in the figure are not actual tests, but rather interpolated points from the curve fit in Figure 5-4a. The data show that humidification results in increased $\mathrm{SO}_{2}$ removals at higher sorbent feed rates $(2 \mathrm{Na} / \mathrm{S}$ ratios in excess of 1.0). At a nominal $2 \mathrm{Na} / \mathrm{S}$ ratio of 2.0 , the removals are increased from approximately 70 to 90 percent with an approach to saturation temperature of nominally $60^{\circ} \mathrm{F}$. Data obtained at a lower $2 \mathrm{Na} / \mathrm{S}$ ratio of 1.0 indicate that humidification had a smaller effect on $\mathrm{SO}_{2}$ removal with increases that ranged from 5 to $15 \%$. As normal data scatter is in the range of 5 to $10 \%$ net $\mathrm{SO}_{2}$ removal at a given $2 \mathrm{Na} / \mathrm{S}$ ratio, the data indicate that humidification has an increasing improvement as injection rate increases.

Figure 5-15 shows the compartment-by-compartment gaseous emissions measurements made during one of the humidification tests at an approach temperature of approximately $60^{\circ} \mathrm{F}$ with a nominal $2 \mathrm{Na} / \mathrm{S}$ ratio of 1.0 . The distribution of $\mathrm{SO}_{2}$ removal within the FFDC is quite different than that for the no humidification case shown in Figure 5-8. With humidification, the removals in each compartment are relatively equal. In contrast, there was a marked difference in the $\mathrm{SO}_{2}$ removals among the compartments when operating without humidification. The non-humidification data indicate that each compartment collected varying amounts of the sodium sorbent. With humidification, the $\mathrm{SO}_{2}$ removals are relatively equal among the compartments suggesting that more of the $\mathrm{SO}_{2}$ removal occurred in the duct upstream of the FFDC. Moisture becoming associated with the sodium particles during the humidification process would be expected to increase the overall reactivity with $\mathrm{SO}_{2}$, thus allowing more of the $\mathrm{SO}_{2}$ removal process to occur ahead of the FFDC.

\subsubsection{Sodium Sesquicarbonate Injection at the Air Heater Inlet}

Although injection at the FFDC inlet was the main focus of the sodium sesquicarbonate tests, two days of tests were also run at the hotter air heater inlet location. Figure 5-16 presents the results of the first day of testing, where $\mathrm{SO}_{2}$ removal was determined as a function of $2 \mathrm{Na} / \mathrm{S}$ ratio. The average results from tests at the FFDC inlet are also shown for comparison. Although the two sets of data indicate that there is little difference in the 
Test 665, Bag comp, $60^{\circ} \mathrm{F}$ approach, $2 \mathrm{Na} / \mathrm{S}=1.0 \mathrm{NaSC}$ 9/20/93

Compartment Gas Measurement

\# O2 SO2 nO NOC SOze sO2n $\Delta \mathrm{SO} 2$

\%dry ppm ppm ppmc ppmc - \%

$\begin{array}{llllllll}1 \text { sw } & 5.50 & 119 & 219 & 254 & 138 & 0.81 & 67.5\end{array}$

$\begin{array}{llllllll}2 W & 5.40 & 109 & 224 & 258 & 126 & 0.73 & 70.4\end{array}$

$\begin{array}{llllllll}3 W & 5.20 & 148 & 222 & 253 & 169 & 0.98 & 60.4\end{array}$

$\begin{array}{llllllll}4 W & 5.25 & 157 & 219 & 250 & 179 & 1.05 & 57.8\end{array}$

$\begin{array}{llllllll}5 w & 5.20 & 185 & 222 & 253 & 211 & 1.23 & 50.5\end{array}$

$\begin{array}{llllllll}6 \mathrm{nw} & 5.44 & 172 & 225 & 260 & 199 & 1.16 & 53.2\end{array}$

$\begin{array}{llllllll}7 \text { se } & 5.18 & 112 & 211 & 240 & 127 & 0.74 & 70.0\end{array}$

$\begin{array}{lllllllll}8 \theta & 5.15 & 133 & 214 & 243 & 151 & 0.88 & 64.5\end{array}$

$\begin{array}{llllllll}9 \theta & 5.20 & 158 & 218 & 248 & 180 & 1.05 & 57.7\end{array}$

$\begin{array}{lllllllll}10 \theta & 5.50 & 177 & 223 & 259 & 206 & 1.20 & 51.7\end{array}$

$\begin{array}{llllllll}11 \theta & 5.35 & 162 & 217 & 250 & 186 & 1.09 & 56.2\end{array}$

$\begin{array}{lllllllll}12 \mathrm{ne} & 5.32 & 162 & 217 & 249 & 186 & 1.08 & 56.3\end{array}$

Ave 5.31

West 5.33

East 5.28

$\begin{array}{llll}252 & 172 & 1.00 & 59.7\end{array}$

$\begin{array}{llll}255 & 170 & 0.99 & 60.0\end{array}$

$\begin{array}{llll}248 & 173 & 1.01 & 59.4\end{array}$

Average Inlet/Outlet Gas Measurements

$\mathrm{O} 2 \mathrm{SO}_{2} \mathrm{NO} \mathrm{H}_{2} \mathrm{O}$ NOC $\mathrm{SO} 2 \mathrm{C} \quad \mathrm{SO}_{2}$

— \%wet ppm ppm \% ppmcdepmed \%

Inlet $4.30 \quad 350 \quad 208 \quad 9.00 \quad 253 \quad 425$

$\begin{array}{llllllll}\text { Stack } & 4.82 & 133 & 185 & 12.1 & 244 & 176 & 58.7\end{array}$
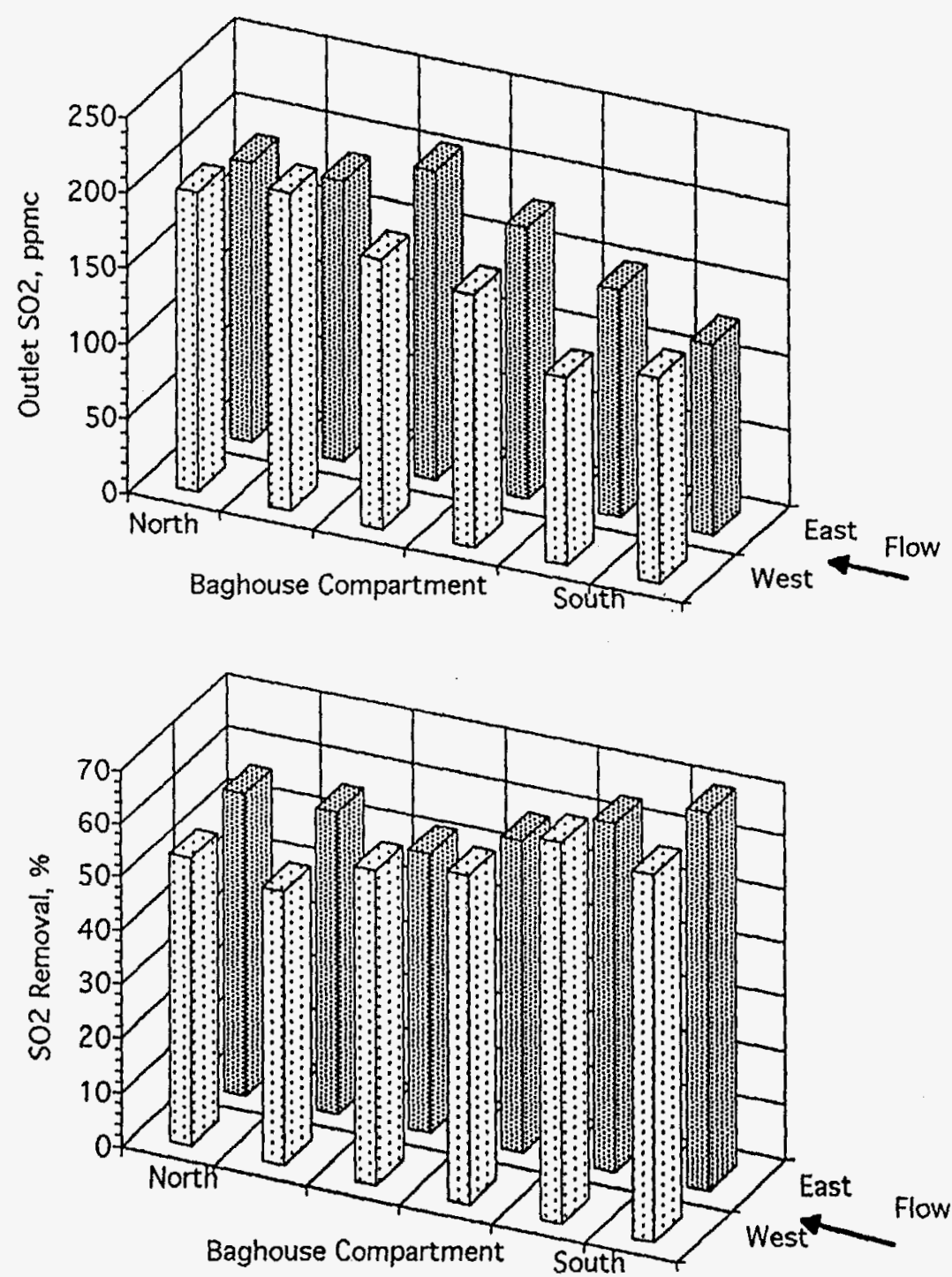

Figure 5-15. Compartment-by-Compartment Gaseous Measurements for Sodium Sesquicarbonate Injection with Humidification Ahead of the FFDC (Test 665) 


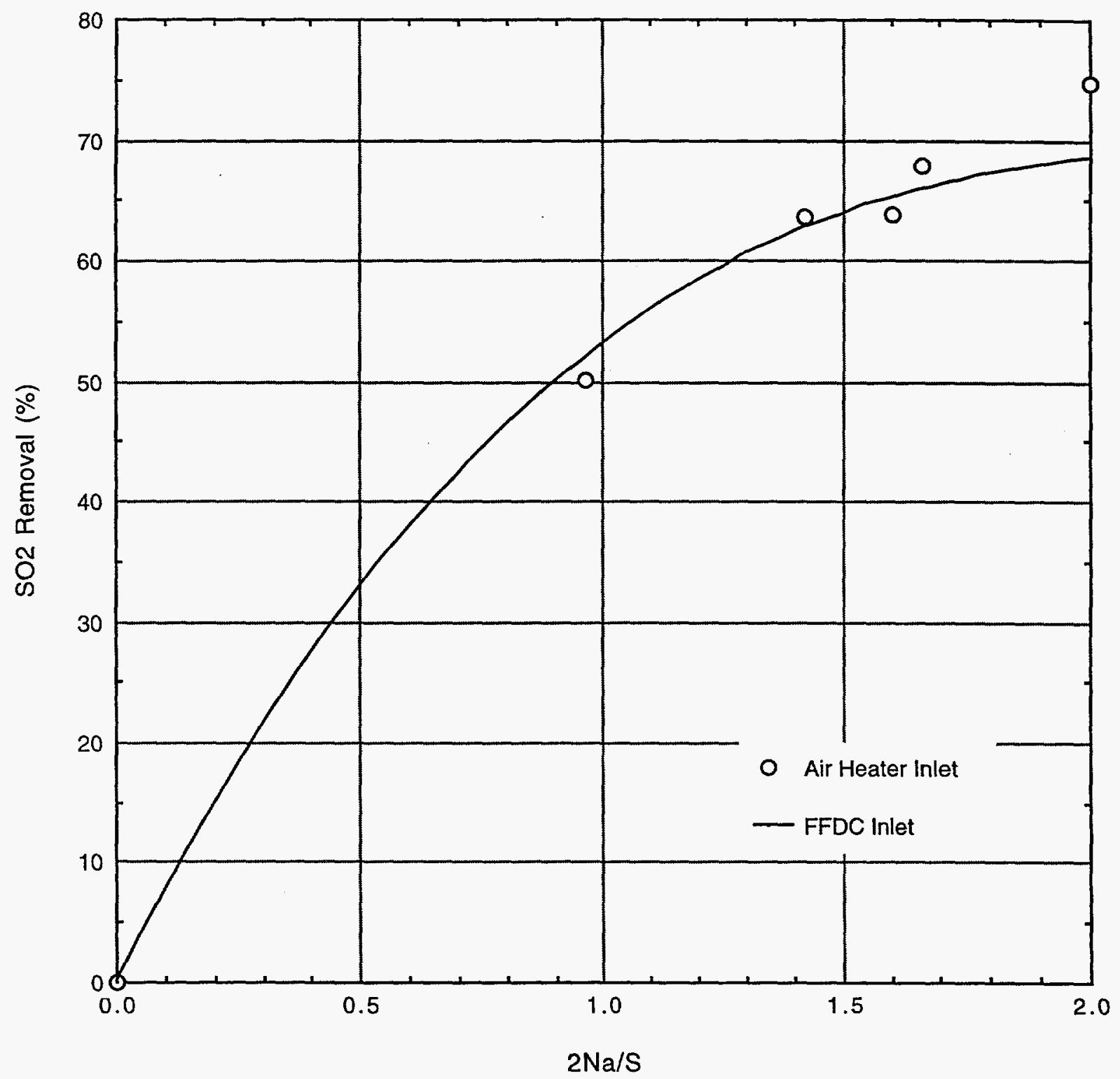

Figure 5-16. Comparison of $\mathrm{SO}_{2}$ Removals for Sodium Sesquicarbonate Injection at Air Heater Inlet and FFDC Inlet Locations 
steady-state $\mathrm{SO}_{2}$ removals, the removals as a function of time for the two injection locations were found to be quite different. Figure 5-17 compares the $\mathrm{SO}_{2}$ removal versus time traces for a test run at each injection location at a nominal $2 \mathrm{Na} / \mathrm{S}$ ratio of 1.5 . The data show that the initial response time at the air heater location is much longer, and the level to which the $\mathrm{SO}_{2}$ removal drops after a cleaning cycle is much lower. These observations indicate that the response time of the overall $\mathrm{SO}_{2}$ removal process is slower when injecting at the air heater inlet. Although the steady-state removals are comparable (roughly 65 percent), the slower response time will result in a lower time-averaged $\mathrm{SO}_{2}$ removal when injecting at the air-heater inlet.

The $\mathrm{NO}_{2}$ emissions resulting from sodium sesquicarbonate injection ahead of the air heater were presented along with the results for injection ahead of the FFDC in Figure 5-12. There does not appear to be any major difference in the $\mathrm{NO}_{2}$ levels produced between these two injection locations.

\subsection{Sodium Bicarbonate}

The sodium bicarbonate tests were performed at two different injection locations. The original test plan called for injection ahead of the FFDC, at the same location utilized for the sodium sesquicarbonate tests. In addition, a series of tests were also run with a second set of injectors located ahead of the air heater. The results of the tests at the two locations are discussed separately in the following subsections.

\subsubsection{Injection of Sodium Bicarbonate at the FFDC Inlet}

A. $\mathrm{SO}_{2}$ Removal. The previous work at Cameo (Muzio, et al., 1984) showed that the $\mathrm{SO}_{2}$ removal process with sodium bicarbonate injection upstream of a fabric filter was highly temperature dependent. At temperatures below approximately $290^{\circ} \mathrm{F}$, the reaction kinetics are slowed significantly, which results in an overall decrease in $\mathrm{SO}_{2}$ removal. Figure 5-18 shows the $\mathrm{SO}_{2}$ removal versus time trace for one of the first tests run with sodium bicarbonate injection upstream of the Arapahoe Unit 4 FFDC. The decrease in $\mathrm{SO}_{2}$ removal seen mid-way through the test is due to a FFDC cleaning cycle which started 


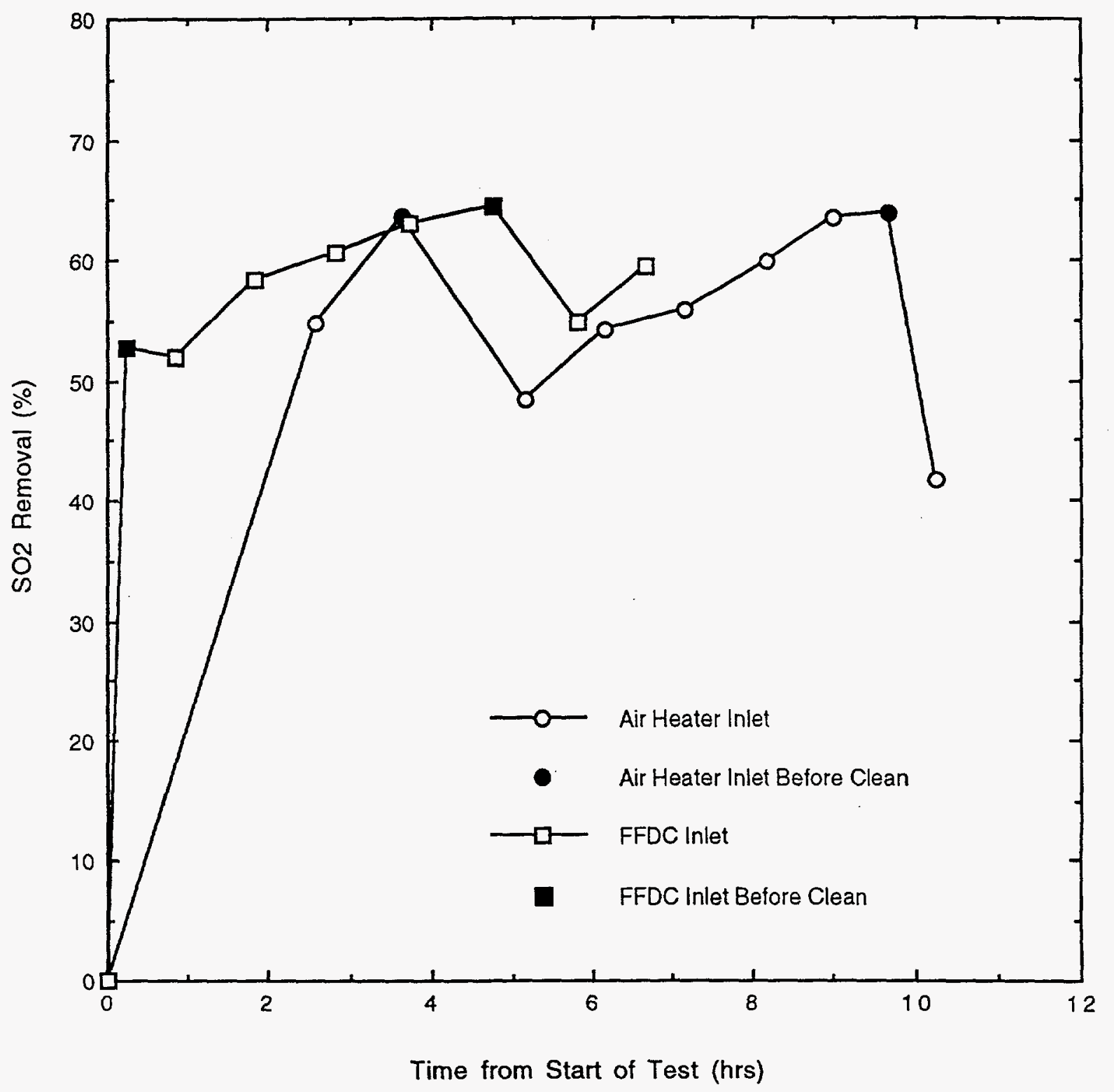

Figure 5-17. Comparison of Response Time Characteristics for Sodium Sesquicarbonate Injection at Air Heater Inlet and FFDC Inlet Locations $(2 \mathrm{Na} / \mathrm{S}=1.5)$ 


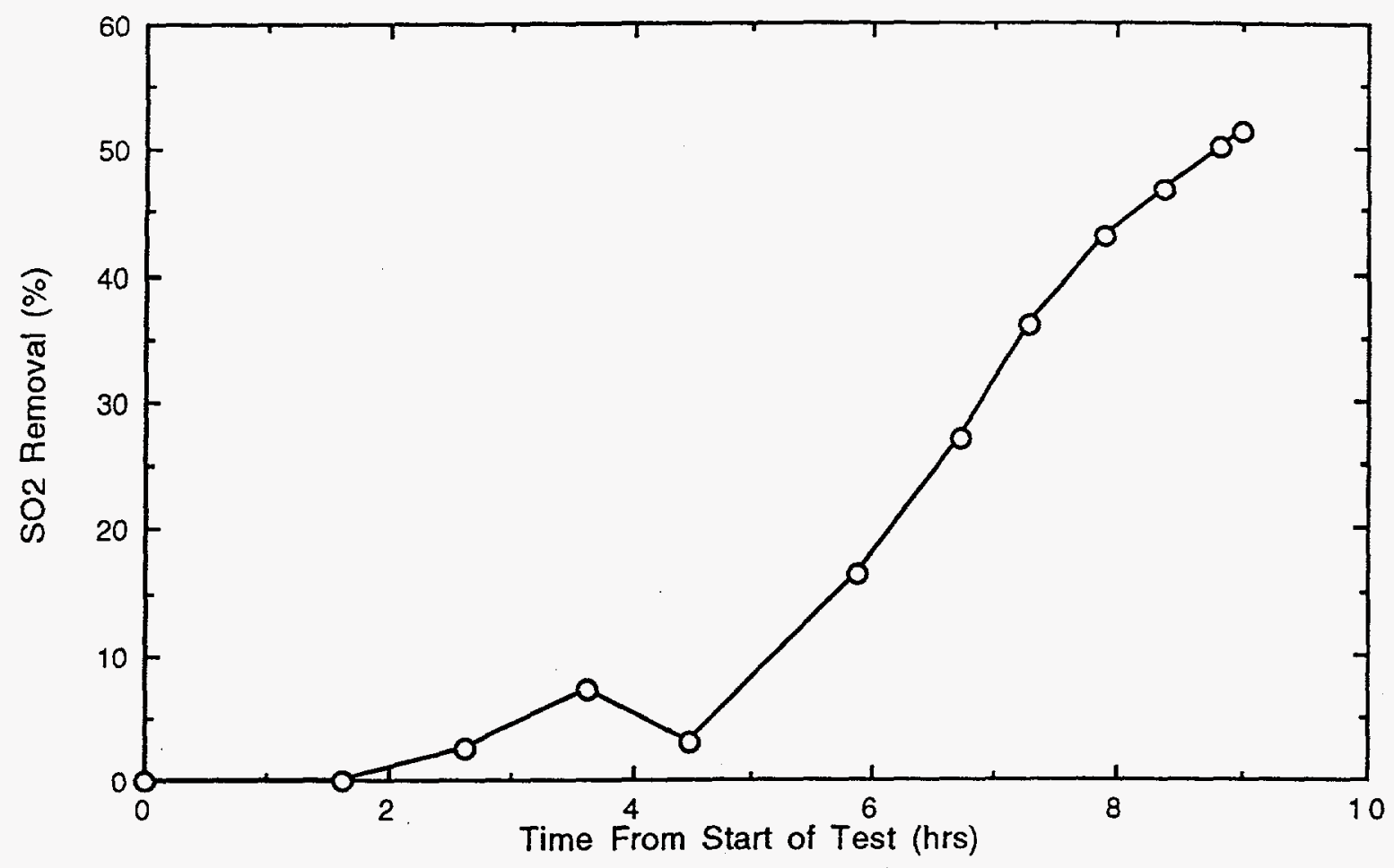

Figure 5-18. $\mathrm{SO}_{2}$ Removal Versus Time for Sodium Bicarbonate Injection Ahead of the FFDC (Test 640)

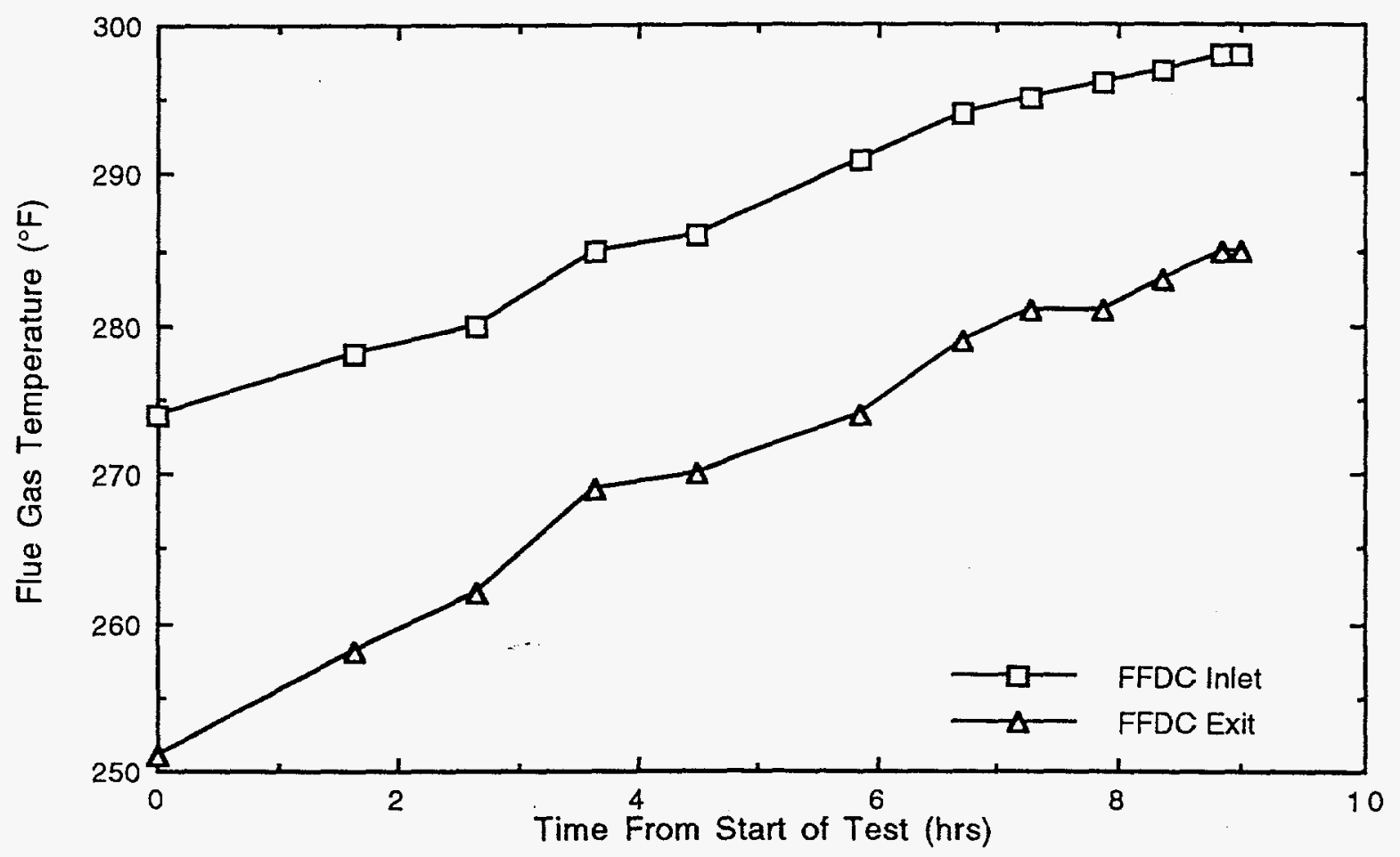

Figure 5-19. Inlet and Exit FFDC Temperatures Versus Time for Sodium Bicarbonate Injection Ahead of the FFDC (Test 640) 
approximately $3-1 / 2$ hours into the test. The data show that nearly nine hours were required for the $\mathrm{SO}_{2}$ removal to reach 50 percent, and at the point when the sorbent feed was shut down at the end of the day, the removals had still not reached a steady-state level. Flue gas temperatures entering and exiting the FFDC slowly climbed throughout the day as shown in Figure 5-19, but the exit gas temperature never exceeded $285^{\circ} \mathrm{F}$. The slowly increasing trend in $\mathrm{SO}_{2}$ removal seen during this test was likely due to a combination of the slow increase in FFDC temperature throughout the day and the accumulation of unreacted sodium on the bags.

The FFDC exit temperature at Arapahoe Unit 4 normally ranges from 230 to $270^{\circ} \mathrm{F}$, and seldom reaches $290^{\circ} \mathrm{F}$. In order to better understand the dynamics of the $\mathrm{SO}_{2}$ removal process at these lower temperatures, the sorbent injection system was run 24 hours a day for a period of five days. Arapahoe Unit 4 is normally used by the PSCo dispatch center for system regulation; therefore, boiler load normally changes suddenly and frequently. During these five days of testing, the boiler was used for regulation, but with the agreement that when load was changed, it would be left constant for a period of three to four hours. Gaseous emission data was collected on an hourly basis during this test, so at least three to four sets of data could be collected at each new load point before another change was made. The sorbent injection rate was manually controlled throughout the duration of the test to maintain a nominal $2 \mathrm{Na} / \mathrm{S}$ ratio of 1.0 . Figure $5-20$ shows the $\mathrm{SO}_{2}$ removal, $2 \mathrm{Na} / \mathrm{S}$ ratio, and FFDC exit temperature trends for the duration of the test. The FFDC was allowed to clean on its normal cycle during the test (automatically when reaching a pressure drop of 4.0 inches of $\mathrm{H}_{2} \mathrm{O}$ ), and a set of data was collected immediately before and after each cleaning cycle in order to accurately characterize the time required for the $\mathrm{SO}_{2}$ removal to recover. The dark symbols on the $\mathrm{SO}_{2}$ removal trace denote the removals recorded just before each cleaning cycle. The results show that the $\mathrm{SO}_{2}$ removals drop dramatically after each cleaning cycle. Although, there were some initial problems encountered in maintaining a constant $2 \mathrm{Na} / \mathrm{S}$ ratio, the first ten hours showed a slow increase in $\mathrm{SO}_{2}$ removal similar to that seen in Figure 5-18. However, after the FFDC had been "conditioned" through a few cleaning cycles, the recovery time after a cleaning was 
SO2 Removal (\%), Temp. ( $\left.{ }^{\circ} \mathrm{F}-200\right), 2 \mathrm{Na} / \mathrm{S}(x 100)$

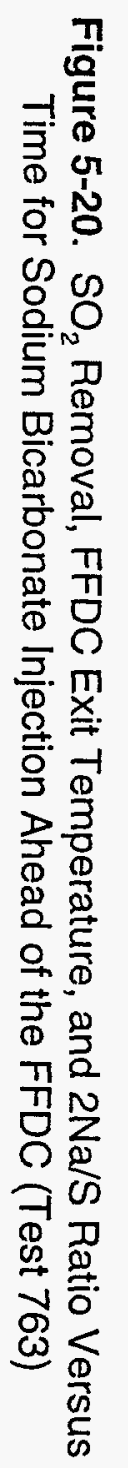

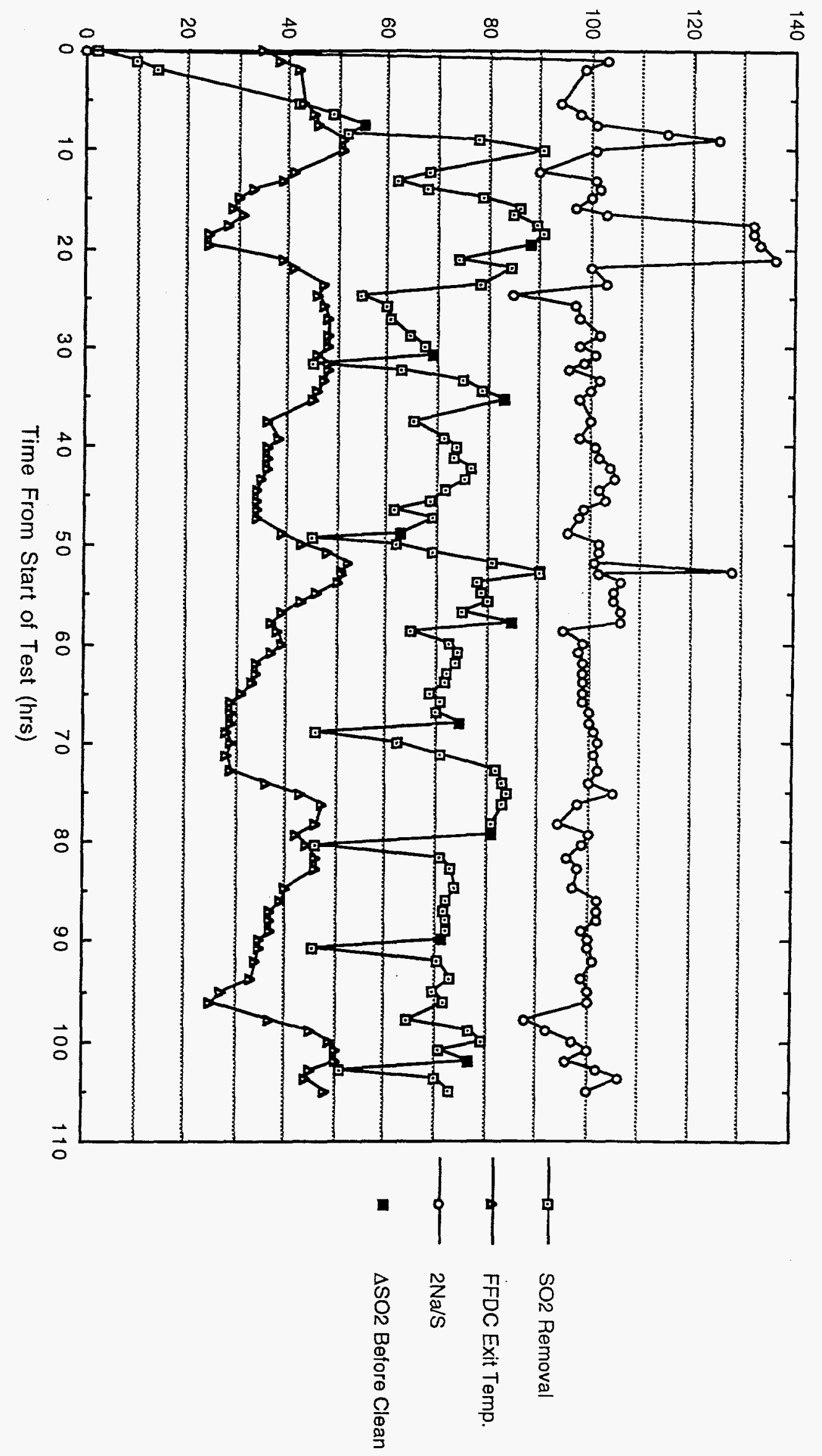


significantly reduced, and the $\mathrm{SO}_{2}$ removals often reached a relatively steady state after only a couple of hours.

Although the $2 \mathrm{Na} / \mathrm{S}$ ratio varied over only a narrow range during the five-day test, the "steady-state" data collected before each cleaning cycle provide an indication of the effect of $2 \mathrm{Na} / \mathrm{S}$ ratio on $\mathrm{SO}_{2}$ removal (Figure 5-21). At a nominal $2 \mathrm{Na} / \mathrm{S}$ ratio of $1.0, \mathrm{SO}_{2}$ removals ranged from 65 to 85 percent. These results are slightly below, but comparable to, those seen in the earlier demonstrations at Cameo and Nixon (Muzio, et al., 1984; Fuchs et al., 1989) where removals ranged from 70 to 90 percent at a nominal $2 \mathrm{Na} / \mathrm{S}$ ratio of 1.0. The sorbent utilizations computed for the data from the current series of tests (Figure 5-22) range from 65 to 85 percent.

In the automatic control mode, the DSI system is set to maintain a specified level of $\mathrm{SO}_{2}$ removal, and the feed rate is constantly varied in order to achieve that goal. The combination of the time required for the $\mathrm{SO}_{2}$ removal to respond to a change in feed rate, the time required to recover after a cleaning cycle, and the variability of the steady-state removals made trying to control the DSI system with bicarbonate injection in this manner impractical. An attempt was made to "tune" the DSI control system to compensate for the slow response of the $\mathrm{SO}_{2}$ removal, but it was unsuccessful.

On the final day of the five-day test, gaseous emission measurements were made at the exit of each FFDC compartment in order to characterize the distribution of sorbent in the baghouse. The results of the compartment-by-compartment measurements are shown in Figure 5-23. At the time that the measurements were performed, compartment Number 11 was out of service for maintenance. The results show high levels of $\mathrm{SO}_{2}$ removal $(80$ to 90 percent) in the first three compartments on each side of the baghouse. This is followed by a rapid decrease down to levels of only 10 to 20 percent in the rear compartments. Clearly, the majority of the sorbent is deposited in the front half of the FFDC. This compartment-by-compartment distribution of $\mathrm{SO}_{2}$ removal is similar to that seen for sodium 


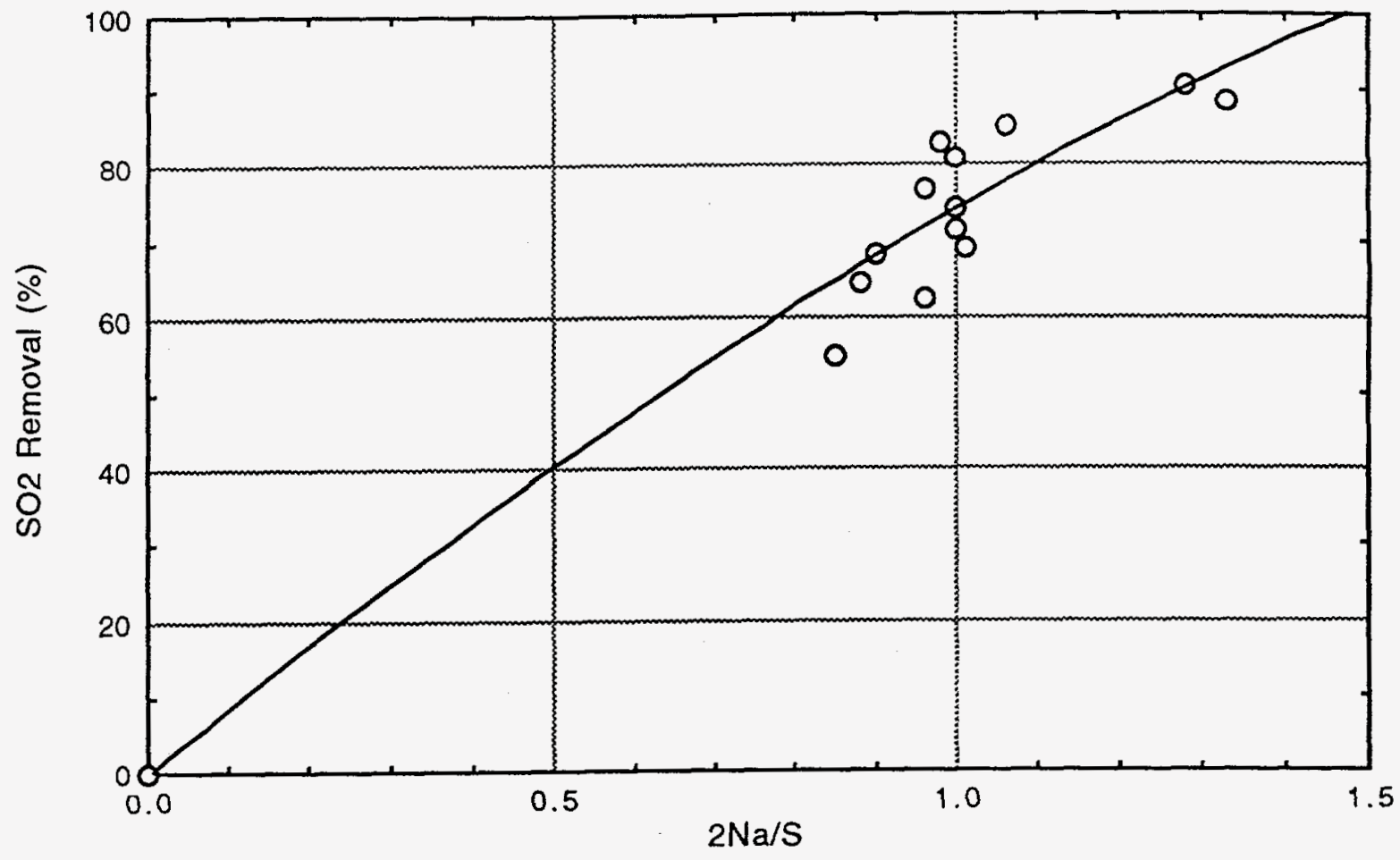

Figure 5-21. $\mathrm{SO}_{2}$ Removal as a Function of $2 \mathrm{Na} / \mathrm{S}$ Ratio for Sodium Bicarbonate Injection Ahead of the FFDC (Test 763)

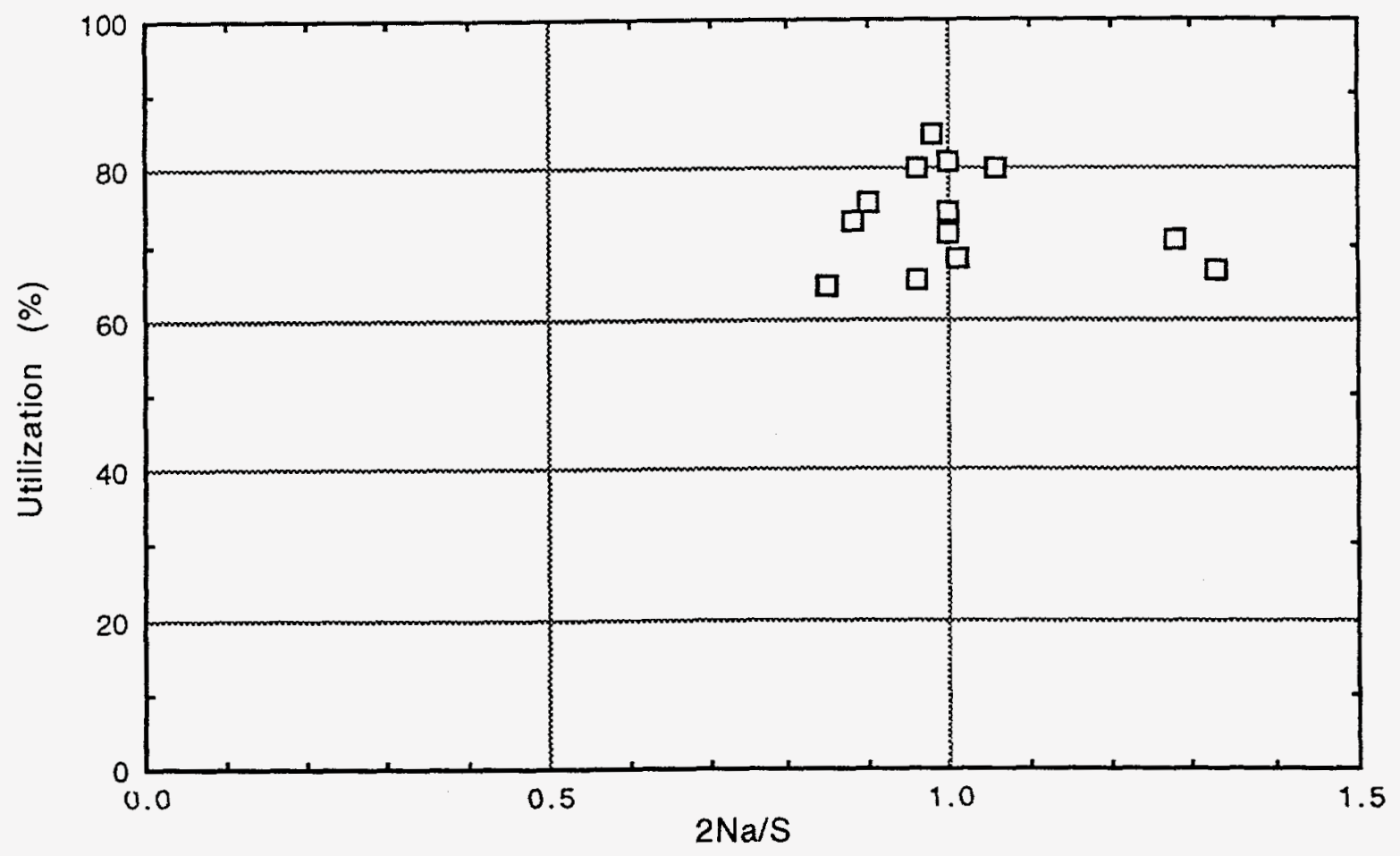

Figure 5-22. Utilization as a Function of $2 \mathrm{Na} / \mathrm{S}$ Ratio for Sodium Bicarbonate Injection Ahead of the FFDC (Test 763) 
Test 763, 2/4/93, 2Na/S=1.0 NaBicarb

Bag Compartment Gas Protile

Compartment Gas Measurement

\# $\mathrm{O}_{2} \mathrm{SO} 2 \mathrm{NO}$ NOc SO2c SO2n $\triangle \mathrm{SO}_{2}$

\%dry ppm ppm ppmc ppmc - \%

$\begin{array}{lllllllllll}1 \mathrm{sW} & 6.80 & 43 & 193 & 245 & 55 & 0.35 & 88.3\end{array}$

$\begin{array}{llllllll}2 w & 6.90 & 36 & 185 & 236 & 46 & 0.29 & 90.1\end{array}$

$\begin{array}{lllllllll}3 w & 6.95 & 46 & 190 & 243 & 59 & 0.37 & 87.4\end{array}$

$\begin{array}{llllllll}4 w & 6.65 & 104 & 211 & 265 & 130 & 0.83 & 72.0\end{array}$

$\begin{array}{lllllllll}5 W & 7.40 & 175 & 219 & 290 & 232 & 1.47 & 50.3\end{array}$

$\begin{array}{llllllll}6 \mathrm{nw} & 9.55 & 235 & 190 & 299 & 369 & 2.35 & 20.8\end{array}$

$\begin{array}{llllllll}7 \mathrm{se} & 5.40 & 85 & 210 & 242 & 98 & 0.62 & 79.0\end{array}$

$\begin{array}{llllllll}8 \theta & 5.50 & 55 & 190 & 221 & 64 & 0.41 & 86.3\end{array}$

$\begin{array}{lllllllll}9 \theta & 5.70 & 79 & 200 & 235 & 93 & 0.59 & 80.1\end{array}$

$\begin{array}{llllllll}10 \theta & 6.00 & 137 & 215 & 268 & 164 & 1.04 & 64.8\end{array}$

110

$\stackrel{\varphi}{\omega}$

$\begin{array}{llllllll}12 \mathrm{ne} & 7.75 & 310 & 219 & 298 & 421 & 2.68 & 9.7\end{array}$

$\begin{array}{cccccc}\text { Ave } & 6.78 & 257 & 157 & 1.00 & 66.3 \\ \text { West } & 7.38 & 263 & 148 & 0.94 & 68.2 \\ \text { East } & 6.07 & 251 & 168 & 1.07 & 64.0\end{array}$

Average Inlet/Outlet Gas Measurements

$22 \mathrm{SO} 2 \mathrm{NO} \mathrm{H} 2 \mathrm{O} \mathrm{NOC} \mathrm{SO} 2 \mathrm{C} \mathrm{SO}_{2}$

\%owet ppm ppm \% ppmedppmed \%

$\begin{array}{lllllll}\text { Inlet } & 4.90 & 370 & 230 & 8.67 & 290 & 466\end{array}$

$\begin{array}{llllllll}\text { Stack } & 6.30 & 120 & 180 & 7.81 & 248 & 165 & 64.5\end{array}$
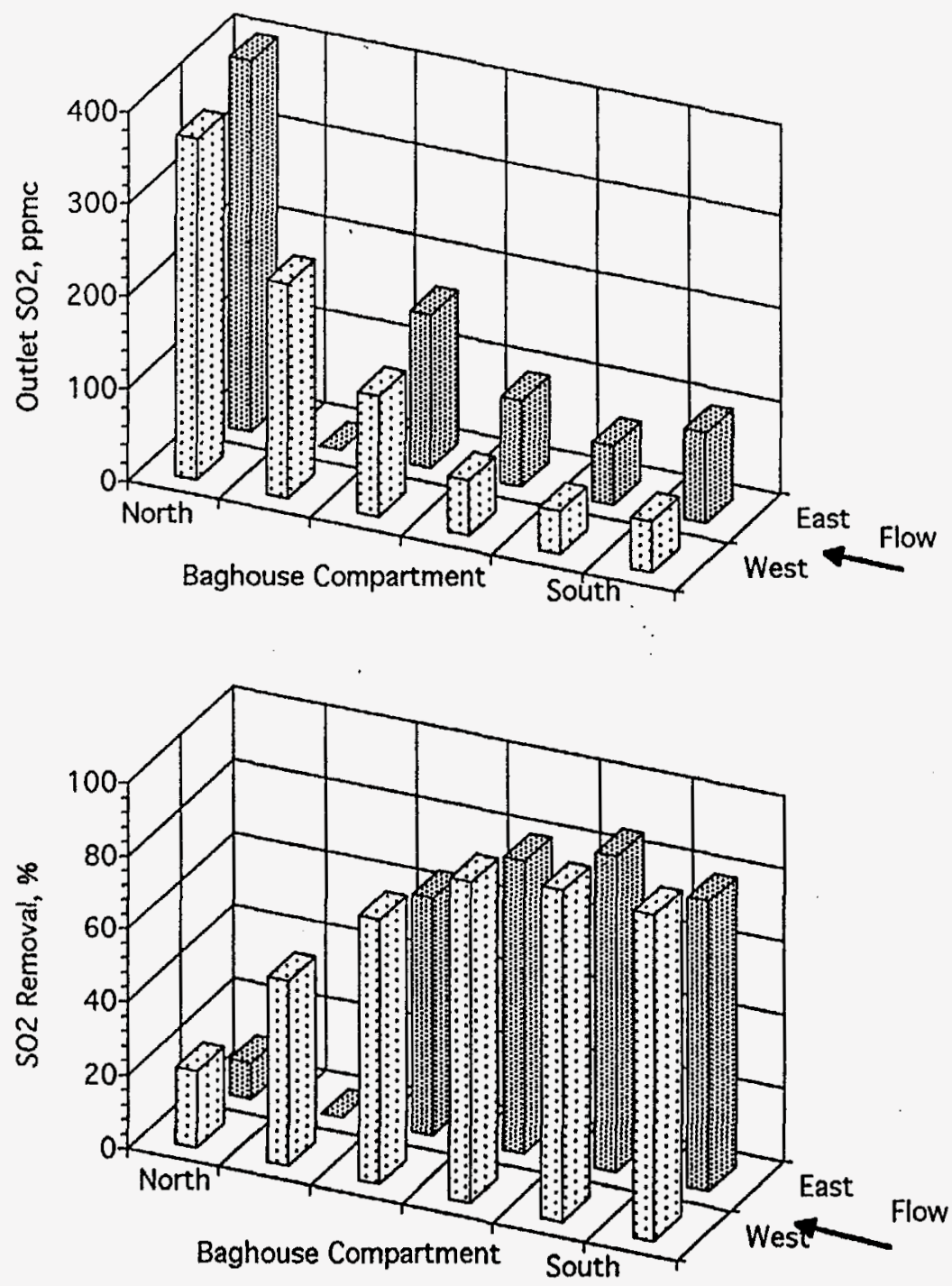

Figure 5-23. Compartment-by-Compartment $\mathrm{SO}_{2}$ Removals for Sodium Bicarbonate Injection Ahead of the FFDC (Test 763) 
sesquicarbonate injection (Figure 5-8), although the peak removals are greater with sodium bicarbonate. The results in Figure 5-23 also show that the removals in the east compartments are nominally ten percent lower than the removals in the corresponding compartments on the west side. This difference is likely due to a bias in the injection system which resulted from a partial plugging of some of the east side injectors.

B. $\mathrm{NO}_{x}$ Removal and $\mathrm{NO}_{2}$ Emissions. Figure 5-24 shows the $\mathrm{NO}_{\mathrm{x}}$ removal and $\mathrm{NO}_{2}$ emission traces recorded over the five-day test period. The average $\mathrm{NO}_{x}$ removal for the test was 10 percent. Although this result is consistent with the levels reported in the earlier work at Cameo (Muzio, et al., 1984), the individual measurements varied widely (from 0 to 25 percent) with no discernable correlation with either FFDC cleaning cycle or temperature. The $\mathrm{NO}_{2}$ emissions also varied widely during the test, ranging from 6 to 33 $\mathrm{ppm}$, with an average of $16 \mathrm{ppm}$. Two trends can be seen in the $\mathrm{NO}_{2}$ data shown in Figure 5-24. First, in all instances but one, there is a sharp increase in $\mathrm{NO}_{2}$ emissions after a FFDC cleaning cycle, followed by a slow decrease in emissions until the next cleaning. This is currently thought to be again due to an interaction between $\mathrm{NO}_{2}$ and the carbon in the fly ash on the bags. When the FFDC is cleaned, there is less fly ash on the bags and, therefore, less absorption. As the cake builds back up after a cleaning cycle, the absorption of $\mathrm{NO}_{2}$ increases. Secondly, the data indicate that there was a general increasing trend in $\mathrm{NO}_{2}$ emissions throughout the duration of the five-day test. The $\mathrm{NO}_{2}$ emissions for the first 40 hours were quite variable. This was partially due to variations in load and $2 \mathrm{Na} / \mathrm{S}$ ratio. However, after 40 hours a continuously increasing trend in $\mathrm{NO}_{2}$ can be seen. Again, no explanation can be offered for this observation.

\subsubsection{Sodium Bicarbonate Injection at the Air Heater Inlet}

A. $\mathrm{SO}_{2}$ Removal. The previous demonstration at PSCo's Cameo station (Muzio et al., 1984) showed that the reduced reactivity of sodium bicarbonate at low baghouse temperatures, can be compensated for by injecting the material at higher temperatures, such as those encountered at the air heater inlet. After it became apparent that flue gas temperatures at the Arapahoe Unit 4 FFDC inlet were limiting the process performance 


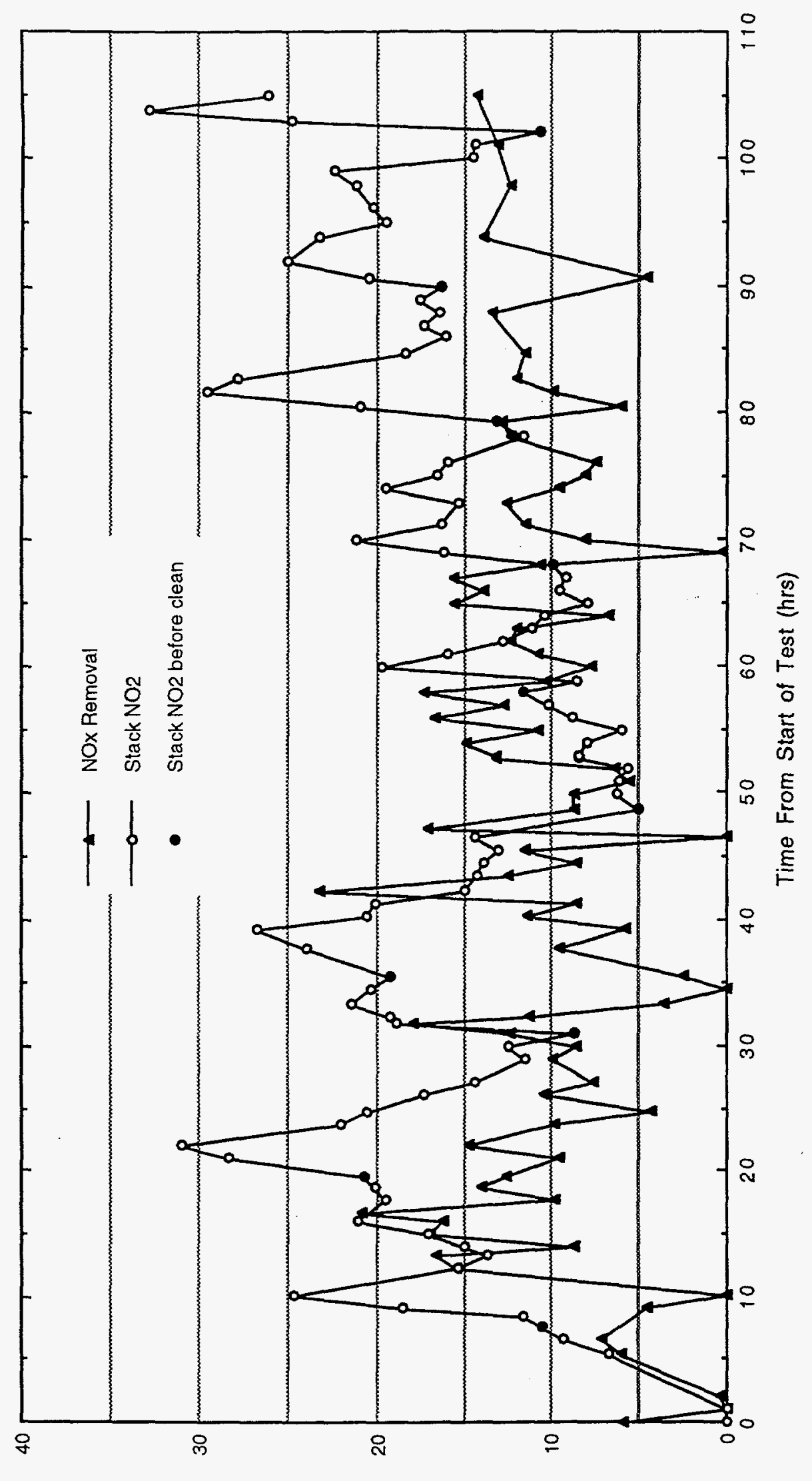

ह

ถั ถึ

$\Phi$

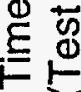

응

这

동

등 흥

올 뭄

шั

일

즘 읗

ธั

조

을

ह

ᄄᄄ

$\mathrm{O}^{\times} \cdot \frac{\bar{d}}{\mathrm{n}}$

竎

(mdd) ZON YOEIS '(\%) je^OUAY XON 
with sodium bicarbonate, the sorbent injection location was moved to a higher temperature zone ahead of the air heater. Four injectors were installed in the top of the duct between the economizer and air heater, as described previously (Section 3.4.3).

Figure 5-25 shows $\mathrm{SO}_{2}$ removals measured at both the baghouse inlet and stack for a test run with the new injection location and a nominal $2 \mathrm{Na} / \mathrm{S}$ ratio of 1.0. The stack data show that the $\mathrm{SO}_{2}$ removal response time is much improved over that seen previously for injection at the FFDC inlet (Figures 5-18 and 5-20). The initial response time was reduced to approximately $1-1 / 2$ hours, and the recovery time after subsequent cleaning cycles was reduced to less than 30 minutes. These results indicate that the endothermic decomposition of sodium bicarbonate (recall Equation 3-1 in Section 3.1) is likely responsible for the temperature sensitivity discussed previously for sorbent injection at the FFDC inlet.

Gaseous measurements at the baghouse inlet show that $\mathrm{SO}_{2}$ removals of nominally 20 percent occur in the "entrained" phase before the sorbent is deposited on the bags. However, it must be noted that these measurements were made through sampling probes with in-duct filters, and although the filters were "blown-back" with compressed air before each measurement, the sorbent which collects on the filters during sampling could scrub $\mathrm{SO}_{2}$ from the sample stream. Therefore, it is possible that the $\mathrm{SO}_{2}$ removals measured at this location are biased slightly on the high side. Irrespective of whether the entrained removals are 20 percent, or less, the results indicate that the majority of the sulfation reaction occurs within the baghouse.

Figures 5-26 and 5-27 show the effect of $2 \mathrm{Na} / \mathrm{S}$ ratio on $\mathrm{SO}_{2}$ removal and sorbent utilization for injection ahead of the air heater. Data for both the 4000 and $5000 \mathrm{rpm}$ pulverizer speeds are plotted in each figure. The data in Figure 5-26 show that at $2 \mathrm{Na} / \mathrm{S}$ ratios up to approximately 1.0, pulverizer speed has little effect on $\mathrm{SO}_{2}$ removal. However, at $2 \mathrm{Na} / \mathrm{S}$ ratios in excess of 1.5 , the $\mathrm{SO}_{2}$ removals at $5000 \mathrm{rpm}$ continue to increase, while the $4000 \mathrm{rpm}$ data begins to level out. If the pulverized reagent size increased as the feed rate decreased from 5000 to $4000 \mathrm{rpm}$, an explanation of this data would be possible; 


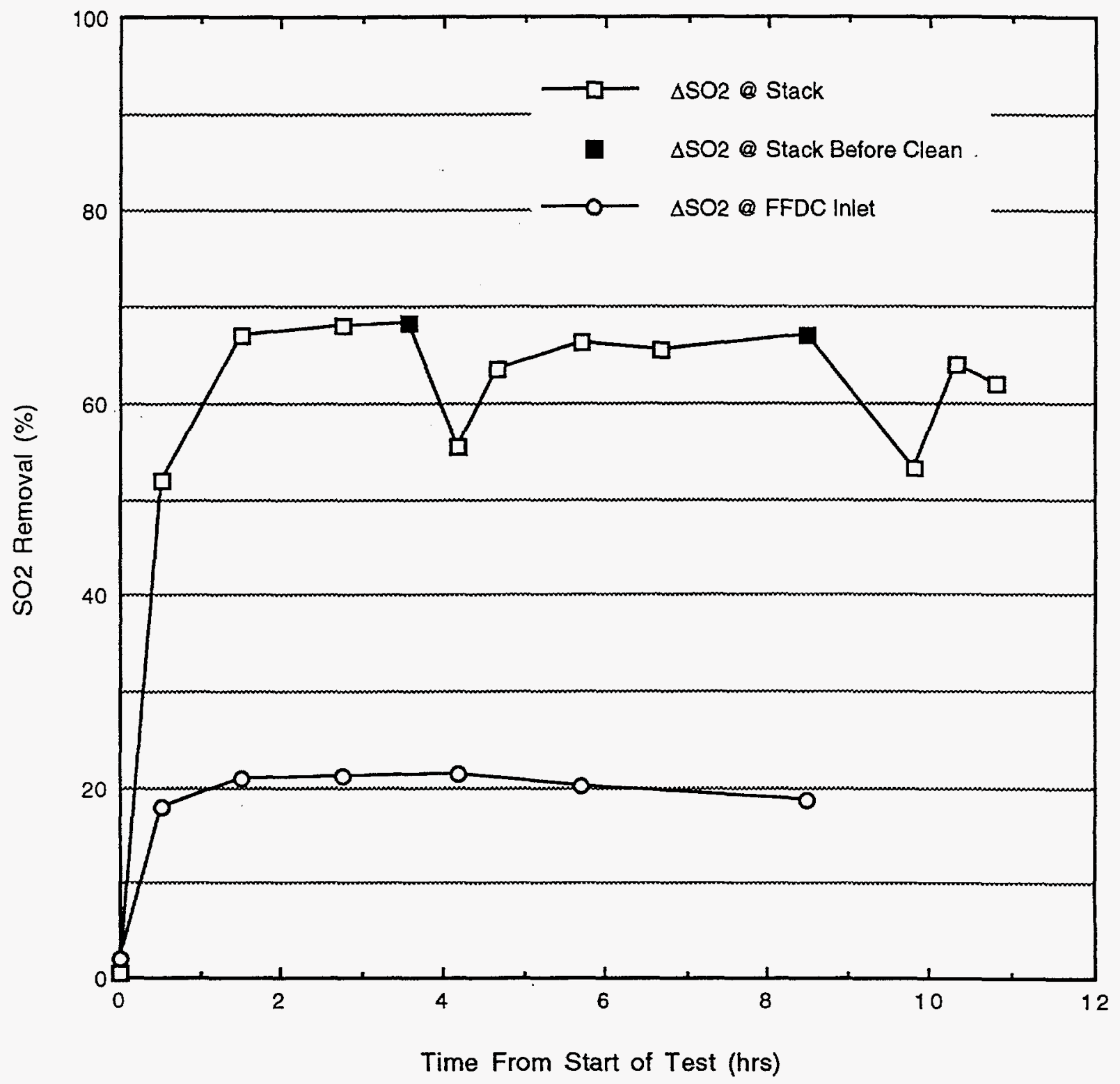

Figure 5-25. $\mathrm{SO}_{2}$ Removal Versus Time for Sodium Bicarbonate Injection Ahead of the Air Heater (Test $792,2 \mathrm{Na} / \mathrm{S}=1.0$ ) 


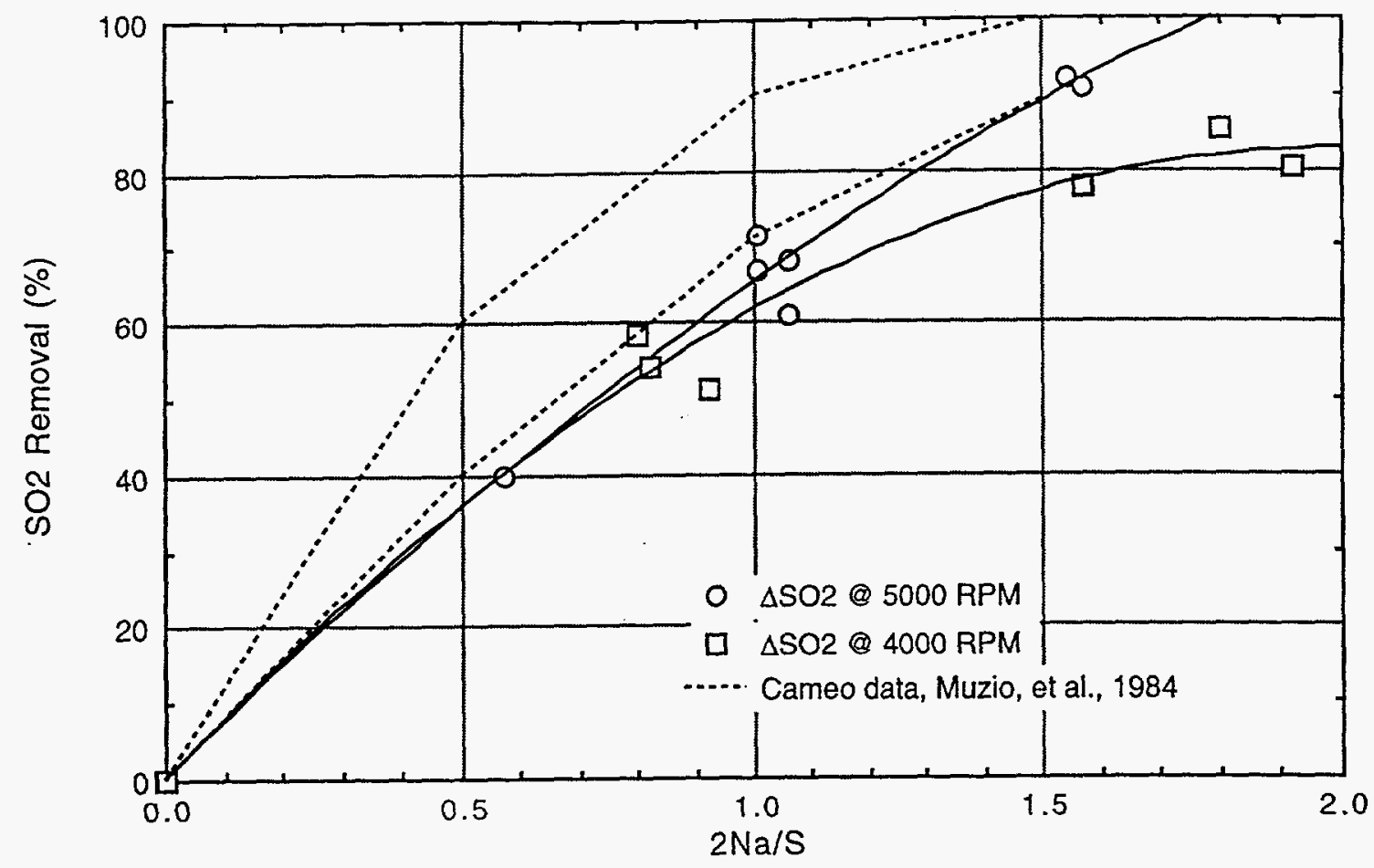

Figure 5-26. $\mathrm{SO}_{2}$ Removal as a Function of $2 \mathrm{Na} / \mathrm{S}$ Ratio for Sodium Bicarbonate Injection Ahead of the Air Heater

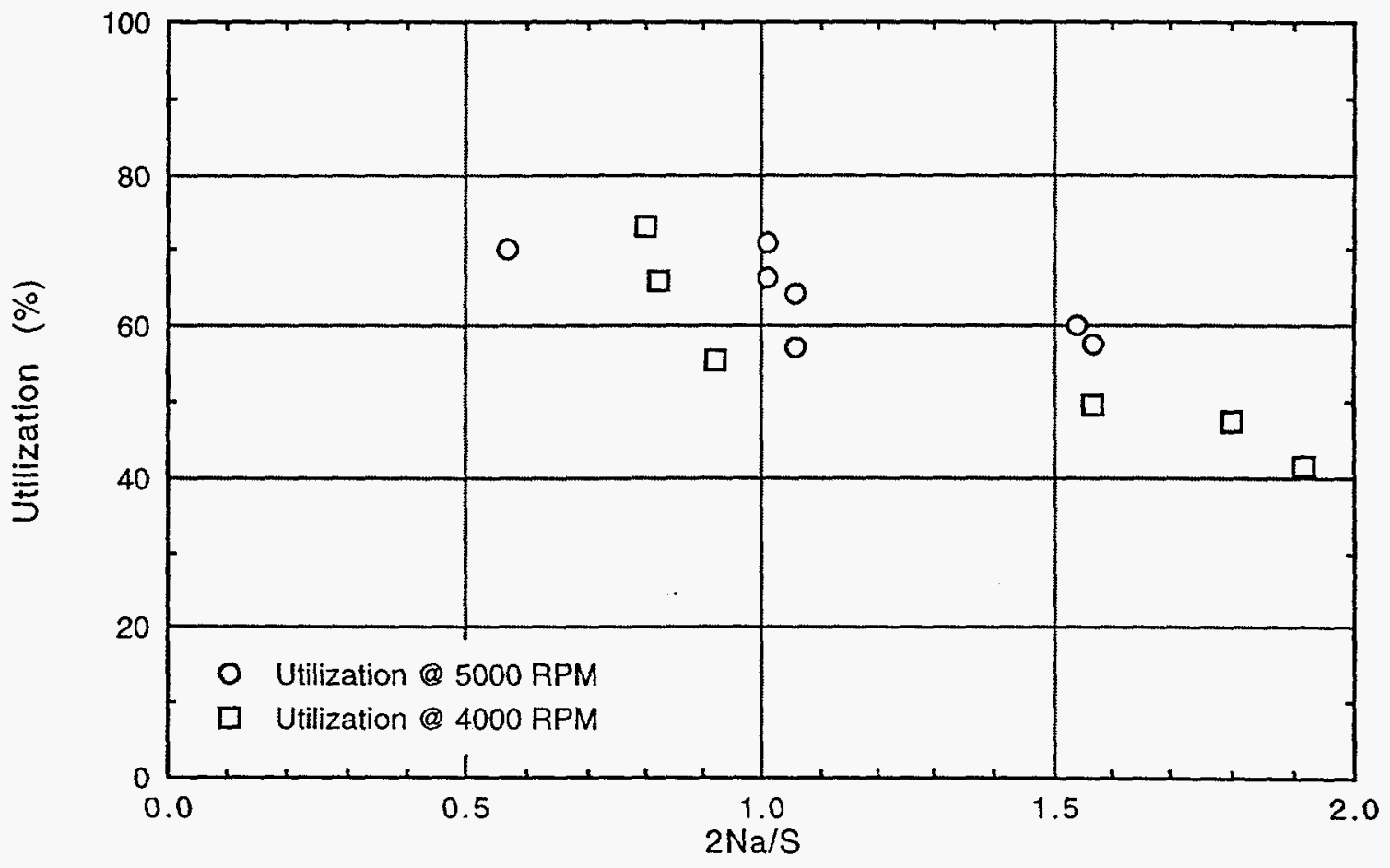

Figure 5-27. Utilization as a Function of $2 \mathrm{Na} / \mathrm{S}$ Ratio for Sodium Bicarbonate Injection Ahead of the Air Heater 
since larger particles are less reactive. However, the data presented previously in Table 5-2 for sodium sesquicarbonate indicated only a minor change in particle size with increased loading at $4000 \mathrm{rpm}$. It is possible that the large raw product size of sodium bicarbonate ( $60 \mu$ for sodium bicarbonate versus $28 \mu$ for sodium sesquicarbonate) would exhibit a larger feed rate effect, but this was not tested.

The $\mathrm{SO}_{2}$ removal data shown previously for sodium bicarbonate injection at the FFDC inlet (Figure 5-21) were collected at the $5000 \mathrm{rpm}$ pulverizer speed. Comparison of this data to the $5000 \mathrm{rpm}$ data in Figure 5-26 shows that the $\mathrm{SO}_{2}$ removals for injection at the FFDC inlet are slightly higher than those for injection ahead of the air heater (approximately 75 percent versus 66 percent at a nominal $2 \mathrm{Na} / \mathrm{S}$ ratio of 1.0 ). It is believed that the higher removals are due to the difference in the rate of endothermic decomposition of sodium bicarbonate. During decomposition, the evolution of $\mathrm{H}_{2} \mathrm{O}$ and $\mathrm{CO}_{2}$ creates a high surface area $\mathrm{Na}_{2} \mathrm{CO}_{3}$ particle. When injecting ahead of the air heater, the decomposition reaction occurs quickly at the higher temperatures, and the sorbent is more decomposed by the time it reaches the bags. As the sulfation reaction progresses, it becomes limited by the diffusion rate of $\mathrm{SO}_{2}$ through the $\mathrm{Na}_{2} \mathrm{SO}_{4}$ product layer which forms on the particle surface. Without further decomposition to expose additional unreacted sorbent, utilization is decreased. When the sorbent is injected at the inlet of the FFDC, however, the decomposition and sulfation reactions occur simultaneously. It is hypothesized that the release of $\mathrm{H}_{2} \mathrm{O}$ and $\mathrm{CO}_{2}$ from the interior of the particle provides a means of maintaining an open pore structure during the sulfation process, which allows the $\mathrm{SO}_{2}$ to reach the unreacted $\mathrm{Na}_{2} \mathrm{CO}_{3}$ more readily. Thus, the sulfation process is less limited by diffusion across the solid $\mathrm{Na}_{2} \mathrm{SO}_{4}$ product layer.

Figure 5-28 shows the compartment-by-compartment gaseous emission measurements for sodium bicarbonate injection ahead of the air heater measured during the test depicted in Figure 5-25. These compartment-by-compartment data were collected between hours 7 and 8 of the test (i.e., just before the second fabric filter cleaning cycle). The front-toback distribution of $\mathrm{SO}_{2}$ removal is similar, but somewhat improved, to that seen for injection at the FFDC inlet (Figure 5-23), indicating that the majority of the sorbent is 

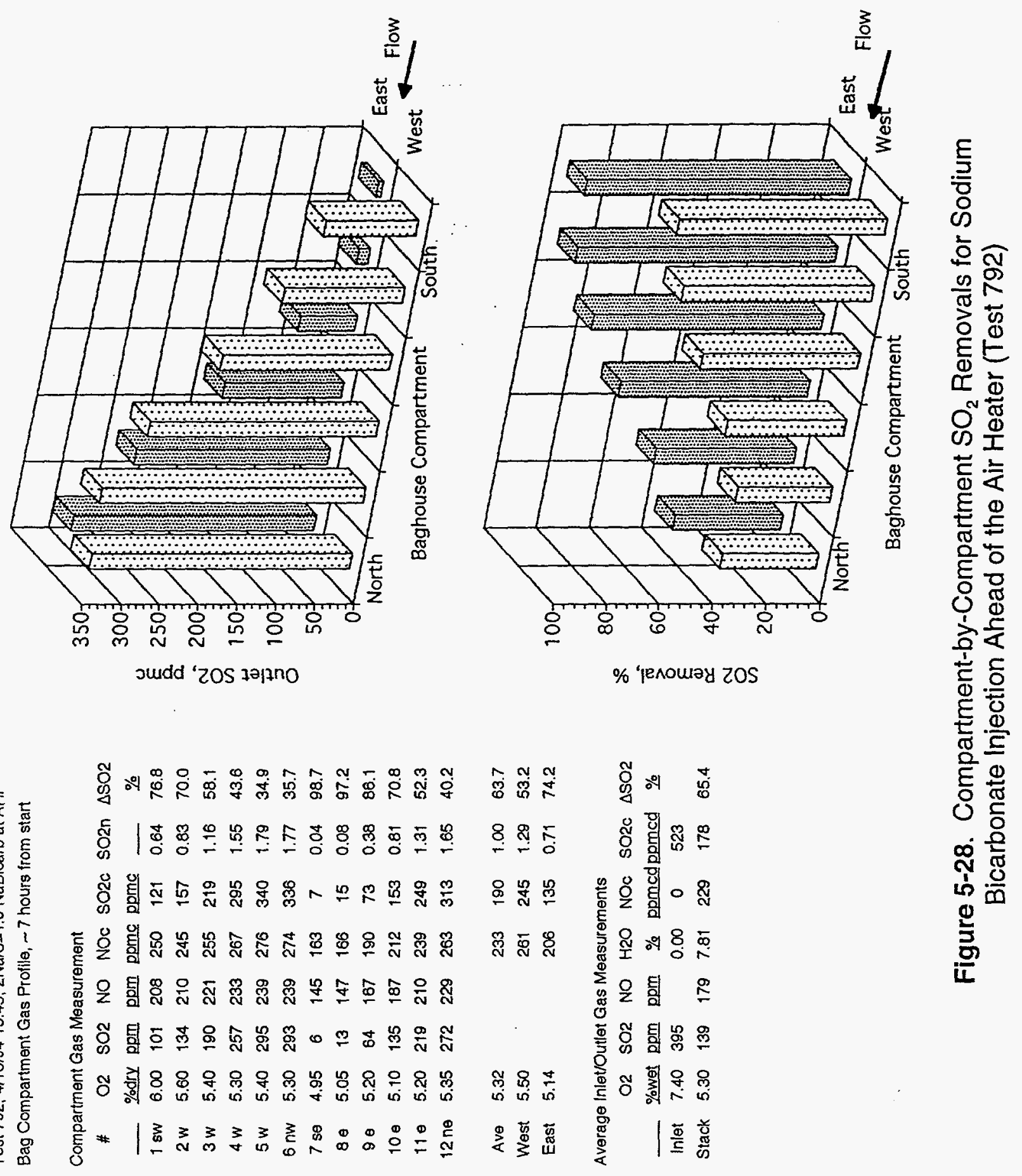
deposited in the forward compartments. The improvement in $\mathrm{SO}_{2}$ removal in the back compartments is due to the additional $\mathrm{SO}_{2}$ removal that occurred in the duct due to the higher temperature and longer residence time. The differences in $\mathrm{SO}_{2}$ removal on the east and west sides are again likely due to an imbalance in the sorbent distribution between individual injectors. All four injectors were supplied by a single distributor located on the east side of the boiler. Four individual hoses ran from the distributor, up the side of the boiler, and across the top of the horizontal duct between the economizer and air heater. Thus, the hoses feeding the two injectors on the west side had long horizontal runs from the east edge of the duct and high pressure drop. This created an imbalance in flow that would also affect reagent distribution.

As discussed previously, it can be noted that the arithmetic average of the compartmentby-compartment $\mathrm{SO}_{2}$ removals is in good agreement with the overall $\mathrm{SO}_{2}$ removal measured across the fabric filter. This indicates that the gas flow rates to each compartment were approximately equal.

B. $\mathrm{NO}_{x}$ Removals and $\mathrm{NO}_{2}$ Emissions. Figure 5-29a shows the $\mathrm{NO}_{x}$ removal and $\mathrm{NO}_{2}$ emission traces for the 11-hour test depicted in Figure 5-25. As was seen with sorbent injection ahead of the FFDC, the $\mathrm{NO}_{2}$ emissions increase after each cleaning cycle. However, unlike the behavior seen at the low-temperature injection location, the $\mathrm{NO}_{2}$ levels were relatively constant between cleaning cycles. Again, on the average, the $\mathrm{NO}_{2}$ emission level continued to increase throughout the duration of the test. The average $\mathrm{NO}_{\mathrm{x}}$ removal for the entire test is 8 percent, but the removals decrease with time in accordance with the increases in $\mathrm{NO}_{2}$ emissions. Initially, the $\mathrm{NO}_{x}$ removal was relatively constant at 12 percent, with $\mathrm{NO}_{2}$ emissions averaging $14 \mathrm{ppm}$. After the first cleaning cycle, the average $\mathrm{NO}_{x}$ removal decreased to 7 percent, and $\mathrm{NO}_{2}$ emissions increased to nominally $27 \mathrm{ppm}$. After the second cleaning cycle, average $\mathrm{NO}_{x}$ removals and $\mathrm{NO}_{2}$ emissions were 4 percent and $39 \mathrm{ppm}$, respectively.

Figure 5-29b shows how the change in NO due to the reactions with sodium is partitioned between $\mathrm{NO}_{2}$ and $\mathrm{NO}_{\mathrm{x}}$ removal. The trend seen for this test with sodium bicarbonate is somewhat different than the data shown in Figure 5-9b. 


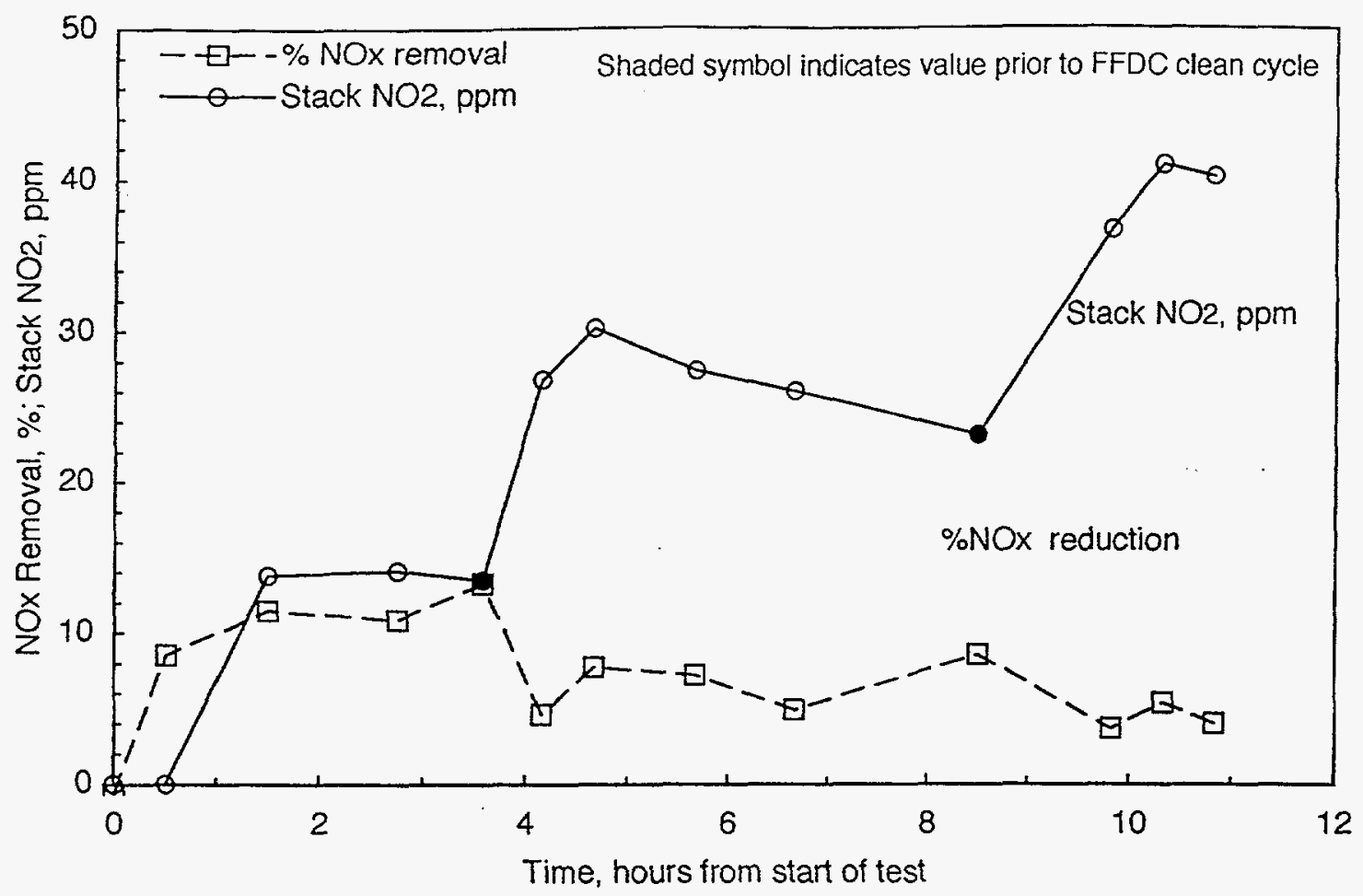

(a) $\mathrm{NO}_{2}, \mathrm{ppm} ; \Delta \mathrm{NO}_{\mathrm{x}} \%$

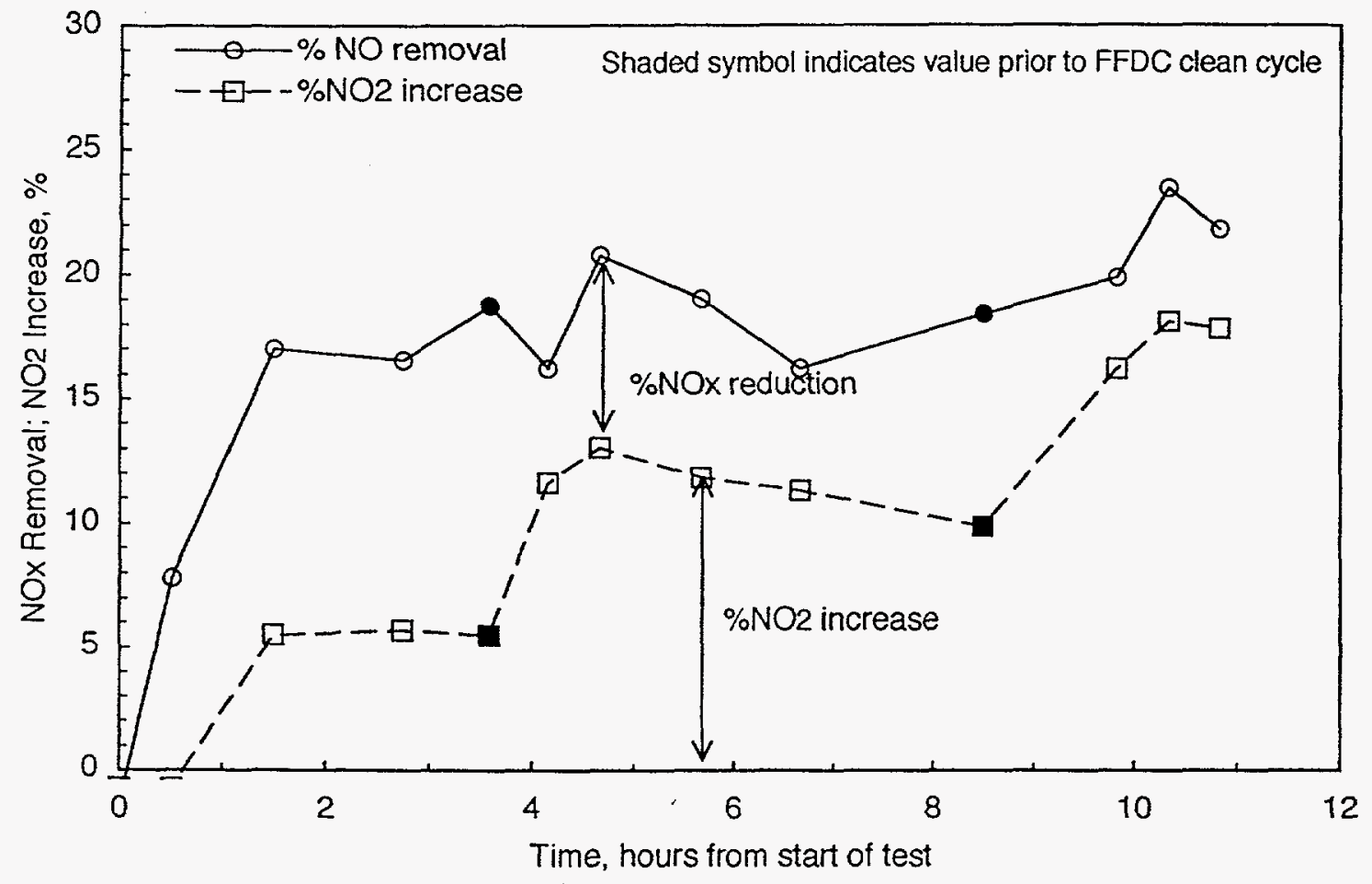

(b) Partitioning of $\triangle \mathrm{NO}$ between $\mathrm{NO}_{2}$ and $\Delta \mathrm{NO}_{\mathrm{x}}$

Figure 5-29. $\mathrm{NO}_{\mathrm{x}}$ Removal and $\mathrm{NO}_{2}$ Emissions Versus Time for Sodium Bicarbonate Injection Ahead of the Air Heater (Test 792) 
With sodium sesquicarbonate, the majority of the change in $\mathrm{NO}$ showed up as $\mathrm{NO}_{\mathrm{x}}$ removal and this trend remained fairly constant with time. However, with sodium bicarbonate, injection ahead of the air preheaters, Figure 5-29b shows that early in the test, $\mathrm{NO}_{\mathrm{x}}$ removal is favored over $\mathrm{NO}_{2}$ emissions. As the test proceeded through two fabric cleaning cycles, the trend shifted and the majority of the $\mathrm{NO}$ that reacted showed up as $\mathrm{NO}_{2}$ with a much lesser fraction as $\mathrm{NO}_{x}$ removal.

Further insight into the $\mathrm{NO}_{\mathrm{x}}$ removal and $\mathrm{NO}_{2}$ formation processes can be gained by looking at the compartment-by-compartment measurements. Figure 5-28 showed the $\mathrm{SO}_{2}$ removals occurring in the individual compartments for sodium bicarbonate injection ahead of the air heater. Figure 5-30 shows the compartment-by-compartment $\mathrm{NO}_{x}$ removals and $\mathrm{NO}_{2}$ emissions for the same test. In general, the $\mathrm{NO}_{x}$ removal and $\mathrm{NO}_{2}$ levels were higher on the east side of the fabric filter where the $\mathrm{SO}_{2}$ removals were also higher. However, there are some observations that are noteworthy. For instance, the $\mathrm{NO}_{x}$ removal and $\mathrm{NO}_{2}$ emissions do not necessarily directly correlate with the $\mathrm{SO}_{2}$ removals. This can be illustrated by looking at the compartment-by-compartment data in terms of what fraction of the NO that reacts appears as $\mathrm{NO}_{2}$, or results in $\mathrm{NO}_{x}$ removal. The data in Figure 5-30 have been replotted on this basis in Figure 5-31 where the total height of the bars represent the percentage of the NO that has reacted in each compartment. The dark shading represents the $\mathrm{NO}_{2}$ and the lighter shading represents the $\mathrm{NO}_{x}$ removal. On the west side, the majority of the $\mathrm{NO}$ that reacted showed up as $\mathrm{NO}_{2}$ with little $\mathrm{NO}_{x}$ removal. On the east side, the compartments near the entrance exhibited more $\mathrm{NO}_{2}$ than $\mathrm{NO}_{\mathrm{x}}$ removal. It is interesting to compare compartments that exhibited similar levels of $\mathrm{SO}_{2}$ removal. For instance, compartments 2 (west) and 10 (east) both had $\mathrm{SO}_{2}$ removals of $70 \%$. Yet, the $\mathrm{NO}_{2}$ level and $\mathrm{NO}_{x}$ removals in compartment 10 were almost double that of 44 and $40 \%$, respectively. For compartment 4 , there was essentially no $\mathrm{NO}_{\mathrm{x}}$ removal and the $\mathrm{NO}_{2}$ levels were $16 \mathrm{ppm}$, whereas for compartment 12 , there was $14 \% \mathrm{NO}_{\mathrm{x}}$ removal and $\mathrm{NO}_{2}$ levels of $32 \mathrm{ppm}$.

It has been observed that the two compartments at the entrance to the fabric filter (Numbers 1 and 7) collect the largest amount of ash. These compartments also capture 
Test 792, 4/18/94 15:45, 2Na/S=1.0 NaBicarb at AHI Bag Compartment Gas Profile, $\sim 7$ hours trom start

Compartment Gas Measurement

\# $\quad 02$ NO NO2 NOx NOxc NOxn $\triangle \% N O x \Delta \% N O 2$

- \%dry ppm ppm ppm ppmc _ \% \%

$\begin{array}{lllllllll}1 \mathrm{sw} & 6.00 & 208 & 22.7 & 231 & 277 & 1.1 & 1.2 & 9.7\end{array}$

$\begin{array}{lllllllll}2 w & 5.60 & 210 & 17.5 & 228 & 266 & 1.0 & 5.1 & 7.3\end{array}$

$\begin{array}{lllllllll}3 w & 5.40 & 221 & 12.4 & 233 & 269 & 1.0 & 3.9 & 5.1\end{array}$

$\begin{array}{lllllllll}4 \mathrm{~W} & 5.30 & 233 & 10.6 & 244 & 279 & 1.1 & 0.3 & 4.3\end{array}$

$\begin{array}{lllllllll}5 W & 5.40 & 239 & 9.3 & 248 & 287 & 1.1 & -2.3 & 3.8\end{array}$

$\begin{array}{lllllllll}6 n w & 5.30 & 239 & 6.8 & 246 & 282 & 1.1 & -0.6 & 2.8\end{array}$

$\begin{array}{lllllllll}7 \mathrm{se} & 4.95 & 145 & 30.4 & 175 & 197 & 0.8 & 29.8 & 12.2\end{array}$

$\begin{array}{lllllllll}88 & 5.05 & 147 & 39.8 & 187 & 211 & 0.8 & 24.8 & 16.0\end{array}$

$\begin{array}{lllllllll}9 \theta & 5.20 & 167 & 44.0 & 211 & 240 & 0.9 & 14.2 & 17.9\end{array}$

$\begin{array}{lllllllll}10 \theta & 5.10 & 187 & 38.8 & 226 & 256 & 1.0 & 8.8 & 15.7\end{array}$

$\begin{array}{lllllllll}11 \theta & 5.20 & 210 & 25.4 & 235 & 268 & 1.0 & 4.3 & 10.3\end{array}$

or

$\begin{array}{lllllllll}12 \text { ne } & 5.35 & 229 & 12.2 & 241 & 277 & 1.1 & 1.0 & 5.0\end{array}$

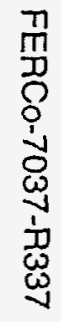
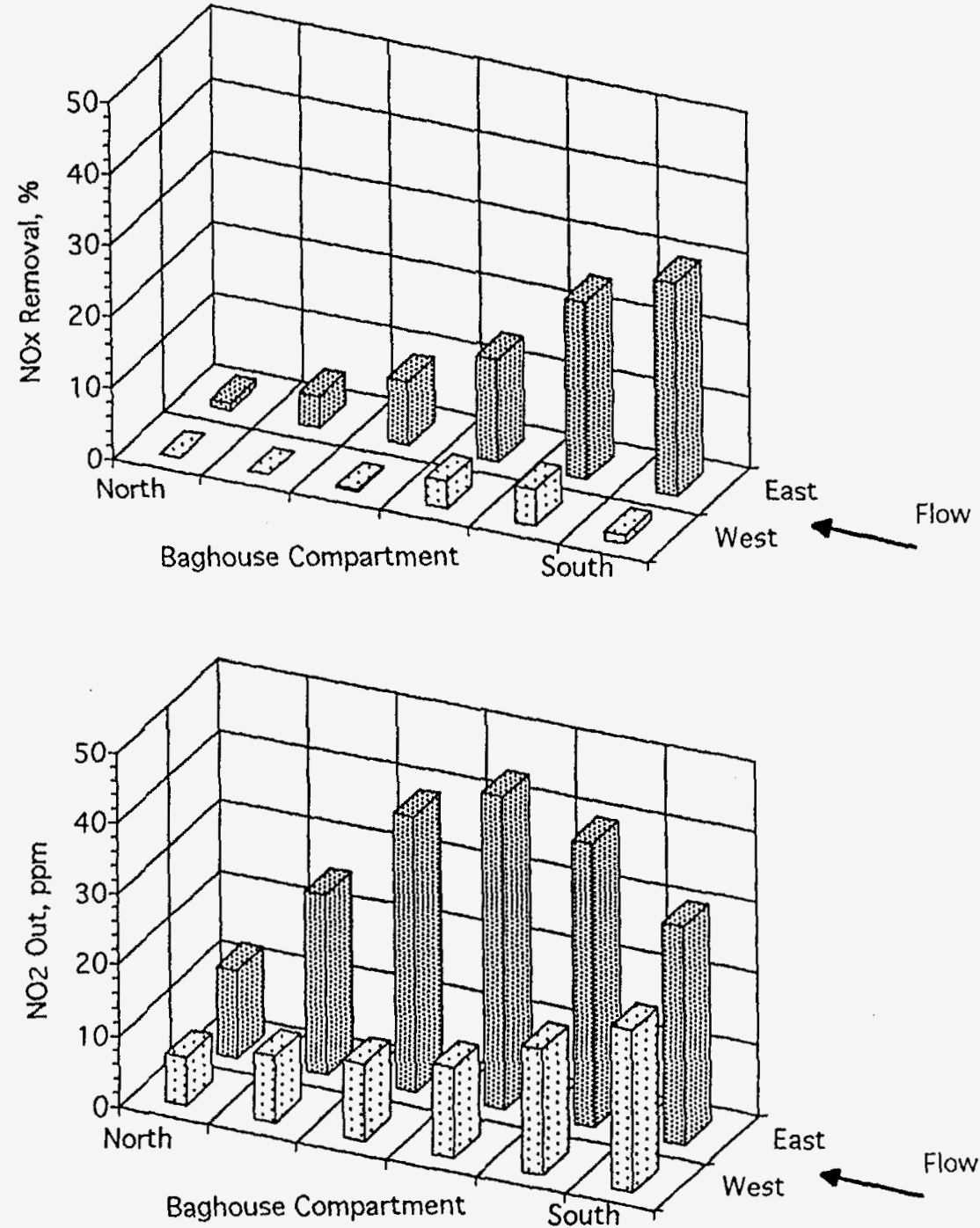

Figure 5-30. Compartment-by-Compartment NO Removals for Sodium Bicarbonate Injection Ahead of the Air Heater (Test 792) 


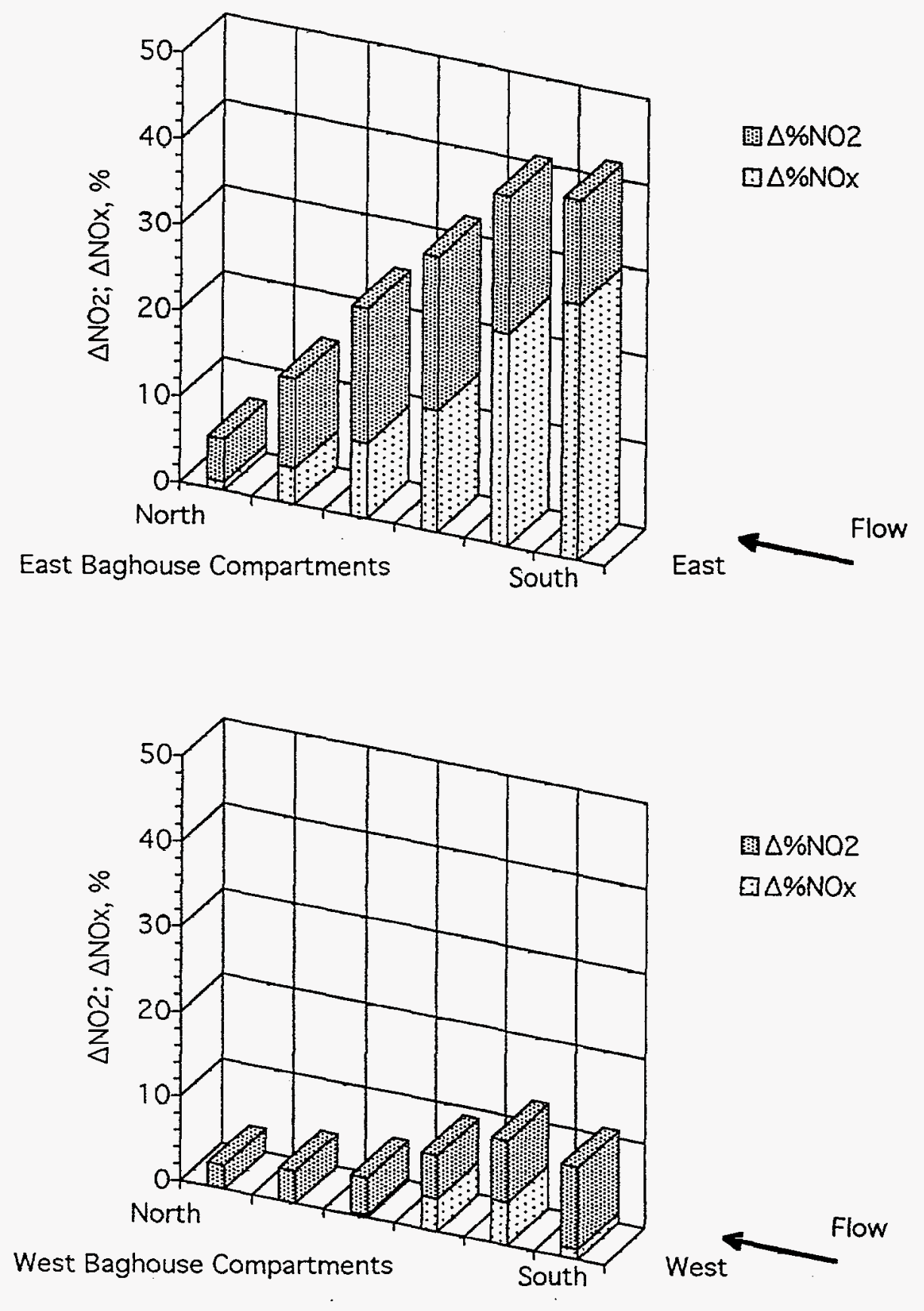

Figure 5-31. Compartment-by-Compartment $\mathrm{NO}_{2}$ Emissions for Sodium Bicarbonate Injection Ahead of the Air Heater (Test 792) 
the largest particles, which will also tend to have the highest carbon content. This could account for the lower $\mathrm{NO}_{2}$ emissions even though the $\mathrm{SO}_{2}$ and $\mathrm{NO}_{x}$ removals are highest in these two compartments.

The differences in the trends between Figure 5-9b and Figure 5-29b, the differences in compartment-by-compartment in Figures 5-30 and 5-31 coupled with the increases in $\mathrm{NO}_{2}$ following a fabric filter cleaning cycle illustrate the complexity of the sodium/ $/ \mathrm{SO}_{2} / \mathrm{NO}_{x}$ chemistry. The reader is referred to Appendix A which reports on the laboratory work completed to better understand the chemistry of $\mathrm{NO}_{2}$ generation and $\mathrm{NO}_{\mathrm{x}}$ reduction that is obtained during sodium injections. However, in addition to the detailed chemistry between sodium, $\mathrm{SO}_{2}$, and $\mathrm{NO}$, there appears to be an interaction with fly ash carbon that is currently not well understood.

The $\mathrm{NO}_{2}$ emissions for all of the sodium bicarbonate tests at both injection locations (ahead of the FFDC and ahead of the air heater) are summarized in Figure 5-32. Again, the scatter in the data is attributable to the dependence of $\mathrm{NO}_{2}$ emissions not only on the amount of sodium injected, but also on the fly ash and FFDC cleaning cycle. As seen with sodium sesquicarbonate, there is no clear difference in the amount of $\mathrm{NO}_{2}$ produced at each injection location. The data in the $2 \mathrm{Na} / \mathrm{S}$ range of 0.9 to 1.1 have been replotted in Figure 5-33 as a function of the time from the end of a fabric filter cleaning cycle. This presentation of the data shows that the large variations in $\mathrm{NO}_{2}$ shown in Figure 5-32 occur just after a cleaning cycle. At longer time periods after a cleaning cycle, the $\mathrm{NO}_{2}$ levels trend toward a steady state level of 10-20 ppm.

The $\mathrm{NO}_{x}$ removals with sodium bicarbonate are summarized in Figure 5-34 for both injection ahead of the FFDC and ahead of the air heater. Again, a fair amount of scatter in the data is seen, which is attributed to the process dynamics. Overall $\mathrm{NO}_{\mathrm{x}}$ removals at a nominal $2 \mathrm{Na} / \mathrm{S}$ ratio of 1.0 ranged from 0 to 20 percent, and averaged roughly 10 percent. These levels of $\mathrm{NO}_{\mathrm{x}}$ removal are consistent with previously reported results from the Cameo and Nixon demonstrations (Muzio, et al., 1984; Fuchs, et al., 1989). 


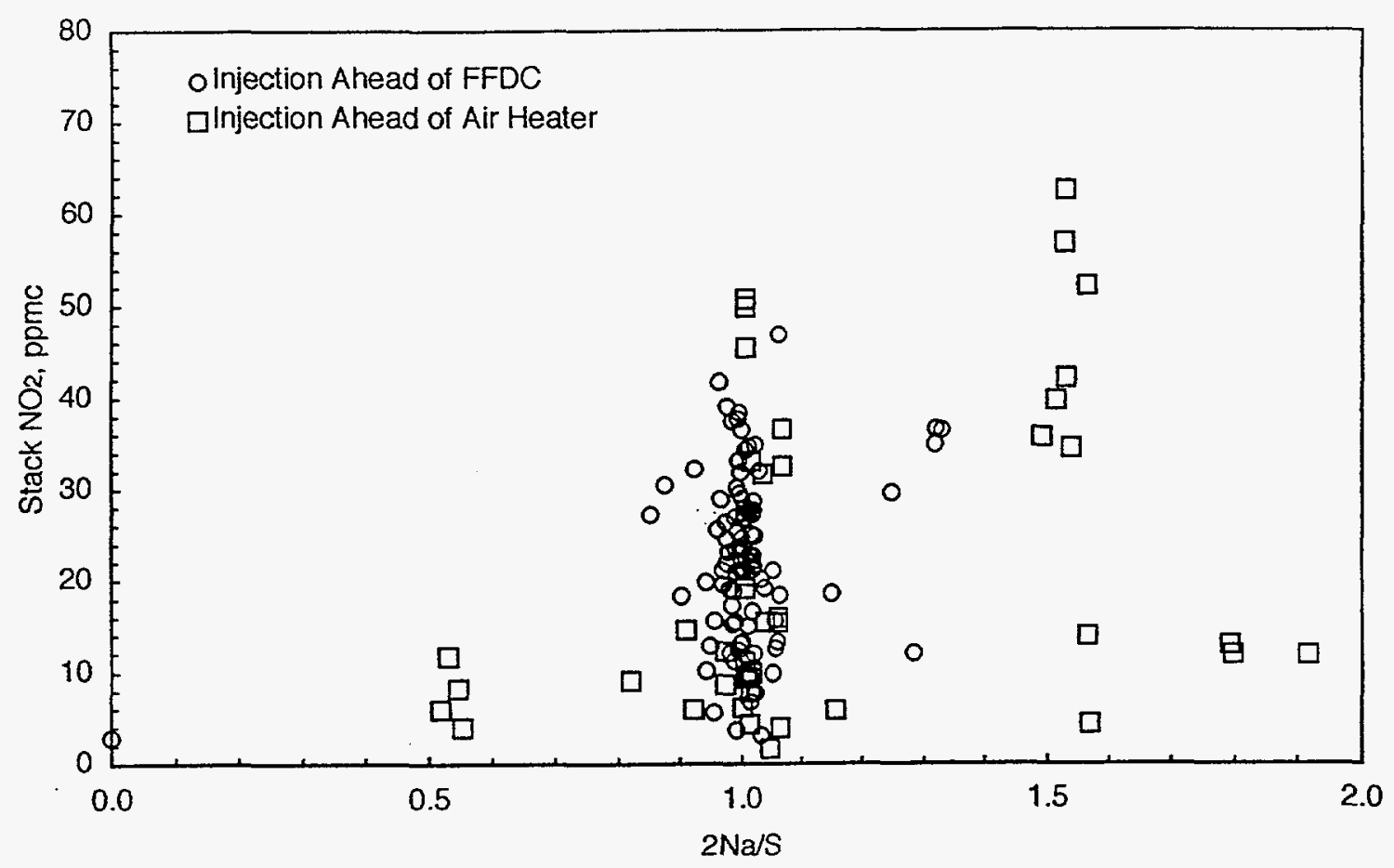

Figure 5-32. Summary of $\mathrm{NO}_{2}$ Emissions with Sodium Bicarbonate Injection

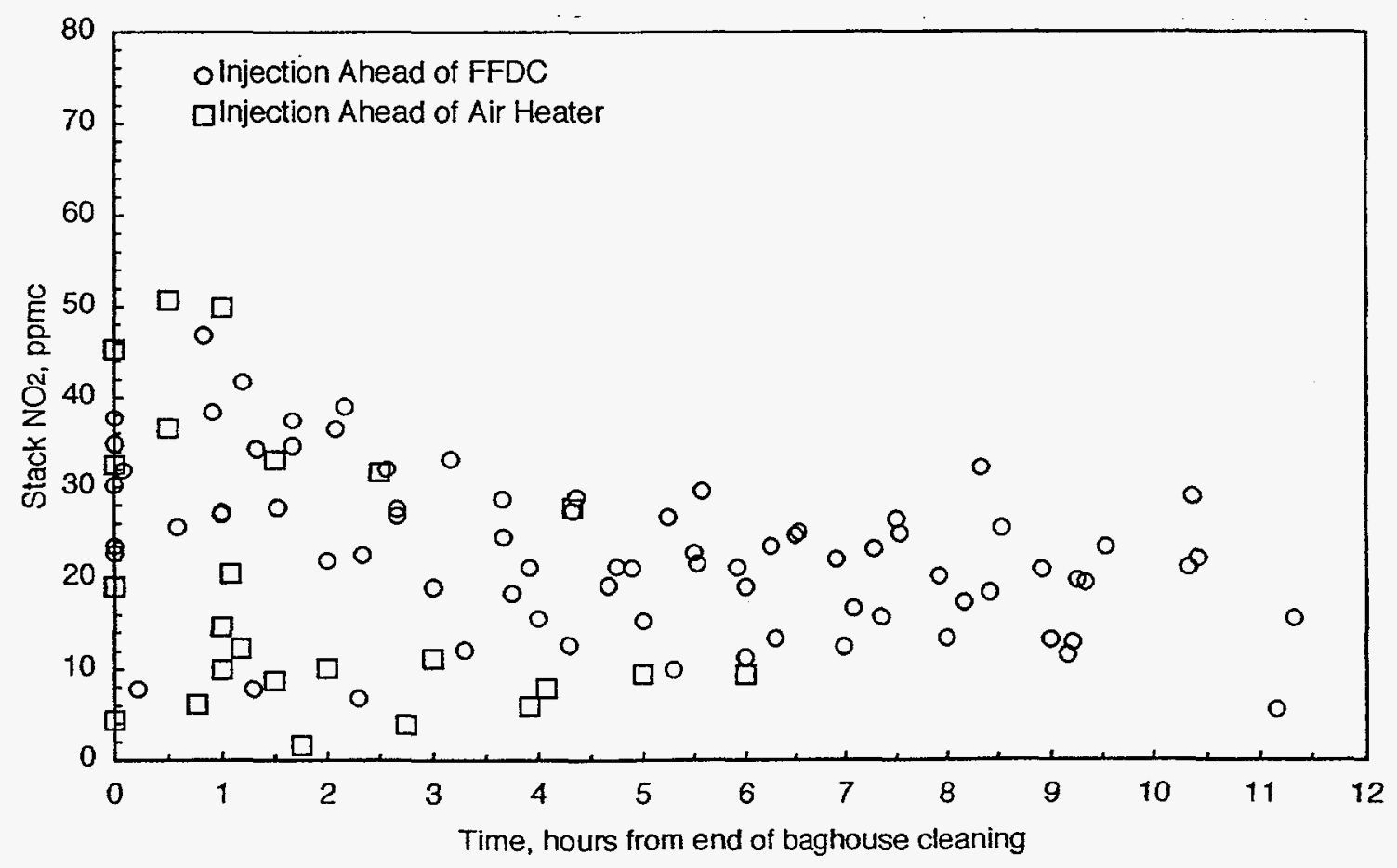

Figure 5-33. $\mathrm{NO}_{2}$ Emissions and FFDC Cleaning with Sodium Bicarbonate (2Na/S 0.9 to 1.1$)$ 


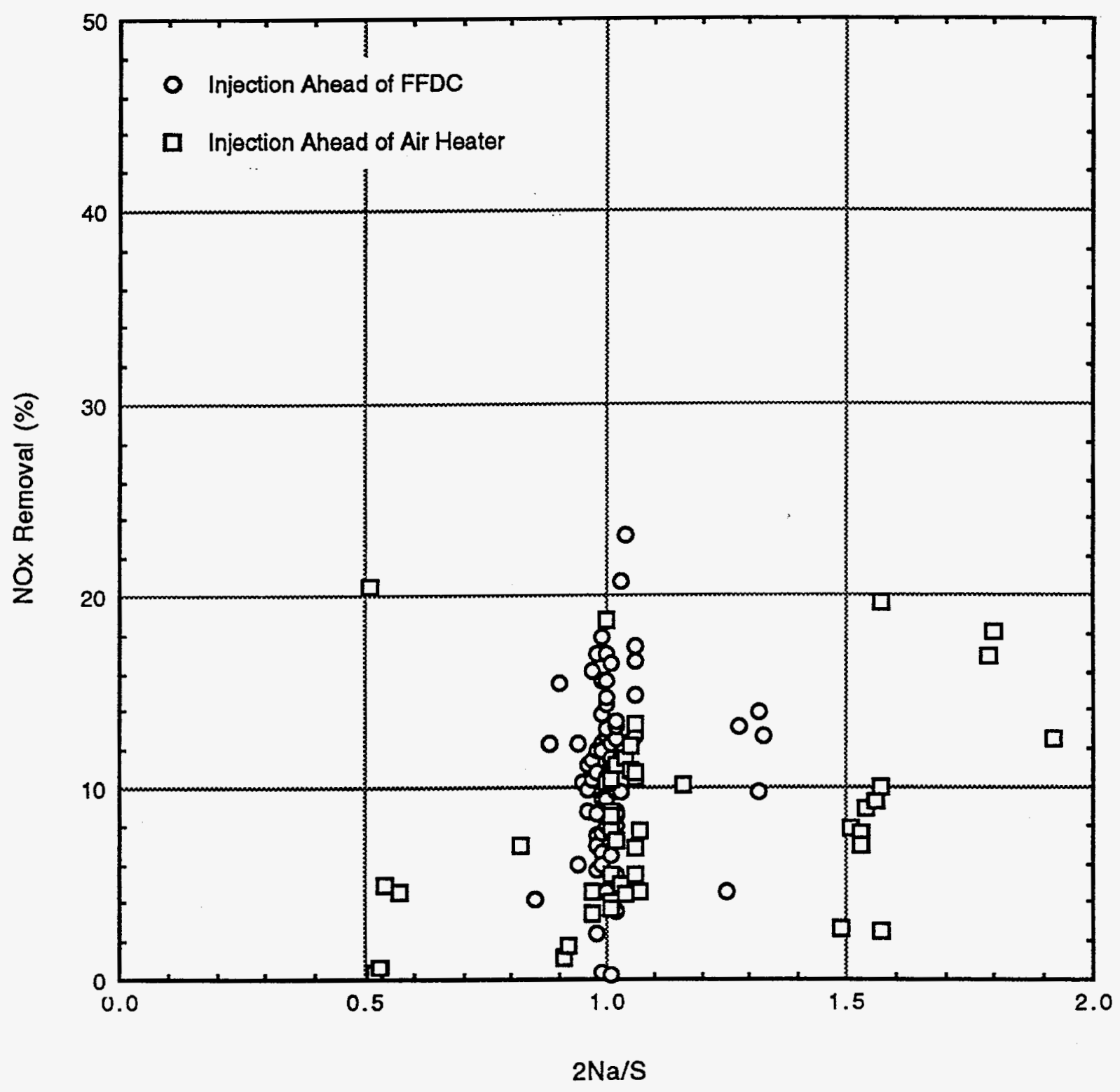

Figure 5-34. Summary of $\mathrm{NO}_{x}$ Reductions with Sodium Bicarbonate Injection 


\subsection{Solids Analysis}

Samples of the sorbent and fly ash mixture were collected on a number of occasions during the current series of tests. The samples were collected separately from the individual FFDC compartments in an effort to assess any variation in sorbent distribution and utilization within the fabric filter. The solids analysis would also provide a means to check the $2 \mathrm{Na} / \mathrm{S}$ ratio calculated from the sorbent feed rate. Samples were collected during both the sodium sesquicarbonate and sodium bicarbonate injection tests; these results are discussed separately in the following subsections.

In general, there are many concerns with the solid sample analysis. Solids analysis would be accurate if the ash and reagent products were equally distributed throughout the hoppers and sampling techniques were perfected. At Arapahoe, the FFDC has twelve compartments arranged in two rows of six (Figure 3-3) with the front compartments Number 1 on the west and 7 on the east. There is a good indication by observing an ash pull that the ash is severely non-uniform. The front compartments collect the greatest quantity of ash, while the last compartments collect the least, even though the gas flow through the FFDC is uniformly distributed. A similar problem occurs with the reagent distribution in the FFDC. The reagent may or may not be distributed in the same manner as the ash. If the reagent distribution is different than the ash distribution, then the solids analyses are difficult to interpret in terms of the dry sorbent process parameters. In addition to the distribution concerns, it is also very difficult to obtain a representative sample of such a large quantity of waste within an individual hopper. Thus, additional unknowns are introduced, although a number of different sampling techniques were tried to minimize the potential problems. Because of these problems, solids analysis should be used only for general observations and not absolute data analysis.

\subsubsection{Sodium Sesquicarbonate}

During the initial tests with sodium sesquicarbonate injection ahead of the FFDC, a set of solid samples was collected in order to determine if a significant amount of the sorbent was falling out of suspension and dropping into the hoppers before reaching the bags. The samples were analyzed to determine what difference, if any, in utilization occurred between 
the material on the bags and that in the hoppers. The samples were collected on a day when the injection system was running at a constant $2 \mathrm{Na} / \mathrm{S}$ ratio of approximately 0.85 . At the beginning of the day, sorbent injection was started, and a cleaning cycle was initiated manually in order to clean the fabric filter of the fly ash collected during the previous night. The hoppers were then evacuated after the cleaning cycle was complete. The injection condition was held constant until the pressure drop approached 4 inches (at which point a cleaning cycle would be initiated automatically). The FFDC control was put into manual in order to prevent the cleaning, and a sample was collected from all twelve compartments through a 4-inch port located near the bottom of each hopper. These samples should be biased toward the material that fell directly into the hopper before reaching the bags. The hoppers were then evacuated, and the FFDC allowed to clean automatically. Immediately after the cleaning, but before the hoppers were evacuated again, another set of single samples from each hopper were collected. These samples should be representative of the material that resided on the bags. Again, the "before clean" and "after clean" samples should be indicative of the material "in the hoppers" and "on the bags", respectively.

Portions of all 24 samples were sent to PSCo Applied Sciences Laboratory for analysis for sodium and sulfate. As very little of the captured sulfur was expected to be in the form of sulfite, only four of the samples were analyzed for this component. Sodium content was determined via an ICP (induced coupled plasma) analysis (EPA Method 200.7). Sulfate and sulfite were determined via ion chromatography (EPA Method 300.1) and titration (ASTM Method 4500), respectively. The sulfite analyses confirmed that only a small amount (generally less than 2 percent) of the sulfur was in the form of $\mathrm{Na}_{2} \mathrm{SO}_{3}$.

Figures $5-35 a$ and $5-35 b$ show the results of the sodium analyses for the samples taken before and after cleaning the FFDC, respectively. Recall that the fabric filter has twelve compartments arranged in two rows of six (Figure 3-3), and that compartment Number 1 is the first one on the west side, while Number 7 is the first one on the east side. The results indicate that the sodium concentration is lower in the first compartments on each 


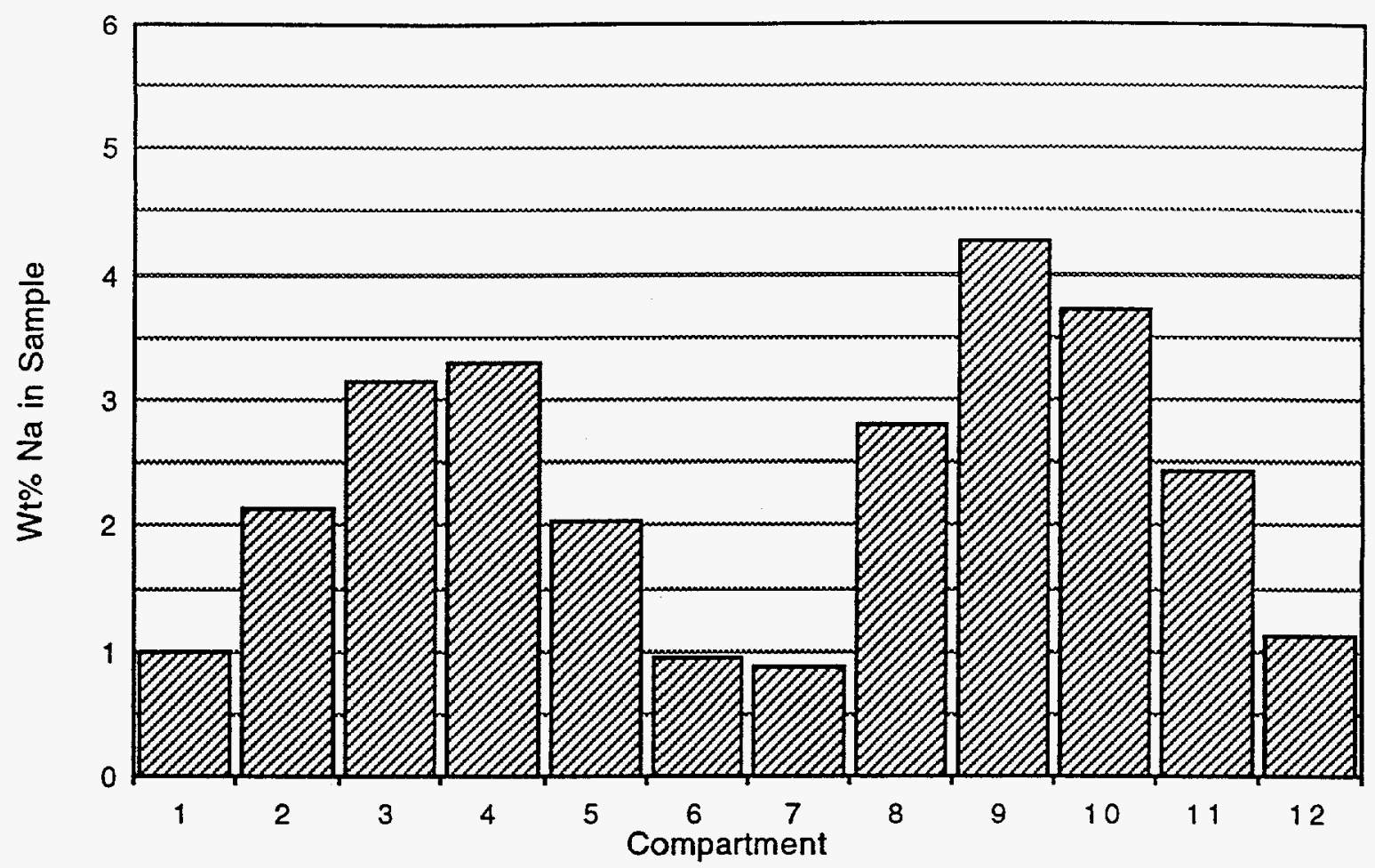

Figure 5-35a. Sodium Analysis Results for Sodium Sesquicarbonate Injection Samples Collected Before FFDC Cleaning (Test 641)

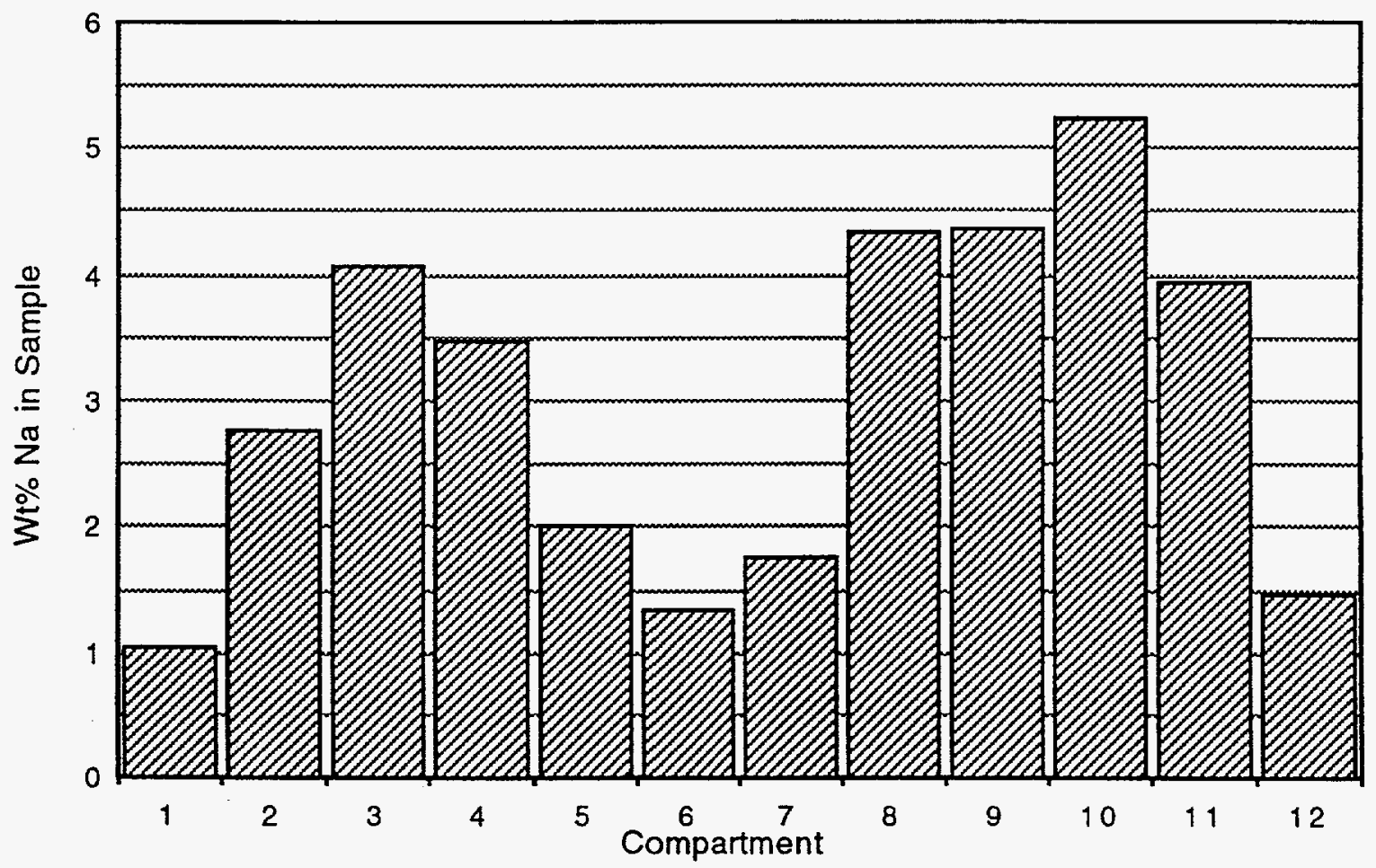

Figure 5-35b. Sodium Analysis Results for Sodium Sesquicarbonate Injection Samples Collected After FFDC Cleaning (Test 641) 
side, increases to a maximum in the central compartments, and then decreases in the rear compartments.

The results of the sulfate analyses (Figures 5-36a and 5-36b) show a compartment-bycompartment distribution of sulfate which is similar to that seen for the sodium. However, there is substantially more sulfur in the material on the bags than in the material which is deposited in the hoppers. Cursory review of the data seem to indicate that the sodium is biased to the middle compartments of the FFDC. If the ash was uniformly distributed within the compartments, then this initial indication would be true. As discussed in the introduction to this section, ash and reagent are not uniformly distributed. In addition to these concerns related to distribution between the FFDC compartments, similar concerns also exist for the sample obtained before cleaning the FFDC. However, using the observation that higher ash collections occur in the front compartments, some observations can be made. The front compartments show low sodium, but the front compartments also contain higher quantities of ash. With more ash, the sodium concentration is "diluted". While no conclusion can be made, it appears that the sodium reagent is also preferentially deposited in the forward compartments, although not to the extent of the fly ash, and the back compartments have significantly less reagent. This is supported by the compartment $\mathrm{SO}_{2}$ removal traverse data in Figure 5-8 which shows approximately equal $\mathrm{SO}_{2}$ removal in the first compartments with a gradual reduction in $\mathrm{SO}_{2}$ removal toward the rear compartments. Another observation is that both the pre- and post-cleaning samples have approximately the same sodium weight percentage in each hopper. This likely indicates that approximately the same amount of reagent and ash fall out in the hoppers. As the particle size for both reagent and ash are approximately equal, this is not unexpected.

A measure of the utilization of the sodium in each sample may be determined from the molar ratios of sodium and sulfur. Since two moles of sodium are required to completely react with a single mole of sulfur, a molar sulfur-to-sodium ratio of 0.5 would indicate complete sodium utilization. Thus, dividing the $\mathrm{S} / \mathrm{Na}$ ratio found in each sample by 0.5 , provides a measure of the sodium utilization in that sample. Figures $5-37 \mathrm{a}$ and $5-37 \mathrm{~b}$ show these calculated utilizations for samples collected both before and after the FFDC 


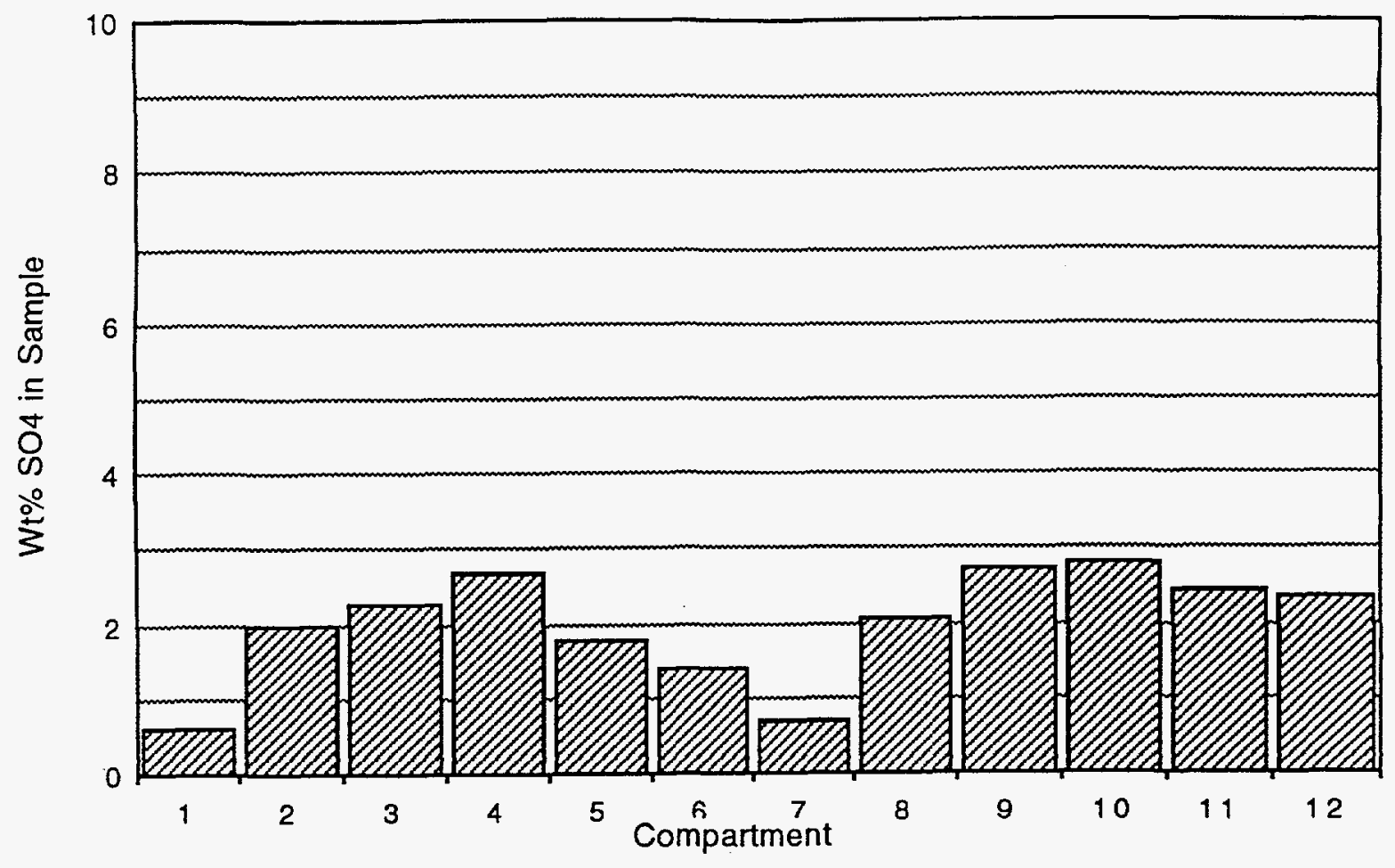

Figure 5-36a. Sulfate Analysis Results for Sodium Sesquicarbonate Injection Samples Collected Before FFDC Cleaning (Test 641)

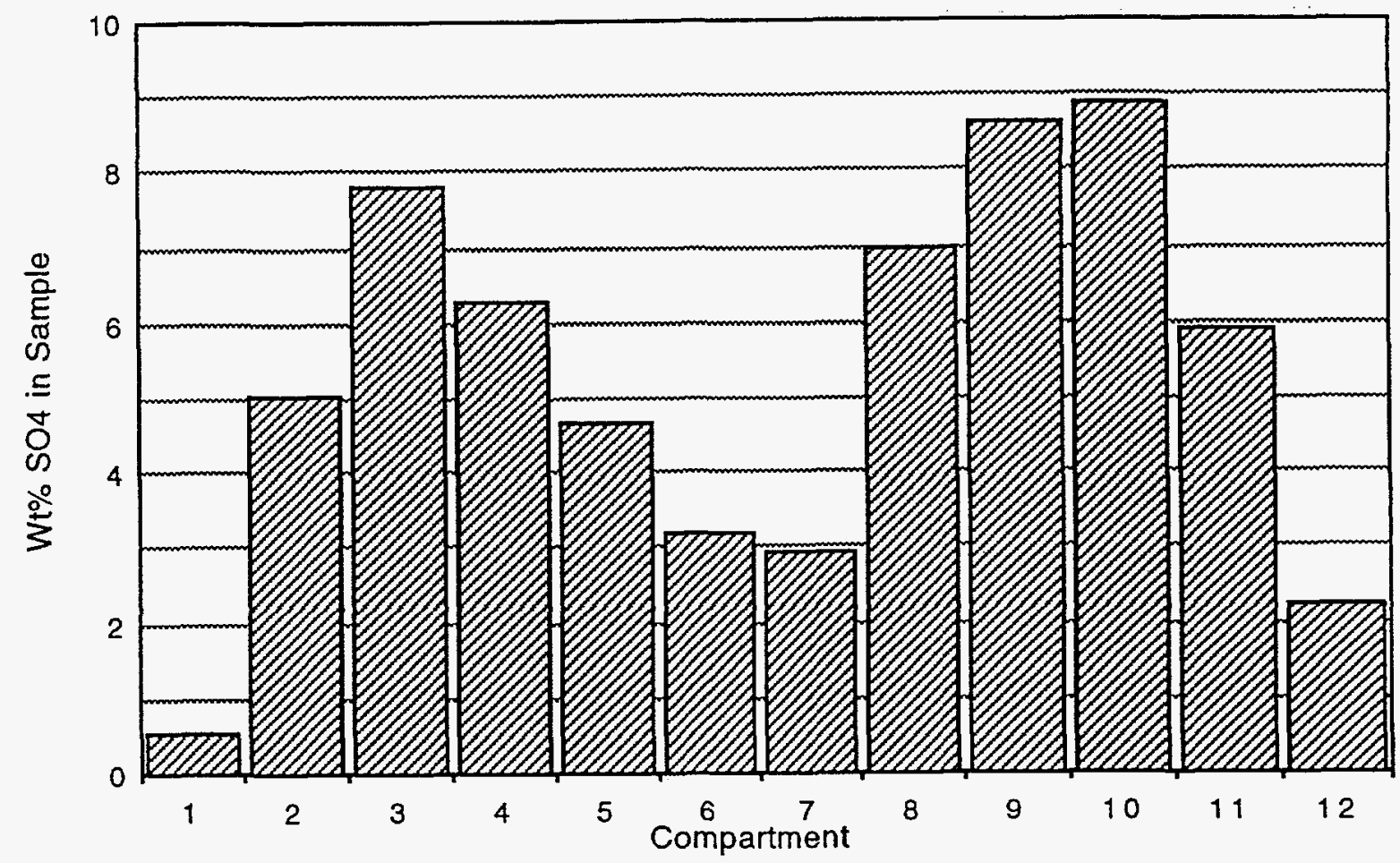

Figure 5-36b. Sulfate Analysis Results for Sodium Sesquicarbonate Injection Samples Collected After FFDC Cleaning (Test 641) 


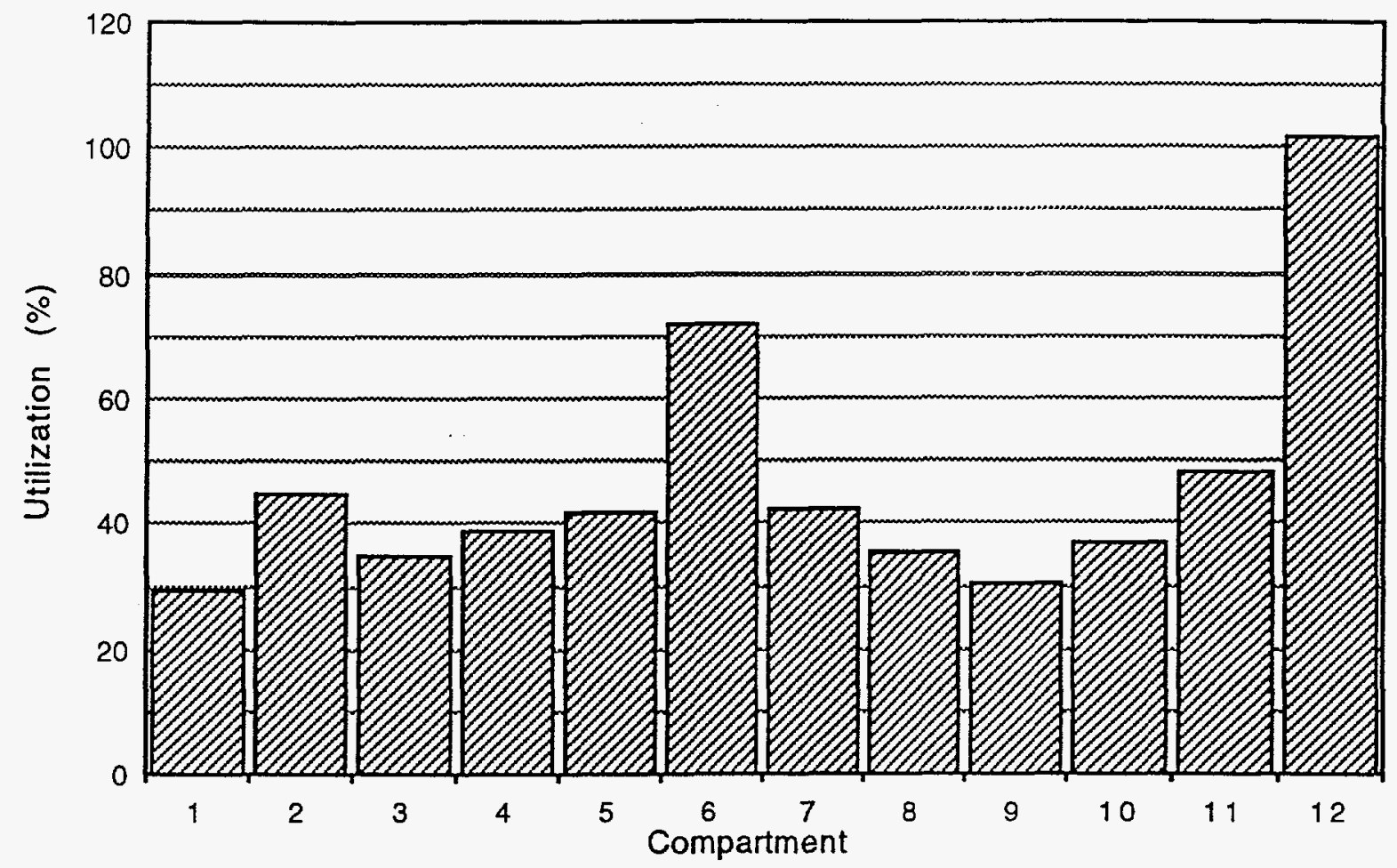

Figure 5-37a. Utilization Calculations for Sodium Sesquicarbonate Injection Samples Collected Before FFDC Cleaning (Test 641)

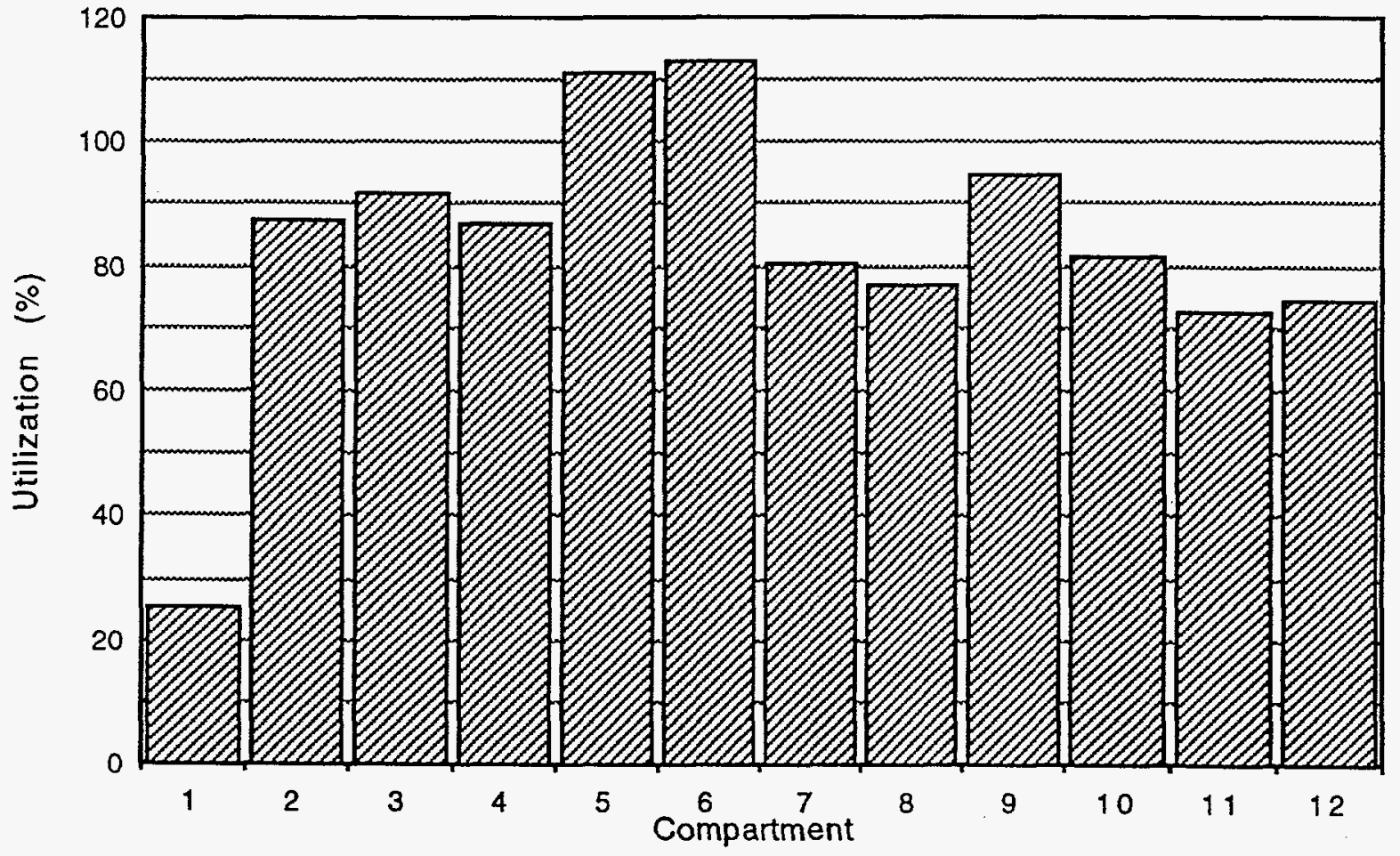

Figure 5-37b. Utilization Calculations for Sodium Sesquicarbonate Injection Samples Collected After FFDC Cleaning (Test 641) 
cleaning. Although, utilizations in excess of 100 percent are unrealistic (and are likely due to small inaccuracies in the solids analyses), the results confirm that the material which collects on the bags is much more highly utilized than the material which falls out of suspension and into the hoppers. The very low utilization of the post-cleaning sample for compartment 1 was not expected and is likely due to a sampling inconsistency. As discussed above, only general observations can be made due to the non-uniform ash and reagent distribution. As the sodium percentages were approximately equal in the pre- and post-cleaning samples, it would be expected that the sulfate quantities would also be equal if the reagent reacted the same. As the post-cleaning sample has significantly more sulfate, it is more fully reacted. This shows that the reagent that drops out in the hoppers obtains less $\mathrm{SO}_{2}$ capture. This is consistent with the gaseous $\mathrm{SO}_{2}$ results which indicated that the majority of the $\mathrm{SO}_{2}$ removal is obtained while the material is in the FFDC.

Another objective of performing the solids analyses was to confirm the $2 \mathrm{Na} / \mathrm{S}$ ratio calculated from the sorbent feed rate and the gaseous $\mathrm{SO}_{2}$ measurements. Since the results discussed above show a large difference in utilization between the material on the bags and that in the hoppers, and the relative quantities of waste before and after cleaning are not known, it was necessary to revise the sampling method. The new method consisted of collecting a single sample having average properties which were representative of the material deposited throughout each compartment. This was accomplished by allowing the baghouse to clean in a normal fashion, and then evacuating the hoppers one at a time while taking a sample from the bottom of the hopper at regular (one minute) intervals. This method provided a series of small samples which were representative of the vertical distribution of material in the hopper.

Once the hopper was completely evacuated, the individual samples were composited together into a single sample for that particular compartment. Samples were collected from all twelve compartments in a similar manner.

The new method was used to collect samples for three tests which were run with similar operating conditions during the final phase of air toxics testing performed in October, 1993. 
The three tests were run at a boiler load of $100 \mathrm{MWe}$, with nominal $2 \mathrm{Na} / \mathrm{S}$ ratios of 1.5 . The overall $\mathrm{SO}_{2}$ removals measured for each test were similar, ranging from 61 to 65 percent. This corresponds to overall utilizations of 40 to 43 percent based on the sorbent feed rate and $\mathrm{SO}_{2}$ removal. The compartment-by-compartment utilizations calculated for all three tests are shown in Figure 5-38.

In general, all three sets of data indicate that the material deposited in the rear compartments is more highly utilized than that deposited in the front. However, the results also show that the utilization calculated for a single compartment can vary greatly, depending on the particular test. It is believed that this variability is due to test-to-test variations in how the sorbent is deposited in the FFDC, and in the ability to obtain a representative sample from each compartment hopper.

If the sorbent was evenly distributed among the twelve FFDC compartments, calculating an overall $2 \mathrm{Na} / \mathrm{S}$ ratio from the compartment-by-compartment solids analyses would be a simple matter of dividing the overall $\mathrm{SO}_{2}$ removal by the arithmetic average of the compartment utilizations. However, the data previously presented in Figures 5-35a and $5-35 b$ indicate that more of the sorbent is deposited in the front compartments than in the rear. Therefore, the $2 \mathrm{Na} / \mathrm{S}$ ratios must be calculated separately for each compartment, and then averaged, in order to provide an accurate overall value. To do so requires the measurement of the $\mathrm{SO}_{2}$ removal in each compartment, in addition to the utilization calculation.

Compartment-by-compartment $\mathrm{SO}_{2}$ removals were measured during the final air toxics test with sodium sesquicarbonate injection (Test 704). These results, as well as the calculated utilizations based on the solid samples for each compartment, are shown in Figure 5-39. The peak $\mathrm{SO}_{2}$ removals occur in the central compartments of each side of the baghouse in a pattern which is similar to that seen for the sodium distribution (Figures 5-35a and 5-35b). The arithmetic average of the $\mathrm{SO}_{2}$ removal data was 55.9 percent. This compares to an overall $\mathrm{SO}_{2}$ removal of 60.8 percent measured across the fabric filter. Since the arithmetic average of the compartment-by-compartment $\mathrm{SO}_{2}$ removal measurements is in 


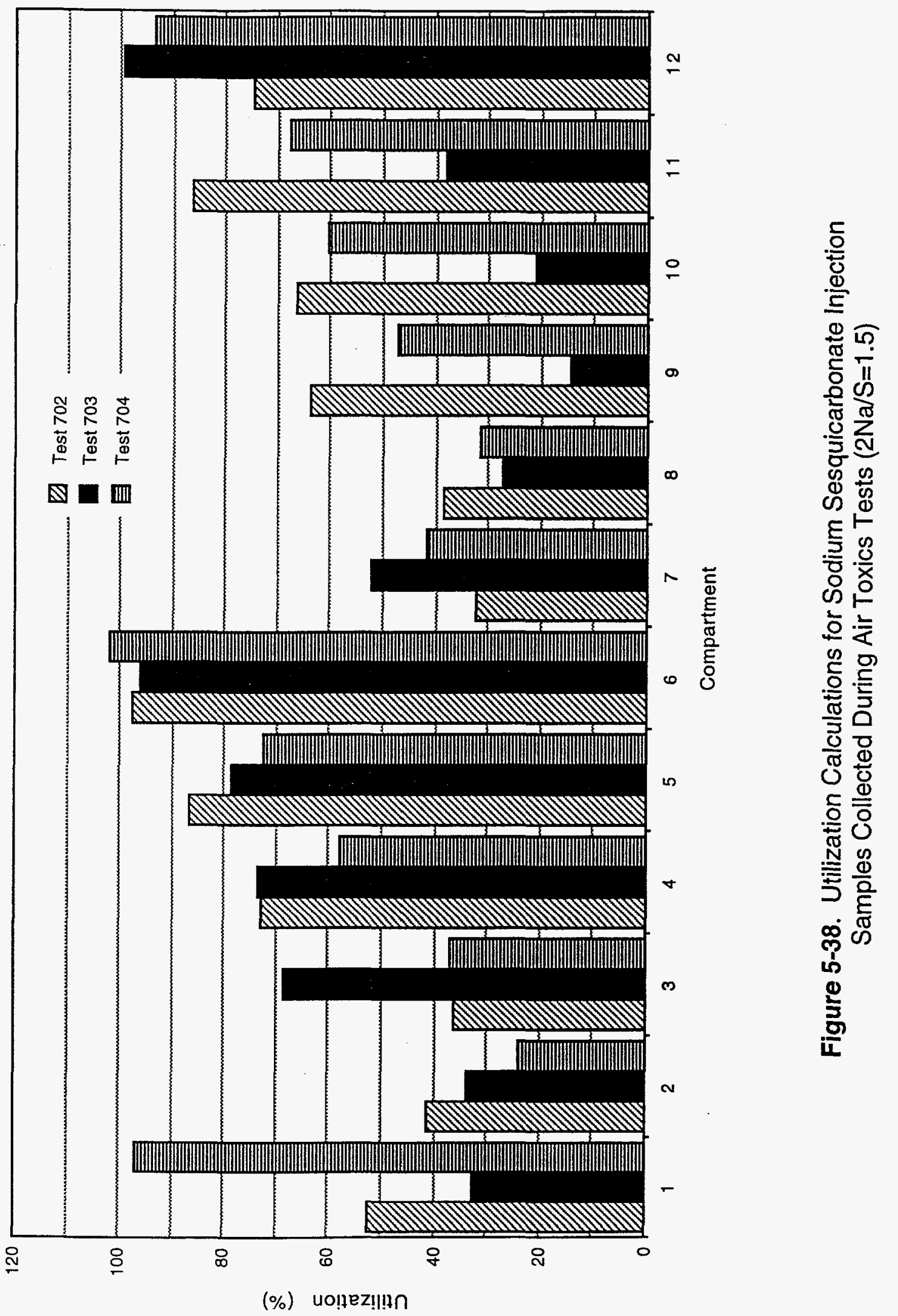




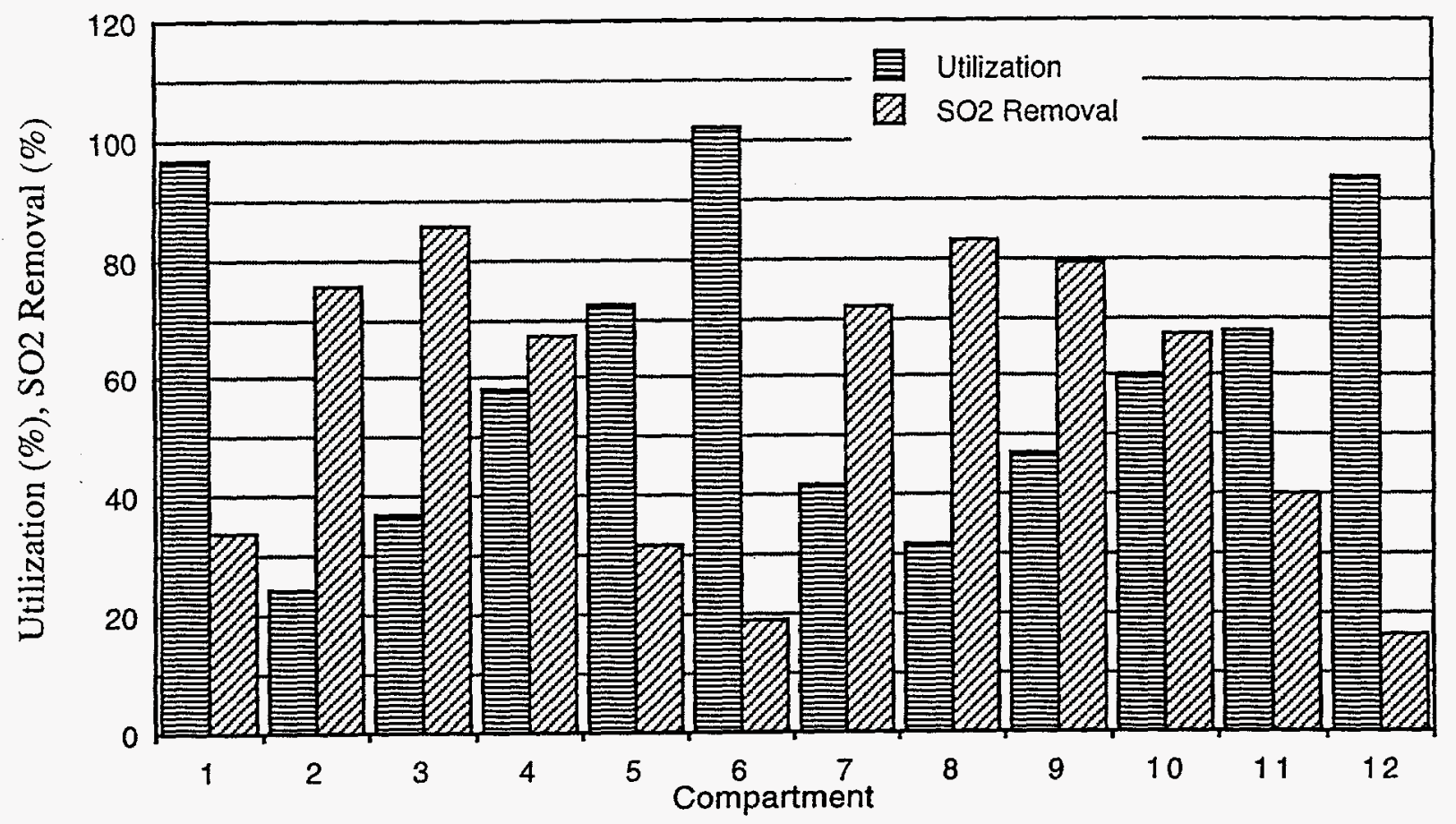

Figure 5-39. Compartment-by-Compartment Utilization Calculations and $\mathrm{SO}_{2}$ Removals for Sodium Sesquicarbonate Injection (Test 704)

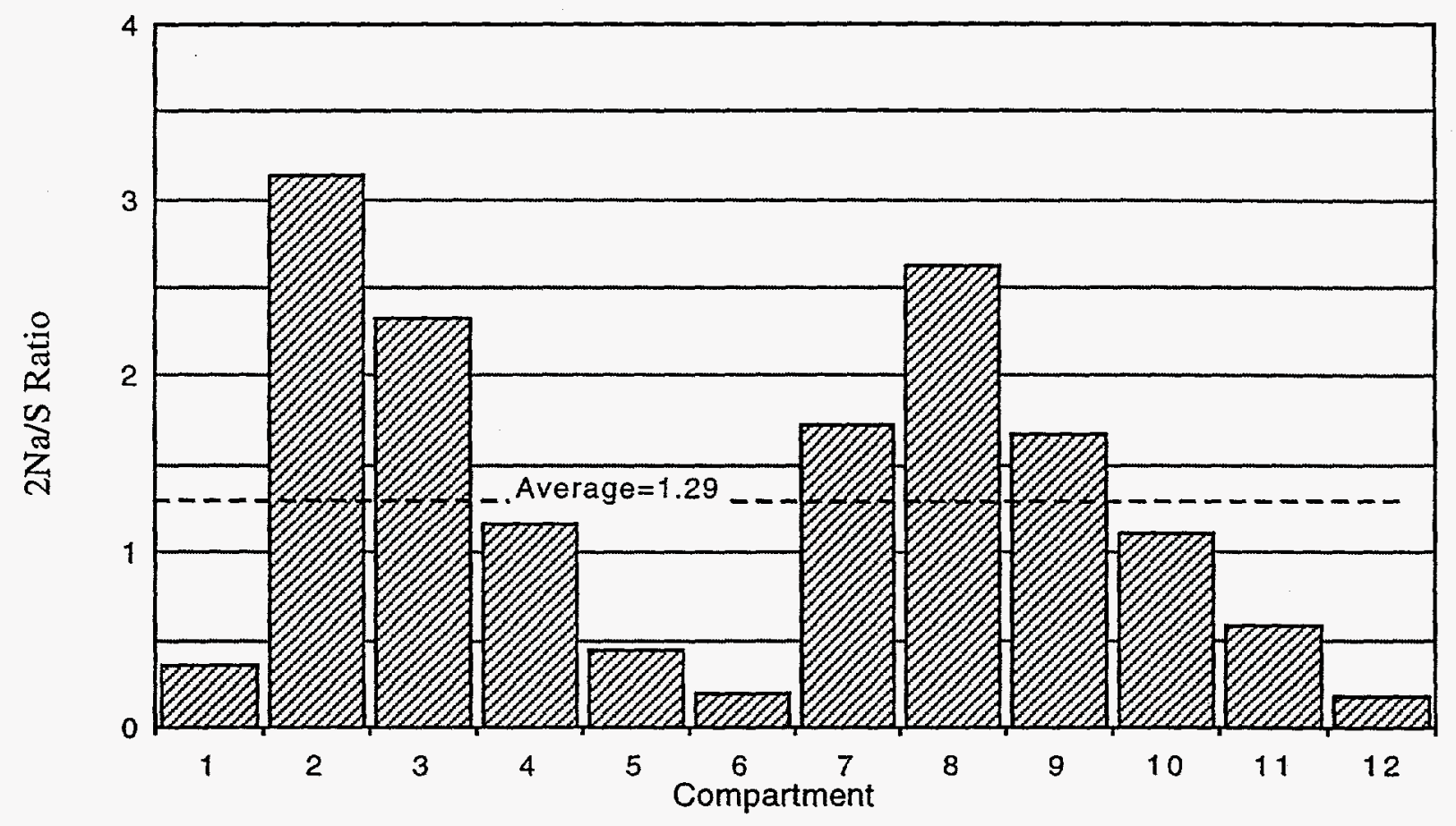

Figure 5-40. Compartment-by-Compartment 2Na/S Ratio Calculations for Sodium Sesquicarbonate Injection (Test 704) 
good agreement with the overall measurement across the fabric filter, each compartment has essentially the same gas flow. Figure 5-40 shows the $2 \mathrm{Na} / \mathrm{S}$ ratio calculated on a compartment-by-compartment basis. These results further support the previous observation that most of the sodium is deposited in the center compartments, and very little reaches the back compartments (Numbers 11 and 12). The arithmetic average of this data $(2 \mathrm{Na} / \mathrm{S}=1.29)$ is in reasonable agreement with the feed rate calculation $(2 \mathrm{Na} / \mathrm{S}=1.53)$.

At the conclusion of the air toxics testing performed during October 1993, a series of twelve fly ash/sorbent samples were collected from a location upstream of the FFDC in an effort to assess the sorbent distribution uniformity inside the duct. The samples were collected at a location approximately 60 feet downstream of the sorbent injection grid, through a set of four ports occupied by the original humidification thermocouple grid (recall Figure 3-2). Three separate samples were collected through each port at depths of $0.25,0.50$, and 0.75 of the total duct depth at that location. All sampling was performed in accordance with EPA Method 17 in order to assure a representative sample. Figures 5-41 and 5-42 show the flue gas velocity and total particulate concentration (fly ash and sorbent) results of these tests. Although the velocity profile is nearly uniform across the duct, the particulate concentration was skewed, with the lowest loading at the top west corner and an increasing trend across the diagonal to the highest concentration at the lower east corner. A sodium analysis of the filter catch from each test (Figure 5-43) also showed high levels of sodium in the samples from the lower east comer. The sodium concentration ( $\mathrm{mg} / \mathrm{Nm}^{3}$ ) at each sample point was computed from the total particulate concentration and the sodium content of the sample. The normalized results (Figure 5-44) show a large maldistribution of sorbent, with most of the material being found in the lower east quadrant of the duct.

As discussed previously, sorbent injector plugging was a recurring problem during the program. Since the pulverizer added a significant amount of heat to the sorbent/air mixture, it was rather easy to locate an injector which was totally plugged by simply touching the pipe upstream of the injector. A warm pipe was flowing, while a cold pipe indicated that the injector was plugged. Unfortunately, this method would not locate a 


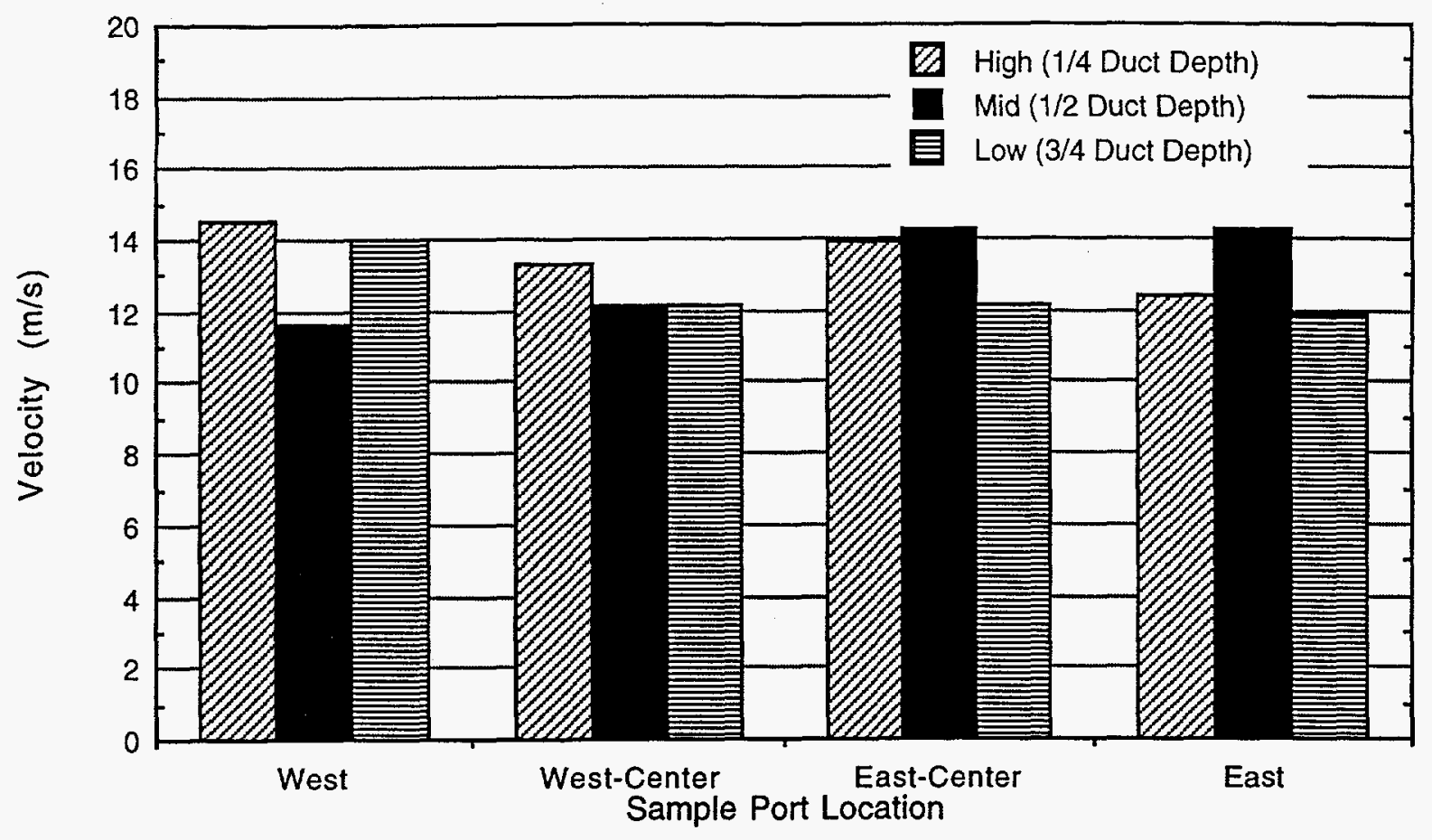

Figure 5-41. Flue Gas Velocity Distribution at FFDC Inlet for Sodium Sesquicarbonate Injection (Test 705)

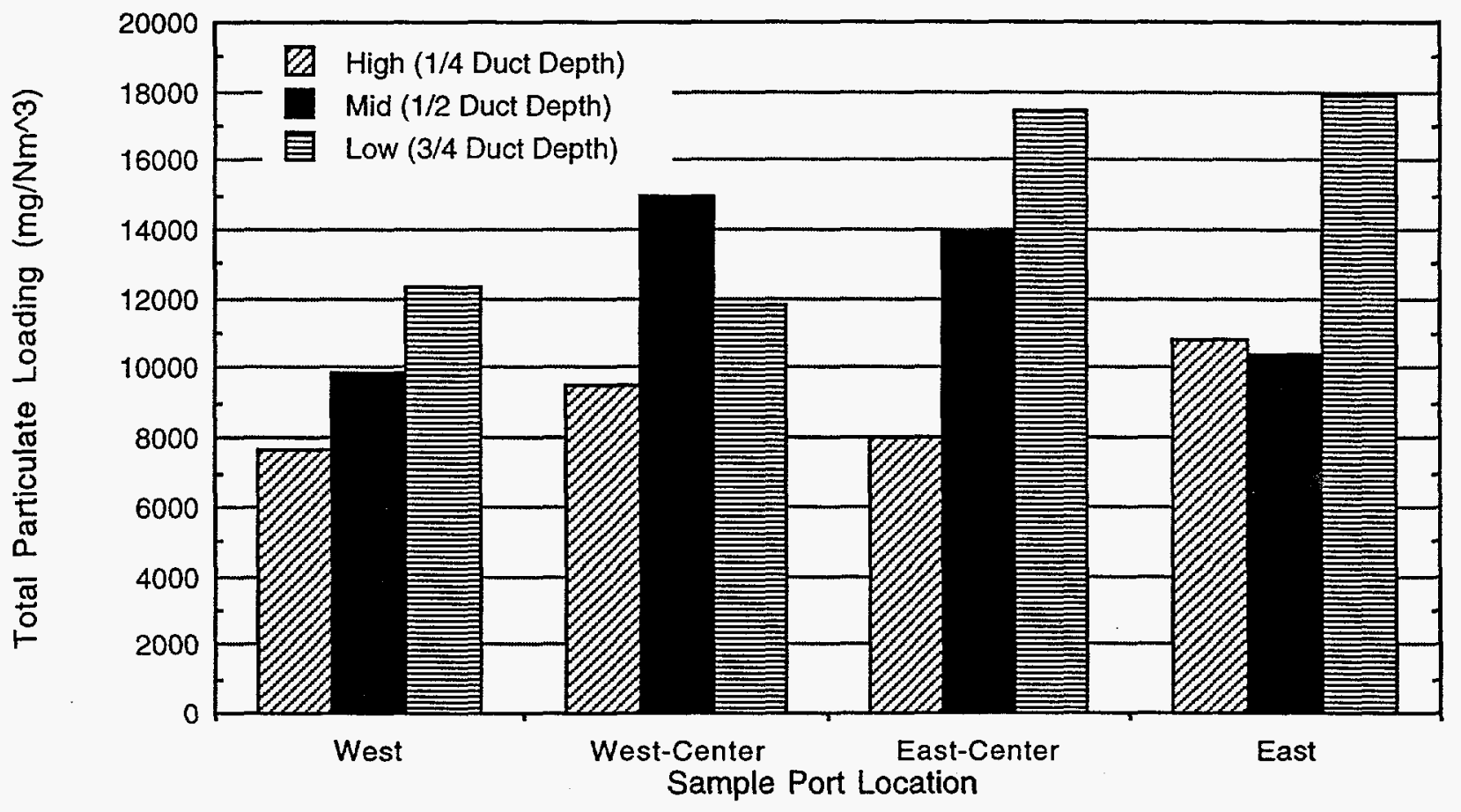

Figure 5-42. Total Particulate Loading (Flyash and Sorbent) at FFDC Inlet for Sodium Sesquicarbonate Injection (Test 705) 


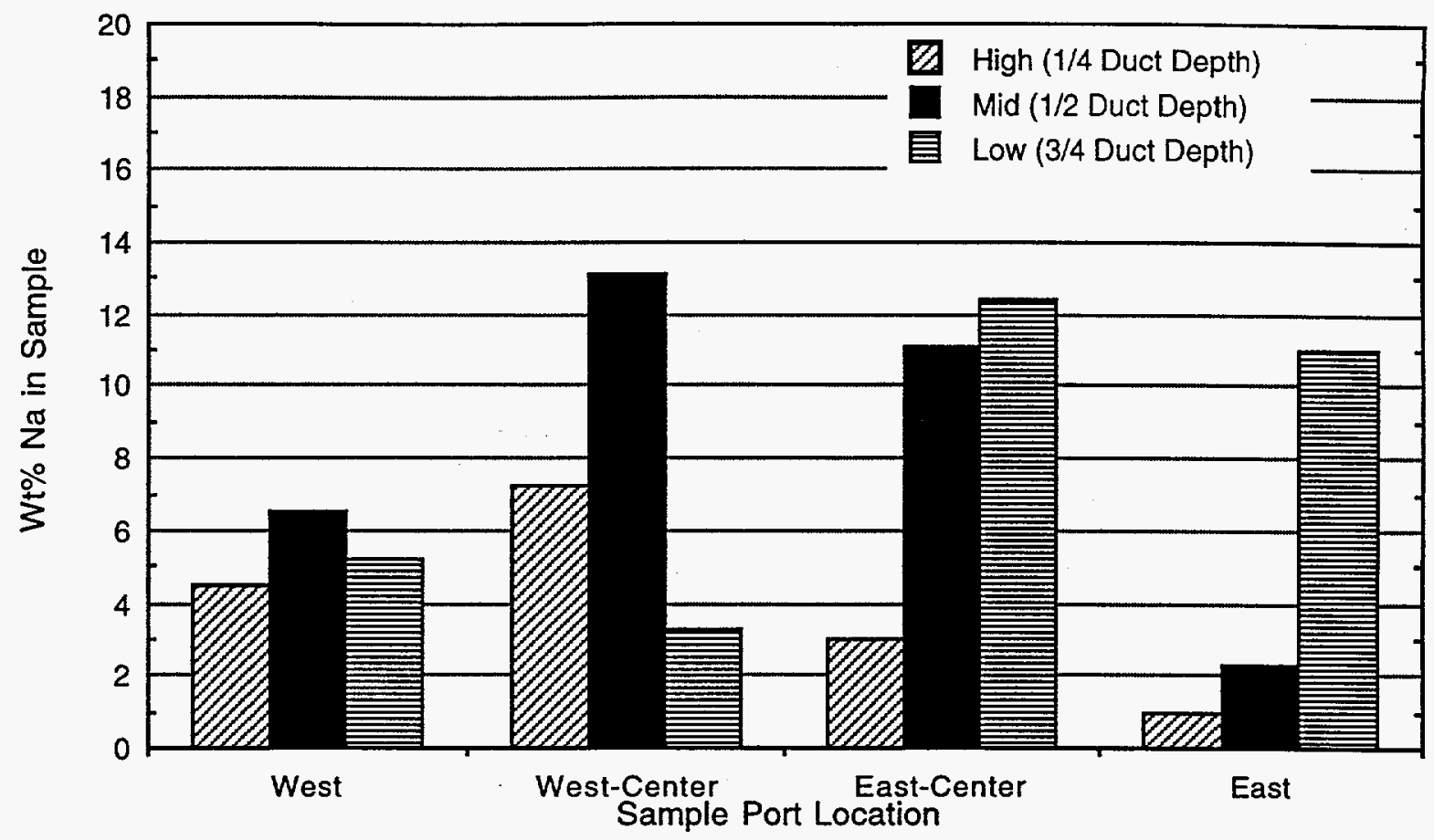

Figure 5-43. Sodium Analysis Results for Samples Collected at FFDC Inlet for Sodium Sesquicarbonate Injection (Test 705)

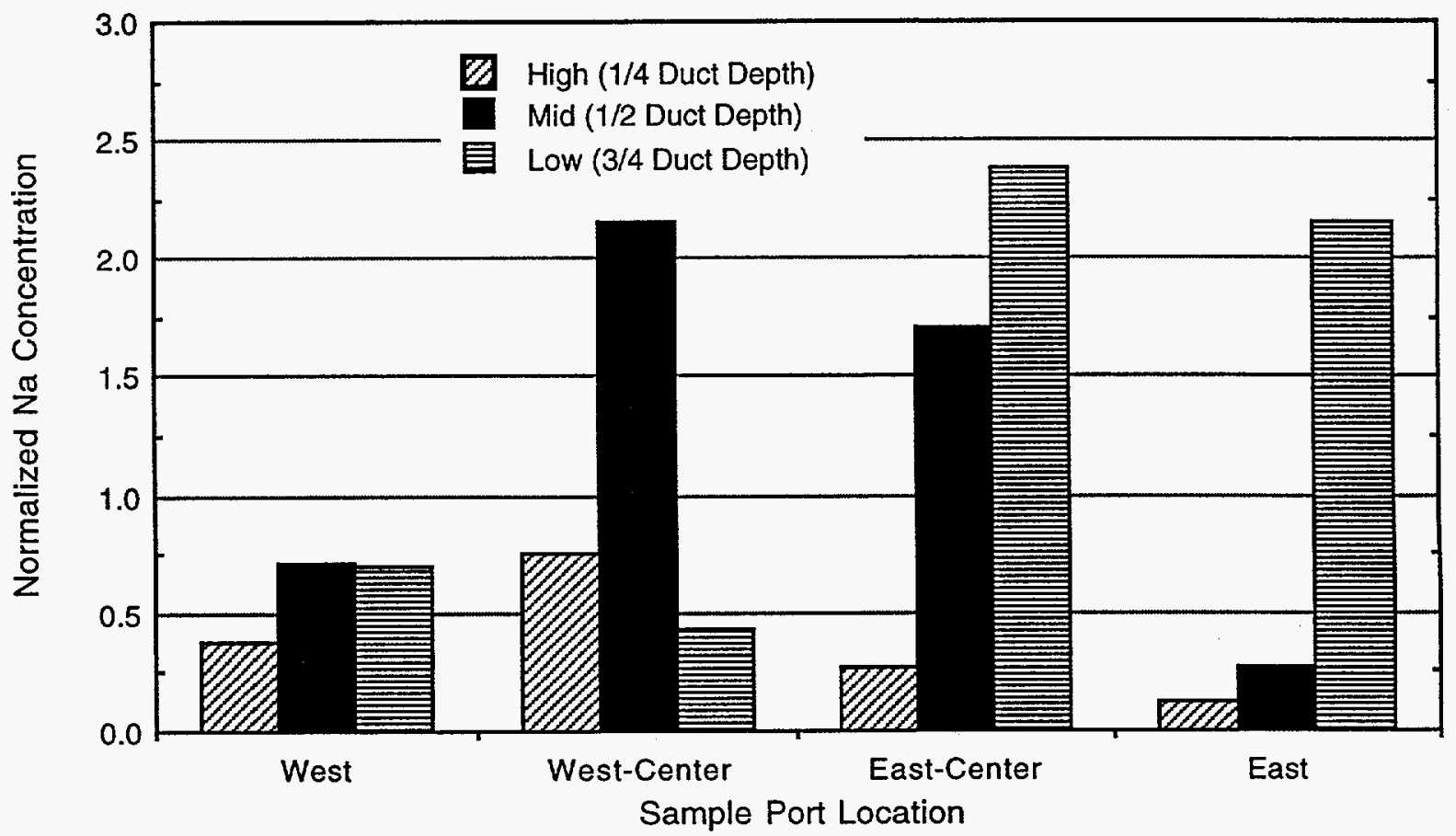

Figure 5-44. Calculated Sodium Distribution at FFDC Inlet for Sodium Sesquicarbonate Injection (Test 705) 
partially blocked injector until it had become totally plugged. It is likely that the distribution of sorbent shown in Figure 5-44 is the result of a particular pattern of partially plugged injectors. It is also believed that the pattern, and therefore the distribution of sorbent in the duct as well as in the baghouse, could change on a day-to-day basis. The variability of the utilizations calculated for a single compartment shown in Figure 5-38 can at least be partially attributed to this behavior of the injection system. Fortunately, this variability does not seem to affect the overall process performance, as the $\mathrm{SO}_{2}$ removals measured at the stack for these three tests were consistent, ranging from 61 to 65 percent.

\subsubsection{Sodium Bicarbonate}

As discussed previously, the $\mathrm{SO}_{2}$ removal process with sodium bicarbonate injection ahead of the FFDC was found to be highly temperature dependent and difficult to control. Solid samples were collected at the end of the five-day test shown in Figure 5-20, in order to provide a set of samples which was representative of the "long-term" process performance. In order to evaluate the extent to which the sorbent was being utilized when deposited on the bags, individual compartment samples were collected. The procedure which collected multiple samples from each hopper at one minute intervals during ash pulling was used. The compartment hoppers were evacuated prior to cleaning the bags and collecting the samples, so that only material on the bags was collected.

Portions of the samples were sent to the PSCo Applied Sciences Laboratory and analyzed for sodium, sulfate and sulfite. As was seen for sodium sesquicarbonate injection, sulfite was found in only negligible amounts. However, the compartment-by-compartment utilizations calculated from the sodium and sulfate results ranged from 140 to 170 percent. Although a review of the laboratory procedures did not indicate any analytical problems, a second portion of each sample was submitted for analysis as a check.

The sodium and sulfate results for the two sets of analyses are compared in Figures 5-45a and $5-45 \mathrm{~b}$, respectively. Note that compartment 11 was out of service for maintenance on the day that the samples were collected. In each figure, there are compartments where 


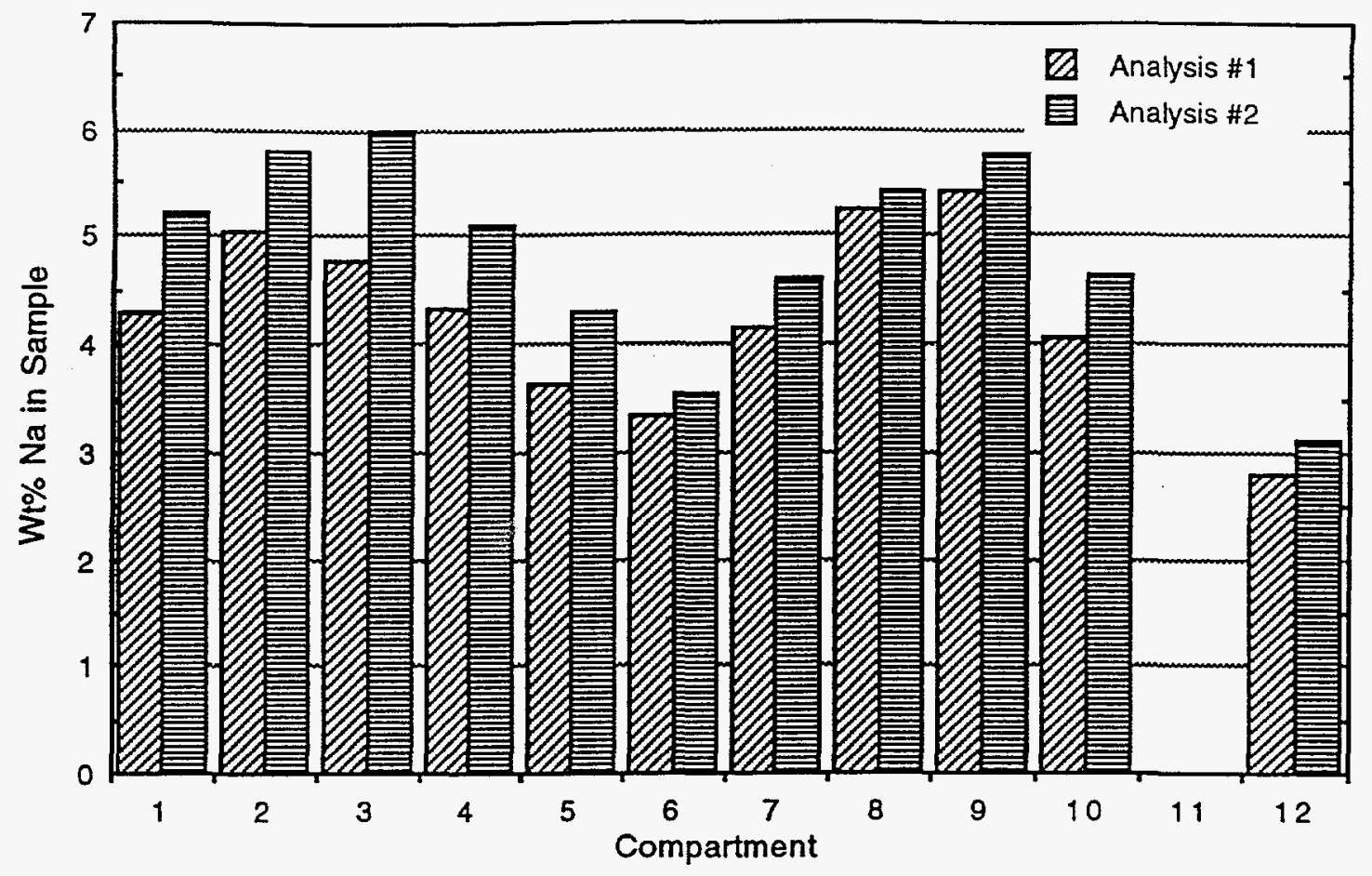

Figure 5-45a. Sodium Analysis Results for Sodium Bicarbonate Injection Ahead of the FFDC (Test 763)

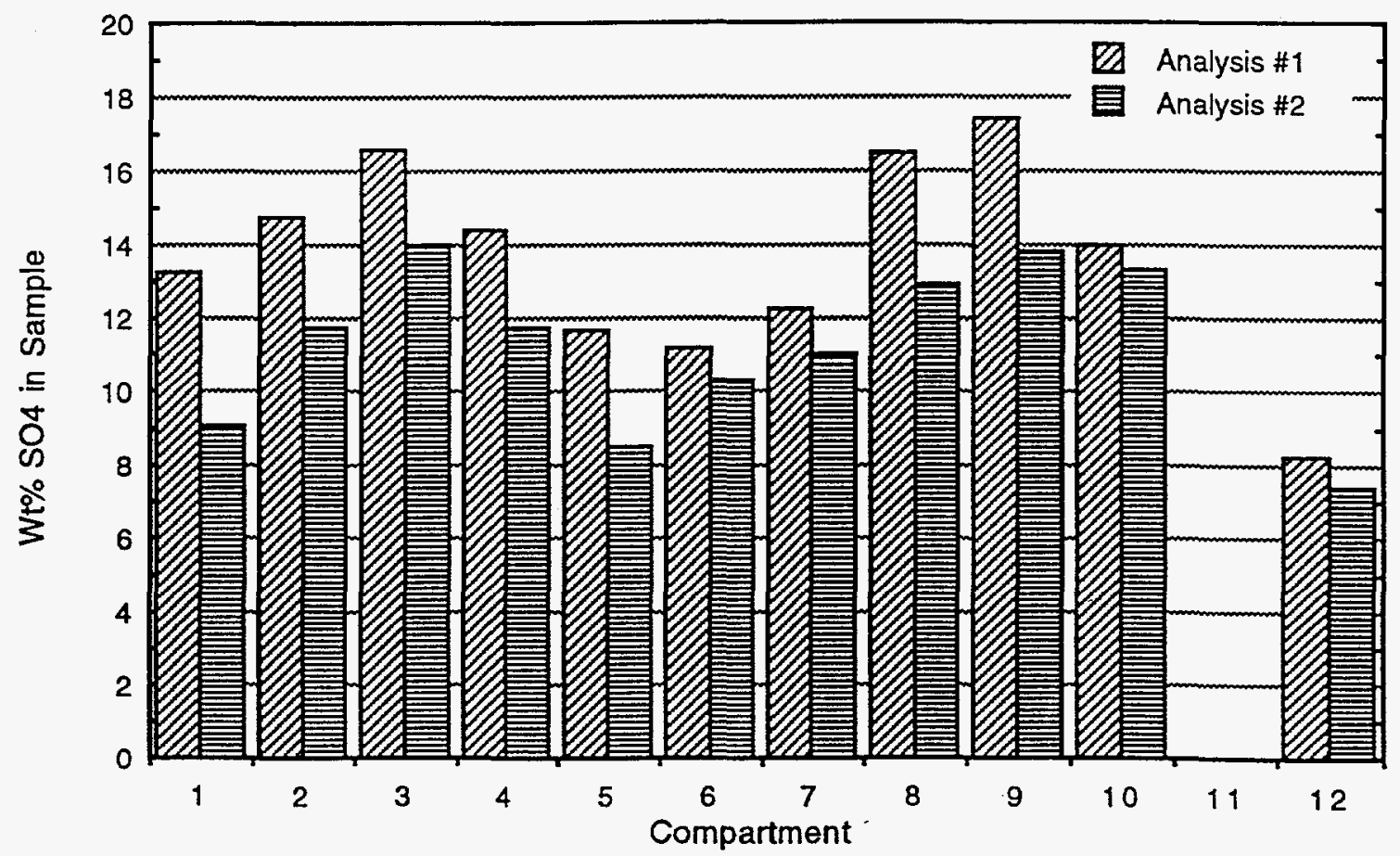

Figure 5-45b. Sulfate Analysis Results for Sodium Bicarbonate Injection Ahead of the FFDC (Test 763) 
the two sets of data are in good agreement, and then others where there are relatively large differences. It is notable, however, that in each compartment, the sodium results from the second analysis are higher than the first, while the sulfate results are lower. Although a small random variability in the results may be explained by the nonhomogeneous nature of the large sample from which the two smaller samples were taken, the "shift" between the two sets of results may also indicate an analysis problem.

Figure 5-46 shows that, in general, the compartment-by-compartment utilizations calculated from the second set of analyses are lower than the first. However, there are still two compartment analyses which are questionable with utilizations of nearly 140 percent. These two notwithstanding, the second set of utilizations fall within the range of 85 to 115 percent, indicating that the sorbent becomes highly utilized when deposited on the bags. This compares to an average utilization of $72 \%$ calculated from the measured $\mathrm{SO}_{2}$ removal and sorbent feed rate. 


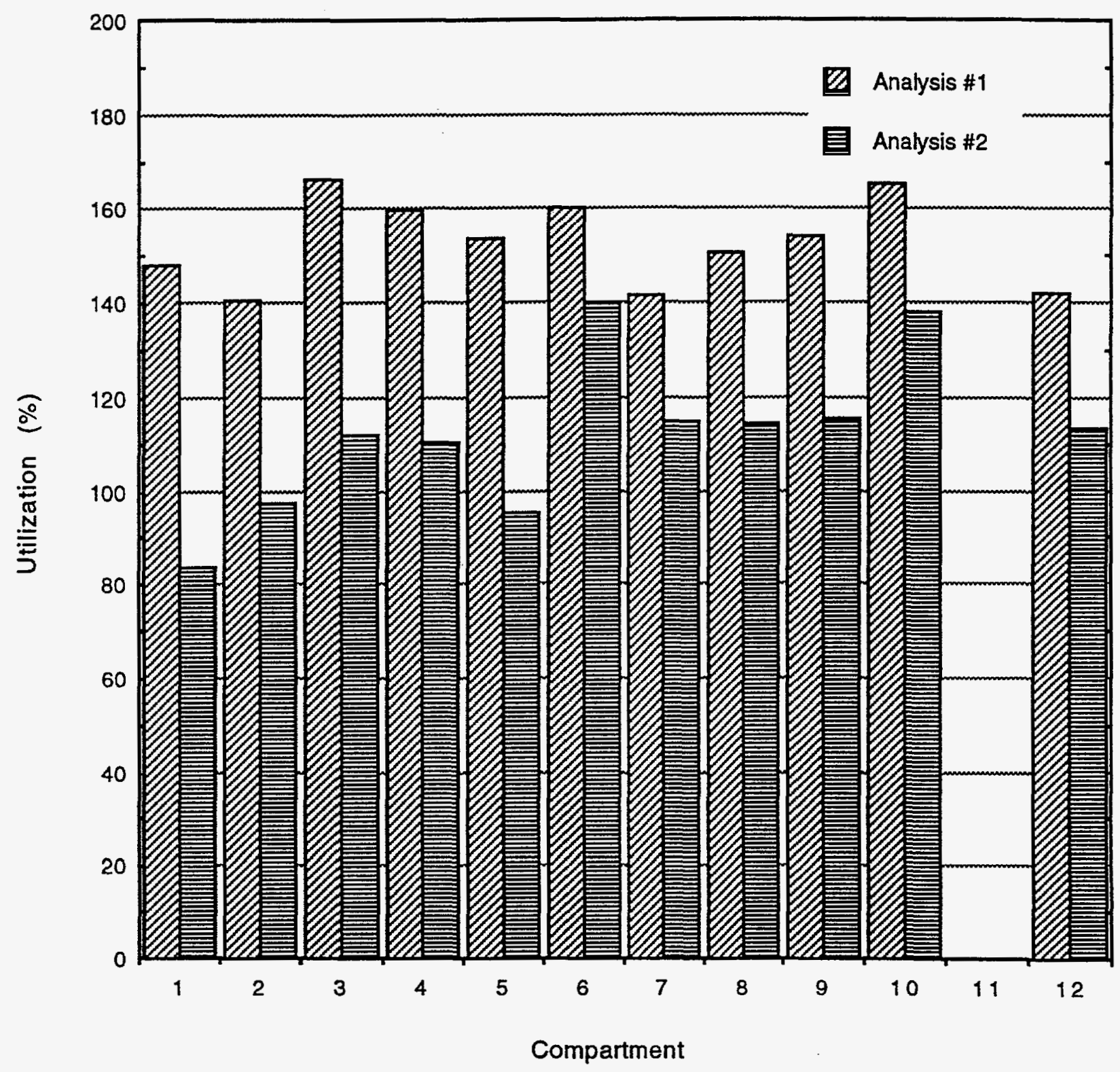

Figure 5-46. Utilization Calculations for Sodium Bicarbonate Injection Ahead of the FFDC (Test 763) 


\subsection{LONG TERM LOAD FOLLOWING TEST RESULTS}

After completion of the short term parametric tests reported in the previous section, a long term test of nominally four months duration was begun with sodium sesquicarbonate injection ahead of the fabric filter. During this test, the boiler was run in the normal load following manner as dictated by the PSCo system dispatch center. The DSI system was run in the automatic control mode, with the goal of maintaining an average $\mathrm{SO}_{2}$ removal of 40 percent for the first two months, and then 70 percent for the remaining two months.

The test began on November 14, 1994, and during the first month, both the " $\mathrm{A}$ " and " $\mathrm{B}$ " injection systems experienced repeated problems with plugging of the piping downstream of the splitter valves. The "A" system piping was redesigned in December, and all of the long radius elbows were replaced with five radius elbows. After the modifications to the piping external to the flue gas duct, the injectors still plugged at the $90^{\circ}$ bend inside the duct every three to five days. To solve this problem, the in-duct elbows were removed, and the sorbent injected perpendicular to, rather than concurrent with, the flue gas flow. The " $A$ " system was then run for two weeks in order to ensure that the modifications corrected the plugging problem, and then the " $\mathrm{B}$ " system was modified similarly. These modifications were completed in early February, 1995, at the time when it was planned to increase the target $\mathrm{SO}_{2}$ removal from 40 to 70 percent.

In February, 1995, a number of equipment problems occurred such that only one injection system was available for much of the time. The two major problems were a screw feeder bearing failure, and a high vibration problem on the "A" pulverizer. The bearing failure resulted in the loss of one system for nearly a week due to problems in locating parts and scheduling a maintenance crew. Additionally, during an attempt to remove the "A" pulverizer rotor disk and investigate the vibration problem, the disk was damaged. Since the delivery time on a new rotor from the manufacturer was eight weeks, an attempt to repair the disk was made in-house by the PSCo machine shop. The repairs were not completed by the time the long-term test ended on March 14, 1995. Due to these 
equipment problems, 70 percent $\mathrm{SO}_{2}$ removal testing was not possible for the entire two month planned period.

Figures 6-1 through 6-5 show the hourly averages of $\mathrm{SO}_{2}$ removal and $\mathrm{NO}_{2}$ emissions as a function of time for the months of November, 1994 through March, 1995, respectively. Throughout the four-month test, the rolling average $\mathrm{SO}_{2}$ removal was easily maintained at, or above, 40 percent. However, the hourly average $\mathrm{SO}_{2}$ removal data show that there were brief periods when the sodium injection system was off-line due to minor problems of line plugging or system maintenance requirements. Normally, when these occur, the backup pulverizer and injection system would be brought on-line. Unfortunately, as noted above, during much of this test period one of the two systems was down for either maintenance or repairs, and thus no back-up system was available.

In Figures 6-1 through 6-5, the timing of the fabric filter cleaning cycles is shown by the "FFDC clean time", which indicates the number of hours which have passed since the last cleaning cycle. As was seen previously during the short term tests (Figure 5-9), the $\mathrm{NO}_{2}$ emissions during the long term test increased sharply after each cleaning cycle, and then slowly decreased until the next cleaning cycle. Although the $\mathrm{NO}_{2}$ emissions are generally low (usually less than $10 \mathrm{ppm}$ ), it is difficult to visually assess an "average" level due to the large spikes after each cleaning cycle.

Figure 6-6 shows the daily average $\mathrm{SO}_{2}$ removals and $\mathrm{NO}_{2}$ emissions as a function of time for the entire duration of the four-month test. These results show that on a daily basis, the $\mathrm{NO}_{2}$ emissions were usually below $10 \mathrm{ppm}$ (the average for the duration of the test was 6.7 ppm). However, there were three periods of time when the levels approached or exceeded $20 \mathrm{ppm}$. All three of these cases correspond to times when the sodium sesquicarbonate feed rate was higher than normal. Figures 6-1 and 6-2 show that the first two cases (late November and late December) correspond to periods when the inlet $\mathrm{SO}_{2}$ levels were higher than usual. The main fuel source for the Arapahoe Station is a Cyprus Yampa 


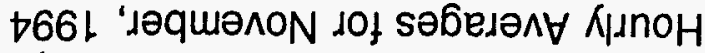

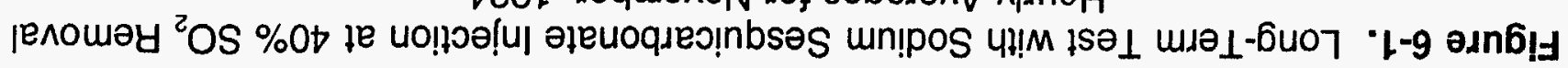

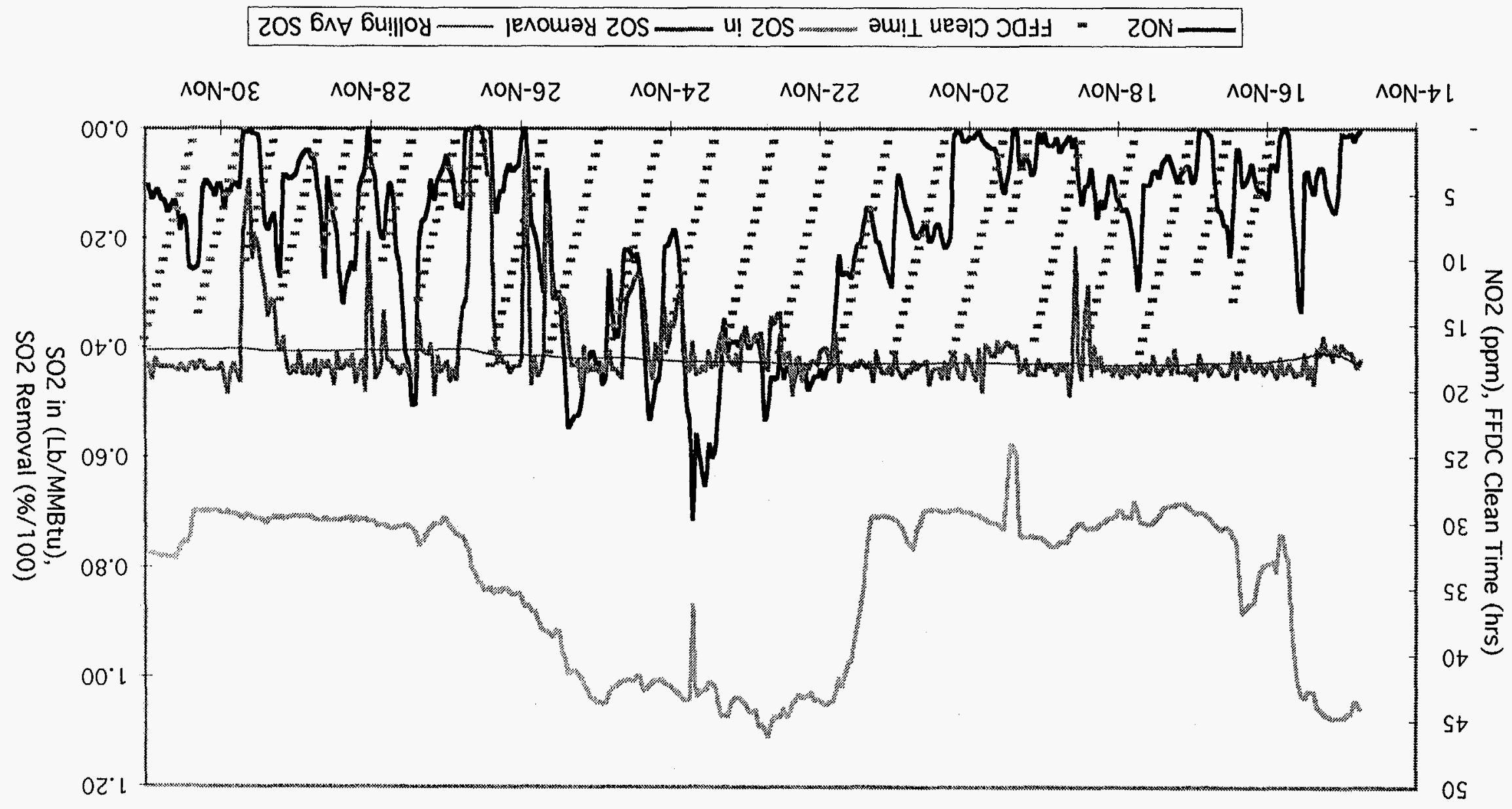

?. 
(00 L/\%) एе^oməy zOS

'(mgWW/q7) u! ZOS

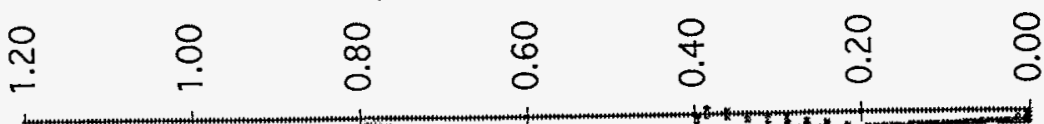

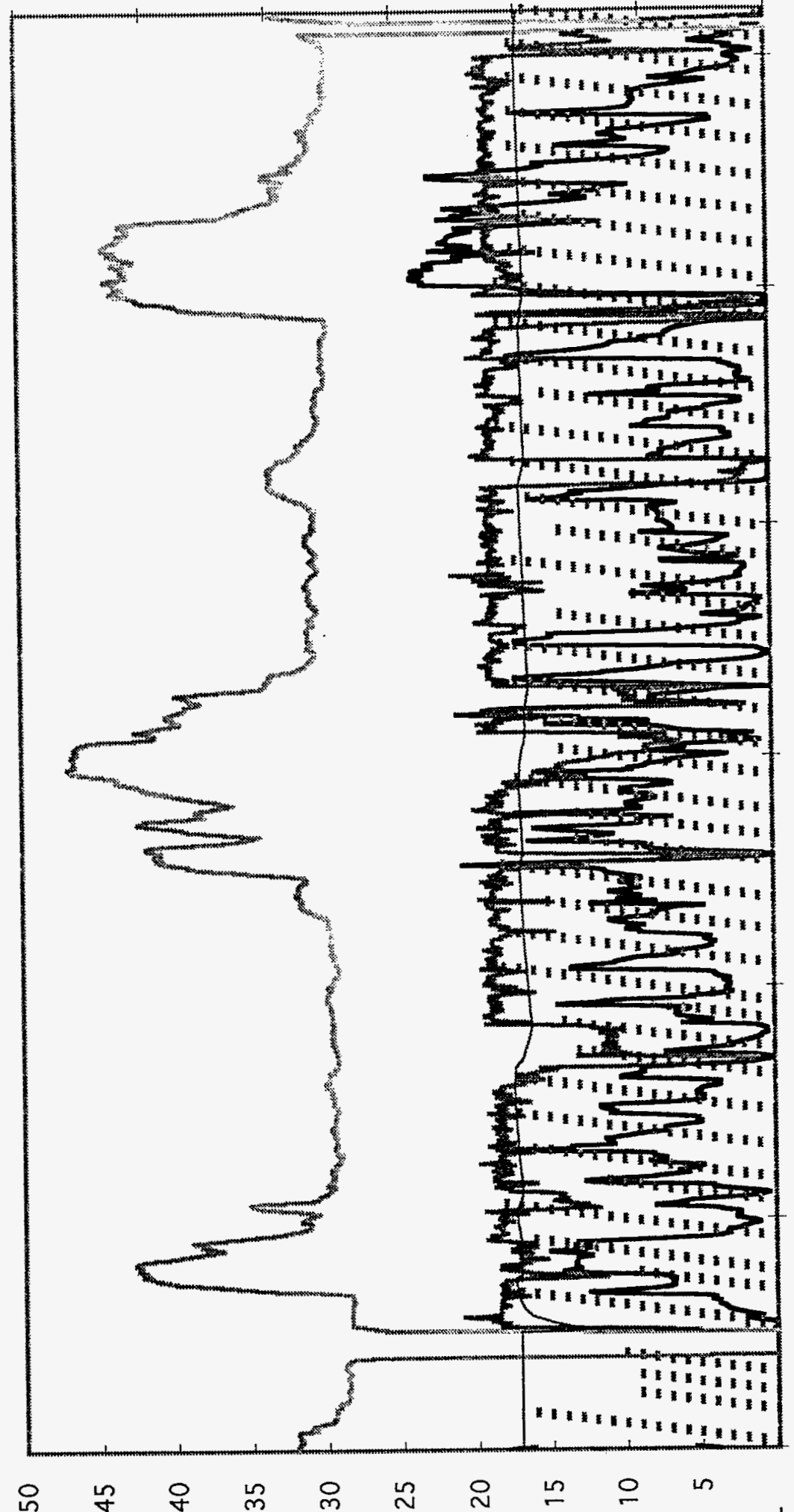

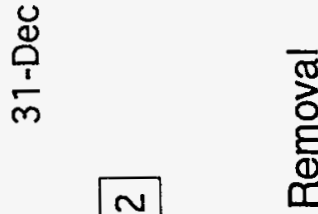

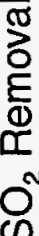

닝

ஓे

$\bar{\sigma}$

은

.

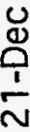

트 ᄋ

壳 ัँ்

등 으

叒

.잉

흉

क क

E

흥

क खे

突

호

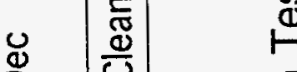

$\varepsilon$

ह

ㄴ

造

$\frac{1}{1}$

옹

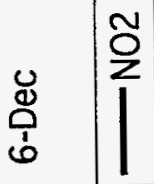

ช่

bे

是

足

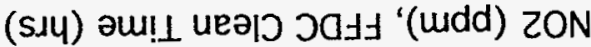




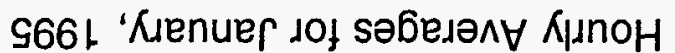

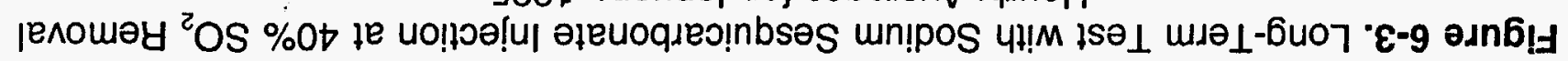

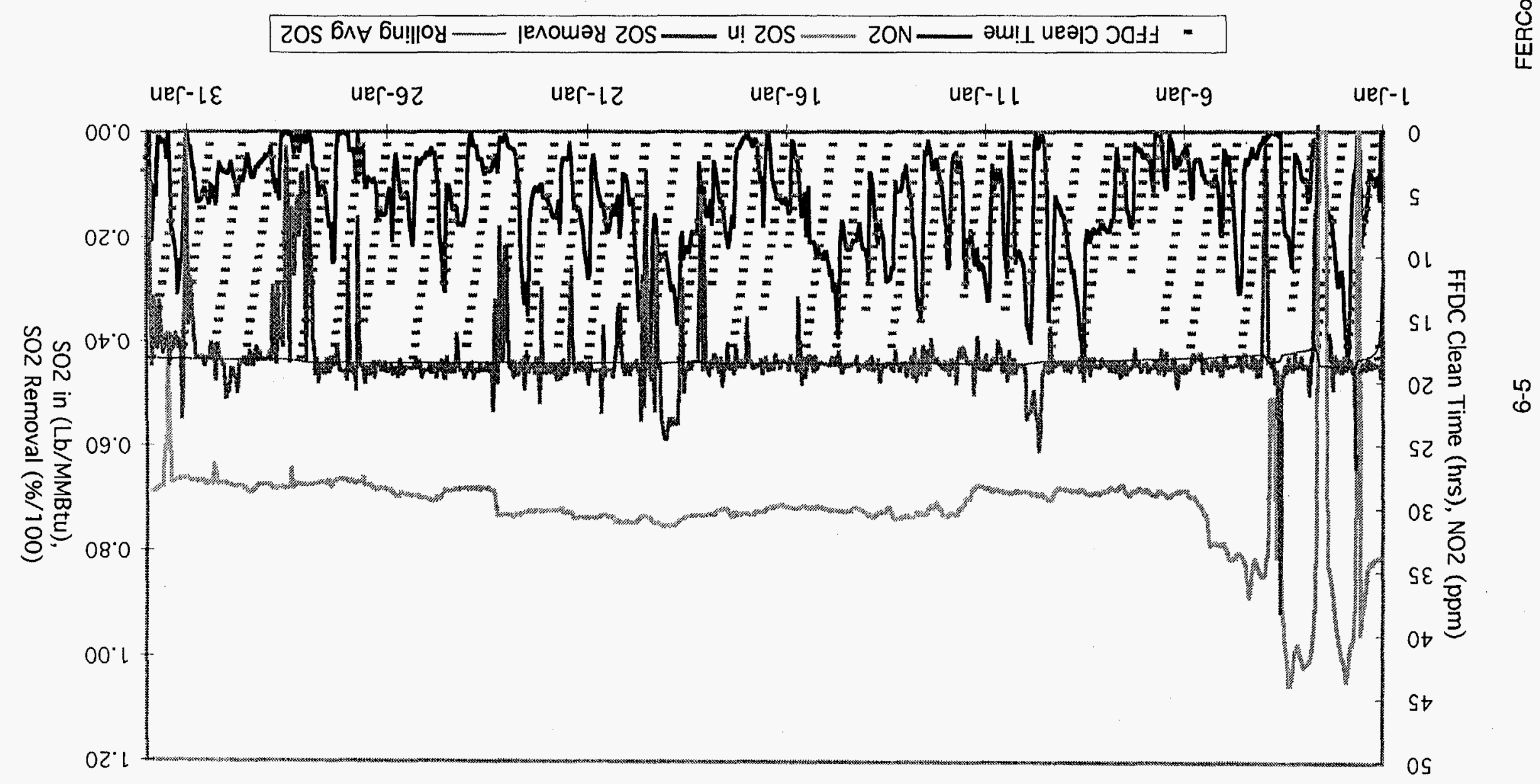




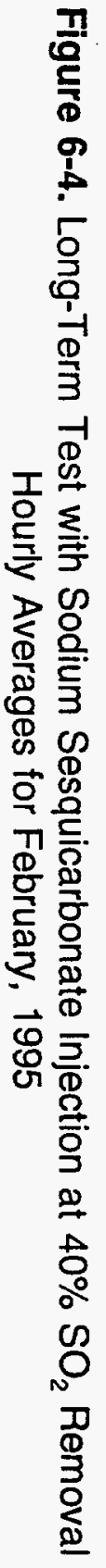

FFDC Clean Time (hrs), NO2 (ppm)

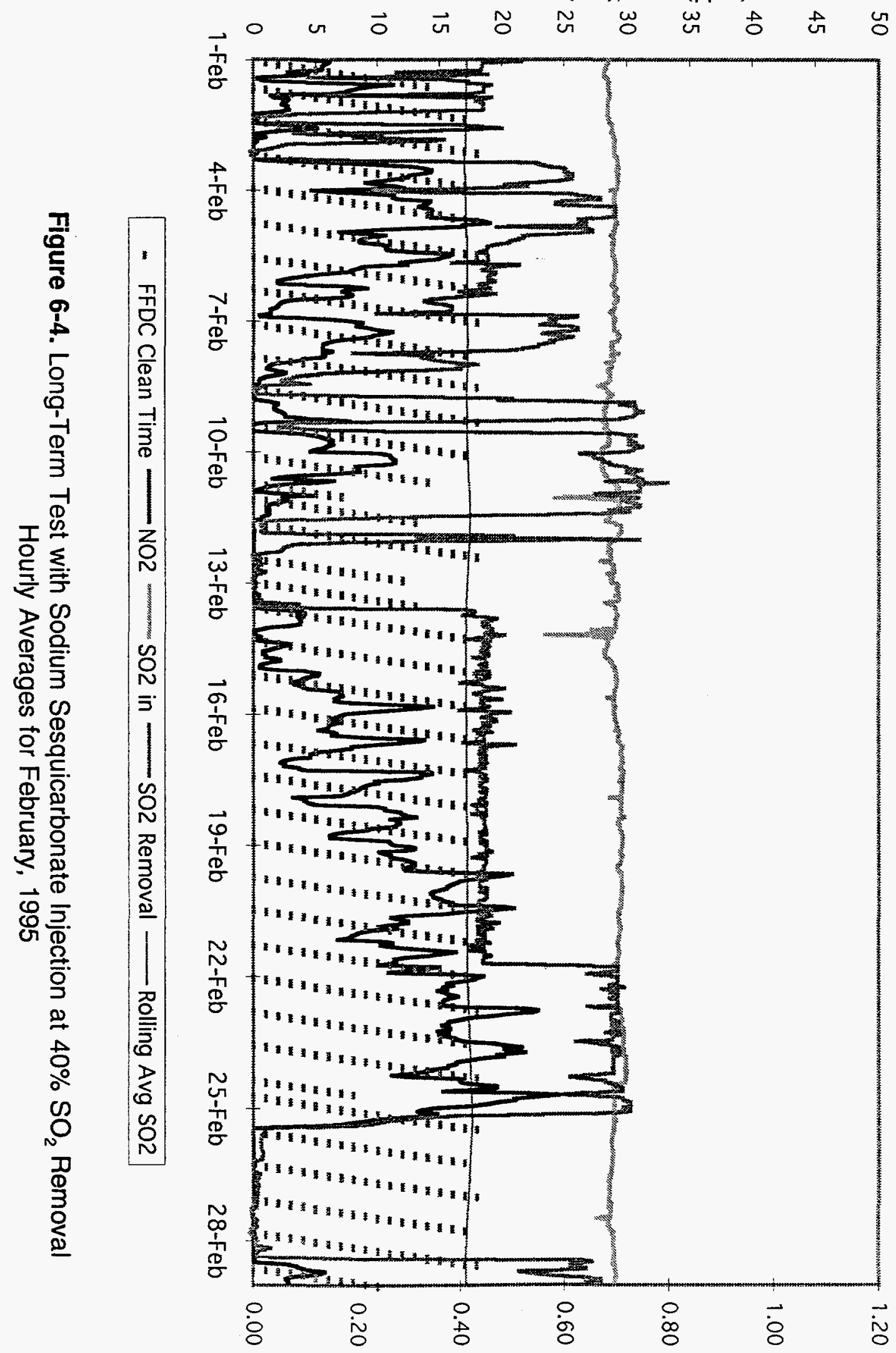

$\mathrm{SO} 2$ in (Lb/MMBtu),

SO2 Removal (\%/100) 
(00 L/\%) ןе^oway zOS

'(ngww/q7) u! zOS

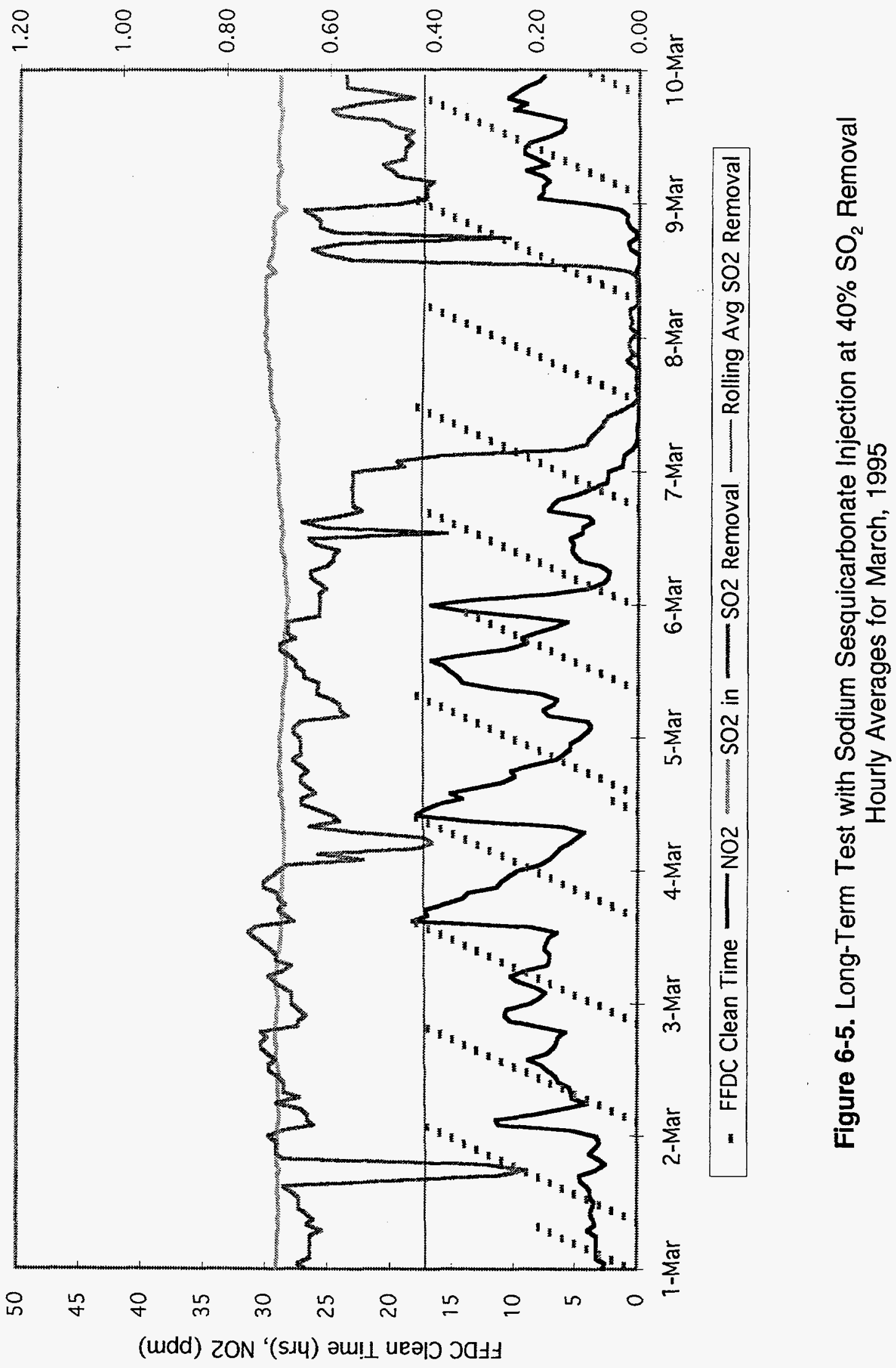


(mdd) ZON

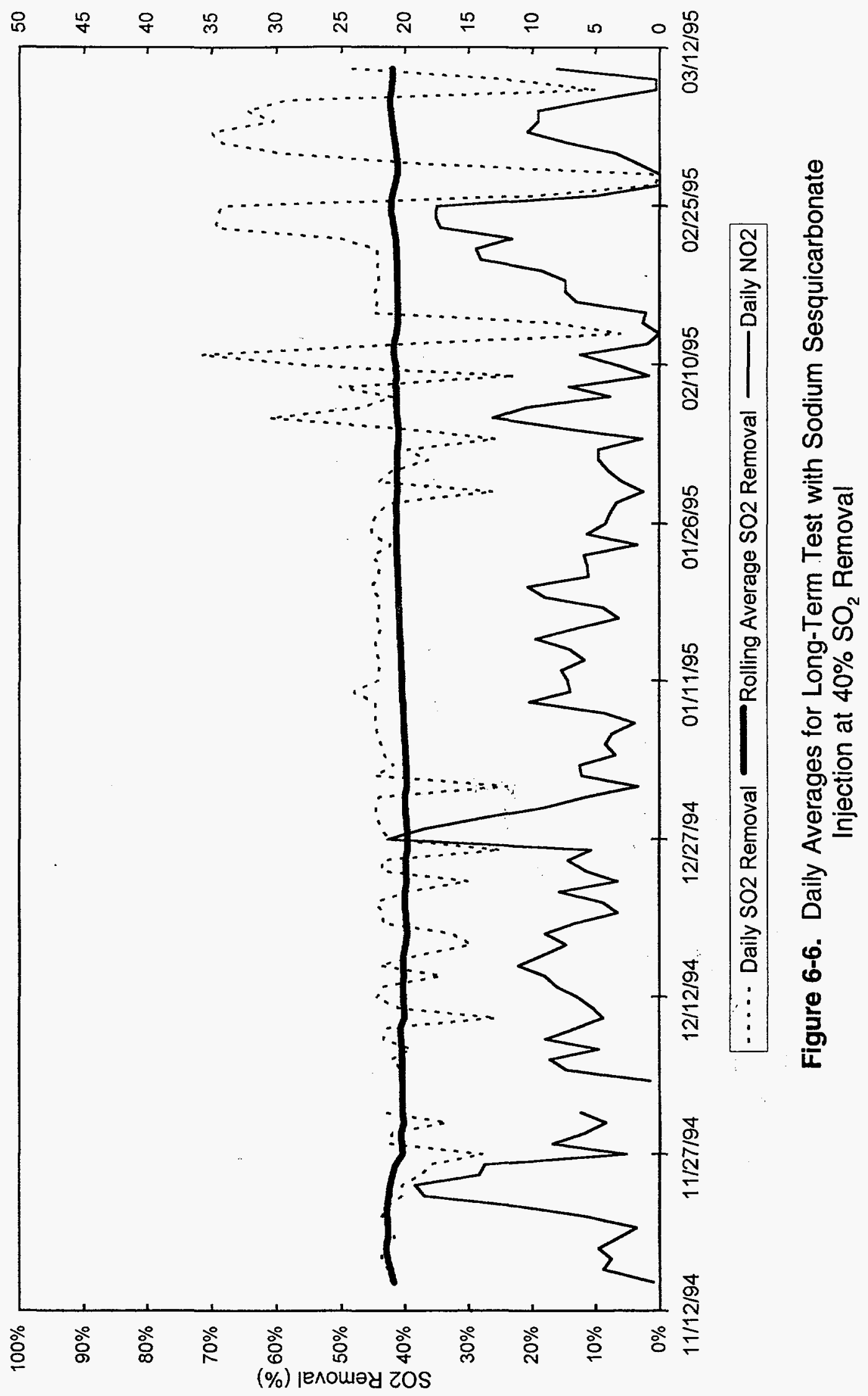


Valley coal. On occasion, coal from a different source (Edna mine) is utilized. The two coals are very similar, with the major difference being the sulfur content. When buming the Yampa coal, the baseline $\mathrm{SO}_{2}$ emissions are usually in the range of $0.7 \mathrm{lb} / \mathrm{MMBtu}$. With the Edna coal, the baseline levels can reach $1.1 \mathrm{lb} / \mathrm{MMBtu}$ as seen in Figures 6-1 to 6-3. These higher $\mathrm{SO}_{2}$ levels require higher reagent feed rates to maintain the 40 percent removal setpoint, and thus the $\mathrm{NO}_{2}$ levels into the fabric filter are also higher. These increased levels are seen at the stack immediately after each cleaning cycle when the bags are relatively clean and there is little fly ash (i.e., carbon) available to interact with the $\mathrm{NO}_{2}$.

The third time when the daily average $\mathrm{NO}_{2}$ emissions approached $20 \mathrm{ppm}$ was near the end of the four-month test. This occurrence was in late February, 1995, during one of the aborted attempts to run the system at 70 percent $\mathrm{SO}_{2}$ removal (Figure 6-4). The higher $\mathrm{NO}_{2}$ emissions are again attributed to an increased reagent feed rate. There were three other occasions during the test when attempts were made to operate the system at 70 percent $\mathrm{SO}_{2}$ removal for a short period of time (two in February and one in March, 1995). Figures 6-4 and 6-5 show that the $\mathrm{NO}_{2}$ emissions tended to increase during these times as well. However, problems with maintaining a consistent reagent feed rate during these attempts precluded generating $\mathrm{NO}_{2}$ emissions which approached or exceeded $20 \mathrm{ppm}$.

The repairs to the " $A$ " pulverizer were completed on June 26,1995 . On June 30,1995 , a second long-term test was started with the goal of maintaining an average $\mathrm{SO}_{2}$ removal of 70 percent. This test ended on July 29, 1995, when Arapahoe Unit 4 was taken off-line for a scheduled 10-week outage. Figure 6-7 shows the hourly averages of $\mathrm{SO}_{2}$ removal and $\mathrm{NO}_{2}$ emissions as a function of time for the duration of the four-week test. At the end of the test, the rolling average $\mathrm{SO}_{2}$ removal was 67.9 percent, just short of the goal of 70 percent. A number of mechanical problems resulted in a system availability of only 94 percent for the four weeks. Bearing failures in both the " $A$ " and " $B$ " screw feeders 
(00 L/\%) ןелоmay $20 S$

'(n+gWW/q7) u! 205

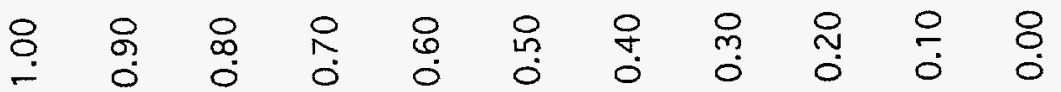

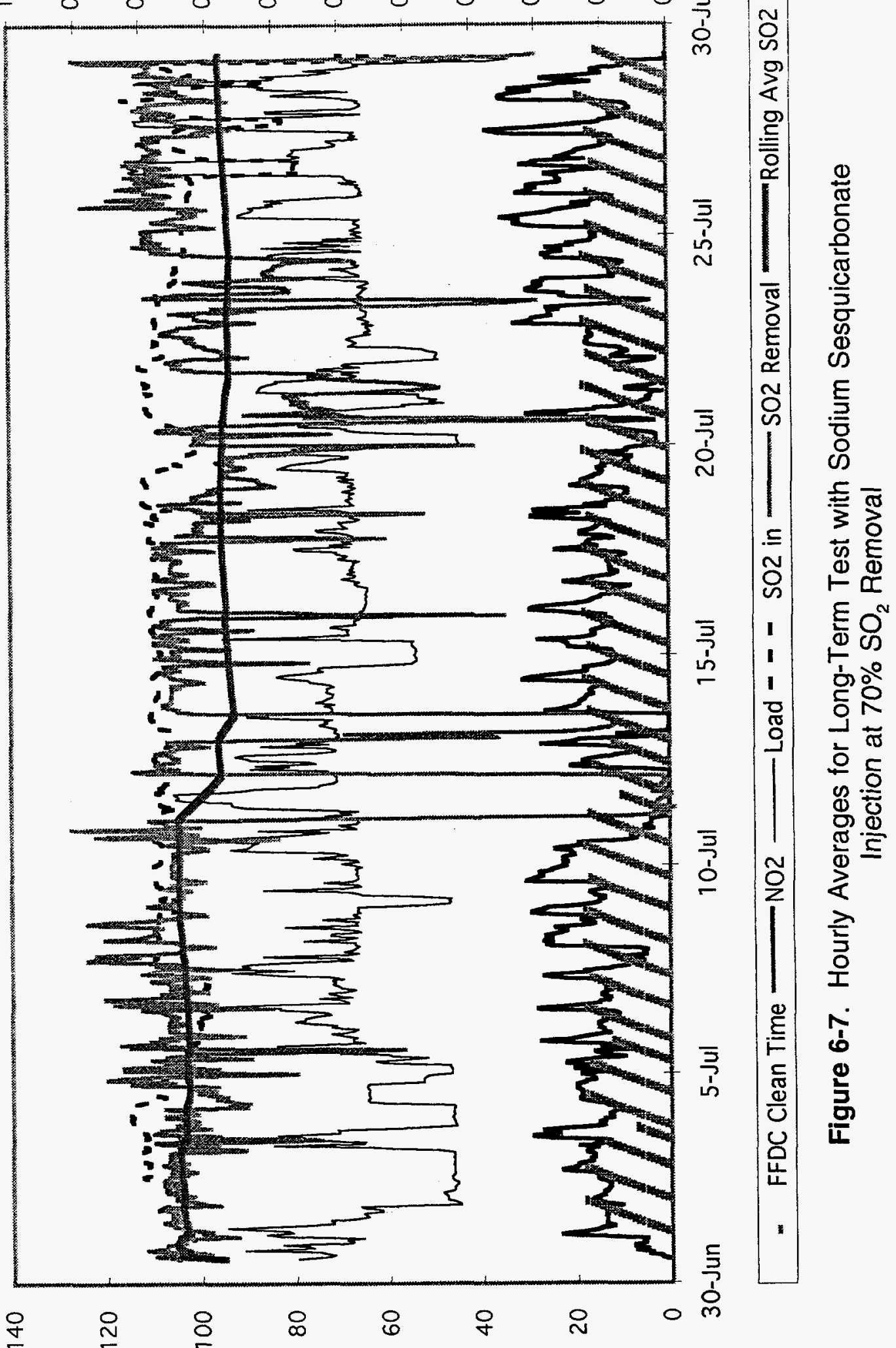

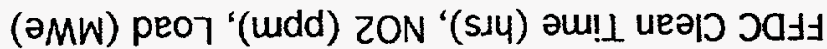


occurred, and there were continued problems with maintaining consistent reagent feed rates. The "A" silo was not emptied after the pulverizer disk was damaged during the $\mathbf{4 0}$ percent $\mathrm{SO}_{2}$ removal test. Four months of no material movement resulted in compaction of the reagent in the silo, and the material became very difficult to feed during the subsequent 70 percent $\mathrm{SO}_{2}$ removal test. It was decided not to add any additional reagent to the "A" silo until it was empty to ensure that all of the compacted material was removed. The silo emptied on July 11,1995 , and the screw feeder bearing on the "B" system failed three hours later. Thirty-two of the total 33 hours of downtime during the four-week test were related to the bearing failure and the lack of reagent in the operating silo.

It is believed that all of the screw feeder bearing failures experienced during both the 40 and 70 percent removal tests were related to excessive air leakage through the rotary airlocks. The leakage from the transport line below the airlock pressurizes the screw feeder and forces reagent into the bearing lubrication material. Lack of proper lubrication then causes failure of the bearing. The possibility of replacing the airlocks before completion of the testing of the integrated system is currently under investigation.

During the long-term test at 40 percent $\mathrm{SO}_{2}$ removal, $\mathrm{NO}_{2}$ emissions averaged $6.7 \mathrm{ppm}$ and there were no occurrences of a brown plume at the stack. The average $\mathrm{NO}_{2}$ emissions during the 70 percent test increased to $15.2 \mathrm{ppm}$, and a faint $\mathrm{NO}_{2}$ plume was visible on some occasions. The plume and $\mathrm{NO}_{2}$ emissions were more prevalent during long periods of low load operation. This again may be related to an ash carbon effect with lower carbon levels at low loads.

After the 10-week outage, further modifications were made to both DSI injection systems. These modifications primarily involved insulating the pipes which transported the material from the sorbent preparation area to the splitter valves at the FFDC injection location. Depending on the sorbent loading, the pulverizers can impart a significant amount of heat to the transport air stream (pulverizer exit temperatures can reach nearly $200^{\circ} \mathrm{F}$ without any sorbent flow). It was believed that some of the plugging problems experienced during 
the previous long-term tests were due to condensation forming in the transport lines as the carrier air/sorbent mixture cooled on the way to the injection location.

A third long-term test was begun on January 2, 1996, with the goal of maintaining a rollingaverage $\mathrm{SO}_{2}$ removal in excess of 70 percent until the long-term testing of the integrated system (simultaneous DSI and SNCR) was to begin in mid February. The $\mathrm{SO}_{2}$ removal setpoint was actually set at 75 percent during this time. The long-term DSI-only test had been running for only 10 days when the new fly ash removal system plugged, and both DSI systems had to be shut down. The rolling-average $\mathrm{SO}_{2}$ removal at the end of the 10-day period was 74 percent. The problem with the fly ash removal system was attributed to some residual effects of a Powder River Basin coal test burn run two months before. It was not believed that the plugging was a direct result of the sodium-injection test, although the increased solids loading may have accelerated the rate of plugging.

The fly ash removal system was brought back on-line on January 19, and both DSI systems were re-started. At this time, a problem with the "B" DSI system developed where the screw feeder would trip off-line randomly, for no apparent reason. This problem was resolved on January 28 , with the installation of a new variable-speed drive controller. At higher boiler loads, both DSI systems are necessary to achieve 70 percent $\mathrm{SO}_{2}$ removal. Since the "B" system was often off-line during the period of January 19 to 28 , the rollingaverage $\mathrm{SO}_{2}$ removal fell well below 70 percent.

The long-term DSI-only test continued until February 12, when the new Unit 4 fly ash removal system again failed. At this time, the fluidizing stones at the base of the ash storage silo (which keep the ash in a fluidized state during the unloading process) had become so plugged with ash, that air could no longer pass through them, and the silo could not be emptied. Both DSI systems were taken off-line at this point in time, and the longterm test ended. During the period from January 28 to February 12, the combination of increasing air leakage rates through the rotary airlocks and minor plugging problems resulted in a rolling-average $\mathrm{SO}_{2}$ removal of less than 70 percent. 
Figures 6-8a and $b$ show the hourly averages of $\mathrm{SO}_{2}$ removal and $\mathrm{NO}_{2}$ emissions, respectively, for the first 10 days of the long-term test begun on January 2, 1996. As mentioned previously, the rolling-average $\mathrm{SO}_{2}$ removal for this time period was 74 percent. Figure $6-8 \mathrm{~b}$ shows that, while the $\mathrm{NO}_{2}$ emissions varied over the range of approximately 0 to $30 \mathrm{ppm}$, the rolling-average for the 10-day period was $11.9 \mathrm{ppm}$. This level is lower than that seen during the month-long 70 percent $\mathrm{SO}_{2}$ removal test shown in Figure 6-7 (June 30 to July 29, 1995). Although the difference is believed to be due to the shorter duration of the test performed in January, 1996, there is no conclusive evidence to support this hypothesis. Another attempt to maintain a rolling-average $\mathrm{SO}_{2}$ removal in excess of 70 percent with DSI alone (for a period of approximately four weeks) will be made after the conclusion of the integrated tests in late 1996. The results of this test will provide a better indication of the average long-term $\mathrm{NO}_{2}$ emissions. 


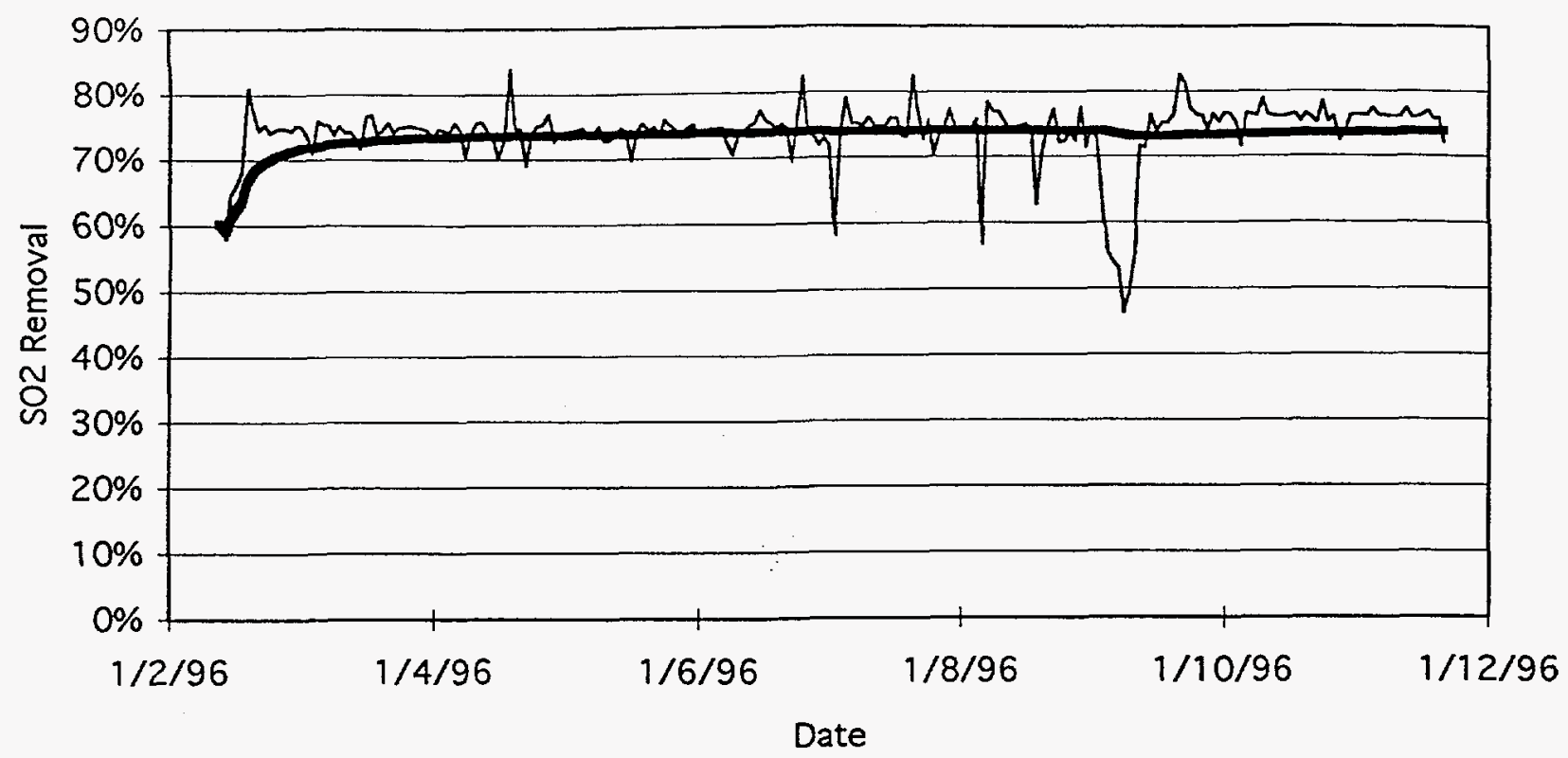

- Rolling Average SO2 Removal —-Hourly SO2 Removal

a) $\mathrm{SO}_{2}$ Removal

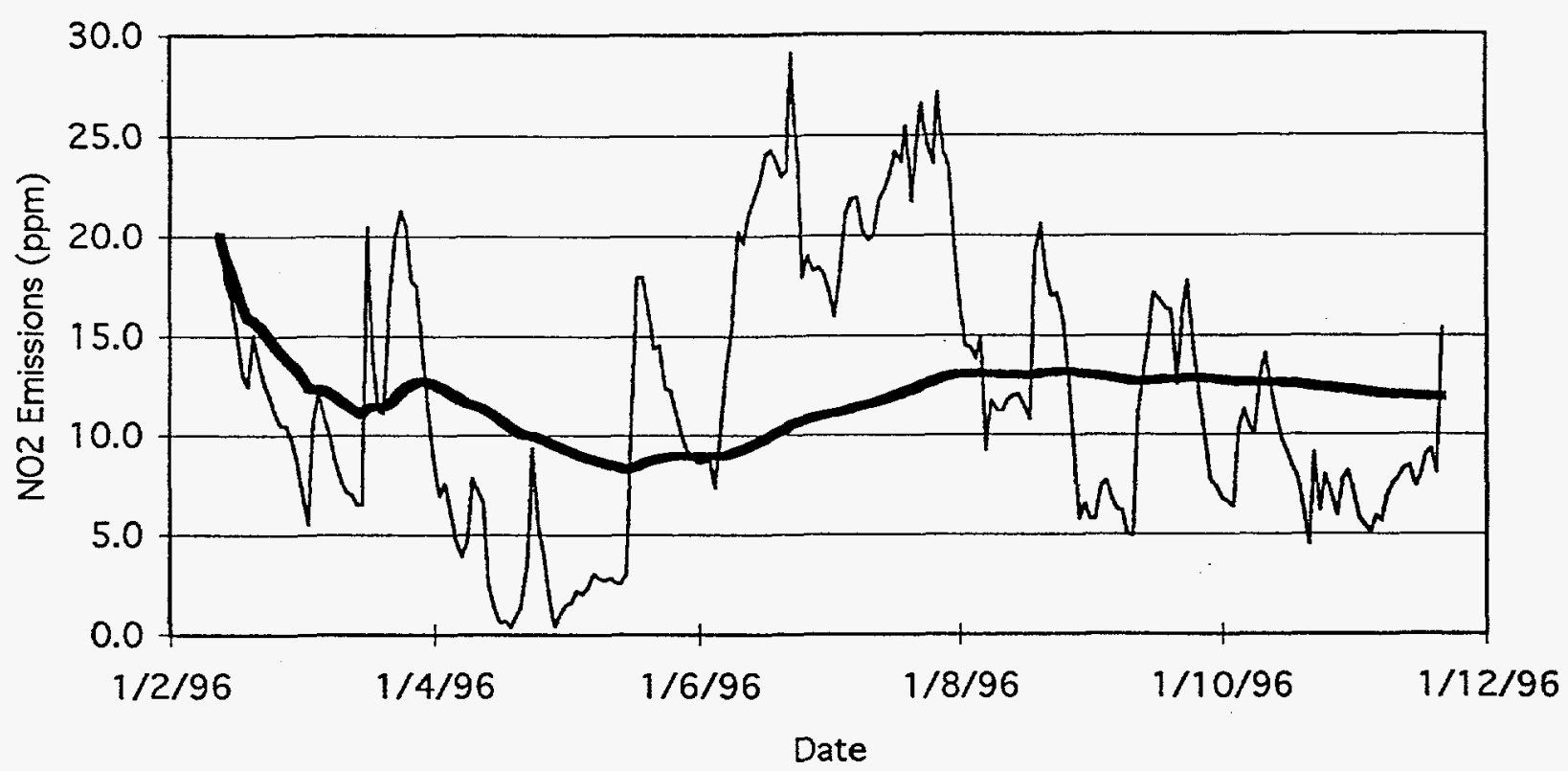

Rolling Average NO2 Emissions - Hourly NO2 Emissions

b) $\mathrm{NO}_{2}$ Emissions

Figure 6-8. Hourly Averages for Long-Term Test with Sodium Sesquicarbonate Injection at $75 \% \mathrm{SO}_{2}$ Removal (January 2-11, 1996) 


\subsection{DISCUSSIONS AND CONCLUSIONS}

During this test phase, sodium sesquicarbonate and sodium bicarbonate were evaluated in terms of $\mathrm{SO}_{2}$ removal, $\mathrm{NO}_{2}$ production and $\mathrm{NO}_{x}$ removal. Of particular concern was the production of $\mathrm{NO}_{2}$ and the possibility of plume coloration. In general, the results were consistent with those reported from previous full-scale dry sodium injection demonstrations (Fuchs, et al., 1989; Muzio, et al., 1984).

One of the more interesting observations from the current test program was the process dynamics of $\mathrm{NO}_{2}$ formation with sodium injection. Time resolved measurements showed that $\mathrm{NO}_{2}$ emissions were not only dependent on the amount of sodium injected, but also on the fabric filter cleaning cycle. With both sodium sesquicarbonate and sodium bicarbonate, $\mathrm{NO}_{2}$ emissions were found to increase markedly just after a cleaning cycle. This suggests that there is an interaction between the $\mathrm{NO}_{2}$ and the fly ash on the bags; and more specifically, the fly ash carbon. This was further confirmed by compartment-bycompartment measurements which showed that the $\mathrm{NO}_{2}$ levels were not just a function of the $\mathrm{SO}_{2}$ removal in each compartment, but also appear to be related to the amount of fly ash collected in each compartment. This phenomena accounts for the high degree of variability in $\mathrm{NO}_{2}$ emissions and $\mathrm{NO}_{x}$ removals reported not only in this test program, but in the previous demonstrations.

Specific conclusions from the dry sodium injection tests are listed below.

1. Both sodium sesquicarbonate and sodium bicarbonate were able to achieve $70 \%$ $\mathrm{SO}_{2}$ removal. Sodium bicarbonate exhibits a higher utilization of sodium than sodium sesquicarbonate. As such, sodium bicarbonate can achieve $70 \% \mathrm{SO}_{2}$ removal at a lower molar ratio of sodium to $\mathrm{SO}_{2}$ than sodium sesquicarbonate. The primary factor controlling $\mathrm{SO}_{2}$ removal was the sorbent injection rate, or normalized stoichiometric ratio $(2 \mathrm{Na} / \mathrm{S})$. Seventy percent $\mathrm{SO}_{2}$ removal was achieved at the following $2 \mathrm{Na} / \mathrm{S}$ ratios for the two sorbents:

$$
\begin{array}{lll}
\text { Sodium Sesquicarbonate } & : & 2 \mathrm{Na} / \mathrm{S}=1.9 \\
\text { Sodium Bicarbonate } & : & 2 \mathrm{Na} / \mathrm{S}=0.9
\end{array}
$$

2. Boiler load had no effect on $\mathrm{SO}_{2}$ removal, or sodium utilization over the load range investigated (60 to $100 \mathrm{MWe}$ ). 
3. Flue gas temperature had some effect on the $\mathrm{SO}_{2}$ removal process and was different for the two reagents. There was no effect on $\mathrm{SO}_{2}$ removal, or utilization, over the investigated temperature range of $220^{\circ} \mathrm{F}$ to $280^{\circ} \mathrm{F}$ for sodium sesquicarbonate injection ahead of the FFDC. When sodium sesquicarbonate was injected ahead of the air heater at approximately $650^{\circ} \mathrm{F}$, the $\mathrm{SO}_{2}$ reaction rate was slower than injection at the FFDC inlet. This resulted in the same steady-state level of $\mathrm{SO}_{2}$ removal but a slightly lower time-averaged $\mathrm{SO}_{2}$ removal. Sodium bicarbonate injection at the FFDC provided a very slow $\mathrm{SO}_{2}$ reaction and was not effective at the flue gas temperatures available at Arapahoe. Injection ahead of the air heater, at approximately $650^{\circ} \mathrm{F}$, increased the reaction rate and allowed good process control and reactivity. Minor temperature changes at both locations did not significantly affect the process.

4. Particle size affected the $\mathrm{SO}_{2}$ removal and utilization of both products. No significant change to $\mathrm{SO}_{2}$ removal, or utilization, was noted when sodium sesquicarbonate was pulverized within the range of 15 to 20 microns MMD. Injection of non-pulverized sodium sesquicarbonate, with a 28 micron MMD, reduced $\mathrm{SO}_{2}$ removal, at an equivalent reagent feed rate, by a significant 10 to $15 \%$.

No significant change to $\mathrm{SO}_{2}$ removal, or utilization, was noted when sodium bicarbonate was pulverized to a range of 18 to 25 microns MMD and injected to yield $\mathrm{SO}_{2}$ removals of $70 \%$ or less. At higher feed rates which provided up to $90 \%$ $\mathrm{SO}_{2}$ removal, reagent in the size range of 25 microns degraded $\mathrm{SO}_{2}$ removal by a net $10 \% . \mathrm{SO}_{2}$ removal and utilization were not affected by the feed rate through the mill with either reagent, when the mill was operated at a set speed.

5. Minor changes in the distribution of the reagent at the injection location, due to injector plugging, or modifications to the injection system, did not affect the overall $\mathrm{SO}_{2}$ removal or utilization for either reagent. However, changes in distribution did change the distribution of $\mathrm{SO}_{2}$ removal within the FFDC.

6. Humidification of the flue gas to a $60^{\circ} \mathrm{F}$ approach to saturation temperature increased $\mathrm{SO}_{2}$ removal by up to $20 \%$ when injecting sodium sesquicarbonate (at the same $2 \mathrm{Na} / \mathrm{S}$ ratios).

7. Both sodium sesquicarbonate and sodium bicarbonate also produce some $\mathrm{NO}_{\mathrm{x}}$ reduction. At a $70 \% \mathrm{SO}_{2}$ removal approximately $10 \% \mathrm{NO}_{\mathrm{x}}$ reduction is obtained with both reagents. The $\mathrm{NO}_{x}$ reduction varies due to unexplainable conditions and cannot be controlled. However, over time, an average $\mathrm{NO}_{x}$ removal of $10 \%$ could be consistently achieved with both reagents at Arapahoe Unit 4 when obtaining $70 \% \mathrm{SO}_{2}$ removal.

8. Both sodium reagents oxidize $\mathrm{NO}$ to $\mathrm{NO}_{2}$. While the total $\mathrm{NO}_{x}$ emissions are reduced, the $\mathrm{NO}_{2}$ emission will increase. There is significant variation in $\mathrm{NO}_{2}$ emissions, but the reagent feed rate, FFDC cleaning cycle, and the ash composition 
are all important variables affecting $\mathrm{NO}_{2} . \mathrm{NO}_{2}$ is a visible gas and thus can create a visible plume. At Arapahoe Unit 4, a net $\mathrm{NO}_{2}$ emission of approximately $35 \mathrm{ppm}$ will create a visible stack plume.

9. Sodium sesquicarbonate creates less $\mathrm{NO}_{2}$ than sodium bicarbonate at the same $\mathrm{SO}_{2}$ removal level. $\mathrm{NO}_{2}$ emissions increased up to $30 \mathrm{ppm}$ with sodium sesquicarbonate and up to $50 \mathrm{ppm}$ with sodium bicarbonate injection.

10. A four week test of sodium sesquicarbonate easily met the 40 percent $\mathrm{SO}_{2}$ removal goal with an average $\mathrm{NO}_{2}$ emission of $7 \mathrm{ppm}$. There were no occurrences of a visible brown plume.

11. A four week test of sodium sesquicarbonate fell just short of the $70 \%$ removal goal, with an average $\mathrm{SO}_{2}$ removal of $68 \%$. $\mathrm{NO}_{2}$ emissions during this test averaged $15 \mathrm{ppm}$ and a faint brown plume was visible on some occasions. The goal was not achieved due to equipment problems that prevented any sodium injection for a 32-hour period during the test. 


\subsection{REFERENCES}

Fuchs, M.R., et al., Full-Scale Demonstration of a Utility Dry Sodium Injection FGD Facility, EPRI Final Report, Project RP-16826, December 1989.

Keener, T., et al., Thermal Decomposition of Sodium Bicarbonate, Chem. Eng. Commun., Vol. 33, pp. 93-105, 1985.

Lai, Y., et al., Simultaneous Removal of $\mathrm{SO}_{2}$ and $\mathrm{NO}_{x}$ By Selected Sodium-Based Dry Sorbents, Final Technical Report to Public Service Co., Colorado School of Mines, 1994.

Lindau, L., $\mathrm{NO}_{2}$ Effect on Flue Gas Opacity, J. Air Waste Management Assoc., Vol. 41, No. 8, August 1991.

Muzio, L.J., et al., Dry $\mathrm{SO}_{2}$ Particulate Removal for Coal-Fired Boilers, Volume 2: 22-MW Demonstration Using Nacholite, Trona, and Soda Ash, EPRI Report CS-2894, June 1984.

Shiomoto, G.H., et al., Integrated Dry $\mathrm{NO}_{x} / \mathrm{SO}_{2}$ Emissions Control System: Baseline Test Report, DOE Contract Number DE-FCC22-91PC90550, NTIS:

DE92040528, March 1992.

Shiomoto, G.H., et al., Integrated Dry $\mathrm{NO}_{x} / \mathrm{SO}_{2}$ Emissions Control System: CalciumBased Dry Sorbent Injection Test Report, DOE Contract Number DE-FCC2291PC90550, December 1994.

Smith, R.A., et al., Integrated Dry NO/ $\mathrm{SO}_{2}$ Emissions Control System: Baseline SNCR Test Report, DOE Contract Number DE-FCC22-91PC90550, NTIS: DE94005035, September 1993.

Smith, R.A., et al., Integrated Dry NO $\mathrm{N}_{x} \mathrm{SO}_{2}$ Emissions Control System: Low-NO Combustion System Retrofit Test Report, DOE Contract Number DE-FCC2291PC90550, NTIS: DE94014532, October 1994a.

Smith, R.A., et al., Integrated Dry NO $\mathrm{N}_{x} \mathrm{SO}_{2}$ Emissions Control System: Low-NO Combustion System SNCR Test Report, DOE Contract Number DE-FCC2291PC90550, NTIS: DE94017370, November 1994b.

Smith, R.A., et al., Calcium Injection Upstream of an Electrostatic Precipitator and a Fabric Filter for Simultaneous $\mathrm{SO}_{2}$ and Particulate Removal: Pilot- and BenchScale Results, EPRI Report EPRI TR-100260, January 1992.

Verlaetent, J., et al., Combined $\mathrm{SO}_{2} / \mathrm{NO}_{x}$ Abatement by Sodium Bicarbonate Dry Injection, 1050 Brussels, Belgium, 1993. 


\section{APPENDICES}




\section{APPENDIX A}

Synopsis of the

Colorado School of Mines

Bench-Scale Study of Sodium/ $/ \mathrm{SO}_{2} / \mathrm{NO}_{x}$ Chemistry Entitled:

"SIMULTANEOUS REMOVAL OF $\mathrm{SO}_{2}$ AND $\mathrm{NO}_{x} \mathrm{BY}$ SELECTED SODIUM-BASED DRY SORBENTS"

by

Y. Lai

and

V. F. Yesavage 


\section{A. FUNDAMENTAL STUDY OF SODIUM/SO $/ \mathrm{NO}_{x}$ CHEMISTRY}

\section{A.1 Background}

A fundamental study of sodium $/ \mathrm{SO}_{2} / \mathrm{NO}_{x}$ chemistry was conducted by Colorado School of Mines to support the full-scale integrated $\mathrm{NO}_{x}$ and $\mathrm{SO}_{2}$ removal work at Arapahoe Unit 4 (Lai, et al., 1994). The goal of the study was to gain a better understanding of the detailed chemistry in terms of $\mathrm{SO}_{2}$ and $\mathrm{NO}_{x}$ removal, as well as $\mathrm{NO}_{2}$ formation. The study involved both bench-scale experiments and chemical kinetic modeling. Since little work was previously published on the fundamental chemistry associated with $\mathrm{NO}_{x}$ removal and $\mathrm{NO}_{2}$ production with sodium compounds, the study emphasized this aspect of the chemistry.

Before discussing the specific experiments that were conducted, it is of value to briefly review the potential detailed mechanisms associated with $\mathrm{NO}_{2}$ formation and $\mathrm{NO}_{x}$ removal. Two different mechanisms have been proposed for the production of $\mathrm{NO}_{2}$ during the sodium $/ \mathrm{SO}_{2}$ reactions. One was developed by work supported by EPRI (EPRI, 1990) and the other is work done by Solvay (Verlaeten, et al., 1993). The mechanisms shown below are for sodium bicarbonate, although parallel mechanisms could be written for sodium sesquicarbonate.

\section{A.2 EPRI Mechanism}

Sodium Bicarbonate Decomposition

$$
2 \mathrm{NaHCO}_{3} \rightarrow \mathrm{Na}_{2} \mathrm{CO}_{3}+\mathrm{CO}_{2}+\mathrm{H}_{2} \mathrm{O}
$$

$\underline{S O}_{2}$ Removal

$$
\mathrm{Na}_{2} \mathrm{CO}_{3}+\mathrm{SO}_{2}+1 / 2 \mathrm{O}_{2} \rightarrow \mathrm{Na}_{2} \mathrm{SO}_{4}+\mathrm{CO}_{2}
$$

NO Removal and $\mathrm{NO}_{2}$ Formation

$$
\begin{aligned}
& \mathrm{NO}+1 / 2 \mathrm{O}_{2} \stackrel{\mathrm{Na} / \mathrm{SO}_{2} \mathrm{NO}_{2}}{\rightarrow} \\
& \mathrm{Na}_{2} \mathrm{CO}_{3}+3 \mathrm{NO}_{2} \rightarrow 2 \mathrm{NaNO}_{3}+\mathrm{NO}+\mathrm{CO}_{2}
\end{aligned}
$$


The EPRI mechanism proposes sodium carbonate $\left(\mathrm{Na}_{2} \mathrm{CO}_{3}\right)$ as the primary reactant leading to $\mathrm{SO}_{2}$ removal. $\mathrm{NO}_{2}$ is proposed to form via reaction (3) along with some undetermined intermediate steps. $\mathrm{NO}_{\mathrm{x}}$ removal then occurs by a reaction between sodium carbonate and $\mathrm{NO}_{2}$ forming sodium nitrate, reaction (4).

\section{A.3 Solvay Mechanism}

The Solvay mechanism incorporates a direct reaction between the undecomposed sodium materials and $\mathrm{SO}_{2}$.

$$
\begin{aligned}
& \mathrm{SO}_{2} \text { Removal } \\
& \mathrm{NaHCO}_{3}+\mathrm{SO}_{2} \rightarrow \mathrm{NaHSO}_{3}+\mathrm{CO}_{2} \\
& 2 \mathrm{NaHSO}_{3} \rightarrow \mathrm{Na}_{2} \mathrm{~S}_{2} \mathrm{O}_{5}+\mathrm{H}_{2} \mathrm{O}
\end{aligned}
$$

With the sodium sulfite $\left(\mathrm{NaHSO}_{3}\right)$ and sodium pyrosulfite $\left(\mathrm{Na}_{2} \mathrm{~S}_{2} \mathrm{O}_{5}\right)$ intermediates, Solvay has an alternate interpretation of the $\mathrm{NO}_{\mathrm{x}}$ removal mechanism which involves the intermediate sodium pyrosulfite compound.

\section{NO Removal}

$$
\begin{aligned}
& \mathrm{Na}_{2} \mathrm{~S}_{2} \mathrm{O}_{5}+2 \mathrm{NO}+\mathrm{O}_{2} \rightarrow \mathrm{NaNO}_{2}+\mathrm{NaNO}_{3}+2 \mathrm{SO}_{2} \\
& 2 \mathrm{NaHSO}_{3}+2 \mathrm{NO}+\mathrm{O}_{2} \rightarrow \mathrm{NaNO}_{2}+\mathrm{NaNO}_{3}+2 \mathrm{SO}_{2}+2 \mathrm{H}_{2} \mathrm{O}
\end{aligned}
$$

In the Solvay mechanism the $\mathrm{NO}_{2}$ would be formed by decomposition of one of the products from reactions (7) and/or (8).

Both of the above mechanisms were investigated through a series of chemical kinetic models. 


\section{A.4 Experimental Approach}

The experiments were conducted in a bench-scale batch-fed reactor. The apparatus consists of $\mathrm{SO}_{2}$ and $\mathrm{NO}$ feed systems, a neutralization bottle containing $1 \mathrm{M} \mathrm{NaOH}$ solution designed for trapping $\mathrm{SO}_{2}$ and $\mathrm{NO}_{x}$ acid gases, a system for feeding background gas containing $3 \%$ oxygen and $97 \%$ nitrogen, a batch reactor with a heating control unit, and a gas analysis system.

A schematic of the bench-scale system is shown in Figure A-1. The basic components are a $12864 \mathrm{ml}$ batch reactor which contains the reaction mixtures, the dry sorbent, a heating control unit which keeps the reaction temperature constant between 100 to $300^{\circ} \mathrm{C}$, and a propeller-type mixer designed to eliminate temperature and mass transfer gradients surrounding the dry sorbent particles.

The batch reactor is made of stainless steel with four fittings on the top cover. The first fitting, V2, is a control valve with a 1/2" opening utilized for either gas or sorbent powder injection. The second fitting, $V 3$, is a control valve with a $1 / 4$ " opening used for draining the unreacted acid gases to a neutralization bottle containing a $1 \mathrm{M} \mathrm{NaOH}$ solution. The third fitting, $\mathrm{P} 1$, is a sampling port with a $1 / 2^{\prime \prime}$ opening which is a swagelock fitting equipped with a 9 millimeter diameter septum. The fourth fitting, $P 2$, is an injection port with a 1/2" opening and septum, which is identical to the sampling port (P1). Both, sampling and injection ports, are used for withdrawing the reacting gas mixtures and for injecting acid gases, $\mathrm{SO}_{2}$ and $\mathrm{NO}$.

A Haake Buchler stirring motor, $M 1$, is used to drive a propeller type mixing device with a variable speed ranging from 0 to $700 \mathrm{rpm}$. A teflon-type Conax sealant is used to seal the rotating rod at ambient pressure in the reactor.

The major components in the heating control unit are a 1800 Watt Watlow mica band heater, and an Omega Series 920 temperature controller. 


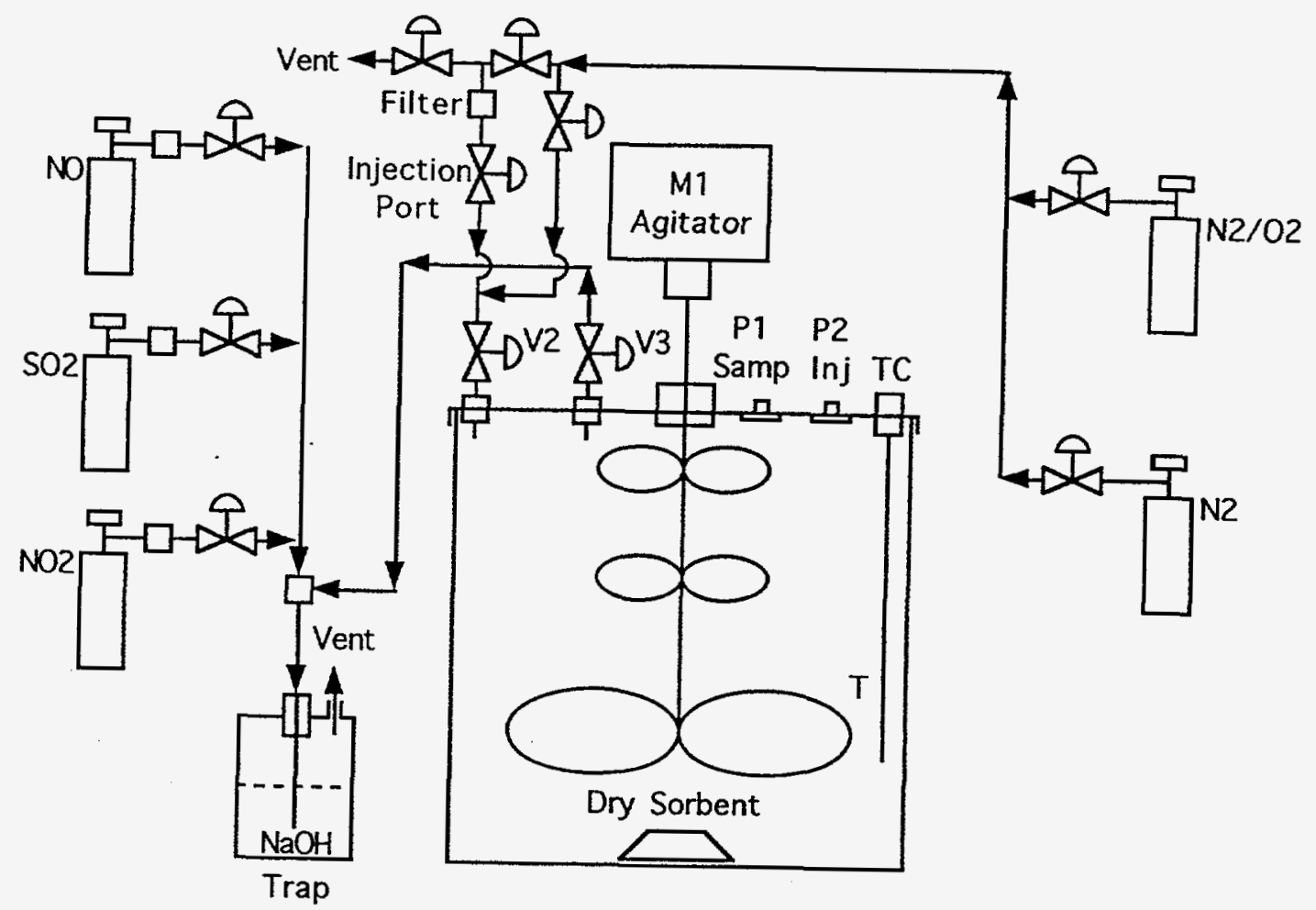

Figure A-1. Dry Sorbent Scrubber System

Bench-Scale Apparatus 
Analytical techniques include the analysis of syringe samples for $\mathrm{SO}_{2}, \mathrm{NO}_{x}$ and $\mathrm{NO} . \mathrm{SO}_{2}$ was measured using a Hewlett Packard model 5890 gas chromatograph equipped with a thermal conductivity detector, and a $8^{\prime} \times 1 / 4^{\prime \prime}$ Supelco Porapak $Q$ glass column with particle size $80 / 100$ at a temperature of $70^{\circ} \mathrm{C}$. During the course of the study, it was shown that this gas chromatographic procedure lead to errors in the $\mathrm{SO}_{2}$ concentration due to the presence of $\mathrm{NO}_{x}$ in the reaction mixture. This effect was thought to be due to a reaction sequence that forms $\mathrm{N}_{2} \mathrm{O}$ (Muzio, et al., 1988). To eliminate the effect, it was necessary to perform the $\mathrm{SO}_{2}$ analysis immediately upon obtaining a sample.

A chemiluminescent $\mathrm{NO}-\mathrm{NO}_{\mathrm{x}}$ analyzer (TECO model 44), was used to measure concentrations of $\mathrm{NO}$ and $\mathrm{NO}_{x} ; \mathrm{NO}_{2}$ was determined by difference. The chemiluminescent instrument is normally used in a continuous sampling mode. For this study, a technique was developed to use the chemiluminescent analyzer to analyze batch syringe samples from the reactor tests (Lai, et al., 1994).

\section{A.5 Experimental Results}

Two basic types of experiments were conducted during the study. The first group of experiments were conducted to develop overall reaction rate data for $\mathrm{SO}_{2}$ reactions with sodium bicarbonate and sodium sesquicarbonate. These experiments were conducted with the following range of parameters:

Sodium Sorbents

: sodium bicarbonate, sodium sesquicarbonate

Particle Size

: $53 \mu \mathrm{m}<\mathrm{d}<63 \mu \mathrm{m}$

Stoichiometric Ratio (2Na/S) : $0,0.85,3.4,13.5$

Temperature

: $260^{\circ} \mathrm{F}, 300^{\circ} \mathrm{F}$

$\mathrm{SO}_{2}$

: $1800 \mathrm{ppm}$

NO

: $1800 \mathrm{ppm}$

$\mathrm{H}_{2} \mathrm{O}, \mathrm{O}_{2}$

: $5 \%, 3 \%$, (balance $\mathrm{N}_{2}$ )

Reaction Times

: $0-30$ minutes

During these tests, time resolved histories of $\mathrm{SO}_{2}, \mathrm{NO}$, and $\mathrm{NO}_{\mathrm{x}}$ were measured. 
The next group of experiments sought to provide a better understanding of the detailed chemistry between $\mathrm{SO}_{2}, \mathrm{NO}_{\mathrm{x}}$ and the sodium compounds. These latter experiments involved varying the water content of the gas; as well as studying the reaction of potential intermediate compounds (i.e., $\mathrm{NaHSO}_{3}$ and $\mathrm{Na}_{2} \mathrm{~S}_{2} \mathrm{O}_{5}$ ) with $\mathrm{SO}_{2}$ and $\mathrm{NO}_{x}$.

In this section, the basic experimental results with sodium bicarbonate and sodium sesquicarbonate will be presented. This will be followed by a discussion of the results of the experiments addressing the detailed chemistry.

\section{A.6 Overall Reaction Rates}

The $\mathrm{SO}_{2}$ time histories for both sodium bicarbonate and sodium sesquicarbonate are shown in Figure A-2. As expected, $\mathrm{SO}_{2}$ removals increase with increasing stoichiometric ratios $(2 \mathrm{Na} / \mathrm{S})$ for both sorbents. The short time data (i.e., less than 5 minutes) are consistent with the full-scale data in that the sesquicarbonate reacts faster than the bicarbonate. At $2 \mathrm{Na} / \mathrm{S}$ ratios of 0.85 and 3.4 , the overall level of $\mathrm{SO}_{2}$ removal are similar for both sorbents. This is quite surprising, particularly at the lower value of 0.85 . The current full-scale tests at Arapahoe, as well as previous full-scale demonstrations (Fuchs, et al., 1989; Muzio, et al., 1984) show that sodium bicarbonate will yield higher overall $\mathrm{SO}_{2}$ removal than sodium sesquicarbonate for a given amount of sodium. This may have been the case had the experiments shown in Figure A-2 been extended to longer time periods.

The $\mathrm{NO}_{2}$ production as a function of reaction time is shown in Figure A-3 for both sodium bicarbonate $(2 \mathrm{Na} / \mathrm{S}=0.85,3.4,13.5)$ and sodium sesquicarbonate $(2 \mathrm{Na} / \mathrm{S}=0.85,3.4)$. For both sorbents, there does not appear to be a strong effect of stoichiometric ratio on the amount of $\mathrm{NO}_{2}$ produced, as the scatter in the data is as great as any perceptible $2 \mathrm{Na} / \mathrm{S}$ effect. The $\mathrm{NO}_{2}$ results do indicate that sodium bicarbonate (solid symbols) tends to produce higher levels of $\mathrm{NO}_{2}$ than sodium sesquibicarbonate (open symbols) which is consistent with the full-scale test results at Arapahoe. With sodium sesquicarbonate the $\mathrm{NO}_{2}$ levels were generally $200 \mathrm{ppm}$ ( $11 \%$ of the initial NO levels). 


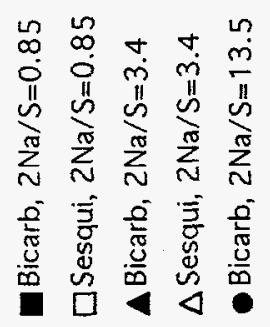

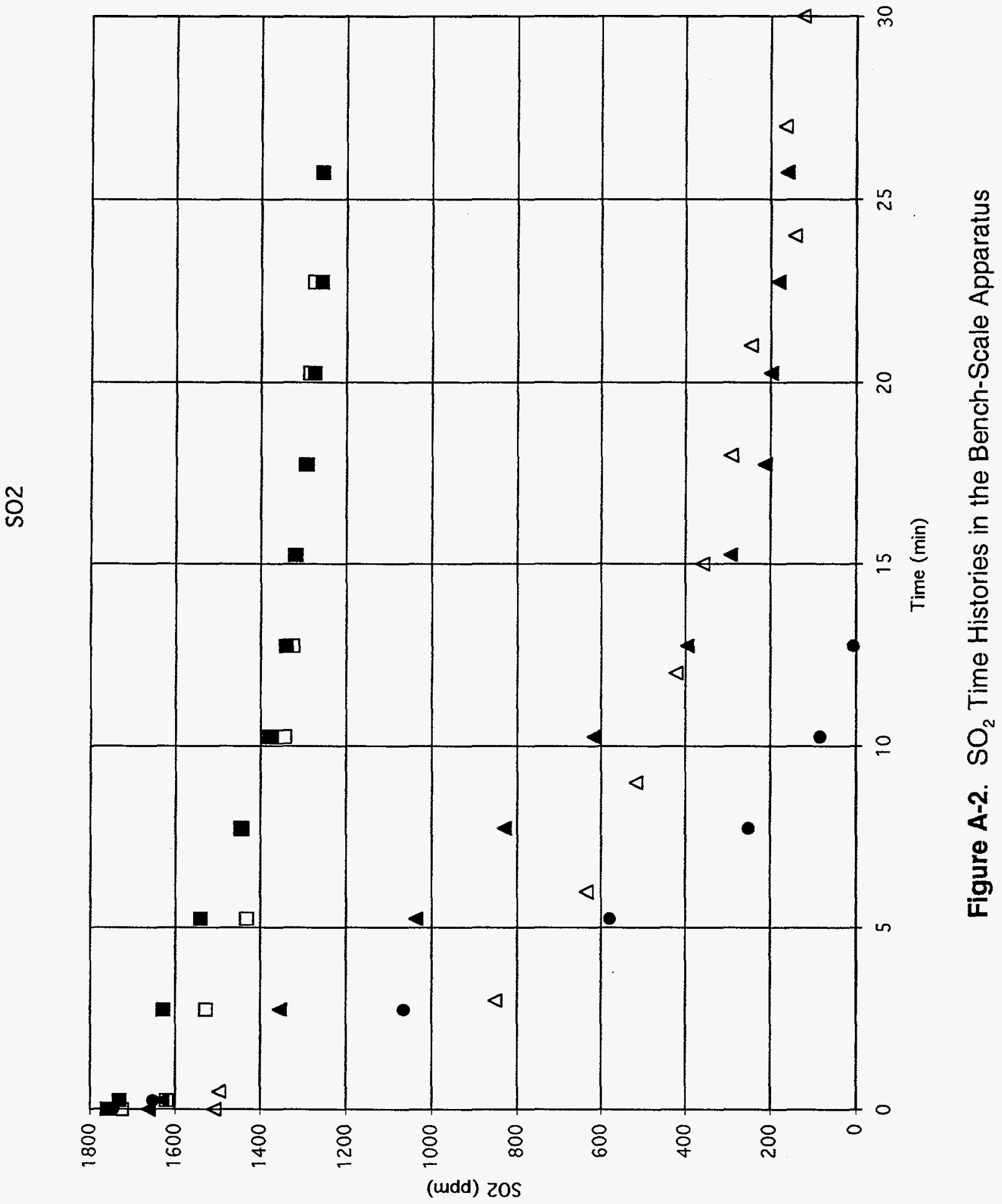



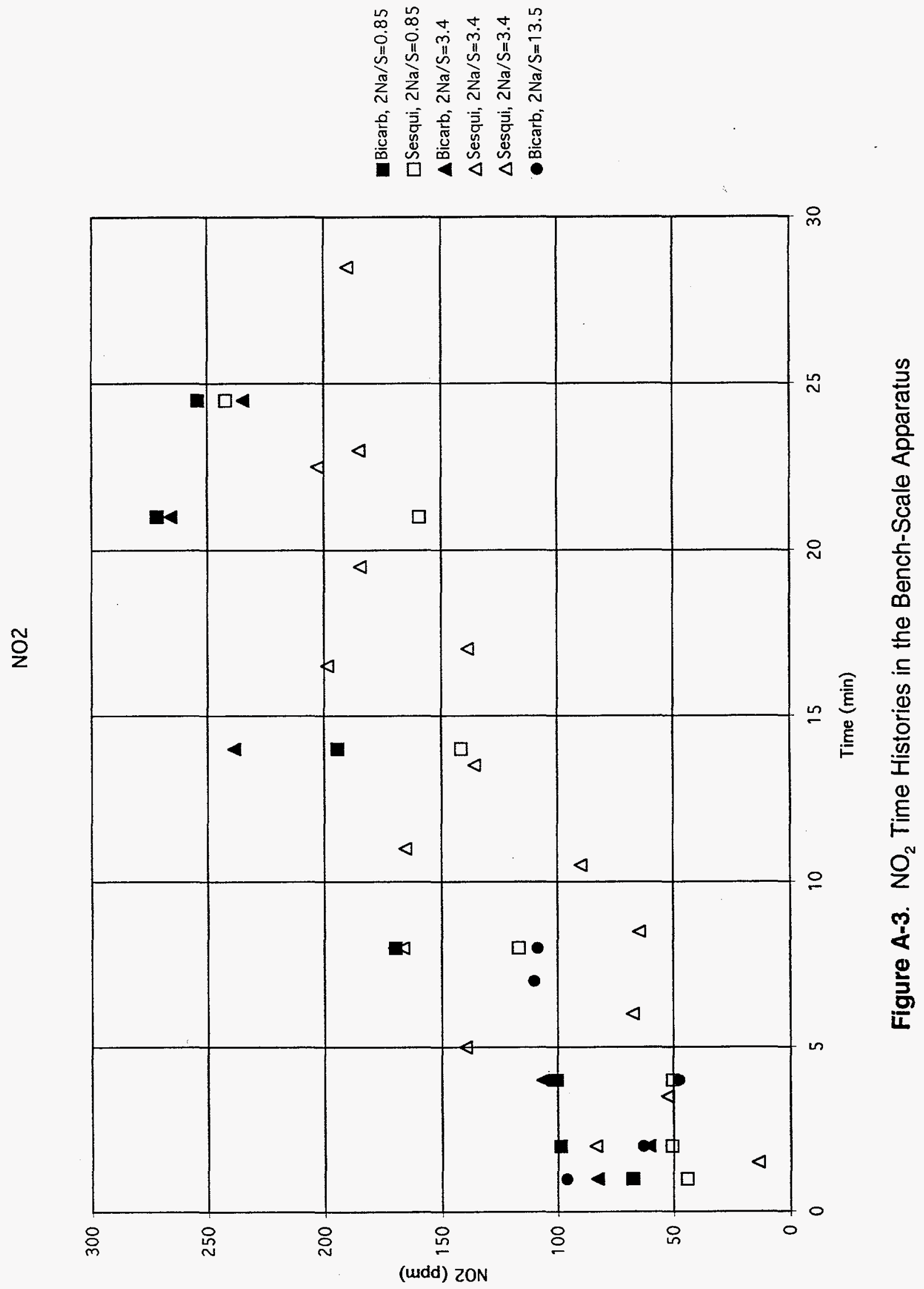
The effect of temperature on $\mathrm{NO}_{2}$ formation with sodium sesquicarbonate is shown in Figure A-4, where the open symbols are tests conducted at $260^{\circ} \mathrm{F}$, and the solid symbols are tests results for temperatures of $300^{\circ} \mathrm{F}$. While there is quite a large amount of scatter in the data presented in Figure $\mathrm{A}-4$, the results suggest that the level of $\mathrm{NO}_{2}$ decreased, somewhat, as the temperature was increased from $260^{\circ} \mathrm{F}$ to $300^{\circ} \mathrm{F}$.

The time histories of $\mathrm{NO}_{x}$ removal are shown in Figure $\mathrm{A}-5$ for both sodium bicarbonate and sodium sesquicarbonate. The open symbols are data for sodium bicarbonate and the solid symbols for sodium sesquicarbonate. For both sorbents, the level of $\mathrm{NO}_{x}$ removal increases with increasing stoichiometric ratio. At $2 \mathrm{Na} / \mathrm{S}$ ratios of 0.85 and 3.4 , the ultimate level of $\mathrm{NO}_{\mathrm{x}}$ removal was similar for both sorbents. Although consistent with the initially higher reaction rate of sodium sesquicarbonate, the rate of $\mathrm{NO}_{x}$ removal with sodium sesquicarbonate at $2 \mathrm{Na} / \mathrm{S}=3.4$ was faster than for sodium bicarbonate. The overall $\mathrm{NO}_{\mathrm{x}}$ removal of $10 \%$ at $2 \mathrm{Na} / \mathrm{S}=0.85$ is also consistent with the $\mathrm{NO}_{x}$ removals achieved at full-scale.

The next group of tests were conducted to determine if the intermediate compounds proposed by Solvay (Verlaetent, et al., 1993); $\mathrm{NaHSO}_{3}$ or $\mathrm{Na}_{2} \mathrm{~S}_{2} \mathrm{O}_{5}$ were important intermediates. Figure A-6 shows the results of the reaction between $3000 \mathrm{ppm}$ sodium pyrosulfite $\left(\mathrm{Na}_{2} \mathrm{~S}_{2} \mathrm{O}_{5}\right)$ and a gas mixture of $1500 \mathrm{ppm} \mathrm{NO}, 3 \% \mathrm{O}_{2}$ and $5 \% \mathrm{H}_{2} \mathrm{O}$ at a temperature of $260^{\circ} \mathrm{F}$. The results show the $\mathrm{NO}_{\mathrm{x}}$ removal is about equal to the amount of $\mathrm{SO}_{2}$ produced; consistent with reaction (7). This would suggest that sodium pyrosulfite may indeed be an intermediate in the removal of $\mathrm{NO}_{x}$ by sodium-based sorbents.

A comparable test was conducted with sodium sulfite $\left(\mathrm{NaHSO}_{3}\right)$ at a stoichiometric ratio of 2 (i.e., 4 moles of $\mathrm{NaHSO}_{3}$ per mole of $\mathrm{NO}$, per reaction (8)) and a temperature of $260^{\circ} \mathrm{F}$. During this test, there was no production of $\mathrm{SO}_{2}$ and the change in $\mathrm{NO}_{x}$ was minimal. This would suggest that while $\mathrm{NaHSO}_{3}$ may be an intermediate in the chemistry, it is the formation of $\mathrm{NaS}_{2} \mathrm{O}_{5}$ from the $\mathrm{NaHSO}_{3}$ (i.e., reaction (6)) that is important, and not reaction (8) in terms of $\mathrm{NO}_{x}$ removal or $\mathrm{NO}_{2}$ production. 
NO2(Effect of Temperature)

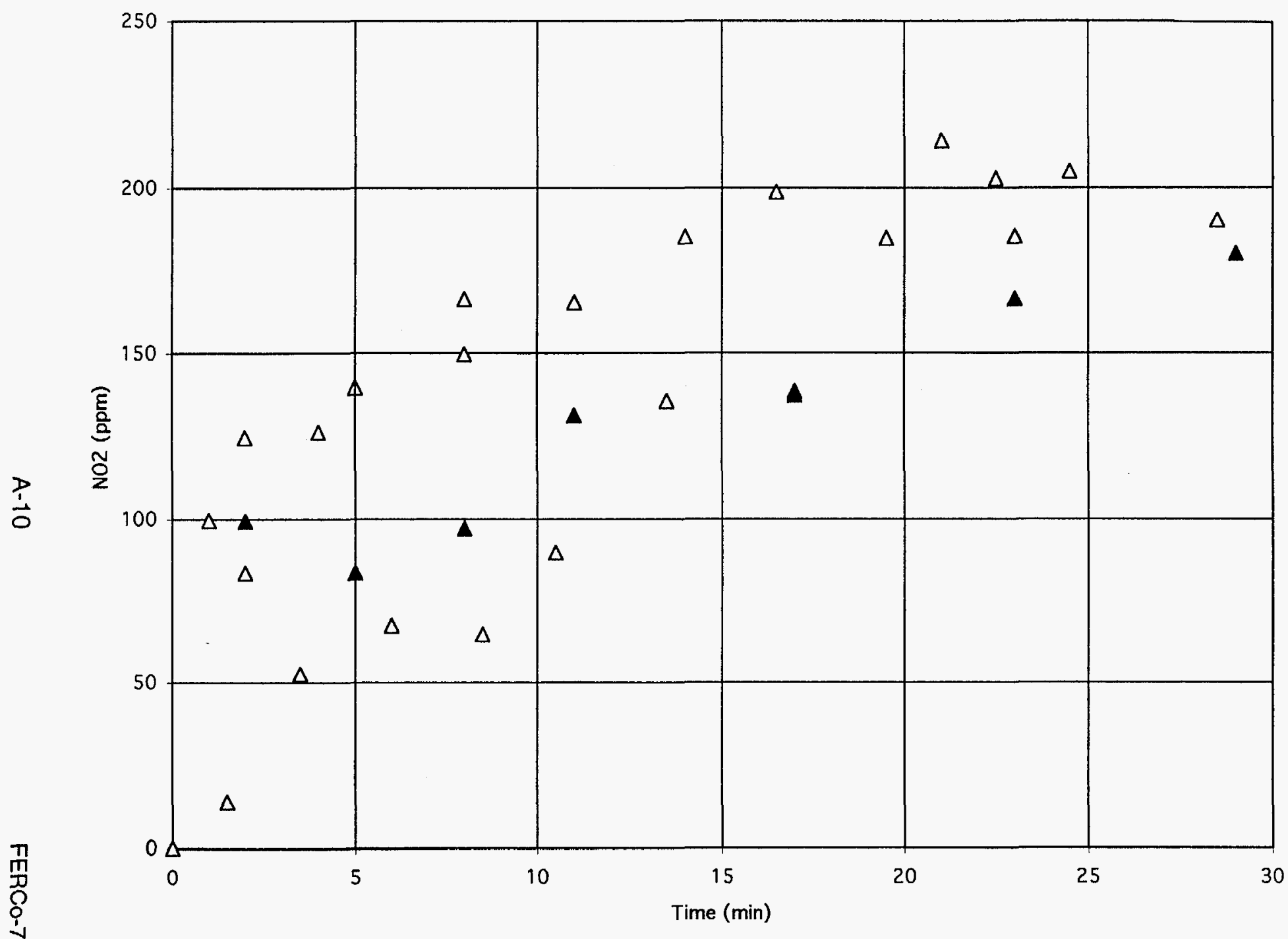

A Sesqui, $2 \mathrm{Na} / \mathrm{S}=3.4,300^{\circ} \mathrm{F}$ $\triangle$ Sesqui, $2 \mathrm{Na} / \mathrm{S}=3.4,260^{\circ} \mathrm{F}$ $\triangle$ Sesqui, $2 \mathrm{Na} / \mathrm{S}=3.4,260^{\circ} \mathrm{F}$ $\Delta$ Sesqui, $2 \mathrm{Na} / \mathrm{S}=3.4,260^{\circ} \mathrm{F}$

Figure A-4. Effect of Temperature on the $\mathrm{NO}_{2}$ Time Histories with Sodium Sesquicarbonate in the Bench-Scale Apparatus 


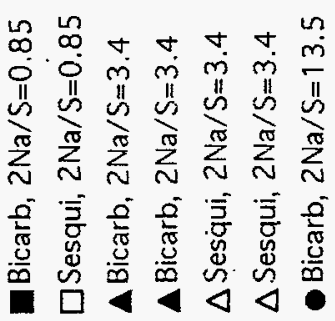

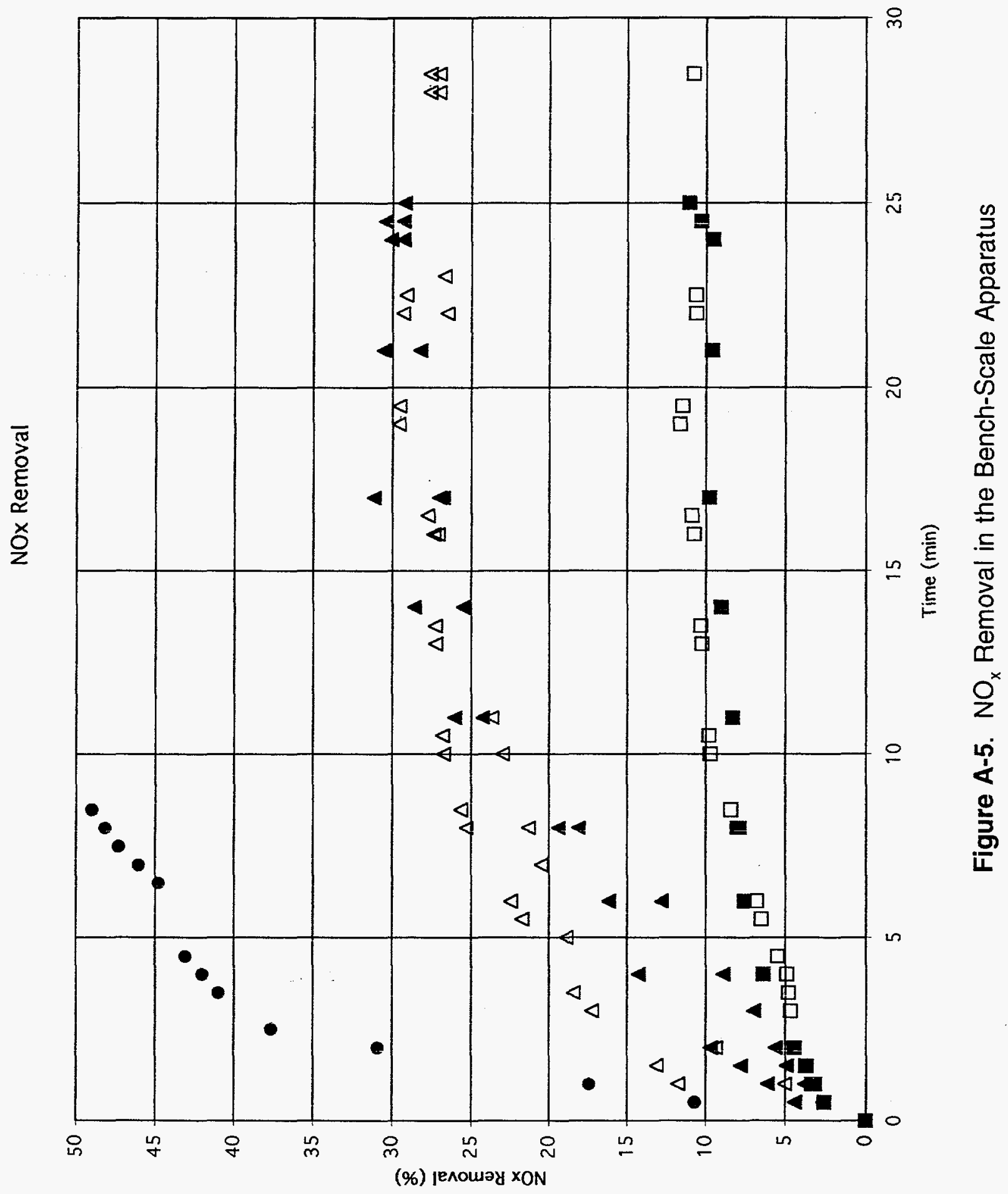




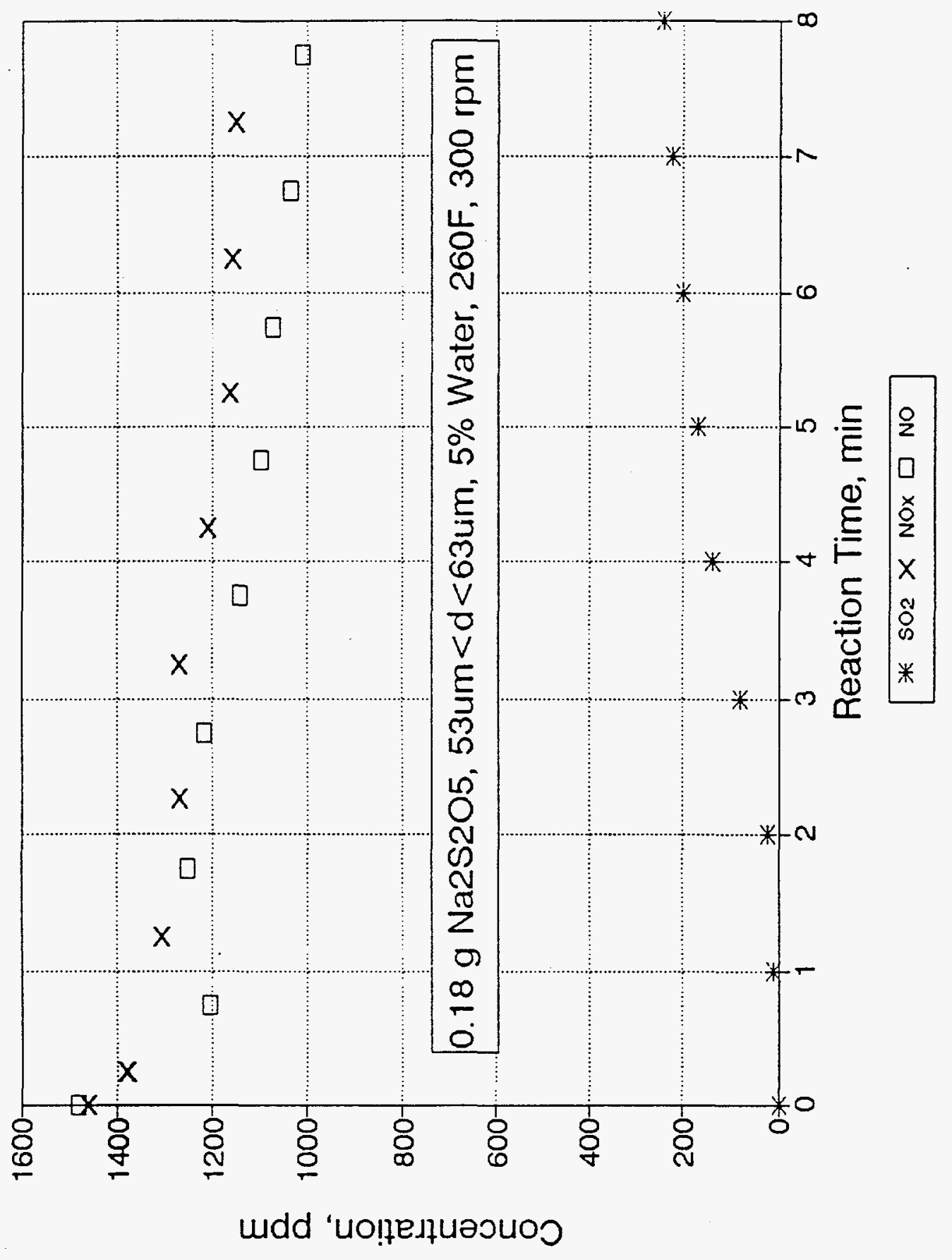

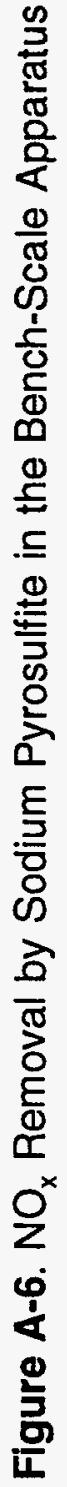




\section{A.7 Chemical Kinetic Modeling}

Chemical kinetic modeling was used to evaluate the two detailed mechanisms discussed previously. A shrinking core model was used and the rate constants for the individual reactions were extracted from the bench-scale data. The modeling effort suggested that the sodium $/ \mathrm{SO}_{2} / \mathrm{NO}_{x}$ chemistry is better predicted using the Solvay mechanism. This subsection will briefly summarize the detailed mechanism and average rate constants extracted from the data.

The detailed Solvay mechanism was simplified assuming that the intermediate compounds $\left(\mathrm{Na}_{2} \mathrm{~S}_{2} \mathrm{O}_{5}\right.$ and $\left.\mathrm{NaHSO}_{3}\right)$ have short lifetimes. With this assumption, the shrinking core model of the Solvay mechanism reduces to the following set of equations.

$$
\begin{aligned}
& \frac{\mathrm{d}\left[\mathrm{SO}_{2}\right]}{\mathrm{dt}}=-\mathrm{Sk}_{\mathrm{A}}\left[\mathrm{SO}_{2}\right] \\
& \frac{\mathrm{d}[\mathrm{NO}]}{\mathrm{dt}}=-\mathrm{Sk}_{\mathrm{B}}\left[\mathrm{SO}_{2}\right][\mathrm{NO}] \\
& \frac{\mathrm{d}\left[\mathrm{NO}_{2}\right]}{\mathrm{dt}}=-\frac{\mathrm{x}}{2} \mathrm{Sk}_{\mathrm{B}}\left[\mathrm{SO}_{2}\right][\mathrm{NO}] \\
& \frac{d R}{d t}=-\frac{1}{2} \frac{M}{D}\left\{k_{A}\left[\mathrm{SO}_{2}\right]+\frac{1}{2}\left(1-\frac{x}{2}\right) k_{B}\left[\mathrm{SO}_{2}\right][\mathrm{NO}]\right\} \\
& S=\frac{6(\mathrm{NSR})\left[\mathrm{SO}_{2}\right]_{0}}{\mathrm{DR}_{0}^{3}} \mathrm{M}^{\prime} \mathrm{R}^{2} \\
& k_{A}, k_{B}=\text { rate constants, }\left(\mathrm{mole} / \mathrm{cm}^{3} \mathrm{~min}\right. \text { ) } \\
& M \quad=\text { molecular weight of the sodium compound } \\
& \text { D = particle density } \\
& \mathrm{R}_{0} \quad=\text { mean initial radius of the sodium sorbent particle } \\
& \mathrm{x} \quad=\text { empirical stoichiometric constant that varies between } 0 \text { and } 1 \\
& S=\text { reactive surface area per unit volume } \\
& \mathrm{NSR}=\text { normalized stoichiometric ratio }(2 \mathrm{Na} / \mathrm{S})
\end{aligned}
$$


$\left[\mathrm{SO}_{2}\right]_{0}=$ initial concentration of $\mathrm{SO}_{2}$

$\mathrm{M}^{\prime} \quad=$ mass of sodium sorbent per mole of sodium

Table A-1 summarizes the rate constants and parameter $\mathrm{x}$ obtained from the data.

Table A-1

Summary of the Model Parameters

\begin{tabular}{|l|c|c|c|}
\hline Sorbent & $\begin{array}{c}\mathrm{k}_{\mathrm{A}} \\
(\mathrm{cm} / \mathrm{min})\end{array}$ & $\begin{array}{c}\mathrm{k}_{\mathrm{B}} \\
\left(\mathrm{cm}^{4} / \mathrm{min} \mathrm{mol}\right)\end{array}$ & $\mathrm{x}$ \\
\hline $\begin{array}{l}\text { Sodium } \\
\text { Sesquicarbonate }\end{array}$ & 8.77 & $1.24 \times 10^{8}$ & 0.55 \\
\hline $\begin{array}{l}\text { Sodium } \\
\text { Bicarbonate }\end{array}$ & 7.65 & $1.06 \times 10^{8}$ & 0.70 \\
\hline
\end{tabular}

Figure A-7 shows the model predictions (curves) relative to the bench-scale data (points) for sodium bicarbonate at $2 \mathrm{Na} / \mathrm{S}=3.37$ and temperature of $260^{\circ} \mathrm{F}$.

\section{A.8 References}

Evaluation of Dry Sodium Sorbent Utilization in Combustion Gas $\mathrm{SO}_{x} / \mathrm{NO}_{x}$ Reduction, Final Report, EPRI Project 1682-2, EPRI GS-6850, May 1990.

Fuchs, M.R., et al., Full-Scale Demonstration of a Utility Dry Sodium Injection FGD Facility, EPRI Final Report, Project RP-16826, December 1989.

Lai, Y, Yesavage V.F., Simultaneous Removal of $\mathrm{SO}_{2}$ and $\mathrm{NO}_{x} \mathrm{By}$ Selected Sodium Based Dry Sorbents, Final Technical Report to Public Service Company of Colorado, 1994.

Muzio, L.J., et al., Dry SO $\mathrm{S}_{2}$ Particulate Removal for Coal-Fired Boilers, Volume 2: 22-MW Demonstration Using Nacholite, Trona, and Soda Ash, EPRI Report CS-2894, June 1984.

Muzio, L.J., Kramlich, J.C., An Artifact in The Measurement of $\mathrm{N}_{2} \mathrm{O}$ from Combustion Systems, Geophysical Research Letters, Vol. 15, No. 12, 1369-1372, November 1988.

Verlaeten, J., De Soele, G.C., Ninane, L., Blondel, J.M., Combined $\mathrm{SO}_{2} / \mathrm{NO}_{2}$ Abatement by Sodium Bicarbonate Dry Injection, 1050 Brussels, Belgium, 1993. 


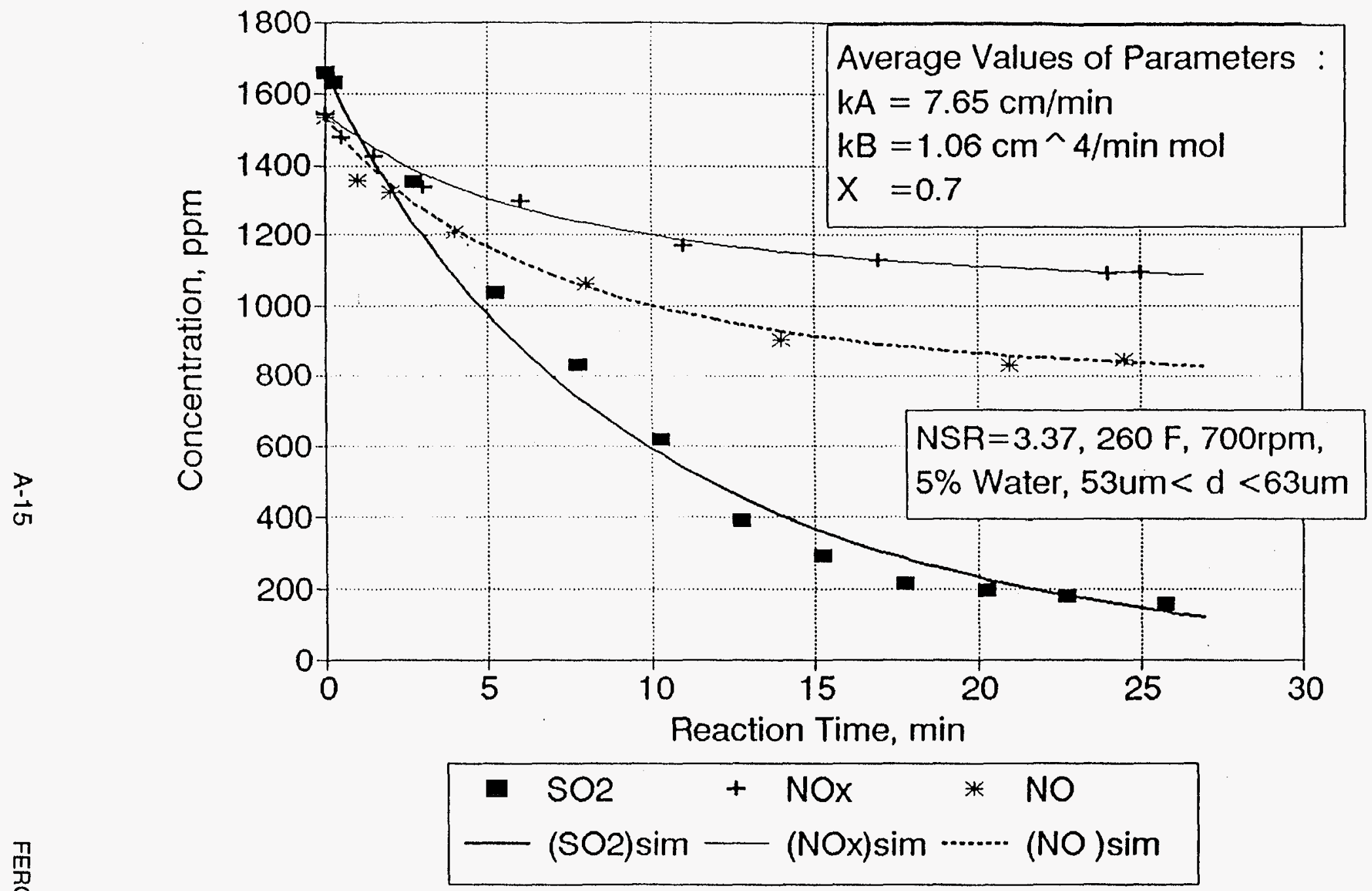

Figure A-7. $\mathrm{SO}_{2}$ and $\mathrm{NO}_{x}$ Removal by Sodium Bicarbonate Comparison of the Bench-Scale Results and Chemical Kinetic Model for Sodium Bicarbonate 


\section{APPENDIX B}

Detailed Data Summary for Parametric Tests 
PSCC Arapahoe Unit 4 Sodium Injection Summary

Calcs based on: Sodium Bicarbonate (b)

$.274 \mathrm{Naw}$

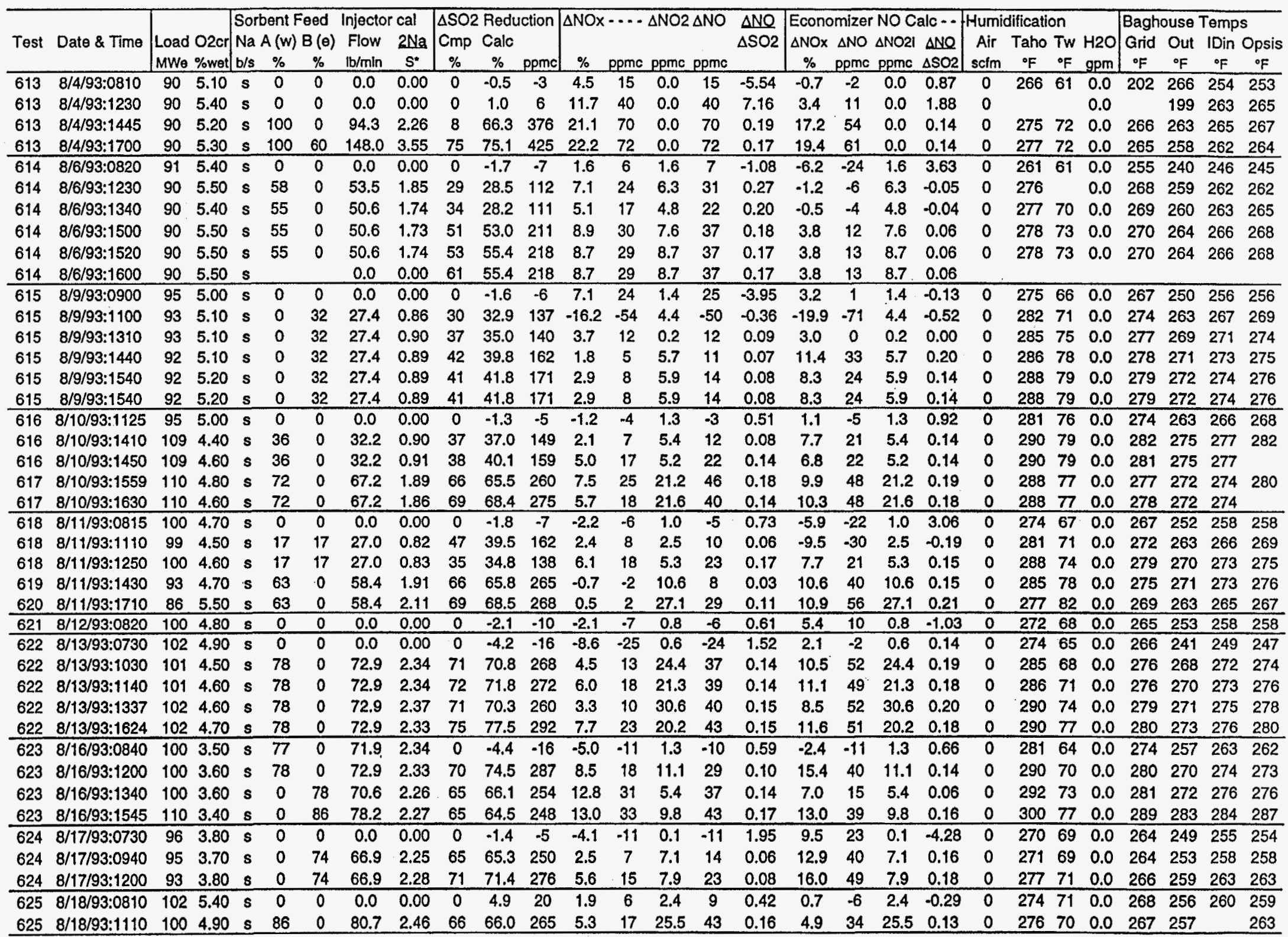




\begin{tabular}{|c|c|c|c|c|c|c|c|c|c|c|c|c|c|c|c|c|c|c|c|c|c|c|}
\hline \multirow[b]{2}{*}{ Test } & \multirow[b]{2}{*}{ Date \& Time } & \multicolumn{6}{|c|}{ Economizer Exit, dry (1-12) } & \multicolumn{7}{|c|}{ Baghouse Inlet Gas Analysis, wet } & \multicolumn{7}{|c|}{ Stack Gas Analysis, wet } & \multirow[b]{2}{*}{ Comments } \\
\hline & & $\begin{array}{l}\text { NO } \\
\mathrm{ppm}\end{array}$ & $\begin{array}{l}\mathrm{CO} \\
\mathrm{ppm}\end{array}$ & $\begin{array}{l}\mathrm{SO} 2 \\
\mathrm{ppm}\end{array}$ & $\begin{array}{l}\mathrm{NO} 2 \\
\mathrm{ppm}\end{array}$ & $\begin{array}{c}\mathrm{CO} 2 \\
\%\end{array}$ & $\begin{array}{c}02 \\
\% \text { dry }\end{array}$ & $\begin{array}{l}\text { NO } \\
\mathrm{ppm}\end{array}$ & $\begin{array}{l}\mathrm{CO} \\
\mathrm{ppm}\end{array}$ & $\begin{array}{l}\mathrm{SO} 2 \\
\mathrm{Ppm}\end{array}$ & $\begin{array}{l}\mathrm{NO} 2 \\
\mathrm{ppm}\end{array}$ & $\begin{array}{c}\mathrm{CO} 2 \\
\% \\
\end{array}$ & $\begin{array}{c}\mathrm{H} 2 \mathrm{O} \\
\%\end{array}$ & $\begin{array}{l}\mathrm{O} 2 \\
\%\end{array}$ & $\begin{array}{l}\text { NO } \\
\text { ppm }\end{array}$ & $\begin{array}{l}\text { CO } \\
\text { ppm }\end{array}$ & $\begin{array}{l}\mathrm{SO} 21 \\
\mathrm{ppm}\end{array}$ & $\begin{array}{l}\mathrm{NO2} \\
\mathrm{ppm}\end{array}$ & $\begin{array}{c}\mathrm{CO} 2 \\
\%\end{array}$ & $\begin{array}{c}\mathrm{H} 2 \mathrm{O} \\
\%\end{array}$ & $\begin{array}{l}\mathrm{O} 2 \\
\%\end{array}$ & \\
\hline 613 & $8 / 4 / 93: 0810$ & 236 & 519 & 427 & & 11.50 & 7.30 & 222 & 49 & 380 & & 10.44 & 8.21 & 7.05 & 211 & 417 & 379 & & 10.36 & 8.16 & 7.15 & \\
\hline 613 & $8 / 4 / 93: 1230$ & 240 & 720 & 430 & & 11.72 & 7.25 & 232 & 42 & 372 & & 10.24 & 8.18 & 7.15 & 207 & 489 & 372 & & 10.35 & 8.06 & 7.05 & \\
\hline 613 & 8/4/93:1445 & 240 & 720 & 430 & & 11.72 & 7.25 & 230 & 27 & 395 & & 10.65 & 8.60 & 6.65 & 177 & 282 & 130 & & 10.61 & 8.62 & 6.95 & \\
\hline 613 & $8 / 4 / 93: 1700$ & 240 & 720 & 430 & & 11.72 & 7.25 & 224 & 22 & 390 & & 10.66 & 8.33 & 6.85 & 171 & 231 & 95 & & 10.55 & 8.29 & 7.15 & \\
\hline 614 & $8 / 6 / 93: 0820$ & 242 & 32 & 292 & -1 & 11.14 & 7.20 & 243 & 13 & 281 & -5 & 10.72 & 8.68 & 6.55 & 223 & 24 & 268 & -4 & 10.22 & 8.27 & 7.42 & 2xfeed sprocket \\
\hline 614 & $8 / 6 / 93: 1230$ & 242 & 32 & 292 & -1 & 11.14 & 7.20 & 242 & 14 & 270 & -7 & 10.37 & 8.10 & 6.90 & 214 & 73 & 187 & -2 & 10.31 & 7.97 & 7.32 & \\
\hline 614 & $8 / 6 / 93: 1340$ & 242 & 32 & 292 & -1 & 11.14 & 7.20 & 244 & 15 & 282 & -6 & 10.76 & 8.39 & 6.36 & 214 & 66 & 190 & -3 & 10.29 & 7.93 & 7.25 & \\
\hline 614 & 8/6/93:1500 & 242 & 32 & 292 & -1 & 11.14 & 7.20 & 240 & 13 & 281 & -6 & 10.55 & 8.27 & 6.55 & 200 & 43 & 124 & -1 & 10.12 & 7.87 & 7.45 & \\
\hline 614 & $8 / 6 / 93: 1520$ & 242 & 32 & 292 & -1 & 11.14 & 7.20 & 241 & 13 & 280 & -6 & 10.64 & 8.37 & 6.45 & 201 & 36 & 117 & 0 & 10.23 & 7.89 & 7.35 & \\
\hline 614 & $8 / 6 / 93: 1600$ & 242 & 32 & 292 & -1 & 11.14 & 7.20 & 241 & 13 & 280 & -6 & 10.64 & 8.37 & 6.45 & 201 & 36 & 117 & 0 & 10.23 & 7.89 & 7.35 & Post cal feed \\
\hline 615 & $8 / 9 / 93: 0900$ & 255 & 324 & 335 & 2 & 12.15 & 6.70 & 245 & 32 & 293 & -6 & 10.77 & 8.65 & 6.40 & 221 & 271 & 290 & -5 & 10.53 & 8.53 & 6.75 & add $\mathrm{B}$ cal \\
\hline 615 & 8/9/93:1100 & 255 & 324 & 335 & 2 & 12.15 & 6.70 & 242 & 37 & 295 & -6 & 10.77 & 8.63 & 6.45 & 266 & 161 & 190 & -3 & 10.49 & 8.45 & 7.00 & feed erratic \\
\hline 615 & 8/9/93:1310 & 255 & 324 & 335 & 2 & 12.15 & 6.70 & 237 & 37 & 284 & -5 & 10.81 & 8.55 & 6.40 & 204 & 278 & 165 & -4 & 9.63 & 7.92 & 7.90 & rratic, B mill \\
\hline 615 & 8/9/93:1440 & 241 & 28 & 331 & 1 & 12.25 & 6.70 & 213 & 15 & 309 & -5 & 11.55 & 8.90 & 5.45 & 188 & 22 & 171 & -1 & 10.80 & 8.34 & 6.68 & \\
\hline 615 & 8/9/93:1540 & 241 & 28 & 331 & 1 & 12.25 & 6.70 & 220 & 10 & 307 & -5 & 11.45 & 8.71 & 5.66 & 192 & 19 & 164 & 0 & 10.64 & 8.11 & 6.90 & \\
\hline 615 & $8 / 9 / 93: 1540$ & 241 & 28 & 331 & 1 & 12.25 & 6.70 & 220 & 10 & 307 & -5 & 11.45 & 8.71 & 5.66 & 192 & 19 & 164 & 0 & 10.64 & 8.11 & 6.90 & pos \\
\hline 616 & $8 / 10 / 93: 1125$ & 272 & 27 & 329 & 2 & 12.25 & 6.40 & 261 & 15 & 322 & -6 & 11.78 & 9.58 & 5.08 & 237 & 21 & 294 & -4 & 10.77 & 8.87 & 6.60 & $\mathrm{dcal}$ \\
\hline 616 & 8/10/93:1410 & 272 & 27 & 329 & 2 & 12.25 & 6.40 & 250 & 19 & 308 & -5 & 11.64 & 9.51 & 5.20 & 223 & 2 & 180 & -1 & 10.99 & 9.02 & 6.30 & \\
\hline 616 & 8/10/93:1450 & 273 & 57 & 335 & 0 & 12.71 & 6.35 & 256 & 14 & 302 & -5 & 11.57 & 9.35 & 5.32 & 223 & 38 & 169 & -1 & 10.96 & 8.92 & 6.32 & \\
\hline 617 & 8/10/93:1559 & 273 & 57 & 335 & 0 & 12.71 & 6.35 & 251 & 14 & 300 & -5 & 11.45 & 9.79 & 5.35 & 203 & 50 & 97 & 11 & 10.94 & 9.29 & 6.30 & \\
\hline 617 & 8/10/93:1630 & 272 & 49 & 335 & 0 & 12.68 & 6.35 & 247 & 13 & 305 & -6 & 11.52 & 9.78 & 5.30 & 202 & 45 & 90 & 10 & 10.91 & 9.32 & 6.32 & \\
\hline 618 & $8 / 11 / 93: 0815$ & 241 & 91 & 365 & 0 & 13.26 & 5.65 & 225 & 19 & 305 & -5 & 11.51 & 9.46 & 5.50 & 217 & 34 & 295 & -4 & 10.97 & 9.09 & 6.25 & load \\
\hline 618 & 8/11/93:1110 & 241 & 91 & 365 & 0 & 13.26 & 5.65 & 259 & 18 & 329 & -5 & 12.09 & 9.75 & 4.52 & 223 & 27 & 177 & -3 & 10.96 & 8.85 & 6.30 & \\
\hline 618 & $8 / 11 / 93: 1250$ & 249 & 46 & 339 & 0 & 12.70 & 6.15 & 230 & 15 & 300 & -6 & 11.30 & 9.31 & 5.45 & 202 & 32 & 188 & -2 & 10.98 & 8.89 & 6.20 & \\
\hline 619 & 8/11/93:1430 & 269 & 27 & 334 & -1 & 12.35 & 6.40 & 244 & 42 & 328 & -5 & 12.37 & 9.89 & 4.25 & 210 & 27 & 99 & 3 & 11.00 & 8.92 & 6.18 & \\
\hline 620 & $8 / 11 / 93: 1710$ & 259 & 20 & 322 & 0 & 11.98 & 6.90 & 233 & 17 & 300 & -5 & 11.60 & 9.99 & 5.10 & 185 & 17 & 83 & 14 & 10.40 & 8.96 & 7.00 & \\
\hline 621 & $8 / 12 / 93: 0820$ & 276 & 19 & 380 & 2 & 12.67 & 6.40 & 257 & 13 & 360 & -6 & 12.14 & 9.95 & 4.65 & 235 & 14 & 330 & -4 & 10.98 & 9.06 & 6.30 & \\
\hline 622 & $8 / 13 / 93: 0730$ & 259 & 22 & 310 & 2 & 12.43 & 6.25 & 230 & 23 & 293 & -6 & 11.74 & 9.73 & 4.90 & 224 & 16 & 275 & .5 & 10.78 & 9.02 & 6.45 & dinj sys \\
\hline 622 & $8 / 13 / 93: 1030$ & 257 & 35 & 326 & -1 & 12.77 & 6.12 & 234 & 21 & 299 & -5 & 11.95 & 9.48 & 4.80 & 187 & 26 & 80 & 13 & 11.15 & 8.80 & 6.15 & $\mathrm{Na}$ flow? \\
\hline 622 & 8/13/93:1140 & 256 & 28 & 323 & -2 & 12.80 & 6.20 & 229 & 19 & 287 & -6 & 11.50 & 9.26 & 5.40 & 187 & 24 & 76 & 9 & 11.02 & 8.83 & 6.30 & \\
\hline 622 & 8/13/93:1337 & 256 & 28 & 323 & -2 & 12.80 & 6.20 & 229 & 17 & 283 & -5 & 11.50 & 8.85 & 5.40 & 184 & 23 & 78 & 17 & 11.00 & 8.46 & 6.45 & \\
\hline 622 & 8/13/93:1624 & 256 & 28 & 323 & -2 & 12.80 & 6.20 & 230 & 16 & 285 & -5 & 11.33 & 8.86 & 5.50 & 185 & 25 & 60 & 9 & 10.78 & 8.43 & 6.45 & \\
\hline 623 & $8 / 16 / 93: 0840$ & 208 & 154 & 352 & 0 & 13.84 & 4.60 & 196 & 88 & 322 & -6 & 12.94 & 10.11 & 3.50 & 188 & 89 & 309 & -4 & 12.02 & 9.30 & 4.90 & \\
\hline 623 & 8/16/93:1200 & 208 & 154 & 352 & 0 & 13.84 & 4.60 & 187 & 53 & 331 & -5 & 12.98 & 9.76 & 3.45 & 150 & 126 & 78 & 4 & 12.27 & 9.19 & 4.72 & \\
\hline 623 & 8/16/93:1340 & 208 & 154 & 352 & 0 & 13.84 & 4.60 & 210 & 30 & 322 & .5 & 12.66 & 9.53 & 3.90 & 168 & 75 & 102 & .1 & 12.08 & 8.93 & 5.00 & abort \\
\hline 623 & 8/16/93:1545 & 236 & 98 & 363 & -2 & 14.04 & 4.50 & 225 & 33 & 331 & -5 & 12.88 & 9.59 & 3.50 & 175 & 113 & 109 & 3 & 12.18 & 9.02 & 4.75 & higher load \\
\hline 624 & $8 / 17 / 93: 0730$ & 258 & 40 & 338 & -1 & 13.40 & 5.60 & 229 & 46 & 329 & -5 & 12.89 & 10.11 & 3.45 & 210 & 40 & 294 & -5 & 11.52 & 9.07 & 5.50 & \\
\hline 624 & 8/17/93:0940 & 258 & 40 & 338 & .1 & 13.40 & 5.60 & 229 & 49 & 319 & -6 & 12.56 & 10.01 & 3.90 & 193 & 36 & 98 & 0 & 11.38 & 9.05 & 5.82 & \\
\hline 624 & $8 / 17 / 93: 1200$ & 258 & 40 & 338 & -1 & 13.40 & 5.60 & 227 & 45 & 319 & -6 & 12.27 & 9.66 & 4.10 & 187 & 37 & 82 & 0 & 11.29 & 8.82 & 5.80 & \\
\hline 625 & $8 / 18 / 93: 0810$ & 269 & 44 & 250 & 2 & 13.06 & 5.75 & 256 & 21 & 318 & -6 & 11.65 & 10.15 & 5.00 & 235 & 29 & 285 & -4 & 11.13 & 9.67 & 5.90 & \\
\hline 625 & 8/18/93:1110 & 270 & 31 & 345 & 1 & 12.92 & 5.95 & 260 & 17 & 315 & -5 & 11.94 & 9.99 & 4.80 & 205 & 22 & 97 & 13 & 11.08 & 9.25 & 6.30 & \\
\hline
\end{tabular}


PSCC Arapahoo Unit 4 Sodium Injection Summary

Calcs based on: Sodium Bicarbonate (b)

$.274 \mathrm{Na} \mathrm{wt}$

Sodium Sesquicarbonate (s)

$.297 \mathrm{Na} w t$

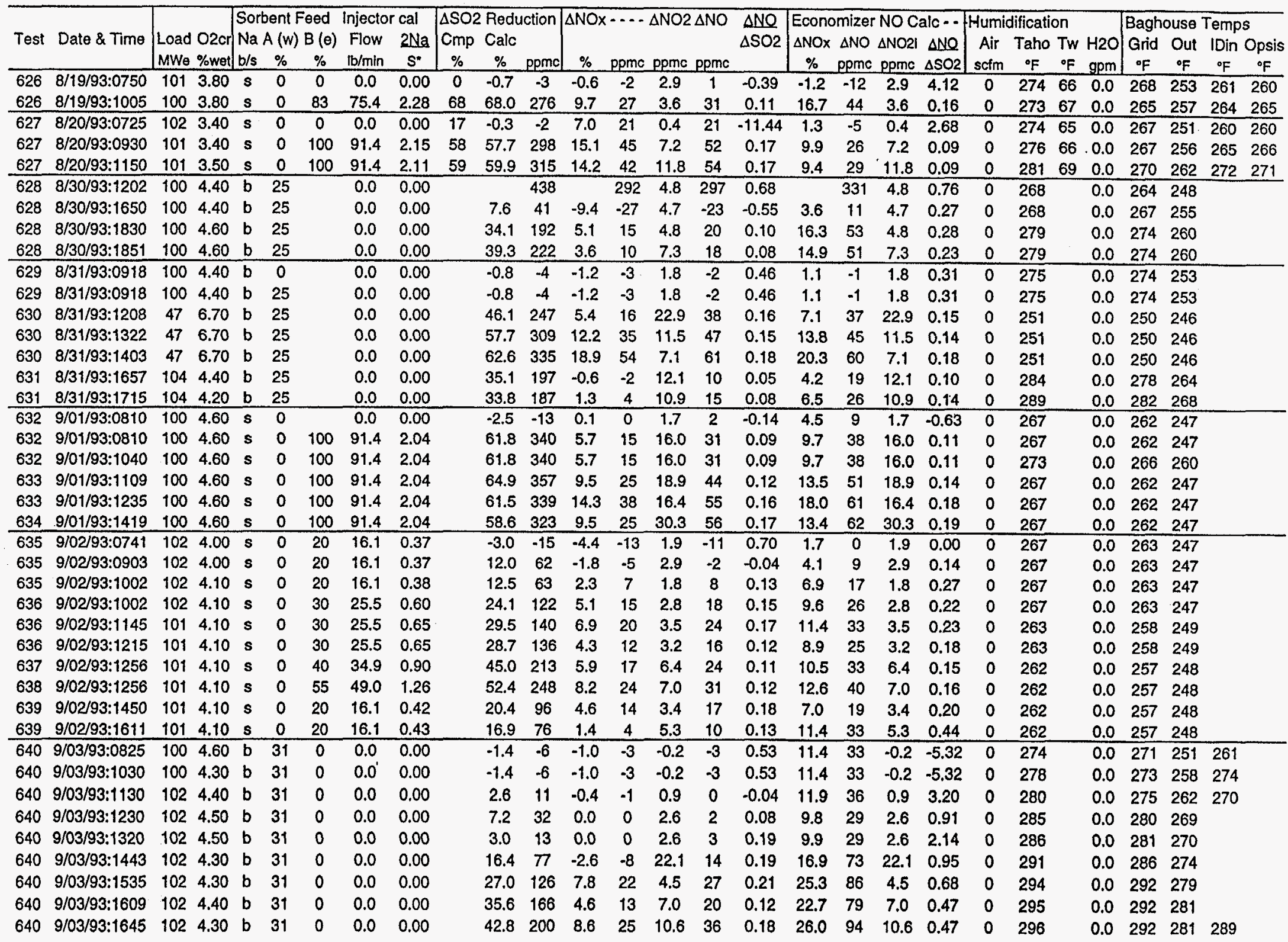




\begin{tabular}{|c|c|c|c|c|c|c|c|c|c|c|c|c|c|c|c|c|c|c|c|c|c|c|}
\hline Test & Date \& Time & $\begin{array}{c}\text { Econon } \\
\text { NO } \\
\text { ppm }\end{array}$ & $\begin{array}{c}\text { nizer } \\
\text { CO } \\
\text { ppm }\end{array}$ & $\begin{array}{l}\text { Exit, dry } \\
\text { SO2 } \\
\text { ppm }\end{array}$ & $\begin{array}{l}(1-1 \\
\text { NO2 } \\
\text { ppm }\end{array}$ & $\begin{array}{l}\text { 2) } \\
\mathrm{CO} 2 \\
\%\end{array}$ & $\begin{array}{c}\mathrm{O} 2 \\
\% \mathrm{dry}\end{array}$ & $\begin{array}{c}\text { Baghou } \\
\text { NO } \\
\text { ppm }\end{array}$ & $\begin{array}{l}\text { sse In } \\
\text { co } \\
\text { ppm }\end{array}$ & $\begin{array}{c}\text { let Ga } \\
\mathrm{SO} 2 \\
\mathrm{ppm}\end{array}$ & $\begin{array}{l}\text { s Ana } \\
\text { NO2 } \\
\text { ppm }\end{array}$ & $\begin{array}{c}\text { alysis, w } \\
\mathrm{CO} 2 \\
\%\end{array}$ & $\begin{array}{c}\mathrm{H} 2 \mathrm{O} \\
\%\end{array}$ & $\begin{array}{l}\mathrm{O} 2 \\
\%\end{array}$ & $\begin{array}{r}\text { Stack } C \\
\text { NO } \\
\text { ppm }\end{array}$ & $\begin{array}{l}\text { Gas An } \\
\text { CO } \\
\text { pom }\end{array}$ & $\begin{array}{l}\text { alysis, } \\
\text { SO2 } \\
\text { ppm }\end{array}$ & $\begin{array}{l}\text { wet } \\
\text { NO2 } \\
\text { ppm }\end{array}$ & $\begin{array}{c}\mathrm{CO} 2 \\
\%\end{array}$ & $\begin{array}{c}\mathrm{H} 2 \mathrm{O} \\
\%\end{array}$ & $\begin{array}{l}\mathrm{O} 2 \\
\%\end{array}$ & Comments \\
\hline 626 & $8 / 19 / 93: 0750$ & 255 & 458 & 350 & 2 & 12.73 & 5.65 & 242 & 57 & 320 & -6 & 11.54 & 9.67 & 5.00 & 232 & 270 & 310 & -4 & 11.50 & 9.57 & 5.55 & \\
\hline 626 & 8/19/93:1005 & 255 & 458 & 350 & 2 & 12.73 & 5.65 & 229 & 60 & 325 & -6 & 12.24 & 10.30 & 4.40 & 195 & 280 & 99 & -3 & 11.78 & 9.99 & 5.15 & \\
\hline 627 & $8 / 20 / 93: 0725$ & 242 & 1140 & 475 & 3 & 13.38 & 5.20 & 237 & 181 & 420 & -5 & 11.95 & 9.84 & 4.80 & 217 & 1175 & 415 & -4 & 11.71 & 9.68 & 5.05 & SO2 out DL bad \\
\hline 627 & 8/20/93:0930 & 242 & 1140 & 475 & 3 & 13.38 & 5.20 & 235 & 174 & 403 & -4 & 11.78 & 9.79 & 4.90 & 192 & 1025 & 168 & 1 & 11.66 & 9.73 & 5.10 & \\
\hline 627 & 8/20/93:1150 & 242 & 1140 & 475 & 3 & 13.38 & 5.20 & 233 & 119 & 407 & -5 & 11.79 & 9.77 & 5.00 & 190 & 1053 & 162 & 4 & 11.63 & 9.79 & 5.10 & \\
\hline 628 & $8 / 30 / 93: 1202$ & 272 & 43 & 372 & 1 & 13.18 & 6.20 & 252 & 225 & 372 & -4 & 13.08 & 9.54 & 3.70 & & & & & & & & Sodium bicarb cal \\
\hline 628 & 8/30/93:1650 & 272 & 43 & 372 & 1 & 13.18 & 6.20 & 244 & 55 & 442 & -4 & 12.80 & 9.09 & 4.30 & 220 & & 342 & & & 9.10 & 6.70 & \\
\hline 628 & 8/30/93:1830 & 272 & 43 & 372 & 1 & 13.18 & 6.20 & 242 & 144 & 459 & -4 & 13.00 & 9.26 & 4.40 & 210 & 105 & 281 & 0 & 11.87 & 8.46 & 5.60 & stm \\
\hline 628 & 8/30/93:1851 & 272 & 43 & 372 & 1 & 13.18 & 6.20 & 242 & 144 & 459 & -4 & 13.00 & 9.26 & 4.40 & 210 & 55 & 257 & 2 & 11.88 & 8.46 & 5.70 & $\operatorname{stm}$ \\
\hline 629 & $8 / 31 / 93: 0918$ & 255 & 110 & 405 & $\overline{1}$ & 13.47 & 5.80 & 247 & 116 & 375 & -5 & 12.72 & 9.29 & 4.30 & 230 & 101 & 350 & -3 & 11.87 & 8.76 & 5.50 & baseline \\
\hline 629 & 8/31/93:0918 & 255 & 110 & 405 & 1 & 13.47 & 5.80 & 247 & 116 & 375 & -5 & 12.72 & 9.29 & 4.30 & 230 & 101 & 350 & -3 & 11.87 & 8.76 & 5.50 & not st \\
\hline 630 & 8/31/93:1208 & 198 & 31 & 376 & 0 & 10.74 & 8.80 & 194 & 25 & 353 & -5 & 10.25 & 7.73 & 7.50 & 156 & 29 & 176 & 10 & 7.23 & 7.20 & 8.50 & nots \\
\hline 630 & 8/31/93:1322 & 198 & 31 & 376 & 0 & 10.74 & 8.80 & 194 & 25 & 353 & -5 & 10.25 & 7.73 & 7.50 & 151 & 27 & 138 & 3 & 9.55 & 7.20 & 8.50 & not \\
\hline 630 & 8/31/93:1403 & 198 & 31 & 376 & 0 & 10.74 & 8.80 & 194 & 25 & 353 & -5 & 10.25 & 7.73 & 7.50 & 142 & & 122 & & & 7.20 & 8.50 & not \\
\hline 631 & 8/31/93:1657 & 262 & 167 & 487 & 1 & 13.56 & 5.80 & 246 & 127 & 458 & -4 & 12.71 & 9.53 & 4.30 & 218 & 93 & 273 & 6 & 11.65 & 9.08 & 5.60 & state \\
\hline 631 & 8/31/93:1715 & 262 & 167 & 487 & 1 & 13.56 & 5.80 & 249 & 101 & 461 & -4 & 12.67 & 9.59 & 4.00 & 215 & 130 & 277 & $5:$ & 11.77 & 9.02 & 5.50 & steady state \\
\hline 632 & $9 / 01 / 93: 0810$ & 238 & 154 & 490 & 0 & 13.59 & 5.60 & 224 & 100 & 449 & -4 & 12.62 & 9.29 & 4.20 & 206 & 166 & 426 & -3 & 11.95 & 8.81 & 5.40 & baseline \\
\hline 632 & 9/01/93:0810 & 238 & 154 & 490 & 0 & 13.59 & 5.60 & 222 & 56 & 450 & -4 & 12.50 & 8.99 & 4.40 & 182 & 39 & 159 & 8 & 11.80 & 8.48 & 5.60 & \\
\hline 632 & 9/01/93:1040 & 238 & 154 & 490 & 0 & 13.59 & 5.60 & 222 & 56 & 450 & -4 & 12.50 & 8.99 & 4.40 & 182 & 39 & 159 & 8 & 11.80 & 8.48 & 5.60 & \\
\hline 633 & 9/01/93:1109 & 238 & 154 & 490 & 0 & 13.59 & 5.60 & 222 & 56 & 450 & -4 & 12.50 & 8.99 & 4.40 & 173 & 54 & 147 & 11 & 11.87 & 8.55 & 5.50 & \\
\hline 633 & $9 / 01 / 93: 1235$ & 238 & 154 & 490 & 0 & 13.59 & 5.60 & 222 & 56 & 450 & -4 & 12.50 & 8.99 & 4.40 & 166 & 102 & 162 & 9 & 11.76 & 8.25 & 5.50 & \\
\hline 634 & 9/01/93:1419 & 238 & 154 & 490 & 0 & 13.59 & 5.60 & 222 & 56 & 450 & -4 & 12.50 & 8.99 & 4.40 & 164 & 68 & 173 & 19 & 11.59 & 8.26 & 5.60 & \\
\hline 635 & $9 / 02 / 93: 0741$ & 262 & 39 & 454 & 1 & 13.52 & 5.60 & 244 & 35 & 428 & -5 & 12.73 & 9.20 & 4.20 & 233 & 36 & 406 & -3 & 11.82 & 8.59 & 5.50 & \\
\hline 635 & 9/02/93:0903 & 262 & 39 & 454 & 1 & 13.52 & 5.60 & 244 & 35 & 428 & -5 & 12.73 & 9.20 & 4.20 & 226 & 32 & 346 & -3 & 11.75 & 8.50 & 5.55 & \\
\hline 635 & 9/02/93:1002 & 262 & 39 & 454 & 1 & 13.52 & 5.60 & 243 & 44 & 414 & -4 & 12.50 & 9.08 & 4.40 & 219 & 43 & 336 & -3 & 11.78 & 8.43 & 5.60 & \\
\hline 636 & 9/02/93:1002 & 262 & 39 & 454 & 1 & 13.52 & 5.60 & 243 & 44 & 414 & -4 & 12.50 & 9.08 & 4.40 & 213 & 49 & 293 & -2 & 11.87 & 8.58 & 5.50 & \\
\hline 636 & 9/02/93:1145 & 262 & 39 & 454 & 1 & 13.52 & 5.60 & 245 & 58 & 390 & -4 & 12.69 & 9.35 & 4.20 & 210 & 74 & 256 & -1 & 12.00 & 8.97 & 5.30 & \\
\hline 636 & $9 / 02 / 93: 1215$ & 262 & 39 & 454 & 1 & 13.52 & 5.60 & 245 & 58 & 390 & -4 & 12.69 & 9.35 & 4.20 & 212 & 50 & 254 & -2 & 11.87 & 8.79 & 5.60 & \\
\hline 637 & 9/02/93:1256 & 262 & 39 & 454 & 1 & 13.52 & 5.60 & 245 & 58 & 390 & -4 & 12.69 & 9.35 & 4.20 & 209 & 70 & 199 & 1 & 11.91 & 8.80 & 5.40 & \\
\hline 638 & $9 / 02 / 93: 1256$ & 262 & 39 & 454 & 1 & 13.52 & 5.60 & 245 & 58 & 390 & -4 & 12.69 & 9.35 & 4.20 & 206 & 69 & 174 & 1 & 12.10 & 8.96 & 5.20 & \\
\hline 639 & $9 / 02 / 93: 1450$ & 262 & 39 & 454 & 1 & 13.52 & 5.60 & 252 & 57 & 389 & -4 & 12.71 & 9.44 & 4.10 & 222 & 63 & 289 & -1 & 12.06 & 8.90 & 5.20 & \\
\hline 639 & 9/02/93:1611 & 279 & 35 & 411 & 2 & 13.63 & 6.10 & 257 & 33 & 372 & .7 & 12.45 & 9.20 & 4.30 & 232 & 40 & 288 & -2 & 12.00 & 8.80 & 5.40 & \\
\hline 640 & 9/03/93:0825 & 294 & 34 & 387 & 2 & 13.65 & 6.10 & 265 & 33 & 362 & -4 & 12.67 & 8.81 & 4.20 & 245 & 33 & 336 & -4 & 11.80 & 8.18 & 5.60 & \\
\hline 640 & 9/03/93:1030 & 294 & 34 & 387 & 2 & 13.65 & 6.10 & 265 & 33 & 362 & -4 & 12.67 & 8.81 & 4.20 & 245 & 33 & 336 & -4 & 11.80 & 8.18 & 5.60 & \\
\hline 640 & 9/03/93:1130 & 294 & 34 & 387 & 2 & 13.65 & 6.10 & 265 & 33 & 362 & -4 & 12.67 & 8.81 & 4.20 & 243 & 25 & 323 & -3 & 11.72 & 8.15 & 5.60 & \\
\hline 640 & $9 / 03 / 93: 1230$ & 294 & 34 & 387 & 2 & 13.65 & 6.10 & 270 & 29 & 360 & -5 & 12.51 & 8.72 & 4.40 & 247 & 26 & 308 & -3 & 11.69 & 8.03 & 5.70 & \\
\hline 640 & $9 / 03 / 93: 1320$ & 294 & 34 & 387 & 2 & 13.65 & 6.10 & 270 & 29 & 360 & -5 & 12.51 & 8.72 & 4.40 & 251 & 31 & 327 & -3 & 11.87 & 7.92 & 5.50 & \\
\hline 640 & 9/03/93:1443 & 294 & 34 & 387 & 2 & 13.65 & 6.10 & 234 & 29 & 369 & -5 & 12.17 & 8.51 & 5.00 & 218 & 34 & 302 & 12 & 11.99 & 8.00 & 5.40 & \\
\hline 640 & 9/03/93:1535 & 294 & 34 & 387 & 2 & 13.65 & 6.10 & 234 & 29 & 369 & -5 & 12.17 & 8.51 & 5.00 & 207 & 40 & 262 & -2 & 11.86 & 7.94 & 5.50 & \\
\hline 640 & 9/03/93:1609 & 294 & 34 & 387 & 2 & 13.65 & 6.10 & 234 & 29 & 369 & -5 & 12.17 & 8.51 & 5.00 & 212 & 31 & 231 & 0 & 11.97 & 8.01 & 5.50 & \\
\hline 640 & 9/03/93:1645 & 294 & 34 & 387 & 2 & 13.65 & 6.10 & 234 & 29 & 369 & -5 & 12.17 & 8.51 & 5.00 & 203 & 36 & 208 & 3 & 12.00 & 8.03 & 5.30 & \\
\hline
\end{tabular}


PSCC Arapahoe Unit 4 Sodium Injection Summary

Calcs based on: Sodium Bicarbonate (b)

$.274 \mathrm{Na} w t$

Sodium Sesquicarbonate (s) $\quad .297 \mathrm{Nawt}$

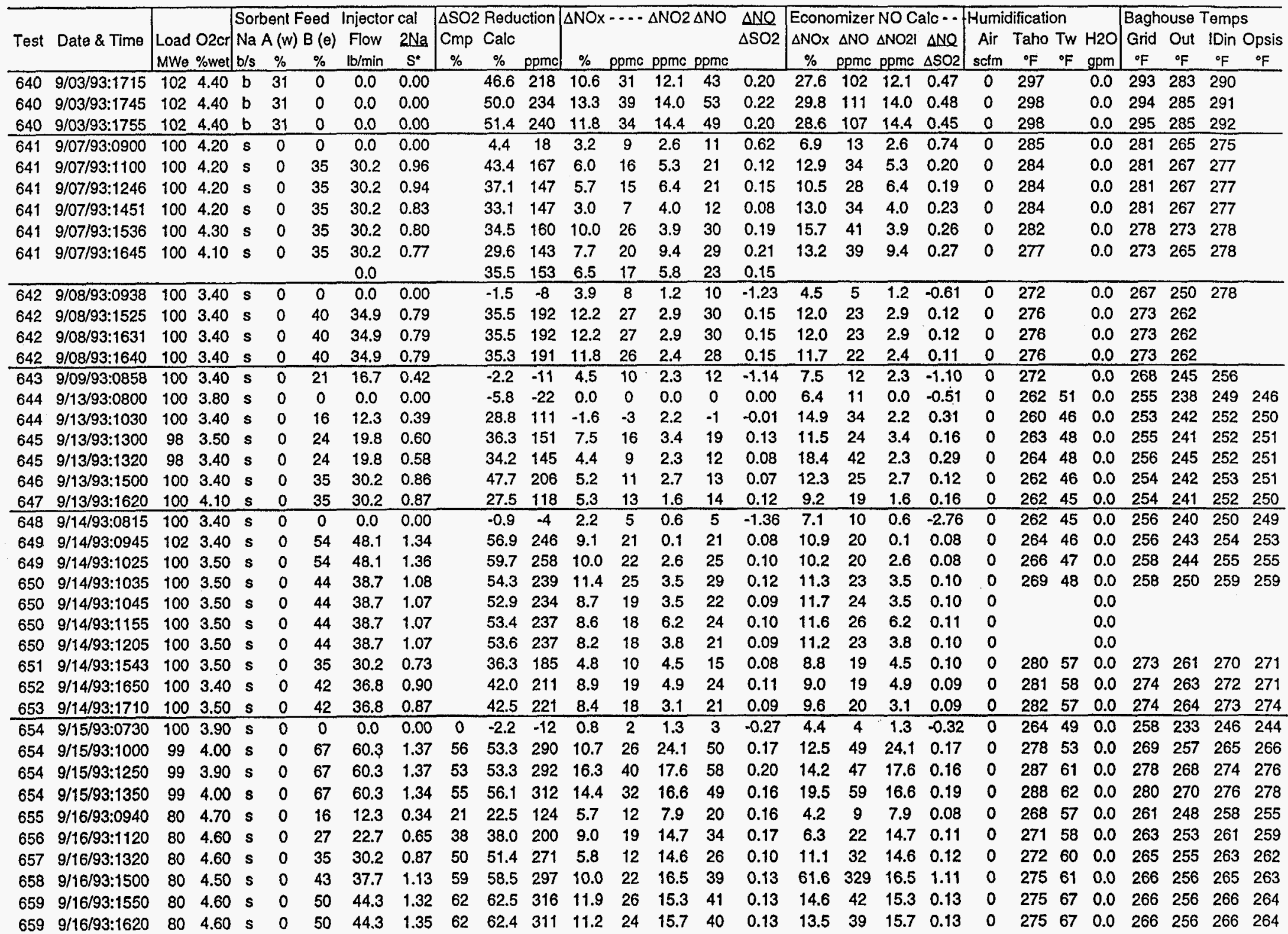




\begin{tabular}{|c|c|c|c|c|c|c|c|c|c|c|c|c|c|c|c|c|c|c|c|c|c|c|}
\hline \multirow[b]{2}{*}{ Test } & \multirow[b]{2}{*}{ Date \& Time } & \multicolumn{6}{|c|}{ Economizer Exit, dry (1-12) } & \multicolumn{7}{|c|}{ Baghouse Inlet Gas Analysis, wet } & \multicolumn{7}{|c|}{ Stack Gas Analysis, wet } & \multirow[b]{2}{*}{ Comments } \\
\hline & & $\begin{array}{l}\text { NO } \\
\text { ppm }\end{array}$ & $\begin{array}{l}\mathrm{CO} \\
\mathrm{ppm}\end{array}$ & $\begin{array}{l}\mathrm{SO} 2 \\
\text { ppm }\end{array}$ & $\begin{array}{l}\mathrm{NO} 2 \\
\mathrm{ppm}\end{array}$ & $\mathrm{CO}_{\%}$ & $\begin{array}{c}\mathrm{O} 2 \\
\% d r y\end{array}$ & $\begin{array}{l}\text { NO } \\
\mathrm{ppm}\end{array}$ & $\begin{array}{l}\text { CO } \\
\text { ppm }\end{array}$ & $\begin{array}{l}\mathrm{SO} 2 \\
\mathrm{ppm}\end{array}$ & $\begin{array}{l}\mathrm{NO2} \\
\mathrm{ppm}\end{array}$ & $\begin{array}{c}\mathrm{CO} 2 \\
\%\end{array}$ & $\begin{array}{c}\mathrm{H} 2 \mathrm{O} \\
\%\end{array}$ & $\begin{array}{l}\mathrm{O} 2 \\
\%\end{array}$ & $\begin{array}{l}\text { NO } \\
\text { ppm }\end{array}$ & $\begin{array}{l}\mathrm{CO} \\
\mathrm{ppm}\end{array}$ & $\mathrm{SO} 2$ & $\begin{array}{l}\mathrm{NO} 2 \\
\mathrm{ppm}\end{array}$ & $\mathrm{CO} 2$ & $\mathrm{H} 2 \mathrm{O}$ & $\mathrm{O} 2$ & \\
\hline 640 & 9/03/93:1715 & 294 & 34 & 387 & 2 & 13.65 & 6.10 & 234 & 29 & 369 & -5 & 12.17 & 8.51 & 5.00 & 197 & 43 & 194 & 4 & 12.03 & 8.15 & 5.30 & \\
\hline 640 & $9 / 03 / 93: 1745$ & 294 & 34 & 387 & 2 & 13.65 & 6.10 & 234 & 29 & 369 & -5 & 12.17 & 8.51 & 5.00 & 191 & 37 & 183 & 6 & 12.10 & 8.10 & 5.20 & \\
\hline 640 & 9/03/93:1755 & 294 & 34 & 387 & 2 & 13.65 & 6.10 & 234 & 29 & 369 & -5 & 12.17 & 8.51 & 5.00 & 194 & 49 & 178 & 6 & 12.12 & 8.13 & 5.20 & \\
\hline 641 & 9/07/93:0900 & 237 & 143 & 383 & 0 & 14.02 & 5.70 & 213 & 19 & 318 & -6 & 11.75 & 9.23 & 5.20 & 199 & 97 & 296 & -4 & 11.74 & 9.55 & 5.50 & \\
\hline 641 & 9/07/93:1100 & 237 & 143 & 383 & 0 & 14.02 & 5.70 & 208 & 46 & 302 & -5 & 11.96 & 9.53 & 4.90 & 185 & 111 & 165 & -1 & 11.83 & 9.48 & 5.40 & \\
\hline 641 & 9/07/93:1246 & 237 & 143 & 383 & 0 & 14.02 & 5.70 & 209 & 94 & 303 & -6 & 12.02 & 9.46 & 5.20 & 189 & 227 & 187 & -1 & 11.77 & 9.25 & 5.50 & \\
\hline 641 & 9/07/93:1451 & 237 & 143 & 383 & 0 & 14.02 & 5.70 & 206 & 31 & 358 & -5 & 12.19 & 9.32 & 4.60 & 188 & 184 & 229 & -2 & 11.93 & 9.05 & 5.30 & \\
\hline 641 & 9/07/93:1536 & 237 & 143 & 383 & 0 & 14.02 & 5.70 & 210 & 34 & 365 & -5 & 12.09 & 9.43 & 4.90 & 184 & 223 & 236 & -2 & 12.08 & 9.35 & 5.10 & \\
\hline 641 & 9/07/93:1645 & 238 & 266 & 455 & 1 & 14.25 & 5.50 & 211 & 29 & 382 & -5 & 12.15 & 9.87 & 4.70 & 183 & 205 & 262 & 2 & 12.09 & 9.68 & 5.10 & \\
\hline 642 & 08/93:0938 & 204 & 738 & 518 & 1 & 15.05 & 4.20 & 188 & 99 & 435 & -5 & 12.98 & 9.78 & 3.80 & 176 & 614 & 432 & -4 & 12.75 & 9.68 & 4.15 & \\
\hline 642 & 9/08/93:1525 & 204 & 738 & 518 & 1 & 15.05 & 4.20 & 191 & 61 & 459 & -5 & 12.93 & 9.68 & 3.70 & 163 & 512 & 291 & -2 & 12.83 & 9.50 & 4.00 & \\
\hline 642 & $9 / 08 / 93: 1631$ & 204 & 738 & 518 & 1 & 15.05 & 4.20 & 191 & 61 & 459 & .5 & 12.93 & 9.68 & 3.70 & 163 & 512 & 291 & -2 & 12.83 & 9.50 & 4.00 & \\
\hline 642 & 9/08/93:1640 & 204 & 738 & 518 & 1 & 15.05 & 4.20 & 191 & 61 & 459 & -5 & 12.93 & 9.68 & 3.70 & 162 & 524 & 288 & -2 & 12.75 & 9.46 & 4.20 & \\
\hline 643 & 9/09/93:0858 & 206 & 511 & 484 & 1 & 14.86 & 4.50 & 187 & 61 & 407 & .5 & 12.80 & 9.78 & 4.00 & 173 & 344 & 407 & -3 & 12.62 & 9.64 & 4.35 & Erratic feed all day \\
\hline 644 & $9 / 13 / 93: 0800$ & 218 & 65 & 367 & 0 & 14.62 & 5.00 & 197 & 35 & 320 & -4 & 12.83 & 9.07 & 3.90 & 187 & 92 & 320 & -3 & 12.42 & 8.77 & 4.80 & \\
\hline 644 & 9/13/93:1030 & 218 & 65 & 367 & 0 & 14.62 & 5.00 & 180 & 177 & 330 & -4 & 13.13 & 9.22 & 3.65 & 170 & 198 & 221 & -2 & 12.64 & 8.86 & 4.65 & \\
\hline 645 & 9/13/93:1300 & 200 & 363 & 388 & 0 & 14.50 & 4.60 & 182 & 170 & 352 & -4 & 13.04 & 9.31 & 3.80 & 160 & 310 & 216 & -1 & 12.79 & 9.04 & 4.40 & \\
\hline 645 & 9/13/93:1320 & 218 & 65 & 367 & 0 & 14.62 & 5.00 & 183 & 124 & 362 & -4 & 13.05 & 9.33 & 3.70 & 165 & 212 & 227 & -2 & 12.69 & 8.93 & 4.50 & \\
\hline 646 & 9/13/93:1500 & 205 & 289 & 430 & 1 & 14.94 & 4.45 & 182 & 141 & 372 & -4 & 13.35 & 9.52 & 3.50 & 162 & 234 & 185 & -1 & 12.91 & 9.14 & 4.35 & \\
\hline 647 & 9/13/93:1620 & 215 & 58 & 410 & 1 & 14.30 & 5.20 & 197 & 28 & 350 & -4 & 12.60 & 9.07 & 4.35 & 178 & 40 & 244 & -3 & 12.32 & 8.77 & 5.00 & mill off \\
\hline 648 & $9 / 14 / 93: 0815$ & 198 & 475 & 420 & 1 & 14.81 & 4.45 & 180 & 182 & 373 & -4 & 13.14 & 9.64 & 3.55 & 168 & 260 & 360 & -3 & 12.67 & 9.24 & 4.30 & \\
\hline 649 & 9/14/93:0945 & 207 & 285 & 403 & 0 & 14.50 & 4.85 & 192 & 49 & 358 & -4 & 12.65 & 9.28 & 4.15 & 170 & 172 & 150 & -4 & 12.44 & 9.10 & 4.60 & \\
\hline 649 & 9/14/93:1025 & 202 & 556 & 425 & 0 & 14.72 & 4.55 & 191 & 101 & 366 & -4 & 13.01 & 9.52 & 3.75 & 162 & 218 & 141 & -1 & 12.55 & 9.12 & 4.55 & \\
\hline 650 & 9/14/93:1035 & 200 & 316 & 430 & 0 & 14.48 & 4.65 & 190 & 78 & 370 & -4 & 12.80 & 9.40 & 3.90 & 160 & 300 & 163 & -1 & 12.65 & 9.09 & 4.50 & \\
\hline 650 & 9/14/93:1045 & 200 & 316 & 430 & 0 & 14.48 & 4.65 & 185 & 81 & 375 & -4 & 12.78 & 9.34 & 3.80 & 160 & 290 & 170 & -1 & 12.52 & 9.02 & 4.45 & \\
\hline 650 & $9 / 14 / 93: 1155$ & 200 & 316 & 430 & 0 & 14.48 & 4.65 & 185 & 81 & 375 & -4 & 12.78 & 9.34 & 3.80 & 158 & 245 & 168 & 1 & 12.57 & 9.01 & 4.45 & \\
\hline 650 & 9/14/93:1205 & 200 & 316 & 430 & 0 & 14.48 & 4.65 & 185 & 81 & 375 & -4 & 12.78 & 9.34 & 3.80 & 160 & 255 & 167 & -1 & 12.59 & 9.00 & 4.50 & \\
\hline 651 & 9/14/93:1543 & 200 & 230 & 488 & 0 & 14.26 & 4.55 & 185 & 104 & 440 & -4 & 13.09 & 9.70 & 3.45 & 163 & 209 & 265 & 0 & 12.61 & 9.24 & 4.40 & \\
\hline 652 & $9 / 14 / 93: 1650$ & 198 & 381 & 505 & 0 & 14.50 & 4.45 & 188 & 70 & 428 & -4 & 12.89 & 9.60 & 3.60 & 160 & 200 & 237 & 0 & 12.62 & 9.29 & 4.35 & \\
\hline 653 & 9/14/93:1710 & 198 & 381 & 505 & 0 & 14.50 & 4.45 & 185 & 66 & 445 & -3 & 12.91 & 9.67 & 3.60 & 160 & 203 & 245 & -1 & 12.52 & 9.22 & 4.35 & \\
\hline 654 & 9/15/93:0730 & 209 & 185 & 490 & 3 & 13.70 & 5.24 & 194 & 36 & 445 & -4 & 12.49 & 9.47 & 4.35 & 184 & 119 & 437 & -3 & 12.15 & 9.17 & 4.98 & base \\
\hline 654 & 9/15/93:1000 & 215 & 161 & 496 & 1 & 13.52 & 5.40 & 196 & 16 & 430 & -4 & 12.15 & 8.96 & 4.90 & 150 & 121 & 192 & 14 & 11.77 & 8.55 & 5.60 & $\# 6 \& 12 \mathrm{BH}$ \\
\hline 654 & 9/15/93:1250 & 211 & 109 & 502 & 0 & 13.90 & 5.12 & 201 & 18 & 440 & -4 & 12.34 & 8.96 & 4.65 & 146 & 80 & 194 & 10 & 11.78 & 8.52 & 5.55 & \\
\hline 654 & 9/15/93:1350 & 211 & 109 & 502 & 0 & 13.90 & 5.12 & 190 & 40 & 460 & -4 & 12.55 & 9.19 & 4.20 & 140 & 111 & 190 & 10 & 12.07 & 8.69 & 5.20 & \\
\hline 655 & 9/16/93:0940 & 177 & 39 & 455 & 1 & 12.77 & 6.15 & 170 & 8 & 415 & -4 & 11.61 & 8.79 & 5.58 & 150 & 45 & 312 & 2 & 11.50 & 8.63 & 6.02 & $80 \mathrm{MWe}$ \\
\hline 656 & 9/16/93:1120 & 176 & 174 & 458 & 0 & 13.02 & 6.00 & 169 & 23 & 399 & -5 & 11.49 & 8.76 & 5.55 & 140 & 116 & 242 & 6 & 11.52 & 8.65 & 5.87 & \\
\hline 657 & $9 / 16 / 93: 1320$ & 182 & 80 & 452 & 0 & 13.23 & 5.85 & 164 & 26 & 410 & -5 & 11.96 & 9.04 & 5.10 & 138 & 59 & 192 & 6 & 11.61 & 8.65 & 5.70 & \\
\hline 658 & 9/16/93:1500 & 435 & 74 & 435 & 0 & 13.02 & 5.95 & 172 & 15 & 380 & -6 & 11.37 & 8.61 & 5.70 & 140 & 37 & 154 & 7 & 11.40 & 8.56 & 6.00 & \\
\hline 659 & 9/16/93:1550 & 187 & 39 & 435 & 0 & 13.12 & 5.95 & 172 & 17 & 390 & -5 & 11.71 & 8.94 & 5.25 & 137 & 40 & 143 & 7 & 11.61 & 8.73 & 5.62 & \\
\hline 659 & 9/16/93:1620 & 187 & 39 & 435 & 0 & 13.12 & 5.95 & 173 & 16 & 383 & -6 & 11.64 & 8.84 & 5.30 & 138 & 37 & 140 & 6 & 11.59 & 8.69 & 5.73 & \\
\hline
\end{tabular}


PSCC Arapahoe Unit 4 Sodium Injection Summary

Calcs based on: Sodium Bicarbonate (b) $\quad 274 \mathrm{Nawt}$

Sodium Sesquicarbonate (s) $\quad .297 \mathrm{Nawt}$

\begin{tabular}{|c|c|c|c|c|c|c|c|c|c|c|c|c|c|c|c|c|c|c|c|c|c|c|c|c|c|c|c|c|}
\hline \multirow{3}{*}{ Test } & \multirow{3}{*}{ Date \& Time } & \multirow{3}{*}{$\begin{array}{l}\text { Load } \\
\text { MWe }\end{array}$} & \multirow{3}{*}{ O2cr } & \multirow{2}{*}{\multicolumn{3}{|c|}{$\begin{array}{l}\text { Sorbent Feed } \\
\mathrm{Na} \mathrm{A}(w) \mathrm{B}(\theta)\end{array}$}} & \multirow{2}{*}{\multicolumn{2}{|c|}{$\begin{array}{l}\text { Injector cal } \\
\text { Flow } 2 \mathrm{Na}\end{array}$}} & \multirow{2}{*}{\multicolumn{3}{|c|}{$\begin{array}{l}\triangle \mathrm{SO} 2 \text { Reduction } \\
\mathrm{Cmp} \text { Calc }\end{array}$}} & \multirow{2}{*}{\multicolumn{4}{|c|}{$\Delta \mathrm{NOX} \cdots \cdot-\Delta \mathrm{NO} 2 \Delta \mathrm{NO}$}} & \multirow{3}{*}{$\frac{\triangle \mathrm{NO}}{\Delta \mathrm{SO} 2}$} & \multirow{2}{*}{\multicolumn{4}{|c|}{$\begin{array}{l}\text { Economizer NO Calc }- \\
\triangle N O x \triangle N O \triangle N O 2 I \Delta N O\end{array}$}} & \multicolumn{4}{|c|}{ Humidification } & \multicolumn{4}{|c|}{ Baghouse Temps } \\
\hline & & & & & & & & & & & & & & & & & & & & & & & & & Grid & Out & & \\
\hline & & & & $\mathrm{b} / \mathrm{s}$ & $\%$ & $\%$ & $\mathrm{lb} / \mathrm{min}$ & & $\%$ & $\%$ & ppme & $\%$ & nom & & & & & & & & & & & & & & & \\
\hline & & 60 & 6.50 & s & 0 & 15 & & 53 & 35 & & 152 & & -1 & & & & 7.2 & & 2.3 & 0.10 & & 253 & 59 & 0.0 & & & & \\
\hline 00 & & & & s & 0 & & & & 4 & & & & & & & & & & & & & & & & & & & \\
\hline 662 & & & 40 & s & 0 & 2 & & & 51 & & 224 & & & & & & & & & .14 & & & & 0.0 & & & & \\
\hline 663 & & 60 & 6.50 & s & 0 & 32 & & 1.15 & 56 & & 259 & 5.6 & & & & & 12 & & 2. & 0.12 & & & & 0.0 & & & & \\
\hline 664 & 18/93:1650 & 60 & 6.40 & s & 0 & 46 & 40.5 & 1.57 & 56 & 55.9 & 283 & 4.1 & 11 & 26.0 & 37 & 0.13 & 11.8 & 52 & 26.0 & 0.18 & 0 & 254 & 55 & 0.0 & 247 & 237 & 240 & \\
\hline
\end{tabular}

$\begin{array}{llllllllllllllllllllllllllllll}673 & 9 / 24 / 93: 0850 & 110 & 4.00 & \text { s } & 0 & 0 & 0.0 & 0.00 & 0 & -3.4 & -14 & -11.0 & -26 & 1.9 & -25 & 1.78 & 5.3 & 8 & 1.9 & -0.57 & 0 & 277 & 56 & 0.0 & 271 & 244 & 256 & 260\end{array}$ $\begin{array}{llllllllllllllllllllllllllllll}673 & 9 / 24 / 93: 1150 & 110 & 3.90 & \mathrm{~s} & 0 & 80 & 72.5 & 2.04 & 64 & 63.6 & 253 & 5.0 & 13 & 12.5 & 26 & 0.10 & 10.1 & 34 & 12.5 & 0.14 & 0 & 279 & 57 & 0.0 & 272 & 262 & 271 & 274\end{array}$ $\begin{array}{lllllllllllllllllllllllllllll}674 & 9 / 24 / 93: 1300 & 110 & 3.90 & s & 40 & 41 & 71.9 & 2.03 & 77 & 77.1 & 305 & 8.1 & 22 & 12.3 & 34 & 0.11 & 11.8 & 40 & 12.3 & 0.13 & 0 & 280 & 57 & 0.0 & 271 & 264 & 271 & 275\end{array}$ $\begin{array}{llllllllllllllllllllllllllllll}675 & 9 / 24 / 93: 1450 & 110 & 4.00 & \text { s } & 76 & 0 & 71.0 & 2.01 & 72 & 72.1 & 284 & 7.4 & 21 & 21.0 & 42 & 0.15 & 9.7 & 42 & 21.0 & 0.15 & 0 & 281 & 59 & 0.0 & 273 & 264 & 272 & 275\end{array}$ $\begin{array}{llllllllllllllllllllllllllllll}676 & 9 / 27 / 93: 0840 & 99 & 4.00 & \mathrm{~s} & 0 & 0 & 0.0 & 0.00 & 0 & -1.9 & -9 & -2.8 & -7 & 1.6 & -5 & 0.64 & 8.4 & 18 & 1.6 & -2.14 & 0 & 268 & 58 & 0.0 & 264 & 245 & 256 & 256\end{array}$ $\begin{array}{lllllllllllllllllllllllllllll}676 & 9 / 27 / 93: 1240 & 99 & 3.90 & s & 40 & 41 & 71.9 & 2.09 & 64 & 67.5 & 287 & 12.6 & 37 & 27.0 & 64 & 0.22 & 10.7 & 53 & 27.0 & 0.18 & 0 & 282 & 59 & 0.0 & 275 & 266 & 274 & 274\end{array}$ $\begin{array}{lllllllllllllllllllllllllllllll}677 & 9 / 27 / 93: 1750 & 99 & 4.00 & \text { s } & 44 & 45 & 79.5 & 2.04 & 64 & 62.7 & 303 & -2.8 & -5 & 16.1 & 12 & 0.04 & 1.8 & 13 & 16.1 & 0.04 & 0 & 290 & 66 & 0.0 & 283 & 278 & 283 & 287\end{array}$ $\begin{array}{lllllllllllllllllllllllllllll}677 & 9 / 27 / 93: 1805 & 99 & 4.00 & s & 44 & 45 & 79.5 & 2.04 & 64 & 66.1 & 319 & -0.6 & -1 & 16.5 & 16 & 0.05 & 3.9 & 17 & 16.5 & 0.05 & 0 & 290 & 66 & 0.0 & 283 & 278 & 283 & 287\end{array}$ $\begin{array}{llllllllllllllllllllllllllllll}680 & 10 / 04 / 93: 1450 & 100 & 3.20 & s & 24 & 0 & 20.0 & 0.61 & 24 & 23.3 & 94 & -3.0 & -7 & 3.0 & -4 & -0.04 & 0.9 & -1 & 3.0 & -0.02 & 0 & 291 & 67 & 0.0 & 284 & 273 & 281 & 282\end{array}$ $\begin{array}{lllllllllllllllllllllllllllll}681 & 10 / 05 / 93: 0800 & 100 & 3.40 & \mathrm{~s} & 0 & 0 & 0.0 & 0.00 & 24 & -3.7 & -21 & -9.5 & -21 & -0.9 & -22 & 1.05 & 5.4 & 8 & -0.9 & -0.38 & 0 & 266 & 58 & 0.0 & 262 & 250 & 258 & 258\end{array}$ $\begin{array}{llllllllllllllllllllllllllllll}682 & 10 / 05 / 93: 1010 & 100 & 3.50 & s & 26 & 0 & 22.5 & 0.49 & 24 & 16.8 & 95 & -10.9 & -25 & 2.7 & -22 & -0.24 & 6.4 & 14 & 2.7 & 0.14 & 0 & 267 & 60 & 0.0 & 261 & 249 & 257 & 259\end{array}$

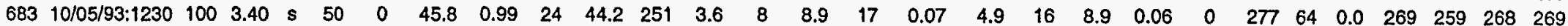
$\begin{array}{lllllllllllllllllllllllllllll}684 & 10 / 05 / 93: 1400 & 100 & 3.30 & \mathrm{~s} & 73 & 0 & 68.1 & 1.49 & 24 & 58.8 & 329 & 6.6 & 15 & 11.2 & 27 & 0.08 & 16.2 & 48 & 11.2 & 0.15 & 0 & 279 & 69 & 0.0 & 271 & 266 & 272 & 274\end{array}$ $\begin{array}{lllllllllllllllllllllllllllllll}685 & 10 / 05 / 93: 1540 & 100 & 3.20 & \mathrm{~s} & 96 & 0 & 90.4 & 2.02 & 24 & 70.2 & 385 & 11.3 & 26 & 9.5 & 35 & 0.09 & 18.5 & 50 & 9.5 & 0.13 & 0 & 282 & 70 & 0.0 & 272 & 267 & 274 & 276\end{array}$

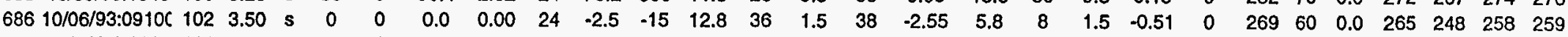

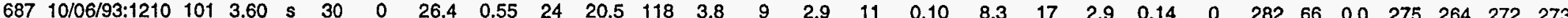

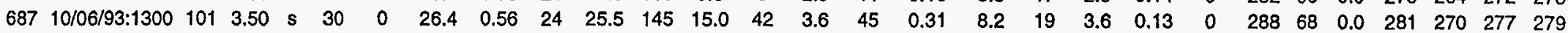
$\begin{array}{lllllllllllllllllllllllllllllll}688 & 10 / 06 / 93: 1510 & 101 & 3.50 & \mathrm{~s} & 50 & 0 & 45.8 & 1.04 & 24 & 46.6 & 248 & 17.1 & 48 & 9.9 & 57 & 0.23 & 11.5 & 34 & 9.9 & 0.14 & 0 & 285 & 71 & 0.0 & 278 & 272 & 278 & 282\end{array}$ $\begin{array}{llllllllllllllllllllllllllllll}689 & 10 / 06 / 93: 1800 & 101 & 3.50 & \text { s } & 65 & 0 & 60.3 & 1.49 & 24 & 60.2 & 296 & 18.6 & 52 & 13.4 & 65 & 0.22 & 12.3 & 40 & 13.4 & 0.14 & 0 & 281 & 72 & 0.0 & 272 & 269 & 274 & 276\end{array}$

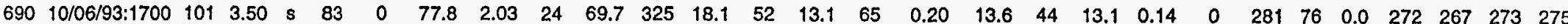
$\begin{array}{lllllllllllllllllllllllllllll}691 & 10 / 07 / 93: 0750 & 101 & 4.20 & \mathrm{~s} & 0 & 0 & 0.0 & 0.00 & 24 & -4.1 & -18 & 11.6 & 38 & 3.0 & 41 & -2.37 & 3.6 & 4 & 3.0 & -0.26 & 0 & 265 & 58 & 0.0 & 260 & 245 & 255 & 256\end{array}$

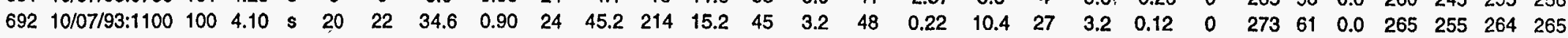
$\begin{array}{llllllllllllllllllllllllllllll}693 & 10 / 07 / 93: 1400 & 100 & 4.10 & s & 32 & 34 & 57.5 & 1.56 & 24 & 66.2 & 299 & 18.8 & 53 & 13.5 & 66 & 0.22 & 14.4 & 45 & 13.5 & 0.15 & 0 & 269 & 63 & 0.0 & 263 & 256 & 264 & 266\end{array}$ $\begin{array}{lllllllllllllllllllllllllllll}694 & 10 / 07 / 93: 1500 & 100 & 4.10 & \mathrm{~s} & 40 & 42 & 72.8 & 2.02 & 24 & 75.6 & 335 & 21.2 & 61 & 11.5 & 72 & 0.22 & 15.8 & 47 & 11.5 & 0.14 & 0 & 270 & 62 & 0.0 & 262 & 255 & 263 & 265\end{array}$ $\begin{array}{llllllllllllllllllllllllllllll}695 & 10 / 07 / 93: 1600 & 100 & 4.10 & \text { s } & 48 & 51 & 89.1 & 2.45 & 24 & 79.8 & 355 & 19.0 & 54 & 11.3 & 65 & 0.18 & 14.7 & 44 & 11.3 & 0.13 & 0 & 272 & 62 & 0.0 & 263 & 256 & 264 & 265\end{array}$ $\begin{array}{lllllllllllllllllllll}696 & 10 / 07 / 93: 1750 & 99 & 4.30 & \mathrm{~s} & 35 & 37 & 63.3 & 1.78 & 24 & 71.8 & 316 & 5.5 & 14 & 18.3 & 32 & 0.10 & 15.2 & 55 & 18.3 & 0.17\end{array}$

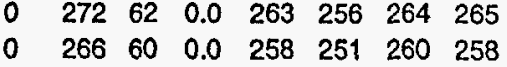

$\begin{array}{llllllllllllllllllllllllllllll}722 & 11 / 4 / 93 & 9: 10 & 110 & 3.20 & \text { s } & 0 & 0 & 0.0 & 0.00 & 0 & 0.0 & 0 & 2.8 & 6 & 2.2 & 8 & & 8.7 & 16 & 2.2 & & 0 & 269 & 67 & 0.0 & 269 & 251 & 259 & 260\end{array}$ $\begin{array}{llllllllllllllllllllllllllllll}723 & 11 / 4 / 93 & 11: 40 & 110 & 3.20 & \mathrm{~s} & 26 & 0 & 23.0 & 0.49 & 30 & 30.8 & 160 & 4.7 & 11 & 2.7 & 13 & 0.08 & 3.3 & 4 & 2.7 & 0.02 & 0 & 270 & 51 & 0.0 & 264 & 256 & 262 & 266\end{array}$ $\begin{array}{llllllllllllllllllllllllllllllll}724 & 11 / 4 / 93 & 14: 40 & 110 & 3.30 & \mathrm{~s} & 0 & 29 & 24.5 & 0.50 & 28 & 30.0 & 164 & 1.7 & 4 & 3.0 & 7 & 0.04 & 9.1 & 18 & 3.0 & 0.11 & 0 & 263 & 49 & 0.0 & 267 & 255 & 261 & 262\end{array}$

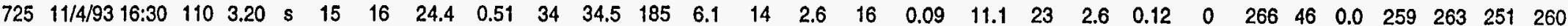
$\begin{array}{llllllllllllllllllllllllllllll}726 & 11 / 5 / 93 & 8: 00 & 109 & 3.40 & \mathrm{~s} & 0 & 0 & 0.0 & 0.00 & 0 & -0.7 & -4 & 4.4 & 10 & 2.8 & 12 & -3.30 & 2.1 & 0 & 2.8 & 0.00 & 0 & 262 & 43 & 0.0 & 256 & 238 & 262 & 244\end{array}$

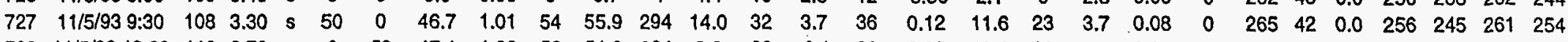
$\begin{array}{llllllllllllllllllllllllllllll}728 & 11 / 5 / 93 & 13: 00 & 110 & 3.70 & \mathrm{~s} & 0 & 53 & 47.1 & 1.02 & 50 & 51.3 & 264 & 8.9 & 20 & -0.1 & 20 & 0.08 & 10.6 & 22 & -0.1 & 0.08 & 0 & 266 & 43 & 0.0 & 258 & 249 & 265 & 259\end{array}$ $\begin{array}{llllllllllllllllllllllllllllll}729 & 11 / 5 / 93 & 14: 40 & 110 & 3.50 & \mathrm{~s} & 26 & 28 & 46.6 & 0.99 & 56 & 54.8 & 289 & 7.2 & 17 & 3.8 & 21 & 0.07 & 9.0 & 21 & 3.8 & 0.07 & 0 & 260 & 44 & 0.0 & 252 & 248 & 258 & 257\end{array}$ 


\begin{tabular}{|c|c|c|c|c|c|c|c|c|c|c|c|c|c|c|c|c|c|c|c|c|c|c|}
\hline \multirow[b]{2}{*}{ Test } & \multirow[b]{2}{*}{ Date \& Time } & \multicolumn{6}{|c|}{ Economizer Exit, dry $(1-12)$} & \multicolumn{7}{|c|}{ Baghouse Inlet Gas Analysis, wet } & \multicolumn{7}{|c|}{ Stack Gas Analysis, wet } & \multirow[b]{2}{*}{ Comments } \\
\hline & & $\begin{array}{l}\text { NO } \\
\mathrm{ppm}\end{array}$ & $\begin{array}{l}\mathrm{CO} \\
\mathrm{ppm}\end{array}$ & $\begin{array}{l}\mathrm{SO} 2 \\
\mathrm{ppm}\end{array}$ & $\begin{array}{l}\mathrm{NO} 2 \\
\mathrm{ppm}\end{array}$ & $\begin{array}{c}\mathrm{CO} 2 \\
\%\end{array}$ & $\begin{array}{c}02 \\
\% d r y\end{array}$ & $\begin{array}{l}\mathrm{NO} \\
\mathrm{ppm}\end{array}$ & $\begin{array}{l}\text { CO } \\
\text { ppm }\end{array}$ & $\begin{array}{l}\mathrm{SO} 2 \\
\mathrm{ppm}\end{array}$ & $\begin{array}{l}\mathrm{NO2} 2 \\
\mathrm{ppm}\end{array}$ & $\begin{array}{c}\mathrm{CO} 2 \\
\%\end{array}$ & $\begin{array}{c}\mathrm{H} 2 \mathrm{O} \\
\%\end{array}$ & $\begin{array}{c}\mathrm{O} 2 \\
\%\end{array}$ & $\begin{array}{l}\mathrm{NO} \\
\mathrm{ppm}\end{array}$ & $\begin{array}{l}\mathrm{CO} \\
\mathrm{ppm}\end{array}$ & $\begin{array}{l}\mathrm{SO} 2 \\
\mathrm{ppm}\end{array}$ & $\begin{array}{l}\mathrm{NO} 2 \\
\mathrm{ppm}\end{array}$ & $\begin{array}{c}\mathrm{CO} 2 \\
\%\end{array}$ & $\begin{array}{c}\mathrm{H} 2 \mathrm{O} \\
\%\end{array}$ & $\begin{array}{c}\mathrm{O} 2 \\
\%\end{array}$ & \\
\hline 660 & 9/18/93:0900 & 199 & 17 & 301 & 0 & 10.70 & 8.70 & 184 & 18 & 278 & -5 & 1.10 & 7.93 & $\overline{7.40}$ & 169 & 14 & 164 & -3 & 9.58 & 7.50 & 8.38 & $60 \mathrm{Mwe}$ \\
\hline 661 & 9/18/93:1020 & 195 & 16 & 300 & 0 & 10.76 & 8.65 & 180 & 16 & 280 & -6 & 10.17 & 7.87 & 7.35 & 162 & 15 & 137 & -3 & 9.60 & 7.48 & 8.32 & \\
\hline 662 & 9/18/93:1210 & 198 & 12 & 312 & 0 & 10.72 & 8.72 & 180 & 14 & 298 & -5 & 10.20 & 7.84 & 7.22 & 160 & 11 & 135 & -3 & 9.63 & 7.40 & 8.32 & \\
\hline 663 & 9/18/93:1340 & 190 & 13 & 332 & 0 & 10.74 & 8.72 & 178 & 11 & 312 & -5 & 10.15 & 8.15 & 7.20 & 153 & 12 & 127 & -3 & 9.61 & 7.74 & 8.28 & \\
\hline 664 & $9 / 18 / 93: 1650$ & 191 & 11 & 360 & 0 & 10.73 & 8.80 & 181 & 10 & 343 & -5 & 10.36 & 8.01 & 7.12 & 142 & 9 & 138 & 11 & 9.67 & 7.51 & 8.30 & post bh clean? \\
\hline
\end{tabular}

$\begin{array}{llllllll}673 & 9 / 24 / 93: 0850 & 247 & 52 & 362 & 3 & 13.84 & 5.12\end{array}$ $\begin{array}{llllllll}673 & 9 / 24 / 93: 1150 & 248 & 43 & 364 & 1 & 13.88 & 5.20\end{array}$ $\begin{array}{llllllll}674 & 9 / 24 / 93: 1300 & 250 & 36 & 362 & 1 & 13.82 & 5.15\end{array}$ $\begin{array}{llllllll}675 & 9 / 24 / 93: 1450 & 252 & 44 & 358 & 1 & 14.07 & 5.10\end{array}$ $\begin{array}{lllllllll}676 & 9 / 27 / 93: 0840 & 242 & 48 & 390 & 1 & 13.66 & 5.71\end{array}$ $\begin{array}{llllllll}676 & 9 / 27 / 93: 1240 & 244 & 149 & 278 & 1 & 13.48 & 5.76\end{array}$ 677 9/27/93:1750 $\quad 144 \quad 382 \quad 418 \quad 0 \quad 13.26 \quad 6.00$ $\begin{array}{llllllll}677 & 9 / 27 / 93: 1805 & 144 & 382 & 418 & 0 & 13.26 & 6.00\end{array}$ $\begin{array}{llllllll}680 & 10 / 04 / 93: 1450 & 210 & 293 & 383 & 2 & 14.37 & 4.40\end{array}$ $\begin{array}{llllllll}681 & 10 / 05 / 93: 0800 & 222 & 66 & 508 & 3 & 13.75 & 5.30\end{array}$ $\begin{array}{llllllll}682 & 10 / 05 / 93: 1010 & 235 & 75 & 515 & 2 & 14.03 & 5.40\end{array}$ $\begin{array}{llllllll}683 & 10 / 05 / 93: 1230 & 215 & 293 & 528 & 2 & 14.68 & 4.70\end{array}$ $\begin{array}{llllllll}684 & 10 / 05 / 93: 1400 & 228 & 103 & 520 & 2 & 14.55 & 5.05\end{array}$ $\begin{array}{llllllll}685 & 10 / 05 / 93: 1540 & 220 & 153 & 512 & 2 & 14.82 & 4.80\end{array}$ $\begin{array}{llllllll}686 & 10 / 06 / 93: 09106 & 228 & 1265 & 530 & 4 & 13.83 & 5.15\end{array}$ $\begin{array}{llllllll}687 & 10 / 06 / 93: 1210 & 212 & 235 & 555 & 2 & 14.88 & 4.70\end{array}$ 687 10/06/93:1300 $233 \quad 1429 \quad 550 \quad 2 \quad 14.61 \quad 4.60$ $\begin{array}{llllllll}688 & 10 / 06 / 93: 1510 & 233 & 1330 & 500 & 1 & 14.41 & 4.78\end{array}$ $\begin{array}{lllllllll}689 & 10 / 06 / 93: 1800 & 237 & 1062 & 468 & 1 & 14.72 & 4.48\end{array}$ $\begin{array}{lllllllll}690 & 10 / 06 / 93: 1700 & 240 & 886 & 434 & 1 & 14.39 & 4.90\end{array}$ $\begin{array}{llllllll}691 & 10 / 07 / 93: 0750 & 255 & 686 & 385 & 2 & 13.51 & 5.80\end{array}$

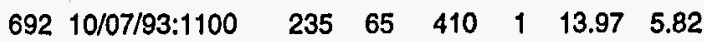
$\begin{array}{llllllll}693 & 10 / 07 / 93: 1400 & 230 & 53 & 410 & 1 & 14.12 & 5.50\end{array}$ $\begin{array}{llllllll}694 & 10 / 07 / 93: 1500 & 233 & 68 & 408 & 1 & 14.38 & 5.20\end{array}$

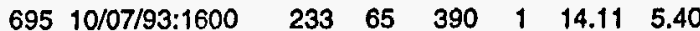

\begin{abstract}
$\begin{array}{llllll}240 & 55 & 240 & 2 & 13.69 & 5.75\end{array}$
\end{abstract}
$\begin{array}{llllll}220 & 942 & 488 & 2 & 14.49 & 3.80\end{array}$ $\begin{array}{lllllll}210 & 1200 & 500 & 2 & 14.47 & 3.65\end{array}$ $\begin{array}{llllll}220 & 722 & 510 & 1 & 14.50 & 4.00\end{array}$ $\begin{array}{llllll}220 & 977 & 520 & 2 & 14.62 & 4.10\end{array}$ $\begin{array}{lllllll}205 & 1444 & 510 & 4 & 14.48 & 3.30\end{array}$ $\begin{array}{lllllll}213 & 1117 & 510 & 2 & 14.77 & 3.60\end{array}$ $\begin{array}{lllllll}218 & 134 & 505 & 1 & 14.61 & 3.70\end{array}$ $\begin{array}{lllllll}228 & 556 & 495 & 1 & 14.55 & 3.90\end{array}$ $\begin{array}{llllllllllllll}210 & 31 & 340 & -5 & 12.93 & 10.13 & 3.60 & 212 & 37 & 323 & -3 & 12.12 & 9.40 & 5.00 \\ & 1.005 e\end{array}$ $\begin{array}{llllllllllllll}222 & 22 & 322 & -5 & 12.38 & 9.60 & 4.40 & 192 & 49 & 112 & 5 & 12.10 & 9.37 & 5.10 \\ B & \text { feeder }\end{array}$ \begin{tabular}{llllllllllllll}
225 & 19 & 320 & -4 & 12.41 & 9.71 & 4.40 & 188 & 31 & 70 & 6 & 12.05 & 9.37 & 5.15 \\
\hline
\end{tabular} $\begin{array}{llllllllllllll}235 & 18 & 325 & -5 & 12.60 & 9.86 & 4.10 & 188 & 27 & 85 & 11 & 12.05 & 9.34 & 5.15 \\ A\end{array}$ $\begin{array}{llllllllllllll}210 & 13 & 354 & -5 & 12.48 & 9.06 & 4.62 & 202 & 39 & 340 & -3 & 11.98 & 8.62 & 5.55 \text { base }\end{array}$ $\begin{array}{lllllllllllllll}233 & 20 & 331 & -4 & 11.99 & 8.60 & 5.20 & 177 & 106 & 104 & 17 & 11.80 & 8.44 & 5.70 & 3 \text { mills }\end{array}$

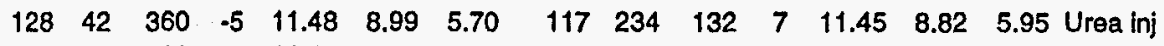

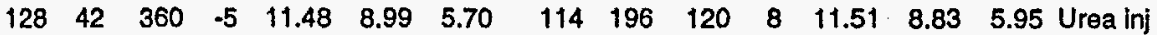
$\begin{array}{llllllllllllll}198 & 143 & 355 & -4 & 13.56 & 9.50 & 3.20 & 187 & 226 & 253 & -1 & 12.99 & 9.10 & 4.40 \\ \text { A feeder, low blr spd }\end{array}$ $\begin{array}{llllllllllllll}190 & 68 & 485 & -2 & 13.19 & 9.62 & 3.75 & 195 & 103 & 470 & -2 & 12.58 & 9.11 & 4.85 \text { Base }\end{array}$ $\begin{array}{llllllllllllll}198 & 41 & 475 & -4 & 13.00 & 9.51 & 3.85 & 202 & 69 & 368 & -1 & 12.52 & 8.98 & 5.00 \\ \text { A feeder }\end{array}$ $\begin{array}{llllllllllllll}202 & 68 & 480 & -2 & 13.09 & 9.42 & 3.80 & 175 & 201 & 250 & 5 & 12.74 & 9.20 & 4.85\end{array}$ $\begin{array}{llllllllllllll}200 & 41 & 475 & -3 & 13.11 & 9.36 & 3.78 & 165 & 69 & 182 & 6 & 12.63 & 8.92 & 4.95\end{array}$ $\begin{array}{llllllllllllll}195 & 44 & 465 & -3 & 13.17 & 9.37 & 3.75 & 155 & 115 & 130 & 5 & 12.89 & 9.08 & 4.75\end{array}$ $\begin{array}{lllllll}225 & 80 & 453 & -3 & 11.99 & 8.77 & 5.15\end{array}$ $\begin{array}{llllllll}190 & 49 & 480 & -3 & 12.85 & 9.47 & 4.05\end{array}$ $\begin{array}{llllllll}222 & 121 & 448 & -4 & 12.21 & 8.85 & 4.99\end{array}$ $\begin{array}{llllllll}220 & 60 & 415 & -4 & 11.98 & 8.70 & 5.15\end{array}$ $\begin{array}{llllllll}225 & 67 & 390 & -3 & 12.20 & 8.84 & 4.85\end{array}$ $\begin{array}{llllllll}228 & 87 & 368 & -4 & 12.09 & 8.78 & 4.95\end{array}$ $\begin{array}{llllllll}255 & 28 & 318 & -5 & 11.48 & 8.61 & 5.65\end{array}$ $\begin{array}{llllllll}235 & 30 & 370 & -3 & 12.08 & 9.04 & 5.00\end{array}$ $\begin{array}{llllllll}230 & 29 & 360 & -5 & 12.30 & 9.51 & 4.65\end{array}$ $\begin{array}{llllllll}233 & 35 & 355 & -4 & 12.21 & 9.55 & 4.55\end{array}$ $\begin{array}{llllllll}233 & 39 & 358 & -5 & 12.27 & 9.64 & 4.50\end{array}$ $\begin{array}{lllllll}208 & 28 & 350 & -4 & 12.26 & 9.57 & 4.65\end{array}$

$\begin{array}{lllllll}190 & 105 & 433 & -4 & 13.02 & 9.57 & 3.55\end{array}$ $\begin{array}{lllllll}200 & 651 & 458 & -4 & 13.43 & 9.77 & 3.10\end{array}$ $\begin{array}{llllllll}190 & 192 & 470 & -4 & 13.21 & 9.70 & 3.50\end{array}$ $\begin{array}{llllllll}199 & 540 & 470 & -3 & 13.30 & 9.81 & 3.20\end{array}$ $\begin{array}{llllllll}195 & 570 & 460 & -4 & 13.27 & 9.45 & 3.20\end{array}$ $\begin{array}{llllllll}200 & 201 & 452 & -4 & 13.06 & 9.31 & 3.60\end{array}$ $\begin{array}{lllllll}198 & 219 & 455 & 0 & 13.37 & 9.55 & 3.10\end{array}$ $\begin{array}{lllllll}203 & 216 & 445 & -3 & 13.09 & 9.43 & 3.80\end{array}$ $\begin{array}{llllllll}200 & 998 & 475 & -2 & 12.32 & 9.17 & 4.75 & \text { Base }\end{array}$

$\begin{array}{llllllll}175 & 280 & 370 & -1 & 13.01 & 9.50 & 4.50 & \text { A feeder }\end{array}$

$\begin{array}{llllllll}190 & 1034 & 340 & -1 & 12.47 & 8.96 & 4.70\end{array}$

$\begin{array}{llllllll}178 & 668 & 225 & 4 & 12.47 & 9.07 & 4.85\end{array}$

$\begin{array}{llllllll}173 & 631 & 155 & 8 & 12.52 & 9.12 & 4.80\end{array}$

$\begin{array}{llllllll}178 & 606 & 112 & 7 & 12.50 & 9.09 & 4.80\end{array}$

$\begin{array}{llllllll}223 & 298 & 330 & -3 & 11.88 & 8.84 & 5.65 & \text { Base }\end{array}$

$\begin{array}{llllllll}190 & 58 & 195 & -1 & 12.14 & 8.94 & 5.55 & \text { A\&B feeders }\end{array}$

$\begin{array}{llllllll}170 & 52 & 117 & 6 & 12.23 & 9.35 & 5.25\end{array}$

$\begin{array}{llllllll}168 & 61 & 83 & 5 & 12.26 & 9.53 & 5.15\end{array}$

$\begin{array}{llllllll}170 & 48 & 68 & 4 & 12.25 & 9.59 & 5.35\end{array}$

$\begin{array}{lllllll}172 & 49 & 93 & 10 & 12.07 & 9.36 & 5.50\end{array}$

$\begin{array}{llllllll}183 & 977 & 433 & -2 & 12.81 & 9.32 & 3.60 & \text { Base, A cal }\end{array}$ $\begin{array}{llllllll}180 & 681 & 303 & -1 & 12.75 & 9.32 & 3.90 & \text { A feeder only }\end{array}$ $\begin{array}{lllllllll}178 & 450 & 318 & -1 & 12.86 & 9.34 & 4.10 & B\end{array}$ $\begin{array}{llllllll}180 & 870 & 300 & -1 & 12.86 & 9.38 & 3.70 & \text { A\&B feeders }\end{array}$ $\begin{array}{llllllll}184 & 137 & 463 & -2 & 13.12 & 9.26 & 3.25 & \text { Base }\end{array}$ $\begin{array}{llllllll}175 & 107 & 215 & 0 & 13.00 & 9.23 & 3.65 & B \text { feeder }\end{array}$ $\begin{array}{lllllll}180 & 673 & 195 & 0 & 12.82 & 9.20 & 4.30\end{array}$ $\begin{array}{lllllll}168 & 916 & 198 & 0 & 12.94 & 9.14 & 3.75 \\ 175 & 107 & \text { A feeder }\end{array}$ 
PSCC Arapahoe Unit 4 Sodium Injection Summary

Calcs based on: Sodium Bicarbonate (b)

$.274 \mathrm{Na} w t$

Sodium Sesquicarbonate (s) $\quad .297 \mathrm{Nawt}$

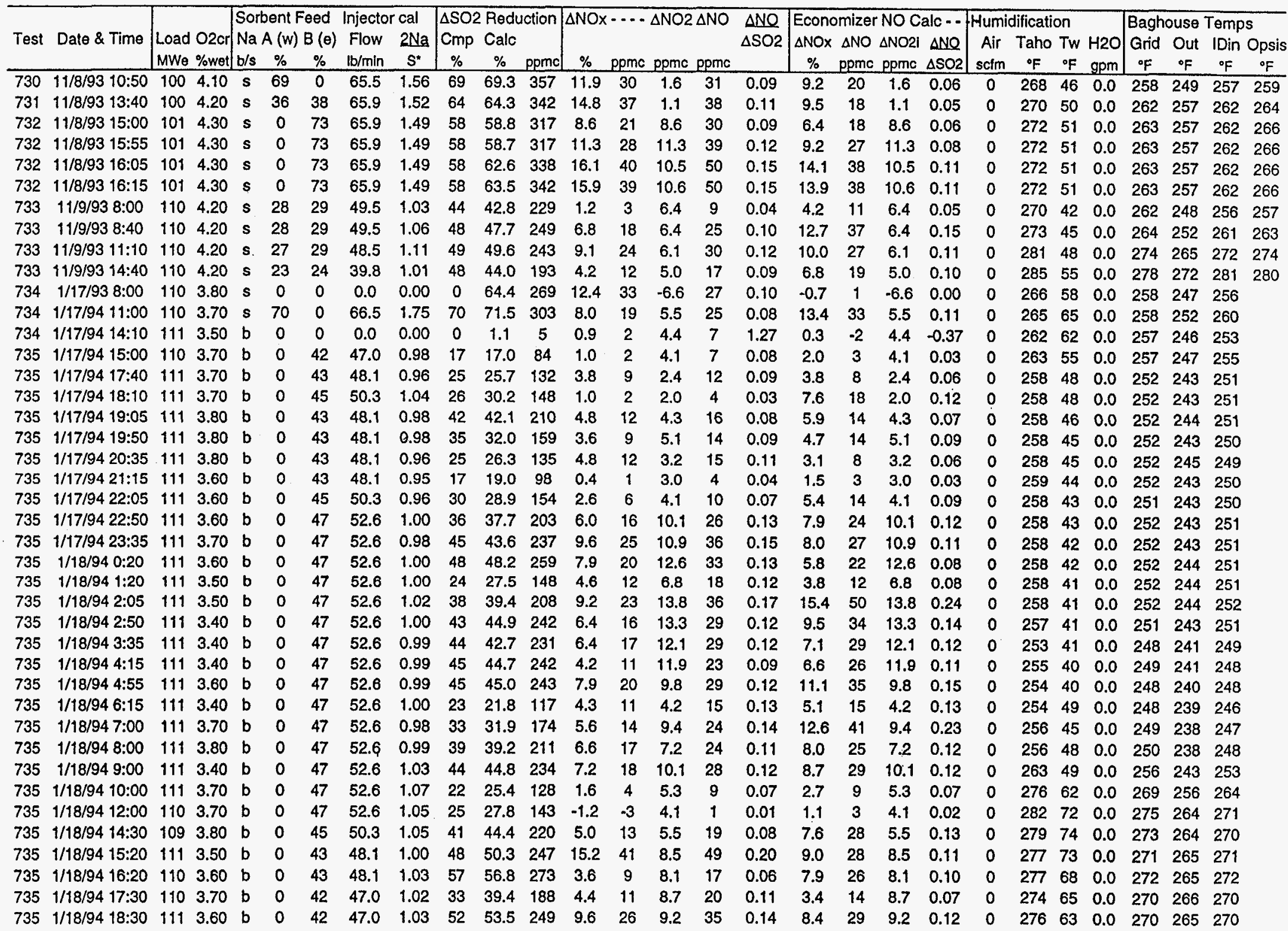




\begin{tabular}{|c|c|c|c|c|c|c|c|c|c|c|c|c|c|c|c|c|c|c|c|c|c|c|}
\hline \multirow[b]{2}{*}{ Test } & \multirow[b]{2}{*}{ Date \& Time } & \multicolumn{6}{|c|}{ Economizer Exit, dry $(1-12)$} & \multicolumn{7}{|c|}{ Baghouse Inlet Gas Analysis, wet } & \multicolumn{7}{|c|}{ Stack Gas Analysis, wet } & \multirow[b]{2}{*}{ Comments } \\
\hline & & $\begin{array}{l}\text { NO } \\
\text { ppm }\end{array}$ & $\begin{array}{l}\mathrm{CO} \\
\mathrm{ppm}\end{array}$ & $\begin{array}{l}\mathrm{SO} 2 \\
\mathrm{ppm}\end{array}$ & $\begin{array}{l}\mathrm{NO} 2 \\
\mathrm{ppm}\end{array}$ & $\begin{array}{c}\mathrm{CO} 2 \\
\%\end{array}$ & $\begin{array}{c}\mathrm{O} 2 \\
\% \mathrm{~d} y\end{array}$ & $\begin{array}{l}\text { NO } \\
\mathrm{ppm}\end{array}$ & $\begin{array}{l}\mathrm{CO} \\
\mathrm{ppm}\end{array}$ & $\begin{array}{c}\mathrm{SO} 2 \\
\mathrm{ppm}\end{array}$ & $\begin{array}{l}\mathrm{NO} 2 \\
\mathrm{ppm}\end{array}$ & $\begin{array}{c}\mathrm{CO} 2 \\
\%\end{array}$ & $\begin{array}{c}\mathrm{H} 2 \mathrm{O} \\
\%\end{array}$ & $\begin{array}{c}\mathrm{O} 2 \\
\%\end{array}$ & $\begin{array}{l}\text { NO } \\
\mathrm{ppm}\end{array}$ & $\begin{array}{l}\mathrm{CO} \\
\mathrm{ppm}\end{array}$ & $\begin{array}{l}\mathrm{SO} 2 \\
\mathrm{ppm}\end{array}$ & $\begin{array}{l}\mathrm{NO2} \\
\mathrm{ppm}\end{array}$ & $\begin{array}{c}\mathrm{CO} 2 \\
\%\end{array}$ & $\begin{array}{c}\mathrm{H} 2 \mathrm{O} \\
\%\end{array}$ & $\begin{array}{c}\mathrm{O} 2 \\
\%\end{array}$ & \\
\hline 730 & $11 / 8 / 9310: 50$ & 215 & 268 & 442 & 1 & 13.28 & 5.05 & 209 & 37 & 423 & -3 & 12.53 & 8.82 & 4.35 & 175 & 237 & 124 & -1 & 12.22 & 8.85 & 5.00 & A \\
\hline 731 & $11 / 8 / 9313: 40$ & 210 & 432 & 480 & 2 & 13.62 & 4.75 & 210 & 46 & 441 & -3 & 12.62 & 8.87 & 4.20 & 170 & 222 & 150 & -2 & 12.32 & 8.64 & 4.95 & $A \& B$ \\
\hline 732 & $11 / 8 / 9315: 00$ & 212 & 603 & 480 & 3 & 13.70 & 4.90 & 208 & 113 & 450 & -3 & 12.73 & 8.93 & 4.10 & 175 & 317 & 177 & 4 & 12.32 & 8.57 & 4.85 & B, BH clean \\
\hline 732 & $11 / 8 / 9315: 55$ & 212 & 603 & 480 & 3 & 13.70 & 4.90 & 208 & 113 & 450 & -3 & 12.73 & 8.93 & 4.10 & 170 & 470 & 180 & 7 & 12.55 & 8.76 & 4.60 & \\
\hline 732 & $11 / 8 / 9316: 05$ & 212 & 603 & 480 & 3 & 13.70 & 4.90 & 208 & 113 & 450 & -3 & 12.73 & 8.93 & 4.10 & 162 & 477 & 164 & 6 & 12.54 & 8.75 & 4.52 & \\
\hline 732 & $11 / 8 / 9$ & 212 & 603 & 480 & 3 & 13.70 & 4.90 & 208 & 113 & 450 & -3 & 12.73 & 8.93 & 4.10 & 162 & 481 & 160 & 6 & 12.54 & 8.73 & 4.55 & \\
\hline 733 & $11 / 9 / 9$ & 241 & 741 & 482 & 3 & 13.94 & 4.80 & 214 & 89 & 430 & -3 & 12.35 & 8.79 & 4.68 & & 602 & 242 & 2 & 12.33 & 8.63 & 4.95 & \\
\hline 733 & $11 / 9 / 938: 40$ & 252 & 677 & 473 & 2 & 13.80 & 5.10 & 225 & 92 & 431 & -3 & 12.80 & 8.99 & 4.25 & 198 & 578 & 218 & 3 & 12.39 & 8.66 & 4.80 & "befo \\
\hline 733 & $11 / 9 / 9311: 10$ & 241 & 998 & 446 & 2 & 14.30 & 4.45 & 226 & 138 & 417 & -2 & 13.12 & 9.09 & 3.75 & 193 & 730 & 202 & 3 & 12.68 & 8.76 & 4.40 & $\mathrm{BH} \mathrm{Cl}$ \\
\hline 733 & $14: 40$ & 254 & 546 & 385 & 2 & 14.07 & 4.70 & 233 & 91 & 368 & -3 & 12.83 & 8.86 & 4.05 & 209 & 400 & 197 & 2 & 12.46 & 8.49 & 4.80 & $\mathrm{BH}$ cle \\
\hline 734 & $8: 00$ & 210 & 575 & 340 & -9 & 12.33 & 5.45 & 225 & 189 & 350 & -1 & 12.88 & 9.19 & 4.00 & 195 & 452 & 120 & -6 & 12.31 & 9.06 & 4.60 & Base \\
\hline 734 & $1 / 17 / s$ & 210 & 445 & 348 & 3 & 12.37 & 6.00 & 210 & 329 & 365 & -3 & 12.98 & 9.34 & 3.55 & 178 & 589 & 98 & & 12.43 & 8.98 & 4.50 & Runr \\
\hline 734 & $1 / 17 /$ & 218 & 622 & 405 & 2 & 12.92 & 5.60 & 222 & 186 & 405 & -4 & 12.63 & 9.17 & 3.90 & 208 & 509 & 385 & 0 & 12.23 & 8.89 & 4.55 & $\mathrm{Ba}$ \\
\hline 735 & $1 / 17$ & 218 & 697 & 420 & 1 & 13.05 & 5.60 & 218 & 192 & 418 & -4 & 13.00 & 9.37 & 3.85 & 202 & 489 & 330 & -1 & 12.23 & 8.88 & 4.70 & Bice \\
\hline 735 & $1 / 17$ & 215 & 672 & 418 & 1 & 13.01 & 5.55 & 218 & 222 & 440 & -2 & 13.14 & 9.50 & 3.55 & 197 & 503 & 310 & 0 & 12.54 & 9.05 & 4.45 & \\
\hline 735 & $1 / 17$ & 215 & 660 & 408 & 2 & 12.84 & 5.75 & 210 & 463 & 430 & -2 & 13.24 & 9.57 & 3.25 & 193 & 506 & 281 & 0 & 12.50 & 8.89 & 4.40 & \\
\hline 735 & $1 / 17$ & 211 & 828 & 425 & 1 & 13.04 & 5.40 & 212 & 313 & 432 & -3 & 12.98 & 9.46 & 3.45 & 190 & 506 & 240 & 1 & 12.47 & 8.95 & 4.20 & \\
\hline 735 & $1 / 17$ & 211 & 829 & 430 & 1 & 13.00 & 5.35 & 210 & 365 & 431 & -1 & 13.10 & 9.46 & 3.45 & 189 & 644 & 280 & 3 & 12.55 & 9.01 & 4.25 & \\
\hline 735 & $1 / 17$ & 211 & 877 & 435 & 2 & 13.10 & 5.40 & 217 & 256 & 442 & -1 & 13.19 & 9.47 & 3.45 & 194 & 664 & 310 & 2 & 12.56 & 8.99 & 4.30 & \\
\hline 735 & $1 / 17 /$ & 214 & 799 & 446 & 2 & 13.02 & 5.35 & 213 & 236 & 446 & -2 & 13.00 & 9.39 & 3.55 & 200 & 576 & 345 & 0 & 12.42 & 8.92 & 4.35 & \\
\hline 735 & $1 / 17 / 5$ & 217 & 962 & 463 & 2 & 13.16 & 5.25 & 213 & 302 & 465 & -2 & 13.16 & 9.46 & 3.35 & 193 & 739 & 313 & 2 & 12.40 & 8.92 & 4.30 & \\
\hline 735 & $1 / 17 / 9422: 50$ & 222 & 670 & 450 & 2 & 12.69 & 5.75 & 224 & 201 & 457 & -4 & 12.62 & 9.12 & 3.80 & 191 & 478 & 269 & 4 & 12.28 & 8.89 & 4.70 & \\
\hline 735 & $1 / 17 / 9423: 35$ & 220 & 541 & 455 & 1 & 12.88 & 5.70 & 225 & 118 & 457 & -3 & 12.74 & 9.22 & 3.95 & 187 & 573 & 248 & 6 & 12.32 & 8.89 & 4.60 & \\
\hline 735 & $0: 20$ & 218 & 905 & 456 & 2 & 13.26 & 5.30 & 221 & 229 & 454 & -3 & 12.63 & 9.12 & 3.85 & 185 & 545 & 225 & 7 & 12.37 & 8.87 & 4.55 & \\
\hline 735 & $1 / 18$ & 215 & 903 & 449 & 2 & 12.93 & 5.55 & 220 & 247 & 460 & -2 & 12.85 & 9.27 & 3.65 & 196 & 672 & 320 & 4 & 12.38 & 8.89 & 4.35 & \\
\hline 735 & 2:05 & 219 & 496 & 436 & 2 & 12.58 & 6.00 & 215 & 172 & 454 & -2 & 12.85 & 9.24 & 3.55 & 175 & 445 & 262 & 9 & 12.44 & 8.92 & 4.35 & \\
\hline 735 & $1 / 18 /$ & 213 & 884 & 452 & 2 & 12.82 & 5.55 & 211 & 312 & 467 & -1 & 13.08 & 9.44 & 3.40 & 175 & 468 & 242 & 10 & 12.31 & 8.82 & 4.45 & \\
\hline 735 & 1/18/94 3:35 & 222 & 803 & 455 & 2 & 12.94 & 5.60 & 220 & 192 & 458 & 0 & 12.88 & 9.28 & 3.80 & 185 & 368 & 248 & 10 & 12.19 & 8.70 & 4.75 & \\
\hline 735 & $44: 15$ & 218 & 555 & 455 & 1 & 13.02 & 5.80 & 218 & 266 & 464 & .1 & 12.97 & 9.38 & 3.60 & 185 & 403 & 239 & 9 & 12.09 & 8.70 & 4.80 & \\
\hline 735 & $1 / 18 / 944: 55$ & 220 & 296 & 451 & 1 & 12.95 & 5.65 & 215 & 151 & 460 & -2 & 13.03 & 9.32 & 3.70 & 180 & 294 & 240 & 7 & 12.34 & 8.83 & 4.60 & \\
\hline 735 & $1 / 18 / 946: 15$ & 220 & 341 & 455 & 2 & 13.13 & 5.65 & 221 & 145 & 460 & -1 & 13.02 & 9.31 & 3.65 & 196 & 267 & 339 & 3 & 12.32 & 8.80 & 4.65 & \\
\hline 735 & $1 / 18 / 947: 00$ & 230 & 385 & 449 & 2 & 12.80 & 6.00 & 220 & 185 & 465 & -1 & 13.00 & 9.27 & 3.75 & 188 & 183 & 298 & 6 & 12.35 & 8.83 & 4.75 & \\
\hline 735 & $1 / 18 / 948: 00$ & 217 & 568 & 462 & 1 & 13.17 & 5.50 & 218 & 242 & 470 & -1 & 13.11 & 9.39 & 3.35 & 185 & 285 & 268 & 5 & 12.43 & 8.83 & 4.45 & \\
\hline 735 & $1 / 18 / 949: 00$ & 220 & 691 & 445 & 1 & 13.03 & 5.35 & 215 & 276 & 445 & -2 & 12.88 & 9.26 & 3.70 & 183 & 507 & 235 & 7 & 12.25 & 8.81 & 4.45 & \\
\hline 735 & $1 / 18 / 9410: 00$ & 218 & 786 & 437 & 1 & 13.10 & 5.45 & 217 & 242 & 431 & -2 & 12.87 & 9.12 & 3.60 & 197 & 486 & 303 & 3 & 12.19 & 8.62 & 4.60 & $\mathrm{BH} \operatorname{cln} 09: 35-10: 00$ \\
\hline 735 & $1 / 18 / 9412: 00$ & 220 & 691 & 428 & 1 & 12.75 & 5.70 & 215 & 76 & 431 & -2 & 12.76 & 9.17 & 4.00 & 205 & 456 & 298 & 1 & 12.20 & 8.63 & 4.75 & \\
\hline 735 & $1 / 18 / 9414: 30$ & 225 & 447 & 405 & 1 & 12.74 & 6.00 & 222 & 176 & 422 & 3 & 12.97 & 9.18 & 3.75 & 195 & 496 & 222 & 7 & 12.24 & 8.59 & 4.70 & $\mathrm{BH}$ cln 12:50-13:25 \\
\hline 735 & $1 / 18 / 9415: 20$ & 211 & 571 & 410 & 1 & 12.87 & 5.75 & 228 & 109 & 415 & -1 & 12.71 & 9.07 & 3.90 & 178 & 464 & 197 & 6 & 12.44 & 8.75 & 4.65 & \\
\hline 735 & $1 / 18 / 9416: 20$ & 220 & 645 & 410 & 1 & 12.88 & 5.70 & 212 & 158 & 408 & -1 & 12.96 & 9.18 & 3.80 & 187 & 359 & 167 & 5 & 12.39 & 8.73 & 4.70 & \\
\hline 735 & $1 / 18 / 9417: 30$ & 212 & 610 & 393 & 1 & 12.98 & 5.70 & 217 & 164 & 405 & -2 & 13.07 & 9.27 & 3.75 & 190 & 437 & 233 & 6 & 12.32 & 8.68 & 4.65 & $6: 55-17: 30$ \\
\hline 735 & $1 / 18 / 9418: 30$ & 220 & 539 & 385 & 1 & 12.75 & 5.85 & 227 & 84 & 395 & -1 & 12.93 & 9.10 & 3.80 & 188 & 323 & 175 & 7 & 12.42 & 8.66 & 4.62 & \\
\hline
\end{tabular}


PSCC Arapahoo Unit 4 Sodium Injection Summary

Calcs based on: Sodium Bicarbonate (b)

$.274 \mathrm{Nawt}$

Sodium Sesquicarbonate (s)

$.297 \mathrm{Na}$ wt

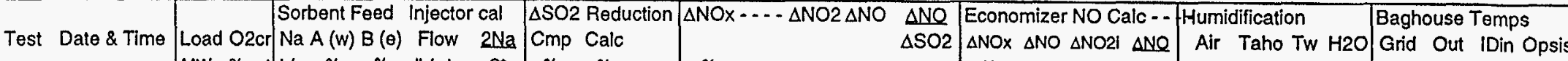

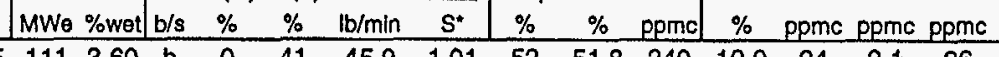

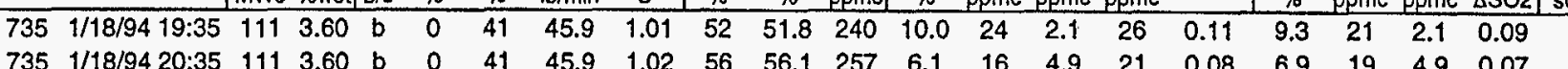

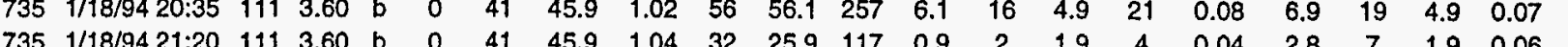
$\begin{array}{lllllllllllllllllllll}735 & 1 / 18 / 9422: 20 & 111 & 3.80 & \text { b } & 0 & 41 & 45.9 & 1.05 & 46 & 48.4 & 216 & 5.5 & 14 & 5.8 & 20 & 0.09 & 8.8 & 26 & 5.8 & 0.12\end{array}$ $\begin{array}{lllllllllllllllllllllll}735 & 1 / 18 / 94 & 23: 15 & 111 & 3.40 & \text { b } & 0 & 39 & 43.7 & 1.01 & 55 & 55.1 & 244 & 8.9 & 24 & 7.0 & 31 & 0.13 & 11.8 & 37 & 7.0 & 0.15 & 0\end{array}$

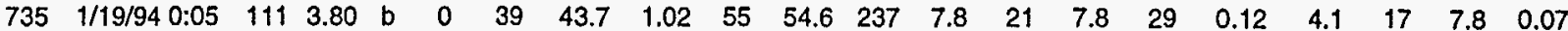

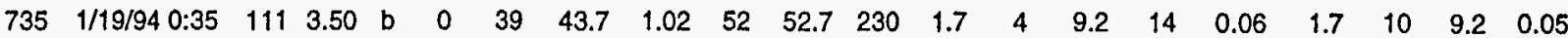
$\begin{array}{lllllllllllllllllllll}735 & 1 / 19 / 941: 10 & 111 & 3.50 & b & 0 & 39 & 43.7 & 1.03 & 20 & 20.1 & 87 & 5.7 & 15 & 2.1 & 17 & 0.20 & 6.9 & 16 & 2.1 & 0.18\end{array}$ $\begin{array}{llllllllllllllllllllll}735 & 1 / 19 / 94 & 4: 35 & 111 & 3.60 & \text { b } & 0 & 38 & 42.6 & 0.99 & 40 & 46.3 & 203 & 17.6 & 46 & 1.9 & 48 & 0.23 & 17.3 & 41 & 1.9 & 0.20\end{array}$ $\begin{array}{llllllllllllllllllllll}735 & 1 / 19 / 94 & 5: 15 & 111 & 3.70 & \text { b } & 0 & 38 & 42.6 & 0.99 & 14 & 15.3 & 67 & 1.5 & 4 & 3.5 & 7 & 0.11 & -0.3 & -2 & 3.5 & -0.03\end{array}$

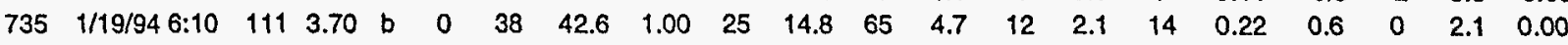

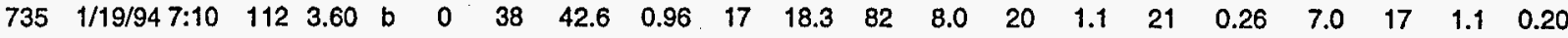

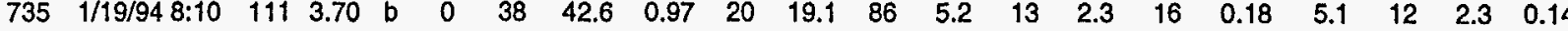
$\begin{array}{lllllllllllllllllllll}735 & 1 / 19 / 949: 00 & 111 & 3.70 & \text { b } & 0 & 38 & 42.6 & 0.97 & 8 & 10.1 & 45 & 2.9 & 7 & 1.9 & 9 & 0.21 & -1.1 & -4 & 1.9 & -0.08\end{array}$ $\begin{array}{llllllllllllllllllllll}735 & 1 / 19 / 94 & 10: 10 & 111 & 3.70 & b & 0 & 38 & 42.6 & 0.97 & 20 & 18.5 & 83 & 7.4 & 19 & 1.8 & 21 & 0.25 & 4.9 & 11 & 1.8 & 0.13\end{array}$

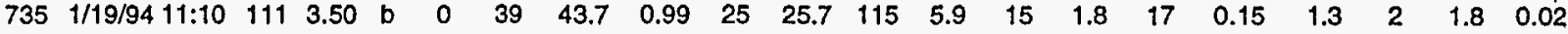
$1 / 19 / 9412: 10 \quad 111 \quad 3.80$ b 735 1/19/94 13.00 1103.90 b $7351 / 19 / 9413: 40 \quad 1113.70$ b 0 $735 \quad 1 / 19 / 94 \quad 14: 10 \quad 111 \quad 3.70$ b:

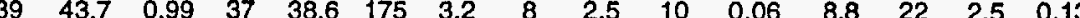
$\begin{array}{lllllllllllllll}39 & 43.7 & 0.99 & 22 & 29.3 & 133 & 2.7 & 7 & 3.3 & 10 & 0.08 & 7.2 & 18 & 3.3 & 0.14\end{array}$ $\begin{array}{llllllllllllllll}39 & 43.7 & 0.98 & 42 & 46.3 & 210 & 7.9 & 21 & 3.4 & 24 & 0.11 & 5.4 & 15 & 3.4 & 0.07\end{array}$ $\begin{array}{ccccccccccccccc}39 & 43.7 & 0.98 & 54 & 56.6 & 257 & 10.7 & 27 & 6.3 & 34 & 0.13 & 9.0 & 27 & 6.3 & 0.10\end{array}$

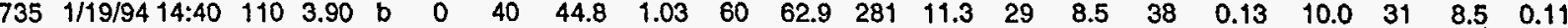

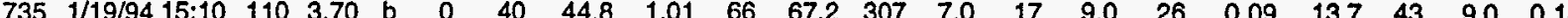
$\begin{array}{llllllllllllllllllllll}735 & 1 / 19 / 94 & 15: 50 & 111 & 3.60 & \text { b } & 0 & 40 & 44.8 & 1.00 & 70 & 71.2 & 326 & 13.2 & 33 & 9.1 & 42 & 0.13 & 17.5 & 52 & 9.1 & 0.16\end{array}$

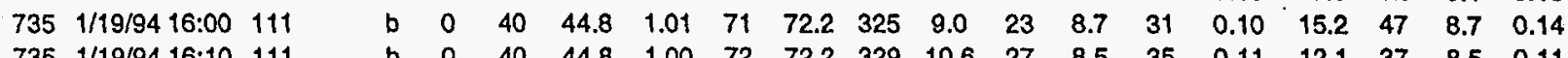

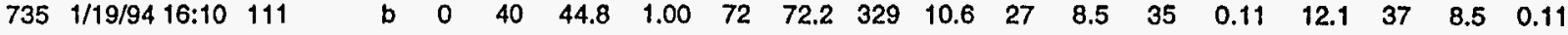

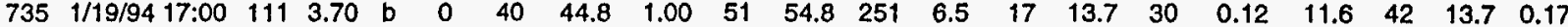

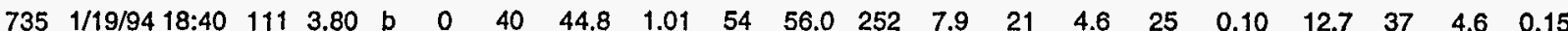

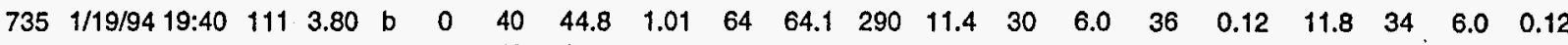

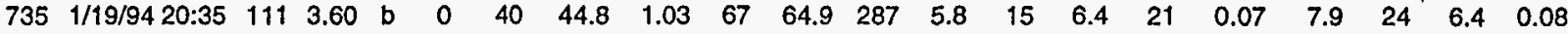

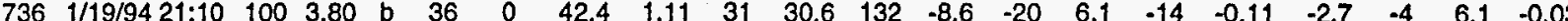

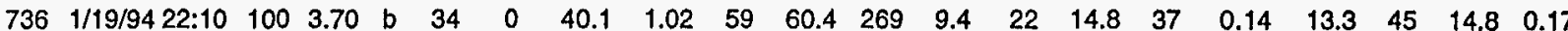

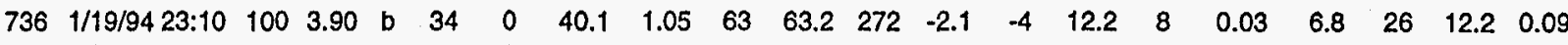

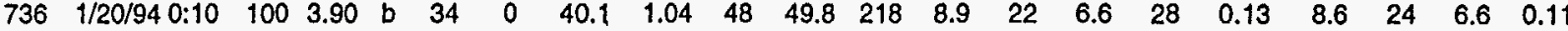

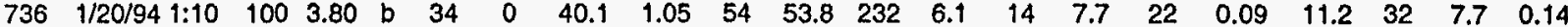

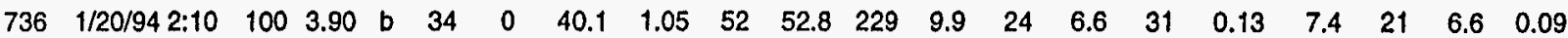
$\begin{array}{lllllll}736 & 1 / 20 / 94 & 3: 10 & 100 & 3.70 & \text { b } & 34\end{array}$ $736 \quad 1 / 20 / 943: 55 \quad 100 \quad 3.80$ b 34 $736 \quad 1 / 20 / 945: 10 \quad 100 \quad 4.00$ b 34 $736 \quad 1 / 20 / 946: 10 \quad 100 \quad 3.90$ b 34

$736 \quad 1 / 20 / 946: 50 \quad 100 \quad 4.00$ b 34

$737 \quad 1 / 20 / 947: 50 \quad 111 \quad 4.20$ b 40

$\begin{array}{cccccccccccccc}40.1 & 1.05 & 52 & 52.8 & 229 & 9.9 & 24 & 6.6 & 31 & 0.13 & 7.4 & 21 & 6.6 & 0.09 \\ 40.1 & 1.06 & 46 & 41.1 & 175 & 1.1 & 2 & 6.3 & 9 & 0.05 & 3.7 & 11 & 6.3 & 0.06\end{array}$ $\begin{array}{llllllllllllllll}40.1 & 1.07 & 28 & 26.4 & 112 & -0.3 & -1 & 6.5 & 6 & 0.05 & 5.6 & 17 & 6.5 & 0.15\end{array}$ $\begin{array}{llllllllllllll}40.1 & 1.05 & 42 & 44.4 & 191 & 0.2 & 0 & 9.9 & 10 & 0.05 & 7.8 & 26 & 9.9 & 0.14\end{array}$ $\begin{array}{llllllllllllll}40.1 & 1.06 & 43 & 48.2 & 206 & 6.5 & 16 & 11.9 & 27 & 0.13 & 12.0 & 39 & 11.9 & 0.19\end{array}$ $\begin{array}{llllllllllllll}40.1 & 1.04 & 48 & 48.7 & 212 & -19.3 & -48 & 13.4 & -34 & -0.16 & -16.7 & -32 & 13.4 & -0.15\end{array}$ $\begin{array}{lllllllllllllll}47.1 & 1.11 & 47 & 47.3 & 205 & 3.4 & 9 & 13.6 & 23 & 0.11 & 4.8 & 24 & 13.6 & 0.12 & 0\end{array}$

$\begin{array}{llllll}277 & 63 & 0.0 & 271 & 264 & 271 \\ 0.0 & & 0\end{array}$ $\begin{array}{llllll}277 & 63 & 0.0 & 271 & 264 & 269\end{array}$ $\begin{array}{llllll}277 & 63 & 0.0 & 271 & 264 & 270\end{array}$ $\begin{array}{llllll}276 & 61 & 0.0 & 271 & 265 & 271\end{array}$ $\begin{array}{llllll}272 & 62 & 0.0 & 267 & 262 & 268\end{array}$ $\begin{array}{llllll}271 & 61 & 0.0 & 265 & 264 & 265\end{array}$ $\begin{array}{llllll}271 & 61 & 0.0 & 266 & 259 & 265\end{array}$ $\begin{array}{llllll}269 & 61 & 0.0 & 264 & 258 & 260\end{array}$ $\begin{array}{llllll}268 & 61 & 0.0 & 263 & 256 & 261\end{array}$ $\begin{array}{llllll}262 & 65 & 0.0 & 258 & 252 & 257\end{array}$ $\begin{array}{lllllll}263 & 66 & 0.0 & 257 & 249 & 256\end{array}$ $\begin{array}{llllll}266 & 71 & 0.0 & 260 & 250 & 258\end{array}$ $\begin{array}{llllll}269 & 73 & 0.0 & 262 & 253 & 260\end{array}$ $\begin{array}{llllll}274 & 73 & 0.0 & 268 & 257 & 265\end{array}$ $\begin{array}{lllll}275 & 76 & 0.0 & 269 & 260\end{array}$ $\begin{array}{llllll}276 & 77 & 0.0 & 270 & 260 & 268\end{array}$ $\begin{array}{llllll}275 & 78 & 0.0 & 269 & 262 & 267\end{array}$ $\begin{array}{llllll}274 & 74 & 0.0 & 268 & 261 & 267\end{array}$ $\begin{array}{llllll}274 & 77 & 0.0 & 269 & 262 & 268\end{array}$ $\begin{array}{llllll}275 & 78 & 0.0 & 269 & 263 & 268\end{array}$ $\begin{array}{llllll}275 & 74 & 0.0 & 270 & 264 & 269\end{array}$ $\begin{array}{llll}274 & 0.0 & 263\end{array}$ $\begin{array}{llll}274 & 72 & 0.0 & 269\end{array}$ 0.0 269 $\begin{array}{llllll}272 & 66 & 0.0 & 266 & 261 & 267\end{array}$ $\begin{array}{llllll}272 & 64 & 0.0 & 265 & 260 & 266\end{array}$ $\begin{array}{llllll}266 & 62 & 0.0 & 261 & 259 & 261\end{array}$ $\begin{array}{lllllll}262 & 60 & 0.0 & 256 & 253 & 257\end{array}$ $\begin{array}{llllll}255 & 58 & 0.0 & 249 & 246 & 252\end{array}$ $\begin{array}{llllll}252 & 56 & 0.0 & 246 & 241 & 247\end{array}$ $\begin{array}{lllllll}253 & 54 & 0.0 & 246 & 239 & 246\end{array}$ $\begin{array}{llllll}253 & 52 & 0.0 & 245 & 237 & 245\end{array}$ $\begin{array}{llllll}252 & 50 & 0.0 & 245 & 237 & 245\end{array}$ $\begin{array}{llllll}251 & 50 & 0.0 & 244 & 236 & 242\end{array}$ $\begin{array}{llllll}250 & 49 & 0.0 & 243 & 236 & 242\end{array}$ $\begin{array}{llllll}249 & 48 & 0.0 & 242 & 233 & 240\end{array}$ $\begin{array}{llllll}248 & 52 & 0.0 & 241 & 232 & 240\end{array}$ $\begin{array}{llllll}241 & 59 & 0.0 & 242 & 233 & 241\end{array}$ $\begin{array}{llllll}257 & 59 & 0.0 & 251 & 238 & 248\end{array}$ 


\begin{tabular}{|c|c|c|c|c|c|c|c|c|c|c|c|c|c|c|c|c|c|c|c|c|c|c|}
\hline \multirow[b]{2}{*}{ Test } & \multirow[b]{2}{*}{ Date \& Time } & \multicolumn{6}{|c|}{ Economizer Exit, dry $(1-12)$} & \multicolumn{7}{|c|}{ Baghouse Inlet Gas Analysis, wet } & \multicolumn{7}{|c|}{ Stack Gas Analysis, wet } & \multirow[b]{2}{*}{ Comments } \\
\hline & & $\begin{array}{l}\text { NO } \\
\text { ppm }\end{array}$ & $\begin{array}{l}\mathrm{CO} \\
\mathrm{ppm}\end{array}$ & $\begin{array}{l}\mathrm{SO} 2 \\
\mathrm{ppm}\end{array}$ & $\begin{array}{l}\mathrm{NO} 2 \\
\mathrm{ppm}\end{array}$ & $\begin{array}{c}\mathrm{CO} 2 \\
\%\end{array}$ & $\begin{array}{c}\mathrm{O} 2 \\
\% \mathrm{dry}\end{array}$ & $\begin{array}{l}\text { NO } \\
\mathrm{ppm}\end{array}$ & $\begin{array}{l}\mathrm{CO} \\
\mathrm{ppm}\end{array}$ & $\begin{array}{l}\mathrm{SO} 2 \\
\mathrm{ppm}\end{array}$ & $\begin{array}{l}\mathrm{NO2} \\
\mathrm{ppm}\end{array}$ & $\begin{array}{c}\mathrm{CO} 2 \\
\%\end{array}$ & $\begin{array}{c}\mathrm{H}_{2} \mathrm{O} \\
\%\end{array}$ & $\begin{array}{l}\mathrm{O} 2 \\
\%\end{array}$ & $\begin{array}{l}\text { NO } \\
\mathrm{ppm}\end{array}$ & $\begin{array}{l}\mathrm{CO} \\
\mathrm{ppm}\end{array}$ & $\begin{array}{l}\mathrm{SO} 2 \\
\mathrm{ppm}\end{array}$ & $\begin{array}{l}\mathrm{NO} 2 \\
\mathrm{ppm}\end{array}$ & $\begin{array}{c}\mathrm{CO} 2 \\
\%\end{array}$ & $\begin{array}{c}\mathrm{H} 2 \mathrm{O} \\
\%\end{array}$ & $\begin{array}{l}\mathrm{O} 2 \\
\%\end{array}$ & \\
\hline 735 & $1 / 18 / 9419: 35$ & 208 & 759 & 395 & 1 & 13.11 & 5.20 & 209 & 164 & 400 & -1 & 13.31 & 9.41 & 3.45 & 180 & 582 & 186 & 0 & 12.66 & 8.90 & 4.10 & \\
\hline 735 & 1/18/94 20:35 & 217 & 577 & 380 & 2 & 12.69 & 5.75 & 221 & 122 & 393 & -2 & 13.06 & 9.17 & 3.65 & 192 & 441 & 163 & 2 & 12.53 & 8.77 & 4.60 & \\
\hline 735 & $1 / 18 / 9421: 20$ & 221 & 506 & 370 & 1 & 13.02 & 5.65 & 220 & 139 & 385 & -1 & 13.03 & 9.14 & 3.65 & 205 & 583 & 270 & 1 & 12.54 & 8.75 & 4.55 & BH cln 20:45-21:20 \\
\hline 735 & $1 / 18 / 9422: 20$ & 222 & 617 & 378 & 1 & 12.77 & 5.70 & 220 & 180 & 385 & -1 & 13.22 & 9.31 & 3.50 & 190 & 347 & 186 & 3 & 12.26 & 8.58 & 4.65 & \\
\hline 735 & 1/18/94 23:15 & 225 & 559 & 360 & 1 & 12.79 & 6.10 & 225 & 103 & 375 & -1 & 12.89 & 9.07 & 3.85 & 189 & 139 & 160 & 5 & 12.46 & 8.70 & 4.70 & NOTE: $1 \%$ Econ \\
\hline 735 & $1 / 19 / 940: 05$ & 220 & 760 & 365 & 1 & 12.97 & 5.85 & 230 & 89 & 365 & -1 & 12.99 & 8.99 & 4.00 & 196 & 400 & 158 & 6 & 12.34 & 8.49 & 4.80 & eak From \\
\hline 735 & $1 / 19 / 940: 35$ & 215 & 523 & 360 & 1 & 12.49 & 5.75 & 220 & 90 & 373 & -2 & 12.92 & 8.98 & 3.70 & 195 & 324 & 165 & 6 & 12.18 & 8.46 & 4.80 & \\
\hline 735 & $1 / 19 / 941: 10$ & 225 & 658 & 370 & 2 & 12.49 & 5.75 & 226 & 86 & 365 & -2 & 12.84 & 8.91 & 3.90 & 203 & 407 & 280 & 0 & 12.23 & 8.44 & 4.60 & $\mathrm{BH} \mathrm{cln}$ \\
\hline 735 & $1 / 19 / 944: 35$ & 215 & 553 & 360 & 3 & 12.85 & 5.85 & 220 & 65 & 368 & -2 & 12.76 & 8.89 & 4.00 & 195 & & 214 & & & & 4.60 & \\
\hline 735 & $1 / 19 / 945: 15$ & 215 & 323 & 355 & 2 & 12.44 & 5.95 & 225 & 73 & 369 & -2 & 12.64 & 8.81 & 3.95 & 208 & 276 & 297 & 1 & 12.23 & 8.34 & 4.80 & \\
\hline 735 & $1 / 19 /$ & 210 & 538 & 372 & 2 & 12.85 & 5.50 & 219 & 81 & 370 & -1 & 12.79 & 8.82 & 3.85 & 199 & 426 & 303 & 1 & 12.34 & 8.54 & 4.50 & \\
\hline 735 & $1 / 19$ & 215 & 491 & 373 & 1 & 12.86 & 5.60 & 218 & 93 & 380 & -1 & 12.88 & 8.94 & 3.80 & 193 & 501 & 300 & 0 & 12.40 & 8.55 & 4.40 & \\
\hline 735 & $1 / 19 /$ & 215 & 711 & 372 & 1 & 12.90 & 5.65 & 217 & 119 & 380 & -2 & 12.89 & 8.91 & 3.85 & 197 & 517 & 297 & 0 & 12.38 & 8.48 & 4.45 & \\
\hline 735 & $1 / 19$ & 212 & 850 & 380 & 1 & 13.02 & 5.30 & 218 & 123 & 380 & -2 & 12.98 & 8.96 & 3.80 & 203 & 599 & 330 & 0 & 12.42 & 8.56 & 4.40 & \\
\hline 735 & $1 / 19$ & 210 & 706 & 373 & 1 & 12.75 & 5.70 & 218 & 101 & 380 & -2 & 12.74 & 8.74 & 3.90 & 195 & 506 & 301 & 0 & 12.47 & 8.58 & 4.35 & \\
\hline 735 & $1 / 19$ & 210 & 769 & 380 & 1 & 13.08 & 5.40 & 217 & 85 & 378 & -2 & 12.74 & 8.67 & 4.00 & 195 & 487 & 270 & 0 & 12.24 & 8.35 & 4.65 & \\
\hline 735 & $1 / 19 /$ & 218 & 416 & 378 & 1 & 12.93 & 5.85 & 212 & 130 & 388 & -2 & 13.15 & 9.09 & 3.65 & 192 & 318 & 225 & 0 & 12.57 & 8.60 & 4.60 & $\mathrm{BH}$ \\
\hline 735 & $1 / 19 /$ & 217 & 273 & 372 & 1 & 12.70 & 6.10 & 212 & 73 & 380 & -2 & 12.75 & 8.92 & 4.10 & 197 & 241 & 260 & 1 & 12.46 & 8.64 & 4.65 & \\
\hline 735 & $1 / 19 /$ & 210 & 245 & 378 & 1 & 12.79 & 6.00 & 220 & 66 & 382 & -1 & 12.99 & 9.03 & 3.95 & 190 & 194 & 195 & 2 & 12.32 & 8.53 & 4.80 & \\
\hline 735 & $1 / 19 /$ & 213 & 424 & 377 & 1 & 12.99 & 5.75 & 217 & 76 & 382 & -1 & 12.83 & 8.98 & 4.00 & 182 & 249 & 160 & 4 & 12.59 & 8.69 & 4.60 & \\
\hline 735 & $1 / 19 /$ & 215 & 488 & 380 & 1 & 12.94 & 5.65 & 220 & 73 & 380 & -2 & 13.02 & 9.06 & 3.80 & 180 & 296 & 135 & 6 & 12.42 & 8.62 & 4.55 & Econ \\
\hline 735 & $1 / 19 /$ & 220 & 382 & 372 & 1 & 12.80 & 6.10 & 213 & 101 & 390 & -1 & 13.02 & 9.13 & 3.70 & 180 & 367 & 121 & 6 & 12.47 & 8.62 & 4.65 & \\
\hline 735 & $1 / 19 /$ & 220 & 382 & 372 & 1 & 12.80 & 6.10 & 215 & 88 & 385 & -2 & 12.82 & 8.99 & 4.00 & 173 & 299 & 107 & 5 & 12.51 & 8.68 & 4.60 & \\
\hline 735 & $1 / 19 / 9416: 00$ & 220 & 382 & 372 & 1 & 12.80 & 6.10 & 212 & 95 & 382 & -1 & 12.95 & 9.07 & 3.85 & 178 & 302 & 102 & 6 & 12.53 & 8.69 & 4.55 & \\
\hline 735 & $1 / 19 / 9416: 10$ & 218 & 467 & 380 & 1 & 12.97 & 5.80 & 218 & 91 & 387 & -1 & 12.96 & 9.07 & 3.80 & 178 & 277 & 102 & 6 & 12.47 & 3.60 & 4.70 & \\
\hline 735 & $1 / 19 / 9417: 00$ & 220 & 443 & 373 & 1 & 12.65 & 6.15 & 215 & 107 & 385 & -1 & 12.84 & 9.02 & 3.95 & 182 & 293 & 167 & 10 & 12.33 & 8.55 & 4.65 & \\
\hline 735 & $1 / 19 / 9418: 40$ & 222 & 261 & 360 & 2 & 12.63 & 6.35 & 220 & 122 & 380 & -1 & 13.02 & 9.17 & 3.90 & 190 & 280 & 160 & 3 & 12.35 & 8.55 & 4.70 & \\
\hline 735 & $1 / 19 / 9419: 40$ & 220 & 255 & 375 & 2 & 12.93 & 5.90 & 225 & 68 & 386 & -1 & 13.06 & 9.13 & 3.75 & 185 & 200 & 132 & 4 & 12.35 & 8.61 & 4.60 & \\
\hline 735 & $1 / 19 / 9420: 35$ & 220 & 356 & 372 & 1 & 13.11 & 5.80 & 220 & 104 & 378 & -1 & 13.12 & 9.27 & 3.70 & 190 & 284 & 125 & 4 & 12.48 & 8.79 & 4.70 & \\
\hline 736 & $1 / 19$ & 205 & 229 & 369 & 2 & 12.68 & 6.25 & 200 & 68 & 360 & -1 & 12.55 & 8.99 & 4.10 & 195 & 198 & 230 & 4 & 12.12 & 8.52 & 5.40 & $\mathrm{BH} \operatorname{cin} 20: 3$ \\
\hline 736 & $1 / 19 /$ & 207 & 274 & 369 & 1 & 12.92 & 6.05 & 200 & 56 & 369 & -1 & 12.78 & 9.06 & 4.20 & 162 & 278 & 140 & 10 & 12.35 & 8.72 & 4.90 & \\
\hline 736 & $1 / 19 / 9$ & 197 & 354 & 370 & 2 & 13.01 & 5.90 & 184 & 133 & 365 & -1 & 12.95 & 9.22 & 3.80 & 165 & 133 & 125 & 9 & 12.15 & 8.59 & 5.00 & \\
\hline 736 & $1 / 20 / 940: 10$ & 201 & 310 & 368 & 2 & 13.16 & 6.00 & 207 & 84 & 369 & -1 & 13.04 & 9.29 & 3.90 & 173 & 182 & 175 & 4 & 12.33 & 8.76 & 4.85 & \\
\hline 736 & $1 / 20 / 941: 10$ & 201 & 210 & 365 & 2 & 12.85 & 6.15 & 200 & 108 & 369 & -1 & 13.12 & 9.26 & 3.65 & 169 & 189 & 159 & 5 & 12.44 & 8.74 & 4.80 & \\
\hline 736 & $1 / 20 / 942: 10$ & 199 & 296 & 365 & 2 & 13.13 & 5.90 & 205 & 84 & 360 & -1 & 12.82 & 9.08 & 4.15 & 171 & 214 & 162 & 4 & 12.54 & 8.83 & 4.90 & \\
\hline 736 & $1 / 20 / 943: 10$ & 205 & 501 & 380 & 2 & 13.89 & 5.00. & 194 & 125 & 362 & -2 & 13.02 & 9.16 & 3.80 & 175 & 241 & 200 & 3 & 12.42 & 8.69 & 4.85 & O2 Leak Fixed \\
\hline 736 & $1 / 20 / 943: 55$ & 215 & 260 & 375 & 2 & 13.55 & 5.25 & 196 & 73 & 355 & -1 & 12.78 & 8.95 & 4.10 & 180 & 173 & 246 & 4 & 12.26 & 8.57 & 5.05 & \\
\hline 736 & $1 / 20 / 945: 10$ & 215 & 200 & 375 & 2 & 13.73 & 5.10 & 190 & 119 & 360 & -1 & 13.07 & 9.18 & 4.05 & 172 & 152 & 190 & 7 & 12.32 & 8.58 & 4.95 & \\
\hline 736 & $1 / 20 / 946: 10$ & 221 & 223 & 372 & 2 & 13.50 & 5.30 & 202 & 88 & 358 & -1 & 12.88 & 9.05 & 4.05 & 169 & 209 & 175 & 9 & 12.16 & 8.55 & 5.00 & \\
\hline 736 & $1 / 20 / 946: 50$ & 220 & 167 & 381 & 2 & 13.64 & 5.15 & 205 & 75 & 360 & -1 & 12.88 & 9.16 & 4.20 & 220 & 213 & 174 & 9 & 12.35 & 8.68 & 5.15 & \\
\hline 737 & $1 / 20 / 947: 50$ & 240 & 87 & 380 & 2 & 13.79 & 5.30 & 225 & 48 & 355 & -1 & 12.83 & 9.01 & 4.35 & 195 & 124 & 177 & 10 & 12.20 & 8.55 & 5.25 & \\
\hline
\end{tabular}


PSCC Arapahoe Unit 4 Sodjum Injection Summary

Calcs based on: Sodium Bicarbonate (b)

$.274 \mathrm{Nawt}$

Sodium Sesquicarbonate (s) $\quad .297 \mathrm{Nawt}$

\begin{tabular}{|c|c|c|c|c|c|c|c|c|c|c|c|c|c|c|c|c|c|c|c|c|c|c|}
\hline \multirow{3}{*}{\multicolumn{2}{|c|}{ Test Date \& Time }} & & \multicolumn{3}{|c|}{ Sorbent Feed Injector cal } & \multirow{2}{*}{\multicolumn{3}{|c|}{$\begin{array}{l}\triangle \mathrm{SO} 2 \text { Reduction } \\
\mathrm{Cmp} \mathrm{Calc}\end{array}$}} & \multirow{3}{*}{\multicolumn{2}{|c|}{$\Delta \mathrm{NO} \cdots \cdots \Delta \mathrm{NO} 2 \Delta \mathrm{NO} \frac{\Delta \mathrm{NQ}}{\Delta \mathrm{SO} 2}$}} & \multicolumn{8}{|c|}{ Economizer NO Calc - - Humidification } & \multicolumn{4}{|c|}{ Baghouse Temps } \\
\hline & & Load $\mathrm{O} 2 \mathrm{cr}$ & $\mathrm{NaA}(w) B(e)$ & Flow & $2 \mathrm{Na}$ & & & & & & $\triangle N O X$ & $\triangle N O$ & $\triangle N O 2 i$ & $\Delta N Q$ & Air & Taho & $T w$ & $\mathrm{H} 2 \mathrm{O}$ & Grid & Out & & in Opsis \\
\hline & & $M W_{\theta} \%$ wet & $b / s$ & $\mathrm{lb} / \mathrm{min}$ & $s^{*}$ & $\%$ & $\%$ & ppme & & & $\%$ & ppme & ppme & $\triangle \mathrm{SO} 2$ & scfm & ${ }^{\circ} \mathrm{F}$ & ${ }^{\circ} \mathrm{F}$ & gpm & ${ }^{\circ} \mathrm{F}$ & ${ }^{\circ} \mathrm{F}$ & & ${ }^{\circ} \mathrm{F}$ \\
\hline
\end{tabular}

$7371 / 20 / 9411: 10111420$ b 29

737 1/20/94 11:50 1114.20 b 28

$737 \quad 1 / 20 / 9413: 00 \quad 1114.40$ b 28

$\begin{array}{lllll}5.4 & 24 & 13.1 & 0.18 & 0 \\ 0.4 & 27 & 12.2 & 0.19 & 0\end{array}$

\begin{tabular}{llllllllllllllll}
\hline & 0 & 33.1 & 0.84 & 38 & 38.5 & 154 & 1.0 & 3 & 10.7 & 13 & 0.09 & 6.7 & 27 & 10.7 & 0.17
\end{tabular}

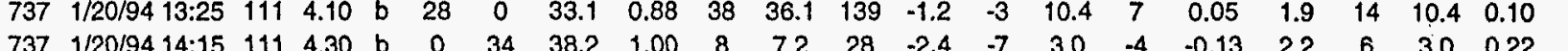

$\begin{array}{lllllllllllllllllllllll}737 & 1 / 20 / 94 & 15: 40 & 111 & 4.20 & b & 0 & 34 & 38.2 & 1.00 & 25 & 28.0 & 109 & -3.3 & -9 & 5.4 & -4 & -0.03 & 6.4 & 21 & 5.4 & 0.19 & 0\end{array}$

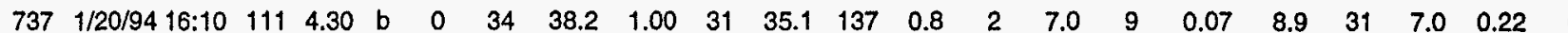

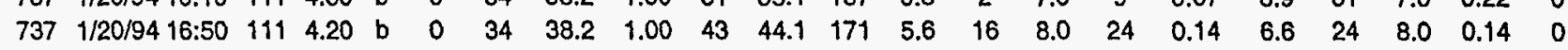

$\begin{array}{lllllllllllllllllllllll}737 & 1 / 20 / 94 & 18: 40 & 111 & 4.20 & \text { b } & 0 & 34 & 38.2 & 1.02 & 57 & 58.3 & 222 & 27.3 & 76 & 14.4 & 90 & 0.41 & 27.1 & 86 & 14.4 & 0.39 & 0\end{array}$

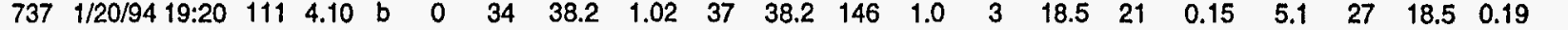

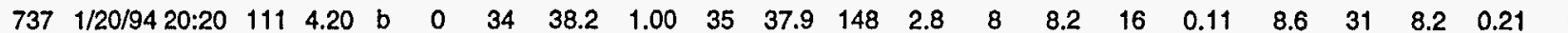

$\begin{array}{lllllllllllllllllllllll}737 & 1 / 20 / 94 & 21: 20 & 111 & 3.90 & b & 0 & 34 & 38.2 & 1.01 & 47 & 49.4 & 190 & 3.7 & 9 & 8.7 & 18 & 0.10 & 7.0 & 25 & 8.7 & 0.13 & 0\end{array}$

$\begin{array}{lllllllllllllllllllllll}737 & 1 / 20 / 94 & 22: 20 & 111 & 3.70 & b & 0 & 34 & 38.2 & 1.02 & 52 & 53.3 & 203 & 4.7 & 12 & 10.1 & 22 & 0.11 & 8.3 & 28 & 10.1 & 0.14 & 0\end{array}$

$\begin{array}{lllllllllllllllllllllll}737 & 1 / 20 / 94 & 23: 20 & 111 & 3.70 & \text { b } & 0 & 34 & 38.2 & 1.03 & 57 & 55.5 & 209 & 7.1 & 18 & 7.8 & 26 & 0.12 & 9.4 & 29 & 7.8 & 0.14 & 0\end{array}$

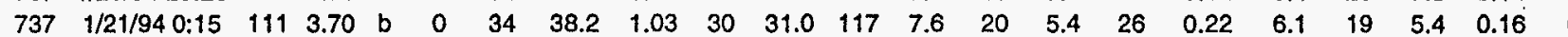

$\begin{array}{llllllllllllllllllllllll}737 & 1 / 21 / 94 & 1: 15 & 111 & 3.60 & \text { b } & 0 & 34 & 38.2 & 1.05 & 46 & 47.5 & 176 & 5.8 & 15 & 10.4 & 25 & 0.14 & 7.7 & 29 & 10.4 & 0.16 & 0\end{array}$

$737 \quad 1 / 21 / 942: 15 \quad 1113.70$ b

$\begin{array}{llllllllllllllll}37.1 & 1.02 & 51 & 50.0 & 186 & 4.4 & 11 & 9.2 & 21 & 0.11 & 2.7 & 13 & 9.2 & 0.07 & 0\end{array}$

$\begin{array}{llllll}7 / 21 / 94 & 3: 15 & 111 & 3.80 & b & 0\end{array}$

$737 \quad 1 / 21 / 944: 05 \quad 1113.70 \quad b \quad 0$

$737 \quad 1 / 21 / 944: 40 \quad 1113.70$ b

$7371 / 21 / 945: 40 \quad 1113.70$ b $\quad 0$

$737 \quad 1 / 21 / 947: 00 \quad 111 \quad 3.80$ b

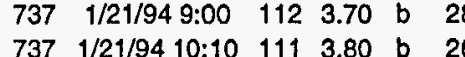

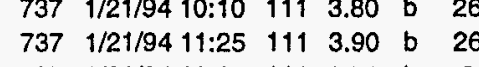

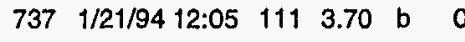

$737 \quad 1 / 21 / 9413: 05 \quad 1113.70$ b

$737 \quad 1 / 21 / 9414: 05 \quad 111 \quad 3.60 \quad b \quad 0$

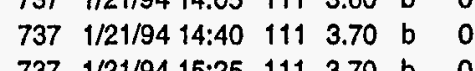

$\begin{array}{lllllllllllllll}37.1 & 1.03 & 53 & 52.3 & 192 & 4.0 & 10 & 8.8 & 19 & 0.10 & 3.5 & 14 & 8.8 & 0.07 & 0.4\end{array}$

$\begin{array}{llllllllllllllll}33 & 37.1 & 1.02 & 53 & 51.4 & 190 & 6.1 & 16 & 6.4 & 22 & 0.12 & 3.4 & 12 & 6.4 & 0.06 & 0\end{array}$

$\begin{array}{cccccccccccccccc}33 & 37.1 & 1.01 & 33 & 32.9 & 123 & 1.7 & 4 & 6.0 & 10 & 0.08 & 2.0 & 7 & 6.0 & 0.06 & 0\end{array}$

$\begin{array}{llllllllllllllll}33 & 37.1 & 1.01 & 33 & 35.5 & 132 & 2.4 & 6 & 5.1 & 11 & 0.08 & 4.6 & 14 & 5.1 & 0.11 & 0\end{array}$

$\begin{array}{lllllllllllllll}0 & 33.1 & 0.86 & 15 & 17.9 & 69 & 2.0 & 5 & 2.2 & 7 & 0.10 & 1.3 & 2 & 2.2 & 0.02\end{array}$

$\begin{array}{lllllllllllllll}0 & 30.7 & 0.82 & 20 & 22.1 & 84 & 0.6 & 1 & 3.2 & 5 & 0.06 & 2.2 & 5 & 3.2 & 0.06\end{array}$

$\begin{array}{lllllllllllllll}30.7 & 0.83 & 25 & 34.9 & 132 & 14.2 & 37 & 1.1 & 38 & 0.29 & 17.0 & 44 & 1.1 & 0.33 & 0\end{array}$

$\begin{array}{lllllllllllllllll}3 & 37.1 & 1.01 & 22 & 21.4 & 80 & 0.8 & 2 & 3.7 & 6 & 0.07 & 3.9 & 10 & 3.7 & 0.13 & 0\end{array}$

$\begin{array}{lllllllllllllllll}37.1 & 1.02 & 43 & 46.7 & 173 & 6.1 & 16 & 6.5 & 22 & 0.13 & 7.7 & 24 & 6.5 & 0.14 & 0\end{array}$

$21 / 9415: 25 \quad 1113.70$ b

$\begin{array}{lllllllllllllll}37.1 & 1.04 & 66 & 68.5 & 249 & 5.4 & 13 & 12.3 & 26 & 0.10 & 10.1 & 34 & 12.3 & 0.14 \\ 35.9 & 1.02 & 75 & 76.6 & 275 & 6.4 & 16 & 13.7 & 29 & 0.11 & 8.8 & 33 & 13.7 & 0.12\end{array}$

$\begin{array}{llllllllllllll}35.9 & 1.01 & 81 & 80.2 & 290 & 15.6 & 41 & 14.2 & 55 & 0.19 & 13.9 & 47 & 14.2 & 0.16\end{array}$

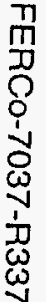

see NaSum763 


\begin{tabular}{|c|c|c|c|c|c|c|c|c|c|c|c|c|c|c|c|c|c|c|c|c|c|c|}
\hline \multirow[b]{2}{*}{ Test } & \multirow[b]{2}{*}{ Date \& Time } & \multicolumn{6}{|c|}{ Economizer Exit, dry (1-12) } & \multicolumn{7}{|c|}{ Baghouse Inlet Gas Analysis, wet } & \multicolumn{7}{|c|}{ Stack Gas Analysis, wet } & \multirow[b]{2}{*}{ Comments } \\
\hline & & $\begin{array}{l}\text { NO } \\
\mathrm{ppm}\end{array}$ & $\begin{array}{l}\text { CO } \\
\text { ppm }\end{array}$ & $\begin{array}{l}\mathrm{SO} 2 \\
\mathrm{ppm}\end{array}$ & $\begin{array}{l}\mathrm{NO} 2 \\
\mathrm{ppm}\end{array}$ & $\begin{array}{c}\mathrm{CO} 2 \\
\%\end{array}$ & $\begin{array}{c}\text { O2 } \\
\% d r y\end{array}$ & $\begin{array}{l}\text { NO } \\
\mathrm{ppm}\end{array}$ & $\begin{array}{l}\mathrm{co} \\
\mathrm{ppm}\end{array}$ & $\begin{array}{l}\mathrm{SO} 2 \\
\mathrm{ppm}\end{array}$ & $\begin{array}{l}\mathrm{NO} 2 \\
\mathrm{ppm}\end{array}$ & $\begin{array}{c}\mathrm{CO} 2 \\
\%\end{array}$ & $\begin{array}{c}\mathrm{H} 2 \mathrm{O} \\
\% \\
\end{array}$ & $\begin{array}{l}\mathrm{O} 2 \\
\% \\
\end{array}$ & $\begin{array}{l}\text { NO } \\
\mathrm{ppm}\end{array}$ & $\begin{array}{l}\mathrm{CO} \\
\mathrm{ppm}\end{array}$ & $\begin{array}{l}\mathrm{SO} 2 \\
\mathrm{ppm}\end{array}$ & $\begin{array}{l}\mathrm{NO} 2 \\
\mathrm{ppm}\end{array}$ & $\begin{array}{c}\mathrm{CO} 2 \\
\%\end{array}$ & $\begin{array}{c}\mathrm{H} 2 \mathrm{O} \\
\%\end{array}$ & $\begin{array}{l}\mathrm{O} 2 \\
\%\end{array}$ & \\
\hline 737 & $1 / 20 / 9410: 40$ & 247 & 149 & 350 & 2 & 13.47 & 5.60 & 223 & 49 & 342 & -2 & 12.59 & 8.91 & 4.50 & 203 & 115 & 218 & 8 & 12.12 & 8.57 & 5.40 & BH $\operatorname{cln} 8: 45-9: 15$ \\
\hline 737 & 1/20/94 11:10 & 247 & 183 & 359 & 2 & 13.66 & 5.45 & 223 & 54 & 338 & .1 & 12.75 & 9.02 & 4.35 & 200 & 112 & 210 & 8 & 12.08 & 8.52 & 5.30 & \\
\hline 737 & $1 / 20 / 9411: 50$ & 227 & 96 & 352 & 2 & 13.48 & 5.65 & 227 & 50 & 330 & -1 & 12.72 & 9.10 & 4.40 & 205 & 77 & 200 & 9 & 11.86 & 8.40 & 5.60 & \\
\hline 737 & $1 / 20 / 9413: 00$ & 252 & 124 & 342 & 2 & 13.36 & 5.50 & 230 & 57 & 330 & -1 & 12.84 & 9.15 & 4.25 & 205 & 109 & 190 & 7 & 12.18 & 8.63 & 5.30 & \\
\hline 737 & $1 / 20 / 9413: 25$ & 250 & 117 & 340 & 2 & 13.47 & 5.40 & 233 & 46 & 318 & 0 & 12.87 & 9.17 & 4.20 & 210 & 103 & 188 & 8 & 12.07 & 8.54 & 5.45 & $\mathrm{BH} \mathrm{cl}$ \\
\hline 737 & $1 / 20 / 9414: 15$ & 248 & 165 & 337 & 2 & 13.70 & 5.20 & 225 & 84 & 320 & -1 & 12.70 & 9.08 & 4.25 & 215 & 141 & 280 & 1 & 12.13 & 8.57 & 5.20 & \\
\hline 737 & $1 / 20 / 9415: 00$ & 250 & 122 & 338 & 2 & 13.49 & 5.50 & 227 & 53 & 320 & -1 & 12.68 & 8.99 & 4.35 & 220 & 89 & 242 & 1 & 12.02 & 8.52 & 5.40 & \\
\hline 737 & $1 / 20 / 9415: 40$ & 252 & 94 & 330 & 1 & 13.40 & 5.60 & 222 & 67 & 320 & -1 & 12.78 & 9.04 & 4.30 & 210 & 94 & 215 & 3 & 11.99 & 8.48 & 5.40 & \\
\hline 737 & $1 / 20 / 9416: 10$ & 260 & 62 & 322 & 2 & 13.18 & 5.70 & 232 & 50 & 318 & -2 & 12.65 & 8.96 & 4.45 & 212 & 78 & 195 & 4 & 12.00 & 8.51 & 5.35 & \\
\hline 737 & $1 / 20 / 9416: 50$ & 243 & 131 & 338 & 2 & 13.54 & 5.40 & 232 & 47 & 321 & -1 & 12.82 & 9.08 & 4.20 & 199 & 80 & 168 & 6 & 12.09 & 8.58 & 5.25 & \\
\hline 737 & $1 / 20 / 9418: 40$ & 240 & 117 & 330 & 2 & 13.63 & 5.30 & 228 & 61 & 311 & -1 & 12.66 & 9.07 & 4.40 & 145 & 79 & 122 & 10 & 11.96 & 8.45 & 5.40 & \\
\hline 737 & $1 / 20 / 9419: 20$ & 245 & 140 & 338 & 2 & 13.84 & 5.30 & 225 & 55 & 312 & -3 & 12.27 & 8.84 & 4.40 & 195 & 150 & 181 & 12 & 12.35 & 8.82 & 5.30 & 19:20 \\
\hline 737 & $1 / 20 / 9420: 20$ & 251 & 170 & 339 & 2 & 13.64 & 5.40 & 222 & 39 & 315 & 0 & 12.43 & 9.03 & 4.55 & 201 & 126 & 188 & 7 & 12.10 & 8.60 & 5.20 & $\operatorname{Max}$ \\
\hline 737 & $1 / 20 / 9421: 20$ & 239 & 265 & 349 & 2 & 14.15 & 4.65 & 218 & 163 & 327 & 0 & 13.21 & 9.43 & 3.70 & 192 & 232 & 157 & 7 & 12.65 & 8.94 & 4.60 & \\
\hline 737 & 1/20/94 22:20 & 238 & 280 & 345 & 2 & 13.99 & 4.80 & 220 & 95 & 324 & -2 & 13.11 & 9.33 & 3.75 & 190 & 178 & 143 & 6 & 12.48 & 8.82 & 4.70 & \\
\hline 737 & 1/20/94 23:20 & 237 & 232 & 347 & 2 & 14.06 & 4.60 & 217 & 78 & 318 & -1 & 13.00 & 9.24 & 3.85 & 186 & 234 & 135 & 6 & 12.45 & 8.79 & 4.65 & \\
\hline 737 & $1 / 21 / 940: 15$ & 237 & 357 & 340 & 2 & 14.16 & 4.65 & 226 & 85 & 319 & 0 & 13.04 & 9.20 & 3.85 & 195 & 229 & 210 & 4 & 12.30 & 8.69 & 4.65 & $\mathrm{BH} \operatorname{cln} 23: 40-00: 15$ \\
\hline 737 & $41: 15$ & 240 & 270 & 338 & 2 & 13.94 & 4.70 & 223 & 71 & 315 & 0 & 13.04 & 9.16 & 3.75 & 190 & 162 & 156 & 8 & 12.44 & 8.73 & 4.70 & \\
\hline 737 & $42: 15$ & 230 & 601 & 339 & 2 & 14.45 & 4.45 & 219 & 110 & 316 & -1 & 13.19 & 9.23 & 3.75 & 190 & 297 & 149 & 7 & 12.62 & 8.87 & 4.70 & \\
\hline 737 & $43: 15$ & 230 & 545 & 339 & 2 & 14.06 & 4.50 & 217 & 115 & 311 & -2 & 13.03 & 9.17 & 3.80 & 191 & 225 & 141 & 6 & 12.50 & 8.74 & 4.65 & \\
\hline 737 & $1 / 21 /$ & 229 & 532 & 340 & 2 & 14.13 & 4.40 & 219 & 111 & 313 & -1 & 13.01 & 9.09 & 3.85 & 191 & 269 & 145 & 4 & 12.47 & 8.67 & 4.65 & \\
\hline 737 & $1 / 21 /$ & 242 & 315 & 336 & 1 & 13.95 & 4.55 & 215 & 111 & 315 & 0 & 13.26 & 9.21 & 4.00 & 203 & 345 & 240 & 2 & 12.70 & 8.78 & 4.65 & $\mathrm{BH} \operatorname{cin} 04: 05-04: 40$ \\
\hline 737 & $1 / 21 / s$ & 230 & 525 & 342 & 2 & 14.29 & 4.30 & 214 & 111 & 320 & -1 & 13.20 & 9.19 & 3.70 & 195 & 448 & 204 & 4 & 12.75 & 8.83 & 4.55 & \\
\hline 737 & $1 / 21 /$ & 235 & 464 & 340 & 2 & 14.17 & 4.55 & 218 & 136 & 320 & -1 & 13.28 & 9.24 & 3.60 & 197 & 459 & 195 & 4 & 12.64 & 8.75 & 4.55 & \\
\hline 737 & $49: 00$ & 230 & 553 & 352 & 2 & 14.19 & 4.45 & 212 & 147 & 320 & -1 & 12.76 & 8.98 & 4.20 & 200 & 371 & 255 & 1 & 12.36 & 8.67 & 4.70 & 07:50-08:25 \\
\hline 737 & $1 / 21 / 9$ & 238 & 505 & 240 & 2 & 14.05 & 4.65 & 218 & 108 & 317 & -1 & 12.88 & 9.03 & 4.10 & 208 & 356 & 240 & 1 & 12.46 & 8.70 & 4.60 & BagCal @10:30 \\
\hline 737 & $1 / 21 / 9411: 25$ & 240 & 433 & 330 & 2 & 14.04 & 4.80 & 218 & 132 & 315 & -1 & 13.23 & 9.29 & 4.00 & 200 & & 220 & & & & 4.85 & gave $29.3 \mathrm{lb} / \mathrm{min} \mid$ \\
\hline 737 & 1/21/94 12:05 & 240 & 442 & 337 & 2 & 14.12 & 4.80 & 220 & 62 & 315 & -1 & 12.88 & 9.04 & 4.00 & 205 & 272 & 236 & 2 & 12.34 & 8.62 & 4.80 & \\
\hline 737 & $1 / 21 / 9413: 05$ & 235 & 367 & 339 & 2 & 14.08 & 4.60 & 218 & 99 & 315 & -1 & 13.08 & 9.24 & 3.75 & 190 & 378 & 160 & 5 & 12.59 & 8.82 & 4.55 & BagCal $=40.31 \mathrm{~b} / \mathrm{min}$ \\
\hline 737 & $1 / 21 / 9414: 05$ & 230 & 573 & 335 & 2 & 14.06 & 4.70 & 210 & 213 & 311 & -2 & 12.91 & 9.13 & 3.70 & 177 & 409 & 92 & 8 & 12.43 & 8.73 & 4.70 & \\
\hline 737 & $1 / 21 / 9414: 40$ & 230 & 544 & 335 & 2 & 14.25 & 4.40 & 213 & 156 & 311 & $\cdot 1$ & 13.06 & 9.19 & 3.50 & 175 & 326 & 68 & 10 & 12.44 & 8.69 & 4.65 & \\
\hline 737 & $1 / 21 / 9415: 25$ & 235 & 549 & 335 & 2 & 14.18 & 4.50 & 222 & 64 & 303 & -1 & 12.85 & 9.07 & 4.00 & 170 & 389 & 58 & 10 & 12.58 & 8.78 & 4.55 & $\mathrm{BH}$ cln 15:35-16:05 \\
\hline 737 & $\begin{array}{c}\text { 1/21/94 16:10 } \\
\text { see NaSum763 }\end{array}$ & 238 & 324 & 330 & 1 & 14.04 & 4.75 & 218 & 121 & 313 & -2 & 12.88 & 9.03 & 3.85 & 187 & 310 & 120 & 13 & 12.18 & 8.53 & 4.75 & \\
\hline
\end{tabular}


PSCC Arapahoe Unit 4 Sodium Injection Summary

Calcs based on: Sodium Bicarbonate (b) $.274 \mathrm{Nawt}$

Sodium Sesquicarbonate (s) $\quad .297 \mathrm{Na}$ wt

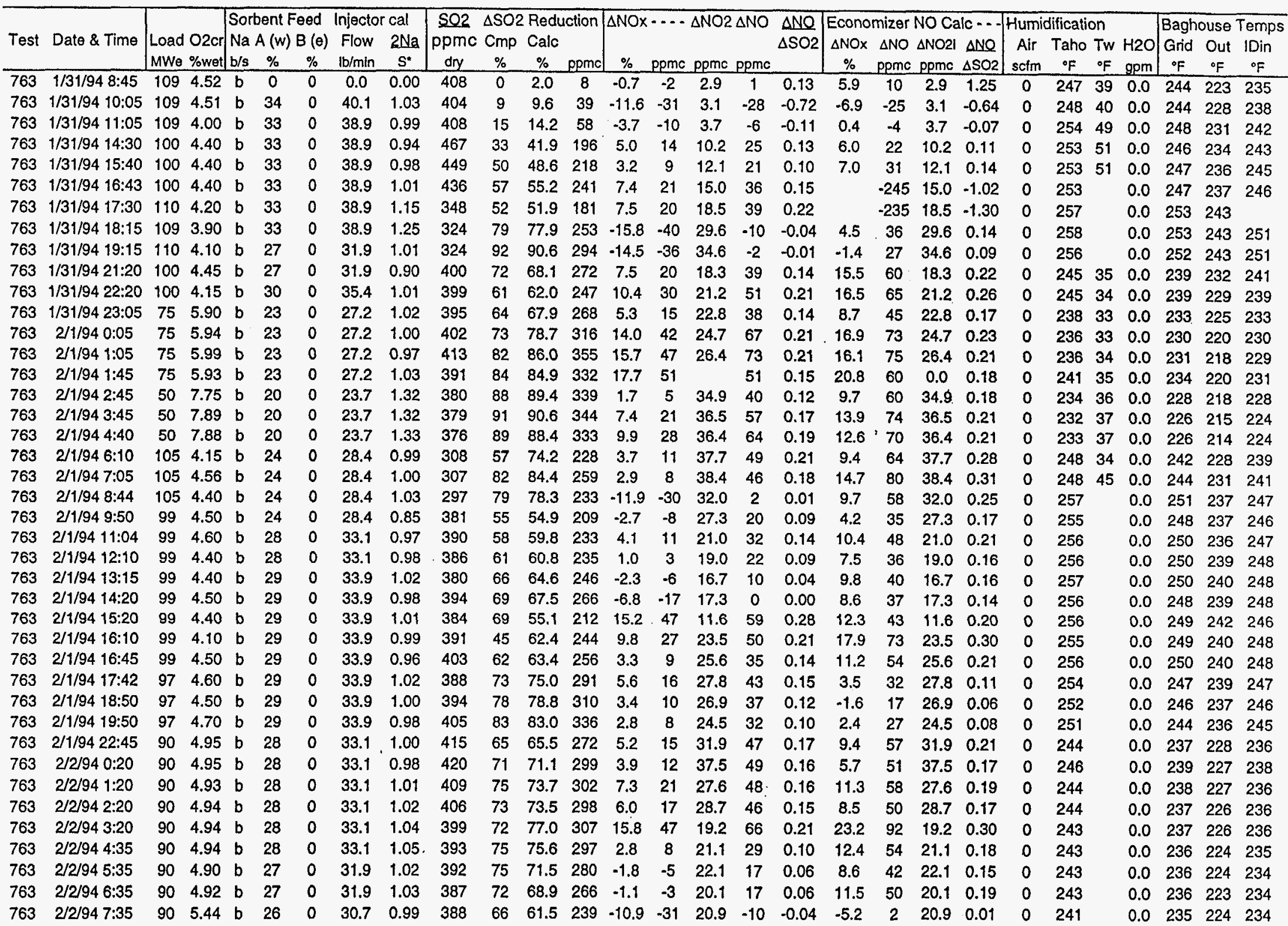




\begin{tabular}{|c|c|c|c|c|c|c|c|c|c|c|c|c|c|c|c|c|c|c|c|c|c|c|}
\hline Test & Date \& Time & $\begin{array}{c}\text { Econon } \\
\text { NO } \\
\text { ppm }\end{array}$ & $\begin{array}{c}\text { mizer } \\
\text { CO } \\
\text { ppm }\end{array}$ & $\begin{array}{l}\text { Exit, d } \\
\text { SO2 } \\
\text { ppm }\end{array}$ & $\begin{array}{l}y(1- \\
\text { NO2 } \\
\text { ppm }\end{array}$ & $\begin{array}{c}-12) \\
\mathrm{CO} 2 \\
\%\end{array}$ & $\begin{array}{c}02 \\
\% \mathrm{dry}\end{array}$ & $\begin{array}{r}\text { Baghou } \\
\text { NO } \\
\text { ppm }\end{array}$ & $\begin{array}{l}\text { use in } \\
\text { co } \\
\text { ppm }\end{array}$ & $\begin{array}{l}\text { let Gas } \\
\text { SO2 } \\
\text { ppm }\end{array}$ & $\begin{array}{l}s \text { Ana } \\
\text { NO2 } \\
\text { ppm }\end{array}$ & $\begin{array}{c}\text { alysis, } w \\
\mathrm{CO} 2 \\
\%\end{array}$ & $\begin{array}{c}\text { Het } \\
\%\end{array}$ & $\begin{array}{c}02 \\
\%\end{array}$ & $\begin{array}{c}\text { Stack G } \\
\text { NO } \\
\mathrm{ppm}\end{array}$ & $\begin{array}{c}\text { Gas } A_{1} \\
\text { CO } \\
\text { ppm }\end{array}$ & $\begin{array}{c}\text { nalysis } \\
\text { SO2 } \\
\text { ppm }\end{array}$ & $\begin{array}{l}\text { s, wet } \\
\text { NO2 } \\
\text { ppm }\end{array}$ & $\begin{array}{c}\mathrm{CO} 2 \\
\%\end{array}$ & $\begin{array}{c}\mathrm{H} 2 \mathrm{O} \\
\%\end{array}$ & $\begin{array}{c}02 \\
\%\end{array}$ & Comments \\
\hline 763 & $1 / 31 / 948: 45$ & 250 & 46 & 335 & 5 & 12.62 & 5.00 & 220 & 23 & 330 & -4 & 11.34 & 10.74 & 4.20 & 210 & 40 & 310 & -1 & 11.06 & 11.01 & 4.75 & AMill OOS,Bmrs \\
\hline 763 & $1 / 31 / 94 \quad 10: 05$ & 242 & 37 & 335 & 5 & 12.48 & 5.10 & 218 & 22 & 325 & .3 & 11.31 & 10.51 & 4.30 & 225 & 24 & 275 & 0 & 10.84 & 10.41 & 5.25 & 2,3,7 Firing Gas \\
\hline 763 & $1 / 31 / 9411: 05$ & 245 & 42 & 341 & 4 & 12.67 & 4.80 & 219 & 27 & 331 & -3 & 11.58 & 10.67 & 4.15 & 213 & & 270 & & & 11.00 & 4.80 & \\
\hline 763 & $1 / 31 / 9414: 30$ & 251 & 466 & 421 & 3 & 13.60 & 5.20 & 242 & 141 & 392 & -1 & 12.90 & 9.04 & 4.00 & 207 & 164 & 213 & 7 & 12.10 & 8.48 & 5.10 & Nat. Ga \\
\hline 763 & $1 / 31 / 9415: 40$ & 260 & 310 & 421 & 2 & 14.12 & 4.90 & 243 & 302 & 388 & 0 & 13.15 & 9.30 & 3.50 & 204 & 197 & 181 & 9 & 12.20 & 8.50 & 5.10 & \\
\hline 763 & $1 / 31 / 9416: 43$ & & & & & & & 242 & 262 & 376 & -2 & 13.02 & 9.45 & 3.50 & 197 & 499 & 157 & 11 & 12.38 & 8.71 & 4.70 & $\mathrm{BH}$ cln 17:00. \\
\hline 763 & $1 / 31 / 9417: 30$ & & & & & & & 220 & 26 & 279 & -3 & 11.42 & 10.79 & 4.30 & 183 & 36 & 130 & 12 & 11.10 & 10.99 & 4.70 & \\
\hline 763 & $1 / 31 / 9418: 15$ & 273 & 34 & 280 & 2 & 12.55 & 4.90 & 208 & 25 & 261 & -4 & 11.60 & 10.99 & 4.20 & 203 & 20 & 54 & 19 & 10.80 & 10.50 & 5.20 & \\
\hline 763 & $1 / 31 / 9419: 15$ & 249 & 36 & 274 & 1 & 12.65 & 5.00 & 206 & 30 & 266 & -2 & 11.65 & 11.16 & 3.90 & 191 & 20 & 23 & 25 & 10.75 & 10.49 & 5.20 & \\
\hline 763 & $1 / 31 / 9421: 20$ & 260 & 461 & 355 & 4 & 13.91 & 4.90 & 235 & 445 & 349 & 0 & 13.29 & 9.41 & 3.30 & 188 & 316 & 104: & 15 & 12.45 & 8.66 & 4.50 & Nat. Gas Off \\
\hline 763 & $1 / 31 / 9422: 20$ & 271 & 84 & 350 & 3 & 13.87 & 5.10 & 250 & 145 & 340 & -4 & 12.87 & 9.03 & 3.75 & 195 & 374 & 122 & 14 & 12.58 & 8.75 & 4.65 & \\
\hline 763 & $1 / 31 / 9423: 05$ & 240 & 64 & 314 & 2 & 12.57 & 6.60 & 230 & 40 & 310 & -1 & 12.10 & 8.43 & 5.10 & 181 & 34 & 90 & 15 & 11.17 & 7.69 & 6.60 & \\
\hline 763 & 2/1/94 0:05 & 244 & 40 & 310 & 2 & 12.48 & 6.70 & 230 & 53 & 308 & -1 & 12.39 & 8.62 & 5.40 & 169 & 40 & 62 & 17 & 11.31 & 7.78 & 6.35 & \\
\hline 763 & 2/1/94 1:05 & 240 & 45 & 320 & 2 & 12.68 & 6.55 & 230 & 54 & 320 & 2 & 12.32 & 8.61 & 5.25 & 162 & 50 & 42 & 21 & 11.40 & 7.87 & 6.30 & \\
\hline 763 & $2 / 1 / 941: 45$ & 239 & 39 & 317 & 2 & 12.64 & 6.60 & 229 & & 309 & & & 8.43 & 5.00 & 170 & & 42 & & & 7.69 & 6.55 & \\
\hline 763 & $2 / 1 / 942: 45$ & 197 & 24 & 250 & 2 & 10.15 & 9.40 & 190 & 25 & 253 & -2 & 10.36 & 7.19 & 7.50 & 146 & 21 & 24 & 19 & 9.41 & 6.44 & 8.95 & \\
\hline 763 & $2 / 1 / 943: 45$ & 198 & 23 & 250 & 2 & 10.29 & 9.20 & 189 & 19 & 251 & -2 & 10.27 & 7.10 & 7.60 & 135 & 19 & 21 & 20 & 9.37 & 6.44 & 9.00 & \\
\hline 763 & $2 / 1 / 944: 40$ & 191 & 25 & 248 & 1 & 10.34 & 9.20 & 190 & 19 & 250 & -1 & 10.32 & 7.12 & 7.55 & 132 & 19 & 26 & 21 & 9.41 & 6.47 & 8.92 & \\
\hline 763 & $2 / 1 / 946: 10$ & 270 & 36 & 251 & 3 & 12.24 & 5.60 & 241 & 33 & 247 & 0 & 11.57 & 10.53 & 4.35 & 190 & 27 & 60 & 28 & 10.55 & 10.02 & 5.30 & Nat. \\
\hline 763 & $2 / 1 / 947: 05$ & 270 & 42 & 250 & 2 & 12.30 & 5.55 & 225 & 38 & 246 & -2 & 11.39 & 10.39 & 4.40 & 201 & 22 & 41 & 31 & 10.44 & 9.84 & 3.50 & \\
\hline 763 & $2 / 1 / 948: 44$ & 270 & 42 & 250 & 2 & 12.30 & 5.55 & 212 & 38 & 244 & -2 & 11.57 & 10.64 & 4.00 & 191 & 24 & 48 & 22 & 10.52 & 10.08 & 5.50 & \\
\hline 763 & $2 / 1 / 949: 50$ & 260 & 76 & 340 & 4 & 13.52 & 5.20 & 238 & 56 & 322 & -1 & 12.90 & 8.91 & 3.90 & 202 & 46 & 132 & 21 & 11.88 & 8.11 & 5.40 & \\
\hline 763 & $2 / 1 / 9411: 04$ & 260 & 76 & 340 & 4 & 13.52 & 5.20 & 238 & 97 & 331 & 1 & 13.16 & 9.11 & 3.80 & 196 & 121 & 124 & 17 & 12.21 & 8.39 & 5.00 & \\
\hline 763 & 2/1/94 12:10 & 260 & 76 & 340 & 4 & 13.52 & 5.20 & 238 & 97 & 326 & -1 & 12.97 & 8.96 & 3.90 & 203 & 65 & 118 & 14 & 11.97 & 8.26 & 5.20 & \\
\hline 763 & $2 / 1 / 94$ 13:15 & 260 & 76 & 340 & 4 & 13.52 & 5.20 & 228 & 109 & 325 & -2 & 12.93 & 9.15 & 3.70 & 196 & 86 & 103 & 11 & 11.88 & 8.29 & 5.50 & \\
\hline 763 & $2 / 1 / 9414: 20$ & 260 & 76 & 340 & 4 & 13.52 & 5.20 & 224 & 155 & 340 & -1 & 13.22 & 9.28 & 3.50 & 205 & 106 & 101 & 12 & 12.19 & 8.45 & 5.00 & \\
\hline 763 & $2 / 1 / 94$ 15:20 & 260 & 76 & 340 & 4 & 13.52 & 5.20 & 253 & 40 & 312 & -1 & 12.43 & 9.06 & 4.50 & 201 & 125 & 137 & 9 & 12.18 & 8.57 & 4.90 & \\
\hline 763 & 2/1/94 16:10 & 260 & 76 & 340 & 4 & 13.52 & 5.20 & 233 & 380 & 333 & 0 & 13.13 & 9.34 & 3.70 & 176 & 311 & 116 & 19 & 12.37 & 8.60 & 5.00 & \\
\hline 763 & $2 / 1 / 9416: 45$ & 260 & 76 & 340 & 4 & 13.52 & 5.20 & 229 & 99 & 333 & -1 & 13.00 & 9.21 & 4.20 & 189 & 177 & 115 & 19 & 11.92 & 8.45 & 5.20 & \\
\hline 763 & $2 / 1 / 94 \quad 17: 42$ & 238 & 105 & 342 & 4 & 13.53 & 5.10 & 238 & 54 & 329 & 0 & 12.95 & 9.19 & 3.80 & 186 & 51 & 76 & 21 & 12.07 & 8.49 & 5.10 & \\
\hline 763 & $2 / 1 / 9418: 50$ & 238 & 105 & 342 & 4 & 13.53 & 5.10 & 239 & 38 & 326 & -1 & 12.63 & 9.06 & 4.20 & 195 & 40 & 65 & 20 & 11.97 & 8.47 & 5.30 & \\
\hline 763 & $2 / 1 / 9419: 50$ & 238 & 105 & 342 & 4 & 13.53 & 5.10 & 233 & 54 & 343 & 0 & 12.98 & 9.27 & 3.80 & 189 & 41 & 54 & 19 & 12.02 & 8.47 & 5.20 & \\
\hline 763 & $2 / 1 / 9422: 45$ & 248 & 43 & 341 & 3 & 12.84 & 6.10 & 230 & 47 & 330 & 0 & 12.56 & 8.95 & 4.80 & 181 & 43 & 107 & 24 & 11.42 & 8.11 & 5.85 & \\
\hline 763 & $2 / 2 / 940: 20$ & 248 & 48 & 349 & 3 & 13.14 & 5.95 & 235 & 45 & 335 & -1 & 12.44 & 8.83 & 4.80 & 180 & 34 & 89 & 27 & 11.64 & 8.20 & 6.10 & \\
\hline 763 & $2 / 2 / 941: 20$ & 246 & 47 & 348 & 2 & 13.10 & 5.85 & 229 & 45 & 332 & 0 & 12.44 & 8.80 & 4.55 & 176 & 42 & 81 & 21 & 11.67 & 8.16 & 5.75 & \\
\hline 763 & $2 / 2 / 942: 20$ & 246 & 61 & 345 & 2 & 13.49 & 5.85 & 230 & 50 & 324 & -1 & 12.36 & 8.76 & 4.80 & 180 & 36 & 80 & 20 & 11.42 & 8.00 & 5.95 & \\
\hline 763 & $2 / 2 / 943: 20$ & 260 & 44 & 325 & 3 & 12.80 & 6.45 & 231 & 309 & 312 & 0 & 11.86 & 8.46 & 5.15 & 175 & 81 & 70 & 15 & 12.09 & 8.47 & 5.50 & \\
\hline 763 & $2 / 2 / 944: 35$ & 245 & 60 & 325 & 2 & 12.17 & 6.10 & 221 & 66 & 321 & -2 & 12.27 & 8.61 & 4.50 & 182 & 46 & 72 & 14 & 11.44 & 7.96 & 5.80 & \\
\hline 763 & $2 / 2 / 945: 35$ & 245 & 49 & 327 & 2 & 13.01 & 6.15 & 222 & 47 & 320 & -3 & 12.21 & 8.56 & 4.50 & 185 & 37 & 81 & 14 & 11.43 & 7.96 & 6.25 & \\
\hline 763 & $2 / 2 / 946: 35$ & 250 & 45 & 322 & 3 & 12.39 & 6.25 & 222 & 65 & 315 & -2 & 12.11 & 8.49 & 4.55 & 189 & 35 & 89 & 13 & 11.20 & 7.75 & 6.05 & \\
\hline 763 & $2 / 2 / 947: 35$ & 246 & 44 & 331 & 2 & 12.85 & 6.00 & 226 & 32 & 310 & 0 & 11.86 & 8.20 & 4.90 & 204 & 26 & 104 & 14 & 10.93 & 7.44 & 6.90 & \\
\hline
\end{tabular}


PSCC Arapahoe Unit 4 Sodium Injection Summary

Calcs based on: Sodium Bicarbonate (b)

$.274 \mathrm{Nawt}$

Sodium Sesquicarbonate (s)

$.297 \mathrm{Na}$ wt

\begin{tabular}{|c|c|c|c|c|c|c|c|c|c|c|c|c|c|c|c|c|c|c|c|c|c|c|c|c|c|c|c|c|}
\hline \multirow{3}{*}{ Test } & \multirow{3}{*}{ Date \& Time } & \multirow{3}{*}{$\begin{array}{l}\text { Load } \\
\text { MWe }\end{array}$} & \multirow{3}{*}{$\begin{array}{l}\text { O2cr } \\
\text { \%wet }\end{array}$} & \multicolumn{5}{|c|}{ Sorbent Feed Injector cal } & \multirow{2}{*}{\multicolumn{4}{|c|}{$\begin{array}{l}\mathrm{SO} 2 \\
\text { ppmc } \mathrm{Cmp} \text { Calc Reduction }\end{array}$}} & \multirow{3}{*}{\multicolumn{5}{|c|}{$=\begin{array}{c}\Delta \mathrm{NOx} \cdots \cdots \\
\% \quad \text { ppme ppme ppme }\end{array}$}} & \multicolumn{8}{|c|}{ Economizer NO Calc - - - Humidification } & \multicolumn{3}{|c|}{ Baghouse Temps } \\
\hline & & & & & & & Flow & $2 \mathrm{Na}$ & & & & & & & & & & $\triangle N O x$ & & & $\triangle N Q$ & Air & & & $\mathrm{H} 2 \mathrm{O}$ & Grid & Out & IDin \\
\hline & & & & $\mathrm{b} / \mathrm{s}$ & $\%$ & $\%$ & & $\mathrm{~s}^{*}$ & $d r y$ & $\%$ & & ppme & & & & & & $\%$ & ppmc & ppme & $\triangle \mathrm{SO} 2$ & & ${ }^{\circ} \mathrm{F}$ & & gpm & $\circ F$ & ${ }^{\circ} \mathrm{F}$ & \\
\hline 63 & $2 / 2 / 948: 20$ & 90 & & $\mathrm{~b}$ & 26 & 0 & 30.7 & 0.98 & 392 & 69 & 69.0 & 271 & 10.1 & 28 & & 28 & 0.10 & 17.0 & 48 & 0.0 & 0.18 & 0 & 241 & & 0.0 & 235 & 224 & 234 \\
\hline 63 & $2 / 2 / 949: 50$ & 100 & 4.12 & b & 28 & 0 & 33.1 & 0.96 & 392 & 61 & 62.6 & 245 & -9.9 & -21 & 5.6 & -16 & -0.06 & 8.7 & 26 & 5.6 & 0.10 & 0 & 251 & & 0.0 & 244 & 229 & 239 \\
\hline 63 & $2 / 2 / 94$ 10:55 & 100 & 4.20 & b & 29 & 0 & 34.2 & 1.02 & 380 & 56 & 62.3 & 237 & -10.0 & -21 & 7.8 & -14 & -0.06 & 8.6 & 27 & 7.8 & 0.11 & 0 & 254 & & 0.0 & 248 & 233 & 243 \\
\hline 63 & 2/2/94 12:00 & 100 & 4.10 & b & 29 & 0 & 34.2 & 1.02 & 379 & 68 & 68.7 & 260 & 3.5 & 9 & 7.8 & 17 & 0.06 & 5.5 & 18 & 7.8 & 0.07 & 0 & 258 & & 0.0 & 252 & 239 & 248 \\
\hline 763 & $2 / 2 / 941$ & 100 & 4.10 & $b$ & 29 & 0 & 34.2 & 1.01 & 382 & 79 & 81.0 & 309 & 8.6 & 23 & 6.8 & 29 & 0.09 & 6.4 & 20 & 6.8 & 0.07 & 0 & 263 & & 0.0 & 255 & 242 & 252 \\
\hline 763 & 2/2/94 & 80 & 6.00 & $b$ & 29 & 0 & 34.2 & 1.28 & 372 & 90 & 90.4 & 336 & 19.4 & 64 & 12.0 & 76 & 0.23 & 13.2 & 47 & 12.0 & 0.14 & 0 & 260 & & 0.0 & 253 & 243 & 251 \\
\hline 763 & $2 / 2 / 9414: 00$ & 80 & 6.00 & b & 23 & 0 & 27.2 & 1.02 & 372 & & 90.4 & 336 & 19.4 & 64 & 12.0 & 76 & 0.23 & 13.2 & 47 & 12.0 & 0.14 & 0 & & & 0.0 & & & \\
\hline 763 & $2 / 2 / 94 \quad 15: 00$ & 80 & 6.00 & b & 24 & 0 & 28.4 & 1.06 & 375 & 78 & 77.6 & 291 & 4.6 & 13 & 12.6 & 25 & 0.09 & 14.8 & 51 & 12.6 & 0.18 & 0 & 256 & & 0.0 & 251 & 242 & 250 \\
\hline 763 & $2 / 2 / 94$ 16:00 & 80 & 6.00 & b & 24 & 0 & 28.4 & 1.05 & 377 & 81 & 78.9 & 297 & -2.0 & -5 & 9.9 & 5 & 0.02 & 10.7 & 36 & 9.9 & 0.12 & 0 & 252 & & 0.0 & 248 & 242 & 246 \\
\hline 763 & $2 / 2 / 94$ 17:00 & 80 & 6.00 & $b$ & 24 & 0 & 28.4 & 1.06 & 374 & 81 & 79.5 & 297 & 0.2 & 0 & 13.3 & 14 & 0.05 & 16.6 & 58 & 13.3 & 0.20 & 0 & 248 & & 0.0 & 243 & 237 & 243 \\
\hline 763 & $2 / 2 / 9418: 03$ & 80 & 6.00 & b & 24 & 0 & 28.4 & 1.06 & 375 & 75 & 74.7 & 280 & 3.3 & 9 & 15.7 & 25 & 0.09 & 12.7 & 48 & 15.7 & 0.17 & 0 & 246 & & 0.0 & 240 & 232 & 239 \\
\hline 763 & 2/2/94 19:07 & 80 & 6.00 & b & 24 & 0 & 28.4 & 1.06 & 372 & 82 & 84.9 & 316 & 2.8 & 8 & 18.4 & 26 & 0.08 & 17.3 & 68 & 18.4 & 0.21 & 0 & 244 & & 0.0 & 239 & 229 & 237 \\
\hline 763 & 2/2/94 19:55 & 90 & 4.92 & b & 24 & 0 & 28.4 & 0.95 & 374 & 59 & 64.7 & 242 & -6.1 & -14 & 12.9 & -2 & -0.01 & 10.2 & 37 & 12.9 & 0.15 & 0 & 247 & & 0.0 & 240 & 233 & 238 \\
\hline 763 & $2 / 2 / 9$ & 90 & 4.93 & b & 25 & 0 & 29.5 & 0.99 & 374 & 71 & 72.7 & 272 & -1.2 & -3 & 27.0 & 24 & 0.09 & 7.6 & 46 & 27.0 & 0.17 & 0 & 247 & & 0.0 & 241 & 231 & 239 \\
\hline 763 & $2 / 2 /$ & 90 & 4.92 & b & 25 & 0 & 29.5 & 0.98 & 379 & 74 & 74.2 & 281 & 2.4 & 6 & 21.9 & 28 & 0.10 & 10.7 & 49 & 21.9 & 0.17 & 0 & 245 & & 0.0 & 239 & 229 & 237 \\
\hline 763 & $2 / 2 / 5$ & 90 & 4.91 & b & 25 & 0 & 29.5 & 0.99 & 375 & 76 & 73.9 & 277 & 1.5 & 4 & 18.9 & 23 & 0.08 & 12.3 & 49 & 18.9 & 0.18 & 0 & 245 & & 0.0 & 237 & 226 & 234 \\
\hline 763 & 2/3/94 0:05 & 90 & 4.90 & b & 25 & 0 & 29.5 & 0.99 & 374 & 75 & 72.0 & 269 & 4.5 & 12 & 15.6 & 27 & 0.10 & 11.9 & 46 & $15.6^{\circ}$ & 0.17 & 0 & 244 & & 0.0 & 237 & 225 & 234 \\
\hline 763 & $2 / 3 / 941: 05$ & 90 & 4.89 & b & 25 & 0 & 29.5 & 0.99 & 375 & 72 & 71.5 & 268 & 1.2 & 3 & 15.3 & 18 & 0.07 & 6.6 & 30 & 15.3 & 0.11 & 0 & 243 & & 0.0 & 237 & 224 & 233 \\
\hline 763 & $2 / 3 / 4$ & 90 & 4.92 & b & 25 & 0 & 29.5 & 0.99 & 374 & 70 & 68.7 & 257 & 4.3 & 11 & 11.2 & 22 & 0.09 & 15.6 & 53 & 11.2 & 0.20 & 0 & 241 & & 0.0 & 235 & 222 & 231 \\
\hline 763 & $2 / 3 /$ & 90 & 4.92 & b & 25 & 0 & 29.5 & 0.99 & 372 & 69 & 71.0 & 264 & 2.3 & 6 & 12.4 & 18 & 0.07 & 13.8 & 49 & 12.4 & 0.19 & 0 & 239 & & 0.0 & 232 & 220 & 229 \\
\hline 763 & $2 / 3 / 5$ & 90 & 4.90 & b & 25 & 0 & 29.5 & 1.00 & 369 & 69 & 70.2 & 259 & 2.5 & 6 & 13.3 & 20 & 0.08 & 15.6 & 56 & 13.3 & 0.22 & 0 & 239 & & 0.0 & 233 & 220 & 229 \\
\hline 763 & $2 / 3 / 5$ & 90 & 4.88 & b & 25 & 0 & 29.5 & 1.00 & 371 & 75 & 74.5 & 276 & 0.0 & 0 & 13.2 & 13 & 0.05 & 10.5 & 41 & 13.2 & 0.15 & 0 & 239 & & 0.0 & 233 & 220 & 229 \\
\hline 763 & $2 / 3 / 5$ & 90 & 4.90 & b & 25 & 0 & 29.5 & 1.01 & 365 & 39 & 46.0 & 168 & -8.4 & -22 & 22.7 & 0 & 0.00 & 0.3 & 20 & 22.7 & 0.12 & 0 & 240 & & 0.0 & 233 & 220 & 228 \\
\hline 763 & $2 / 3 / 5$ & 90 & 4.82 & b & 25 & 0 & 29.5 & 1.02 & 364 & 62 & 62.5 & 227 & -0.3 & -1 & 27.3 & 27 & 0.12 & 8.0 & 48 & 27.3 & 0.21 & 0 & 239 & & 0.0 & 233 & 220 & 229 \\
\hline 763 & $8: 25$ & 90 & 4.90 & b & 25 & 0 & 29.5 & 1.01 & 367 & 70 & 70.7 & 259 & 1.1 & 3 & 22.5 & 25 & 0.10 & 11.5 & 49 & 22.5 & 0.19 & 0 & 240 & & 0.0 & 232 & 218 & 228 \\
\hline 763 & $2 / 3 / 9$ & 90 & 4.90 & b & 0 & 26 & 29.3 & 1.02 & 360 & 80 & 81.7 & 294 & 5.8 & 14 & 21.2 & 35 & 0.12 & 12.5 & 51 & 21.2 & 0.17 & 0 & 240 & & 0.0 & 234 & 221 & 229 \\
\hline 763 & $2 / 3 /$ & 90 & 5.40 & b & 0 & 26 & 29.3 & 1.00 & 365 & 83 & 83.1 & 304 & 3.7 & 10 & 26.7 & 37 & 0.12 & 9.4 & 50 & 26.7 & 0.17 & 0 & 252 & & 0.0 & 243 & 227 & 236 \\
\hline 763 & $2 / 3 /$ & 90 & 5.40 & b & 0 & 26 & 29.3 & 1.00 & 366 & 84 & 84.7 & 310 & 3.1 & 9 & 23.5 & 32 & 0.10 & 8.0 & 42 & 23.5 & 0.14 & 0 & 256 & & 0.0 & 249 & 234 & 243 \\
\hline 763 & 2/3/94 13 & 90 & 5.40 & b & 0 & 26 & 29.3 & 0.98 & 375 & 83 & 82.6 & 310 & 2.8 & 8 & 23.2 & 31 & 0.10 & 7.5 & 40 & 23.2 & 0.13 & 0 & 258 & & 0.0 & 253 & 239 & 247 \\
\hline 763 & $2 / 3 / 94$ 15:20 & 70 & 6.62 & b & 0 & 20 & 22.7 & 0.94 & 378 & 81 & 79.9 & 302 & -2.9 & -8 & 19.8 & 12 & 0.04 & 12.3 & 50 & 19.8 & 0.17 & 0 & 253 & & 0.0 & 248 & 241 & 246 \\
\hline 763 & $2 / 3 / 94$ 16:30 & 70 & 6.60 & b & 0 & 21 & 23.8 & 1.00 & 374 & 81 & 80.7 & 302 & 2.2 & 6 & 22.2 & 28 & 0.09 & 12.7 & 57 & 22.2 & 0.19 & 0 & 250 & & 0.0 & 245 & 236 & 242 \\
\hline 763 & 2/3/94 17:33 & 90 & 5.40 & b & 0 & 27 & 30.4 & 0.99 & 384 & 46 & 50.7 & 195 & -2.3 & -7 & 30.2 & 24 & 0.12 & 5.9 & 45 & 30.2 & 0.23 & 0 & 256 & & 0.0 & 248 & 240 & 244 \\
\hline 763 & $2 / 3 / 94 \quad 18: 45$ & 90 & 5.40 & b & 0 & 27 & 30.4 & 0.96 & 395 & 68 & 71.1 & 281 & 0.9 & 3 & 41.7 & 44 & 0.16 & 9.9 & 70 & 41.7 & 0.25 & 0 & 257 & & 0.0 & 250 & 239 & 246 \\
\hline 763 & 2/3/94 19:43 & 90 & 5.50 & b & 0 & 28 & 31.5 & 0.98 & 404 & 73 & 73.5 & 297 & 2.7 & 8 & 39.0 & 47 & 0.16 & 11.9 & 73 & 39.0 & 0.25 & 0 & 255 & & 0.0 & 249 & 238 & 246 \\
\hline 763 & 2/3/94 21:55 & 90 & 5.43 & $b$ & 0 & 28 & 31.5 & 0.97 & 409 & 75 & 74.0 & 303 & 4.3 & 12 & 28.9 & 41 & 0.14 & 11.4 & 56 & 28.9 & 0.18 & 0 & 249 & & 0.0 & 243 & 233 & 240 \\
\hline 763 & $2 / 3 / 9423: 05$ & 90 & 5.39 & b & 0 & 29 & 32.6 & 1.02 & 401 & 71 & 72.1 & 289 & 10.5 & 31 & 21.6 & 52 & 0.18 & & & & & 0 & 248 & & 0.0 & 242 & 231 & 239 \\
\hline 763 & $2 / 4 / 940: 05$ & 90 & 5.41 & b & 0 & 29 & 32.6 & 1.02 & 402 & 71 & 71.6 & 288 & 3.6 & 10 & 25.1 & 35 & 0.12 & & & & & 0 & 247 & & 0.0 & 240 & 229 & 237 \\
\hline 763 & $2 / 4 / 941: 05$ & 90 & 5.39 & b & 0 & 29 & 32.6 & 1.02 & 400 & 69 & 72.1 & 289 & 4.5 & 13 & 24.9 & 37 & 0.13 & 13.4 & 58 & 24.9 & 0.20 & 0 & 247 & & 0.0 & 240 & 228 & 237 \\
\hline 763 & 2/4/94 2:05 & 90 & 5.38 & b & 0 & 29 & 32.6 & 0.99 & 411 & 71 & 72.3 & 297 & -0.2 & -1 & 25.5 & 25 & 0.08 & & & & & 0 & 246 & & 0.0 & 239 & 227 & 237 \\
\hline 763 & $2 / 4 / 943: 05$ & 90 & 5.42 & b & 0 & 29 & 32.6 & 1.00 & 409 & 71 & 71.4 & 292 & 3.7 & 10 & 23.5 & 34 & 0.12 & & & & & 0 & 245 & & 0.0 & 238 & 226 & 235 \\
\hline 763 & $2 / 4 / 943: 45$ & 90 & 5.41 & $b$ & 0 & 29 & 32.6 & 1.00 & 409 & 46 & 45.5 & 186 & -4.3 & -12 & 29.0 & 17 & 0.09 & 4.5 & 38 & 29.0 & 0.20 & 0 & 246 & & 0.0 & 238 & 229 & 235 \\
\hline
\end{tabular}




\begin{tabular}{|c|c|c|c|c|c|c|c|c|c|c|c|c|c|c|c|c|c|c|c|c|c|c|}
\hline Test & Date \& Time & $\begin{array}{c}\text { Econom } \\
\text { NO } \\
\text { ppm }\end{array}$ & $\begin{array}{l}\text { nizer } \\
\mathrm{CO} \\
\mathrm{ppm} \\
\end{array}$ & $\begin{array}{l}\text { Exit, d } \\
\text { SO2 } \\
\text { ppm }\end{array}$ & $\begin{array}{l}\text { ly (1-1 } \\
\text { NO2 } \\
\text { ppm }\end{array}$ & $\begin{array}{c}\text { 12) } \\
\mathrm{CO} 2 \\
\% \\
\end{array}$ & $\begin{array}{c}\mathrm{O} 2 \\
\% \mathrm{dry}\end{array}$ & $\begin{array}{c}\text { Baghou } \\
\text { NO } \\
\text { ppm }\end{array}$ & $\begin{array}{l}\text { Jse In! } \\
\text { CO } \\
\mathrm{ppm} \\
\end{array}$ & $\begin{array}{l}\text { let } \mathrm{Ga} \\
\mathrm{SO} 2 \\
\mathrm{ppm}\end{array}$ & $\begin{array}{l}\text { s Anal } \\
\text { NO2 } \\
\text { ppm }\end{array}$ & $\begin{array}{c}\text { alysis, } w \\
\mathrm{CO} 2 \\
\%\end{array}$ & $\begin{array}{c}\mathrm{H} 2 \mathrm{O} \\
\% \\
\end{array}$ & $\begin{array}{c}\mathrm{O} 2 \\
\% \\
\end{array}$ & $\begin{array}{c}\text { Stack } G \\
\text { NO } \\
\text { ppm }\end{array}$ & $\begin{array}{l}\text { Gas } \mathrm{Ar} \\
\mathrm{CO} \\
\mathrm{ppm}\end{array}$ & $\begin{array}{l}\text { nalysis } \\
\mathrm{SO} 2 \\
\mathrm{ppm}\end{array}$ & $\begin{array}{l}\mathrm{s}_{1} \text { wet } \\
\mathrm{NO} 2 \\
\mathrm{ppm}\end{array}$ & $\begin{array}{c}\mathrm{CO} 2 \\
\%\end{array}$ & $\begin{array}{c}\mathrm{H} 2 \mathrm{O} \\
\% \\
\end{array}$ & $\begin{array}{c}\mathrm{O} 2 \\
\%\end{array}$ & Comments \\
\hline 763 & $2 / 2 / 948: 20$ & 246 & 44 & 331 & 2 & 12.85 & 6.00 & 221 & & 315 & & & 8.76 & 4.70 & 181 & & 89 & & & 8.20 & 6.10 & \\
\hline 763 & $2 / 2 / 949: 50$ & 225 & 472 & 345 & 3 & 13.26 & 5.10 & 194 & 680 & 356 & 1 & 13.33 & 9.50 & 2.65 & 180 & 403 & 115 & 5 & 11.80 & 8.37 & 5.10 & BHcln 1007-1042 \\
\hline 763 & $2 / 2 / 9410: 55$ & 225 & 472 & 345 & 3 & 13.26 & 5.10 & 195 & 829 & 346 & 0 & 13.30 & 9.58 & 2.60 & 180 & 488 & 113 & 6 & 11.86 & 8.44 & 5.00 & \\
\hline 763 & $2 / 2 / 9412: 00$ & 225 & 472 & 345 & 3 & 13.26 & 5.10 & 223 & 211 & 334 & 0 & 13.18 & 9.20 & 3.20 & 193 & 224 & $97:$ & 6 & 12.34 & 8.54 & 4.50 & \\
\hline 763 & $2 / 2 / 9413: 00$ & 225 & 472 & 345 & 3 & 13.26 & 5.10 & 228 & 166 & 330 & 0 & 13.12 & 9.25 & 3.50 & 191 & 346 & 59 & 6 & 12.37 & 8.74 & 4.50 & \\
\hline 763 & $2 / 2 / 9413: 48$ & 231 & 37 & 286 & 4 & 11.59 & 7.10 & 251 & 37 & 285 & 0 & 11.52 & 8.16 & 5.50 & 178 & 33 & 25 & 8 & 10.76 & 7.55 & 6.70 & \\
\hline 763 & $2 / 2 / 9414: 00$ & 231 & 37 & 286 & 4 & 11.59 & 7.10 & 251 & 37 & 285 & 0 & 11.52 & 8.16 & 5.50 & 178 & 33 & 25 & 8 & 10.76 & 7.55 & 6.70 & \\
\hline 763 & $2 / 2 / 9415: 00$ & 231 & 37 & 286 & 4 & 11.59 & 7.10 & 212 & 48 & 291 & -1 & 11.73 & 8.22 & 5.30 & 179 & 37 & 61 & 8 & 10.94 & 7.64 & 6.40 & \\
\hline 763 & $2 / 2 / 9416: 00$ & 231 & 37 & 286 & 4 & 11.59 & 7.10 & 208 & 41 & 293 & -1 & 11.98 & 8.54 & 5.20 & 182 & 36 & 55 & 6 & 10.95 & 7.77 & 6.90 & \\
\hline 763 & $2 / 2 / 9417: 00$ & 231 & 37 & 286 & 4 & 11.59 & 7.10 & 206 & 87 & 302 & -1 & 12.25 & 8.79 & 4.60 & 172 & 49 & 55 & 9 & 11.01 & 7.89 & 6.50 & \\
\hline 763 & $2 / 2 / 9418: 03$ & 231 & 37 & 286 & 4 & 11.59 & 7.10 & 213 & 35 & 290 & -1 & 11.73 & 8.49 & 5.30 & 174 & 28 & 66 & 10 & 10.68 & 7.67 & 6.90 & \\
\hline 763 & $2 / 2 / 9419: 07$ & 243 & 25 & 286 & 3 & 11.84 & 7.20 & 213 & 35 & 288 & -1 & 11.75 & 8.45 & 5.30 & 173 & 27 & 39 & 12 & 10.73 & 7.63 & 6.90 & \\
\hline 763 & $2 / 2 / 9419: 55$ & 235 & 64 & 323 & 2 & 13.21 & 5.60 & 200 & 151 & 317 & -1 & 12.82 & 9.29 & 3.80 & 180 & 71 & 100 & 9 & 11.40 & 8.16 & 5.65 & $\mathrm{BH}$ \\
\hline 763 & $2 / 2 / 9421: 05$ & 241 & 62 & 312 & 2 & 12.91 & 5.80 & 218 & 99 & 310 & 0 & 12.77 & 9.20 & 4.15 & 178 & 29 & 76 & 20 & 11.33 & 8.15 & 5.90 & \\
\hline 763 & $2 / 2 / 9422: 05$ & 241 & 62 & 324 & 2 & 13.37 & 5.45 & 216 & 94 & 317 & -1 & 12.96 & 9.24 & 4.00 & 174 & 59 & 74 & 16 & 11.67 & 8.31 & 5.65 & \\
\hline 763 & $2 / 2 / 9423: 05$ & 237 & 87 & 321 & 2 & 13.22 & 5.55 & 212 & 128 & 318 & -2 & 12.90 & 9.19 & 3.80 & 172 & 84 & 74 & 13 & 11.65 & 8.21 & 5.65 & \\
\hline 763 & $2 / 3 / 940: 05$ & 240 & 73 & 320 & 2 & 13.18 & 5.50 & 217 & 64 & 312 & -1 & 12.70 & 8.93 & 4.10 & 178 & 81 & 80 & 11 & 11.71 & 8.24 & 5.50 & \\
\hline 763 & 2/3/94 1:05 & 227 & 92 & 320 & 2 & 13.16 & 5.55 & 214 & 79 & 317 & -1 & 12.73 & 9.00 & 3.90 & 176 & 63 & 80 & 10 & 11.59 & 8.14 & 5.80 & \\
\hline 763 & 2/3/94 2:05 & 240 & 64 & 319 & 2 & 13.25 & 5.60 & 211 & 75 & 316 & -1 & 12.88 & 9.10 & 3.90 & 173 & 78 & 89 & 8 & 11.59 & 8.05 & 5.65 & \\
\hline 763 & $2 / 3 / 943: 05$ & 237 & 166 & 319 & 2 & 12.97 & 5.65 & 211 & 142 & 318 & 0 & 13.07 & 9.09 & 3.70 & 174 & 66 & 82 & 10 & 11.73 & 8.15 & 5.60 & \\
\hline 763 & $2 / 3 / 944: 05$ & 246 & 42 & 308 & 2 & 12.68 & 6.15 & 216 & 65 & 305 & -1 & 12.44 & 8.73 & 4.30 & 180 & 42 & 82 & 9 & 11.26 & 7.85 & 5.95 & \\
\hline 763 & $2 / 3 / 945: 05$ & 238 & 45 & 314 & 2 & 13.07 & 5.70 & 212 & 59 & 311 & 0 & 12.54 & 8.77 & 4.05 & 180 & 50 & 71 & 10 & 11.48 & 7.95 & 5.80 & \\
\hline 763 & $2 / 3 / 946: 05$ & 240 & 36 & 309 & 2 & 12.72 & 5.85 & 219 & 48 & 301 & -1 & 12.36 & 8.57 & 4.35 & 195 & 30 & 145 & 16 & 11.16 & 7.64 & 6.15 & \\
\hline 763 & $2 / 3 / 947: 05$ & 235 & 45 & 319 & 2 & 13.06 & 5.55 & 209 & 48 & 301 & 1 & 12.51 & 8.62 & 4.30 & 169 & 40 & 102 & 21 & 11.48 & 7.85 & 5.90 & \\
\hline 763 & $2 / 3 / 948: 25$ & 223 & 65 & 315 & 3 & 13.04 & 5.75 & 200 & 107 & 307 & -1 & 12.72 & 8.77 & 4.10 & 161 & 64 & 81 & 16 & 11.51 & 7.90 & 5.80 & \\
\hline 763 & $2 / 3 / 9410: 00$ & 223 & 65 & 315 & 3 & 13.04 & 5.75 & 209 & 86 & 304 & -1 & 12.62 & 8.74 & 4.00 & 161 & 59 & 50 & 15 & 11.67 & 7.97 & 5.70 & \\
\hline 763 & $2 / 3 / 9411: 20$ & 240 & 39 & 305 & 3 & 12.57 & 6.00 & 220 & 43 & 293 & 0 & 12.16 & 8.43 & 4.80 & 173 & 30 & 45 & 19 & 11.10 & 7.63 & 6.30 & \\
\hline 763 & $2 / 3 / 94 \quad 12: 20$ & 240 & 39 & 305 & 3 & 12.57 & 6.00 & 223 & 33 & 293 & -1 & 12.06 & 8.39 & 4.80 & 179 & 31 & 41 & 17 & 11.09 & 7.62 & 6.30 & \\
\hline 763 & $2 / 3 / 9413: 22$ & 240 & 39 & 305 & 3 & 12.57 & 6.00 & 225 & 29 & 302 & -1 & 12.06 & 8.54 & 4.70 & 179 & 28 & 47 & 16 & 11.10 & 7.69 & 6.40 & \\
\hline 763 & $2 / 3 / 9415: 20$ & 222 & 28 & 282 & 3 & 11.30 & 7.60 & 200 & 46 & 291 & -2 & 11.62 & 8.34 & 5.40 & 166 & 31 & 51 & 12 & 10.25 & 7.33 & 7.40 & \\
\hline 763 & $2 / 3 / 9416: 30$ & 225 & 24 & 275 & 2 & 11.12 & 8.00 & 210 & 28 & 278 & -2 & 11.28 & 8.16 & 5.90 & 165 & 21 & 47 & 13 & 10.00 & 7.21 & 7.80 & \\
\hline 763 & $2 / 3 / 94 \quad 17: 33$ & 257 & 30 & 317 & 3 & 12.57 & 6.30 & 237 & 29 & 310 & -1 & 12.27 & 8.90 & 4.60 & 194 & 24 & 136 & 21 & 10.96 & 7.93 & 6.40 & \\
\hline 763 & $2 / 3 / 94 \quad 18: 45$ & 257 & 30 & 317 & 3 & 12.57 & 6.30 & 235 & 32 & 321 & 0 & 12.36 & 8.98 & 4.50 & 176 & 24 & 82 & 30 & 11.07 & 7.99 & 6.40 & \\
\hline 763 & $2 / 3 / 9419: 43$ & 257 & 30 & 317 & 3 & 12.57 & 6.30 & 231 & 31 & 324 & 0 & 12.29 & 8.87 & 4.70 & 173 & 28 & 77 & 28 & 11.16 & 8.05 & 6.40 & \\
\hline 763 & $2 / 3 / 9421: 55$ & 250 & 37 & 330 & 5 & 12.61 & 6.35 & 229 & 35 & 320 & -3 & 11.73 & 8.64 & 5.10 & 180 & 41 & 76 & 18 & 11.33 & 8.16 & 6.40 & \\
\hline 763 & $2 / 3 / 9423: 05$ & & & & & & & 229 & 38 & 317 & 0 & 12.25 & 8.84 & 4.90 & 174 & 41 & 82 & 16 & 11.37 & 8.09 & 6.10 & \\
\hline 763 & $2 / 4 / 940: 05$ & & & & & & & 228 & 38 & 321 & -1 & 12.10 & 8.78 & 4.80 & 180 & 34 & 82 & 17 & 11.19 & 7.93 & 6.40 & \\
\hline 763 & $2 / 4 / 941: 05$ & 246 & 36 & 339 & 5 & 12.62 & 6.35 & 225 & 34 & 320 & -2 & 11.95 & 8.63 & 4.80 & 177 & 35 & 81 & 16 & 11.04 & 7.84 & 6.30 & \\
\hline 763 & $2 / 4 / 942: 05$ & & & & & & & 224 & 48 & 333 & -1 & 12.49 & 8.97 & 4.55 & 181 & 32 & 82 & 18 & 11.17 & 7.95 & 6.40 & \\
\hline 763 & 2/4/94 3:05 & & & & & & & 225 & 52 & 328 & -1 & 12.56 & 8.95 & 4.70 & 179 & 30 & 85 & 16 & 11.15 & 7.86 & 6.30 & \\
\hline 763 & $2 / 4 / 943: 45$ & 250 & 33 & 331 & 4 & 12.31 & 6.30 & 229 & 39 & 328 & -1 & 12.40 & 8.81 & 4.70 & 193 & 46 & 160 & 20 & 11.12 & 7.93 & 6.40 & \\
\hline
\end{tabular}


PSCC Arapahoe Unit 4 Sodium Injection Summary

Calcs based on: Sodium Bicarbonate (b)

$.274 \mathrm{Nawt}$

Sodium Sesquicarbonate (s) $.297 \mathrm{Na} \mathrm{wt}$

\begin{tabular}{|c|c|c|c|c|c|c|c|c|c|c|c|c|c|c|c|c|c|c|c|c|c|c|c|c|c|c|c|c|}
\hline \multirow{3}{*}{ Test } & \multirow{3}{*}{ Date \& Time } & \multirow{3}{*}{$\begin{array}{l}\text { Load } \\
\text { MWe }\end{array}$} & \multirow{3}{*}{$\begin{array}{l}\text { O2cr } \\
\text { \%wet }\end{array}$} & \multicolumn{5}{|c|}{ Sorbent Feed Injector cal } & \multirow{2}{*}{\multicolumn{4}{|c|}{$\begin{array}{l}\mathrm{SO} 2 \Delta \mathrm{SO} 2 \text { Reduction } \\
\text { ppmc cmp Calc }\end{array}$}} & \multirow{2}{*}{\multicolumn{4}{|c|}{$\triangle N O X \cdots \Delta N O 2 \triangle N O$}} & \multirow{3}{*}{$\frac{\Delta \mathrm{NO}}{\Delta \mathrm{SO} 2}$} & \multicolumn{8}{|c|}{ Economizer NO Calc..--Humidification } & \multicolumn{3}{|c|}{ Baghouse Temps } \\
\hline & & & & & & & & $2 \mathrm{Na}$ & & & & & & & & & & $\triangle N O x$ & & & $\Delta N O$ & 1 & & & $\mathrm{H} 2 \mathrm{O}$ & Grid & Out & \\
\hline & & & & $\mathrm{b} / \mathrm{s}$ & $\%$ & $\%$ & $\mathrm{lb} / \mathrm{min}$ & $s^{*}$ & $d y$ & $\%$ & $\%$ & ppme & $\%$ & $8 \mathrm{~s}$ & & Ppmc & & & & & & scfm & ${ }^{\circ} \mathrm{F}$ & & & ${ }^{\circ} \mathrm{F}$ & ${ }^{\circ} \mathrm{F}$ & \\
\hline 763 & & 90 & 5.24 & $\mathrm{~b}$ & 0 & 29 & 32. & 1 & 66 & 70 & 70.3 & 285 & 12.6 & 37 & 34.3 & 71 & 0.25 & & & & & 0 & 245 & & 0.0 & 238 & 226 & \\
\hline 6. & & & & $b$ & $c$ & & & & & 69 & & 300 & -2.4 & & & 27 & & 13.8 & 72 & 33.2 & & 0 & & & .0 & & 24 & \\
\hline 6 & & & & $b$ & c & & & & & 69 & & 284 & 5.3 & 15 & & 42 & & & & & & 0 & & & .0 & & & \\
\hline 6 & & 90 & & b & c & & & 1.00 & 410 & 72 & & 295 & 4 & & & 43 & & & & & & 0 & & & 0.0 & & & \\
\hline & & 9 & & b & $c$ & & & 0.88 & 466 & 64 & & 301 & & 11 & & 42 & & 12.3 & 64 & 30.5 & 0.21 & & & & 0.0 & & 29 & \\
\hline & & 8 & 25 & $b$ & c & & & 0.92 & 502 & 75 & 0.8 & 386 & -1.3 & -4 & & 29 & 7 & & & & & & & & 0.0 & & 38 & \\
\hline & & 5 & 92 & b & c & & & .97 & 521 & 80 & 79.2 & 413 & 5.9 & 16 & & 35 & & & & & & & & & .0 & & 243 & \\
\hline 763 & & 90 & 4.90 & b & 0 & & 41. & 0.96 & 543 & 78 & 77.0 & 418 & -0.5 & -1 & 15.6 & 15 & 0. & & & & & 0 & & & .0 & 53 & 44 & 50 \\
\hline 763 & & 90 & 4.88 & b & 0 & & 43 & 1.02 & 536 & 50 & 50.9 & 273 & -2.7 & -7 & 34 & 28 & & & & & & & & & .0 & 48 & 44 & 245 \\
\hline 763 & $14 / 9416: 50$ & 90 & 4.89 & $b$ & 0 & & 43.7 & 1.06 & 515 & 67 & 70.2 & 362 & -5.0 & -12 & 46.8 & 34 & & & & & & & & & 0.0 & 246 & 240 & 244 \\
\hline 763 & 2/4/94 18:05 & 90 & 4.88 & b & 0 & 37 & 41.5 & 1.00 & 519 & 76 & 73.0 & 379 & -5.3 & -13 & 36.5 & 24 & 0.06 & 14.3 & 72 & 36.5 & 0.19 & 0 & 258 & & 0.0 & 252 & 242 & 248 \\
\hline
\end{tabular}


PSCC Arapahoe Uni

Calcs based on:

\begin{tabular}{|c|c|c|c|c|c|c|c|c|c|c|c|c|c|c|c|c|c|c|c|c|c|c|}
\hline \multirow[b]{2}{*}{ Test } & \multirow[b]{2}{*}{ Date \& Time } & \multicolumn{6}{|c|}{ Economizer Exit, dry (1-12) } & \multicolumn{7}{|c|}{ Baghouse Inlet Gas Analysis, wet } & \multicolumn{7}{|c|}{ Stack Gas Analysis, wet } & \multirow[b]{2}{*}{ Comments } \\
\hline & & $\begin{array}{c}\text { NO } \\
\mathrm{ppm}\end{array}$ & $\begin{array}{l}\text { CO } \\
\text { ppm }\end{array}$ & $\begin{array}{l}\mathrm{SO} 2 \\
\mathrm{ppm}\end{array}$ & $\begin{array}{l}\mathrm{NO} 2 \\
\mathrm{ppm}\end{array}$ & $\begin{array}{c}\mathrm{CO} 2 \\
\%\end{array}$ & $\begin{array}{c}\mathrm{O} 2 \\
\% \mathrm{dry}\end{array}$ & $\begin{array}{l}\text { NO } \\
\text { ppm }\end{array}$ & $\begin{array}{l}\mathrm{CO} \\
\mathrm{ppm}\end{array}$ & $\begin{array}{l}\mathrm{SO} 2 \\
\mathrm{ppm}\end{array}$ & $\begin{array}{l}\mathrm{NO} 2 \\
\mathrm{ppm}\end{array}$ & $\begin{array}{c}\mathrm{CO} 2 \\
\% \\
\end{array}$ & $\begin{array}{c}\mathrm{H} 2 \mathrm{O} \\
\% \\
\end{array}$ & $\begin{array}{l}\mathrm{O} 2 \\
\% \\
\end{array}$ & $\begin{array}{l}\text { NO } \\
\mathrm{ppm}\end{array}$ & $\begin{array}{l}\mathrm{co} \\
\mathrm{ppm}\end{array}$ & $\begin{array}{l}\text { SO2 } \\
\text { ppm }\end{array}$ & $\begin{array}{l}\text { NO2 } \\
\text { ppm }\end{array}$ & $\begin{array}{c}\mathrm{CO} 2 \\
\%\end{array}$ & $\begin{array}{c}\mathrm{H} 2 \mathrm{O} \\
\%\end{array}$ & $\begin{array}{l}\mathrm{O} 2 \\
\%\end{array}$ & \\
\hline 763 & $2 / 4 / 945: 05$ & & & & & & & 233 & 30 & 326 & 0 & 12.21 & 8.77 & 4.70 & 162 & 41 & 89 & 25 & 11.37 & 8.08 & 6.00 & \\
\hline 763 & $2 / 4 / 946: 55$ & 260 & 34 & 335 & 4 & 12.53 & 6.30 & 219 & 44 & 329 & .1 & 12.10 & 8.47 & 4.80 & 180 & 53 & 81 & 23 & 11.77 & 8.19 & 6.15 & \\
\hline 763 & $2 / 4 / 948: 05$ & & & & & & & 231 & 41 & 330 & -1 & 12.48 & 8.68 & 4.60 & 177 & 39 & 90 & 20 & 11.62 & 7.99 & 6.20 & \\
\hline 763 & $2 / 4 / 949: 20$ & & & & & & & 237 & 35 & 328 & 0 & 12.47 & 8.64 & 4.80 & 175 & 27 & 80 & 20 & 10.77 & 7.42 & 7.00 & \\
\hline 763 & $2 / 4 / 9411: 00$ & 255 & 32 & 380 & 3 & 12.87 & 6.30 & 230 & 32 & 370 & -1 & 12.45 & 8.67 & 4.90 & 180 & 29 & 120 & 21 & 11.34 & 7.81 & 6.30 & \\
\hline 763 & $2 / 4 / 94 \quad 12: 05$ & & & & & & & 227 & 52 & 408 & -1 & 12.54 & 8.93 & 4.50 & 179 & 32 & 83 & 22 & 11.28 & 7.87 & 6.50 & \\
\hline 763 & $2 / 4 / 9413: 05$ & & & & & & & 220 & 174 & 430 & 0 & 12.69 & 9.05 & 4.25 & 171 & 75 & 80 & 15 & 11.82 & 8.34 & 5.95 & \\
\hline 763 & $2 / 4 / 9414: 05$ & 249 & 79 & 425 & 4 & 13.08 & 5.95 & 216 & 187 & 429 & -1 & 12.94 & 9.26 & 4.20 & 179 & 184 & 111 & 14 & 11.66 & 8.22 & 6.05 & \\
\hline 763 & $2 / 4 / 9415: 05$ & & & & & & & 210 & 191 & 459 & -1 & 13.03 & 9.26 & 3.85 & 176 & 151 & 94 & 11 & 11.95 & 8.41 & 5.70 & \\
\hline 763 & $2 / 4 / 9416: 00$ & & & & & & & 216 & 149 & 441 & -1 & 12.91 & 9.20 & 4.25 & 174 & 91 & 195 & 25 & 11.73 & 8.31 & 5.90 & \\
\hline 763 & $2 / 4 / 9416: 50$ & & & & & & & 211 & 186 & 430 & -2 & 13.09 & 9.45 & 4.00 & 161 & 94 & 113 & 33 & 11.76 & 8.30 & 6.00 & \\
\hline 763 & $2 / 4 / 9418: 05$ & 243 & 54 & 431 & 4 & 13.32 & 5.80 & 204 & 138 & 442 & -1 & 13.35 & 9.65 & 3.65 & 160 & 37 & 104 & 26 & 11.69 & 8.36 & 5.90 & \\
\hline
\end{tabular}


PSCC Arapahoe Unit 4 Sodium Injection Summary--Air Toxics Testing

Calcs based on: Sodium Bicarbonate (b) .274 Na wt

Sodium Sesquicarbonate (s) $\quad .297 \mathrm{Na} w$

\begin{tabular}{|c|c|c|c|c|c|c|c|c|c|c|c|c|c|c|c|c|c|c|c|c|c|c|c|c|c|c|c|c|c|c|c|}
\hline \multirow{3}{*}{ Test } & \multirow{3}{*}{ Date \& Time } & \multirow{3}{*}{$\begin{array}{l}\text { Load } \\
\text { MWe }\end{array}$} & \multirow{3}{*}{$\begin{array}{l}\mathrm{O} 2 \mathrm{cr} \\
\% \mathrm{wet}\end{array}$} & \multirow{2}{*}{\multicolumn{5}{|c|}{$\begin{array}{l}\text { Sorbent Feed Injector cal } \\
\mathrm{Na} \mathrm{A}(w) \mathrm{B}(\theta) \text { Flow } 2 \mathrm{Na}\end{array}$}} & \multirow{2}{*}{\multicolumn{3}{|c|}{\begin{tabular}{|l|}
$\Delta \mathrm{SO} 2$ Reduction \\
$\mathrm{Cmp}$ Calc
\end{tabular}}} & \multirow{2}{*}{\multicolumn{4}{|c|}{$\Delta \mathrm{NOX} \cdots \cdot-. . \mathrm{NO2} \triangle \mathrm{NO}$}} & \multirow{3}{*}{$\frac{\Delta \mathrm{NO}}{\Delta \mathrm{SO} 2}$} & \multirow{3}{*}{$\begin{array}{c}\mathrm{SO} 2 \\
\text { inlet } \\
\text { ppmcd }\end{array}$} & \multicolumn{4}{|c|}{ Economizer NO Calc - - } & & idifica & & & & & & & & \\
\hline & & & & & & & & & & & & & & & & & & & & & $\triangle N Q$ & & Taho & & & & $\mathrm{H} 2 \mathrm{Oe}$ & Grid & Out & & Opsis \\
\hline & & & & & $\%$ & $\%$ & & $s^{*}$ & $\%$ & $\%$ & ppme & $\%$ & ppme & ppmc & ppme & & & & ppme & ppmc & $\triangle \mathrm{SO}_{2}$ & sctm & 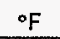 & ${ }^{\circ} \mathrm{F}$ & $\mathrm{gpm}$ & ${ }^{\circ} \mathrm{F}$ & $\% \mathrm{w}$ & of & ${ }^{\circ} \mathrm{F}$ & ${ }^{\circ} \mathrm{F}$ & ${ }^{\circ} \mathrm{F}$ \\
\hline 699 & $10 / 11 / 93: 1330$ & 75 & 6.00 & & 0 & 0 & 0.0 & 0.00 & 0 & -7.8 & -33 & -9.8 & -28 & 1.2 & -27 & 0.81 & 423 & 10.1 & 29 & 1.2 & -0.88 & 0 & 272 & 61 & 0.0 & & 7.32 & 267 & 248 & 258 & 259 \\
\hline 699 & 10/11/93:1431 & 75 & 6.00 & & 0 & 0 & 0.0 & 0.00 & 0 & -7.7 & -33 & -7.2 & -21 & 1.8 & -19 & 0.58 & 426 & 4.5 & 10 & 1.8 & -0.29 & 0 & 275 & 61 & 0.0 & & 7.61 & 270 & 254 & 261 & 263 \\
\hline 699 & 10/11/93:1530 & 76 & 5.90 & & 0 & 0 & 0.0 & 0.00 & 0 & -7.3 & -31 & -7.6 & -22 & 1.1 & -21 & 0.68 & 426 & 6.2 & 15 & 1.1 & -0.48 & 0 & 277 & 62 & 0.0 & & 63 & 72 & 257 & 264 & 267 \\
\hline 699 & 10/11/93:1630 & 76 & 6.00 & & 0 & 0 & 0.0 & 0.00 & 0 & -8.8 & -37 & -12.1 & -34 & 1.6 & -33 & 0.89 & 420 & 5.2 & 11 & 1.6 & -0.31 & 0 & 277 & 62 & 0.0 & & .58 & 272 & 259 & 266 & 269 \\
\hline 699 & $10 / 11 / 93: 1710$ & & & & 0 & 0 & 0.0 & & & -1.4 & -6 & -2.3 & -6 & 2.4 & -4 & $0: 70$ & 397 & 4.8 & & 2.4 & -1.68 & 0 & & & 0.0 & & 7.31 & & & & \\
\hline 699 & 10/11/93: & 76 & 5.90 & & 0 & 0 & 0.0 & 0.00 & 0 & -1.1 & -4 & -3.7 & -10 & 1.6 & -8 & 1.89 & 398 & 6.0 & 12 & 1.6 & -2.84 & 0 & 277 & 62 & 0.0 & & 7.27 & 272 & 260 & 267 & 270 \\
\hline 699 & $10 / 11$ & 76 & 5.90 & & 0 & 0 & 0.0 & 0.00 & 0 & -1.3 & -5 & -4.2 & -11 & 1.6 & -9 & 1.81 & 392 & 3.9 & & 1.6 & -1.19 & & 276 & 61 & 0.0 & & 7.34 & 271 & 260 & 266 & 270 \\
\hline 699 & 10/11/93:1930 & 76 & 5.90 & & 0 & 0 & 0.0 & 0.00 & 0 & -1.9 & -7 & -2.8 & -7 & 1.1 & -6 & 0.87 & 391 & 5.9 & 12 & 1.1 & -1.63 & 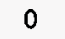 & 27.4 & 60 & 0.0 & & 7.51 & 270 & 258 & 265 & 269 \\
\hline 700 & $10 / 12 / 93: 1200$ & 75 & 5.30 & & 0 & 0 & 0.0 & 0.00 & 0 & -1.3 & -5 & 0.2 & 0 & 0.7 & 1 & -0.23 & 389 & 6.3 & 11 & 0.7 & -2.18 & 0 & 266 & & 0.0 & & 7.92 & 261 & 243 & 253 & 251 \\
\hline 0 & 10/12/93:1300 & 75 & 5.30 & & 0 & 0 & 0.0 & 0.00 & 0 & -2.0 & -8 & 0.8 & 2 & 0.9 & 3 & -0.36 & 386 & 3.2 & 3 & 0.9 & -0.43 & 0 & 269 & 60 & 0.0 & & 8.00 & 264 & 247 & 57 & 55 \\
\hline 0 & 10/12/93:1400 & 75 & 5.30 & & 0 & 0 & 0.0 & 0.00 & 0 & -2.0 & -8 & -3.0 & -7 & 0.1 & -7 & 0.88 & 383 & 2.2 & 0 & 0.1 & -0.04 & 0 & 271 & 60 & 0.0 & & 8.03 & 265 & 250 & 259 & 257 \\
\hline 700 & $10 / 12 / 9$ & 75 & 5.30 & & 0 & 0 & 0.0 & 0.00 & 0 & -2.2 & -8 & -2.9 & -7 & 0.5 & -6 & 0.75 & 381 & 5.4 & 9 & 0.5 & -1.14 & 0 & 272 & 61 & 0.0 & & 7.83 & 266 & 251 & 261 & 259 \\
\hline 700 & $10 / 1$ & 75 & 5.30 & & 0 & 0 & 0.0 & 0.00 & 0 & -2.0 & -8 & -3.4 & -8 & 0.8 & -7 & 0.94 & 379 & 3.7 & 5 & 0.8 & -0.67 & 0 & 272 & 61 & 0.0 & & 7.87 & 268 & 254 & 262 & 261 \\
\hline 700 & 700 & 75 & 5.30 & & 0 & 0 & 0.0 & 0.00 & 0 & -2.2 & -9 & -0.9 & -2 & 1.0 & -1 & 0.15 & 382 & 4.3 & 6 & 1.0 & -0.75 & & 272 & 61 & 0.0 & & 7.82 & 267 & 254 & 262 & 261 \\
\hline 700 & $10 /$ & 74 & 5.40 & & 0 & 0 & 0.0 & 0.00 & 0 & -2.4 & -9 & 3.1 & 7 & 0.8 & 8 & -0.90 & 376 & 6.4 & 11 & 0.8 & -1.21 & & 271 & 60 & 0.0 & & 7.88 & 266 & 253 & 262 & 260 \\
\hline 700 & 10/12/93:1900 & 75 & 5.30 & & 0 & 0 & 0.0 & 0.00 & 0 & -2.1 & -8 & -0.9 & -2 & 1.1 & -1 & 0.12 & 375 & 2.0 & 0 & 1.1 & -0.05 & & 271 & 59 & 0.0 & & 7.87 & 265 & 253 & 261 & 261 \\
\hline 700 & 10/12/93:2000 & 75 & 5.30 & & 0 & 0 & 0.0 & 0.00 & 0 & -3.3 & -12 & -0.8 & -2 & 1.5 & 0 & 0.04 & 377 & 5.4 & 9 & 1.5 & $-0: 72$ & & 70 & 59 & 0.0 & & 7.86 & 265 & 252 & 261 & 261 \\
\hline 701 & 10/13/93:0810 & 75 & 5.20 & & 0 & 0 & 0.0 & 0.00 & 0 & -3.8 & -15 & -2.8 & -7 & -1.2 & -8 & 0.52 & 390 & 6.4 & 12 & -1.2 & -0.78 & & 56 & 48 & 0.0 & & 7.83 & 50 & 235 & & 42 \\
\hline 701 & $10 / 13 /$ & 75 & 5.40 & & 0 & 0 & 0.0 & 0.00 & 0 & -2.4 & -9 & -1.8 & -4 & 1.0 & -3 & 0.38 & 381 & 2.8 & 3 & 1.0 & -0.33 & 0 & 258 & 50 & 0.0 & & .92 & 53 & 236 & 246 & 43 \\
\hline 1 & & 75 & 5.40 & & 0 & 0 & 0.0 & 0.00 & 0 & -2.0 & -8 & -5.2 & .12 & 1.1 & -11 & 1.45 & 380 & 1.6 & 0 & 1.1 & 0.03 & 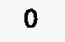 & 264 & 53 & 0.0 & & 8.01 & 258 & 240 & 252 & 248 \\
\hline 701 & $10 /$ & 74 & 5.30 & & 0 & 0 & 0.0 & 0.00 & 0 & -2.1 & -8 & -3.7 & -9 & 1.3 & -7 & 0.91 & 381 & 4.3 & 7 & 1.3 & -0.80 & & 267 & 55 & 0.0 & & 7.90 & 262 & 245 & 255 & 252 \\
\hline 701 & $10 /$ & 76 & 5.20 & & 0 & 0 & 0.0 & 0.00 & 0 & -1.8 & -7 & -5.4 & -13 & 0.6 & -12 & 82 & 377 & 2.7 & 2 & 0.6 & -0.35 & & 270 & 58 & 0.0 & & 7.99 & 265 & 248 & 258 & 256 \\
\hline 701 & $10 / 13 / 4$ & 74 & 5.30 & & 0 & 0 & 0.0 & 0.00 & 0 & -2.0 & -7 & -0.5 & -1 & 1.1 & 0 & 02 & 377 & 6.0 & 11 & 1.1 & -1.53 & & 273 & 61 & 0.0 & & 7.87 & 267 & 251 & 261 & 259 \\
\hline 701 & 10/13/93:1410 & 75 & 5.30 & & 0 & 0 & 0.0 & 0.00 & 0 & -2.5 & -10 & -4.9 & -1 & 0.8 & -10 & 1.09 & 378 & 5.2 & 9 & 0.8 & -0.89 & & 273 & 61 & 0.0 & & 7.72 & 269 & 254 & 263 & 261 \\
\hline 701 & 10/13/93:1500 & 75 & 5.30 & & 0 & 0 & 0.0 & 0.00 & 0 & -2.7 & -10 & -3.5 & -8 & 0.3 & -8 & 0.79 & 378 & 5.6 & 10 & 0.3 & -1.03 & & 274 & 62 & 0.0 & & 7.77 & 269 & 255 & 264 & 263 \\
\hline 701 & 10/13/93:1600 & 75 & 5.20 & & 0 & $\mathbf{0}$ & 0.0 & 0.00 & 0 & -1.4 & -5 & -2.2 & -5 & 0.5 & -5 & 0.87 & 380 & 5.1 & 8 & 0.5 & -1.44 & & 274 & 63 & 0.0 & & .85 & 270 & 256 & 265 & 63 \\
\hline 702 & $1: 0830$ & 100 & 4.40 & $\mathbf{s}$ & 50 & 0 & 46.7 & 1.50 & 61 & 61.8 & 236 & 4.7 & 11 & 12.0 & 23 & 0.10 & 381 & 13.3 & 37 & 12.0 & 0.16 & & 263 & 49 & 0.0 & & 8.58 & 257 & 243 & 254 & 253 \\
\hline 702 & $10 / 14 / 93: 0930$ & 101 & 4.30 & $\mathbf{s}$ & 50 & 0 & 46.7 & 1.47 & 66 & 65.1 & 251 & 1.3 & 3 & 12.5 & 16 & 0.06 & 385 & 9.5 & 31 & 12.5 & 0.13 & & 266 & 50 & 0.0 & & 8.62 & 258 & 245 & 256 & 256 \\
\hline 702 & & 100 & 4.40 & s & 50 & 0 & 46.7 & 1.52 & 64 & 64.3 & 242 & 6.9 & 1) & 8.8 & 26 & 0.11 & 377 & 14.3 & 43 & 8.8 & 0.18 & & 268 & 52 & 0.0 & & 8.58 & 260 & 247 & 159 & 259 \\
\hline 702 & & 99 & 4.40 & s & 50 & 0 & 46.7 & 1.54 & 63 & 63.6 & 240 & 6.5 & 1 & 9.4 & 26 & 0 & 377 & 10.3 & 31 & 9.4 & 0.13 & & 271 & 55 & 0.0 & & 3.66 & 263 & 251 & 261 & 263 \\
\hline 702 & 10/14/93:1230 & 100 & 4.30 & $\mathbf{s}$ & 50 & 0 & 46.7 & 1.55 & 67 & & 247 & 6.9 & 1 & 7.9 & 24 & & 370 & 14.0 & 39 & 7.9 & 0.16 & 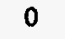 & 274 & 59 & 0.0 & & 8.66 & 267 & 255 & & 266 \\
\hline 702 & 10/14/93:1330 & 99 & 4.30 & $\mathbf{s}$ & 50 & 0 & 46.7 & 1.56 & 63 & 55.3 & 206 & 6.4 & 1 & 12.7 & 28 & 14 & 372 & 12.4 & 39 & 12.7 & 0.19 & $\mathbf{U}$ & 277 & 60 & 0.0 & & 8.51 & 270 & 261 & 269 & 268 \\
\hline 703 & 10/14/93:1700 & 99 & 4.40 & $\mathbf{s}$ & 0 & 53 & 47.1 & 1.53 & 63 & 63.9 & 244 & 4.8 & 11 & 7.9 & 19 & 0.08 & 381 & 14.7 & 41 & 7.9 & 0.17 & 0 & 271 & 58 & 0.0 & & 8.59 & 265 & 257 & 264 & 265 \\
\hline 703 & 10/14/93:1800 & 99 & 4.50 & $\mathbf{s}$ & 0 & 53 & 47.1 & 1.53 & 65 & 65.5 & 250 & 3.1 & 7 & 8.3 & 16 & 0.06 & 381 & 7.9 & 22 & 8.3 & 0.09 & & 271 & 58 & 0.0 & & 3.73 & 263 & 256 & 264 & 266 \\
\hline 703 & 10/14/93:1900 & 99 & 4.50 & $\mathbf{s}$ & 0 & 53 & 47.1 & 1.53 & 65 & 64.6 & 246 & 10.0 & 25 & 8.1 & 33 & 0.14 & 381 & 13.9 & 35 & 8.1 & 0.16 & & 270 & 57 & 0.0 & & 8.77 & 263 & 255 & 263 & 265 \\
\hline 703 & 10/14/93:2000 & 99 & 4.30 & $\mathbf{s}$ & 0 & 53 & 47.1 & 1.501 & 65 & 63.9 & 248 & 9.3 & 24 & 6.4 & 30 & 0.12 & 388 & 14.3 & 40 & 6.4 & 0.16 & & 271 & 55 & 0.0 & & 8.73 & 264 & 254 & 263 & 266 \\
\hline 703 & 10/14/93:2105 & 99 & 4.20 & $\mathbf{s}$ & 0 & 53 & 47.1 & 1.46 & 66 & 65.2 & 260 & 8.9 & 22 & 6.5 & 29 & 0.11 & 399 & 16.2 & 46 & 6.5 & 0.18 & 0 & 270 & 55 & 0.0 & & 8.78 & 262 & 254 & 262 & 265 \\
\hline 703 & 10/14/93:2200 & 99 & 4.40 & $\mathbf{s}$ & 0 & 53 & 47.1 & 1.53 & 67 & 66.1 & 252 & 7.3 & 19 & 6.3 & 25 & 0.10 & 381 & 10.8 & 31 & 6.3 & 0.12 & 0 & 270 & 55 & 0.0 & & 99 & 263 & 253 & 262 & 265 \\
\hline 704 & 0830 & 100 & 4.20 & $\mathbf{s}$ & 0 & 53 & 47.1 & 1.56 & 57 & 57.7 & 214 & 5.8 & 14 & 9. & 24 & 0.11 & 371 & 16.5 & 51 & 9.8 & 0.24 & 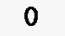 & 260 & 48 & 0.0 & & 8.84 & 253 & 240 & 250 & 250 \\
\hline 704 & & 100 & 4.10 & $\mathbf{s}$ & 0 & 53 & 47.1 & 1.51 & 59 & 63.0 & 241 & 3.5 & 8 & 8.3 & 17 & 0.07 & 383 & 7.7 & 2. & 8.3 & 0.09 & & & 52 & 0.0 & & 9.20 & 255 & 243 & 253 & 254 \\
\hline 704 & 10/15/93:1030 & 100 & 4.30 & s & 0 & 53 & 47.1 & 1.48 & 59 & 60.8 & 237 & 4.8 & 11 & 6.1 & 17 & 0.07 & 390 & 13.9 & 37 & 6.1 & 0.16 & 0 & 265 & 53 & 0.0 & & 9.09 & 257 & 246 & 256 & 256 \\
\hline 704 & 10/15/93:1130 & 99 & 4.20 & $\mathbf{s}$ & 0 & 53 & 47.1 & 1.53 & 62 & 58.9 & 224 & 1.3 & 3 & 8.0 & 11 & 0.05 & 381 & 9.0 & 25 & 8.0 & 0.11 & 0 & 267 & 56 & 0.0 & & 8.96 & 260 & 248 & 258 & 259 \\
\hline 704 & 10/15/93:1230 & 99 & 4.20 & $\mathbf{s}$ & 0 & 53 & 47.1 & 1.55 & 63 & 63.4 & 239 & 9.8 & 24 & 7.1 & 31 & 0.13 & 376 & 14.0 & 37 & 7.1 & 0.15 & 0 & 270 & 57 & 0.0 & & 8.94 & 263 & 251 & 261 & 262 \\
\hline
\end{tabular}


PSCC Arapahoe Uni

Calcs based on:

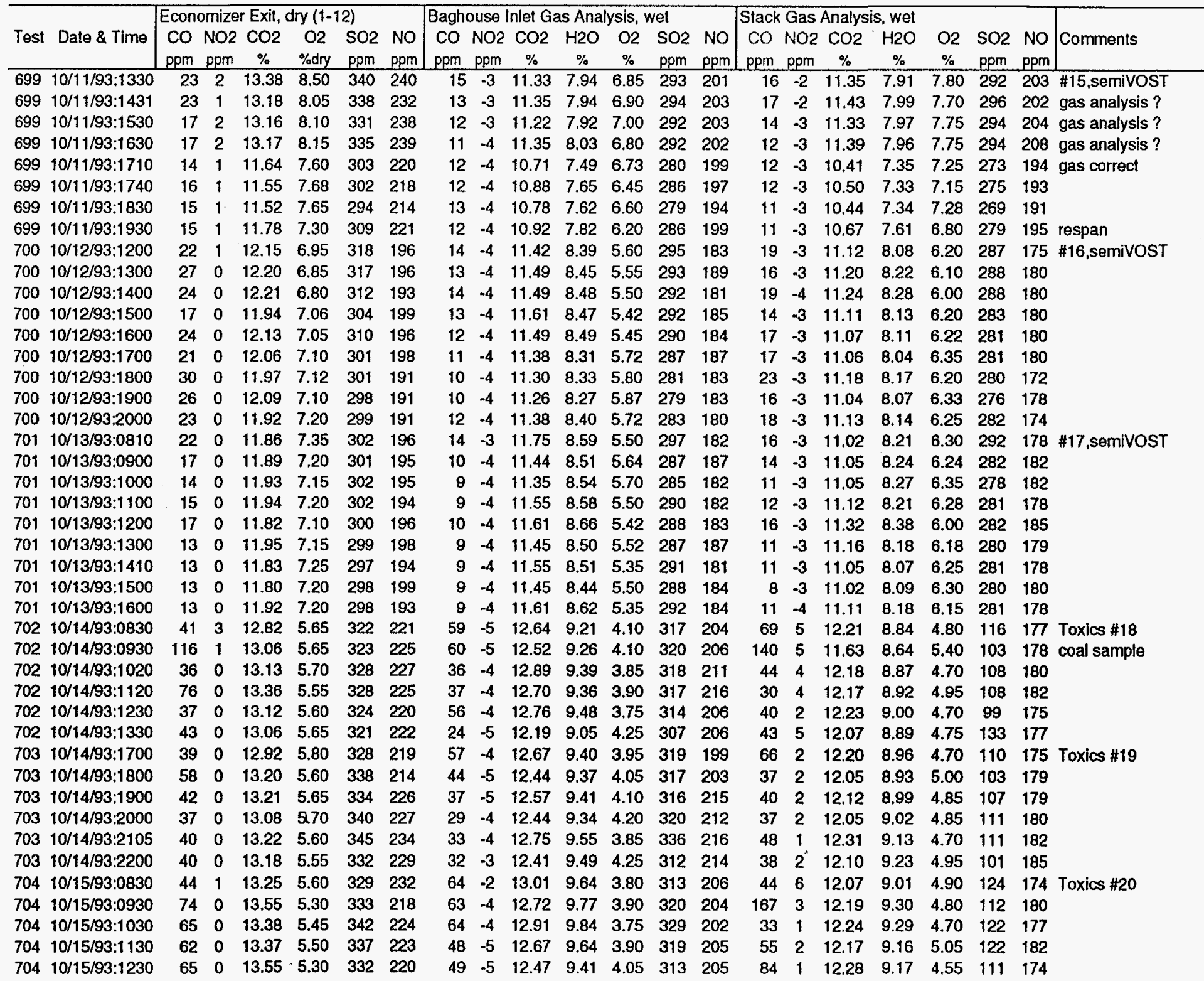


PSCC Arapahoe Unit 4 Sodium Injection Summary--Sodium/Humidification Tests $\begin{array}{lll}\text { Calcs based on: } & \text { Sodium Bicarbonate (b) } & 0.274 \mathrm{Nawt} \\ & \text { Sodium Sesquicarbonate (s) } & 0.297 \mathrm{Nawt}\end{array}$

\begin{tabular}{|c|c|c|c|c|c|c|c|c|c|c|c|c|c|c|c|c|c|c|c|c|c|c|c|c|c|c|c|c|c|c|}
\hline \multirow{3}{*}{ Test } & \multirow{3}{*}{ Date \& Time } & \multirow{3}{*}{\multicolumn{2}{|c|}{$\begin{array}{l}\text { Load O2cr } \\
\text { MWO \% Wet }\end{array}$}} & \multirow{2}{*}{\multicolumn{5}{|c|}{$\begin{array}{l}\text { Sorbent Feed Injector cal } \\
\mathrm{Na} \mathrm{A}(\mathrm{w}) \mathrm{B}(e) \text { Flow 2Na }\end{array}$}} & \multirow{2}{*}{\multicolumn{3}{|c|}{$\begin{array}{l}\Delta \text { SO2 Reduction } \\
\text { Cmp Calc }\end{array}$}} & \multirow{2}{*}{\multicolumn{4}{|c|}{$\Delta N O x \cdots \Delta N O 2 \Delta N O$}} & \multirow{3}{*}{$\frac{\Delta \mathrm{NO}}{\Delta \mathrm{SO} 2}$} & \multirow{2}{*}{\multicolumn{7}{|c|}{ 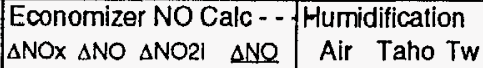 }} & \multirow{2}{*}{\multicolumn{3}{|c|}{ Humid calc }} & \multicolumn{4}{|c|}{ Baghouse Temps } \\
\hline & & & & & & & & & & & & & & & & & & & & & & & & & & & & & & Ops \\
\hline & & & & b/s & $\%$ & $\%$ & $\mathrm{Ib} / \mathrm{m} / \mathrm{n}$ & $S^{*}$ & $\%$ & $\%$ & ppmc & $\%$ & ppmc & ppme & ppme & & $\%$ & $\mathrm{ppmc}$ & ppmc & & & ${ }^{\circ} \mathrm{F}$ & ${ }^{\circ} \mathrm{F}$ & & & $\% w$ & & & ${ }^{\circ} \mathrm{F}$ & \\
\hline 665 & 9/20/93:0810 & 100 & 3.60 & $\mathbf{s}$ & 0 & 41 & 35.8 & 0.99 & 0 & -0.2 & -1 & 0.8 & 2 & -0.5 & 2 & -2.11 & 6.9 & 14 & -0.5 & -18.82 & 3855 & 279 & 64 & 47.0 & 183 & 8.88 & 177 & 181 & 183 & 185 \\
\hline 665 & /20/93:1410 & 100 & 3.60 & $\mathbf{s}$ & 0 & 41 & 35.8 & 1.03 & 60 & 58.7 & 250 & 3.4 & 9 & 0.1 & 8 & 0.03 & 4.5 & 5 & 0.1 & 0.02 & 3843 & 285 & 63 & 49.0 & 183 & 90 & 176 & 178 & 180 & 181 \\
\hline 665 & 40 & 100 & 3.60 & $\mathbf{s}$ & 0 & 41 & 5.8 & 1.07 & 64 & 64.2 & 264 & 5.5 & 14 & -0.3 & 13 & .05 & 11.1 & 23 & -0.3 & 0.09 & 3823 & 288 & 63 & 50.0 & 186 & 86 & 176 & 179 & 181 & 182 \\
\hline 666 & 30 & 99 & 4.00 & $s$ & 0 & 37 & 2.1 & 1.03 & 0 & 7.2 & 105 & 2.5 & 6 & 3.1 & 9 & .09 & 6.1 & 11 & 3.1 & 1.10 & 0 & 275 & 56 & 0.0 & 275 & 9.00 & 267 & 247 & 256 & 255 \\
\hline 666 & 1/21/93:1040 & 100 & 4.50 & $\mathbf{s}$ & 0 & 38 & & 1.02 & 42 & & 172 & 3.7 & 10 & & 17 & .10 & 4.3 & 12 & .0 & 0.07 & 0 & 286 & 64 & 0.0 & 186 & 8.54 & 279 & 267 & 276 & 278 \\
\hline 666 & $/ 21 / 93: 1300$ & 100 & 4.50 & $\mathbf{s}$ & 0 & 38 & 33.0 & 0.99 & 40 & 42.0 & 172 & 3.3 & 9 & & 15 & .09 & 3.7 & 10 & 6.0 & 0.06 & 4261 & 289 & 67 & 0.0 & 289 & 8.50 & 281 & 273 & 280 & 32 \\
\hline 666 & $: 1420$ & 100 & 4.60 & s & 0 & 38 & 33.0 & 1.00 & 51 & 49.6 & 201 & 1.2 & & 2.3 & 5 & 0.03 & 4.7 & 8 & 2.3 & .04 & 11 & 288 & 67 & 23.0 & 241 & 8.67 & 234 & 242 & 42 & 247 \\
\hline 666 & 530 & 101 & 4.40 & $s$ & 0 & 38 & 33.0 & 1.02 & 52 & 49.9 & 196 & 2.5 & & 1. & & 0.04 & 5.6 & 11 & 1.2 & 0.06 & 3968 & 291 & 67 & 34.0 & 223 & 8.89 & 217 & 223 & 23 & \\
\hline 66 & 630 & 101 & 4.50 & s & 0 & 38 & 33.0 & 1.03 & 63 & 56.9 & 222 & 0.6 & & 0.5 & 2 & 0.01 & 6.5 & 12 & 0.5 & 0.05 & 3747 & 290 & 65 & 56.0 & 180 & 8.90 & 179 & 193 & 88 & 8 \\
\hline & & 00 & 3.90 & $\mathbf{s}$ & 0 & & & 0.00 & & -3.5 & -17 & 5.9 & 14 & 0.5 & 15 & -0.86 & 8.6 & 14 & 0.9 & -0.78 & 0 & 274 & 58 & 0.0 & 274 & 9.74 & 269 & 249 & 259 & 257 \\
\hline 667 & 30 & 03 & 3.90 & $\mathbf{s}$ & 0 & 2 & .8 & 0.49 & & 28.8 & 144 & 2 & 1 & & 1 & 09 & 6.5 & & & 0.07 & 844 & 276 & 63 & 47.0 & 184 & 9.66 & 179 & 184 & 186 & 188 \\
\hline 38 & & 03 & 3.40 & $\mathbf{s}$ & 0 & 50 & 4.3 & 1.04 & 69 & 68.5 & 347 & 5.0 & 11 & 1. & 12 & .04 & 3.7 & 3 & 1.3 & .01 & 830 & 278 & 62 & 48.0 & 178 & 10.21 & 174 & 175 & 178 & 180 \\
\hline 669 & 0 & 103 & 3.30 & s & 0 & 71 & .1 & 1.57 & 72 & 7 & 351 & -1.2 & -3 & & -1 & 00 & 6.6 & 12 & 1.4 & .03 & 810 & 279 & 62 & 50.0 & 180 & 9.53 & 173 & 174 & 178 & 179 \\
\hline 670 & $9 / 22$ & 103 & 3.30 & $\mathbf{s}$ & 0 & 90 & 1.9 & 2.12 & 9 & 90 & 415 & 2.5 & 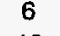 & & 7 & 0.02 & 10.0 & 20 & & 05 & & 277 & 61 & 50.0 & 178 & 9.54 & 174 & 173 & 176 & 177 \\
\hline 671 & $9 / 22 / 93$ & 103 & 3.50 & $\mathbf{s}$ & 0 & 44 & 38.7 & 1.03 & 62 & 61.2 & 275 & 5.7 & 13 & 0.7 & 14 & 0.05 & 9.6 & 19 & 0.7 & 0.07 & 3822 & 277 & 63 & 49.0 & 179 & 9.62 & 174 & 173 & 176 & 177 \\
\hline 672 & 100 & 103 & 3.50 & $\mathbf{s}$ & 0 & 43 & 37.7 & 1.06 & 63 & 66.4 & 282 & 2.7 & & 0.5 & 7 & 0.02 & 5.7 & 8 & 0.5 & 0.03 & 3786 & 275 & 61 & 53.0 & 169 & 9.71 & 164 & 165 & 168 & 169 \\
\hline 678 & & 00 & 4.00 & $\mathbf{s}$ & 0 & 0 & & 0.00 & & 4.1 & -19 & 5.6 & & & 18 & -0.92 & 2.4 & 0 & & & & 255 & 52 & 0.0 & 55 & .18 & 250 & 231 & 41 & 23 \\
\hline 679 & & 101 & 3.90 & $\mathbf{s}$ & 0 & 0 & & 0.00 & c & -5.0 & -20 & 8.4 & 25 & 0 & 26 & -1.28 & 5.3 & 9 & 0.5 & -0.42 & 4087 & 264 & 63 & 25.5 & 213 & 8.78 & 207 & 222 & 224 & 228 \\
\hline 679 & 9/29/ & 100 & 4.00 & $\mathbf{s}$ & 0 & 39 & 33.9 & 1.08 & 5 & 45. & 177 & 6.8 & 2 & & 25 & 0.14 & 1.8 & 1 & 3.5 & 0.01 & 4004 & 277 & 63 & 32.5 & 211 & 8.80 & 208 & 207 & 209 & 211 \\
\hline 679 & 9/29/93:1240 & 99 & 4.00 & $\mathbf{s}$ & 0 & 39 & 33.9 & 1.09 & 4 & 46.9 & 182 & 11.6 & 3 & 0 & 38 & 0.21 & 8.0 & 19 & 0.7 & 0.10 & 3977 & 282 & 65 & 34.5 & 212 & 8.41 & 208 & 206 & 208 & 211 \\
\hline 679 & 9/29/93:1500 & 99 & 4.10 & s & 0 & 39 & 33.9 & 1.09 & 56 & 58.9 & 227 & 10.0 & 32 & 1 & 34 & 0.15 & 3 & 5 & & 0.6 & 3948 & 286 & 65 & 37.0 & 210 & 8.60 & 207 & 205 & 208 & 212 \\
\hline 697 & 10/08/93:0740 & 102 & 3.70 & $\mathbf{s}$ & 0 & 0 & 0.0 & 0.00 & 0 & -4.6 & -21 & -0.9 & -2 & & -2 & 0.10 & 3.9 & 11 & 0.4 & -0.51 & & 266 & 48 & 0.0 & 266 & 9.23 & 260 & 241 & 252 & 250 \\
\hline 698 & 10/08/93:1040 & 101 & 3.80 & $s$ & 22 & 24 & 38.4 & 1.01 & 68 & 66.4 & 306 & -0.1 & 0 & 0.9 & 1 & 0.00 & -8.2 & -20 & 0.9 & -0.06 & 3868 & 269 & 61 & 43.0 & 183 & 9.12 & 173 & 181 & 182 & 184 \\
\hline
\end{tabular}


PSCC Arapahoe Uni

Calcs based on:

\begin{tabular}{|c|c|c|c|c|c|c|c|c|c|c|c|c|c|c|c|c|c|c|c|c|c|c|}
\hline \multirow[b]{2}{*}{ Test } & \multirow[b]{2}{*}{ Date \& Time } & \multicolumn{6}{|c|}{ Economizer Exit, dry (1-12) } & \multicolumn{7}{|c|}{ Baghouse Inlet Gas Analysis, wet } & \multicolumn{7}{|c|}{ Stack Gas Analysis, wet } & \multirow[b]{2}{*}{ Comments } \\
\hline & & $\begin{array}{l}\text { NO } \\
\text { ppm }\end{array}$ & $\begin{array}{l}\mathrm{CO} \\
\mathrm{ppm}\end{array}$ & $\begin{array}{l}\mathrm{SO} 2 \\
\mathrm{ppm}\end{array}$ & $\begin{array}{l}\mathrm{NO} 2 \\
\mathrm{ppm}\end{array}$ & $\begin{array}{c}\mathrm{CO} 2 \\
\%\end{array}$ & $\begin{array}{c}02 \\
\% \text { dry }\end{array}$ & $\begin{array}{l}\text { NO } \\
\text { ppm }\end{array}$ & $\begin{array}{l}\mathrm{CO} \\
\mathrm{ppm}\end{array}$ & $\begin{array}{l}\mathrm{SO} 2 \\
\mathrm{ppm}\end{array}$ & $\begin{array}{l}\mathrm{NO} 2 \\
\mathrm{ppm}\end{array}$ & $\begin{array}{c}\mathrm{CO} 2 \\
\%\end{array}$ & $\begin{array}{c}\mathrm{H} 2 \mathrm{O} \\
\% \\
\end{array}$ & $\begin{array}{l}\mathrm{O} 2 \\
\%\end{array}$ & $\begin{array}{l}\text { NO } \\
\text { ppm }\end{array}$ & $\begin{array}{l}\text { CO } \\
\text { ppm }\end{array}$ & $\begin{array}{l}\mathrm{SO} 2 \\
\mathrm{ppm}\end{array}$ & $\begin{array}{l}\mathrm{NOE} \\
\mathrm{ppm}\end{array}$ & $\begin{array}{c}\mathrm{CO} 2 \\
\%\end{array}$ & $\begin{array}{c}\mathrm{H} 2 \mathrm{O} \\
\%\end{array}$ & $\begin{array}{l}\mathrm{O} 2 \\
\%\end{array}$ & \\
\hline 665 & 9/20/93:0810 & 240 & 47 & 410 & 0 & 14.13 & 5.05 & 212 & 29 & 363 & -4 & 12.48 & 9.03 & 4.35 & 193 & 59 & 333 & -4 & 11.70 & 11.99 & 4.97 & Humid only \\
\hline 665 & 9/20/93:1410 & 223 & 180 & 393 & 0 & 14.09 & 4.90 & 208 & 27 & 350 & -5 & 12.48 & 9.00 & 4.30 & 185 & 142 & 133 & -5 & 11.87 & 12.14 & 4.82 & $\mathrm{NaSC}$ \\
\hline 665 & 9/20/93:1640 & 231 & 118 & 385 & 0 & 14.00 & 5.20 & 208 & 21 & 338 & -4 & 12.53 & 9.14 & 4.27 & 179 & 78 & 110 & -4 & 11.71 & 12.36 & 4.95 & \\
\hline 666 & 9/21/93:0730 & 229 & 86 & 353 & 2 & 13.70 & 5.15 & 211 & 29 & 316 & -5 & 12.31 & 9.17 & 4.40 & 190 & 84 & 215 & -2 & 11.88 & 8.73 & 5.45 & Base \\
\hline 666 & 9/21/93:1040 & 233 & 56 & 350 & 1 & 13.43 & 5.70 & 217 & 16 & 310 & -5 & 11.76 & 8.54 & 5.22 & 198 & 56 & 171 & 1 & 11.76 & 8.38 & 5.65 & \\
\hline 666 & 9/21/93:1300 & 225 & 86 & 357 & 1 & 13.33 & 5.80 & 213 & 26 & 320 & -4 & 11.90 & 8.63 & 5.08 & 190 & 46 & 175 & 1 & 11.38 & 8.28 & 5.95 & \\
\hline 666 & 9/21/93:1420 & 225 & 57 & 350 & 1 & 13.32 & 5.65 & 205 & 26 & 318 & -5 & 11.84 & 8.74 & 5.05 & 188 & 54 & 150 & -3 & 11.44 & 10.03 & 5.68 & \\
\hline 666 & 9/21/93:1530 & 237 & 86 & 352 & 0 & 13.60 & 5.50 & 215 & 23 & 310 & -5 & 12.08 & 8.96 & 4.90 & 195 & 85 & 145 & -3 & 11.44 & 10.83 & 5.45 & \\
\hline 666 & 9/21/93:1630 & 238 & 64 & 353 & 1 & 13.62 & 5.50 & 213 & 26 & 312 & -5 & 12.10 & 9.09 & 4.70 & 192 & 52 & 122 & -4 & 11.16 & 12.06 & 5.40 & \\
\hline 667 & 9/22/93:0720 & 215 & 212 & 463 & 2 & 13.91 & 5.07 & 193 & 40 & 397 & -5 & 12.09 & 9.63 & 4.75 & 180 & 258 & 408 & -4 & 12.34 & 9.61 & 4.85 & \\
\hline 667 & 9/22/93:0930 & 213 & 247 & 472 & 1 & 14.03 & 5.18 & 197 & 41 & 402 & -4 & 12.42 & 9.78 & 4.50 & 173 & 167 & 265 & -4 & 11.70 & 12.52 & 5.00 & \\
\hline 668 & 9/22/93:1150 & 205 & 715 & 490 & 1 & 14.81 & 4.15 & 187 & 123 & 415 & -4 & 12.74 & 9.93 & 4.15 & 165 & 259 & 122 & -3 & 12.02 & 12.96 & 4.50 & \\
\hline 669 & 9/22/93:1300 & 215 & 234 & 453 & 1 & 13.97 & 5.15 & 190 & 74 & 400 & -4 & 12.64 & 9.88 & 4.12 & 174 & 231 & 101 & -3 & 11.84 & 12.86 & 4.82 . & \\
\hline 670 & 9/22/93:1420 & 215 & 236 & 421 & 0 & 14.05 & 5.10 & 191 & 91 & 383 & -4 & 12.79 & 9.97 & 3.95 & 170 & 286 & 35 & -3 & 11.96 & 12.80 & 4.60 & \\
\hline 671 & 9/22/93:1550 & 215 & 222 & 407 & 0 & 14.11 & 4.90 & 195 & 70 & 372 & -4 & 12.83 & 9.90 & 4.00 & 168 & 207 & 132 & -3 & 11.90 & 12.81 & 4.65 & \\
\hline 672 & 9/22/93:1700 & 215 & 309 & 397 & 1 & 14.20 & 4.85 & 197 & 65 & 352 & -4 & 12.79 & 9.94 & 4.02 & 173 & 152 & 107 & -3 & 11.73 & 13.01 & 4.80 & \\
\hline 678 & 9/29/93:0815 & 250 & 226 & 419 & 3 & 13.91 & 5.45 & 239 & 21 & 361 & -4 & 12.05 & 9.09 & 5.10 & 220 & 175 & 367 & -3 & 11.97 & 8.99 & 5.45 & base/abort \\
\hline 679 & 9/29/93:0800 & 250 & 491 & 372 & 2 & 13.59 & 5.50 & 239 & 24 & 312 & -4 & 11.67 & 8.67 & 5.20 & 207 & 405 & 310 & -4 & 11.37 & 10.37 & 5.60 & base \\
\hline 679 & 9/29/93:1100 & 258 & 363 & 349 & 1 & 13.62 & 5.45 & 248 & 26 & 298 & -5 & 11.67 & 8.57 & 5.35 & 216 & 254 & 153 & -2 & 11.21 & 10.60 & 5.70 & \\
\hline 679 & 9/29/93:1240 & 260 & 409 & 345 & 1 & 13.34 & 5.70 & 250 & 52 & 298 & -4 & 11.60 & 8.31 & 5.40 & 211 & 464 & 151 & -3 & 11.15 & 10.59 & 5.55 & \\
\hline 679 & 9/29/93:1500 & 256 & 607 & 347 & 1 & 13.43 & 5.45 & 254 & 32 & 301 & -4 & 11.74 & 8.50 & 5.15 & 212 & 377 & 115 & -2 & 11.30 & 10.76 & 5.65 & \\
\hline 697 & 10/08/93:0740 & 245 & 66 & 430 & -3 & 14.54 & 5.15 & 215 & 41 & 375 & -3 & 12.53 & 9.37 & 4.45 & 210 & 52 & 380 & -3 & 12.71 & 9.37 & 4.90 & Base \\
\hline 698 & 10/08/93:1040 & 220 & 24 & 373 & -3 & 14.83 & 5.25 & 220 & 24 & 373 & -3 & 12.58 & 9.30 & 4.48 & 198 & 32 & 113 & -2 & 12.02 & 12.19 & 5.30 & \\
\hline
\end{tabular}



Inlet StK $\Delta B$ h Util Overall $\mathrm{Na}$ ANOx Inout

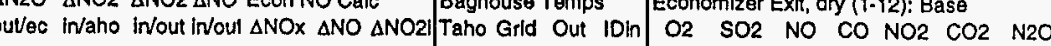

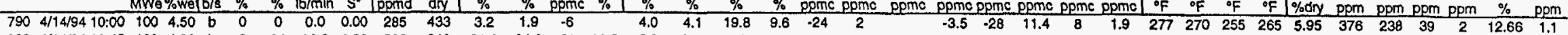

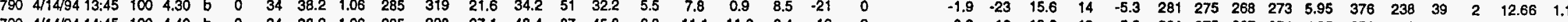

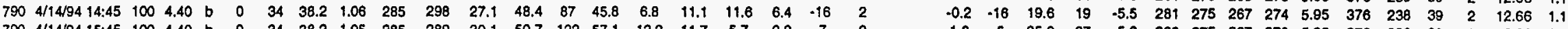

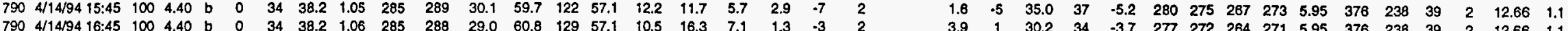

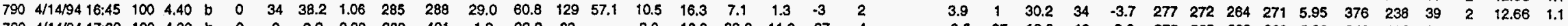

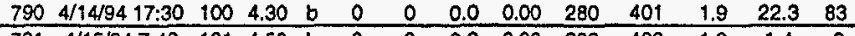

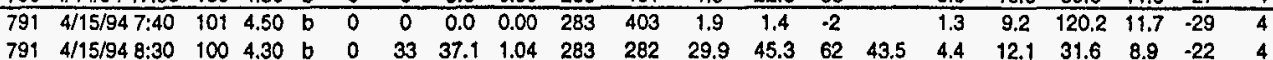
$\begin{array}{llllllllllllllllllllll}791 & 4 / 15 / 94 & 8: 30 & 100 & 4.30 & b & 0 & 33 & 37.1 & 1.04 & 283 & 282 & 29.9 & 45.3 & 62 & 43.5 & 4.4 & 12.1 & 31.6 & 8.9 & -22 & 4 \\ 791 & 4 / 15 / 94 & 9.15 & 100 & 4.40 & b & 0 & 32 & 35.9 & 1.01 & 283 & 271 & 32.4 & 43.4 & 44 & 42.9 & 7.8 & 11.5 & 18.8 & 4.2 & -11 & 4\end{array}$ $\begin{array}{llllllllllllllllllllll}791 & 4 / 15 / 94 & 9: 15 & 100 & 4.40 & \text { b } & 0 & 32 & 35.9 & 1.01 & 283 & 271 & 32.4 & 43.4 & 44 & 42.9 & 7.8 & 11.5 & 18.8 & 4.2 & -11 & 4 \\ 791 & 4 / 15 / 94 & 10: 15 & 100 & 4.40 & \text { b } & 0 & 32 & 35.9 & 1.02 & 283 & 263 & 34.2 & 58.0 & 87 & 55.1 & 11.2 & 16.4 & 11.4 & 1.1 & -3 & 4\end{array}$

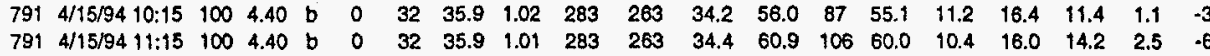
$\begin{array}{lllllllllllllllllllll}791 & 4 / 15 / 94 & 11: 15 & 100 & 4.40 & b & 0 & 32 & 35.9 & 1.01 & 283 & 263 & 34.4 & 60.9 & 106 & 60.0 & 10.4 & 16.0 & 14.2 & 2.5 & -6 \\ 791 & 4 / 15 / 94 & 12.15 & 100 & 4.40 & b & 0 & 32 & 35.9 & 1.00 & 283 & 273 & 32.6 & 64.8 & 130 & 64.4 & 18.7 & 24.2 & 4.6 & -7.0 & 17\end{array}$

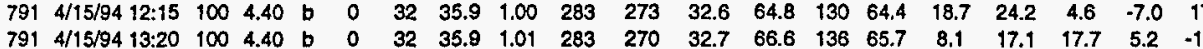
$\begin{array}{lllllllllllllllllllll}791 & 4 / 15 / 94 & 13: 20 & 100 & 4.40 & b & 0 & 32 & 35.9 & 1.01 & 283 & 270 & 32.7 & 66.6 & 136 & 65.7 & 8.1 & 17.1 & 17.7 & 5.2 & -1 \\ 791 & 4 / 15 / 94 & 14: 15 & 100 & 4.40 & b & 0 & 32 & 35.9 & 1.01 & 283 & 270 & 33.3 & 69.7 & 147 & 69.3 & 8.7 & 17.1 & 17.5 & 3.1 & -8\end{array}$ $\begin{array}{lllllllllllllllllllll}791 & 4 / 15 / 94 & 14: 15 & 100 & 4.40 & b & 0 & 32 & 35.9 & 1.01 & 283 & 270 & 33.3 & 69.7 & 147 & 69.3 & 8.7 & 17.1 & 17.5 & 3.1 & -8 \\ 791 & 4 / 15 / 94 & 15: 15 & 100 & 4.50 & b & 0 & 32 & 35.9 & 1.01 & 283 & 263 & 34.8 & 71.5 & 148 & 70.9 & 7.9 & 21.2 & 16.2 & 2.9 & -7\end{array}$

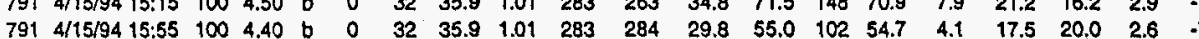

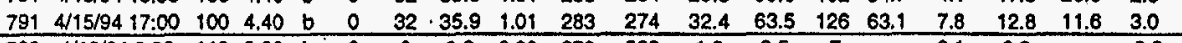

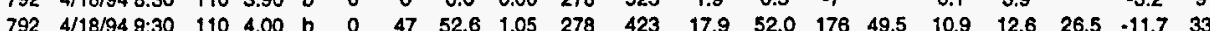

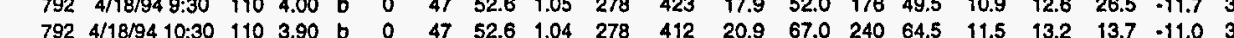

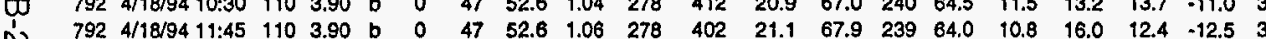

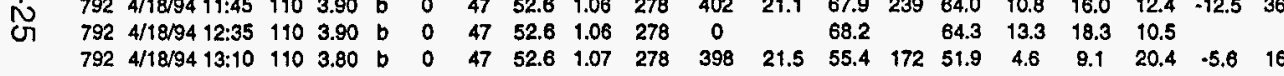

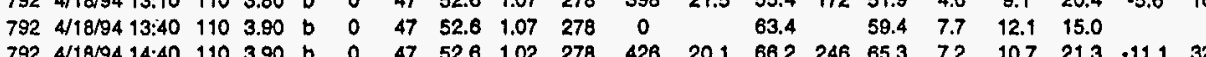

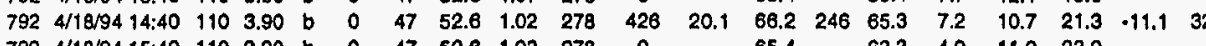

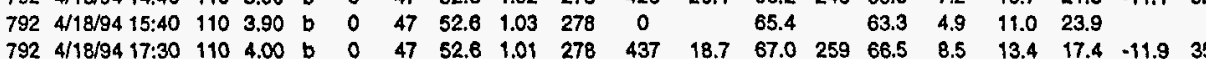

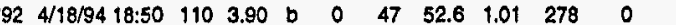

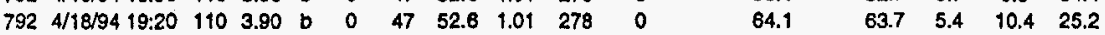

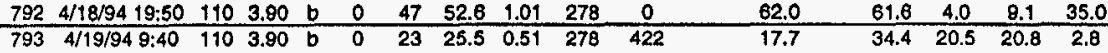

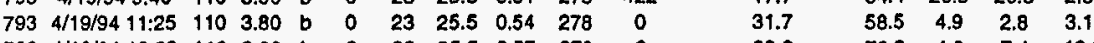

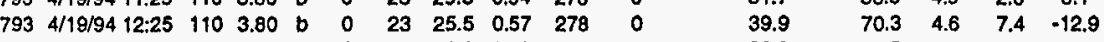

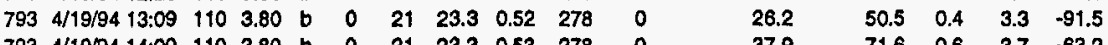

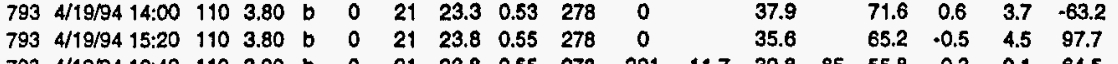

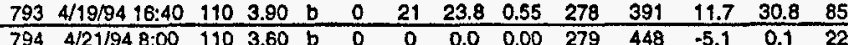
$\begin{array}{lllllllllllllllllllllll}794 & 4 / 21 / 94 & 10: 00 & 110 & 3.60 & 0 & 0 & 55 & 61.7 & 1.57 & 283 & 283 & 30.1 & 88.1 & 227 & 54.8 & 2.6 & -4.2 & -1.1 & -7.0 & 22\end{array}$ $\begin{array}{lllllllllllllllll}794 & 4 / 21 / 94 & 10: 35 & 110 & 3.60 & \text { b } & 0 & 55 & 61.7 & 1.57 & 283 & 0 & 90.6 & 57.7 & 19.6 & 19.9 & 2.3\end{array}$

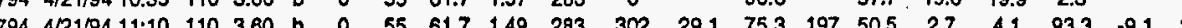

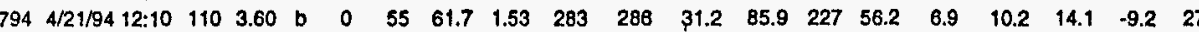

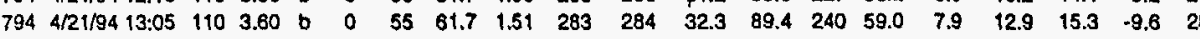

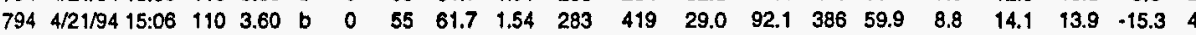

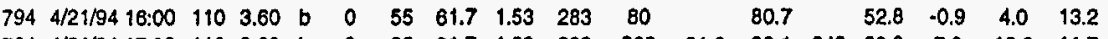

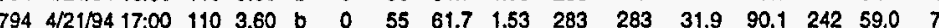

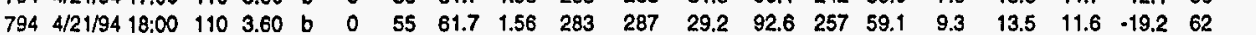

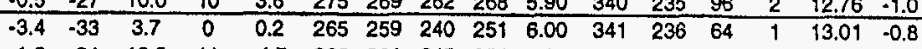
$\begin{array}{lllllllllllllllll}-1.5 & -24 & 12.5 & 11 & -4.7 & 268 & 261 & 245 & 254 & 6.00 & 341 & 238 & 84 & 1 & 13.01 & -0.8\end{array}$ $\begin{array}{cccccccccccccccc}4.3 & -6 & 22.2 & 26 & -4.3 & 267 & 261 & 250 & 256 & 6.00 & 341 & 236 & 64 & 1 & 13.01 & -0.8\end{array}$ $\begin{array}{lllllllllllllllll}9.9 & 7 & 31.9 & 42 & -0.8 & 269 & 264 & 25 & 259 & 6.00 & 341 & 236 & 64 & 1 & 13.01 & 0.0 .8\end{array}$ $\begin{array}{llllllllllllllll}10.1 & 4 & 29.7 & 40 & 1.2 & 273 & 267 & 254 & 281 & 6.00 & 341 & 236 & 64 & 1 & 13.01 & 0.8\end{array}$ $\begin{array}{llllllllllllllll}11.1 & 28 & 53.3 & 64 & 2.1 & 275 & 268 & 257 & 264 & 8.00 & 341 & 236 & 64 & 1 & 13.01 & -0.8\end{array}$ $\begin{array}{llllllllllllllll}7.9 & -5 & 22.9 & 31 & 0.0 & 27 & 271 & 259 & 261 & 6.00 & 341 & 236 & 64 & 1 & 13.01 & -0.8\end{array}$

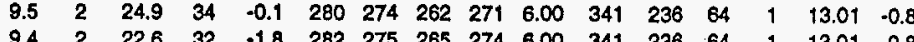

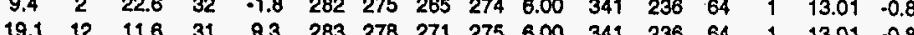
\begin{tabular}{cccccccccccccccc}
20.5 & 13 & 22.1 & 43 & 10.6 & 285 & 278 & 271 & 277 & 6.00 & 341 & 236 & 84 & 1 & 13.01 & -0.8 \\
\hline-4.1 & 5 & 0.2 & -4 & -3.9 & 286 & 281 & 263 & 274 & 5.40 & 466 & 241 & 56 & 2 & 13.49 & -1.6
\end{tabular} $\begin{array}{rlllllllllllllll}-8.7 & 24 & 30.6 & 22 & -13.7 & 291 & 285 & 272 & 281 & 5.40 & 466 & 241 & 56 & 2 & 13.49 & -1.6 \\ 15.5 & 46 & 32.2 & 48 & 9.8 & 294 & 289 & 28 & 287 & 5.40 & 46 & 241 & 56 & 2 & 13.49 & 1.6\end{array}$

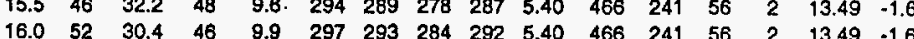

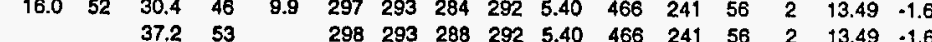

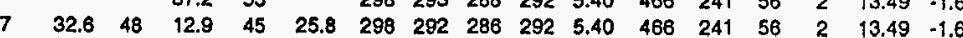
$\begin{array}{llllllllllllllll}7 & 21.8 & 58 & 298 & 294 & 288 & 293 & 5.40 & 468 & 241 & 56 & 2 & 13.49 & 1.6\end{array}$ $\begin{array}{lllllllllllllllll}7 & 33.1 & 65 & 20.1 & 53 & 26.9 & 297 & 293 & 286 & 294 & 5.40 & 466 & 241 & 56 & 2 & 13.49 & 1.6\end{array}$ $\begin{array}{llllllllllllllllll} & & & & 13.7 & 45 & & 296 & 294 & 289 & 294 & 5.40 & 468 & 241 & 56 & 2 & 1.49 & 1.6 \\ & 27.7 & 23.9 & 52 & 19.6 & 295 & 292 & 288 & 293 & 5.40 & 466 & 241 & 56 & 2 & 13.49 & -1.6\end{array}$ $\begin{array}{llllllllllllll}23.9 & 52 & 19.6 & 295 & 292 & 288 & 293 & 5.40 & 486 & 241 & 58 & 2 & 13.49 & -1.6\end{array}$

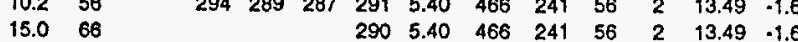
\begin{tabular}{llllllll}
289 & 5.40 & 466 & 241 & 58 & 2 & 13.49 & -1.8 \\
\hline 278 & 5.40 & 439 & 241 & 42 & 2 & 13.58 & 2.0
\end{tabular} $\begin{array}{lllllllllllllll}58.7 & 55 & 287 & 281 & 267 & 276 & 5.40 & 439 & 24 & 42 & 2 & 13.58 & 2.0 \\ 13.8 & 12 & 291 & 285 & 274 & 283 & 5.40 & 439 & 241 & 42 & 2 & 13.58 & 2.0\end{array}$ $\begin{array}{lllllllllllll}13.8 & 12 & 291 & 285 & 274 & 283 & 5.40 & 439 & 241 & 42 & 2 & 13.58 & 2.0 \\ 12.9 & 13 & 294 & 289 & 276 & 285 & 5.40 & 439 & 241 & 42 & 2 & 13.58 & 2.0\end{array}$ $\begin{array}{lllllllllllll}1.3 & 7 & 294 & 289 & 276 & 285 & 5.40 & 439 & 241 & 42 & 2 & 13.58 & 2.0\end{array}$ $\begin{array}{lllllllllllll}1.8 & 14 & 295 & 290 & 280 & 286 & 5.40 & 439 & 241 & 42 & 2 & 13.58 & 2.0 \\ -1.5 & 7 & & 294 & 289 & 279 & 280 & 5.40 & 439 & 241 & 42 & 2 & 1350\end{array}$ $\begin{array}{llllllllllllll}-1.5 & 7 & & 294 & 289 & 279 & 280 & 5.40 & 439 & 241 & 42 & 2 & 13.58 & 2.0\end{array}$ 
PSCC Arapahoo Uni

\begin{tabular}{|c|c|c|c|c|c|c|c|c|c|c|c|c|c|c|c|c|c|c|c|c|c|c|c|c|c|c|c|}
\hline Test & Date \& Time & $\mid \begin{array}{c}E c o n \\
O 2 \\
\% d r y\end{array}$ & $\begin{array}{l}\text { Exil, o } \\
\text { so2 } \\
\text { pom }\end{array}$ & $\begin{array}{l}\text { dy (1,2. } \\
\text { No } \\
\text { ppm }\end{array}$ & $\begin{array}{c}2,11,1 \\
\mathrm{Co} \\
\mathrm{ppm}\end{array}$ & $\begin{array}{l}\text { 2): } \mathrm{w} / \mathrm{s} \\
\mathrm{NO2} \\
\mathrm{pgm}\end{array}$ & $\begin{array}{c}\text { Sorb } \\
\text { CO2 } \\
\%\end{array}$ & $\begin{array}{l}\mathrm{N} 2 \mathrm{O} \\
\mathrm{ppm}\end{array}$ & $\begin{array}{l}\text { Bagho } \\
02 \\
\%\end{array}$ & $\begin{array}{l}\text { ouse in } \\
\text { SO2 } \\
\text { ppm }\end{array}$ & $\begin{array}{c}\text { nlet Ga } \\
\text { No } \\
\text { ppm }\end{array}$ & $\begin{array}{c}\text { Ias Anal } \\
\text { CO } \\
\mathrm{ppm}\end{array}$ & $\begin{array}{l}1 \text { alysis, } \\
\text { NO2 } \\
\mathrm{ppm}\end{array}$ & $\begin{array}{l}\text { 3) wel (AHC } \\
\begin{array}{l}\mathrm{CO} \\
\%\end{array}\end{array}$ & $\begin{array}{l}401-6 \\
420 \\
\%\end{array}$ & $\begin{array}{l}\text { 6. if dn } \\
\text { N2O } \\
\text { pom }\end{array}$ & $\begin{array}{l}\mathrm{iry}) \\
\mathrm{NH3} \\
\mathrm{ppm}\end{array}$ & \begin{tabular}{|l} 
Stack \\
02 \\
$\%$
\end{tabular} & $\begin{array}{l}\text { GasA } \\
\text { sO2 } \\
\text { ppm }\end{array}$ & $\begin{array}{l}\text { Analysi } \\
\text { NO } \\
\text { ppm }\end{array}$ & $\begin{array}{c}\text { sis, wet } \\
\text { co : } \\
\text { ppm }\end{array}$ & $\begin{array}{l}\mathrm{NO} \\
\mathrm{NO} 2 \\
\mathrm{pPm}\end{array}$ & $\begin{array}{c}\mathrm{CO} 2 \\
\%\end{array}$ & $\begin{array}{c}\mathrm{H} 2 \mathrm{O} \\
\%\end{array}$ & $\begin{array}{l}\mathrm{N} 20 \\
\mathrm{ppm}\end{array}$ & $\begin{array}{l}\mathrm{NH} 3 \\
\mathrm{ppm}\end{array}$ & Comments \\
\hline 790 & $4 / 14 / 9410: 00$ & 7.70 & 331 & 211 & $\frac{26}{26}$ & 1 & 11.22 & 1.0 & 4.60 & 351 & 206 & 51 & -3 & 11.918 & 8.69 & 2.9 & 1.0 & 5.65 & 332 & 209 & 28 & -1 & 11.35 & 8.36 & 2.7 & $\frac{1.1}{1.1}$ & baseline, blcarb on 1330 \\
\hline 790 & 4/14/94 13:45 & 7.75 & 300 & 215 & 23 & 1 & 11.21 & 0.8 & 4.45 & 260 & 199 & 103 & 4 & 12.129 & 9.05 & 2.7 & 2.4 & 5.60 & 203 & 205 & 59 & 0 & 11.41 & 8.48 & 1.1 & 2.4 & FFDC clean 1325-1400 \\
\hline 790 & 4/14/94 14:45 & 7.75 & 301 & 219 & 23 & 2 & 11.18 & 0.8 & 4.50 & 242 & 198 & 99 & 6 & 12.018 & 8.95 & 1.8 & 3.0 & 5.65 & 159 & 200 & 54 & 1 & 11.44 & 8.47 & 2.7 & 2.7 & \\
\hline 790 & 4/14/94 15:45 & 7.70 & 305 & 209 & 23 & 1 & 11.26 & 0.8 & 4.40 & 235 & 192 & 178 & 7 & $12.28 \mathrm{~g}$ & 9.25 & 6.1 & 3.2 & 5.55 & 126 & 188 & 66 & 3 & 11.53 & 8.61 & 2.5 & 3.2 & \\
\hline 790 & $4 / 14 / 94+16: 45$ & 8.05 & 292 & 219 & 17 & 1 & 10.93 & 0.4 & 4.55 & 233 & 197 & 118 & 8 & 12.169 & 9.02 & 3.4 & 2.0 & 5.60 & 120 & 189 & 51 & 4 & 11.48 & 8.67 & 2.6 & 3.2 & \\
\hline 790 & 4/14/94 17:30 & 8.20 & 291 & 229 & 15 & 1 & 10.92 & -0.4 & 4.20 & 331 & 204 & 197 & -2 & 12.349 & 9.31 & 7.0 & 0.6 & 5.55 & 241 & 205 & 89 & 1 & 11.46 & 8.63 & 2.1 & 1.3 & Bicarb off 1700 \\
\hline 791 & $4 / 15 / 947: 40$ & 8.35 & 289 & 216 & 20 & 2 & 11.05 & .0 .4 & 4.40 & 329 & 207 & 102 & -2 & 12.509 & 9.11 & 2.9 & 0.0 & 5.85 & 301 & 210 & 56 & -1 & 11.62 & 8.52 & 2.6 & 0.5 & baseline, blcarb on 0800 \\
\hline 791 & $4 / 15 / 948: 30$ & 8.05 & 290 & 221 & 21 & 2 & 11.14 & 0.4 & 4.50 & 229 & 199 & 99 & 4 & $12.38 \mathrm{~g}$ & 9.06 & 2.3 & 3.3 & 6.00 & 162 & 200 & 42 & 0 & 11.57 & 8.46 & 2.2 & 2.5 & FFDC clean 0840-0915 \\
\hline 791 & 4/15/94 9:15 & 8.05 & 289 & 212 & 29 & 2 & 11.13 & 0.5 & 4.50 & 220 & 196 & 58 & 8 & 12.459 & 9.08 & 3.4 & 3.5 & 5.75 & 170 & 192 & 43 & 5 & 11.62 & 8.51 & 2.4 & 3.7 & \\
\hline 791 & $4 / 15 / 9410: 15$ & 8.00 & 289 & 217 & 22 & 2 & 11.21 & 0.2 & 4.30 & 216 & 195 & 103 & 10 & 12.639 & 9.23 & 5.1 & 2.7 & 5.70 & 132 & 181 & 50 & 9 & 11.70 & 8.59 & 2.0 & 2.5 & \\
\hline 791 & $4 / 15 / 9411: 15$ & 8.30 & 283 & 213 & 21 & 1 & 10.82 & 0.0 & 4.35 & 215 & 195 & 164 & 9 & 12.589 & 9.20 & 3.7 & 2.9 & 5.90 & 116 & 180 & 70 & 9 & 11.53 & 8.52 & 2.4 & 2.8 & \\
\hline 791 & $4 / 15 / 9412: 15$ & 8.20 & 288 & 216 & 21 & 1 & 11.18 & 0.3 & 4.60 & 220 & 192 & 92 & 9 & 12.369 & 9.01 & 4.0 & 3.0 & 8.00 & 105 & 161 & 47 & 9 & 11.48 & 8.36 & 1.1 & 2.8 & \\
\hline 791 & $4 / 15 / 94$ 13:20 & 8.45 & 280 & 219 & 18 & $i$ & 11.01 & 0.6 & 4.60 & 218 & 193 & 90 & 8 & 12.259 & 9.00 & 4.3 & 2.5 & 5.80 & 100 & 188 & 51 & 7 & 11.59 & 8.54 & 2.3 & 2.0 & \\
\hline 791 & $4 / 15 / 9414: 15$ & 8.40 & 283 & 218 & 22 & 1 & 10.92 & -0.2 & 4.70 & 216 & 193 & 86 & 9 & 12.238 & 8.95 & 3.8 & 2.1 & 6.00 & 90 & 183 & 51 & 8 & 11.40 & 8.39 & 2.5 & 2.7 & \\
\hline 791 & 4/15/94 15:15 & 8.55 & 279 & 229 & 14 & 1 & 10.80 & $\cdot 0.8$ & 4.45 & 214 & 197 & 76 & 11 & 12.428 & 8.07 & 1.6 & 3.0 & 5.90 & 85 & 188 & 46 & 8 & 11.54 & 8.45 & 2.0 & 2.2 & FFDC clean 1520-1555 \\
\hline 791 & $4 / 15 / 9415: 55$ & 8.55 & 280 & 228 & 14 & 1 & 10.86 & -1.0 & 4.95 & 224 & 201 & 59 & 9 & 12.058 & 8.83 & 2.2 & 2.9 & 5.90 & 935 & 187 & 41 & 15 & 11.49 & 8.43 & 1.0 & 2.3 & \\
\hline 791 & 4/15/94 17:00 & 8.10 & 290 & 215 & 23 & 1 & 11.14 & -0.4 & 4.65 & 220 & 196 & 49 & $\theta$ & 12.258 & 8.91 & 1.5 & 2.9 & 5.85 & 110 & 179 & 35 & 17 & 11.50 & 8.37 & 1.2 & 2.3 & \\
\hline 792 & $4 / 18 / 948: 30$ & 7.50 & 400 & 217 & 55 & 1 & 11.88 & .0 .5 & 5.35 & 455 & 250 & 34 & 2 & 13.490 & 0.00 & -1.0 & 1.2 & 5.20 & 410 & 218 & 33 & -2 & 11.97 & 8.99 & 1.1 & -0.8 & Baseline, blcarb on 0900 \\
\hline 792 & 4/18/94 8:30 & 7.40 & 389 & 214 & 54 & 2 & 11.54 & 0.0 & 5.25 & 370 & 241 & 55 & 6 & 13.560 & 0.00 & 0.4 & 1.0 & 5.10 & 193 & 200 & 51 & .5 & 11.59 & 8.80 & 4.9 & 3.2 & \\
\hline 792 & $4 / 18 / 94 \quad 10: 30$ & 7.35 & 395 & 215 & 45 & 2 & 12.00 & .0 .8 & 5.10 & 364 & 239 & 88 & 7 & 13.580 & 0.00 & -1.6 & 1,2 & 4.95 & 135 & 189 & 51 & 14 & 12.35 & 9.10 & 2.0 & 2.2 & \\
\hline 792 & $4 / 18 / 9411: 45$ & 7.55 & 381 & 221 & 41 & 1 & 91.62 & 1.1 & 5.30 & 351 & 242 & 65 & 7 & 13.520 & 0.00 & 0.7 & 9.1 & 5.10 & 128 & 181 & 42 & 14 & 12.01 & 8.78 & 1.5 & 2.5 & \\
\hline 792 & 4/18/94 12:35 & 7.55 & 381 & 221 & 41 & 1 & 11.62 & 1.1 & & & & & & & & & & 5.20 & 125 & 174 & 38 & 14 & 12.09 & 9.10 & 1.6 & 2.9 & FFDC clean $1235-1310$ \\
\hline 782 & $4 / 18 / 94+3 ; 10$ & 7.50 & 380 & 219 & 53 & 2 & 11.84 & 1.9 & 5.25 & 348 & 240 & 62 & 8 & 13.600 & 0.00 & 0.2 & 0.9 & 5.20 & 175 & 180 & 49 & 27 & 12.14 & 8.92 & 0.6 & 2.8 & \\
\hline 792 & $4 / 18 / 9413: 40$ & 7.50 & 380 & 218 & 53 & 2 & 11.84 & 1.9 & & & & & & & & & & 5.05 & 145 & 172 & 49 & 30 & 12.18 & 8.91 & 1.1 & 2.2 & \\
\hline 792 & $4 / 18 / 94 / 14: 40$ & 7.35 & 404 & 220 & 69 & 1 & 11.89 & 1.8 & 5.35 & 370 & 247 & 48 & 7 & 13.480 & 0.00 & -0.4 & 9.6 & 5.10 & 140 & 175 & 63 & 27 & 12.26 & 8.99 & 1.9 & 1.5 & \\
\hline 792 & 4/18/94 15:40 & 7.40 & 395 & 225 & 34 & 1 & 11.63 & -0.1 & & & & & & & & & & 5.30 & 139 & 179 & 22 & 26 & 12.01 & 8.81 & 1.1 & 2.8 & \\
\hline 792 & $4 / 18 / 9417: 30$ & 7.20 & 412 & 225 & 21 & 2 & 11.84 & 0.3 & 5.30 & 381 & 245 & 33 & 9 & 13.360 & 0.00 & 0.7 & 1.2 & 5.15 & 138 & 176 & 26 & 23 & 12.10 & 8.87 & 1.8 & 3.3 & \\
\hline 792 & $4 / 18 / 9418: 50$ & 7.20 & 412 & 225 & 21 & 2 & 11.84 & 0.3 & & & & & & & & & & 5.15 & 195 & 172 & 22 & 37 & 12.13 & 9.20 & 1.3 & 2.4 & FFDC clean 1815-1850 \\
\hline 792 & 4/18/94 19:20 & 7.20 & 412 & 225 & 21 & 2 & 11.84 & 0.3 & & & & & & & & & & 5.10 & 150 & 165 & 28 & 41 & 12.08 & 0.12 & 1.5 & 3.0 & \\
\hline 792 & $4 / 18 / 94 \quad 19: 50$ & 7.20 & 412 & 225 & 21 & 2 & 11,84 & 0.3 & & & & & & & & & & 5.15 & 158 & 168 & 31 & 40 & 12.17 & 8.13 & 1.8 & 1.9 & \\
\hline 793 & $4 / 19 / 849: 40$ & 7.10 & 396 & 216 & 25 & 2 & 12.32 & -1.2 & & & & & & & & & & 2.00 & 400 & 213 & 42 & -2 & 12.30 & 9.28 & 0.1 & 1.5 & \\
\hline 793 & 4/19/94 11:25 & 7.00 & 377 & 212 & 257 & 2 & 12.19 & 2.9 & & & & & & & & & & 5.20 & 255 & 205 & 131 & 1 & 12.20 & 8.20 & 2.1 & 2.4 & \\
\hline 793 & 4/19/94 12:25 & 7.20 & 355 & 220 & 31 & 2 & 11.89 & -0.8 & & & & & & & & & & 5.15 & 215 & 205 & 48 & 2 & 12.17 & 9.27 & 0.5 & 3.3 & \\
\hline 793 & 4/19/94 13:09 & 7.20 & 355 & 220 & 31 & 2 & 11.89 & -0.8 & & & & & & & & & & 5.10 & 265 & 210 & 32 & 7 & 12.15 & 8.26 & 0.8 & 2.3 & FFOC cclean 1230.1305 \\
\hline 793 & 4/19/94 14:00 & 7.10 & 350 & 222 & 32 & 2 & 12.20 & .0 .5 & & & & & & & & & & 5.10 & 218 & 205 & 25 & 11 & 12.13 & 0.28 & 0.9 & 3.5 & \\
\hline 793 & $4 / 19 / 9415: 20$ & 7.20 & 344 & 225 & 27 & 1 & 11,95 & -0.8 & & & & & & & & & & 6.00 & 211 & 198 & 23 & 8 & 11.53 & 8.88 & 0.8 & 2.8 & \\
\hline 793 & 4/19/94 16:40 & 7.50 & 332 & 230 & 22 & 2 & 11.66 & 0.4 & 5.30 & 341 & 255 & 28 & 5 & 13.640 & 0.00 & -0.3 & 2.1 & 5.20 & 235 & 211 & 27 & 5 & 12.07 & 9.44 & 1.4 & 3.6 & \\
\hline 794 & $4 / 21 / 948: 00$ & 6.70 & 339 & 222 & 58 & 1 & 12.69 & 0.0 & 5.10 & 396 & 275 & 65 & 3 & 14.960 & 0.00 & -2.1 & 1.7 & 4.90 & 333 & 230 & 52 & .2 & 12.52 & 9.71 & 0.1 & 1.0 & Start feod 10900 \\
\hline 794 & $4 / 21 / 94$ 10:00 & 6.90 & 317 & 222 & 44 & 2 & 11.92 & 1.0 & 4.90 & 253 & 244 & 71 & 10 & 13.990 & 0.00 & 0.3 & 2.0 & 4.90 & 44 & 196 & 71 & 5 & 12.52 & 9.59 & 1.3 & 4.4 & \\
\hline 794 & $4 / 21 / 94 \quad 10: 35$ & 6.90 & 317 & 222 & 44 & 2 & 11.82 & 1.0 & & & & & & & & & & 4.85 & 30 & 180 & & & 12.52 & 8.59 & & & FFDC \\
\hline 794 & $4 / 21 / 9411: 10$ & 7.10 & 329 & 222 & 61 & $i$ & 12.45 & 1.8 & 5.20 & 265 & 257 & 62 & 19 & 94.730 & 0.00 & 0.3 & 2.1 & 5.10 & 81 & 185 & 53 & 29 & 12.38 & 8.82 & 4.5 & 3.8 & \\
\hline 794 & $4 / 21 / 9412: 10$ & 7.00 & 323 & 228 & 41 & 2 & 12.10 & Q.6 & 5.00 & 254 & 250 & 45 & 10 & 13.790 & 0.00 & 0.7 & 2.0 & 4.90 & 46 & 173 & 48 & 34 & 12.58 & 9.67 & 1.2 & 4.6 & \\
\hline 794 & $4 / 21 / 9413: 05$ & 7.40 & 317 & 226 & 41 & 2 & 11.90 & 0.1 & 5.10 & 259 & 248 & 40 & 11 & 13.590 & 0.00 & 3.0 & 2.2 & 5.00 & 35 & 172 & 36 & 32 & 12.45 & 9.47 & 1.7 & 3.6 & \\
\hline 794 & $4 / 21 / 9415: 06$ & 7.40 & 312 & 227 & 39 & 2 & 11.78 & 0.9 & 5.40 & 254 & 253 & 44 & 12 & 13.920 & 0.00 & -0.2 & 1.4 & 5.30 & 25 & 171 & 37 & 28 & 12.21 & 9.25 & 1.7 & 4.4 & \\
\hline 794 & $4 / 21 / 94,16: 00$ & 7.40 & 314 & 225 & 33 & 1 & 11.97 & 0.1 & & & & & & & & & & 5.40 & 61 & 170 & 31 & 49 & $\$ 1.99$ & 9.05 & 1.3 & 4.2 & FFDC clean 1520-1555 \\
\hline 794 & $4 / 21 / 9417: 00$ & 7.30 & 317 & 236 & 35 & 2 & 12.02 & .0 .2 & 5.30 & 247 & 250 & 40 & 11 & 13.660 & 0.00 & 0.5 & 1.4 & 5.00 & 32 & 160 & 49 & 46 & 12.38 & 9.18 & 1.0 & 4.4 & \\
\hline 794 & 4/21/94 18:00 & 7.30 & 309 & 226 & 39 & 2 & 11.80 & 0.6 & 5.40 & 249 & 266 & 32 & 12 & 13.910 & 0.00 & 0.0 & 2.4 & 5.10 & 24 & 159 & 34 & 42 & 12.36 & 9.10 & 1.4 & 4.0 & \\
\hline
\end{tabular}




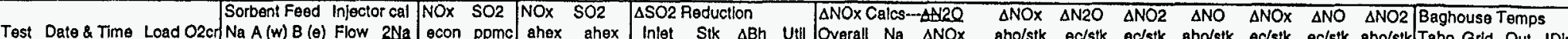

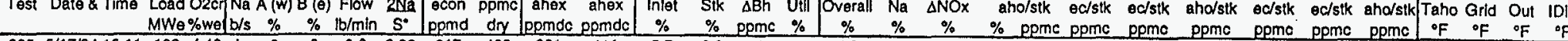

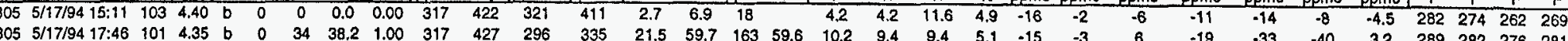

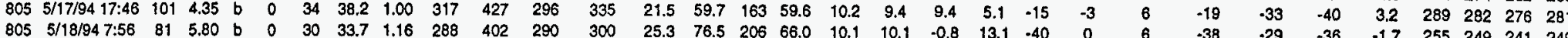

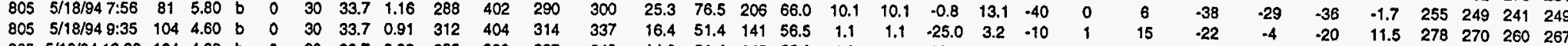

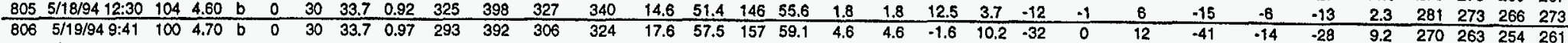

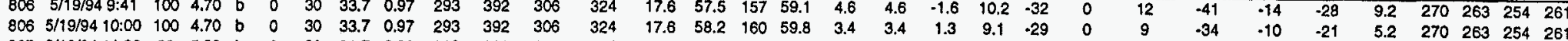

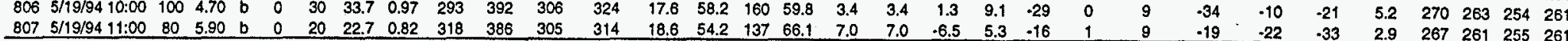

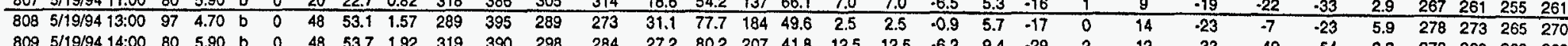

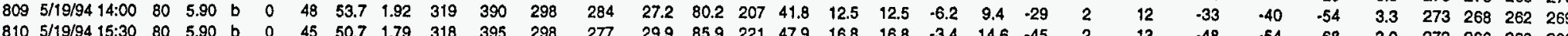

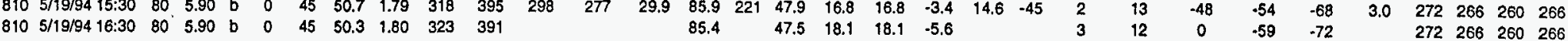

\begin{tabular}{|c|c|c|c|c|c|c|c|c|c|c|c|c|c|c|c|c|c|c|c|c|c|c|c|c|c|c|c|c|c|c|c|}
\hline 812 & 5/23/94 10:38 & 103 & 3.40 & $s$ & 0 & 0 & 0.0 & 0.00 & 277 & 461 & 289 & 457 & 0.8 & 4.8 & 18 & & $\cdot 3.7$ & -3.8 & 2.5 & 0.4 & -1 & 0 & -4 & 3 & 10 & 15 & -4.1 & 281 & 274 & 263 & \\
\hline 812 & & 102 & 3.40 & 8 & 0 & 45 & 39.4 & 0.94 & & 507 & & & & 29.0 & & 31.0 & & -9.4 & & & & 7 & -1 & & & & & 287 & 280 & 271 & 276 \\
\hline 813 & $5 / 23 / 94 \quad 14: 00$ & 110 & 3.90 & $s$ & 0 & 45 & 39.6 & 9.15 & 325 & 385 & 325 & 365 & 5.3 & & & & & & & & & & & & & & $\cdot 1.9$ & 292 & 286 & 278 & \\
\hline 813 & $5 / 23 / 9415: 40$ & 107 & 3.90 & s & 0 & 45 & 39.6 & 1.18 & & 385 & 294 & 311 & 19.2 & 31.2 & 97 & 26.4 & & 9.4 & & 6.0 & .18 & 4 & .2 & -10 & & & -8.5 & 290 & 283 & 276 & \\
\hline 814 & 5/23/94 16:05 & 108 & 3.80 & $s$ & 0 & 75 & 67.6 & 2.00 & & 385 & 308 & 293 & 24.0 & 74.7 & 195 & 37.4 & & 13.8 & & 15.4 & .49 & 2 & $B$ & -47 & & & -2.0 & 288 & 283 & 277 & \\
\hline 815 & $5 / 23 / 94 \quad 17: 45$ & 95 & 4.00 & 8 & 0 & 75 & 67.6 & 1.66 & & 524 & 278 & 402 & 23.3 & 67.9 & 234 & 40.8 & & 10.6 & & 8.9 & -26 & 4 & 14 & -29 & & & 3.1 & 282 & 278 & 275 & \\
\hline 816 & $5 / 23 / 94$ 19:25 & 95 & 4.10 & 3 & 0 & 45 & 39.4 & 0.98 & & 527 & 273 & 441 & 16.3 & 50.1 & 178 & 52.0 & & 8.8 & & 8.7 & -24 & 5 & 11 & -28 & & & 3.2 & 277 & 272 & 269 & 274 \\
\hline 817 & $5 / 24 / 948: 10$ & 106 & 3.90 & 8 & 0 & 0 & 0.0 & 0.00 & & 493 & 298 & 491 & 0.4 & -3.8 & .21 & & & 6.7 & & 5.7 & .17 & 2 & -3 & -13 & & & -4.6 & 277 & 269 & 254 & \\
\hline 817 & $5 / 24 / 9411: 35$ & 105 & 3.90 & s & 0 & 71 & 63.6 & 1.50 & & 496 & 285 & 410 & 17.3 & 54.7 & 188 & 36.5 & & 10.2 & & 6.7 & -20 & 3 & 4 & -13 & & & -6.4 & 285 & $2 \pi$ & 270 & \\
\hline 817 & $5 / 24 / 9412: 40$ & 104 & 4.10 & $\mathbf{s}$ & 0 & 71 & 63.6 & 1.42 & & 529 & 280 & 414 & 21.9 & 63.7 & 221 & 44,9 & & 12.0 & & 9.1 & -28 & 7 & 8 & -25 & & & -1.5 & 286 & 279 & 277 & \\
\hline 817 & $5 / 24 / 94$ 14:11 & 104 & 4.10 & 8 & 0 & 71 & 63.6 & 1.50 & & 501 & 275 & 401 & 20.0 & 48.3 & 142 & 32.2 & & 8.3 & & 4.5 & .13 & 8 & 15 & -20 & & & 6.8 & 284 & 278 & 273 & \\
\hline 817 & $5 / 24 / 94 \quad 15: 09$ & 105 & 4.00 & 8 & 0 & 71 & 63.8 & 1.47 & & 507 & 271 & 387 & 23.7 & 54.9 & 154 & 36.9 & & 8.4 & & 6.2 & -17 & 4 & 12 & -21 & & & 3.5 & 284 & 278 & 273 & \\
\hline 817 & 5/24/94 16:09 & 104 & 3.90 & $s$ & 0 & 71 & 63.6 & 1.55 & & 484 & 281 & 384 & 20.7 & 55.8 & 170 & 36.0 & & 7.5 & & 8.8 & -26 & 5 & 11 & .29 & & & 3.3 & 278 & 272 & 268 & \\
\hline 817 & 5/24/94 17:10 & 105 & 4.10 & s & 0 & 71 & 63.6 & 1.57 & & 473 & 278 & 380 & 19.7 & 59.8 & 190 & 38.0 & & 8.5 & & 8.6 & -28 & 7 & 11 & -31 & & & 3.4 & 280 & 273 & 267 & \\
\hline 817 & $5 / 24 / 94$ 18:00 & 105 & 4.10 & s & 0 & 71 & 63.6 & 1.57 & & 473 & 272 & 373 & 21.2 & 63.4 & 199 & 40.3 & & 11.1 & & 7.1 & .20 & 5 & 10 & -22 & & & 2.3 & 280 & 273 & 268 & 274 \\
\hline 817 & $5 / 24 / 94 \quad 18: 40$ & 105 & 4.10 & s & 0 & 71 & 63,6 & 1.80 & & 465 & & & & B3.9 & & 40.0 & & 6.7 & & & & 5 & 10 & & & & 10.9 & 280 & 273 & 268 & 289 \\
\hline 817 & $5 / 24 / 94 \quad 18: 17$ & 105 & 4.10 & $\mathrm{~s}$ & 0 & 71 & 63.6 & 1.57 & & 473 & & & & 41.6 & & 26.4 & & 7.2 & & & & 4 & 12 & & & & 13.8 & 280 & 273 & 268 & 27 \\
\hline
\end{tabular}




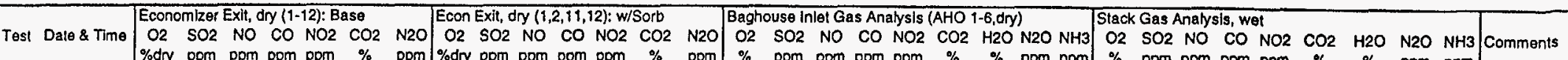

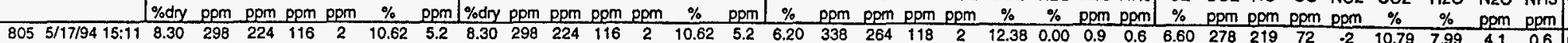

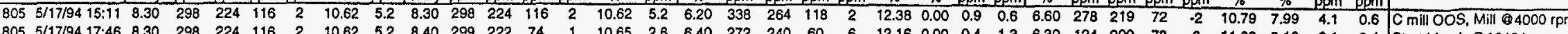

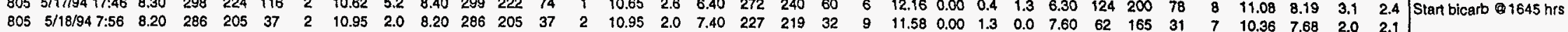

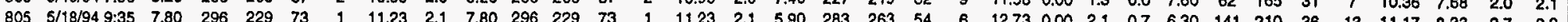

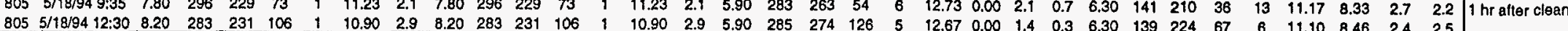

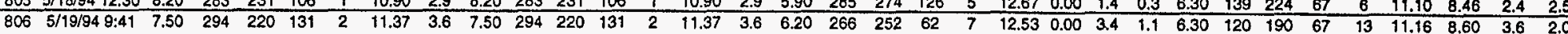

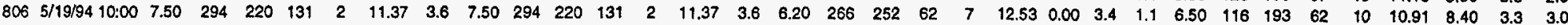

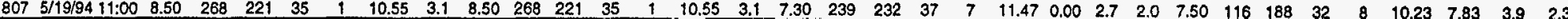
$\begin{array}{llllllllllllllllllllllllllllllllllll}808 & 5 / 19 / 94 & 13: 00 & 7.80 & 290 & 212 & 106 & 1 & 11.29 & 3.7 & 7.80 & 290 & 212 & 106 & 1 & 11.29 & 3.7 & 6.60 & 218 & 231 & 45 & 9 & 12.22 & 0.00 & 2.4 & 1.2 & 6.50 & 62 & 187 & 50 & 12 & 10.97 & 8.71 & 3.6 & 3.1\end{array}$

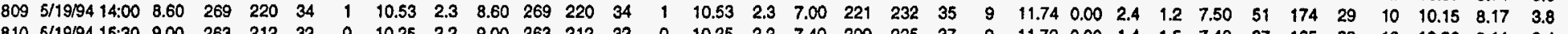

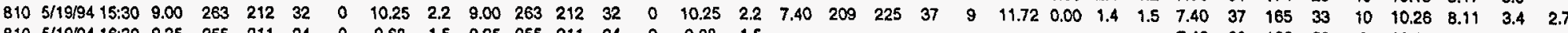
$\begin{array}{llllllllllllllllllllllllllll}810 & 5 / 19 / 94 & 16: 30 & 9.25 & 255 & 211 & 24 & 0 & 9.68 & 1.5 & 9.25 & 25 & 211 & 24 & 0 & 9.68 & 1.5 & 7.40 & 38 & 168 & 30 & 9 & 10.18 & 8.07 & 3.7 & 2.9\end{array}$

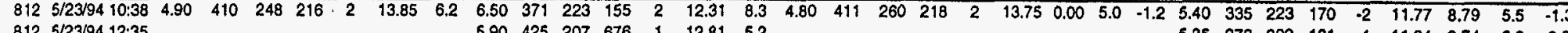

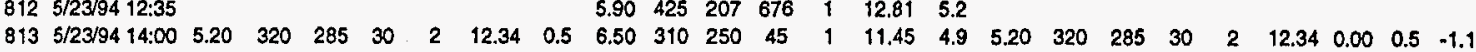

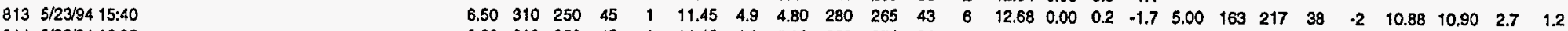

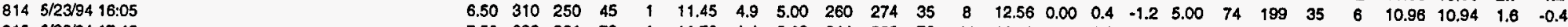

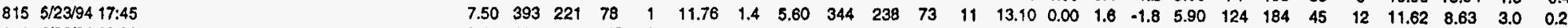

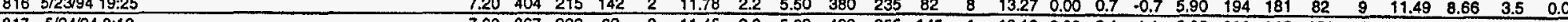

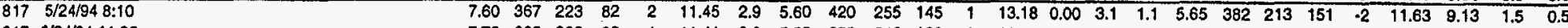
$8175 / 24 / 9411: 35$ $8175 / 249412: 40$ $8175 / 24 / 9414: 11$

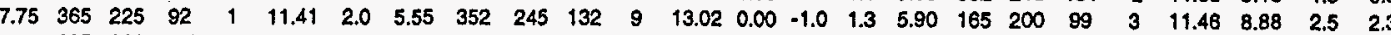

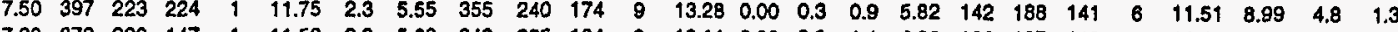

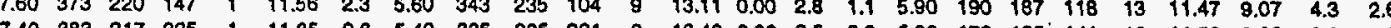

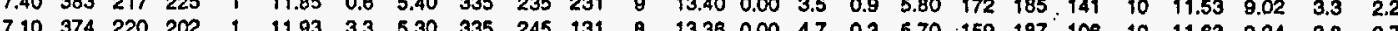

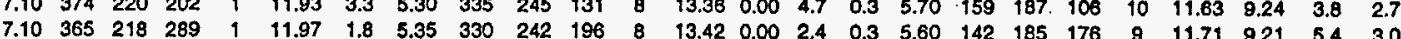

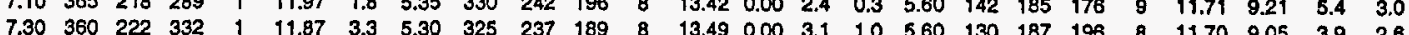
$7.30360202332,11.87$ (3.3 $\begin{array}{llllllllll}5.60 & 128 & 186 & 199 & 8 & 11.10 & 9.00 & 3.8 & 3.0\end{array}$ 


\begin{tabular}{|c|c|c|c|c|c|c|c|c|c|c|c|c|c|c|c|c|c|c|c|c|c|c|c|c|c|c|c|c|c|}
\hline \multirow{3}{*}{ Test } & \multirow{3}{*}{ Date \& Time } & \multirow{3}{*}{\multicolumn{2}{|c|}{$\begin{array}{l}\text { Load O2cr } \\
\text { MWe\%We }\end{array}$}} & \multicolumn{5}{|c|}{ Sorbent Feed Injector cal } & \multirow{3}{*}{\multicolumn{2}{|c|}{$\begin{array}{cc}\text { NOx } & \mathrm{SO} 2 \\
\text { econ } & \text { ppmc } \\
\text { ppmed } & \text { dry }\end{array}$}} & \multirow{3}{*}{$\begin{array}{l}\text { NOx } \\
\text { ahex } \\
\text { ppmed }\end{array}$} & \multirow{3}{*}{$\begin{array}{l}\mathrm{SO} 2 \\
\text { ahex } \\
\text { epmed }\end{array}$} & \multirow{2}{*}{\multicolumn{3}{|c|}{$\begin{array}{l}\triangle S \mathrm{SO} 2 \text { Reduction } \\
\text { Stack UtI }\end{array}$}} & \multirow{2}{*}{\multicolumn{2}{|c|}{$\begin{array}{l}\triangle N O x-\triangle N 2 Q \\
\text { Overall } \triangle N O x\end{array}$}} & \multirow{2}{*}{\multicolumn{2}{|c|}{$\begin{array}{l}\triangle N O X \\
\text { aho/stk }\end{array}$}} & \multirow{3}{*}{$\begin{array}{l}\Delta \mathrm{N} 20 \\
\text { ed/stk } \\
\text { ppmc }\end{array}$} & \multirow{3}{*}{$\begin{array}{l}\triangle \mathrm{NO2} \\
\text { ec/stk } \\
\text { ppmc }\end{array}$} & \multirow{3}{*}{$\begin{array}{c}\Delta N O \\
\text { aho/stk } \\
\text { ppme }\end{array}$} & \multirow{3}{*}{$\begin{array}{l}\Delta N O x \\
\text { ed/stk } \\
\text { ppmc }\end{array}$} & \multirow{3}{*}{$\begin{array}{l}\Delta N O \\
\text { ecistk } \\
\text { ppmc }\end{array}$} & \multirow{3}{*}{$\begin{array}{c}\Delta \mathrm{NO} 2 \\
\text { aho/stk } \\
\mathrm{ppmc}\end{array}$} & \multirow{2}{*}{\multicolumn{4}{|c|}{ Baghouse Temps }} \\
\hline & & & & & & & & $2 \mathrm{Na}$ & & & & & & & & & & & & & & & & & & & & & \\
\hline & & & & & $\%$ & & & & & & & & & & & & & & ppme & & & & & & & ${ }^{\circ} \mathrm{F}$ & & & \\
\hline 818 & $5 / 25 / 9411: 45$ & 108 & 4.40 & s & 0 & 66 & 59.4 & 1.54 & 313 & 439 & 273 & 384 & 1.6 & 7 & 1.1 & 0.0 & & -3.0 & 9 & 0 & -8 & 6 & -0.1 & 8 & 1.0 & 281 & 273 & 262 & 269 \\
\hline 818 & $5 / 25 / 94 \quad 12: 35$ & 108 & 4.50 & $\mathbf{s}$ & 0 & 66 & 59.4 & 1.54 & 313 & 439 & 273 & 384 & 52.8 & 232 & 34.4 & 5.8 & -0.2 & 3.0 & -9 & 0 & -6 & -9 & -18.3 & -13 & 2.8 & 284 & 272 & 266 & 270 \\
\hline 818 & $5 / 25 / 94$ 13:12 & 108 & 4.30 & $\mathbf{s}$ & 0 & 66 & 59.4 & 1.51 & 310 & 447 & 272 & 390 & 52.0 & 233 & 34.5 & 3.4 & -47.8 & 1.3 & -4 & & & -11 & -10.6 & -17 & 15.1 & 284 & 273 & 269 & 273 \\
\hline 818 & 5/25/94 14:10 & 108 & 4.30 & $\mathbf{s}$ & 0 & 66 & 59.4 & 1.51 & 310 & 447 & 272 & 390 & 58.3 & 261 & 38.6 & 10.5 & -17.8 & 8.6 & -26 & c & 8 & -26 & -32.9 & -41 & 16.4 & 284 & 273 & 3269 & 273 \\
\hline 818 & 5/25/94 15:12 & 108 & 4.30 & $\mathbf{s}$ & 0 & 66 & 59.4 & 1.51 & 310 & 447 & 272 & 390 & 60.7 & 272 & 40.2 & 8.7 & -15.7 & 6.7 & -20 & & & -21 & .27 .0 & -32 & 13.1 & 279 & 268 & 263 & 270 \\
\hline 818 & 5/25/94 16:06 & 108 & 4.20 & $s$ & 0 & 66 & 59.4 & 1.51 & 310 & 447 & 272 & 390 & 63.0 & 282 & 41.7 & 7.7 & -30.3 & 5.7 & -17 & & & -19 & -24.1 & -28 & 11.8 & 277 & 266 & 263 & 267 \\
\hline 818 & 4 17:06 & 106 & 4.20 & $\mathbf{s}$ & 0 & 64 & 57.7 & 1.49 & 334 & 448 & $2 \pi$ & 390 & 64.5 & 289 & 43.3 & 12.2 & -7.9 & 4.9 & -15 & 3 & 2 & -15 & -41.1 & .43 & 11.5 & 276 & 266 & 263 & 267 \\
\hline 818 & $5 / 25 /$ & 105 & 4.20 & $s$ & 0 & 64 & 57.7 & 1.51 & 334 & 448 & $2 \pi 7$ & 390 & 54.7 & 245 & 36.3 & 14.9 & -4.0 & 7.8 & -24 & 2 & 14 & -25 & -50.3 & -64 & 22.9 & 275 & 264 & 258 & 265 \\
\hline 818 & 18:53 & 105 & 4.20 & $s$ & 0 & 64 & 57.7 & 1.51 & 334 & 448 & 277 & 390 & 59.4 & 266 & 39.5 & 14.2 & -2.1 & 7.0 & -22 & 1 & 10 & -24 & -47.7 & -57 & 19.0 & 275 & 264 & 258 & 265 \\
\hline 819 & $5 / 26 / 947: 30$ & 105 & 3.90 & $\mathbf{s}$ & 0 & 0 & 0.0 & 0.00 & 298 & 442 & 260 & 386 & 0.3 & 1 & & 2.6 & -8.3 & -0.7 & 2 & 1 & .9 & 2 & -8.0 & 1 & 1.3 & 270 & 261 & 245 & 255 \\
\hline 819 & $5 / 26 / 9410: 05$ & 101 & 4.10 & $\mathbf{s}$ & 0 & 24 & 20.1 & 0.56 & 296 & 435 & 262 & 381 & 33.3 & 145 & 59.2 & 1.6 & .108 & 0.1 & 0 & 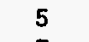 & -5 & 0 & -4.6 & 0 & 3.6 & 273 & 263 & 250 & .260 \\
\hline 820 & & 100 & 4.10 & $\mathbf{s}$ & 0 & 41 & & 1.02 & 296 & 435 & 262 & 381 & 45.8 & 199 & 45.0 & & -29.5 & 6.6 & .19 & & -2 & -14 & -23.5 & -22 & 6.6 & 275 & 265 & 257 & 264 \\
\hline 820 & $5 / 26 / 9413: 00$ & 100 & 4.20 & $\mathbf{s}$ & 0 & 41 & 36.0 & 1.02 & 296 & 435 & 262 & 381 & 48.7 & 212 & 47.9 & 6.9 & -23.7 & 5.6 & -16 & & -3 & -14 & -20.5 & -18 & 5.4 & 274 & 264 & 255 & 264 \\
\hline 820 & $5 / 26 / 9414: 00$ & 100 & 4,00 & $s$ & 0 & 41 & 36.0 & 1.02 & 296 & 435 & 262 & 381 & 50.3 & 219 & 49.5 & 4.2 & -52.4 & 2.8 & -8 & & -4 & .5 & -12.5 & -9 & 4.7 & 274 & 263 & 256 & 263 \\
\hline 821 & & 101 & 4.20 & $\mathbf{s}$ & 0 & 78 & 70.6 & 1.97 & 296 & 435 & 262 & 381 & 67.0 & 291 & 33.9 & 8.1 & -12.8 & 6.8 & -20 & 3 & -2 & -17 & -24.1 & -22 & 6.1 & 275 & 264 & 256 & 264 \\
\hline 821 & $5 / 26 / 94$ 16:08 & 101 & 4.00 & $\mathbf{s}$ & 0 & 78 & 70.6 & 1.97 & 296 & 435 & 262 & 381 & 68.5 & 298 & 34.7 & 7.8 & -8.0 & 6.5 & -19 & 2 & -1 & -17 & -23.2 & -22 & 7.0 & & 65 & 60 & 2 \\
\hline 821 & $5 / 26 / 9$ & 101 & 4.10 & $\mathbf{s}$ & 0 & 78 & 70.6 & 1.97 & 296 & 435 & 262 & 381 & 60.2 & 262 & 30.5 & 2.8 & -71.6 & 1.4 & -4 & & 13 & -19 & -8.3 & -21 & 21.0 & 265 & 265 & 259 & 265 \\
\hline 821 & $5 / 26 / 9417: 00$ & 100 & 3.90 & $s$ & 0 & 78 & 70.6 & 1.99 & 296 & 435 & 262 & 381 & 62.2 & 270 & 31.2 & 6.8 & -22.0 & 5.5 & -16 & & 10 & -24 & -20 & -31 & 18.7 & 265 & 264 & 259 & 265 \\
\hline 821 & 5/26/94 18:00 & 100 & 4.00 & $\mathbf{s}$ & 0 & 78 & 70.6 & 1.99 & 296 & 435 & 262 & 381 & 65.4 & 285 & 32.8 & 7.5 & -30.5 & 6.1 & -18 & 7 & 9 & -24 & -22.2 & -32 & 17.7 & 274 & 263 & 57. & 2 \\
\hline 822 & $5 / 27 / 947: 30$ & 102 & 4.80 & $s$ & 0 & 80 & 72.5 & 2.02 & 331 & 432 & 277 & 377 & 0.5 & 2 & 0.2 & 2.3 & -25.4 & -4.8 & 15 & 2 & -8 & 9 & -7.6 & 0 & 0.8 & 268 & 259 & 240 & 2 \\
\hline 822 & $5 / 27 / 949: 20$ & 102 & 4.80 & $\mathbf{s}$ & 0 & 80 & 72.5 & 2.02 & 331 & 432 & $2 \pi$ & 377 & 57.6 & 249 & 28.5 & 4.6 & -17.8 & -2.3 & 7 & & 13 & -16 & & -28 & 21.3 & & & & \\
\hline 822 & $5 / 27 / 9410: 20$ & 100 & 4.80 & 8 & 0 & 80 & 72.5 & 2.03 & 340 & 438 & 281 & 382 & 64.6 & 283 & 31.8 & 11.7 & -7.6 & 4.2 & -13 & 3 & & -23 & -40.1 & -48 & 16.6 & 279 & 266 & 256 & 265 \\
\hline 822 & & 100 & 4.80 & $\mathbf{s}$ & 0 & 80 & 72.5 & 2.03 & 340 & 438 & 281 & 382 & 65.2 & 285 & 32,0 & 8.7 & -9.3 & 1.0 & -3 & 3 & & -17 & -29.9 & -39 & 17.9 & 279 & 266 & & \\
\hline 822 & 5/27/94 12:20 & 100 & 4.80 & s & 0 & 80 & 72.5 & 2.03 & 340 & 438 & 281 & 382 & 65.3 & 286 & 32.1 & 11.8 & -9.6 & 4.3 & -13 & 4 & 7 & -20 & -40.2 & -47 & 15.4 & 280 & 270 & 261 & \\
\hline
\end{tabular}


Tost

conomizer Exit, dry (1-12): Base 1 Baghouse Inlet Gas Analysis $02 \mathrm{SO}_{2} \mathrm{NO} \mathrm{CO} \mathrm{NO} 2 \mathrm{CO} 2 \mathrm{~N} 2 \mathrm{O} \mathrm{O} 2 \mathrm{SO} 2$ NO $\mathrm{CO}$ NO2 $\mathrm{CO} 2 \mathrm{H} 2 \mathrm{O}$ N2O NH3 O2 SO2 NO

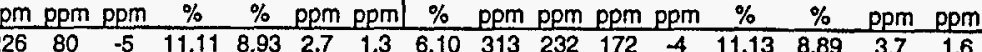

$\begin{array}{llllllllllllllllllllllllllll} & & & \end{array}$

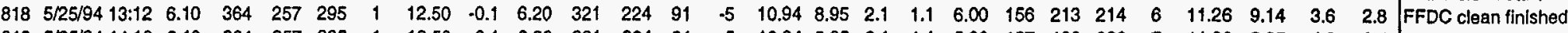

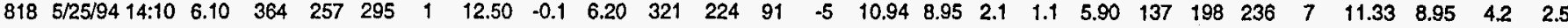

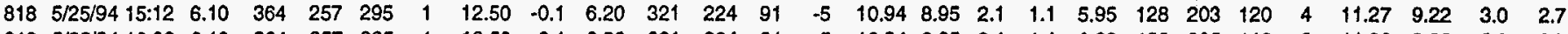

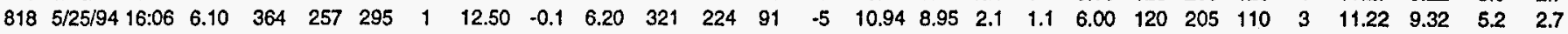

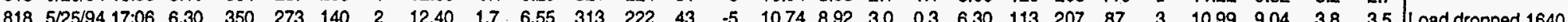

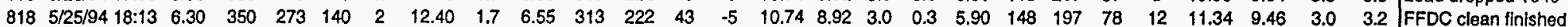
\begin{tabular}{llllllllllllllllllllllllllllll}
818 & $5 / 25 / 94$ & $18: 53$ & 6.30 & 350 & 273 & 140 & 2 & 12.40 & 1.7 & 6.55 & 313 & 222 & 43 & -5 & 10.74 & 8.92 & 3.0 & 0.3 & 6.20 & 130 & 198 & 87 & 9 & 11.14 & 9.19 & 2.2 & 2.5 \\
\hline 8
\end{tabular}

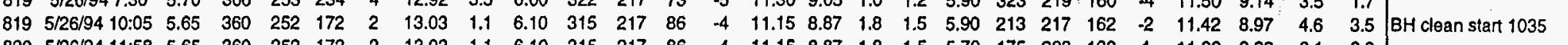

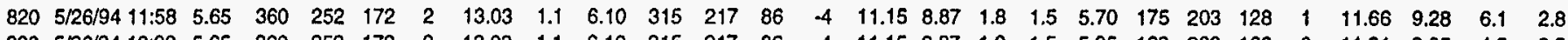

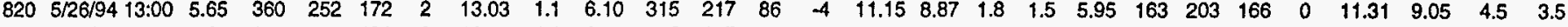

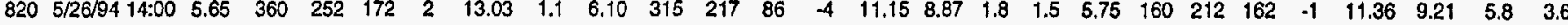
$821 \quad 5 / 26 / 94 \begin{array}{llllllllllllllllllllllllllll}15: 05 & 5.65 & 360 & 252 & 172 & 2 & 13.03 & 1.1 & 6.10 & 315 & 217 & 86 & -4 & 11.15 & 8.87 & 1.8 & 1.5 & 5.90 & 105 & 200 & 209 & 0 & 11.40 & 9.24 & 3.2 & 4.2\end{array}$ \begin{tabular}{llllllllllllllllllllllllllll}
821 & $5 / 26 / 94$ & 16.08 & 5.65 & 360 & 252 & 172 & 2 & 13.03 & 1.1 & 6.10 & 315 & 217 & 86 & -4 & 11.15 & 8.87 & 1.8 & 1.5 & 5.95 & 100 & 200 & 131 & 1 & 11.13 & 9.00 & 2.3 & 3.5 \\
\hline
\end{tabular}

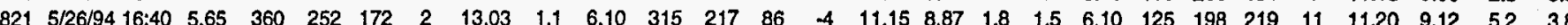

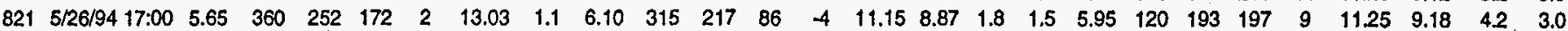

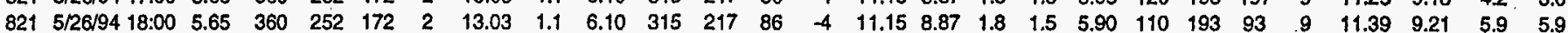

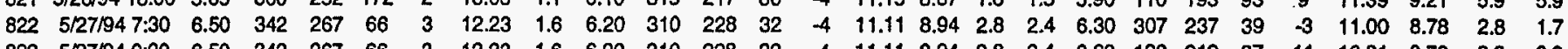

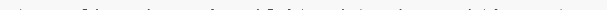
$\begin{array}{lllllllllllll}822 & 5 / 27 / 949: 20 & 6.50 & 342 & 267 & 66 & 3 & 12.23 & 1.6 & 6.20 & 310 & 228 & 32\end{array}$

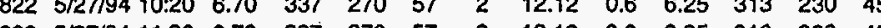

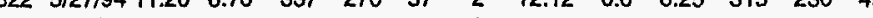
$\begin{array}{lllllllll}-4 & 11.11 & 8.94 & 2.8 & 2.4 & 6.60 & 128 & 212 & 37\end{array}$ $822 \quad 5 / 27 / 94 \quad 12: 20 \quad 6.70 \quad 337 \quad 270 \quad 57$ 